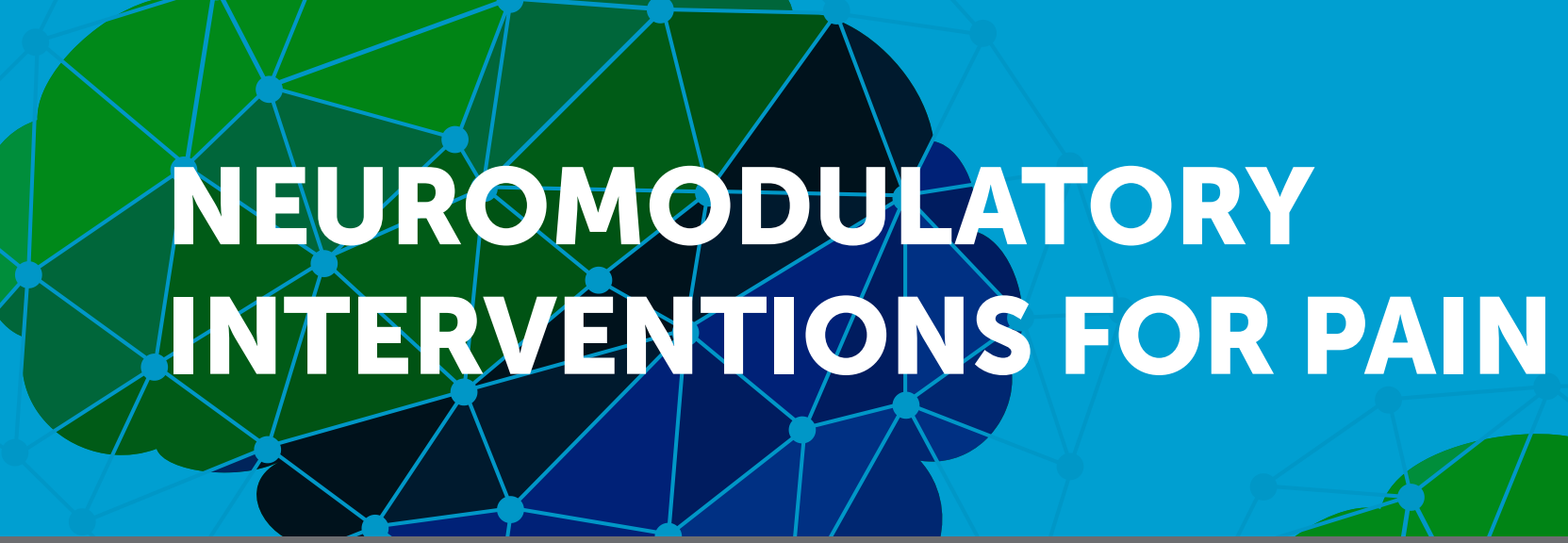

EDITED BY: Trevor Thompson, Marco Solmi, Hannah Madaleine Hobson and Lauren C. Heathcote

PUBLISHED IN: Frontiers in Neuroscience
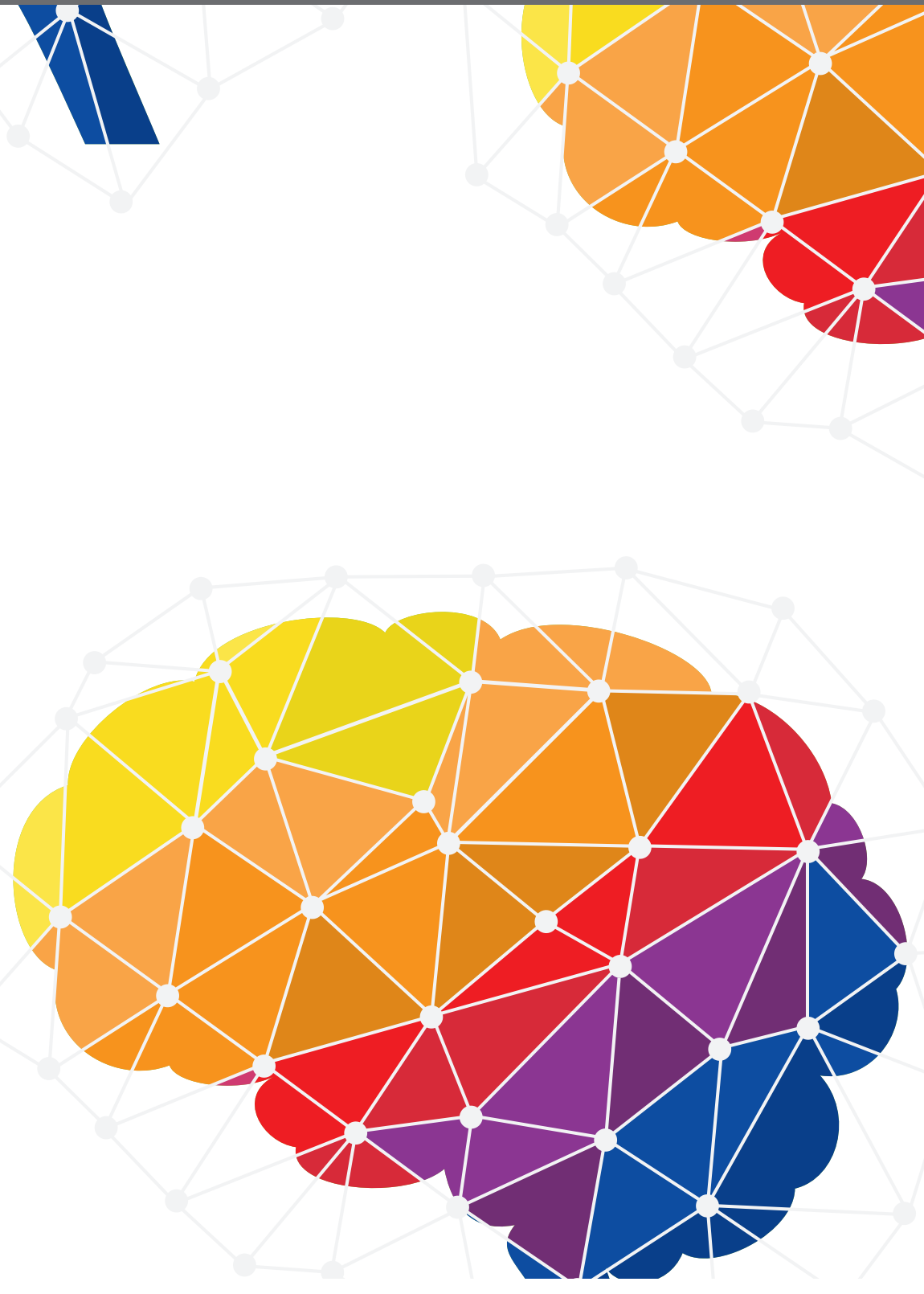


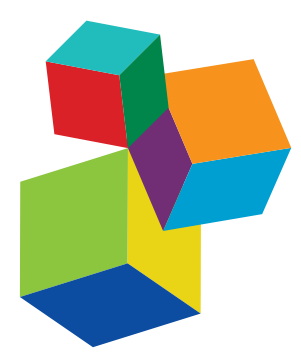

Frontiers eBook Copyright Statement

The copyright in the text of individual articles in this eBook is the property of their respective authors or their respective institutions or funders. The copyright in graphics and images within each article may be subject to copyright of other parties. In both cases this is subject to a license granted to Frontiers.

The compilation of articles constituting this eBook is the property of Frontiers.

Each article within this eBook, and the eBook itself, are published under the most recent version of the Creative Commons CC-BY licence.

The version current at the date of publication of this eBook is CC-BY 4.0. If the CC-BY licence is updated, the licence granted by Frontiers is automatically updated to the new version.

When exercising any right under the CC-BY licence, Frontiers must be attributed as the original publisher of the article or eBook, as applicable.

Authors have the responsibility of ensuring that any graphics or other materials which are the property of others may be included in the

CC-BY licence, but this should be checked before relying on the

CC-BY licence to reproduce those materials. Any copyright notices relating to those materials must be complied with.

Copyright and source acknowledgement notices may not be removed and must be displayed in any copy, derivative work or partial copy which includes the elements in question.

All copyright, and all rights therein, are protected by national and international copyright laws. The above represents a summary only.

For further information please read Frontiers' Conditions for Website

Use and Copyright Statement, and the applicable CC-BY licence.

ISSN 1664-8714

ISBN 978-2-88971-441-4 DOI 10.3389/978-2-88971-441-4

\section{About Frontiers}

Frontiers is more than just an open-access publisher of scholarly articles: it is a pioneering approach to the world of academia, radically improving the way scholarly research is managed. The grand vision of Frontiers is a world where all people have an equal opportunity to seek, share and generate knowledge. Frontiers provides immediate and permanent online open access to all its publications, but this alone is not enough to realize our grand goals.

\section{Frontiers Journal Series}

The Frontiers Journal Series is a multi-tier and interdisciplinary set of open-access, online journals, promising a paradigm shift from the current review, selection and dissemination processes in academic publishing. All Frontiers journals are driven by researchers for researchers; therefore, they constitute a service to the scholarly community. At the same time, the Frontiers Journal Series operates on a revolutionary invention, the tiered publishing system, initially addressing specific communities of scholars, and gradually climbing up to broader public understanding, thus serving the interests of the lay society, too.

\section{Dedication to Quality}

Each Frontiers article is a landmark of the highest quality, thanks to genuinely collaborative interactions between authors and review editors, who include some of the world's best academicians. Research must be certified by peers before entering a stream of knowledge that may eventually reach the public - and shape society; therefore, Frontiers only applies the most rigorous and unbiased reviews.

Frontiers revolutionizes research publishing by freely delivering the most outstanding research, evaluated with no bias from both the academic and social point of view. By applying the most advanced information technologies, Frontiers is catapulting scholarly publishing into a new generation.

\section{What are Frontiers Research Topics?}

Frontiers Research Topics are very popular trademarks of the Frontiers Journals Series: they are collections of at least ten articles, all centered on a particular subject. With their unique mix of varied contributions from Original Research to Review Articles, Frontiers Research Topics unify the most influential researchers, the latest key findings and historical advances in a hot research area! Find out more on how to host your own Frontiers Research Topic or contribute to one as an author by contacting the Frontiers Editorial Office: frontiersin.org/about/contact 


\section{NEUROMODULATORY INTERVENTIONS FOR PAIN}

Topic Editors:

Trevor Thompson, University of Cagliari, Italy

Marco Solmi, University of Connecticut, United States

Hannah Madaleine Hobson, Universitat Jaume I, Spain

Lauren C. Heathcote, Universitat Jaume I, Spain

Citation: Thompson, T., Solmi, M., Hobson, H. M., Heathcote, L. C., eds.

(2022). Neuromodulatory Interventions for Pain. Lausanne: Frontiers Media SA.

doi: 10.3389/978-2-88971-441-4 


\section{Table of Contents}

05 Editorial: Neuromodulatory Interventions for Pain

Trevor Thompson, Lauren C. Heathcote, Hannah Hobson and Marco Solmi

08 The Value of High-Frequency Repetitive Transcranial Magnetic Stimulation of the Motor Cortex to Treat Central Pain Sensitization Associated With Knee Osteoarthritis

Jean-Paul Nguyen, Véronique Dixneuf, Julien Esnaut, Alcira Suarez Moreno, Catherine Malineau, Julien Nizard and Jean-Pascal Lefaucheur

14 Inhibition of GABAergic Neurons and Excitation of Glutamatergic Neurons in the Ventrolateral Periaqueductal Gray Participate in Electroacupuncture Analgesia Mediated by Cannabinoid Receptor He Zhu, Hong-Chun Xiang, Hong-Ping Li, Li-Xue Lin, Xue-Fei Hu, Hong Zhang, Wang-Yang Meng, Lu Liu, Chao Chen, Yang Shu, Ru-Yue Zhang, Pei Zhang, Jun-Qiang Si and Man Li

28 EEG Correlates of Self-Managed Neurofeedback Treatment of Central Neuropathic Pain in Chronic Spinal Cord Injury

Aleksandra Vučković, Manaf Kadum Hussein Altaleb, Matthew Fraser,

Ciarán McGeady and Mariel Purcell

45 Exposure in vivo Induced Changes in Neural Circuitry for Pain-Related Fear: A Longitudinal fMRI Study in Chronic Low Back Pain

Inge Timmers, Jeroen R. de Jong, Mariëlle Goossens, Jeanine A. Verbunt, Rob J. Smeets and Amanda L. Kaas

63 Acupuncture Improves Comorbid Cognitive Impairments Induced by Neuropathic Pain in Mice Jae-Hwan Jang, Yu-Kang Kim, Won-Mo Jung, Hyung-Kyu Kim, Eun-Mo Song, Hee-Young Kim, Ju-Young Oh, Ji-Yeun Park, Yeonhee Ryu, Mi-Yeon Song and Hi-Joon Park

78 Self-Regulation of SMR Power Led to an Enhancement of Functional Connectivity of Somatomotor Cortices in Fibromyalgia Patients

Juan L. Terrasa, Alfonso Barros-Loscertales, Pedro Montoya and Miguel A. Muñoz

92 Neurofeedback for Pain Management: A Systematic Review Rubén Roy, Rocío de la Vega, Mark P. Jensen and Jordi Miró

114 Entraining Alpha Activity Using Visual Stimulation in Patients With Chronic Musculoskeletal Pain: A Feasibility Study

Laura J. Arendsen, James Henshaw, Christopher A. Brown, Manoj Sivan, Jason R. Taylor, Nelson J. Trujillo-Barreto, Alexander J. Casson and Anthony K. P. Jones

131 Non-invasive Brain and Spinal Stimulation for Pain and Related Symptoms in Multiple Sclerosis: A Systematic Review

Chiara Zucchella, Elisa Mantovani, Roberto De Icco, Cristina Tassorelli, Giorgio Sandrini and Stefano Tamburin 
144 Analgesic and Antidepressant Effects of the Clinical Glutamate Modulators Acetyl-L-Carnitine and Ketamine

Ulderico Freo, Viola Brugnatelli, Fabio Turco and Gastone Zanette

157 A Sham-Controlled Study of Neurofeedback for Pain Management

Charlotte Ide-Walters and Trevor Thompson 


\title{
Editorial: Neuromodulatory Interventions for Pain
}

\author{
Trevor Thompson ${ }^{1 *}$, Lauren C. Heathcote ${ }^{2}$, Hannah Hobson ${ }^{3}$ and Marco Solmi ${ }^{4}$ \\ ${ }^{1}$ Centre for Chronic Illness and Ageing, University of Greenwich, London, United Kingdom, ${ }^{2}$ Department of Anesthesiology, \\ Perioperative, and Pain Medicine, Stanford University School of Medicine, Stanford, CA, United States, ${ }^{3}$ Department of \\ Psychology, University of York, York, United Kingdom, ${ }^{4}$ Department of Neuroscience, University of Padova, Padua, Italy
}

Keywords: neuromodulation, pain, neurofeedback, acupuncture, non-invasive brain stimulation, TMS, tDCS

Editorial on the Research Topic

Neuromodulatory Interventions for Pain

The opioid crisis has prompted a renewed interest in non-pharmacological interventions for pain, with recent promising evidence for their effectiveness (Pillai Riddell et al., 2015; Veehof et al., 2016; Thompson et al., 2017, 2019; Franco et al., 2018; Owen et al., 2020). So called neuromodulatory interventions, which include neurofeedback and electrical and magnetic brain stimulation, are a novel class of non-pharmacological treatments that have attracted both intrigue and controversy. Despite a growing research literature, there remains a sense of uncertainty over whether they represent realistic alternatives to pain medication.

The aim of this research topic was to stimulate new, much-needed research evaluating the effectiveness of such treatments and providing insights into their possible mechanisms. We present a collection of 11 articles focused primarily on neurofeedback, non-invasive brain stimulation and

OPEN ACCESS

Edited and reviewed by: Rufin VanRullen, Centre National de la Recherche Scientifique (CNRS), France

*Correspondence:

Trevor Thompson t.thompson@gre.ac.uk

Specialty section:

This article was submitted to

Perception Science,

a section of the journal

Frontiers in Neuroscience

Received: 23 July 2021

Accepted: 26 July 2021

Published: 13 August 2021

Citation:

Thompson T, Heathcote LC, Hobson H and Solmi M (2021)

Editorial: Neuromodulatory Interventions for Pain.

Front. Neurosci. 15:746328. doi: 10.3389/fnins.2021.746328 acupuncture that attempt to address some of these issues.

\section{NEUROFEEDBACK}

A number of studies examined neurofeedback (NFB). This involves providing users with visual or auditory feedback on their brain activity (usually from EEG or fMRI), with the aim of assisting them in self-regulating this activity in a way that will produce favorable effects on pain.

A systematic review of 24 studies by Roy et al. provided a comprehensive summary of the current evidence for NFB across a range of pain conditions. The authors concluded that most studies identified improvements in pain, fatigue, sleep, and mood, but that heterogeneity in study protocols made it impossible to determine an optimal protocol for NFB administration. There was some evidence that regulation of EEG/MRI activity was possible, but this was not consistent across studies. Interestingly, the authors noted that improvement in pain sometimes occurred whether or not the targeted brain activity was successfully changed, suggesting a likely non-specific therapeutic component to NFB. They also found an encouraging improvement in study quality over the last few years, which included the increased use of control groups. Several limitations were nevertheless noted, with infrequent use of sham groups or randomization.

Some of these limitations were tackled in a new primary study by Terrasa et al., which randomized 17 fibromyalgia patients to 6 sessions of sensorimotor rhythm (SMR) training $(n=9)$ or a sham false-feedback procedure $(n=8)$. They found that 4 patients in the SMR training group who showed some ability to modulate SMR ("good" responders) also showed significant reductions in pain, with no such changes observed for "bad" responders or controls. Ide-Walters and Thompson randomized 24 healthy participants to receive $10 \mathrm{x}$ NFB (with real EEG feedback) 
or $10 \times$ sham (with false EEG feedback) sessions and assessed their responses to experimentally-induced cold pain. NFB was based on individualized protocols determined by initial qEEG assessments. While a significant decrease in pain across sessions was found for the NFB group, a near identical decrease was found for the sham group, consistent with the idea that any therapeutic effects of NFB could be at least in part attributable to a non-specific component. Vučković et al. examined the feasibility of self-administering NFB as a home treatment. After initial training in alpha upregulation and theta downregulation, 15 chronic pain patients with spinal cord injuries were asked to practice sessions at home for several weeks. Twelve patients showed statistically significant reductions in pain, with 8 showing clinically significant $(>30 \%)$ reductions. Training was particularly successful when an individualized alpha target frequency was used, based on the participant's dominant alpha peak frequency $(7.6 \mathrm{~Hz})$ rather than a fixed frequency band $(8-12 \mathrm{~Hz})$. Such results suggest NFB could be feasible for selfadministration, providing appropriate training and oversight are given.

An interesting alternative approach to alpha regulation was explored by Arendsen et al. They attempted to directly manipulate alpha activity in 20 chronic musculoskeletal pain patients using a novel visual stimulation procedure. Although no significant impact on pain was found, global alpha power was significantly higher during $10 \mathrm{~Hz}$ (alpha) stimulation than other frequencies. This suggests visual stimulation may be effective at regulating alpha activity, and this method could therefore warrant further investigation.

\section{NON-INVASIVE BRAIN STIMULATION}

Non-invasive brain and spinal stimulation methods have also been used to treat chronic pain. Zucchella et al. conducted a systematic review of studies assessing the effectiveness of these techniques for pain in individuals with multiple sclerosis. They reviewed 9 studies, that included direct current (tDCS) and magnetic stimulation (TMS) applied primarily to the left dorsolateral pre-frontal cortex or primary motor cortex. Consistent improvements in pain were identified, although there was less evidence for beneficial effects for broader well-being symptoms such as depression, fatigue, cognition, and quality of life. These findings suggest that the promising results that have been previously found for brain stimulation in conditions such as neuropathy and fibromyalgia may extend to pain in multiple sclerosis, although the authors caution that better controlled studies that assess longer term outcomes are still needed. A

\section{REFERENCES}

Franco, J. V., Turk, T., Jung, J. H., Xiao, Y. T., Iakhno, S., Garrote, V., et al. (2018). Non-pharmacological interventions for treating chronic prostatitis/chronic pelvic pain syndrome. Cochrane Database Syst. Rev. 5:CD012551. doi: 10.1002/14651858.CD012551.pub2

Owen, P. J., Miller, C. T., Mundell, N. L., Verswijveren, S. J. J. M., Tagliaferri, S. D., Brisby, H., et al. (2020). Which specific modes of exercise training are most novel case study by Nguyen et al. provided preliminary evidence that the potential benefits of rTMS might also apply to knee osteoarthritis. Stimulation of the motor cortex in a 71-year-old woman who exhibited evidence of central sensitization of pain was linked to substantial improvements in pain, sleep and fatigue and these were still evident at the end of treatment almost 1 year after treatment commenced.

\section{ACUPUNCTURE AND COGNITIVE THERAPY}

Several knockout mice studies also examined potential mechanisms that might explain how central nervous system activity linked to pain processing is modulated by interventions such as acupuncture. Zhu et al. used an induced pain paradigm in mice and found a reduction in pain hypersensitivity following electroacupuncture. They also found evidence for bidirectional regulation of GABAergic neurons and glutamatergic neurons via the $\mathrm{CB} 1$ receptors in the ventrolateral periaqueductal gray as a likely key analgesic mechanism. Jang et al. also found that acupuncture reduced pain behavior in mice, and identified an elevation in the expression levels of glutamate receptors in the hippocampus as the likely mechanism. The importance of the modulation of glutamine activity in altering pain processing was corroborated by a review of the two clinically approved glutamate modulators acetyl-L-carnitine and ketamine by Freo et al.

In a different approach, Timmers et al. examined how neuromodulatory changes resulting from a form of cognitivebehavioral therapy to reduce pain-related fear may be beneficial for patients with low back pain. fMRI showed that the changes that occurred in the right posterior insula and medial prefrontal cortex in patients (but not controls) during presentation of painrelated imagery did not occur after treatment. Other observed patterns led the authors to conclude that the neural circuitry for pain-related fear was modulated by the therapy, and that these changes were associated with decreases in pain-related fear.

Our collection brings together a diverse set of research papers on the issue of pain and potential neuromodulatory interventions for its management.

\section{AUTHOR CONTRIBUTIONS}

TT wrote the manuscript. $\mathrm{LH}, \mathrm{HH}$, and MS contributed to the revision, read, and approved the submitted version. All authors contributed to the article and approved the submitted version. 
Thompson, T., Terhune, D. B., Oram, C., Sharangparni, J., Rouf, R., Solmi, M., et al. (2019). The effectiveness of hypnosis for pain relief: a systematic review and meta-analysis of 85 controlled experimental trials. Neurosci. Biobehav. Rev. 99, 298-310. doi: 10.1016/j.neubiorev.2019.02.013

Veehof, M. M., Trompetter, H. R., Bohlmeijer, E. T., and Schreurs, K. M. (2016). Acceptance- and mindfulness-based interventions for the treatment of chronic pain: a meta-analytic review. Cogn. Behav. Ther. 45, 5-31. doi: 10.1080/16506073.2015.109 8724

Conflict of Interest: The authors declare that the research was conducted in the absence of any commercial or financial relationships that could be construed as a potential conflict of interest.
Publisher's Note: All claims expressed in this article are solely those of the authors and do not necessarily represent those of their affiliated organizations, or those of the publisher, the editors and the reviewers. Any product that may be evaluated in this article, or claim that may be made by its manufacturer, is not guaranteed or endorsed by the publisher.

Copyright (๑) 2021 Thompson, Heathcote, Hobson and Solmi. This is an open-access article distributed under the terms of the Creative Commons Attribution License (CC $B Y)$. The use, distribution or reproduction in other forums is permitted, provided the original author(s) and the copyright owner(s) are credited and that the original publication in this journal is cited, in accordance with accepted academic practice. No use, distribution or reproduction is permitted which does not comply with these terms. 
OPEN ACCESS

Edited by:

Trevor Thompson, University of Greenwich,

United Kingdom

Reviewed by:

Antonio Oliviero,

Fundación del Hospital Nacional de Parapléjicos, Spain Veit Mylius,

University of Marburg, Germany

*Correspondence: Jean-Pascal Lefaucheur jean-pascal.lefaucheur@hmn.aphp.fr

Specialty section: This article was submitted to Brain Imaging Methods, a section of the journal

Frontiers in Neuroscience

Received: 13 December 2018 Accepted: 04 April 2019

Published: 18 April 2019

Citation

Nguyen J-P, Dixneuf V, Esnaut J,

Suarez Moreno A, Malineau C Nizard J and Lefaucheur J-P (2019)

The Value of High-Frequency

Repetitive Transcranial Magnetic

Stimulation of the Motor Cortex

to Treat Central Pain Sensitization Associated With Knee Osteoarthritis.

Front. Neurosci. 13:388.

doi: 10.3389/fnins.2019.00388
The Value of High-Frequency Repetitive Transcranial Magnetic Stimulation of the Motor Cortex to Treat Central Pain Sensitization Associated With Knee Osteoarthritis

\author{
Jean-Paul Nguyen', Véronique Dixneuf', Julien Esnaut', Alcira Suarez Moreno', \\ Catherine Malineau ${ }^{1}$, Julien Nizard ${ }^{2}$ and Jean-Pascal Lefaucheur ${ }^{3 *}$ \\ 1 Unité de Stimulation Magnétique, Centre d'Evaluation et de Traitement de la Douleur (CETD), Clinique Bretéché, Groupe \\ ELSAN, Nantes, France, ${ }^{2}$ Centre Fédératif Douleur, Soins Palliatifs et Support, Ethique Clinique et Laboratoire de \\ Thérapeutique, Nantes, France, ${ }^{3}$ Unité de Neurophysiologie Clinique, CHU Henri Mondor, APHP, Faculté de Médecine, \\ UPEC, Créteil, France
}

Aim: Chronic pain associated with knee osteoarthritis may develop in connection with a maladaptive process of pain sensitization in the central nervous system. Repetitive transcranial magnetic stimulation (rTMS) has been proposed to treat various pain syndromes related to central sensitization phenomenon, but was never applied in the context of knee osteoarthritis.

Methods: A 71-year-old woman presenting clinical evidence of central sensitization of pain associated with left knee osteoarthritis underwent monthly sessions of rTMS delivered at $10 \mathrm{~Hz}$ over the right motor cortex.

Results: From the week following the third session, she began to improve on various clinical aspects, including pain. After 10 sessions (i.e., almost one year of follow-up), pain was reduced by $67 \%$, especially regarding neuropathic components, while sleep disorders and fatigue also improved by $57-67 \%$. The central sensitization inventory (CSI) score was reduced by $70 \%$.

Conclusion: This observation suggests that high-frequency motor cortex rTMS could be a therapeutic option to treat neuropathic pain and psychological symptoms associated with central sensitization developing in the context of chronic osteoarthritis of the knee joint.

Keywords: alpha synuclein, Parkinson disease, lipid rafts, prion protein, amyloid precursor protein, metabotropic glutamate receptor 5 , NMDA receptor 


\section{INTRODUCTION}

Pain is the main symptom of knee osteoarthritis (Nguyen et al., 2011). It initially appears as "mechanical," occurring when the joint is solicited, and gradually leads to joint stiffness, which can cause gait disorders and postural imbalance. The management of osteoarthritis of the lower limbs has been the subject of regularly updated recommendations, including those of EULAR in Fernandes et al. (2013). Weight loss in case of overweight and physical therapies can improve pain and peri-articular stiffness, but they are often insufficiently effective. Since pain is also related to inflammation of the joint and surrounding soft tissues, antiinflammatory treatments can be used, such as intra-articular infiltrations of corticosteroids or hyaluronic acid, whose efficacy is also inconstant.

In the medium or long term, the repetition of painful episodes may be at the origin of a phenomenon of sensitization to pain, which develops in the central nervous system. In this case, pain extends beyond the joint and acquires neuropathic features (Lluch Girbés et al., 2016). Central sensitization is also accompanied by exacerbated fatigue, alteration of sleep quality, and mood disorders (Murphy et al., 2011). At this stage, the pain syndrome can become resistant to medical treatment or surgical intervention (Skou et al., 2016) and then other therapeutic approaches must be proposed.

Among these, repetitive transcranial magnetic stimulation (rTMS) of the cerebral cortex has shown interest to treat chronic pain syndrome associated with a central sensitization phenomenon, like fibromyalgia (Passard et al., 2007; Mhalla et al., 2011; Hou et al., 2016; Knijnik et al., 2016). The usual cortical target is the motor cortex (precentral gyrus), and rTMS aims at activating various neural circuits present in this cortical region, which are involved in the sensory, attentional, or emotional control of pain. In clinical practice, rTMS can be an effective therapy in a number of chronic pain syndromes (Lefaucheur, 2016), but has never been used in osteoarthritisassociated pain to our knowledge. In this article, we present the case of a woman suffering from chronic pain related to knee osteoarthritis and resistant to medical treatment. She was not considered eligible for total knee arthroplasty (TKA) surgery and therefore an attempt of rTMS therapeutic trial was proposed. This treatment resulted in a long-lasting analgesic and functional improvement, consistent with a clear relief of the central sensitization phenomenon.

\section{CASE REPORT}

A 71-year-old woman presented bilateral gonalgia predominant on the left side and related to knee osteoarthritis for 20 years. There was a context of overweight (weight: $125 \mathrm{~kg}$; height: $1.60 \mathrm{~m}$; body mass index: $\left.48.8 \mathrm{~kg} / \mathrm{m}^{2}\right)$. Initially, pain had "mechanical" features, occurring only while standing or walking and limiting physical activities. The diagnosis of bilateral, tricompartmental knee osteoarthritis predominant on the left side was confirmed radiologically. However, about 4 years ago (2014), knee pain became more intense, diffuse, and permanent, even at rest. In parallel, the patient developed sleep and mood disorders. Intra-articular injections of corticosteroids and hyaluronic acid had only very modest and transient effects. Then, an attempt to use opioids was undertaken for a period of time (fentanyl transdermal patch $25-50 \mu \mathrm{g} / \mathrm{h} /$ day), associated with paracetamol on demand (1,000-3,000 mg/day). This treatment was also ineffective and the patient had to reduce her physical activity even more. In September 2015, her walking distance was limited to $50 \mathrm{~m}$ with the aid of a cane. However, X-ray examination did not reveal any aggravation of osteoarthritic lesions.

In June 2016, the patient was referred to our center. She was unable to walk and essentially restricted to wheelchair. She scored her average daily pain intensity at $9 / 10$ on a numeric rating scale (NRS), while NRS scores for sleep disorders and fatigue were $7 / 10$ and $6 / 10$, respectively. Anxiety and depression scores were both $12 / 21$ on the hospital anxiety and depression scale (HAD) (Zigmond and Snaith, 1983). The lequesne index of severity for osteoarthritis (LISO) score (Lequesne, 1997) was $20 / 24$, corresponding to an extremely severe handicap. The LISO sub-scores were $7 / 8$ for "pain or discomfort," $7 / 8$ for "maximum walking distance," and 6/8 for "activities of daily living." A rTMS therapeutic trial was proposed, first because total knee replacement surgery was considered too risky for the patient and then because of the clinical arguments in favor of a central sensitization phenomenon. Actually, pain was no more strictly related to joint mobilization, but rather permanent, even present at rest. In addition, pain showed an expanded distribution outside the primarily affected knee joint on the left, as evidenced on pain drawings (Figure 1). This is known to be a reliable way for identifying the occurrence of a central sensitization phenomenon in the context of knee osteoarthritis (Lluch Girbés et al., 2016). There were also burning and tingling sensations, as well as mechanical allodynia and hyperalgesia (but no wind-up phenomenon) in the area of referred pain on the left side. Pain had neuropathic characteristics, as evidenced by a score of $6 / 10$ for the DN4 questionnaire (Bouhassira et al., 2005). A more ambiguous result was observed for the modified painDETECT questionnaire (mPDQ) (Freynhagen et al., 2006), with a score of $13 / 38$, which is the gray zone between improbable neuropathic pain (score $<12$ ) and highly probable neuropathic pain (score $>19$ ). Nevertheless, this mPDQ score was compatible with a central sensitization phenomenon, as the cut-off score was set at $>12$ in a recent study of patients with knee osteoarthritis (Hochman et al., 2013). This was further confirmed on the central sensitization inventory (CSI) (Mayer et al., 2012), which assesses both somatic and emotional complaints related to central sensitization and was validated in the context of knee osteoarthritis (Gervais-Hupé et al., 2018). On a short version of the CSI (Nishigami et al., 2018), the patient had an initial score of $20 / 36$ corresponding to moderate/severe central sensitization. In addition, sleep and mood disorders were in the foreground of the clinical picture and no clear worsening of arthritic lesions was observed on X-ray examination. Conversely, on the right side, pain had lower intensity $(\leq 4 / 10)$, with only "mechanical" features limited to knee joint. 


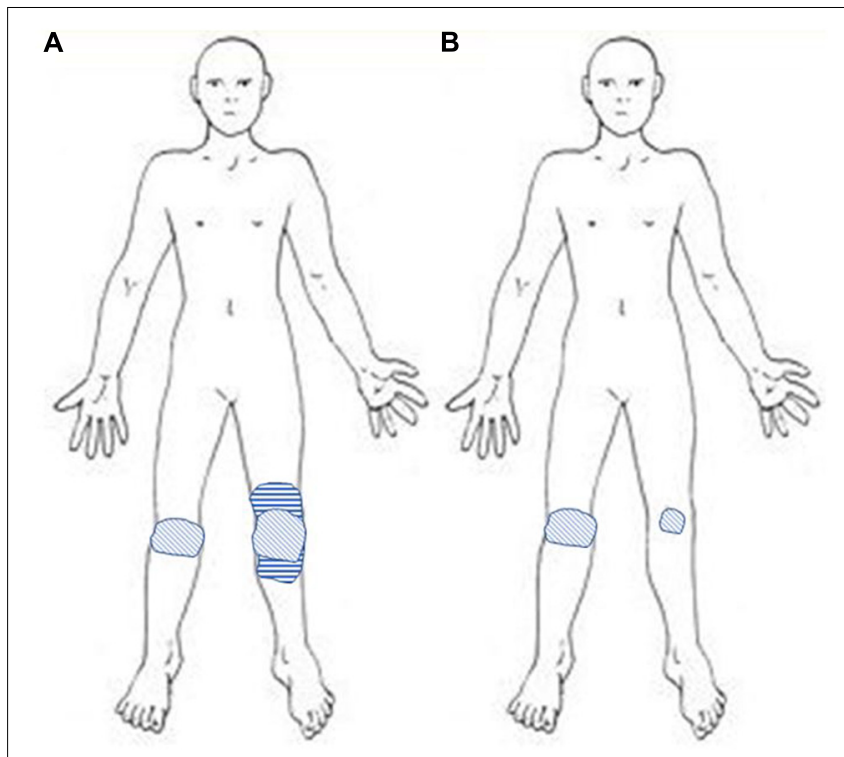

FIGURE 1 | (A) Before rTMS: drawing of the extent of mechanical pain (hatched area), surrounded by a region of exacerbated permanent pain with mechanical allodynia evoking central sensitization on the left side only (horizontal stripes). (B) After 10 rTMS sessions: the area of mechanical pain is reduced and the zone likely related to central sensitization disappeared on the left side, whereas pain remained unchanged on the right side.

A rTMS protocol was applied as for the treatment of focal neuropathic pain (Lefaucheur, 2016). The stimulation was delivered over the motor cortex contralateral to the predominant painful region, i.e., right motor cortex stimulation to treat left knee pain. The stimulation parameters for one session were as follows: 20 trains of 70 rTMS pulses delivered at high frequency of $10 \mathrm{~Hz}$ (train duration: $7 \mathrm{~s}$; inter-train duration: 55 s), i.e., 1,400 pulses for a session lasting about $20 \mathrm{~min}$. The stimulation intensity was set at $80 \%$ of the resting motor threshold, which was determined in a conventional way by means of motor evoked potential recording (Rossini et al., 2015). One rTMS session was performed each month. Assessment was performed during the week after each session (pain, sleep, fatigue) and after the 10th session for all variables, including DN4, mPDQ, CSI, HAD, and LISO.

Pain intensity began to decrease in the week following the third rTMS session, but more clearly after the sixth rTMS session (from $9 / 10$ before to 3/10, 67\% improvement) (Figure 2). After 10 sessions, pain was no longer permanent and only occurred when the patient was rising from a sitting position and for walking distance longer than $200 \mathrm{~m}$. Burning sensations, as well as mechanical allodynia and hyperalgesia, disappeared at the left knee. Neuropathic pain scores decreased by $67 \%$ (DN4, from 6 to $2 / 10$ ) to 85\% (mPDQ, from 13 to $2 / 38$ ), and the CSI score by $70 \%$ (from 20 to 6/36). Thus, at this stage, pain could no longer be considered neuropathic and central sensitization was absent or at most subclinical (Nishigami et al., 2018). Conversely, pain intensity did not change on the right side.
After the 10th session compared to baseline, sleep disorders (NRS score) also improved by $57 \%$ (from 7 to $3 / 10$ ), fatigue (NRS score) by $67 \%$ (from 6 to $2 / 10$ ), anxiety (HAD score) by $50 \%$ (from 12 to 6/21), depression (HAD score) by $42 \%$ (from 12 to $7 / 21$ ), and handicap (LISO score) by $40 \%$ (from 20 to 12/24). The LISO sub-scores improved by $71 \%$ for "pain or discomfort" (from 7 to $2 / 8$ ), $14 \%$ for "maximum walking distance" (from 7 to 6/8), and 33\% for "activities of daily living" (from 6 to 4/8). Analgesic medications were stopped gradually from 3 to 4 months after rTMS therapy initiation to be completely withdrawn at 7 months (after the seventh rTMS session). At this time, body mass index was $48.0 \mathrm{~kg} / \mathrm{m}^{2}$ and returned to its baseline value $\left(48.8 \mathrm{~kg} / \mathrm{m}^{2}\right)$ at 10 months. Pain remains fully controlled to date by monthly rTMS sessions. A written informed consent was obtained from the patient for the publication of this case report.

\section{DISCUSSION}

Chronic pain syndrome may worsen in the context of knee osteoarthritis even though the joint lesions are not evolving (Bedson and Croft, 2008), because of a maladaptive sensitization of the pain pathways in the central nervous system (Lluch et al., 2014). Such phenomenon of central sensitization is common in patients with osteoarthritis (Suokas et al., 2012; Lluch et al., 2014), although its propensity to develop varies among patients (Lee et al., 2011). In patients with osteoarthritis, chronic inflammatory nociceptive inputs can dynamically maintain altered central processing (Latremoliere and Woolf, 2009). Central changes particularly affect the descending mechanisms of pain control (impaired descending inhibition and/or enhanced descending facilitation) (Gebhart, 2004; Zambreanu et al., 2005; ArendtNielsen et al., 2010; Graven-Nielsen et al., 2012). Such a central dysfunction occurs in various chronic pain conditions, like fibromyalgia, bladder pain syndrome, or irritable bowel syndrome. Clinically, the central sensitization phenomenon makes the pain more intense, permanent and diffuse than it should be, with both temporal and spatial summations. It increases the resistance to local treatments and analgesic medications and is negative prognostic factor for the outcome of total knee replacement surgery (Kurien et al., 2018).

Some psychological factors may explain the discordance between the worsening of pain and the absence of radiographic changes at joint level. Actually, central sensitization is characterized by diffuse psychophysical changes, including unusual fatigue, cognitive difficulties, sleep disturbance, depression and catastrophism, associated with neuropathic pain features, including hyperalgesia and allodynia extending outside the affected joint (Murphy et al., 2011; Lluch et al., 2014, 2017). Central sensitization may be difficult to prove in a clinical setting and requires the identification of both psychological factors and neuropathic pain features. Various questionnaires may help, such the CSI and the MPDQ, of which usefulness was demonstrated in patients with chronic knee osteoarthritis (Hochman et al., 2011, 2013; Moreton et al., 2015). More recently, a list of 14 criteria provided by clinical and sensory examination was proposed for making the diagnosis of central 


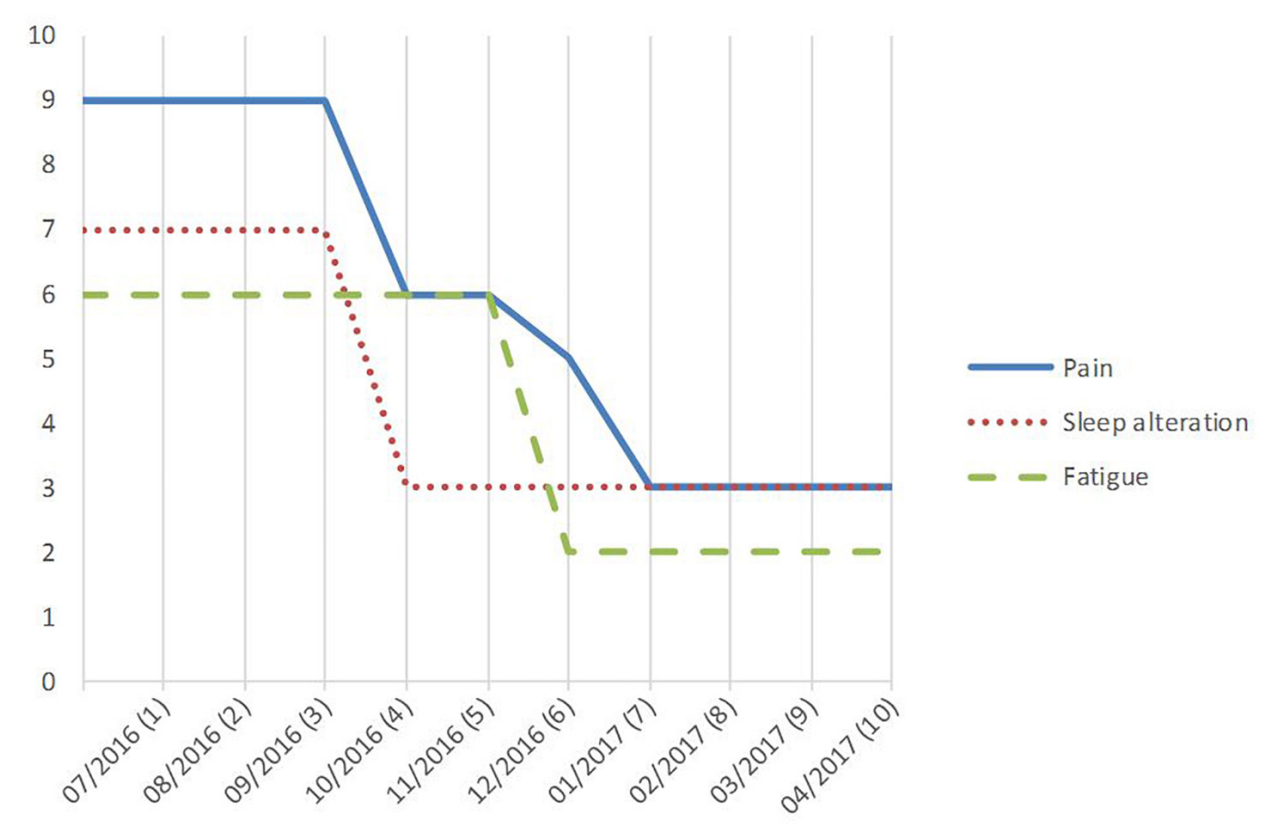

FIGURE 2 | Evolution of average intensity of pain, sleep alteration, and fatigue on a 0-10 numeric rating scale (NRS), between before and the week after 10 monthly sessions (1-10) of repetitive transcranial magnetic stimulation (rTMS) delivered to the right motor cortex.

pain sensitization associated with knee osteoarthritis (Lluch et al., 2017). Our patient initially presented 10 of these criteria, including: pain intensity $>5 / 10$, disproportion between pain intensity and structural damage, disproportionate pain after physical activity, inconsistent response to clinical tests, enlarged areas of pain outside the knee, diffuse tenderness, tactile allodynia and hypoesthesia, poor results with analgesics, and unsuccessful response to local interventions.

A trial of rTMS therapy was attempted according to the presence of central sensitization. In such a condition, rTMS can produce analgesia by activating brain circuits that are able to control the hyperactivity of sensitized pain networks. This objective was reached in our patient, since rTMS improved the clinical symptoms related to central sensitization more than those related to the mechanical "aspects" of knee osteoarthritis. For example, "pain or discomfort" LISO sub-score improved much more than the "maximum walking distance" LISO sub-score. In addition, both psychological aspects and neuropathic pain features were dramatically reduced, as well as the CSI score.

Non-invasive cortical stimulation was rarely used to treat knee osteoarthritis, and only by means of transcranial direct current stimulation (tDCS). For example, tDCS (over M1 and/or DLPFC) was used to reduce pain and opioid consumption in postsurgical time after TKA (Borckardt et al., 2013, 2017; Khedr et al., 2017). Motor cortex tDCS was also combined with exercise (Chang et al., 2015, 2017) or peripheral electrical stimulation (Luz-Santos et al., 2017) to promote pain control in knee osteoarthritis. The main results, observed on both provoked and ongoing spontaneous pain, have been recently reported by Ahn et al. (2017, 2018). They performed five daily sessions of anodal tDCS of M1 in patients with knee osteoarthritis and obtained significant analgesic effects compared to sham condition, with lasting clinical benefit for 3 weeks beyond the time of stimulation.

However, to our knowledge, our observation is the first reported case of using rTMS to treat knee osteoarthritisassociated pain syndrome in the long term. Interestingly, in this clinical condition, it has been shown by means of TMS techniques that the corticospinal system had a reduced excitability, in correlation with the intensity of pain, disability, and impairment of the descending controls of pain (Tarragó Mda et al., 2016). Therefore, high-frequency rTMS of the motor cortex could have restored intracortical inhibitory controls in association with pain relief in our patient, as it was previously shown in neuropathic pain (Lefaucheur et al., 2006, 2012) and fibromyalgia or myofascial pain syndromes (Mhalla et al., 2011; Dall'Agnol et al., 2014). Corticospinal excitability studies, based on single- and paired-pulse TMS techniques, deserve to be investigated in future trials in this context.

Usual rTMS protocols include a phase of "induction," consisting of a daily session for five consecutive days, repeated for one to 2 weeks, followed by one session per week for 4 to 6 weeks (Lefaucheur, 2016). In our patient, we performed only monthly rTMS sessions, especially because the patient was difficult to mobilize. She clearly benefited from this therapy from the third month and one might think that she could have responded well before if the intervals between sessions had been shorter. The benefit continued thereafter throughout the year on various clinical aspects, including daily living activities.

Although a placebo effect cannot be ruled out in the absence of sham procedure, various arguments can be raised against this hypothesis: the absence of previous response 
to medications and invasive treatments (e.g., intra-articular injections), the progressive clinical improvement which settled down as the rTMS sessions were repeated, and the fact that pain relief occurred only on the left side (contralateral to cortical stimulation), leaving the pain of the right knee unchanged.

\section{CONCLUSION}

In conclusion, a therapeutic use of motor cortex rTMS is particularly appealing to treat pain associated with knee osteoarthritis, especially when a central sensitization

\section{REFERENCES}

Ahn, H., Suchting, R., Woods, A. J., Miao, H., Green, C., Cho, R. Y., et al. (2018). Bayesian analysis of the effect of transcranial direct current stimulation on experimental pain sensitivity in older adults with knee osteoarthritis: randomized sham-controlled pilot clinical study. J. Pain Res. 11, 2071-2082. doi: $10.2147 /$ JPR.S173080

Ahn, H., Woods, A. J., Kunik, M. E., Bhattacharjee, A., Chen, Z., Choi, E., et al. (2017). Efficacy of transcranial direct current stimulation over primary motor cortex (anode) and contralateral supraorbital area (cathode) on clinical pain severity and mobility performance in persons with knee osteoarthritis: an experimenter- and participant-blinded, randomized, sham-controlled pilot clinical study. Brain Stimul. 10, 902-909. doi: 10.1016/j.brs.2017.05.007

Arendt-Nielsen, L., Nie, H., Laursen, M. B., Laursen, B. S., Madeleine, P., Simonsen, O. H., et al. (2010). Sensitization in patients with painful knee osteoarthritis. Pain 149, 573-581. doi: 10.1016/j.pain.2010.04.003

Bedson, J., and Croft, P. R. (2008). The discordance between clinical and radiographic knee osteoarthritis: a systematic search and summary of the literature. BMC Musculoskelet Disord. 9:116. doi: 10.1186/1471-2474-9-116

Borckardt, J. J., Reeves, S. T., Milliken, C., Carter, B., Epperson, T. I., Gunselman, R. J., et al. (2017). Prefrontal versus motor cortex transcranial direct current stimulation (tDCS) effects on post-surgical opioid use. Brain Stimul. 10, 10961101. doi: 10.1016/j.brs.2017.09.006

Borckardt, J. J., Reeves, S. T., Robinson, S. M., May, J. T., Epperson, T. I., Gunselman, R. J., et al. (2013). Transcranial direct current stimulation (tDCS) reduces postsurgical opioid consumption in total knee arthroplasty (TKA). Clin. J. Pain 29, 925-928. doi: 10.1097/AJP.0b013e31827e32be

Bouhassira, D., Attal, N., Alchaar, H., Boureau, F., Brochet, B., Bruxelle, J., et al. (2005). Comparison of pain syndromes associated with nervous or somatic lesions and development of a new neuropathic pain diagnostic questionnaire (DN4). Pain 114, 29-36. doi: 10.1016/j.pain.2004.12.010

Chang, W. J., Bennell, K. L., Hodges, P. W., Hinman, R. S., Liston, M. B., and Schabrun, S. M. (2015). Combined exercise and transcranial direct current stimulation intervention for knee osteoarthritis: protocol for a pilot randomised controlled trial. BMJ Open 5:e008482. doi: 10.1136/bmjopen-2015-008482

Chang, W. J., Bennell, K. L., Hodges, P. W., Hinman, R. S., Young, C. L., Buscemi, V., et al. (2017). Addition of transcranial direct current stimulation to quadriceps strengthening exercise in knee osteoarthritis: a pilot randomised controlled trial. PLoS One 12:e0180328. doi: 10.1371/journal.pone.0180328

Dall'Agnol, L., Medeiros, L. F., Torres, I. L., Deitos, A., Brietzke, A., Laste, G., et al. (2014). Repetitive transcranial magnetic stimulation increases the corticospinal inhibition and the brain-derived neurotrophic factor in chronic myofascial pain syndrome: an explanatory double-blinded, randomized, sham-controlled trial. J. Pain 15, 845-855. doi: 10.1016/j.jpain.2014.05.001

Fernandes, L., Hagen, K. B., Bijlsma, J. W., Andreassen, O., Christensen, P., Conaghan, P. G., et al. (2013). EULAR recommendations for the nonpharmacological core management of hip and knee osteoarthritis. Ann. Rheum Dis. 72, 1125-1135. doi: 10.1136/annrheumdis-2012-202745

Freynhagen, R., Baron, R., Gockel, U., and Tölle, T. R. (2006). painDETECT: a new screening questionnaire to identify neuropathic components in patients with back pain. Curr. Med. Res. Opin. 22, 1911-1920. doi: 10.1185/ 030079906x132488 phenomenon is diagnosed and when total knee replacement surgery is risky or even contraindicated. Further sham-controlled study on a large series of patients is of course necessary to confirm this hope.

\section{AUTHOR CONTRIBUTIONS}

J-PN, VD, JE, ASM, CM, JN, and J-PL contributed substantial to the conception or design of the work, acquisition, analysis, or interpretation of data. J-PN and J-PL drafted the manuscript or revised it critically for important intellectual content.

Gebhart, G. F. (2004). Descending modulation of pain. Neurosci. Biobehav. Rev. 27, 729-737.

Gervais-Hupé, J., Pollice, J., Sadi, J., and Carlesso, L. C. (2018). Validity of the central sensitization inventory with measures of sensitization in people with knee osteoarthritis. Clin. Rheumatol. 37, 3125-3132. doi: 10.1007/s10067-0184279-8

Graven-Nielsen, T., Wodehouse, T., Langford, R. M., Arendt-Nielsen, L., and Kidd, B. L. (2012). Normalization of widespread hyperesthesia and facilitated spatial summation of deep-tissue pain in knee osteoarthritis patients after knee replacement. Arthritis Rheum 64, 2907-2916. doi: 10.1002/art.34466

Hochman, J. R., Davis, A. M., Elkayam, J., Gagliese, L., and Hawker, G. A. (2013). Neuropathic pain symptoms on the modified painDETECT correlate with signs of central sensitization in knee osteoarthritis. Osteoarthritis Cartilage 21, 1236-1242. doi: 10.1016/j.joca.2013.06.023

Hochman, J. R., Gagliese, L., Davis, A. M., and Hawker, G. A. (2011). Neuropathic pain symptoms in a community knee OA cohort. Osteoarthritis Cartilage 19, 647-654. doi: 10.1016/j.joca.2011.03.007

Hou, W. H., Wang, T. Y., and Kang, J. H. (2016). The effects of add-on noninvasive brain stimulation in fibromyalgia: a meta-analysis and meta-regression of randomized controlled trials. Rheumatology 55, 1507-1517. doi: 10.1093/ rheumatology/kew205

Khedr, E. M., Sharkawy, E. S. A., Attia, A. M. A., Ibrahim Osman, N. M., and Sayed, Z. M. (2017). Role of transcranial direct current stimulation on reduction of postsurgical opioid consumption and pain in total knee arthroplasty: double randomized clinical trial. Eur. J. Pain 21, 1355-1365. doi: 10.1002/ ejp.1034

Knijnik, L. M., Dussán-Sarria, J. A., Rozisky, J. R., Torres, I. L., Brunoni, A. R., Fregni, F., et al. (2016). Repetitive transcranial magnetic stimulation for fibromyalgia: systematic review and meta-analysis. Pain Pract. 16, 294-304. doi: 10.1111 papr.12276

Kurien, T., Arendt-Nielsen, L., Petersen, K. K., Graven-Nielsen, T., and Scammell, B. E. (2018). Preoperative neuropathic pain-like symptoms and central pain mechanisms in knee osteoarthritis predicts poor outcome 6 months after total knee replacement surgery. J. Pain 19, 1329-1341. doi: 10.1016/j.jpain.2018. 05.011

Latremoliere, A., and Woolf, C. J. (2009). Central sensitization: a generator of pain hypersensitivity by central neural plasticity. J. Pain 10, 895-926. doi: 10.1016/j. jpain.2009.06.012

Lee, Y. C., Nassikas, N. J., and Clauw, D. J. (2011). The role of the central nervous system in the generation and maintenance of chronic pain in rheumatoid arthritis, osteoarthritis and fibromyalgia. Arthritis Res. Ther. 13:211. doi: 10. 1186/ar3306

Lefaucheur, J. P. (2016). Cortical neurostimulation for neuropathic pain: state of the art and perspectives. Pain 157(Suppl. 1), S81-S89. doi: 10.1097/j.pain. 0000000000000401

Lefaucheur, J. P., Ayache, S. S., Sorel, M., Farhat, W. H., Zouari, H. G., Ciampi de Andrade, D., et al. (2012). Analgesic effects of repetitive transcranial magnetic stimulation of the motor cortex in neuropathic pain: influence of theta burst stimulation priming. Eur. J. Pain 16, 1403-1413. doi: 10.1002/j.1532-2149.2012. 00150.x

Lefaucheur, J. P., Drouot, X., Ménard-Lefaucheur, I., Keravel, Y., and Nguyen, J. P. (2006). Motor cortex rTMS restores defective intracortical inhibition in chronic 
neuropathic pain. Neurology 67, 1568-1574. doi: 10.1212/01.wnl.0000242731. $10074.3 \mathrm{c}$

Lequesne, M. G. (1997). The algofunctional indices for hip and knee osteoarthritis. J. Rheumatol. 24, 779-781.

Lluch, E., Nijs, J., Courtney, C. A., Rebbeck, T., Wylde, V., Baert, I., et al. (2017). Clinical descriptors for the recognition of central sensitization pain in patients with knee osteoarthritis. Disabil. Rehabil. 40, 2836-2845. doi: 10.1080/ 09638288.2017.1358770

Lluch, E., Torres, R., Nijs, J., and Van Oosterwijck, J. (2014). Evidence for central sensitization in patients with osteoarthritis pain: a systematic literature review. Eur. J. Pain 18, 1367-1375. doi: 10.1002/j.1532-2149.2014.499.x

Lluch Girbés, E., Dueñas, L., Barbero, M., Falla, D., Baert, I. A., Meeus, M., et al. (2016). Expanded distribution of pain as a sign of central sensitization in individuals with symptomatic knee osteoarthritis. Phys. Ther. 96, 1196-1207. doi: $10.2522 /$ ptj.20150492

Luz-Santos, C., Ribeiro Camatti, J., Barbosa Paixão, A., Nunes Sá, K., Montoya, P., Lee, M., et al. (2017). Additive effect of tDCS combined with peripheral electrical stimulation to an exercise program in pain control in knee osteoarthritis: study protocol for a randomized controlled trial. Trials 18:609. doi: 10.1186/s13063-017-2332-6

Mayer, T. G., Neblett, R., Cohen, H., Howard, K. J., Choi, Y. H., Williams, M. J., et al. (2012). The development and psychometric validation of the central sensitization inventory (CSI). Pain Pract. 12, 276-285. doi: 10.1111/j.1533-2500. 2011.00493.x

Mhalla, A., Baudic, S., Ciampi de Andrade, D., Gautron, M., Perrot, S., Teixeira, M. J., et al. (2011). Long-term maintenance of the analgesic effects of transcranial magnetic stimulation in fibromyalgia. Pain 152, 1478-1485. doi: 10.1016/j.pain.2011.01.034

Moreton, B. J., Tew, V., das Nair, R., Wheeler, M., Walsh, D. A., and Lincoln, N. B. (2015). Pain phenotype in patients with knee osteoarthritis: classification and measurement properties of PainDETECT and self-report leeds assessment of neuropathic symptoms and signs scale in a cross-sectional study. Arthritis Care Res. 67, 519-528. doi: 10.1002/acr.22431

Murphy, S. L., Lyden, A. K., Phillips, K., Clauw, D. J., and Williams, D. A. (2011). Subgroups of older adults with osteoarthritis based upon differing comorbid symptom presentations and potential underlying pain mechanisms. Arthritis Res. Ther. 13:R135. doi: 10.1186/ar3449

Nguyen, U. S., Zhang, Y., Zhu, Y., Niu, J., Zhang, B., and Felson, D. T. (2011). Increasing prevalence of knee pain and symptomatic knee osteoarthritis: survey and cohort data. Ann. Intern. Med. 155, 725-732. doi: 10.7326/0003-4819-15511-201112060-00004

Nishigami, T., Tanaka, K., Mibu, A., Manfuku, M., Yono, S., and Tanabe, A. (2018). Development and psychometric properties of short form of central sensitization inventory in participants with musculoskeletal pain: a cross-sectional study. PLoS One 13:e0200152. doi: 10.1371/journal.pone.0200152

Passard, A., Attal, N., Benadhira, R., Brasseur, L., Saba, G., Sichere, P., et al. (2007). Effects of unilateral repetitive transcranial magnetic stimulation of the motor cortex on chronic widespread pain in fibromyalgia. Brain 130, 2661-2670. doi: 10.1093/brain/awm189

Rossini, P. M., Burke, D., Chen, R., Cohen, L. G., Daskalakis, Z., Di Iorio, R., et al. (2015). Non-invasive electrical and magnetic stimulation of the brain, spinal cord, roots and peripheral nerves: basic principles and procedures for routine clinical and research application. An updated report from an I.F.C.N. Committee. Clin. Neurophysiol. 126, 1071-1107. doi: 10.1016/j.clinph.2015. 02.001

Skou, S. T., Roos, E. M., Simonsen, O., Laursen, M. B., Rathleff, M. S., ArendtNielsen, L., et al. (2016). The effects of total knee replacement and non-surgical treatment on pain sensitization and clinical pain. Eur. J. Pain 20, 1612-1621. doi: $10.1002 /$ ejp. 878

Suokas, A. K., Walsh, D. A., McWilliams, D. F., Condon, L., Moreton, B., Wylde, V., et al. (2012). Quantitative sensory testing in painful osteoarthritis: a systematic review and meta-analysis. Osteoarthritis Cartilage 20, 1075-1085. doi: 10.1016/ j.joca.2012.06.009

Tarragó Mda, G., Deitos, A., Brietzke, A. P., Vercelino, R., Torres, I. L., Fregni, F., et al. (2016). Descending control of nociceptive processing in knee osteoarthritis is associated with intracortical disinhibition: an exploratory study. Medicine 95:e3353. doi: 10.1097/MD. 0000000000003353

Zambreanu, L., Wise, R. G., Brooks, J. C., Iannetti, G. D., and Tracey, I. (2005). A role for the brainstem in central sensitisation in humans: evidence from functional magnetic resonance imaging. Pain 114, 397-407. doi: 10.1016/j.pain. 2005.01.005

Zigmond, A. S., and Snaith, R. P. (1983). The hospital anxiety and depression scale. Acta Psychiatr. Scand. 67, 361-370.

Conflict of Interest Statement: The authors declare that the research was conducted in the absence of any commercial or financial relationships that could be construed as a potential conflict of interest.

Copyright (c) 2019 Nguyen, Dixneuf, Esnaut, Suarez Moreno, Malineau, Nizard and Lefaucheur. This is an open-access article distributed under the terms of the Creative Commons Attribution License (CC BY). The use, distribution or reproduction in other forums is permitted, provided the original author(s) and the copyright owner(s) are credited and that the original publication in this journal is cited, in accordance with accepted academic practice. No use, distribution or reproduction is permitted which does not comply with these terms. 
OPEN ACCESS

Edited by:

Lauren C. Heathcote,

Stanford University, United States

Reviewed by:

Christoph Thoeringer,

Technische Universität

München, Germany

Vijay K. Samineni,

Washington University in St. Louis,

United States

${ }^{*}$ Correspondence: Jun-Qiang Si sijunqiang11@hotmail.com

Man Li

liman73@mails.tjmu.edu.cn

Specialty section: This article was submitted to

Perception Science,

a section of the journal

Frontiers in Neuroscience

Received: 29 January 2019 Accepted: 29 April 2019

Published: 17 May 2019

Citation:

Zhu $H$, Xiang $H-C, L i H-P, L$ in $L-X$ Hu X-F, Zhang $H$, Meng $W-Y$, Liu L, Chen $C$, Shu Y, Zhang R-Y, Zhang $P$,

Si J-Q and Li M (2019) Inhibition of GABAergic Neurons and Excitation of

Glutamatergic Neurons in the Ventrolateral Periaqueductal Gray Participate in Electroacupuncture Analgesia Mediated by Cannabinoid Receptor. Front. Neurosci. 13:484. doi: 10.3389/fnins.2019.00484

\section{Inhibition of GABAergic Neurons and Excitation of Glutamatergic Neurons in the Ventrolateral Periaqueductal Gray Participate in Electroacupuncture Analgesia Mediated by Cannabinoid Receptor}

\author{
He Zhu ${ }^{1,2}$, Hong-Chun Xiang ${ }^{1}$, Hong-Ping Li ${ }^{1}$, Li-Xue Lin ${ }^{1}$, Xue-Fei Hu ${ }^{1}$, Hong Zhang ${ }^{1}$, \\ Wang-Yang Meng ${ }^{1}$, Lu Liu ${ }^{1}$, Chao Chen ${ }^{1}$, Yang Shu ${ }^{3}$, Ru-Yue Zhang ${ }^{1}$, Pei Zhang ${ }^{1}$, \\ Jun-Qiang $\mathrm{Si}^{2 *}$ and Man $\mathrm{Li}^{1 *}$

\footnotetext{
${ }^{1}$ Department of Neurobiology and Key Laboratory of Neurological Diseases of Ministry of Education, School of Basic Medicine, The Institute of Brain Research, Tongji Medical College of Huazhong University of Science and Technology, Wuhan, China, ${ }^{2}$ Department of Physiology, Medical College of Shihezi University, Shihezi, China, ${ }^{3}$ Department of Central Laboratory, Affiliated Hospital of Jiangsu University, Zhenjiang, China
}

Although electroacupuncture (EA) has become a worldwide practice, little is understood about its precise target in the central nervous system (CNS) and the cell type-specific analgesia mechanism. In the present study, we found that EA has significant antinociceptive effects both in inflammatory and neuropathic pain models. Chemogenetic inhibition of GABAergic neurons in the ventrolateral periaqueductal gray (vIPAG) replicated the effects of EA, whereas the combination of chemogenetic activation of GABAergic neurons and chemogenetic inhibition of glutamatergic neurons in the VIPAG was needed to reverse the effects of EA. Specifically knocking out CB1 receptors on GABAergic neurons in the VIPAG abolished the EA effect on pain hypersensitivity, while specifically knocking out $\mathrm{CB} 1$ receptors on glutamatergic neurons attenuated only a small portion of the EA effect. EA synchronously inhibits GABAergic neurons and activates glutamatergic neurons in the VIPAG through CB1 receptors to produce EA-induced analgesia. The CB1 receptors on GABAergic neurons localized in the vIPAG was the basis of the EA effect on pain hypersensitivity. This study provides new experimental evidence that EA can bidirectionally regulate GABAergic neurons and glutamatergic neurons via the CB1 receptors of the vIPAG to produce analgesia effects.

Keywords: pain, electroacupuncture, chemogenetics, GABAergic neuron, glutamatergic neuron, vIPAG

\section{INTRODUCTION}

A series of randomized controlled trials of acupuncture and analgesia have shown that acupuncture has an analgesic effect in the context of chronic pain, such as knee pain, low back pain, migraine, and fibromyalgia (Liu et al., 2018; Mist and Jones, 2018; Musil et al., 2018). However, the specific target sites in the central nervous system (CNS) for electroacupuncture (EA) analgesia are still unclear, which seriously hinders the clinical promotion and application of EA analgesia. 
Studies have confirmed that EA can induce the specific expression of $\mathrm{c}$-fos in the periaqueductal gray (PAG) region (Fusumada et al., 2007). It has been demonstrated that the ventrolateral periaqueductal gray (vlPAG) is an essential part of the neural pathway that mediates pain regulation (Ho et al., 2011). Microinjection of GABA agonists into the vlPAG promotes pain, and microinjection of GABA antagonists produces antinociceptive effects by reducing inhibitory neurotransmission (Bobeck et al., 2014; Takasu et al., 2015). According to the GABA disinhibition hypothesis, tonically active GABAergic interneurons are present within the $P A G$, which release the neurotransmitter GABA that acts via $G_{A B A}$ receptors to inhibit spinally projecting output neurons (Basbaum and Fields, 1984). It was proposed that opioids and cannabinoids activate the descending pathway by indirectly suppressing the inhibitory influence of local GABAergic interneurons, thereby disinhibiting the antinociceptive pathway of the neuronal output to the spinal cord (Meng et al., 1998).

The endocannabinoid system, which is a crucial neuromodulatory system involved in the control of pain transmission and acupuncture analgesia within the CNS (Chen et al., 2009). Cannabinoid-induced antinociception plays a role in the activation of a descending inhibitory pain pathway (Palazzo et al., 2010). Cannabinoid receptor $1(\mathrm{CB} 1)$ are expressed in both nerve endings of GABAergic neurons and glutamatergic neurons in PAG and activation of the $\mathrm{CB} 1$ receptor may regulate GABAergic and glutamatergic neurotransmission (Drew et al., 2008; Tjen-A-Looi et al., 2009). Our previous results have shown that EA reversed the reduced expression of $\mathrm{CB} 1$ receptors and the 2 -arachidonoylglycerol (2-AG) level in the midbrain in chronic pain conditions (Yuan et al., 2018). These results indicated that EA may exert an analgesic effect by acting on the $\mathrm{CB} 1$ receptor. Microinjection of the $\mathrm{CB} 1$ receptor antagonist AM251 into the vlPAG can reverse the EA effect on pain hypersensitivity, which further confirms the mechanism of EA exerting an antinociceptive effect through CB1 receptors in the vlPAG (Yuan et al., 2018). Despite the abundant evidence supporting the hypothesis that the $\mathrm{CB} 1$ receptor is involved in EA analgesia, the exact cell type involved in EA analgesia through vlPAG descending pain modulation has not been directly investigated. In this study, with the help of cell type-specific chemogenetic manipulations in the vlPAG, we attempt to verify the hypothesis that GABAergic neurons and glutamatergic neurons are involved in CB1-mediated EA antinociception.

\section{MATERIALS AND METHODS}

\section{Animals}

All animal experiments were ratified by the Animal Care and Use Committee of Huazhong University of Science and Technology, the procedures conform to the ethical guidelines of the International Association for the Study of Pain (Demers et al., 2006). Eight-weeks-old male C57BL/6 mice (20-25g) were obtained from Beijing Vital River Laboratory Animal Technology Co., Ltd. The mCnrlflox/flox mice were bought from the Cyagen biosciences laboratory (Nanjing). GAD67-GFP mice were kindly provided by Dr. Xiangning Li (Huazhong
University of Science and Technology, Whuhan, China). The mice were individually housed in cages with a 12-h light/dark cycle and had free access to food and water. The mice used for EA experiments in CCI and KOA mice were divided into four groups (control, CCI or KOA, EA, and sham EA). The mice used for chemogenetic manipulation and knockout experiments in CCI and KOA mice were divided into three groups (control, chemogenetic manipulation or knockout, EA).

\section{Viruses Constructs and Surgery}

Adeno-associated viruses (AAV/2-9) were designed to achieve CRE-DIO system-mediated chemogenetic manipulation strategy: rAAV-mDlx-CRE-WPRE-pA combined with rAAV-hSyn-DIO-hM3D(Gq)-mCherry-WPRE-pA or rAAVhSyn-DIO-hM4D(Gi)-mCherry-WPRE-pA were designed to excite or inhibit GABAergic neurons of vlPAG (Dimidschstein et al., 2016). rAAV-hSyn-mCherry-WPRE-pA was selected as control virus. Furthermore, rAAV-CaMKIIa-HA-KORD-IRESmCitrine-WPRE-pA, selecting salvinorin B as DREAD, could inhibit glutaminergic neurons of vlPAG individually on the basis of above combined operations and without interactions.

All viruses used in this study were acquired from the Wuhan BrainVTA scientific and technical corporation. Before surgery, mice were anesthetized with isoflurane and fixed in the stereotaxic apparatus (RWD Instruments, China). Make a $1.5 \mathrm{~cm}$ length longitudinal incision along the midline of the skull, gently remove the periosteum from the exposed surface of the surgical area. Viruses injections were performed using the coordinates of vlPAG as following: $-4.8 \mathrm{~mm}$ from bregma, $-0.4 \mathrm{~mm}$ lateral from midline, and $2.8 \mathrm{~mm}$ ventral to skull. Desired viruses vectors $(150 \mathrm{~nL})$ were injected into the vlPAG at a rate of $50 \mathrm{nl}$ per $60 \mathrm{~s}$ (Samineni et al., 2017). If the virus infection area exceeds the vlPAG area, it is not included in the statistics (Figure S5).

\section{Chemogenetic Manipulation}

Three weeks after viruses injections, mice were intraperitoneally injected with clozapine N-oxide (CNO, Sigma) 60 min before the behavioral assessment. All baselines for thermal and mechanical sensitivity were recorded after the viruses injections and before the CNO administration. We administered $1 \mathrm{mg} / \mathrm{kg}$ CNO for both hM3Dq activation and hM4Di inhibition (Samineni et al., 2017). In rAAV-CaMKIIa-HA-KORD-IRES-mCitrineWPRE-pA operation mice, we individually administered $5 \mathrm{mg} / \mathrm{kg}$ salvinorin B for inhibiting glutaminergic neurons of vlPAG even on the basis of GABAergic neurons have been excited by hM3Dq activation with CNO (Vardy et al., 2015). In the chemogenetic reversing experiment of EA antinociception, $\mathrm{CNO}$ was applied just before EA treatment.

\section{Model}

Neuropathy was induced by chronic constriction injury (CCI) of the sciatic nerve using a similar procedure for rats (Bennett and Xie, 1988) which was adapted for mice (Sommer et al., 1998). Two loosely constrictive ligatures were tied around the left sciatic nerve to slow the blood flow of the sciatic myelin blood vessels without causing acute crushing damage. The sham group animals were anesthetized, their sciatic nerves were only 
exposed without constriction. Sham-operated animals were used as neuropathy controls.

\section{Induction of Knee Osteoarthritis (KOA)}

Intra-articular injection of monosodium iodoacetate (MIA) (Sigma, UK) into the left knee joint was applied to induce KOA model. Five microliters of $5 \mathrm{mg} / \mathrm{ml} \mathrm{MIA}$ in sterile saline (0.9\%) were injected into the joint space of the left knee through the infrapatellar ligament with a 30-gauge needle (La Porta et al., 2013). This method causes histopathology changes in the cartilage (van Osch et al., 1994) and produces obvious joint pain in mice (Harvey and Dickenson, 2009). The sham-operated mice received an intra-articular injection of $5 \mu \mathrm{l}$ of $0.9 \%$ sterile saline.

\section{EA Treatment}

For the CCI model EA treatment group, the mouse received EA on the left "Huantiao" (GB30) and "Yanglingquan" (GB34) once a day, starting from the eighth day after operation. GB30 and GB34 were chosen based on their effect in improving inflammatory pain and neuropathic pain in mouse (Kang et al., 2007; Park et al., 2014; Lee et al., 2018). Two acupuncture needles were inserted 2-3 mm deep into two acupoints corresponding to GB30 and GB34 in humans. EA ( $1 \mathrm{~mA}$ and $0.1 \mathrm{~ms}$ ) was carried out at $2 \mathrm{~Hz}$ for $30 \mathrm{~min}$ (Wu et al., 2013). Current was delivered with a modified constant current Han's Acupoint Nerve Stimulator (LH202, Huawei Co. Ltd., Beijing, China) (Wu et al., 2013).

For the EA treatment group of $\mathrm{KOA}$, mice received EA administration on the left "Neixiyan" (Ex-LE4) and "Dubi" (ST35) for 7 days, starting from the fifteenth day after MIA injection. EA ( $1 \mathrm{~mA}$ and $0.1 \mathrm{~ms}$ ) was carried out at $2 \mathrm{~Hz}$ for $30 \mathrm{~min}$. Ex-LE4 and ST35 were chosen based on the fact that their using frequency is the highest in KOA (Wu et al., 2010).

For sham treatment control, acupuncture needles were inserted into above acupuncture points without electrical stimulation or manual manipulation. Previous studies showed that needles inserted into active acupoints, but no electrical or manual stimulation, do not produce analgesia (Lao et al., 2004). During EA treatment, each mouse was placed in a homemade bag. Control group also received the same method to exclude the stress response. The animals remained still during EA treatment and showed no evident signs of distress.

\section{Nociceptive Behavioral Tests}

Mechanical allodynia and heat hyperalgesia were checked with von Frey filaments and the hot plate, respectively (Fernihough et al., 2004). The animals were habituated to the testing environment for $30 \mathrm{~min}$. The behavioral tests were performed 3 times before model induction and every other day after model induction until the formal experimental operation. The behavioral tests were performed once a day during kinds of experimental operations (Figure S1).

The surface temperature of the hot-plate was maintained at $53^{\circ} \mathrm{C}$. The withdrawal latency started from the mouse was put on the plate and terminated when a quick/prolonged withdrawal or flick of the paw was observed. Twenty seconds was set as a cutoff time for mice to prevent tissue damage (Chen et al., 2017). Thermal stimuli were delivered three times to hindpaw at $10 \mathrm{~min}$ intervals, and the mean value was calculated.
The tactile withdrawal threshold of mice was measured by using the "up-down" method (Chaplan et al., 1994). After an acclimation period of $30 \mathrm{~min}$, we stimulated the plantar surface of the hindpaw vertically with a series of von Frey (Stoelting, Wood Dale, IL) hairs with logarithmically increasing stiffness, and bent the filament for $5 \mathrm{~s}$ to the central plantar surface with sufficient force. Brisk withdrawal or paw flinching was considered as a positive response. The test of tactile withdrawal threshold was repeated two times in each mouse, and the mean value was calculated.

\section{Conditioned Place Preference (CPP) Test}

The test trials consisted of one habituation (1st day), four conditionings (2nd-5th days), and one test (6th day) (Hnasko et al., 2005). In the case of EA treatment, the mice were confined in white box, thereby achieving EA treatment and white box condition matching. On the test day, animals were free to go any parts of the apparatus, and the time spent in bright or dark box was measured. Data were analyzed by the SuperMaze software (Xinsoft SuperMaze Animal Behavior Analysis System, Shanghai).

\section{Immunofluorescence}

Mice under deep anesthesia with $10 \%$ chloralic hydras were transcardially perfused with $37^{\circ} \mathrm{C}$ normal saline followed by $4 \%$ paraformaldehyde in $0.1 \mathrm{M}$ PBS $\left(\mathrm{pH}, 7.4 ; 4^{\circ} \mathrm{C}\right)$. The brain were removed immediately and post-fixed in the same fixative. Then, the tissues were cryoprotected in 20 and $30 \%$ sucrose in $0.1 \mathrm{M}$ PBS for $24 \mathrm{~h}$, respectively, at $4^{\circ} \mathrm{C}$. The OCT embedded blocks were sectioned for $25-\mu \mathrm{m}$ thickness. Sections from each group were rinsed in $0.01 \mathrm{M}$ PBS and blocked for $2 \mathrm{~h}$ with blocking liquid (5\% donkey serum and $0.2 \%$ tween-20 in $0.01 \mathrm{M} \mathrm{PBS}$ ) at room temperature. The sections were probed with the following antibodies: rabbit anti-CB1 (1:200), mouse anti-GAD67 (1:200), guinea pig anti-VGLUT2 (1:100). Subsequently, the free-floating sections were washed with $0.01 \mathrm{M}$ PBS 3 times, and incubated with following secondary antibodies (Jackson ImmunoResearch) for $2 \mathrm{~h}$ : donkey anti-rabbit IgG conjugated with Dylight 594 (1:600), donkey anti-mouse IgG conjugated with Dylight 488 (1:600), donkey anti-guinea pig IgG conjugated with Dylight 488 (1:400). The sections were incubated with DAPI for the nucleus staining for $8 \mathrm{~min}$, and washed 3 times in $0.01 \mathrm{M}$ PBS and then cover-slipped. The samples were studied under a fluorescence microscope (Olympus) for the immunefluorescence staining. Images were analyzed by using NIH Image J software (Bethesda, MD, USA). The layouts of images were based on the Photoshop (ADOBE company, USA).

\section{Statistics}

Results of data are expressed as mean \pm SEM. The thermal latency and withdrawal thresholds between different groups over time were tested with two-way analysis of variance (ANOVA) followed by Bonferroni post hoc tests. Other data were analyzed by One-way ANOVA and Newman-Keuls post hoc test (SPSS, Version 11.0). Results represented as significance based on a value of $P<0.05$. 
A

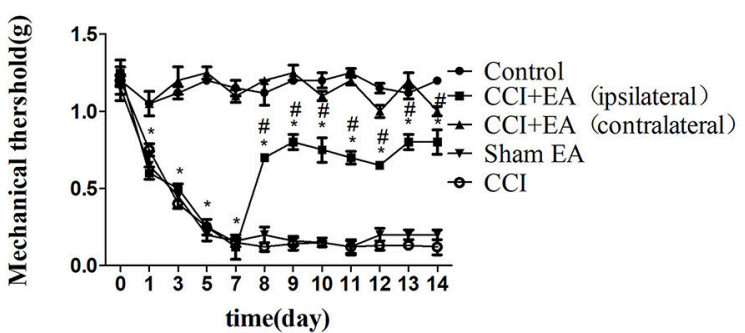

C

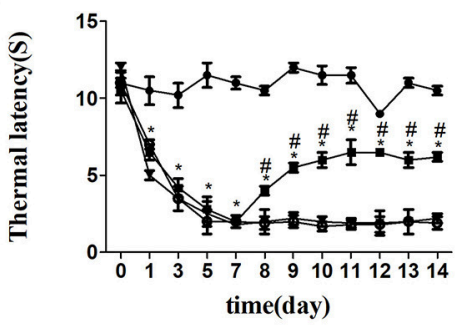

E

Bright box Dark box

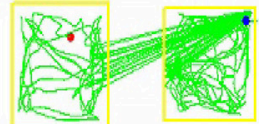

Before EA

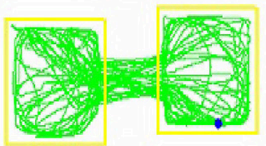

G

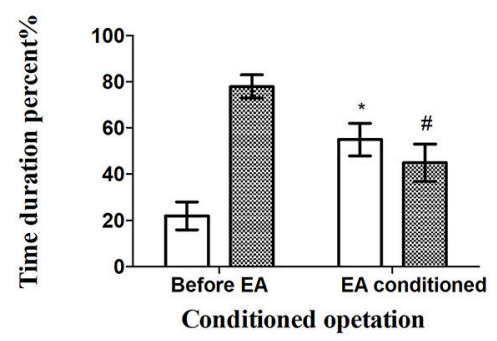

B

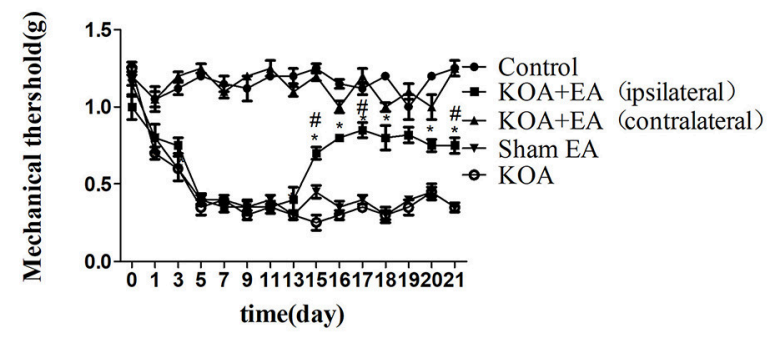

D

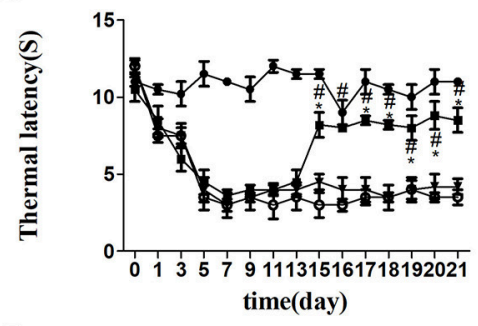

$\mathbf{F}$

Bright box Dark box

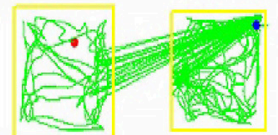

Before EA

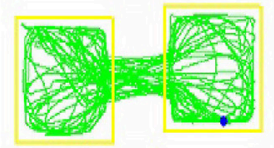

H

EA conditioned

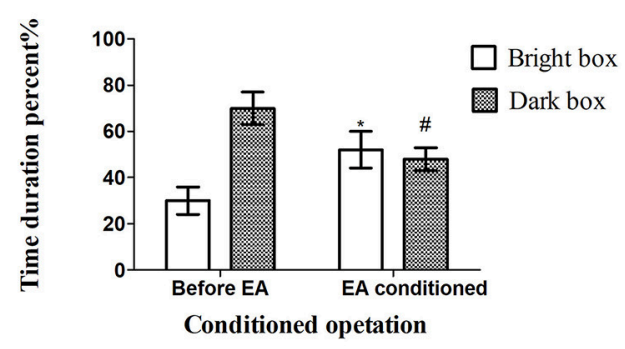

FIGURE 1 | EA effectively reduces pain hypersensitivity in CCl and KOA mice. (A,B) Time course of tactile threshold in response to von Frey filaments. (C,D) The noxious heat stimulus $\left(53^{\circ} \mathrm{C}\right)$ caused a change in the thermal thresholds. The effect of tactile (A) and thermal (C) withdrawal thresholds changes in $\mathrm{CCl}$ mice. The change of tactile (B) and thermal (D) withdrawal thresholds of KOA mice caused by EA. A motion map of the EA-conditioned CPP test in CCI (E) and KOA mice (F). $\mathbf{G}, \mathbf{H}$ are summary values of motion trajectories. EA $(1 \mathrm{~mA}$ and $0.1 \mathrm{~ms})$ at $2 \mathrm{~Hz}$ was administered for $30 \mathrm{~min}$, once a day starting from the 8 th to the 14 th day in the CCl model and starting from the 15th to 21 st day in the KOA model. Control group consisted of sham-operated mice. Data are expressed as the means \pm SEM $(n=12$ in each group). In $\mathbf{A}-\mathbf{D},{ }^{*} p<0.05$, compared with the control group; ${ }^{*} p<0.05$, compared with the model group; In panels $\mathbf{G}, \mathbf{H}$, ${ }^{*} p<0.05$, compared with the bright box time duration percent before EA; $\# p<0.05$, compared with the dark box time duration percent before EA.

\section{RESULTS}

\section{EA Effectively Reduces Pain Hypersensitivity in $\mathrm{CCl}$ and KOA Mice}

CCI or KOA induction significantly reduced mechanical withdrawal thresholds and thermal withdrawal latencies (Figures S2A-D).
The tactile threshold and thermal withdrawal latency were effectively increased by EA in CCI and KOA mice (Figures 1A-D).

The repeated EA treatment also affected the behavior in the CPP test, as the mouse spent more time in the bright box after being paired with EA treatment (Figures 1E-H). 
A

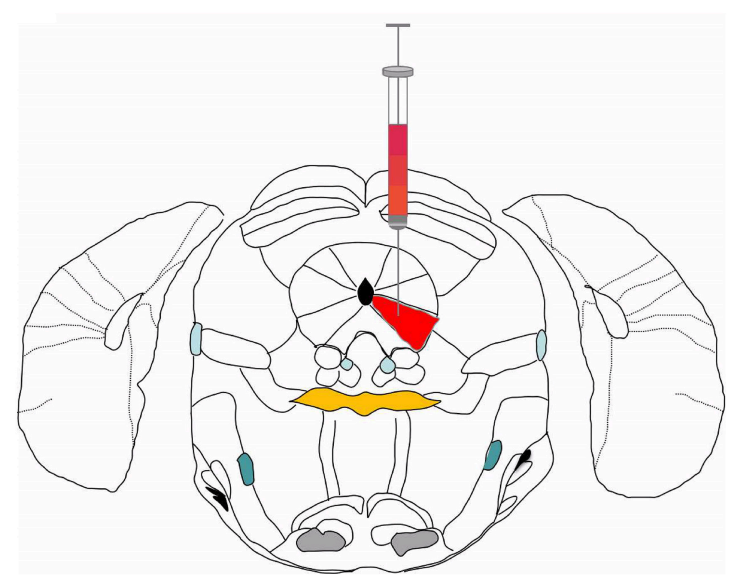

C

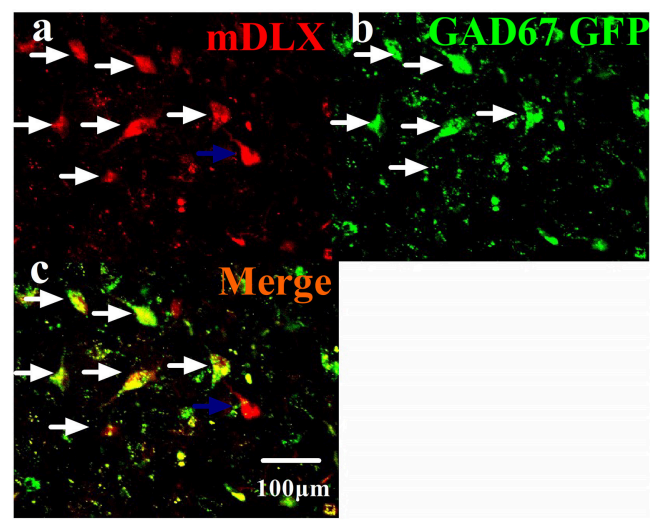

B

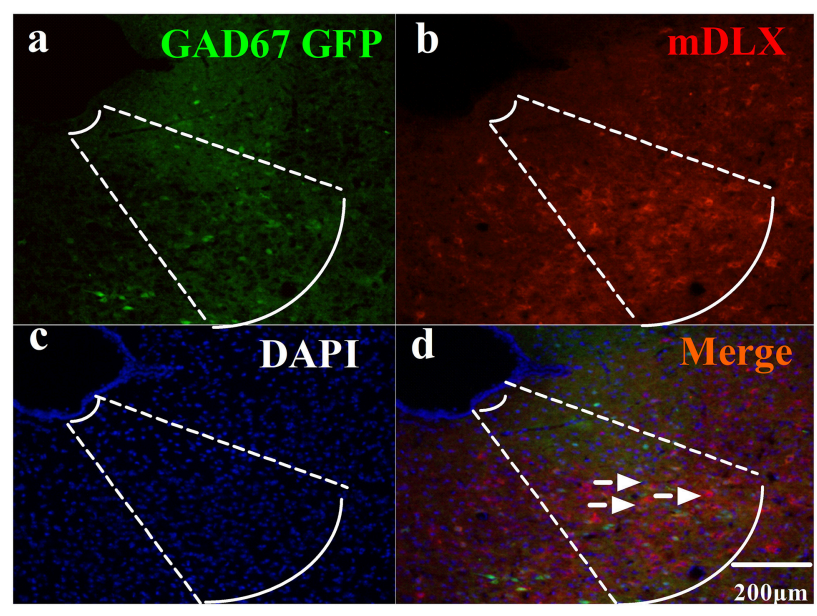

D

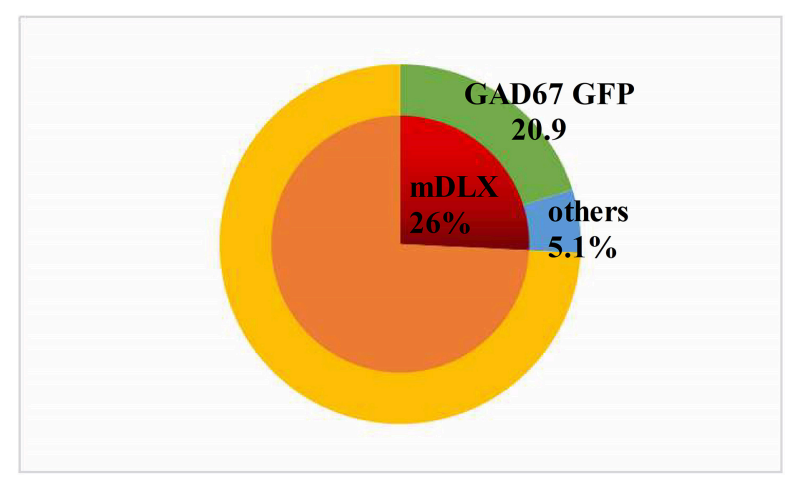

Viral combination lable efficiency

FIGURE 2 | The viruses combination cocktail label efficiency. (A) Pattern map of the viruses injection area. The red color region is the vIPAG. (B) The viruses combination cocktail was injected into the right side of the vIPAG of GAD67 GFP mice. (a) GAD67 GFP cells in the vIPAG (green). (b) Viruses-labeled cells (red). (c) DAPI nuclear staining (blue). (d) Colocalization fluorescence (pink), arrows show typical fluorescent, colocalized cells. Scale bar, $200 \mu \mathrm{m}$. (C) High magnification images, scale bar, $100 \mu \mathrm{m}$. White arrows indicate cells that are co-labeled, and blue arrows indicate cells that are mDLX-labeled but not labeled with GAD67 GFP. (D) Summary data show the percentage of colocalized cells in the area of total viruses-labeled cells. Red sector, viruses-labeled cells; green sector, GAD67 GFP cells; blue sector, viruses-labeled other cells. Data are expressed as the means \pm SEM ( $n=3$ mice in each group).

\section{Effect of Chemogenetic Inhibition of GABAergic Neurons in vIPAG}

Since the PAG is a vital brain region related to the descending pain control system, both cannabinoids and opioids, released by EA, may inhibited GABAergic neurons to produce antinociceptive effect by GABAergic disinhibition mechanism (Osborne et al., 1996; Finn et al., 2003), we applied a chemogenetic strategy to assess this hypothesis.

The viruses combination cocktail of rAAV-mDlx-CREWPRE-pA and rAAV-hSyn-DIO-hM3D(Gi)-mCherryWPRE-pA, designed to inhibit GABAergic neurons, was injected into the right side of the vlPAG (Figure 2A). The viruses combination was injected into the right side of the vlPAG of GAD67 GFP mouse to confirm the viruses combination efficiency (Figures 2B,C). A total of $80.5 \%$ of the viruses combination-tagged neurons in the vlPAG were marked together with GAD67 GFP-positive cells (Figure 2D).

Chemogenetic inhibition of GABAergic neurons reliably replicated the effect of $\mathrm{EA}$, including the antinociceptive effects (Figures 3A-H).

\section{Chemogenetic Activation of GABAergic Neurons in vIPAG Only Partly Attenuated the Effect of EA}

On the basis of chemogenetic inhibition of GABAergic neurons reliably replicating the antinociceptive effect of EA, we considered if chemogenetic activation could abolish the effect 


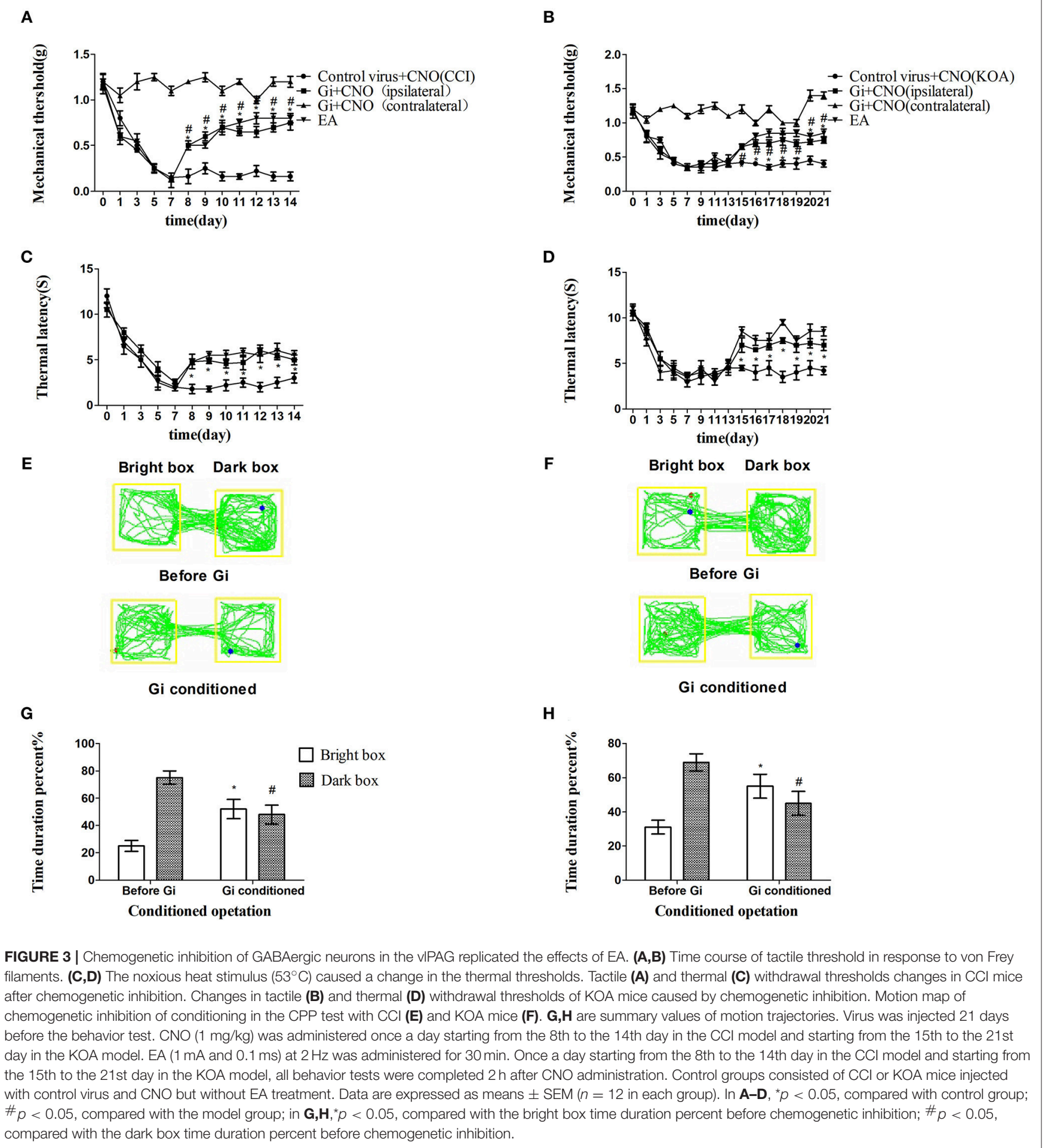

of EA. The viruses combination of rAAV-mDlxCRE-WPRE-pA and rAAV-hSyn-DIO-hM3D(Gq)mCherry-WPRE-pA, designed to activate GABAergic neurons, was injected into the right side of the vlPAG.
We unexpectedly found that chemogenetic activation of GABAergic neurons in the vlPAG only partly decreased the mechanical withdrawal threshold and thermal withdrawal latency in EA-treated mice (Figures 4A-D) while partly decreasing the bright box time in the CPP test (Figures $4 \mathbf{E}-\mathbf{H}$ ). 
A

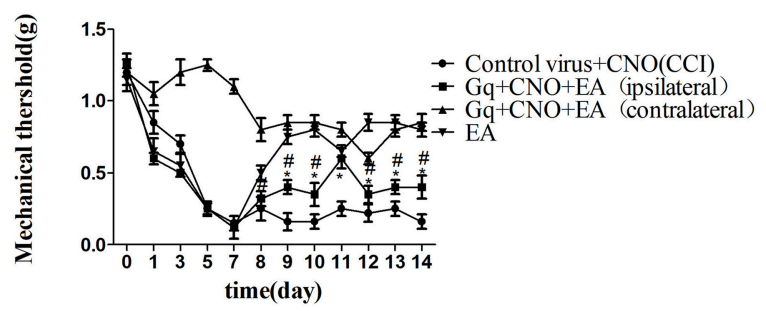

C

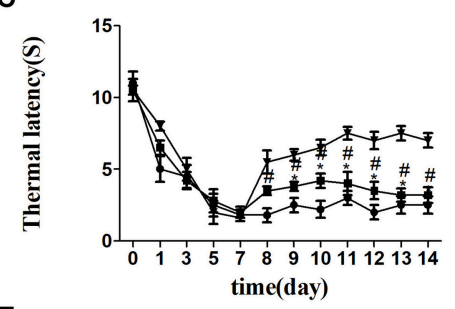

E

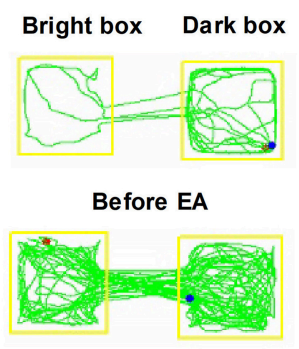

EA conditioned

G

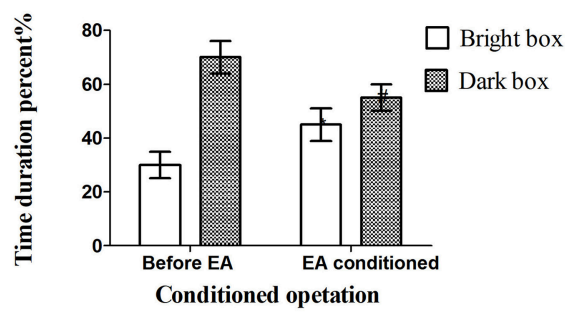

B

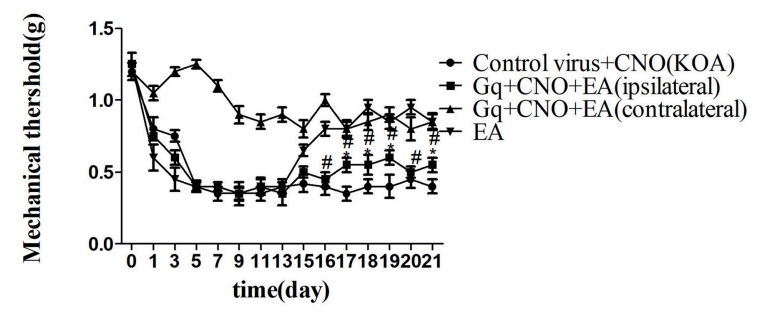

D

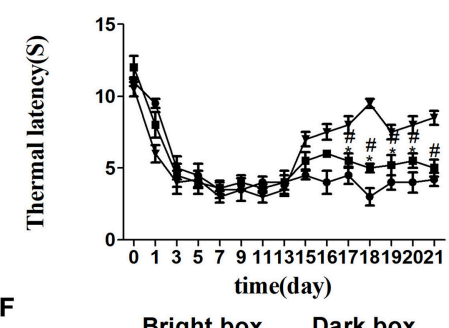

Bright box Dark box

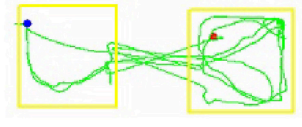

Before EA

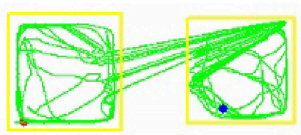

EA conditioned

H

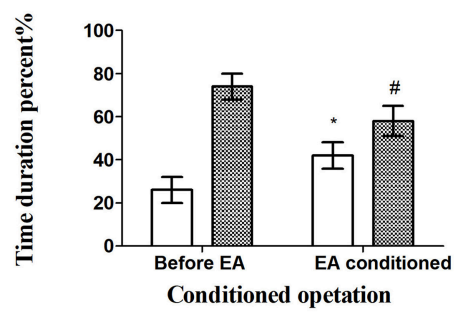

FIGURE 4 | Chemogenetic activation of GABAergic neurons in the vIPAG only partly attenuated the effect of EA. (A,B) Time course of tactile threshold in response to von Frey filaments. (C,D) The noxious heat stimulus $\left(53^{\circ} \mathrm{C}\right)$ caused a change in the thermal thresholds. Tactile $\mathbf{( A )}$ and thermal $(\mathbf{C})$ withdrawal thresholds changes in $\mathrm{CCl}$ mice after chemogenetic activation. Changes in tactile (B) and thermal (D) withdrawal thresholds of KOA mice caused by chemogenetic activation. Motion map of chemogenetic activation of conditioning in the CPP test with $\mathrm{CCl}(\mathbf{E})$ and $\mathrm{KOA}$ mice $\mathbf{( F )}$. G,H are summary values of motion trajectories. Virus was injected 21 days before the behavior test. CNO $(1 \mathrm{mg} / \mathrm{kg})$ was administered once a day starting from the 8th to the 14 th day in the $\mathrm{CCl}$ model and starting from the 15 th to the $21 \mathrm{st}$ day in the KOA model. EA ( $1 \mathrm{~mA}$ and $0.1 \mathrm{~ms})$ at $2 \mathrm{~Hz}$ was administered for $30 \mathrm{~min}$. Once a day starting from the 8th to the 14 th day in the $\mathrm{CCl}$ model and starting from the 15th to the 21st day in the KOA model, all behavior tests were completed $2 \mathrm{~h}$ after CNO administration. Control groups consisted of CCl or KOA mice injected with control virus and CNO but without EA treatment. Data are expressed as means \pm SEM $\left(n=12\right.$ in each group). In $\mathbf{A}-\mathbf{D}$, ${ }^{*} p<0.05$, compared with control group; $\#_{p}<0.05$, compared with the model group; In $\mathbf{G}, \mathbf{H},{ }^{*} p<0.05$, compared with the bright box time duration percent before chemogenetic activation; $\# p<0.05$, compared with the dark box time duration percent before chemogenetic activation.

\section{The Combination of Chemogenetic}

\section{Activation of GABAergic Neurons and} Chemogenetic Inhibition of Glutamatergic Neurons in VIPAG Effectively Attenuated the Effect of EA

A large number of studies have shown that GABAergic neurons and glutamatergic neurons in the vlPAG play an important and complex role in nociceptive processes. Microinjection of glutamate receptor agonists or GABA antagonists into the vlPAG has a significant antinociceptive effect against noxious stimuli (Budai et al., 1998; Morgan et al., 2003). Since activation of GABAergic neurons alone in the vlPAG only partly attenuated the effect of EA, we speculated that glutamatergic and GABAergic neurons were both involved in these effects.

The rAAV-CaMKIIa-HA-KORD-IRES-mCitrine-WPRE-pA virus was applied to selectively inhibit the glutamatergic neurons of the vlPAG (Figure S4), on the basis that GABAergic neurons 
A

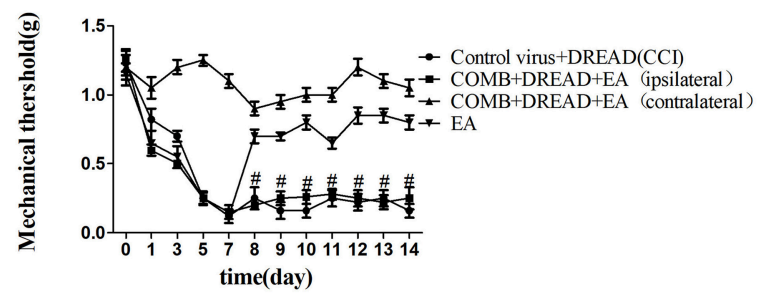

C

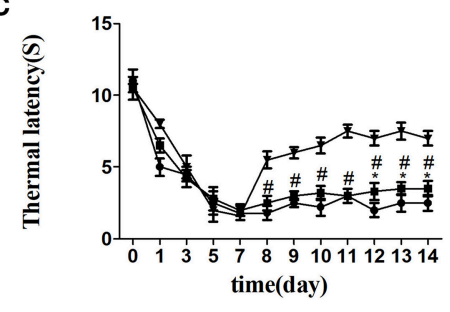

E

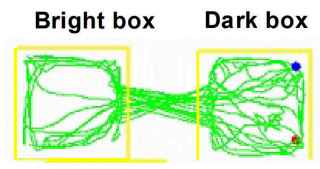

Before EA

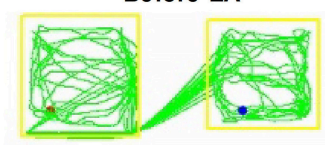

EA conditioned

G

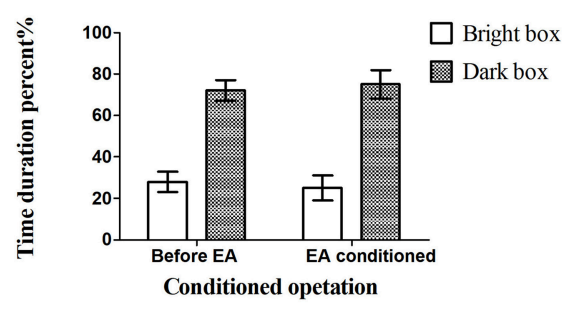

B

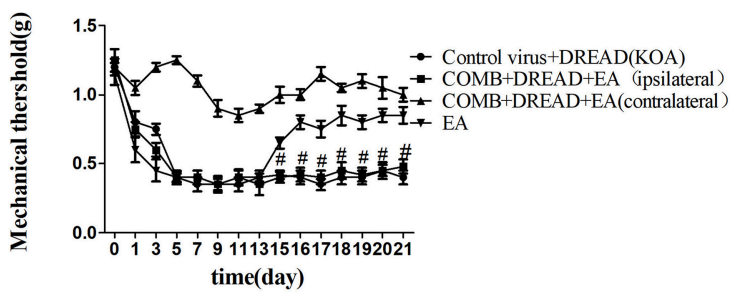

D
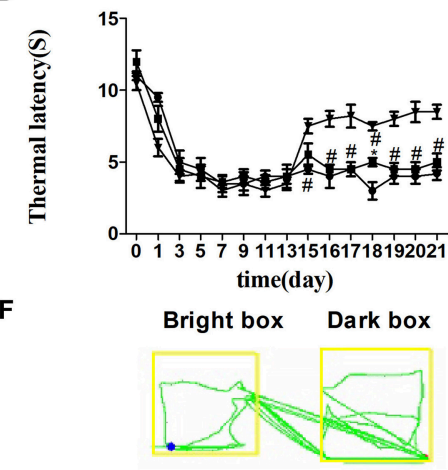

Before EA

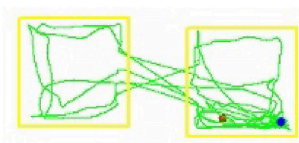

EA conditioned

H

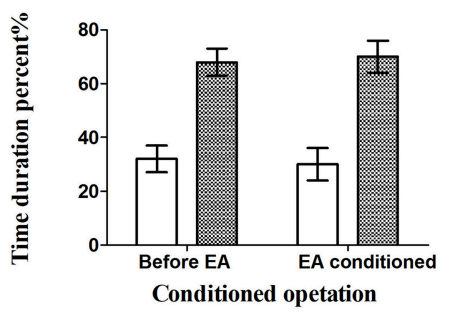

FIGURE 5 | The combination of chemogenetic activation of GABAergic neurons and chemogenetic inhibition of glutamatergic neurons in the vIPAG effectively attenuate the effect of EA. COMB is abstract of combination operation. (A,B) Time course of tactile threshold in response to von Frey filaments. (C,D) The noxious heat stimulus $\left(53^{\circ} \mathrm{C}\right)$ caused a change in the thermal thresholds. Tactile (A) and thermal (C) withdrawal thresholds changes in CCl mice after chemogenetic activation. Changes in tactile (B) and thermal (D) withdrawal thresholds of KOA mice caused by combination operation. Motion map of combination operation of conditioning in the CPP test with $\mathrm{CCl}(\mathbf{E})$ and $\mathrm{KOA}$ mice $\mathbf{( F )}$. G,H are summary values of motion trajectories. Virus was injected 21 days before the behavior test. CNO (1 $\mathrm{mg} / \mathrm{kg})$ and salvinorin $B(5 \mathrm{mg} / \mathrm{kg})$ were administered once a day starting from the 8th to the 14th day in the $\mathrm{CCl}$ model and starting from the 15th to the $21 \mathrm{st}$ day in the $\mathrm{KOA}$ model. EA ( $1 \mathrm{~mA}$ and $0.1 \mathrm{~ms})$ at $2 \mathrm{~Hz}$ was administered for $30 \mathrm{~min}$. Once a day starting from the 8 th to the 14 th day in the $\mathrm{CCl}$ model and starting from the 15 th to the 21 st day in the KOA model, all behavior tests were completed $2 \mathrm{~h}$ after $\mathrm{CNO}$ administration. Control groups consisted of CCl or KOA mice injected with control virus and DREAD but without EA treatment. Data are expressed as means \pm SEM $\left(n=12\right.$ in each group). In $\mathbf{A}-\mathbf{D},{ }^{*} p<0.05$, compared with control group; ${ }^{*} p<0.05$, compared with the model group; In $\mathbf{G}, \mathbf{H},{ }^{*} p<0.05$, compared with the bright box time duration percent before combination operation; $\# p<0.05$, compared with the dark box time duration percent before combination operation.

were excited by hM3Dq and CNO. These two systems were designed to work separately without interactions.

The combination of chemogenetic activation of GABAergic neurons and chemogenetic inhibition of glutamatergic neurons in the vlPAG powerfully decreased the mechanical withdrawal threshold and thermal withdrawal latency (Figures 5A-D) and the bright box time in the CPP test (Figures 5E-H).

\section{CB1 Receptors on GABAergic Neurons Is Involved in the EA Effect on Pain Hypersensitivity}

Studies have found that $\mathrm{CB} 1$ receptors are distributed on the axon terminals of GABAergic and glutamatergic PAG neurons (Tsou et al., 1998; Vaughan et al., 2000).On this basis, it was important to explore whether the $\mathrm{CB} 1$ receptors on GABAergic neurons or 


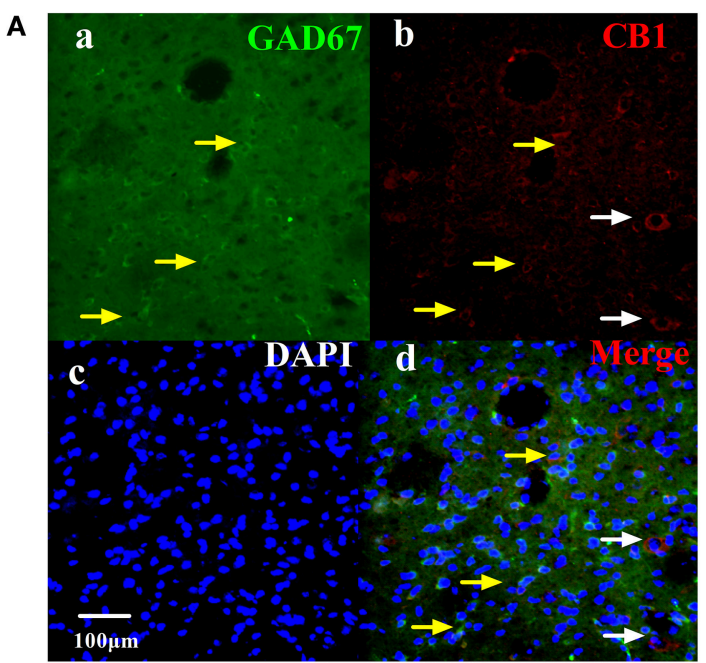

C

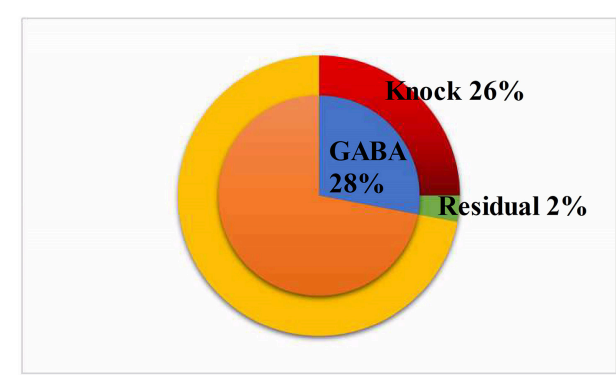

CB1 receptors on GABAergic neurons
B

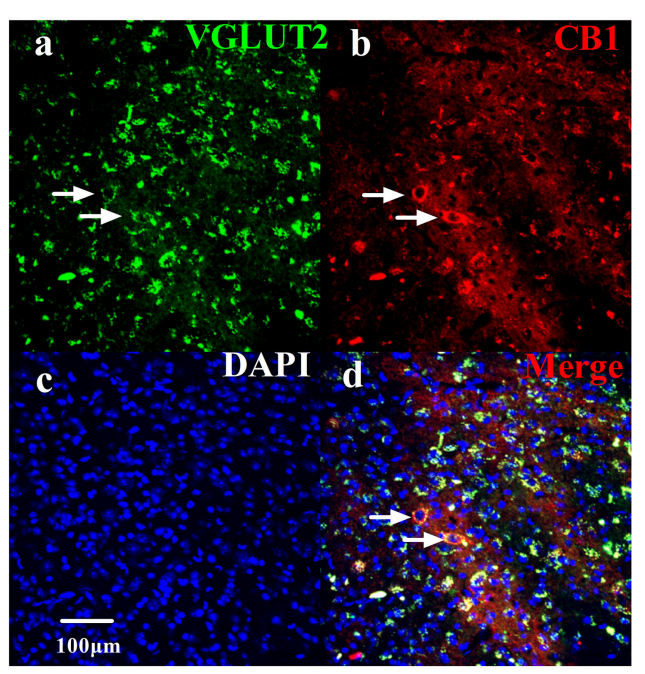

D

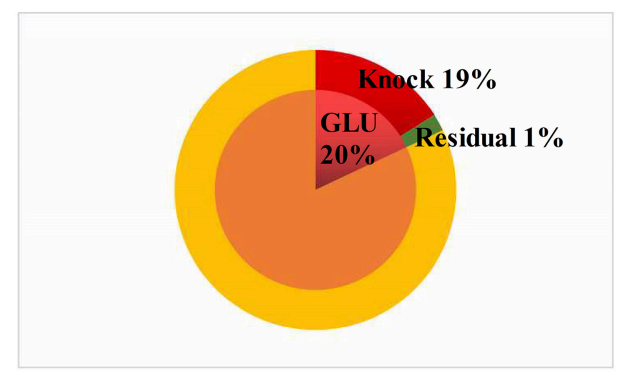

CB1 receptors on glutamatergic neurons

FIGURE 6 | Specific knockout of the CB1 receptor on GABAergic neurons localized in the vIPAG. (A) rAAV-mDlx-CRE-WPRE-pA was injected into the right side of the vIPAG of mCnr1flox/flox mice. (a) GABA-immunoreactive cellsin the vIPAG (green). (b) CB1-immunoreactive cells (red). (c) DAPI nuclear staining (blue). (d) GABA-immunoreactive and DAPI nuclear staining colocalization fluorescence (purple); Yellow arrows show CB1 receptors not knocked out on GABAergic neurons. White arrows show CB1 receptors not knocked out on other large diameter neurons. Scale bar, $100 \mu \mathrm{m}$. (B) rAAV-CaMKII-CRE-WPRE-pA was injected into the right side of the vIPAG of mCnr1flox/flox mice. (a) GLU-immunoreactive cells (green). (b) CB1-immunoreactive cells in the vIPAG (red). (c) DAPI nuclear staining (blue). (d) GLU-immunoreactive and DAPI nuclear staining colocalization fluorescence (purple); White arrows show CB1 receptors not knocked out on glutamatergic neurons. Scale bar, $100 \mu \mathrm{m}$. (C) Summary data show that the percentage of CB1-immunoreactive cells was knocked out in the area of GABA-immunoreactive cells. Blue sector, GABA-immunoreactive cells; red sector, CB1-immunoreactive cells were knocked out; green sector, residual cells. (D) Summary data shows the percentage of CB1-immunoreactive cells knocked out in the area of glutamatergic-immunoreactive cells. Pink sector, glutamatergic-immunoreactive cells; red sector,

CB1-immunoreactive cells been knocked out; green sector, residual cells. Data are expressed as the means \pm SEM ( $n=3$ mice in each group).

the $\mathrm{CB} 1$ receptors on glutamatergic neurons participate in the analgesic action of EA.

We injected rAAV-mDlx-CRE-WPRE-pA into the right side of the vlPAG of $\mathrm{mCnr}^{\text {flox/flox }}$ mice to specifically knock out the $\mathrm{CB} 1$ receptor on GABAergic neurons localized in the vlPAG (Figures 6A,C). Specifically knocking out the $\mathrm{CB1}$ receptor on GABAergic neurons abolished the EA effect on pain hypersensitivity, as it decreased the mechanical withdrawal threshold, thermal withdrawal latency (Figures 7A-D), and the bright box time in the CPP test (Figures 7E-H).

On the other hand, rAAV-CaMKII-CRE-WPRE-pA was injected into the right side of the vlPAG of $\mathrm{mCnr} 1^{\text {flox/flox }}$ mice for the purpose of specifically knocking out the CB1 receptor on glutamatergic neurons localized in the vlPAG (Figures 6B,D) and only slightly decreased the EA effect on pain hypersensitivity
(Figures S3A-H). It seems that the CB1 receptor on GABAergic neurons localized in the vlPAG was the basis of the EA effect on pain hypersensitivity.

\section{DISCUSSION}

The vlPAG, as an essential part of the neural pathway that mediates pain sensation, has been extensively studied (Vaughan et al., 1997; Ho et al., 2013; Tovote et al., 2016). Consistent with these studies, we found that inhibition of vlPAG neurons by chemogenetics can produce significant antinociceptive effects. We also found that chemogenetic activation of vlPAG neurons resulted in noxious hypersensitivity, and the results of this two-way manipulation were consistent with the bidirectional regulation of the vlPAG in nociceptive regulation (Koutsikou 


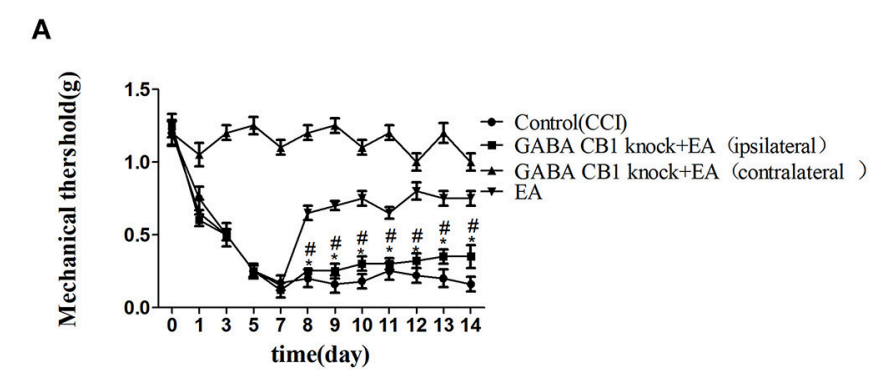

C

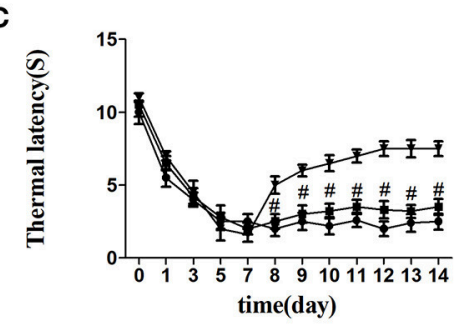

E

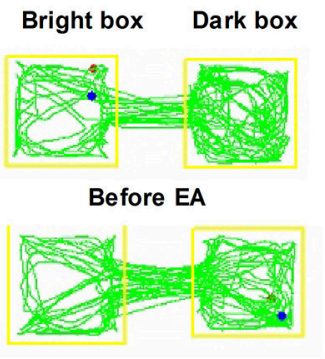

G

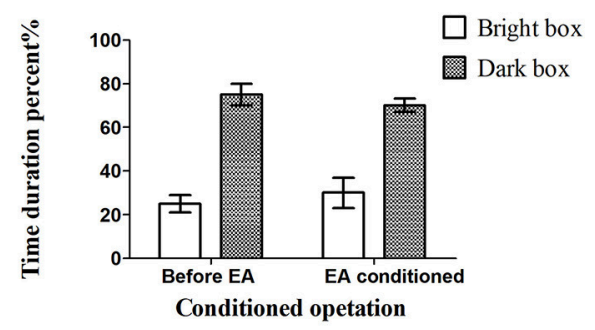

B

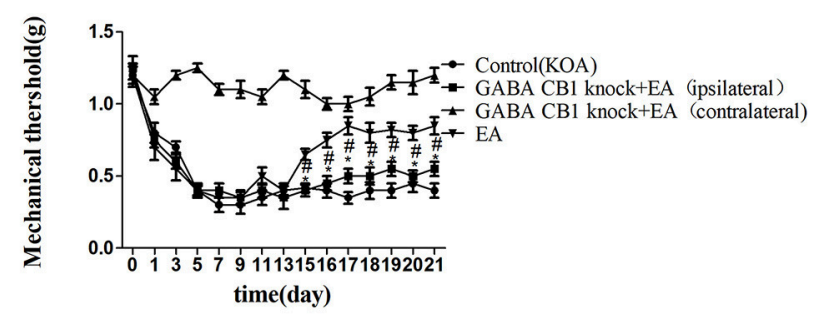

。

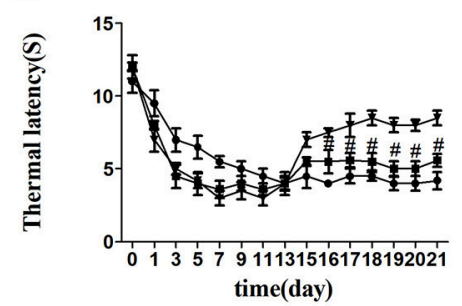

$\mathbf{F}$

Bright box Dark box

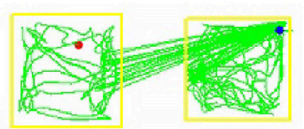

Before EA

H

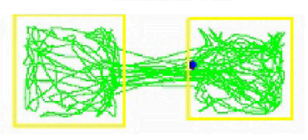

EA conditioned

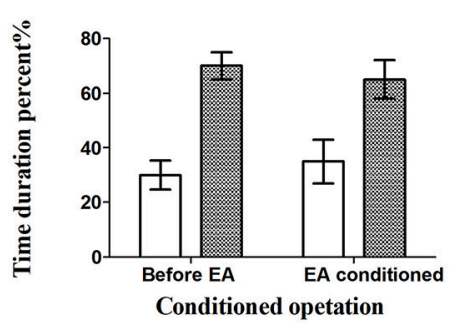

FIGURE 7 | Specifically knocking out CB1 receptor of GABAergic neurons abolished the EA effect on pain hypersensitivity. (A,B) Time course of tactile threshold in response to von Frey filaments. (C,D) The noxious heat stimulus $\left(53^{\circ} \mathrm{C}\right)$ caused a change in the thermal thresholds. Tactile $(\mathbf{A})$ and thermal $\mathbf{( C )}$ withdrawal thresholds changes in $\mathrm{CCl}$ mice. Changes in tactile (B) and thermal (D) withdrawal thresholds of KOA mice. Motion map of EA conditioned CPP test of CCI (E) and KOA mice (F) on the basis of specifically knocking out CB1 receptors of GABAergic neurons. G,H are statistical charts of motion trajectories. The rAAV-mDlx-CRE-WPRE-pA was injected into the right side of vIPAG of $\mathrm{mCnr} 1^{\text {flox/flox }} 21$ days before behavior test. Same volume of saline was injected into the right side of vIPAG of mCnr $1^{\text {flox/flox }}$ as control group. EA (1mA and $0.1 \mathrm{~ms}$ ) at $2 \mathrm{~Hz}$ was administered for $30 \mathrm{~min}$, once a day starting from 8 th day to 14 th in $\mathrm{CCl}$ model and starting from 15 th day to 21 th in KOA model. Control groups are $\mathrm{CCl}$ or KOA mice without EA treatment. Data are expressed as means \pm SEM $\left(n=5\right.$ in each group). In A-D, ${ }^{*} p<0.05$, compared with the control group; ${ }^{\#} p<0.05$, compared with the model group; In $\mathbf{G}, \mathbf{H},{ }^{\star} p<0.05$, compared with the bright box time duration percent before EA; $\# p<0.05$, compared with the dark box time duration percent before EA.

et al., 2015; Hernandez-Leon et al., 2016; Samineni et al., 2017). The vlPAG is critical for the mechanisms of EA-induced analgesia, and we have demonstrated in previous work that during KOA chronic pain, EA exerted an analgesic effect by increasing the levels of $\mathrm{CB} 1$ receptors and 2-AG in the vlPAG that had been significantly reduced (Yuan et al., 2018). In this study, we provided new experimental evidence that chemogenetic inhibition of GABAergic neurons in the vlPAG was able to replicate the antinociceptive effect of EA and accordingly further verify that the vIPAG is essential for EA analgesia.

The cellular mechanisms of analgesia and hyperalgesia, which involve in inhibitory and excitatory neurotransmission in the vlPAG have not been directly evaluated. It is not known how distinct neuron subpopulations in the vlPAG are engaged in the descending pain modulation pathway. For the first time, we showed that chemogenetic inhibition of GABAergic 


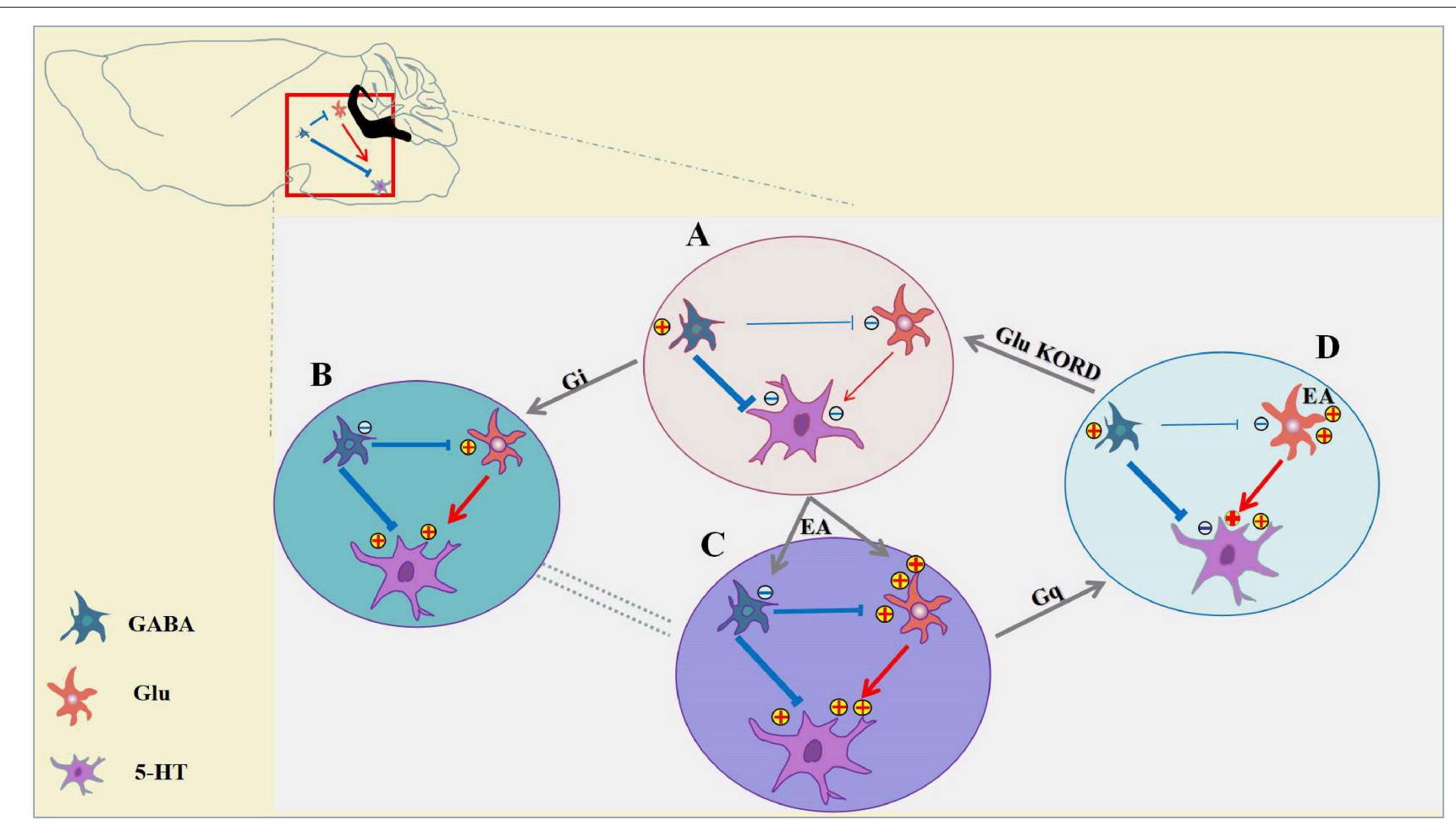

FIGURE 8 | Inhibition of GABAergic neurons and excitation of glutamatergic neurons in the vIPAG participate in EA analgesia. (A) Under pain conditions, GABAergic neurons are excited, resulting in glutamatergic, and serotoninergic neuron inhibition. (B) Chemogenetic inhibition of GABAergic neurons results in activation of glutamatergic neurons and serotoninergic neurons, and the descending inhibitory pain pathway would be activated to relieve the pain. (C) EA synchronously inhibits GABAergic neurons and activates glutamatergic neurons, thereby allowing serotoninergic neurons to be excited sufficiently. (D) With chemogenetic inhibition of GABAergic neurons, glutamatergic neurons are still activated by EA, and serotoninergic neurons in the descending inhibitory pain pathway remained partially activated. Chemogenetic activation of GABAergic neurons and inhibition of glutamatergic neurons is sufficient to attenuate the effects of EA.

vlPAG neurons produced antinociceptive effects, while the combination of chemogenetic activation of GABAergic neurons and chemogenetic inhibition of glutamatergic neurons in vlPAG effectively attenuated the effect of EA. It is hypothesized that GABAergic neurons have a tonic inhibitory effect on vlPAG glutamatergic neurons, while glutamate neurons are output neurons that project to the rostral ventromedial medulla (RVM) (Vaughan et al., 1997; Morgan et al., 2008; Ho et al., 2013). As we can see from Figure 8, chemogenetic inhibition of GABAergic neurons in the vlPAG also produces activation of glutamatergic neurons according to the GABA disinhibition hypothesis, whereas selective activation of GABAergic neurons is not sufficient to attenuate the effect of EA on the condition that some glutamatergic neurons are still directly activated by EA (Figure $\mathbf{8 C}$ ). This is the potential reason why specific chemogenetic inhibition of GABAergic neurons is sufficient to simulate the EA effect, while the reverse of the EA effect requires the combination of chemogenetic activation of GABAergic neurons and chemogenetic inhibition of glutamatergic neurons in the vlPAG. More studies are necessary to determine the neural circuitry of the vlPAG and connectivity with other brain regions. It is also possible that that GABAergic and glutamatergic neurons in the vlPAG have local circuits, such as synaptic connections between GABAergic neurons and glutamatergic neurons in the vlPAG. Future studies should examine the exact physiological function of GABAergic and glutamatergic neurons in the vlPAG local circuits and through synaptic connections between brain regions.

Endogenous cannabinoid ligands and $\mathrm{CB1}$ receptors are widely present in the nociceptive and descending inhibitory pathways (Mitrirattanakul et al., 2006). CB1 receptors are expressed in both nerve endings of GABAergic neurons and glutamatergic neurons in the PAG, which provides histological evidence that the activation of the $\mathrm{CB} 1$ receptor may regulate GABAergic and glutamatergic neurotransmission (Drew et al., 2009; Hu et al., 2014). WIN55212-2 (WIN), a CB1R agonist, modulates various $\mathrm{K}^{+}$and $\mathrm{Ca}^{2+}$ currents that could activate GABAergic and glutamatergic neurons (Straiker et al., 1999; Yazulla et al., 2000; Fan and Yazulla, 2003; Straiker and Sullivan, 2003; Yazulla, 2008; Schwitzer et al., 2016).

Consistent with previous electrophysiology results, we experimentally confirmed that the $\mathrm{CB} 1$ receptors on GABAergic and glutamatergic neurons are involved in the analgesia of EA. Using the Cre/loxP system, we are able to specifically knock out the CB1 receptor on GABAergic neurons or glutamatergic neurons localized in the vlPAG. Knocking out the CB1 receptor on GABAergic neurons abolished most of the EA effect on pain hypersensitivity, while knocking out the $\mathrm{CB} 1$ receptor on 
glutamatergic neurons localized in the vlPAG, only lessened some of the EA effect on pain hypersensitivity. According to our results, EA can simultaneously inhibit GABAergic neurons and excite glutamatergic neurons in the vlPAG via $\mathrm{CB} 1$ receptors. Chemogenic CB1 knock-out on GABAergic neurons abolished the effect of EA while chemogenic activation of GABAergic neurons only partly attenuated the effect of EA. It suggested that the $\mathrm{CB} 1$ receptors on GABAergic neurons may also be required for activation of the $\mathrm{CB} 1$ receptor on glutamatergic neurons localized in the vlPAG, which conforms to the theory of ONand OFF-cells in the vlPAG (Heinricher et al., 1987). According to Wang's research (Wang et al., 2016), a CB1R agonist reduced the mIPSC frequency regardless of whether AMPA receptors were blocked, while it affected the mEPSC frequency when inhibitory inputs were present. It indicated that the activation of GABAergic neurons may be necessary for glutamatergic neuron excitation via $\mathrm{CB} 1$ receptors.

Through chemical genetic manipulations, we have observed for the first time that EA can exert an analgesic effect by simultaneously inhibiting GABAergic neurons and stimulating glutamatergic neurons. We know that when pain occurs, it is accompanied by a functional imbalance between GABAergic neurons and glutamatergic neurons. The relative balance of GABAergic neurons and glutamatergic neurons is critical for the maintenance of homeostasis (Siegfried and de Souza, 1989; Schmidtko et al., 2008). The microinjection of $\mathrm{GABA}_{A}$ receptor antagonists or glutamate agonists into the vlPAG produces antinociceptive effects (Bobeck et al., 2009; Liao et al., 2011). According to recent research by Vijay K, activation of glutamatergic neurons or inhibition of GABAergic neurons by chemical genetics achieves an effective inhibition of nociceptive sensation, in spite of that experiment being performed in normal mice rather than in a mouse pain model, as in the present experiments (Samineni et al., 2017). Despite the support of those experiments, the effect of clinical application of $\mathrm{GABA}_{\mathrm{A}}$ receptor antagonists or glutamate agonists is not optimistic (de Meij et al., 2014; Bruhn et al., 2017). In clinical treatment, it is indeed difficult to find a drug that can simultaneously act on both GABAergic and glutamatergic neurons, as they belong to different neuroreceptor systems with two distinct functions. According to our results, EA may simultaneously inhibit

\section{REFERENCES}

Basbaum, A. I., and Fields, H. L. (1984). Endogenous pain control systems: brainstem spinal pathways and endorphin circuitry. Annu. Rev. Neurosci. 7, 309-338. doi: 10.1146/annurev.ne.07.030184. 001521

Bennett, G. J., and Xie, Y. K. (1988). A peripheral mononeuropathy in rat that produces disorders of pain sensation like those seen in man. Pain 33, 87-107. doi: 10.1016/0304-3959(88)90209-6

Bobeck, E. N., Chen, Q., Morgan, M. M., and Ingram, S. L. (2014). Contribution of adenylyl cyclase modulation of pre- and postsynaptic GABA neurotransmission to morphine antinociception and tolerance. Neuropsychopharmacology 39, 2142-2152. doi: 10.1038/npp.2014.62
GABAergic neurons and activate glutamatergic neurons in the vlPAG via $\mathrm{CB} 1$ receptors to exert antinociceptive effects. The results of this study provide experimental evidence contributing to our understanding of the dual target adjustment mechanism of EA analgesia and homeostasis recovery under pain conditions.

In conclusion, we have demonstrated a novel role of GABAergic neurons and glutamatergic neurons in the vlPAG in EA antinociceptive effects. EA can simultaneously inhibit GABAergic neurons and excite glutamatergic neurons in the vlPAG via $\mathrm{CB} 1$ receptors, thereby exerting antinociceptive effects. Knocking out the $\mathrm{CB} 1$ receptor on GABAergic neurons is sufficient to abolish the EA effect on pain hypersensitivity, while the combination of activation of GABAergic neurons and inhibition of glutamatergic neurons in vlPAG is sufficient to attenuate the effect of EA. EA synchronously regulates the GABAergic system and the glutamatergic system with distinct functions through the $\mathrm{CB} 1$ receptor. This finding may provide new ideas for the clinical treatment of pain and the development of analgesic drugs.

\section{AUTHOR CONTRIBUTIONS}

J-QS and ML designed this experiment. HeZ mainly completed this experiment. H-CX, H-PL, L-XL, and X-FH provided help in brain stereotactic injection work. HoZ, W-YM, LL, CC, YS, and $\mathrm{R}-\mathrm{YZ}$ assisted in the completion of animal behavior experiments. PZ, J-QS, and ML provided help in writing and modifying this paper.

\section{FUNDING}

This work was supported by a grant from the National Natural Science Foundation of China (81473768) and the Natural Science Foundation of Jiangsu Province (No. BK 20160548 to YS).

\section{SUPPLEMENTARY MATERIAL}

The Supplementary Material for this article can be found online at: https://www.frontiersin.org/articles/10.3389/fnins. 2019.00484/full\#supplementary-material

Bobeck, E. N., McNeal, A. L., and Morgan, M. M. (2009). Drug dependent sexdifferences in periaqueducatal gray mediated antinociception in the rat. Pain 147, 210-216. doi: 10.1016/j.pain.2009.09.008

Bruhn, J., Scheffer, G. J., and van Geffen, G. J. (2017). Clinical application of perioperative multimodal analgesia. Curr. Opin. Support. Palliat. Care 11, 106-111. doi: 10.1097/SPC.0000000000000267

Budai, D., Harasawa, I., and Fields, H. L. (1998). Midbrain periaqueductal gray (PAG) inhibits nociceptive inputs to sacral dorsal horn nociceptive neurons through alpha2-adrenergic receptors. J. Neurophysiol. 80, 2244-2254. doi: 10.1152/jn.1998.80.5.2244

Chaplan, S. R., Bach, F. W., Pogrel, J. W., Chung, J. M., and Yaksh, T. L. (1994). Quantitative assessment of tactile allodynia in the rat paw. J. Neurosci. Methods 53, 55-63. doi: 10.1016/0165-0270(94)90144-9 
Chen, G., Kim, Y. H., Li, H., Luo, H., Liu, D. L., Zhang, Z. J., et al. (2017). PD-L1 inhibits acute and chronic pain by suppressing nociceptive neuron activity via PD-1. Nat. Neurosci. 20, 917-926. doi: 10.1038/nn.4571

Chen, L., Zhang, J., Li, F., Qiu, Y., Wang, L., Li, Y. H., et al. (2009). Endogenous anandamide and cannabinoid receptor-2 contribute to electroacupuncture analgesia in rats. J. Pain 10, 732-739. doi: 10.1016/j.jpain.2008. 12.012

de Meij, N., Köke, A., van der Weijden, T., van Kleef, M., and Patijn, J. (2014). Pain treatment facilities: do we need quantity or quality? J. Eval. Clin. Pract. 20, 578-581. doi: 10.1111/jep.12148

Demers, G., Griffin, G., De Vroey, G., Haywood, J. R., Zurlo, J., and Bédard, M. (2006). Animal research. Harmonization of animal care and use guidance. Science 312, 700-701. doi: 10.1126/science.1124036

Dimidschstein, J., Chen, Q., Tremblay, R., Rogers, S. L., Saldi, G. A., Guo, L., et al. (2016). A viral strategy for targeting and manipulating interneurons across vertebrate species. Nat. Neurosci. 19, 1743-1749. doi: 10.1038/nn.4430

Drew, G. M., Lau, B. K., and Vaughan, C. W. (2009). Substance P drives endocannabinoid-mediated disinhibition in a midbrain descending analgesic pathway. J. Neurosci. 29, 7220-7229. doi: 10.1523/JNEUROSCI.4362-08.2009

Drew, G. M., Mitchell, V. A., and Vaughan, C. W. (2008). Glutamate spillover modulates GABAergic synaptic transmission in the rat midbrain periaqueductal grey via metabotropic glutamate receptors and endocannabinoid signaling. J. Neurosci. 28, 808-815. doi: 10.1523/JNEUROSCI.4876-07.2008

Fan, S. F., and Yazulla, S. (2003). Biphasic modulation of voltagedependent currents of retinal cones by cannabinoid CB1 receptor agonist WIN 55212-2. Vis. Neurosci. 20, 177-188. doi: 10.1017/S0952523803 20208X

Fernihough, J., Gentry, C., Malcangio, M., Fox, A., Rediske, J., Pellas, T., et al. (2004). Pain related behaviour in two models of osteoarthritis in the rat knee. Pain 112, 83-93. doi: 10.1016/j.pain.2004.08.004

Finn, D. P., Jhaveri, M. D., Beckett, S. R., Roe, C. H., Kendall, D. A., Marsden, C. A., et al. (2003). Effects of direct periaqueductal grey administration of a cannabinoid receptor agonist on nociceptive and aversive responses in rats. Neuropharmacology 45, 594-604. doi: 10.1016/S0028-3908(03)00235-1

Fusumada, K., Yokoyama, T., Miki, T., Wang, Z. Y., Yang, W., Lee, N. S., et al. (2007). c-Fos expression in the periaqueductal gray is induced by electroacupuncture in the rat, with possible reference to GABAergic neurons. Okajimas Folia Anat. Jpn. 84, 1-9. doi: 10.2535/ofaj.84.1

Harvey, V. L., and Dickenson, A. H. (2009). Behavioural and electrophysiological characterisation of experimentally induced osteoarthritis and neuropathy in C57Bl/6 mice. Mol. Pain 5, 18-18. doi: 10.1186/1744-8069-5-18

Heinricher, M. M., Cheng, Z. F., and Fields, H. L. (1987). Evidence for two classes of nociceptive modulating neurons in the periaqueductal gray. J. Neurosci. 7, 271-278. doi: 10.1523/JNEUROSCI.07-01-00271.1987

Hernandez-Leon, A., Fernández-Guasti, A., and González-Trujano, M. E. (2016). Rutin antinociception involves opioidergic mechanism and descending modulation of ventrolateral periaqueductal grey matter in rats. Eur. J. Pain 20, 274-283. doi: 10.1002/ejp.720

Hnasko, T. S., Sotak, B. N., and Palmiter, R. D. (2005). Morphine reward in dopamine-deficient mice. Nature 438, 854-857. doi: 10.1038/nature 04172

Ho, Y. C., Cheng, J. K., and Chiou, L. C. (2013). Hypofunction of Glutamatergic neurotransmission in the periaqueductal gray contributes to nerve-injury-induced neuropathic pain. J. Neurosci. 33, 7825-7836. doi: 10.1523/JNEUROSCI.5583-12.2013

Ho, Y. C., Lee, H. J., Tung, L. W., Liao, Y. Y., Fu, S. Y., Teng, S. F., et al. (2011). Activation of orexin 1 receptors in the periaqueductal gray of male rats leads to antinociception via retrograde endocannabinoid (2Arachidonoylglycerol)-induced disinhibition. J. Neurosci. 31, 14600-14610. doi: 10.1523/JNEUROSCI.2671-11.2011

Hu, S. S., Ho, Y. C., and Chiou, L. C. (2014). No more pain upon Gq-proteincoupled receptor activation: role of endocannabinoids. Eur. J. Neurosci. 39, 467-484. doi: 10.1111/ejn.12475

Kang, J. M., Park, H. J., Choi, Y. G., Choe, I. H., Park, J. H., Kim, Y. S., et al. (2007). Acupuncture inhibits microglial activation and inflammatory events in the MPTP-induced mouse model. Brain Res. 1131, 211-219. doi: $10.1016 /$ j.brainres.2006.10.089
Koutsikou, S., Watson, T. C., Crook, J. J., Leith, J. L., Lawrenson, C. L., Apps, R., et al. (2015). The periaqueductal gray orchestrates sensory and motor circuits at multiple levels of the neuraxis. J. Neurosci. 35, 14132-14147. doi: 10.1523/JNEUROSCI.0261-15.2015

La Porta, C., Bura, S. A., Aracil-Fernández, A., Manzanares, J., and Maldonado, R. (2013). Role of CB1 and CB2 cannabinoid receptors in the development of joint pain induced by monosodium iodoacetate. Pain 154, 160-174. doi: 10.1016/j.pain.2012.10.009

Lao, L., Zhang, R. X., Zhang, G., Wang, X., Berman, B. M., and Ren, K. (2004). A parametric study of electroacupuncture on persistent hyperalgesia and Fos protein expression in rats. Brain Res. 1020, 18-29. doi: 10.1016/j.brainres.2004.01.092

Lee, Y., Choi, G., Jeon, H., Kim, D., Ryu, S., Koo, S., et al. (2018). Acupuncture stimulation at GB34 suppresses 1-methyl-4-phenyl-1,2,3,6-tetrahydropyridineinduced oxidative stress in the striatum of mice. J. Physiol. Sci. 68, 455-462. doi: 10.1007/s12576-017-0547-7

Liao, H. T., Lee, H. J., Ho, Y. C., and Chiou, L. C. (2011). Capsaicin in the periaqueductal gray induces analgesia via metabotropic glutamate receptormediated endocannabinoid retrograde disinhibition. Br. J. Pharmacol. 163, 330-345. doi: 10.1111/j.1476-5381.2011.01214.x

Liu, L., Zhao, L. P., Zhang, C. S., Zeng, L., Wang, K., Zhao, J., et al. (2018). Acupuncture as prophylaxis for chronic migraine: a protocol for a singleblinded, double-dummy randomised controlled trial. BMJ Open 8:e020653. doi: 10.1136/bmjopen-2017-020653

Meng, I. D., Manning, B. H., Martin, W. J., and Fields, H. L. (1998). An analgesia circuit activated by cannabinoids. Nature 395, 381-383. doi: 10.1038/26481

Mist, S. D., and Jones, K. D. (2018). Randomized controlled trial of acupuncture for women with fibromyalgia: group acupuncture with traditional chinese medicine diagnosis-based point selection. Pain Med. 19, 1862-1871. doi: $10.1093 / \mathrm{pm} / \mathrm{pnx} 322$

Mitrirattanakul, S., Ramakul, N., Guerrero, A. V., Matsuka, Y., Ono, T., Iwase, H., et al. (2006). Site-specific increases in peripheral cannabinoid receptors and their endogenous ligands in a model of neuropathic pain. Pain 126, 102-114. doi: 10.1016/j.pain.2006.06.016

Morgan, M., Nanzer, A., Camacho-Hubner, C., Kaltsas, G. A., and Monson, J. P. (2003). Hypoglycaemia secondary to the secretion of pro-insulin like growth factor II by a metastatic neuroendocrine tumour with sarcomatous differentiation. Hormones 2, 125-129. doi: 10.14310/horm.2002.1192

Morgan, M. M., Whittier, K. L., Hegarty, D. M., and Aicher, S. A. (2008). Periaqueductal gray neurons project to spinally projecting GABAergic neurons in the rostral ventromedial medulla. Pain 140, 376-386. doi: 10.1016/j.pain.2008.09.009

Musil, F., Pokladnikova, J., Pavelek, Z., Wang, B., Guan, X., and Valis, M. (2018). Acupuncture in migraine prophylaxis in Czech patients: an openlabel randomized controlled trial. Neuropsychiatr. Dis. Treat. 14, 1221-1228. doi: 10.2147/NDT.S155119

Osborne, P. B., Vaughan, C. W., Wilson, H. I., and Christie, M. J. (1996). Opioid inhibition of rat periaqueductal grey neurones with identified projections to rostral ventromedial medulla in vitro. J. Physiol. 490, 383-389. doi: 10.1113/jphysiol.1996.sp021152

Palazzo, E., Luongo, L., Novellis, V., Rossi, F., and Maione, S. (2010). The role of cannabinoid receptors in the descending modulation of pain. Pharmaceuticals 3, 2661-2673. doi: 10.3390/ph3082661

Park, J. Y., Park, J. J., Jeon, S., Doo, A. R., Kim, S. N., Lee, H., et al. (2014). From peripheral to central: the role of ERK signaling pathway in acupuncture analgesia. J. Pain 15, 535-549. doi: 10.1016/j.jpain.2014.01.498

Samineni, V. K., Grajales-Reyes, J. G., Copits, B. A., O’Brien, D. E., Trigg, S. L., Gomez, A. M., et al. (2017). Divergent modulation of nociception by glutamatergic and GABAergic neuronal subpopulations in the periaqueductal gray. eNeuro 4:ENEURO.0129-16.2017. doi: 10.1523/ENEURO.0129-16.2017

Schmidtko, A., Luo, C., Gao, W., Geisslinger, G., Kuner, R., and Tegeder, I. (2008). Genetic deletion of synapsin II reduces neuropathic pain due to reduced glutamate but increased GABA in the spinal cord dorsal horn. Pain 139, 632-643. doi: 10.1016/j.pain.2008.06.018

Schwitzer, T., Schwan, R., Angioi-Duprez, K., Giersch, A., and Laprevote, V. (2016). The endocannabinoid system in the retina: from physiology to practical and therapeutic applications. Neural Plast. 2016:2916732. doi: $10.1155 / 2016 / 2916732$ 
Siegfried, B., and de Souza, R. L. (1989). NMDA receptor blockade in the periaqueductal grey prevents stress-induced analgesia in attacked mice. Eur. J. Pharmacol. 168, 239-242. doi: 10.1016/0014-2999(89)90570-0

Sommer, C., Schmidt, C., and George, A. (1998). Hyperalgesia in experimental neuropathy is dependent on the TNF receptor 1. Exp. Neurol. 151, 138-142. doi: 10.1006/exnr.1998.6797

Straiker, A., Stella, N., Piomelli, D., Mackie, K., Karten, H. J., and Maguire, G. (1999). Cannabinoid CB1 receptors and ligands in vertebrate retina: Localization and function of an endogenous signaling system. Proc. Natl. Acad. Sci. U.S.A. 96, 14565-14570. doi: 10.1073/pnas.96.25.14565

Straiker, A., and Sullivan, J. M. (2003). Cannabinoid receptor activation differentially modulates ion channels in photoreceptors of the tiger salamander. J. Neurophysiol. 89, 2647-2654. doi: 10.1152/jn.00268.2002

Takasu, K., Ogawa, K., Nakamura, A., Kanbara, T., Ono, H., Tomii, T., et al. (2015). Enhanced GABAergic synaptic transmission at VLPAG neurons and potent modulation by oxycodone in a bone cancer pain model. Br. J. Pharmacol. 172, 2148-2164. doi: 10.1111/bph.13039

Tjen-A-Looi, S. C., Li, P., and Longhurst, J. C. (2009). Processing cardiovascular information in the vlPAG during electroacupuncture in rats: roles of endocannabinoids and GABA. J. App. Physiol. 106, 1793-1799. doi: $10.1152 /$ japplphysiol.00142.2009

Tovote, P., Esposito, M. S., Botta, P., Chaudun, F., Fadok, J. P., Markovic, M., et al. (2016). Midbrain circuits for defensive behaviour. Nature 534, 206-212. doi: 10.1038 /nature17996

Tsou, K., Nogueron, M. I., Muthian, S., Sañudo-Pena, M. C., Hillard, C. J., Deutsch, D. G., et al. (1998). Fatty acid amide hydrolase is located preferentially in large neurons in the rat central nervous system as revealed by immunohistochemistry. Neurosci. Lett. 254, 137-140. doi: 10.1016/S0304-3940(98)00700-9

van Osch, G. J., van der Kraan, P. M., and van den Berg, W. B. (1994). Site-specific cartilage changes in murine degenerative knee joint disease induced by iodoacetate and collagenase. J. Orthop. Res. 12, 168-175. doi: $10.1002 /$ jor. 1100120204

Vardy, E., Robinson, J. E., Li, C., Olsen, R. H. J., DiBerto, J. F., Giguere, P. M., et al. (2015). A new DREADD facilitates the multiplexed chemogenetic interrogation of behavior. Neuron 86, 936-946. doi: 10.1016/j.neuron.2015.03.065

Vaughan, C. W., Connor, M., Bagley, E. E., and Christie, M. J. (2000). Actions of cannabinoids on membrane properties and synaptic transmission in rat periaqueductal gray neurons in vitro. Mol. Pharmacol. 57, 288-295. doi: $10.1002 /$ bjs.5237
Vaughan, C. W., Ingram, S. L., Connor, M. A., and Christie, M. J. (1997). How opioids inhibit GABA-mediated neurotransmission. Nature 390, 611-614. doi: $10.1038 / 37610$

Wang, X. H., Wu, Y., Yang, X. F., Miao, Y., Zhang, C. Q., Dong, L. D., et al. (2016). Cannabinoid CB1 receptor signaling dichotomously modulates inhibitory and excitatory synaptic transmission in rat inner retina. Brain Struct. Funct. 221, 301-316. doi: 10.1007/s00429-0140908-4

Wu, C. H., Lv, Z. T., Zhao, Y., Gao, Y., Li, J. Q., Gao, F., et al. (2013). Electroacupuncture improves thermal and mechanical sensitivities in a rat model of postherpetic neuralgia. Mol. Pain 9, 18-18. doi: $10.1186 / 1744-8069-9-18$

Wu, M. X., Li, X. H., Lin, M. N., Jia, X. R., Mu, R., Wan, W. R., et al. (2010), Clinical study on the treatment of knee osteoarthritis of Shen -Sui insufficiency syndrome type by electroacupuncture. Chin. J. Integr. Med. 16, 291-297. doi: $10.1007 /$ s11655-010-0513-1

Yazulla, S. (2008). Endocannabinoids in the retina: from marijuana to neuroprotection. Prog. Retin. Eye Res. 27, 501-526. doi: 10.1016/j.preteyeres.2008.07.002

Yazulla, S., Studholme, K. M., McIntosh, H. H., and Fan, S. F. (2000). Cannabinoid receptors on goldfish retinal bipolar cells: electron-microscope immunocytochemistry and whole-cell recordings. Vis. Neurosci. 17, 391-401. doi: $10.1017 /$ S0952523800173079

Yuan, X. C., Zhu, B., Jing, X. H., Xiong, L. Z., Wu, C. H., Gao, F., et al. (2018). Electroacupuncture potentiates cannabinoid receptor-mediated descending inhibitory control in a mouse model of knee osteoarthritis. Front. Mol. Neurosci. 11:112. doi: 10.3389/fnmol.2018.00112

Conflict of Interest Statement: The authors declare that the research was conducted in the absence of any commercial or financial relationships that could be construed as a potential conflict of interest.

Copyright $\odot 2019$ Zhu, Xiang, Li, Lin, Hu, Zhang, Meng, Liu, Chen, Shu, Zhang, Zhang, Si and Li. This is an open-access article distributed under the terms of the Creative Commons Attribution License (CC BY). The use, distribution or reproduction in other forums is permitted, provided the original author $(s)$ and the copyright owner(s) are credited and that the original publication in this journal is cited, in accordance with accepted academic practice. No use, distribution or reproduction is permitted which does not comply with these terms. 
OPEN ACCESS

Edited by:

Lauren C. Heathcote, Stanford University, United States

Reviewed by:

Sarah Prinsloo,

The University of Texas MD Anderson

Cancer Center, United States

Geoffrey Bird,

University of Oxford, United Kingdom

*Correspondence:

Aleksandra Vučković

Aleksandra.vuckovic@glasgow.ac.uk

Specialty section

This article was submitted to

Perception Science,

a section of the journal

Frontiers in Neuroscience

Received: 28 April 2019

Accepted: 09 July 2019

Published: 25 July 2019

Citation:

Vučković A, Altaleb MKH,

Fraser M, McGeady $C$ and Purcell $M$

(2019) EEG Correlates

of Self-Managed Neurofeedback Treatment of Central Neuropathic

Pain in Chronic Spinal Cord Injury.

Front. Neurosci. 13:762.

doi: 10.3389/fnins.2019.00762
EEG Correlates of Self-Managed Neurofeedback Treatment of Central Neuropathic Pain in Chronic Spinal Cord Injury

\author{
Aleksandra Vučković1*, Manaf Kadum Hussein Altaleb ${ }^{1,2}$, Matthew Fraser ${ }^{3}$, \\ Ciarán McGeady ${ }^{1}$ and Mariel Purcell ${ }^{3}$
}

${ }^{1}$ Rehabilitation and Assistive Devices, Biomedical Engineering Division, School of Engineering, University of Glasgow, Glasgow, United Kingdom, ${ }^{2}$ Faculty of Electrical Engineering, Wasit University, Wasit, Iraq, ${ }^{3}$ Queen Elizabeth National Spinal Injuries Unit, Queen Elizabeth University Hospital, Glasgow, United Kingdom

Background: Neurofeedback (NFB) is a neuromodulatory technique that enables voluntary modulation of brain activity in order to treat neurological condition, such as central neuropathic pain (CNP). A distinctive feature of this technique is that it actively involves participants in the therapy. In this feasibility study, we present results of participant self-managed NFB treatment of CNP.

Methods: Fifteen chronic spinal cord injured (SCI) participants (13M, 2F), with chronic CNP equal or greater than 4 on the Visual Numeric Scale, took part in the study. After initial training in hospital (up to 4 sessions), they practiced NF at home, on average 2-3 times a week, over a period of several weeks ( $\min 4$, max 20). The NFB protocol consisted of upregulating the alpha $(9-12 \mathrm{~Hz})$ and downregulating the theta $(4-8 \mathrm{~Hz})$ and the higher beta band (20-30 Hz) power from electrode location $\mathrm{C} 4$, for $30 \mathrm{~min}$. The output measures were pain before and after NFB, EEG before and during NFB and pain questionnaires. We analyzed EEG results and show NFB strategies based on the Power Spectrum Density of each single participant.

Results: Twelve participants achieved statistically significant reduction in pain and in eight participants this reduction was clinically significant (larger than 30\%). The most successfully regulated frequency band during NFB was alpha. However, most participants upregulated their individual alpha band, that had an average dominant frequency at $\alpha_{p}=7.6 \pm 0.8 \mathrm{~Hz}$ (median $8 \mathrm{~Hz}$ ) that is lower than the average of the general population, which is around $10 \mathrm{~Hz}$. Ten out of fifteen participants significantly upregulated their individual alpha power $\left(\alpha_{p} \pm 2 \mathrm{~Hz}\right)$ as compared to 4 participants who upregulated the power in the fixed alpha band $(8-12 \mathrm{~Hz})$. Eight out of the twelve participants who achieved a significant reduction of pain, significantly upregulated their individual alpha band power. There was a significantly larger increase in alpha power $(p<0.0001)$ and decrease of theta power $(p<0.04)$ in participant specific rather than in fixed frequency bands. 
Conclusion: Neurofeedback is a neuromodulatory technique that gives participants control over their pain and can be self-administered at home. Regulation of individual frequency band was related to a significant reduction in pain.

Keywords: central neuropathic pain, spinal cord injury, electroencephalography, neurofeedback, sensory-motor rhythm

\section{INTRODUCTION}

Neuropathic pain is a chronic condition caused by damage to or disease of the somatosensory nervous system (Haanpää et al., 2011), affecting $7-10 \%$ of the general population (Colloca et al., 2017). Although the cause of pain is in the nervous system, it is typically perceived as burning or stinging sensation coming from the body (Siddall et al., 2003). Neuropathic pain may be of a peripheral (i.e., post operative, cancer, and painful diabetic neuropathy) or of a central origin (Seifert and Maihöfner, 2009). Central neuropathic pain (CNP) is caused by an injury to the central nervous system including the brain and the spinal cord, and it has a high prevalence in conditions such a spinal cord injury (SCI) (Siddall et al., 2003; Finnerup, 2013), multiple sclerosis (Osterberg et al., 2005), stroke (Andersen et al., 1995) and Parkinson's disease (Beiske et al., 2009).

In people with SCI, the prevalence of neuropathic pain is around $50 \%$ and it typically occurs within the first year of injury (Siddall et al., 2003). Severe CNP results in reduced quality of life (Middleton et al., 2007) due to its impact on sleep and anxiety (Siddall et al., 2003; Finnerup, 2013) and in more extreme cases also affects employability (Mann et al., 2013).

There are six main mechanisms involved in the chronification of neuropathic pain: (i) activity increase in areas of the pain neuromatrix, (ii) recruitment of additional cortical areas beyond the classical pain neuromatrix, (iii) cortical reorganization and maladaptive neuroplasticity, (iv) alterations in neurochemistry, (v) structural brain changes, and (vi) disruption of the brain default mode network (Seifert and Maihöfner, 2009).

Central neuropathic pain is typically treated with medications, such as antidepressant, anticonvulsants and opioids (Dickenson and Ghandehari, 2007). These medications have multiple side effects, such as drowsiness, nausea, constipation and dry mouth (Bakonja and Rowbotham, 2006) and some of them lead to misuse or even drug-related deaths (Gov.UK, 2019). In addition to having side effects, medications have a limited efficacy (Finnerup et al., 2018). For example, about half the people taking gabapentin experience a moderate reduction in pain $(30 \%)$ (Wiffen et al., 2017).

Neuromodulatory treatment of CNP typically rely on external electrical or magnetic stimulation such as repetitive Transcranial Magnetic Stimulation (rTMS), transcranial Direct Current Stimulation (tDCS), and Cranial Electrotherapy Stimulation (CES) (O'Connell et al., 2018). A recent Cohrane review showed that rTMS on average results in $12 \%$, while tDCS results in $17 \%$ short term relief in pain. There was no evidence of CES effectiveness. O'Connell et al. (2018) suggested $15 \%$ as a clinically relevant reduction in pain, meaning that only tDCS may result in a clinically significant reduction in pain. Typically, tCDS electrodes are applied over the motor cortex while rTMS is applied either over the pre-frontal or central area. It is believed that while both techniques target the central motor area, they indirectly influence cortical areas involved in the pain matrix. For this reason, stimulation sites do not necessarily correspond to the somatotopic location of the part of the body that is perceived as being painful.

Neurofeedback is a neuromodulatory intervention which does not require an external electrical or magnetic filed. It is a type of a biofeedback where users are provided with real time information about their brain activity in a form of a visual, audio or even haptic feedback. Neurofeedback allows users to develop a voluntary control over an unconscious physiological process using a feedback signal (Sherlin et al., 2011; Ros et al., 2014). Thus it enables the implicit control of covert brain activity that may have no direct behavioral correlates.

Neurofeedback has been used for decades to treat various disorders, most successfully attention deficit hyperactivity disorder and epilepsy (Demos, 2005). Development of inexpensive Brain Computer Interface technology contributed to its recent popularity not only for the treatment of neurological conditions but also for the improvement of peak performance in the able bodied people (Gruzelier, 2014). NFB has been used for treatments of various types of pain including complex regional pain syndrome (Jensen et al., 2007) fibromyalgia (Kayiran et al., 2010; Caro and Winter, 2011), trigemina neuralgia (Sime, 2004), cancer pain (Hetkamp et al., 2019), migraine (Siniatchkin et al., 2000), and CNP (Jensen et al., 2013a; Hassan et al., 2015).

The advantage of NFB over other neurostimulation techniques is that it enables the participants to take the active part in therapy, changing the locus of control from the external to the internal (Wood and Kober, 2018). People who use NFB for a prolonged period of time can learn to apply the technique at will without a feedback (Sherlin et al., 2011). Furthermore the technique is less costly than rTMS and is also potentially safer than rTMS, tDCS, and CES. From a research perspective, NFB has an additional advantage over neurostimulation techniques, it records brain activity thus providing direct evidence of neuromodulation during the therapy.

Neurofeedback is often based on operant conditioning, a strategy that increases a preferred behavior and decreases an undesired behavior providing a reward or punishment (Skinner, 1948). The idea behind this is to train a user to promote a desirable response to occur again under the same conditions (Sherlin et al., 2011). Typically in NFB a "reward" and a "penalty" are presented in a visual form, e.g., changes in the color or size of an object on a computer screen. Simple, frequently used forms of reward and penalty are changes in color (i.e., reward corresponds 
to green color while penalty corresponds to red color) or size of on object on a computer screen.

It is believed that NFB tunes brain oscillations toward a homeostatic set-point which affords an optimal balance between network flexibility and stability (i.e., self-organizing criticality) (Ros et al., 2014). It is believed that non-degenerative brain disorders may have a self-tuning impairment, having their dynamic repertoire "trapped" in an abnormal resting state (Ros et al., 2014). In this respect, chronic pain disrupts "a default mode network” (Baliki et al., 2008). NFB facilitates global brain connectivity and establishes a normalized default mode network.

In order to establish a NFB protocol for CNP it was necessary to identify how CNP affects brain oscillatory activity, as measured by EEG. An important factor influencing oscillatory EEG activity is thalamo-cortical dysrhythmia, that serves as a trigger for cortical dysfunction (Llinas et al., 1999; Walton and Llinas, 2010). Thalamo-cortical dysrhythmia is caused by either deafferentation or disinhibition of thalamic nuclei and as such may have a subcortical or supracortical origin such as neuropathic pain, tinnitus, Parkinson's disease and depression (Llinas et al., 1999). In case of CNP caused by SCI, a lesion to the spinal cord causes deafferentation of thalamic nuclei leading to hyperpolarization of these cells. When hyperpolarized, thalamic neurons change from high threshold tonic firing to low threshold theta range oscillatory burst. Such low frequency oscillations entrain corticothalamic loops generating increased coherence between the thalamus and the cortex, and increased power in $4-8 \mathrm{~Hz}$ range in the cortical level, also accompanies by the reduction of the dominant (alpha) frequency (Llinas et al., 1999). At the same time thalamocortical modules in theta mode exert less collateral inhibition to the neighboring modules, which are thereby activated in higher beta and gamma frequency ranges (Llinas et al., 1999; Sarnthein et al., 2006). This abnormal high frequency firing is proposed to generate the positive symptoms of pain and allodynia (Schulman et al., 2005). In people with CNP, increased theta band activity is considered the main signature of CNP (Sarnthein et al., 2006; Stern et al., 2006; Boord et al., 2008; Vuckovic et al., 2014).

Previous EEG studies on CNP in SCI also reported a shift in the dominant alpha frequency toward lower values and a reduced reactivity (reduction of EEG power) to eyes opening, attributed to the altered input from the thalamus (Boord et al., 2008; Jensen et al., 2013b; Vuckovic et al., 2014). Changes in the resting state alpha band power are reported in some studies to be increased (Vuckovic et al., 2014) while others reported decreased alpha band power compared with participants with no pain (Jensen et al., 2013b). Our group also found an increased level of desynchronisation during imagined movements of both painful and non-painful limbs (Vuckovic et al., 2014), supporting results from fMRI studies indicating the over-activity of the sensory-motor cortex due to CNP (Wrigley et al., 2009; Gustin et al., 2010).

A recent review of EEG patterns in chronic pain found that the increased theta and alpha activity at rest and decreased amplitude of evoked potentials to sensory stimulation and cognitive tasks are the main indices of chronic pain (Pinheiro et al., 2016). On the contrary, Camfferman et al. (2017) suggested that the reduced alpha band power is an indicator of chronic pain in general.
A discrepancy with respect to alpha band power in different studies may be partially explained by the way alpha band power was calculated, as in people with CNP peak activity is lower. Thus standard 8-12 Hz band might not reflect the individual alpha band power.

In our recent study we showed that a reduced alpha power and a reduced dominant alpha frequency are predictive asymptomatic markers of CNP in people with SCI (Vuckovic et al., 2018b). These markers appear before pain and are representative features for machine learning systems that can predict the risk of developing pain (Vuckovic et al., 2018a). This indicates that alpha activity may be the most relevant feature for a NFB protocol.

The NFB protocols for treatment of different types of chronic pain show that most protocols target the central or temporal cortex, upregulating alpha $(8-12 \mathrm{~Hz})$ or lower beta $(12-15 \mathrm{~Hz})$ activity and downregulating theta $(4-8 \mathrm{~Hz})$ and higher beta $(20-$ $30 \mathrm{~Hz}$ ) (Kayiran et al., 2010; Caro and Winter, 2011; Jensen et al., 2013a; Hassan et al., 2015). All protocols were based on fixed frequency bands.

In our previous study we tested protocols that increased the alpha $(9-12 \mathrm{~Hz})$ and decreased the theta and higher beta band over the primary motor cortex (C3 and C4) (Hassan et al., 2015). Four out of five participants who received 20 to 40 sessions reported a more than $30 \%$ reduction in pain. The effect was notable up to 1 month following the last session. We also demonstrated that all participants were able to modulate their brain activity in the direction of NFB even without the feedback. On the contrary, with pre-recorded sham feedback provided they were not able to modulate their brain activity. Finally we showed that a week following the last session, their event related desynchronisation over the sensory-motor cortex during imagined movement was reduced, indicating reduced over activity of the motor cortex (Hasan et al., 2016). Their baseline EEG was also reduced in a wider pain matrix including the dorsolateral prefrontal cortex, the anterior cingulate cortex and the insular cortex, in the higher beta band.

In this participant self-managed study, we asked participants with long standing CNP and SCI to practice NFB on their own and monitored their pain level and EEG activity. The advantage of self-managed NFB is that users can decide when to use the $\mathrm{NFB}$, thus potentially maximizing the effect of treatment.

Research questions that we are trying to answer in this paper are:

- Can people learn how to self-manage NFB for CNP?

- Are NFB bands for treatment of CNP fixed or patient specific?

- What is the relation between self-regulated brain activity and the experience of pain?

\section{MATERIALS AND METHODS}

\section{Participant Demographics}

Twenty people with SCI (4 female and 16 male, aged $50.6 \pm 14.1$ years), previously diagnosed with CNP (Mahnig et al., 2016) participated in this study. The American Spinal 
Injury Association (ASIA) Impairment Classification was used to determine the neurological level of SCI (Kirshblum et al., 2011), and the completeness of injury. The level of injury $C$ (cervical) corresponds to tetraplegia while $\mathrm{T}$ (thoracic), and $\mathrm{L}$ (lumbar) to paraplegia. The completeness of injury is defined as: A-sensory and motor complete, B-sensory incomplete and motor complete and C and D-sensory and motor incomplete. Eight participants were able to walk, nine were fulltime wheelchair users who had good hand function and three participants were tetraplegic with poor hand function. Tetraplegics were assisted by their caregivers with NFB setup.

There were no inclusion restrictions with respect to the level or completeness of the injury, as there is no clear evidence of correlation between these factors and the incidence of CNP (Siddall et al., 2003). Table 1 shows participants' demographic information. The inclusion criteria were: intensity of CNP $\geq 4$ on a Visual Numerical Scale (VNS, $0=$ no pain, $10=$ worst pain imaginable), CNP ongoing for at least 6 months (chronic), aged between 18 and 75 years, no self-reported history of brain disease or injury, and basic computer skills. The exclusion criteria were: presence of chronic or acute muscular or visceral pain $\geq 4 \mathrm{VNS}$, traumatic brain injury, stroke, epilepsy, or any other self-reported neurological problem.

All participants had below level pain while participants 3, 8 , and 12 also had pain at the level of injury. The location of the painful region was marked by participants on a body chart (Figure 1). At level pain may occur due to injury to the roots or spinal cord, thus it may have central or peripheral origin. Below

TABLE 1 | Participants' demographic information.

\begin{tabular}{|c|c|c|c|c|c|c|}
\hline $\mathrm{Nr}$ & $\begin{array}{l}\text { ASIA } \\
\text { level }\end{array}$ & $\begin{array}{l}\text { ASIA } \\
\text { comp }\end{array}$ & $\begin{array}{l}\text { Pain } \\
\text { (VNS) }\end{array}$ & $\begin{array}{c}\text { Yeas since } \\
\text { injury }\end{array}$ & $\begin{array}{c}\text { Type } \\
\text { of CNP }\end{array}$ & Med \\
\hline P1 & L3/L4 & $\mathrm{D}$ & 9 & 10 & Un & $P$ \\
\hline P2 & $\mathrm{T} 6 / \mathrm{T} 7$ & D & 7 & 7 & Un & G \\
\hline P3 & $\mathrm{T} 5$ & D & 3 & 7 & At/Un & $\mathrm{T}$ \\
\hline P4 & $\mathrm{T} 4$ & A & 7 & 6 & Un & $\mathrm{T}$ \\
\hline P5 & L3 & D & 5 & 5 & Un & / \\
\hline P6 & $\mathrm{C} 2$ & B & 5 & 8 & Un & $\mathrm{P}$ \\
\hline P7 & $\mathrm{C} 2$ & A & 7 & 5 & Un & / \\
\hline P8 & C3/C5 & D & 3 & 5 & At/Un & / \\
\hline P9 & T5 & A & 7 & 5 & Un & $\mathrm{P}$ \\
\hline P10 & $\mathrm{C} 4$ & D & 15 & 10 & Un & G \\
\hline P11 & $\mathrm{C} 2$ & $A$ & 1 & $4-5$ & Un & $G$ \\
\hline P12 & $\mathrm{T} 6$ & B & 1 & 5 & At/Un & D \\
\hline P13 & T5 & $D$ & 10 & 6 & Un & / \\
\hline P14 & $\mathrm{C6} / \mathrm{C} 7$ & $A$ & 5 & 6 & Un & I \\
\hline P15 & $\mathrm{T} 6 / \mathrm{T} 7$ & A & 30 & $9-10$ & un & $\mathrm{N}$ \\
\hline P16 & L3/4 & $D$ & 7 & 4 & Un & / \\
\hline P17 & C5-C7 & A & 15 & 6 & Un & $\mathrm{B} / \mathrm{P}$ \\
\hline P18 & $\llcorner 4$ & A & 21 & 6 & Un & $\mathrm{B} / \mathrm{P}$ \\
\hline P19 & L2 & $A$ & 13 & 7 & Un & / \\
\hline P20 & $\mathrm{T} 10$ & $A$ & 1 & 8 & Un & $B$ \\
\hline
\end{tabular}

Nr, participant's number; ASIA, Americal Spinal Injury Association; VNS, Visual Numerical Scale, CNP, Central Neuropathic Pain; Med, medications; P, pregabaline; G, gabapentin; T, tramadol; $D$, duloxetine; $N$, nabline.

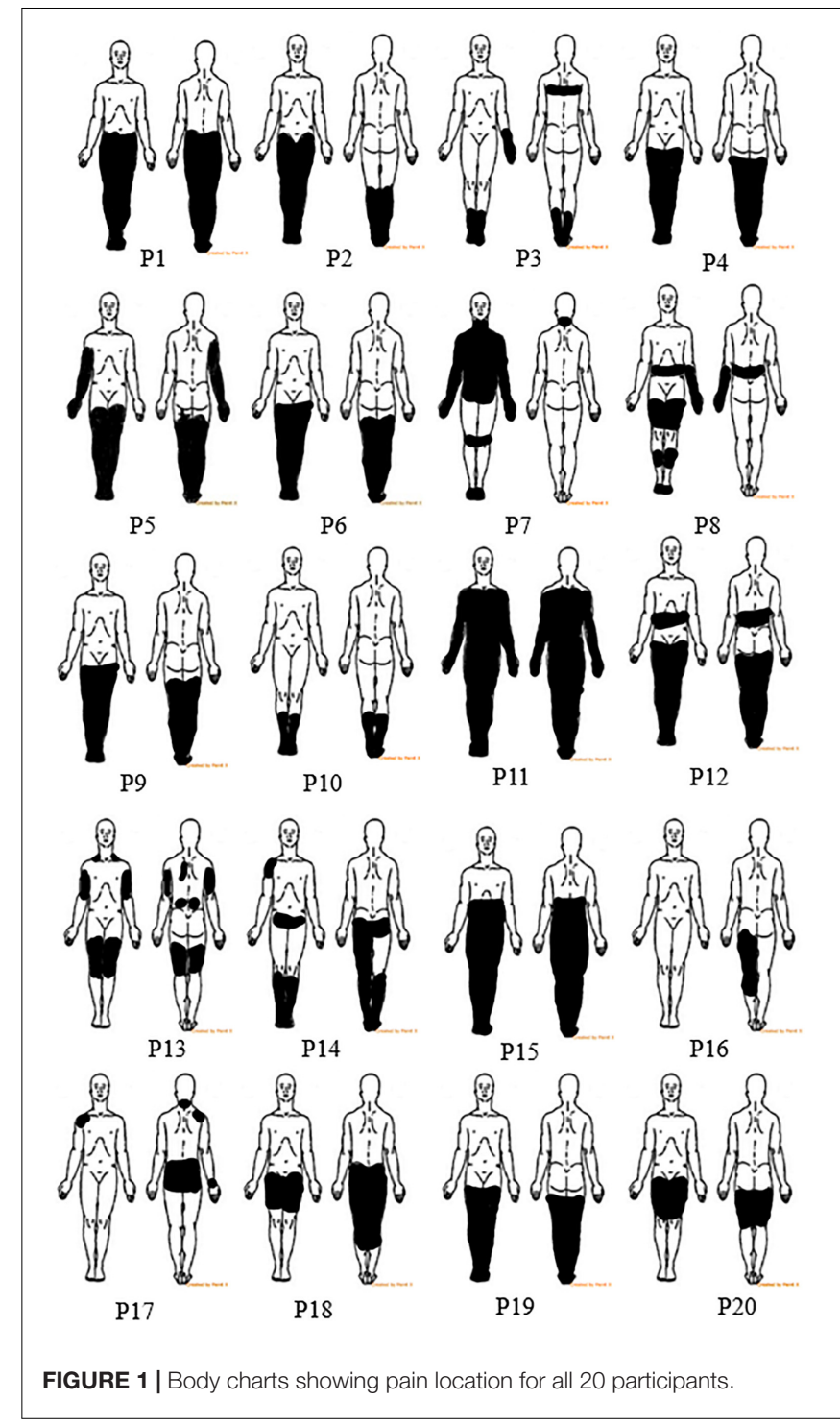

level pain is more common and more persistent; it has a central origin and is caused by the injury to the spinal cord (Bryce et al., 2012; Finnerup et al., 2014). Participants typically described the pain sensation as pins and needles, constant burning or freezing, tingling or squeezing combined with the intermittent electrical shock sensations.

Most of the participants were prescribed CNP medications, such as antidepressants or anticonvulsants. They were asked not to change their medications throughout the study to avoid their interference with the NFB effect on pain. Six patients previously tried non-pharmacological treatments (acupuncture and mindfulness) that were available through the National Healthcare Service, but have not been receiving any nonpharmacological treatment for at least 6 months prior to taking part in the study. During a regular check-up communication, approximately once a fortnight (in person, email, or Skype) there were asked if there were any changes dosage or type of medication. All patient participants and able-bodied participant 
shown in Figure 2 provided written informed consent. The study has been approved by the Greater Glasgow and Clyde National Health Service Ethical Committee. This study is a registered clinical trial NCT02678494.

\section{The Experimental Protocol Initial Assessment}

At the beginning of the study, all participants were asked to fill out a "Brief Pain Inventory" (Tan et al., 2004) and a "Neuropathic Pain Symptom Inventory” (Bouhassira et al., 2004). Participant's initial response to NFB was measured using a laboratory device usbamp (Guger Technology, Austria), as in our previous study (Hassan et al., 2015; Hasan et al., 2016). This devices uses an EEG cap and can therefore precisely define the electrode locations. This is how the location of electrodes for the wearable EEG headset (Epoch, Emotiv, United States) was determined. Based on the literature (Jensen et al., 2013a) and our initial assessment of NFB on able-bodied people (Al-Taleb, 2018), we organized up to four initial assessment sessions in hospital. We were looking for early sensory responses to NFB such as: reduction in pain of at least one point on the VNS, a pleasant warmth replacing the sensation of burning or freezing, tingling in the toes or finger tips (Hassan et al., 2015). Five out of twenty participants decided to withdraw before completing all four NFB sessions, three could not commit to the study and two were lacking any response to NFB.

\section{Participant Training to Use a Wearable BCI}

The remaining fifteen participants and caregivers were trained to use the Epoch EEG (Emotiv, United States) device and custom made software. They were offered up to 4 training sessions before taking the EEG device and tablet with software home to train on their own. To minimize the number of hospital visits, some of the training sessions took place on the same day as the assessment with the usbamp. Participants were instructed to practice the NFB on demand but at least once a week for a period of 2 months; some participants decided to keep the system after 2 months, we followed them up till the end of the study. All participants were also asked to fill out an electronic pain diary before and after training. The intensity of pain was recorded using the VNS ranging from 0 (no pain) to 10 (worst pain imaginable) in integer values. EEG was recorded during NFB sessions from the training electrode. At the end of the training, semi-structured interviews were organized. The results of the interviews with participants will be analyzed in a usability study presented elsewhere.

Because Epoch was not initially designed to record from the central cortex, participants were trained to place the headset on the correct central location, so that the recording electrode was placed approximately in between C2 and C4. The Epoch headset is only available in one size it was therefore not possible to provide a more precise location. Figure 2A shows an example of correct placing of the headset with location for the electrode from which training was provided.

\section{System Description and Neurofeedback Training Protocol}

NFB training was provided approximately from the electrode location C4 (American Clinical Neurophysiology Society [ACNS], 2006), placed over the primary motor cortex of the left arm and hand. The sampling frequency was 128 samples/s and two reference electrodes were placed parietaly, above the ears for CMS/DRL noise cancelation. The impedance was set under $10 \mathrm{k} \Omega$ using Epoch proprietary software. A wireless communication between the EEG device and tablet, was based on proprietary $2.4 \mathrm{~GB}$ wireless technology.

Custom made software written in C++ (Al-Taleb, 2018) consisted of a unit for EEG recording and analysis and a Graphical User Interface (GUI). The GUI had the main screen for neurofeedback training, a screen for EEG parameters setting, and an electronic pain diary.

Neurofeedback sessions always started with 2 min baseline recording. During the baseline recording, participants sat still with their eyes open looking at the center of the screen. NFB training sessions were 5 min long and participants were advised to have 5-6 daily sessions.

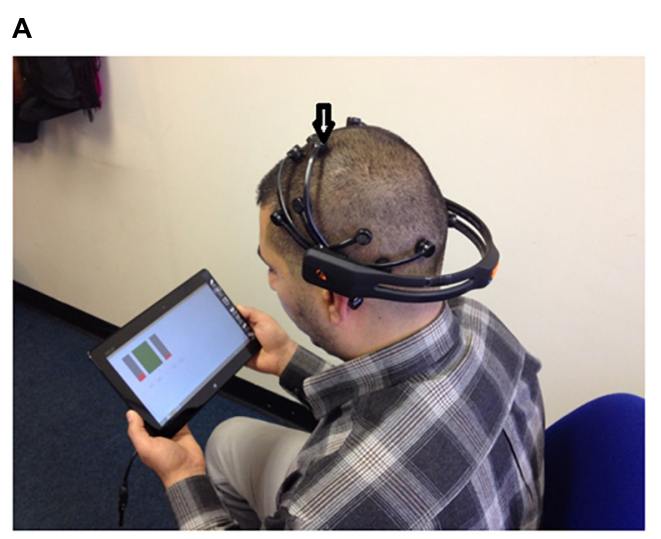

B

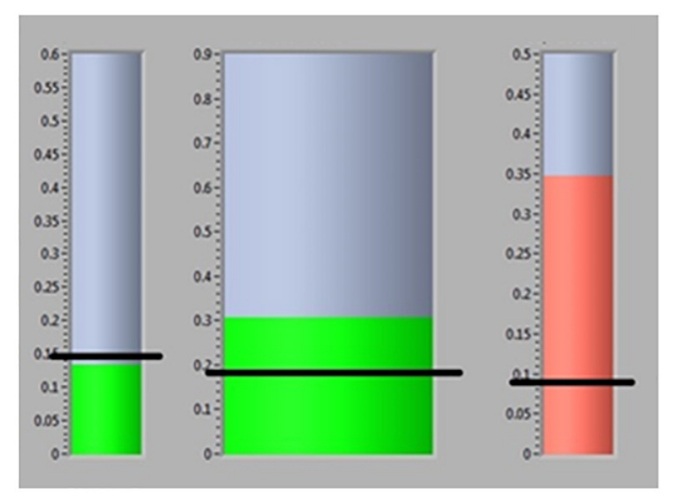

FIGURE 2 | (A) A member of the research team (who consented that his photo is provided) demonstrate the correct location of the EEG headset with an arrow pointing to the electrodes from which the EEG was provided. (B) Neurofeedback GUl; Horizontal black lines present an example of threshold values, they were not shown to users. 
A training protocol was adopted from the previous study by our group (Hassan et al., 2015). During NFB training the power of the EEG signal was calculated over a 0.5 s sliding window. EEG signal was filtered in four frequency bands: wideband 2$30 \mathrm{~Hz}, \Theta$ theta $(4-8 \mathrm{~Hz}), \alpha$ alpha $(9-12 \mathrm{~Hz})$, and higher $\beta$ beta (20-30 Hz) using a 5th order Butterworth filter. The relative power was calculated as a power in a selected band divided by wide band $2-30 \mathrm{~Hz}$ power. Relative power was presented to users during NFB (range from 0 to 1, corresponding to 0 to $100 \%$ of the wide band power).

The values of relative theta, alpha and beta power were shown on a computer screen, in a form of three separate bars (Figure 2B). During NFB training, participants were aiming at increasing the alpha band power and decreasing the theta and beta band power, relative to the baseline power recorded on the same day prior to NFB. A slightly higher alpha range starting from 9 rather than $8 \mathrm{~Hz}$ was selected in order to shift the dominant frequency toward the higher values, as a lower dominant alpha frequency was found to be a signature of CNP (Boord et al., 2008). While the theta and beta bands were related to EEG signatures of pain, these two bands are also related to noise coming from blinking (theta band) and muscle activity (beta band). Thus minimizing theta and beta power also minimized the online noise.

The training threshold was fixed and set to $110 \%$ of the baseline alpha power and to $90 \%$ of the baseline theta and higher beta power. A color of the representative bar was green when the alpha power was above the threshold and was red otherwise. For the theta and beta power the color of the representative bars was green when the power was under the threshold and red otherwise. The bars also changed size proportional to the amplitude of the power.

\section{Off-Line Analysis \\ Removal of artifact}

EEG was visually inspected and signal having an amplitude greater than $100 \mu \mathrm{V}$ or containing electrooculogram EOG (blinking and eye rolling) was manually removed. On average $10-20 \%$ of the signal was removed.

\section{Power spectrum density}

PSD was calculated using Hamming windows over 4 s long recording overlapping for $2 \mathrm{~s}$ and averaging PSD over all windows (Welch periodogram method). A logarithmic PSD was calculated as $10 \log _{10}$ PSD for visualization purposes.

Power was calculated for $(4-8 \mathrm{~Hz}),(8-12 \mathrm{~Hz})$, and $(20-30 \mathrm{~Hz})$ and for individual frequency bands by summing up PSD values over the selected frequency range. In order to determine the individual bands, the frequency of alpha peak $\alpha_{\mathrm{p}}$ was determined first as a maximum of PSD graph and a band power was calculated with respect to that peak. The individual alpha band was defined as $\left(\alpha_{p} \pm 2 \mathrm{~Hz}\right)$, the individual theta band as $\left(\alpha_{\mathrm{p}}-6 \mathrm{~Hz}\right.$ to $\left.\alpha_{\mathrm{p}}-2 \mathrm{~Hz}\right)$ and the individual higher beta band as $\left(\alpha_{\mathrm{p}}+12 \mathrm{~Hz}\right.$ to $\alpha_{\mathrm{p}}+22 \mathrm{~Hz}$ ).

\section{Statistical analysis}

Mann-Whitney $U$-test was used to compare VNS pain intensity before and after neurofeedback and EEG data. A non-parametric
Spearman correlation coefficient was calculated between the intensity of pain and EEG features as well as between pain and patient demographic data. A significance level of $p=0.05$ was adopted in all cases.

\section{RESULTS}

\section{Participants Compliance With the Study}

Out of fifteen participants who took part in the NFB study, seven used the system for 2 months as required. Three out of these seven continued to use the system for additional 6 weeks. Eight participants discontinued the study for the following reasons: unrelated health problems $(N=3)$, new caregiver $(N=1)$, moving home $(N=1)$, too long donning time (>15 $\mathrm{min})$ $(N=2)$, broken device $(N=1)$. Out of seven participants who used the system in their home for 8 weeks or longer, five could walk, one was a sensory and motor complete paraplegic, and one sensory and motor complete tetraplegic. EEG data and pain diaries were collected from all fifteen participants (participants could not start NFB or exit the software application unless filling out the pain diary). Table 2 shows the number of session, frequency of use and the total duration of the study for each participant. Participants were advised to use NFB on demand, when they had time and when they felt most pain. From the frequency of use, which in most participants was about three times per week, it can be concluded that NFB had an effect that lasted several days. This is, however, only an estimate, because participants did not fill out the pain diary on a daily basis and at the same time of the day (participants often reported that pain tends to be higher in the evening than in the morning).

\section{The Ability to Regulate EEG Power}

We analyzed the average ability of each participant to upregulate the alpha and to down regulate the theta and beta band power. Figure 3 shows the average (mean \pm SD) modulation of power, with respect to the baseline power in fixed frequency ranges (theta, alpha, and higher beta) for each participant. Positive values correspond to the upregulation (increase) while negative values correspond to the downregulation (decrease). A dashed line represents $10 \%$ change with respect to the baseline ( $\pm 10 \%$ was also set as a training threshold). In addition, a statistical analysis was performed over all training sessions, to assess whether NFB consistently modulated EEG power in a desired direction (increase of the alpha and decrease for the theta and beta bands). This was a somewhat conservative approach as it also included early sessions while participants were still learning the NFB technique. Only three participants significantly upregulated their alpha band power, but nine participants significantly downregulated either theta or beta band power.

Figure 4 shows the average (mean \pm SD) modulation of power, with respect to the baseline power in participant specific frequency ranges for each participant. Twelve out of fifteen participants increased on average the alpha power for more than $10 \%$ and for nine of them this was statistically significant. Ten reduced their theta band power by more than $10 \%$ while 
TABLE 2 | The number of NFB sessions.

\begin{tabular}{lcccc}
\hline $\begin{array}{l}\text { Part. } \\
\text { code }\end{array}$ & $\begin{array}{c}\text { Nr } \\
\text { sessions }\end{array}$ & $\begin{array}{c}\text { Total } \mathbf{N r} \\
\text { subsess. }\end{array}$ & $\begin{array}{c}\text { Weekly } \\
\text { usage }\end{array}$ & $\begin{array}{c}\text { Duration } \\
\text { (weeks) }\end{array}$ \\
\hline P1 & 3 & 12 & $1-2$ & 2 \\
P2 & 12 & 30 & 1 & 8 \\
P3 & 7 & 24 & $3-5$ & 2 \\
P4 & 40 & 235 & $3-6$ & 12 \\
P5 & 48 & 280 & $3-6$ & 14 \\
P6 & $\backslash$ & $\backslash$ & $\backslash$ & $\backslash$ \\
P7 & 9 & 31 & 3 & 3 \\
P8 & $\backslash$ & $\backslash$ & $\backslash$ & $\backslash$ \\
P9 & $\backslash$ & $\backslash$ & $\backslash$ & $\backslash$ \\
P10 & 14 & 84 & $1-3$ & 8 \\
P11 & 3 & 16 & $1-3$ & 4 \\
P12 & 6 & 29 & $1-3$ & 3 \\
P13 & $\backslash$ & $\backslash$ & $\backslash$ & $\backslash$ \\
P14 & 10 & 42 & $3-4$ & 3 \\
P15 & $\backslash$ & $\backslash$ & $\backslash$ & $\backslash$ \\
P16 & 20 & 108 & $3-5$ & 8 \\
P17 & 31 & 143 & $3-5$ & 9 \\
P18 & 4 & 21 & $1-3$ & 3 \\
P19 & 3 & 12 & 1 & 3 \\
P20 & 3 & 17 & $1-2$ & 2 \\
\hline
\end{tabular}

in seven this downregulation was statistically significant. Six reduced their beta band.

There was a statistically significant difference in the alpha $(p<0.001)$ and theta band power $(p<0.04)$ calculated for fixed and participant specific ranges. For the beta bands this difference was not statistically significant $(p<0.08)$.

To further illustrate why it was necessary to calculate the alpha band power in a participant specific manner, we show a representative example of power spectrum density for each participant during the baseline period and during NFB in Figure 5. Participants average dominant alpha peak is shown in Table 3. All but one participant had a dominant alpha peak $8 \mathrm{~Hz}$ or lower, as opposed to the general population where it is about $10 \mathrm{~Hz}$ (Niedermeyer, 1999). The dominant peak did not change as a result of NFB.

Figure 5 shows that during NFB participants did not shift their alpha peak in the $9-12 \mathrm{~Hz}$ range but increased the power in their own alpha range (see, e.g., P3, P4, P7, andP17). Six participants downregulated their beta band activity (desired direction) while four were increased their beta band activity (undesired direction). The individual theta band in most cases actually overlapped with the delta $(1-4 \mathrm{~Hz})$ band while individual alpha band partially overlapped with the theta $(4-8 \mathrm{~Hz})$ band. Furthermore in five participants (P5, P7, P11, P16, and P17) the dominant peak was barely visible in the relaxed state but emerged during NFB.

The alpha band power normally increases when a person closes their eyes. Engagement in a cognitive task normally results in decrease in alpha and increase in beta band activity (Niedermeyer, 1999). In addition to the wide spread alpha activity, the central cortex is also a source of the sensory motor rhythm, also present at 8-12 Hz (Niedermeyer, 1999). The idea behind NFB from electrode location C4 was to modulate the sensory-motor rhythm. This rhythm drops upon motor

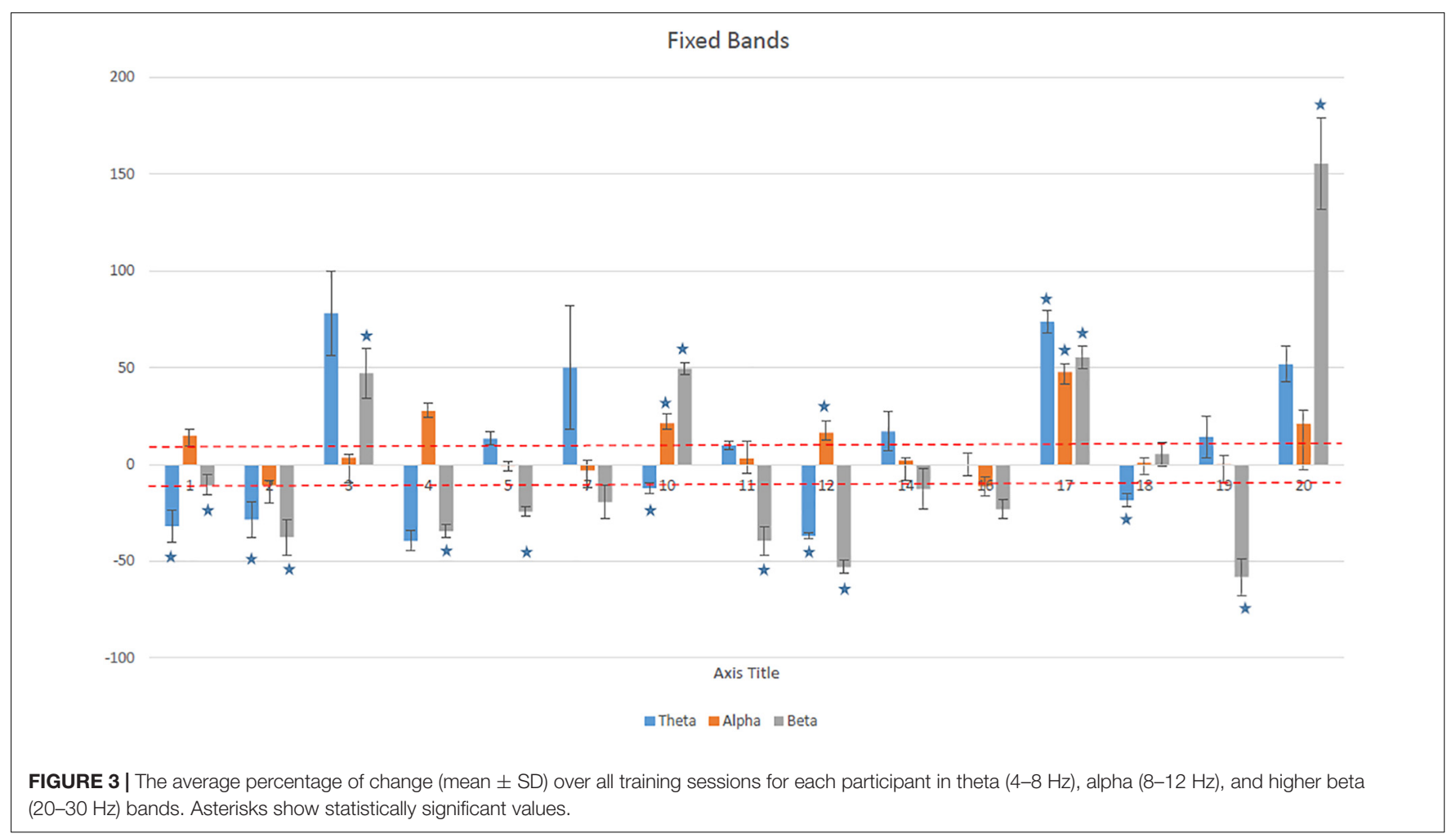




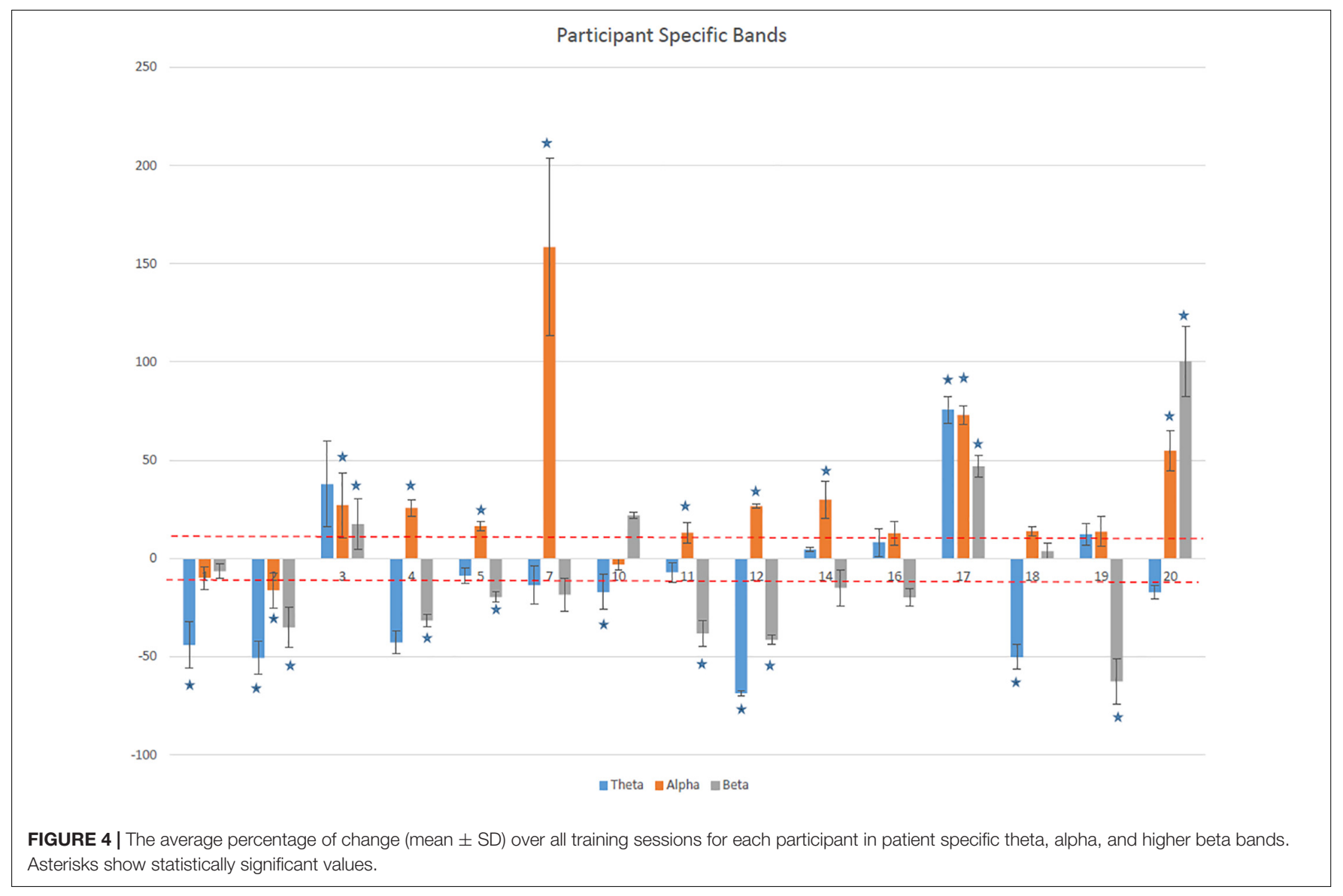

action, and there is no known verbalized strategy to increase it with the eyes opened. For this reason we believe that the increase in alpha (i.e., sensory motor rhythm) activity in this

TABLE 3 | Individual dominant alpha peak.

\begin{tabular}{lcc}
\hline Participant & $\begin{array}{c}\text { Alpha } \\
\text { peak (Hz) }\end{array}$ & $\begin{array}{c}\text { Alpha range with respect to } \\
\text { the dominant peak }(\boldsymbol{\alpha} \pm \mathbf{2}) \mathbf{H z}\end{array}$ \\
\hline 1 & 8.0 & $6.0-10.0$ \\
2 & 6.5 & $4.5-8.5$ \\
3 & 8.0 & $6.0-10.0$ \\
4 & 8.0 & $6.0-10.0$ \\
5 & 8.0 & $6.0-10.0$ \\
7 & 8 & $6.0-10.0$ \\
10 & 6 & $4.0-8.0$ \\
11 & 6 & $4.0-8.0$ \\
12 & 8 & $6.0-10.0$ \\
14 & 8 & $6.0-10.0$ \\
16 & 8 & $6.0-10.0$ \\
17 & 9 & $7.0-11.0$ \\
18 & 7.5 & $5.5-9.5$ \\
19 & 7.5 & $5.5-9.5$ \\
20 & 8 & $6.0-8.0$ \\
Mean \pm SD & $7.6 \pm 0.9$ & \\
Median [Q1,Q3] & $8,[7.5,8]$ &
\end{tabular}

case is due to NFB and not due to general engagement in a cognitive task.

\section{The Effect of NFB on Pain}

Table 4 shows the average level of pain reduction following NFB treatment for each participant. Because the VNS pain ratings are not normally distributed (e.g., the initial pain level in all participants was between 4 and 10) we present them as median and first and third quartile and related statistical results based on non-parametric statistical analysis. We, however, also present the same results as mean $\pm \mathrm{SD}$ in the Appendix, similar to how results are presented in most pain related publications. Both approaches provide similar results.

Twelve out of fifteen participants achieved statistically significant reduction in pain. In eight participants this was also clinically significant (larger than $30 \%$ ). Thirty percent was based on studies reporting on the effectiveness of pharmacological treatments (Wiffen et al., 2017). If we adopt $15 \%$ as a clinically significant value, as in papers reporting on benefit of neurostimulation technology (O'Connell et al., 2018), thirteen participants achieved a clinically significant reduction in pain.

There was no direct correlation between the increase in alpha band power during NFB and decrease in pain $(p=0.4589$, $R=-0.0152$ ). This is, however, not surprising bearing in mind that the NFB tunes the brain toward a homeostatic set point, that 

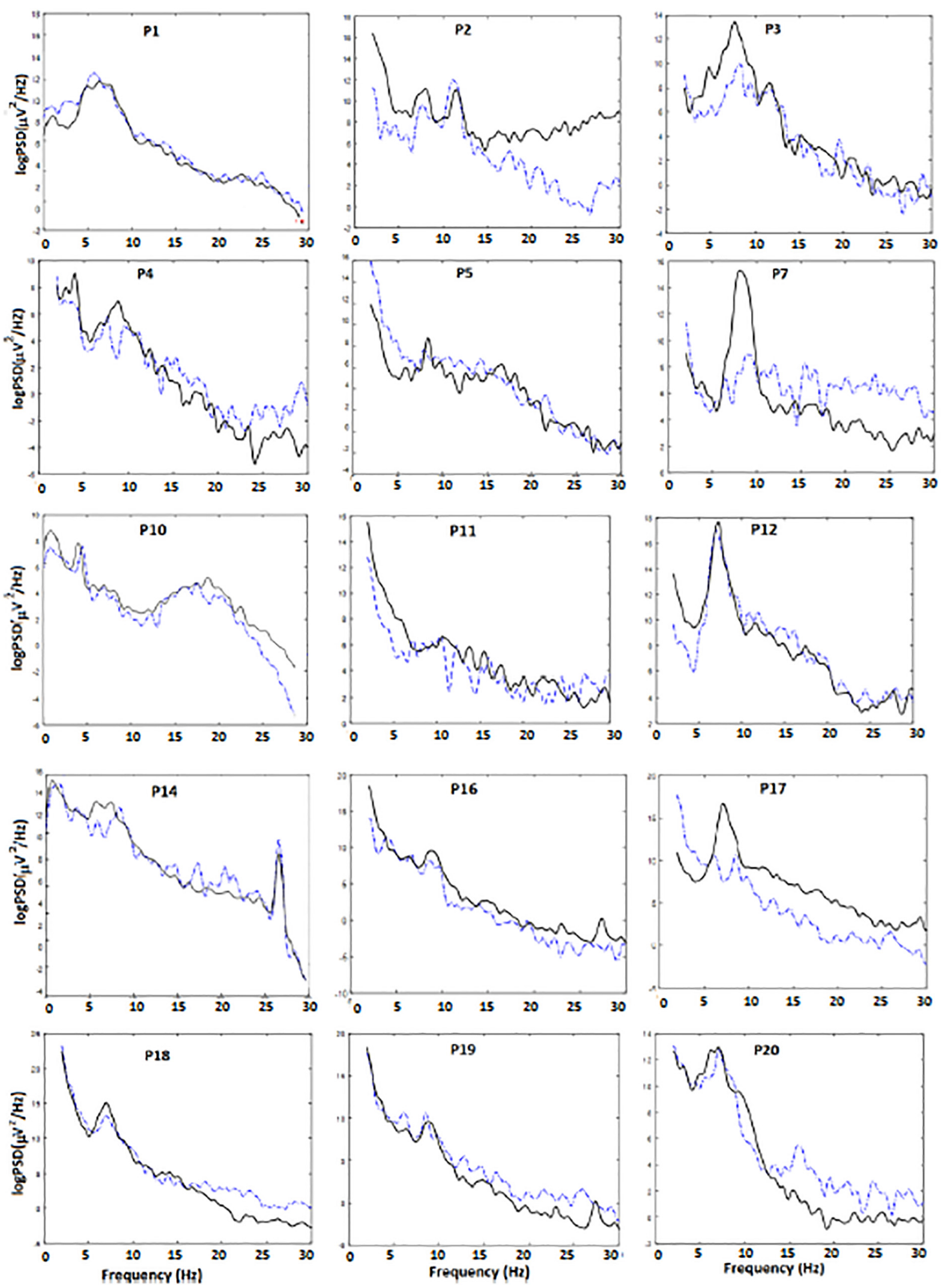

FIGURE 5 | Power spectrum density during baseline (dashed line) and during NFB (solid line) for each single participant. 
TABLE 4 | Pain intensity before and after NF training (median, first and third quartile Q1, Q3).

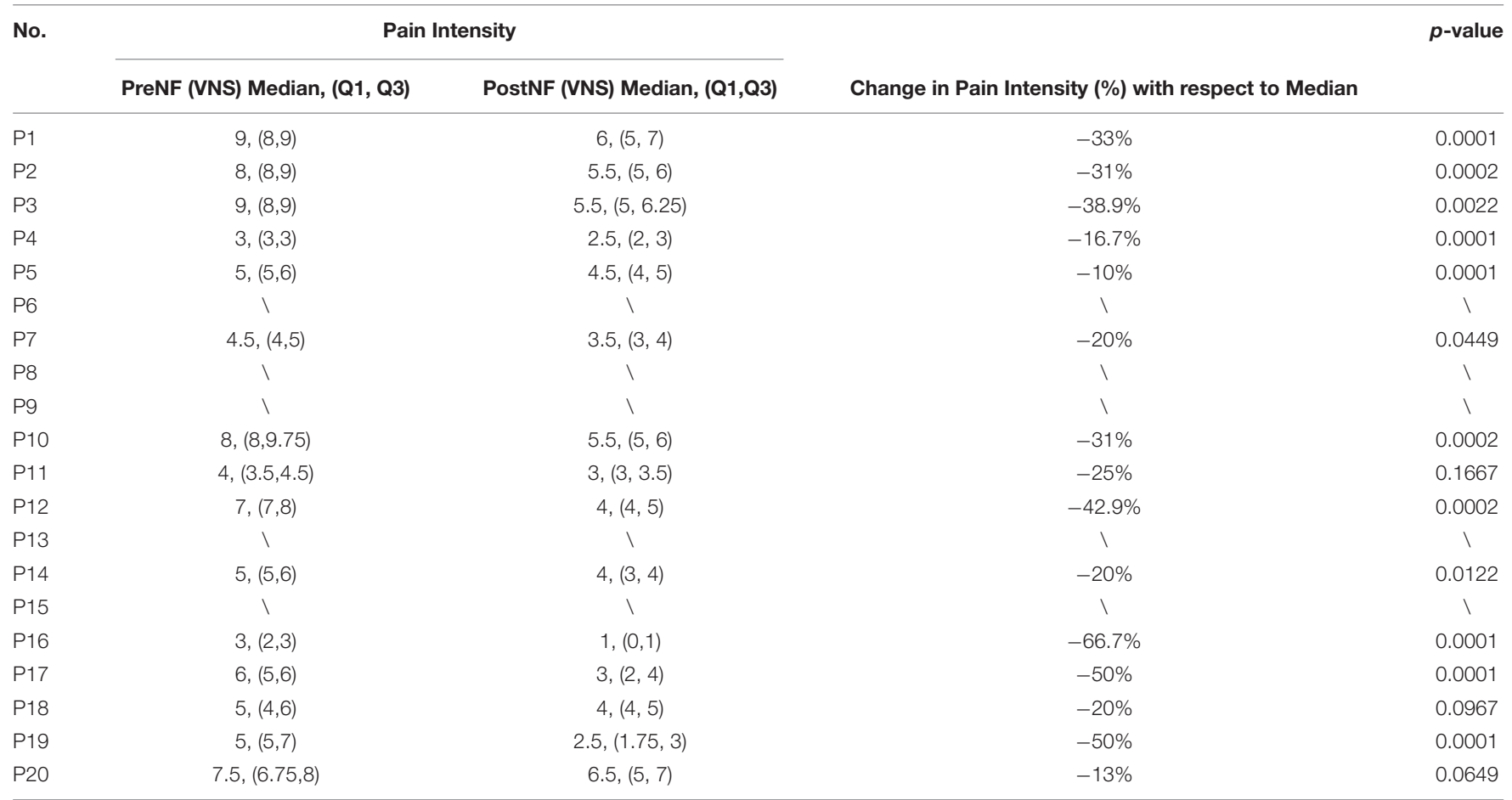

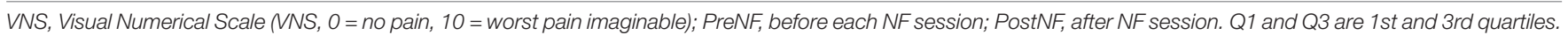

might not be a state with a maximum alpha band power. Baseline preNFB alpha power varies due to the circadian rhythm and was not possible to assess because participants did not necessarily always practice NFB at the same time of the day.

We analyzed how the baseline pain intensity changes over time. There was not a clear trend of Pre NFB pain level decrease, though in some patients relief of pain during NFB was almost compete (Figures 6B,C). This could be attributed to a fact that participants did not practice NFB at regular intervals but only when their pain level increased to the point that they needed pain relief. With that respect some participants consistently practiced NFB when their pain would reach the same level and achied about the same pain relief (Figure 6A) while in the others the pre and post NFB pain level varied, frequently reaching very low level (Figures 6B,C).

\section{A Relationship Between the Modulation of EEG Activity and the Intensity of Pain}

We analyzed the relationship between a significant reduction in pain and a significant upregulation of the alpha power. There were four possible scenarios

(a) Participants significantly increased their individual alpha power and achieved a statistically significant reduction in pain.

(b) Participants significantly increased their alpha power but did not achieve a statistically significant reduction in pain.

(c) Participants did not significantly increase their alpha power but did achieve a statistically significant reduction in pain. (d) Participants did not significantly increase their alpha power and did not achieve a statistically significant reduction in pain.

We showed that eight participants who significantly increased their alpha power also achieved a significant reduction in pain (P3, P4, P5, P7, P10, P12, P14, and P17), while four who did not achieve a significant increase in alpha power also achieved a significant reduction of pain (P1, P2, P16, and P19). However, three of these four had a significant reduction in beta or both theta and beta band power.

Furthermore two participants who achieved a significant increase in the alpha band power did not achieve a significant reduction of pain. One of these (P11) achieved a significant reduction in the beta band power while the other (P20) achieved a significant increase in beta power. Finally one participant who did not significantly increase the alpha band power did not significantly reduce pain (P18), this participant significantly reduced the theta band power.

\section{Relationship Between Pain and Demographic Data}

Our analysis confirmed results from the literature regarding the weak relationship between the intensity of pain and the participants' demographic data (Siddall et al., 2003). Spearman non- parametric rank sum test was applied. There was no significant correlation between the level of pain and the level of injury ( $p=0.6949, r=0.0935)$. The level of pain was not significantly correlated with the time since injury though 
A

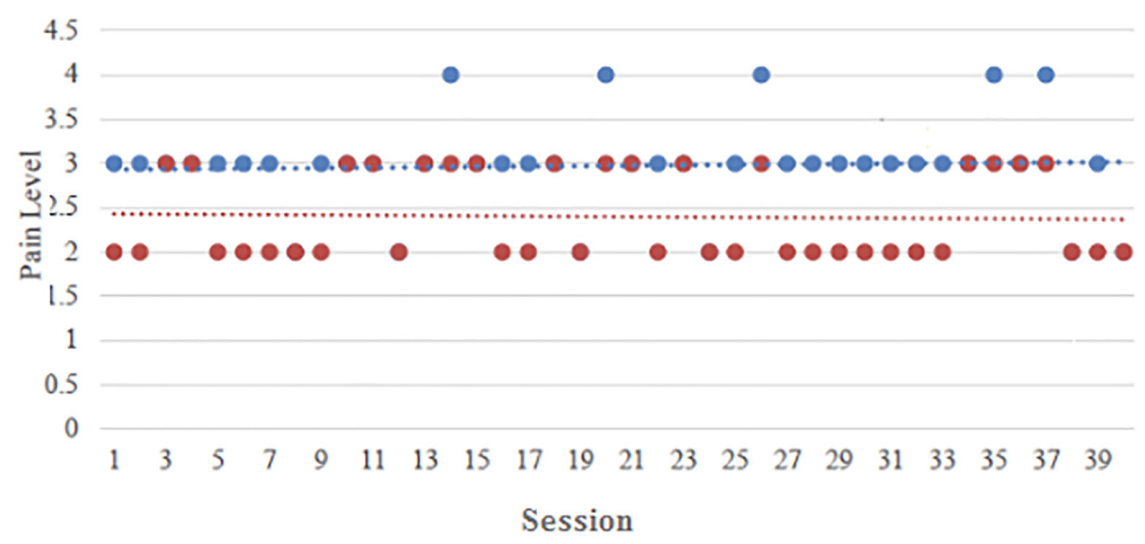

B

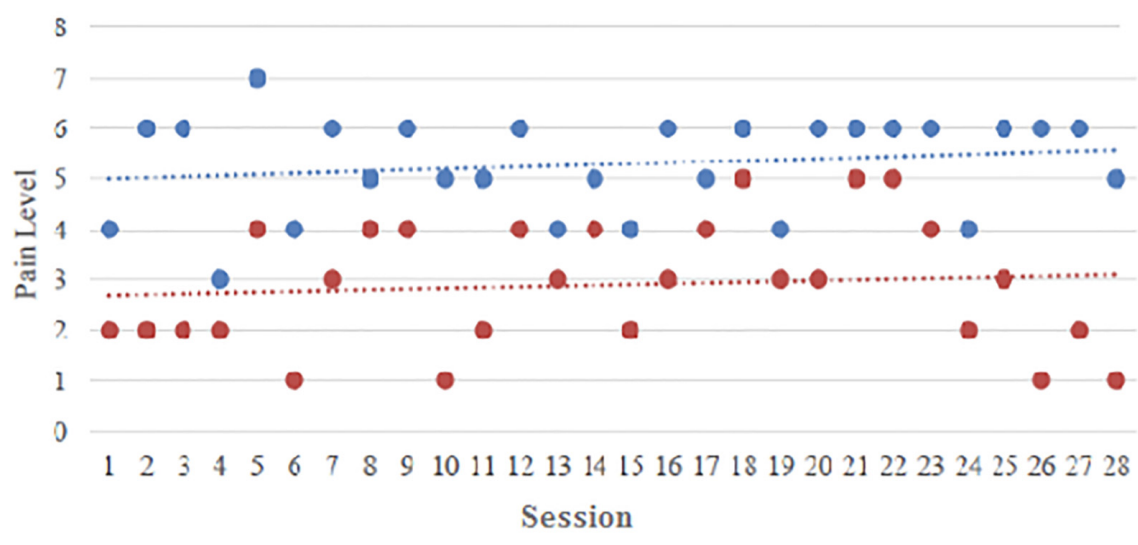

C

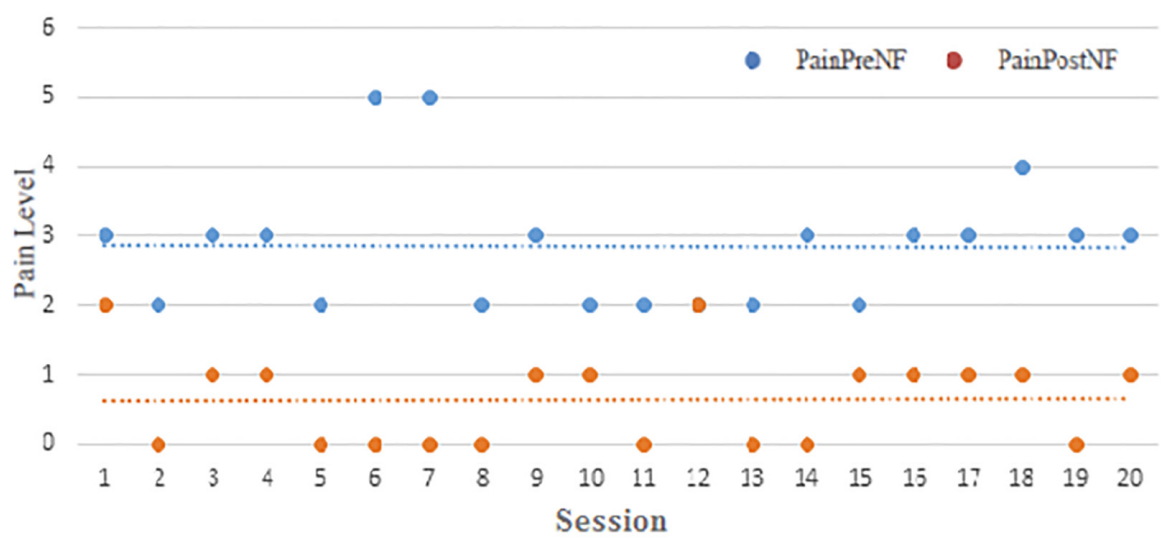

FIGURE 6 | Pain intensity (Visual Numerical Scale) before (blue dots) and after (red dots) NFB over all training sessions for representative participants (A) P4, (B) P17, and (C) P16.

the $p$-value was close to the significance level $(p=0.0631$, $r=0.4231$ ) indicating that pain might get worse over time. No significant correlation was found between the level of pain and the reduction of pain on the VNS $(p=0.81$, $r=0.65)$, the dominant baseline power alpha frequency and the reduction in pain during $\operatorname{NFB}(p=0.9703, r=-0.0105)$, the initial level of pain and the dominant alpha frequency
( $p=0.4522, r=-0.2101)$, and time since injury and the reduction in pain during $\mathrm{NFB}(p=0.9701, r=0.1010)$. Likewise, there was no significant difference in the initial level of pain between walkers (ASIA D) and non-walkers (ASIA A and B) $(p=0.7528)$, nor between participants with incomplete (ASIA B, C, and D) and complete (ASIA A) injury $(p=0.6242)$. 
There was no clear correlation between the duration of the study in weeks and the reduction of pain $(p=0.796, R=0.094)$ and the total number of NFB sessions and reduction of pain ( $p=0.299, R=0.287$ ). This may indicate that NFB may contribute to reduction in pain to a certain level, but that further implementation may only keep this pain level. It also has to be acknowledged that not every NFB session is successful as it depends on the participant's mental state (Demos, 2005).

\section{Incidental Findings and Side Effects}

Similar to our previous study, most participants reported a tingling sensations staring in their toes, or in case of tetraplegics also at the tip of their fingers. One participant that initially had CNP in the form of an excessive cold sensation, reported a sensation of pleasant warmth during NFB; the sensation of a pleasant warmth was also reported by the majority of participants whose pain was experienced as a burning sensation. Three participants reported successful transfer learning, i.e., applying NFB mental strategy without a device (it is possible that more participants had this ability but we did not systematically explored it). One participant said that wearing an audio headset at work produced similar sensation on his head which additionally helped him to imagine doing NFB. Transfer learning is an inherent property of successful NFB independent on a specific application (Sherlin et al., 2011) that might affected regularity of NFB practice with the device. It may be considered as the main advantage of NFB over tDCS or rTCS because it allows a person to apply a NFB strategy even without a device. Experience from other NFB studies (Sherlin et al., 2011), however, show that using NFB device at least once a week is necessary in order to keep the transfer learning ability.

Two participants with limited walking ability due to clonic spasm were spasm free immediately after 20-30 min of training, demonstrated in our laboratory. The spasm subsided for the rest of the day following NFB. Another participants who could walk with poor prioprioception reported returned sensation in his foot sole to the extent that he could feel whether the surface on which he walked was slippery or rough. He also demonstrated that, due to improved proprioception, he could stand still with his eyes closed, that is a challenging task for people with poor proprioception. Participants also occasionally mentioned improved sleep but it was hard to determine whether it was primarily a better nights sleep or better sleep quality due to reduced pain.

Negative side effects were occasional headaches, but it was unclear whether this was due to increased concentration or due to the NFB protocol. Another negative side effect was occasional hypersensitivity in the soles of the feet in the participant who regained proprioception, this was managed by reducing the duration of NFB.

We did not systematically examine participants' mental strategy but on their check-up visit we asked what were they thinking of during NFB. They all mentioned evoking some happy episodic memory, such as thinking of a favorite activity, an episode with a favorite family member or a favorite holiday (e.g., imaging laying on a beach). This might be an indication that they activated default mode network (Sestieri et al., 2011).

\section{DISCUSSION}

With the advent of wearable consumer grade wearable EEG headsets, this technology become available to individual users. Thus neurofeedback becomes competitive with direct current stimulation technique, which has also been used for pain reduction, and can be purchased over the Internet. This prompted us to organize this feasibility participant self-managed NFB study. We demonstrate that after up to 4 training sessions at hospital, people naïve to EEG technology could learn how to use NFB on their own at home, reporting reduction in pain.

The most significant result of this study is the evidence that NFB is based on upregulation of participant specific rather than fixed frequency bands, which are lower than in healthy individuals. We show PSD modulation for each single participant, advancing our understanding of variation in neuromodulation strategies. Our previous study in a controlled hospital environment indicated a relationship between NFB and changes in pain related areas of the cortex (Hassan et al., 2015; Hasan et al., 2016). The NFB protocol, however, had three parameters and the number of participants was too small to establish which of these are most relevant for the reduction in pain. In this study we showed that nine out of fifteen participants achieved a significant modulation of their individual alpha band and that eight of those who achieved significant modulation of the alpha band also achieved a statistically significant reduction in pain. A study by Sarnthein et al. (2006) showed that patients who underwent central lateral thalamoctomy experienced immediate relief of CNP while their alpha peak frequency remained low 3 months post-surgery. Only 12 month post-surgery the frequency of the dominant alpha peak shifted toward higher frequencies (Sarnthein et al., 2006). Schulman et al. (2005) also showed that the dominant alpha peak shifted toward higher frequencies (energy in 9$11 \mathrm{~Hz}$ band increased) only in patients who reported 50\% reduction of pain as a result of spinal cord stimulation. They did not report how long patients were pain free. Our study lasted on average under 3 months, so it is possible that prolonged NFB practice would result in alpha peak shift, under assumption that NFB would cause long term top down thalamic depolarization.

Although theta and beta band have clear relation to thalamocortical dysrhythmia, their relation to the reduction in pain through NFB was less clear than for the alpha band. One of the reasons might be that the bar representing the alpha power was placed in the middle of the GUI and was largest so participants might have perceived it as the most relevant. The other reason might be that the theta and beta bands overlap with eye movement artifact and muscular activity frequency range and could be reduced by minimizing these artifacts. A commercially available NFB software (e.g., NeXus, MindMedia) typically observe one frequency band and monitors the theta and higher beta band as a means of reducing eye movement and muscular artifacts rather than a proper NFB training. They typically present the central (e.g., $8-12 \mathrm{~Hz}, 12-15 \mathrm{~Hz}$ ) band with the largest bar as it reliably presents EEG activity without the artifacts and is most relevant for the NFB protocol. On the other hand, minimizing the 
theta and beta activity also minimized the eye movement (theta) and muscular artifacts (beta).

We showed that 8 out of 15 participants achieved a clinically significant (larger than 30\%) reduction in pain. We did not specifically check for how long the pain subsided, but judging by the frequency of use, the effect lasted from 1 to 3 days. This is comparable to the effect of medication and of other neurostimulation techniques (O'Connell et al., 2018). CNP is a chronic condition, and although NFB has a potential to target EEG features and to presumably restore the default mode network, the cause of CNP is the injury of the spinal cord which cannot be treated.

The main signatures of CNP are increased theta activity and reduced dominant alpha frequency (Sarnthein et al., 2006; Boord et al., 2008; Vuckovic et al., 2014). Reduced alpha activity has also been related to chronic pain in general (Camfferman et al., 2017). More recently we identified reduced alpha activity and reduced dominant alpha frequency as early, predictive markers of CNP (Vuckovic et al., 2018b). In the current study we show that individual alpha band in most participants has shifted toward the theta band frequency range. We also showed that none of the participants who reported reduced pain had increased their individual alpha peaks, which stayed around $8 \mathrm{~Hz}$. From this we may conclude that the level of alpha synchronization, rather than its peak frequency is related to the cause of pain. Reduced dominant frequency has been also reported in people with SCI who do not suffer from CNP (Tran et al., 2004). Increased alpha power in people with SCI related CNP, reported in some previous studies (Jensen et al., 2013b) may be at least partially related to the fact that researchers were looking in too high frequency range.

Peak alpha band frequency varies among healthy individuals and is age dependent (Niedermeyer, 1999). From that reason, some NFB studies are based on individual frequency bands with respect to the individual peak alpha frequency $\alpha_{\mathrm{p}}$ (Quaedflieg et al., 2016; Lavy et al., 2019).

Increasing the alpha power, effectively means increasing the level of synchronization, i.e., bringing the sensory-motor area to an inactive state. Several EEG and fMRI studies indicated that CNP in the SCI population is related to the overactive sensorymotor cortex (Sarnthein et al., 2006; Gustin et al., 2010) and we also showed that prolonged NFB practice relates to decreased event related desynchronization during imagined movements of both painful and non-painful limbs (Hasan et al., 2016).

There are several limitations of this study. First of all we did not have a control group, as this would have been difficult and potentially unethical to organize in a self-managed study taking place over several months and requiring lots of participants' engagement.

The role of placebo in pain treatments should not be underestimated (Cragg et al., 2016), as even the randomized controlled trial with one of the most widely used medication for CNP, pregabalin, showed that while about half of the participants taking gabapentin experienced a moderate reduction in pain $(30 \%)$ and at the same time, one third of people taking placebo also experienced a moderate reduction in pain (Wiffen et al., 2017). The placebo effect might be even higher in NFB due to the fact that participants were given active "control" over their pain.
We could only show that $2 / 3$ of people who actively regulated their alpha brain waves also achieved a significant reduction in pain. On the other hand, due to "transfer learning," i.e., the ability to reduce pain by brining themselves into "neurofeedback" state even without using the device it would be hard to test for placebo in people who are experienced in NFB.

Although NFB is a popular technique for the treatment of various neurological problems, there are few published pain related randomized controlled NFB studies. A randomized controlled studies for the treatment of fibromyalgia, suggested a protocol that upregulated the $12-15 \mathrm{~Hz}$ band and downregulated the theta and higher beta $(22-30 \mathrm{~Hz}$ ) band from $\mathrm{Cz}$ (Caro and Winter, 2011). Similar to our study they selected the motor cortex and had a most relevant frequency band to upregulate and two additional to downregulate. They reported a reduction in pain of $39 \%$ but did not provide any EEG evidence that participants successfully modulated their brain waves. Kayiran et al. (2010) performed a randomized control trial to compare the efficacy of NFB with the efficacy of medication for the treatment of fibromyalgia, using a similar protocol as Caro and Winter (2011). They claimed that NFB that increases a $12-15 \mathrm{~Hz}$ band power appears to facilitate a thalamic inhibitory mechanisms. They reported that the NFB group achieved larger benefits than the control group in terms of reduction in pain and other parameters related to sleep, depression and quality of life. They provided results averaged over all 18 participants about changes in the theta, alpha and beta power over different weeks. The only group who looked at the effectiveness of NFB for the treatment of CNP tested three different protocols (none being the same as ours) over four NFB sessions each and found modest, clinically nonsignificant reduction in pain (Jensen et al., 2013a). The protocols were based on increasing alpha or $10-15 \mathrm{~Hz}$ while decreasing beta or theta and beta bands from several different locations. They reported that this effect persisted at 3 months follow up. However, they later suggested that NFB may be a useful technique to enhance the effect of hypnotic analgesia (Jensen et al., 2016).

Motor imagery (MI) is a self-induced neuromodulation technique based on a verbalized strategy used in SCI both as a therapy and method to reduce CNP (Moseley, 2007). Similarity between MI and our NFB protocol is that both modulate the alpha/sensory-motor rhythm. MI results in the suppression of SMR (movement event related desynchronisation) (Pfurtscheller and Lopes da Silva, 1999) while our protocol rewards the increase of SMR (synchronization). Gustin et al. (2010) showed that prolonged MI in people with CNP due to SCI increases pain. With that respect, one might expect that the opposite, i.e., increase of SMR would reduce CNP as shown in our study.

On the contrary, Moseley (2007) showed that motor imagery combined with a visual illusion reduces neuropathic pain in people with SCI. However, Moseley recruited mostly people with at level pain while in Gustin et al. (2010) participants had pain had under the level of injury CNP. In a case report, Yoshida et al. (2016) also showed that motor imagery training to increase SRM resulted in reduced neuropathic pain in a single participant with SCI who received 4 months of training. A systematic review of application of MI in SCI shows that MI has rehabilitation benefits but that effect on pain is inconclusive (Aikat and Dua, 2016). 
The other limitation of our study is that the total duration and the total number of NFB sessions varied among participants. However, as one of the primary goals was to observe the self-managed usage pattern, this provided us with valuable information about frequency of use of NFB which for most participants was about three times a week for 20-30 min.

In this study most participants were taking drugs, such as antidepressants or anticonvulsants, which in large doses (more than normally prescribed) might increase the frequency content in the theta band (Bauer and Bauer, 2005; Wauquier, 2005). To the best of our knowledge all participants received medications in doses prescribed by a medical specialist.

Finally because it was necessary to reduce the setup time, EEG was recorded from one electrode only, which was sufficient to understand the neuromodulation strategy but did not allow for the exploration of the effect of NFB on wider cortical structures.

A randomized controlled trial with a modified NFB protocol, focused on participant individual alpha band should be performed to confirm the efficiency of the therapy. The analysis of non-oscillatory, scale-free cortical activity (e.g., fractals, long range temporal correlations (Dimitriadis and Linden, 2016; Kesiæ and Spasiæ, 2016) should confirm whether NFB bring the neural system in the state of self-organizing criticality Hernandez-Urbina and Michael Herrmann, 2016) which is considered a state of homeostatic set-point (Ros et al., 2014). A study in controlled conditions would also provide an opportunity to regularly test the mental states at the time of NFB (STAI form Y1-Y2 (Spielberger et al., 1983), BDI-II (Beck et al., 1996) and workload (NTLI NASA, 1986) as it might affect both participants ability to modulate their brain waves and to perceive the benefit of the therapy.

In this study all participants used the same protocol and the same electrode location, irrespective of the location of pain. In our previous study (Hassan et al., 2015) we showed that C3 was also effective in reducing pain but that it occasionally evoked spasms. A Cochrane review of rTMS and rDCS studies also showed that studies that targeted the motor cortex do not necessarily stimulate in a somatotopic manner with respect to the location of perceived pain. We believe taht the underlying mechanism is common in rTMS, tDCS, and NFB. CNP is seen by some researchers as a disrupted homeostatic state, in particular disrupted thermosensory inhibitory process (Craig, 2002). Thus neuromodulatory techniques for treatment of CNP maybe seen as therapies that tune the cortical activity toward a homeostatic setpoint. The main advantages of NFB over the other two modalities are that it potentially has less side effects as it does not apply an external electrical or magnetic stimulation, does not require an additional device and most importantly provides to participants an external locus of control and empowers them to actively contribute toward the reduction in pain. Hypnosis is another neuromodulatory technique which is believed to have a similar neuromodulatory mechanism to NFB, rTMS, and tDCS (Jensen et al., 2016).

Neurofeedback practitioners often set a variable NFB threshold which self-adjusts based on the last several minutes of
NFB with the intention of adjusting the difficulty level for the participant's performance. We used a fixed threshold in this study as it would have been difficult to perform quantitative analysis based on a variable threshold and also because variable threshold could lead to training in the opposite direction of the desired one (Ros et al., 2014).

Self-reported neuromodulation techniques through NFB included some pleasant episodic memories which indicate possible involvement of the default mode network (Sestieri et al., 2011). Our collaborators who used the same NFB equipment and protocol as our group tested the NFB mental strategy in ten participants with SCI and CNP (Anil et al., 2019). They showed that a mental state (e.g., attentiveness) rather than a mental strategy (e.g., imagination), was associated with neuromodulation success. They also showed that people who were unsuccessful in self-regulating their brainwaves also could not differentiate between successful and unsuccessful mental strategies.

In conclusion NFB may provide a clinically significant reduction of pain that lasts up to several days and people experienced with the technique may apply it even without the feedback. During NBF which results in reduction of CNP, people modulate their specific rather than fixed frequency bands. People with CNP can use NFB on their own without help of a trained professional. NFB does not provide cure for CNP and similar to pharmacological treatments would require long term use.

\section{DATA AVAILABILITY}

The datasets generated for this study are available on request to the corresponding author.

\section{AUTHOR CONTRIBUTIONS}

AV designed the study, contributed to the data collection and analysis, and wrote the manuscript. MKHA collected and analyzed the data. MF and MP selected the participants and contributed to the data interpretation, and wrote the manuscript. $\mathrm{CM}$ contributed to the data analysis and wrote the manuscript.

\section{FUNDING}

This study has been funded by the Inspire Foundation United Kingdom, EPSCR DTG EP/N509668/1, and by the Higher Committee for Education Development, Iraq.

\section{ACKNOWLEDGMENTS}

We would like to thank all the participants with SCI who took part in our study. 


\section{REFERENCES}

Aikat, D., and Dua, V. (2016). Mental imagery in spinal cord injury: a systematic review. J Spine 5:310.

Al-Taleb, M. K. H. (2018). Development and Clinical Testing of Home-based Braincomputer Interfaces for Neurofeedback and for Rehabilitation. Ph. D thesis, University of Glasgow, Scotland.

American Clinical Neurophysiology Society [ACNS] (2006). Guideline 5: Guidelines for standard electrode position nomenclature. J. Clin. Neurophysiol. 23, 107-110. doi: 10.1097/00004691-200604000-00006

Andersen, G., Vestergaard, K., Ingeman-Nielsen, M., and Jensen, T. S. (1995). Incidence of central post-stroke pain. Pain 61, 187-193. doi: 10.1016/03043959(94)00144-4

Anil, K., Simpson, D., Demain, S., Vuckovic, A., and Burridge, J. (2019). "Neurofeedback for central neuropathic pain treatment: mental strategies used for successful," in Proceedings of the Neuromodulation Accepted for the 3rd International Congress on NeuroRehabilitation and Neural Repair, Maastricht.

Bakonja, M., and Rowbotham, M. C. (2006). "Pharmacological therapy for neuropathic pain," in Wall and Melzack's Textbook of Pain, eds S. B. McMahom and M. Koltzenburg (Philadelphia, PA: Elsevier), 1075-1083. doi: 10.1016/b0443-07287-6/50073-4

Baliki, M. N., Geha, P. Y., Apkarian, A. V., and Chialvo, D. R. (2008). Beyond feeling: chronic pain hurts the brain, disrupting the default-mode network dynamics. J. Neurosci. 28, 1398-1403. doi: 10.1523/JNEUROSCI.4123-07.2008

Bauer, G., and Bauer, R. (2005). "EEG, drug effects and central neural system poisoning," in E. Electroencephalography, Basic Principle, Clinical Applications and Related Fields, Niedermeyer, ed. L. da Silva (Philadelphia, PA: Lippincott Williams \& Wilkins), 701-723.

Beck, A. T., Steer, R. A., and Brown, G. K. (1996). Manual for the Beck Depression Inventory-II. San Antonio, TX: Psychological Corporation.

Beiske, A. G., Loge, J. H., Rønningen, A., and Svensson, E. (2009). Pain in Parkinson's disease: prevalence and characteristics. Pain 141, 173-177. doi: 10.1016/j.pain.2008.12.004

Boord, P., Siddall, P. J., Tran, Y., Herbert, D., Middleton, J., and Craig, A. (2008). Electroencephalographic slowing and reduced reactivity in neuropathic pain following spinal cord injury. Spinal Cord 46, 118-123. doi: 10.1038/sj.sc. 3102077

Bouhassira, D., Attal, N., Fermanian, J., Alchaar, H., Gautron, M., Masquelier, E., et al. (2004). Development and validation of the neuropathic pain symptom inventory. Pain 108, 248-257. doi: 10.1016/j.pain.2003.12.024

Bryce, T. N., Biering-Sørensen, F., Finnerup, N. B., Cardenas, D. D., Defrin, R., Lundeberg, T., et al. (2012). International spinal cord injury pain classification: Part I. Background and description. Spinal Cord 50, 413-417. doi: 10.1038/sc. 2011.156

Camfferman, D., Moseley, G. L., Gertz, K., Pettet, M. W., and Jensen, M. P. (2017). Waking EEG cortical markers of chronic pain and sleepiness. Pain Med. 18, 1921-1931. doi: 10.1093/pm/pnw294

Caro, X. J., and Winter, E. F. (2011). EEG biofeedback treatment improves certain attention and somatic symptoms in fibromyalgia: a pilot study. Appl. Psychophysiol. Biofeedback 36, 193-200. doi: 10.1007/s10484-0119159-9

Colloca, L., Ludman, T., Bouhassira, D., Baron, R., Dickenson, A. H., Yarnitsky, D., et al. (2017). Neuropathic pain. Nat. Rev. Dis. Primers. 16:17002.

Cragg, J. J., Warner, F. M., Finnerup, N. B., Jensen, M. P., Mercier, C., Richards, J. S., et al. (2016). Meta-analysis of placebo responses in central neuropathic pain: impact of subject, study, and pain characteristics. Pain 157, 530-540. doi: $10.1097 /$ j.pain.0000000000000431

Craig, A. D. (2002). How do you feel? Interoception: the sense of the physiological condition of the body. Nat. Rev. Neurosci. 3, 655-666. doi: 10.1038/nrn894

Demos, J. N. (2005). Getting Started with Neurofeedback. New York, NY: W W Norton \& Co.

Dickenson, A. H., and Ghandehari, J. (2007). Anti-convulsants and antidepressants. Handb. Exp. Pharmacol. 177, 145-177. doi: 10.1007/978-3-54033823-9_6

Dimitriadis, S. I., and Linden, D. (2016). Modulation of brain criticality via suppression of EEG long-range temporal correlations (LRTC) in a closed-loop neurofeedback stimulation. Clin. Neurophysiol. 127, 2882-2889. doi: 10.1016/j. clinph.2016.04.028
Finnerup, N. B. (2013). Pain in participants with spinal cord injury. Pain 154(Suppl. 1), S71-S76.

Finnerup, N. B., Haroutounian, S., Baron, R., Dworkin, R. H., Gilron, I., Haanpaa, M., et al. (2018). Neuropathic pain clinical trials: factors associated with decreases in estimated drug efficacy. Pain 159, 2339-2346. doi: 10.1097/j.pain. 0000000000001340

Finnerup, N. B., Norrbrink, C., Trok, K., Piehl, F., Johannesen, I. L., Sørensen, J. C., et al. (2014). Phenotypes and predictors of pain following traumatic spinal cord injury: a prospective study. J. Pain 15, 40-48. doi: 10.1016/j.jpain.2013.09.008

Gov.UK (2019). Pregabalin (Lyrica), gabapentin (Neurontin) and Risk of Abuse and Dependence: New Scheduling Requirements. Available at: https://www.gov. uk/drug-safety-update/pregabalin-lyrica-gabapentin-neurontin-and-risk-ofabuse-and-dependence-new-scheduling-requirements-from-1-april) accessed (accessed April 27, 2019).

Gruzelier, J. H. (2014). EEG-neurofeedback for optimising performance. I: a review of cognitive and affective outcome in healthy participants. Neurosci. Biobehav. Rev. 44, 124-141. doi: 10.1016/j.neubiorev.2013.09.015

Gustin, S. M., Wrigley, P. J., Henderson, L. A., and Siddall, P. J. (2010). Brain circuitry underlying pain in response to imagined movement in people with spinal cord injury. Pain 148, 438-445. doi: 10.1016/j.pain.2009.12.001

Haanpää, M., Attal, N., Backonja, M., Baron, R., Bennett, M., Bouhassira, D., et al. (2011). NeuPSIG guidelines on neuropathic pain assessment. Pain 152, 14-27. doi: 10.1016/j.pain.2010.07.031

Hasan, M. A., Fraser, M., Conway, B. A., Allan, D. B., and Vuèković, A. (2016). Reversed cortical over-activity during movement imagination following neurofeedback treatment for central neuropathic pain. Clin. Neurophysiol. 127, 3118-3127. doi: 10.1016/j.clinph.2016.06.012

Hassan, M. A., Fraser, M., Conway, B. A., Allan, D. B., and Vuckovic, A. (2015). The mechanism of neurofeedback training for treatment of central neuropathic pain in paraplegia: a.pilot study. BMC Neurol. 13:200. doi: 10.1186/s12883-0150445-7

Hernandez-Urbina, V., and Michael Herrmann, J. (2016). Neuronal avalanches in complex networks. Cogent Phys. 3:1150408.

Hetkamp, M., Bender, J., Rheindorf, N., Kowalski, A., Lindner, M., Knispel, S., et al. (2019). A systematic review of the effect of neurofeedback in cancer patients. Integr. Cancer Ther. 18:1534735419832361. doi: 10.1177/1534735419832361

Jensen, M. P., Gertz, K. J., Kupper, A. E., Braden, A. L., Howe, J. D., Hakimian, S., et al. (2013a). Steps toward developing an EEG biofeedback treatment for chronic pain. Appl. Psychophysiol. Biofeedback 38, 101-108. doi: 10.1007/ s10484-013-9214-9

Jensen, M. P., Sherlin, L. H., Gertz, K. J., Braden, A. L., Kupper, A. E., Gianas, A., et al. (2013b). Brain EEG activity correlates of chronic pain in persons with spinal cord injury: clinical implications. Spinal Cord 51, 55-58. doi: 10.1038/ sc. 2012.84

Jensen, M. P., Gianas, A., George, H. R., Sherlin, L. H., Kraft, G. H., and Ehde, D. M. (2016). Use of neurofeedback to enhance response to hypnotic analgesia in individuals with multiple sclerosis. Int. J. Clin. Exp. Hypn. 64, 1-23. doi: 10.1080/00207144.2015.1099400

Jensen, M. P., Grierson, C., Tracy-Smith, V., Bacigalupi, S. C., and Othmer, S. (2007). Neurofeedback treatment for pain associated with complex regional pain syndrome type I. J. Neurother. 11, 45-53.

Kayiran, S., Dursun, E., Dursun, N., Ermutlu, N., and Karamursel, S. (2010). Neurofeedback intervention in fibromyalgia syndrome; a randomized, controlled, rater blind clinical trial. Appl. Psychophysiol. Biofeedback 35, 293 302. doi: 10.1007/s10484-010-9135-9

Kesiæ, S., and Spasiæ, S. Z. (2016). Application of Higuchi's fractal dimension from basic to clinical neurophysiology: a review. Comput. Methods Prog. Biomed. 133, 55-70. doi: 10.1016/j.cmpb.2016.05.014

Kirshblum, S. C., Burns, S. P., Biering-Sorensen, F., Donovan, W., Graves, D. E., Jha, A., et al. (2011). International standards for neurological classification of spinal cord injury (revised 2011). J. Spinal. Cord Med. 34, 535-546. doi: $10.1179 / 204577211 \times 13207446293695$

Lavy, Y., Dwolatzky, T., Kaplan, Z., Guez, J., and Todder, D. (2019). Neurofeedback improves memory and peak alpha frequency in individuals with mild cognitive impairment. Appl. Psychophysiol. Biofeedback 44, 41-49. doi: 10.1007/s10484018-9418-0

Llinas, R. R., Ribary, U., Jeanmond, D., Kronberg, E., and Mitra, P. P. (1999). Thalamocortical dysrhythmia: a neurological and neurophyhiatric syndrome 
characterized by magnetoencephalography. Proc. Natl. Acad. Sci. U.S.A. 96, 15222-15227. doi: 10.1073/pnas.96.26.15222

Mahnig, S., Landmann, G., Stockinger, L., and Opsommer, E. (2016). Pain assessment according to the international spinal cord injury pain classification in participants with spinal cord injury referred to a multidisciplinary pain center. Spinal Cord 54, 809-815. doi: 10.1038/sc.2015.219

Mann, R., Schaefer, C., Sadosky, A., Bergstrom, F., Baik, R., Parsons, B., et al. (2013). Burden of spinal cord injury-related neuropathic pain in the United States: retrospective chart review and cross-sectional survey. Spinal Cord 51, 564-570. doi: $10.1038 /$ sc.2013.34

Middleton, J., Tran, Y., and Craig, A. (2007). Relationship between quality of life and self-efficacy in persons with spina cord injuries. Arch. Phys. Med. Rehabil. 88, 1643-1648. doi: 10.1016/j.apmr.2007.09.001

Moseley, G. L. (2007). Using visual illusion to reduce at-level neuropathic pain in paraplegia. Pain 130, 294-298. doi: 10.1016/j.pain.2007.01.007

NASA (1986). NASA task load index. Hum. Ment. Workload 1:21.

Niedermeyer, E. (1999). "The Normal EEG of the Waking Man," in Electroencephalography: Basic Principles, Clinical Applications and Related Fields, eds E. Niedermeyer and F. Lopes da Silva (Baltimore, MD: Lippincott Williams \& Wilkins), 149-173.

O'Connell, N. E., Marston, L., Spencer, S., DeSouza, L. H., and Wand, B. M. (2018). Non-invasive brain stimulation techniques for chronic pain. Cochrane Database Syst. Rev. 3:CD008208. doi: 10.1002/14651858.CD008208.pub4

Osterberg, A., Boivie, J., and Thuomas, K. A. (2005). Central pain in multiple sclerosis-prevalence and clinical characteristics. Eur. J. Pain 9, 531-542.

Pfurtscheller, G., and Lopes da Silva, F. H. (1999). Event-related EEG/MEG synchronization and desynchronization: basic principles. Clin. Neurophysiol. 110, 1842-1857. doi: 10.1016/s1388-2457(99)00141-8

Pinheiro, E. S., de Queirós, F. C., Montoya, P., Santos, C. L., do Nascimento, M. A., Ito, C. H., et al. (2016). Electroencephalographic patterns in chronic pain: a systematic review of the literature. PLoS One 11:e0149085. doi: 10.1371/journal. pone. 0149085

Quaedflieg, C. W., Smulders, F. T., Meyer, T., Peeters, F., Merckelbach, H., and Smeets, T. (2016). The validity of individual frontal alpha asymmetry EEG neurofeedback. Soc. Cogn. Affect. Neurosci. 11, 33-43. doi: 10.1093/scan/nsv090

Ros, T., J Baars, B., Lanius, R. A., and Vuilleumier, P. (2014). Tuning pathological brain oscillations with neurofeedback: a systems neuroscience framework. Front. Hum. Neurosci. 8:1008. doi: 10.3389/fnhum.2014.01008

Sarnthein, J., Stern, J., Aufenberg, C., Rousson, V., and Jeanmonod, D. (2006). Increased EEG power and slowed dominant frequency in participants with neurogenic pain. Brain 129, 55-64. doi: 10.1093/brain/awh631

Schulman, J., Ramirez, R., Zonenshayn, M., Ribary, U., and Llinas, R. (2005). Thalamocortical dysrhythmia syndrome: MEG imaging of neuropathic pain. Thal. Rel. Syst. 3, 33-39. doi: 10.1016/j.pain.2010.02.023

Seifert, F., and Maihöfner, C. (2009). Central mechanisms of experimental and chronic neuropathic pain: findings from functional imaging studies. Cell Mol. Life Sci. 66, 375-390. doi: 10.1007/s00018-008-8428-0

Sestieri, C., Corbetta, M., Romani, G. L., and Shulman, G. L. (2011). Episodic memory retrieval, parietal cortex, and the default mode network: functional and topographic analyses. J. Neurosci. 31, 4407-4420. doi: 10.1523/JNEUROSCI. 3335-10.2011

Sherlin, L. H., Arns, M., Lubar, J., Heinrich, H., Kerson, C., Strehl, U., et al. (2011). Neurofeedback and basic learning theory: implications for research and practice. J. Neurother. 15, 292-304. doi: 10.1080/10874208.2011.623089

Siddall, P. J., McClelland, J. M., Rutkowski, S. B., and Cousins, M. J. (2003). A longitudinal study of the prevalence and characteristics of pain in the first 5 years following spinal cord injury. Pain 103, 249-257. doi: 10.1016/s03043959(02)00452-9

Sime, A. (2004). Case study of trigeminal neuralgia using neurofeedback and peripheral biofeedback. J. Neurother. 8, 59-71. doi: 10.1300/j184v08n01_05
Siniatchkin, M., Hierundar, A., Kropp, P., Kuhnert, R., Gerber, W.-D., and Stephani, U. (2000). Self-regulation of slow cortical potentials in children with migraine: an exploratory study. Appl. Psychophysiol. Biofeedback 25, 13-32.

Skinner, B. F. (1948). 'Superstition' in the pigeon. J. Exp. Psychol. 38, 168-172. doi: $10.1037 / \mathrm{h} 0055873$

Spielberger, C. D., Gorsuch, R. L., Lushene, R., Vagg, P. R., and Jacobs, G. A. (1983). Manual for the State-Trait Anxiety (Form Y1 - Y2). Palo Alto, CA: Consulting Psychologists Press, Inc.

Stern, J., Jeanmonod, D., and Sarnthein, J. (2006). Persistent EEG overactivation in the cortical pain matrix of neurogenic pain participants. Neuroimage 31, 721-731. doi: 10.1016/j.neuroimage.2005.12.042

Tan, G., Jensen, M. P., Thornby, J. I., and Shanti, B. F. (2004). Validation of the brief pain inventory for chronic nonmalignant pain. J. Pain 5, 133-137. doi: 10.1016/j.jpain.2003.12.005

Tran, Y., Boord, P., Middleton, J., and Craig, A. (2004). Levels of brain wave activity $(8-13 \mathrm{~Hz})$ in persons with spinal cord injury. Spinal Cord 42, 73-79. doi: $10.1038 /$ sj.sc.3101543

Vuckovic, A., Hasan, M. A., Fraser, M., Conway, B. A., Nasseroleslami, B., and Allan, D. B. (2014). Dynamic oscillatory signatures of central neuropathic pain in spinal cord injury. J. Pain 15, 645-655. doi: 10.1016/j.jpain.2014. 02.005

Vuckovic, A., Gallardo, V. J. F., Jarjees, M., Fraser, M., and Purcell, M. (2018a). Prediction of central neuropathic pain in spinal cord injury based on EEG classifier. Clin. Neurophysiol. 129, 1605-1617. doi: 10.1016/j.clinph.2018. 04.750

Vuckovic, A., Jajrees, M., Purcell, M., Berry, H., and Fraser, M. (2018b). Electroencephalographic predictors of neuropathic pain in subacute spinal cord injury. J Pain 19, 1256.e1-1256.e17. doi: 10.1016/j.jpain.2018.04.011

Walton, K. D., and Llinas, R. R. (2010). "Chapter 13: Central pain as thalamocrotical dysrhythmia. A thalamic efference disconnection?," in Translational Pain Research: From Mouse to Man, eds L. Kruger and A. R. Light (Boca Raton, FL: CRC Press).

Wauquier, A. (2005). "EEG and neuropharmacology," in Electroencephalography, Basic Principle, Clinical Applications and Related Fields, eds E. Niedermeyer and L. da Silva (Philadelphia, PA: Lippincott Williams \& Wilkins), 689-700.

Wiffen, P. J., Derry, S., Bell, R., Rice, A. S. C., Tölle, T., Phillips, T., et al. (2017). Gabapentin for chronic neuropathic pain in adults. Cochrane Database Syst. Rev. 6:CD007938. doi: 10.1002/14651858.CD007938.pub4

Wood, G., and Kober, S. E. (2018). EEG neurofeedback is under strong control of psychosocial factors. Appl. Psychophysiol. Biofeedback 43, 293-300. doi: 10. 1007/s10484-018-9407-3

Wrigley, P. J., Press, S. R., Gustin, S. M., Macefield, V. G., Gandevia, S. C., Cousins, M. J., et al. (2009). Neuropathic pain and primary somatosensory cortex reorganization following spinal cord injury. Pain 141, 52-59. doi: 10. 1016/j.pain.2008.10.007

Yoshida, N., Hashimoto, Y., Shikota, M., and Ota, T. (2016). Relief of neuropathic pain after spinal cord injury by brain-computer interface training. Spinal Cord Ser. Cases. 27:16021. doi: 10.1038/scsandc.2016.21

Conflict of Interest Statement: The authors declare that the research was conducted in the absence of any commercial or financial relationships that could be construed as a potential conflict of interest.

Copyright $\odot 2019$ Vučković, Altaleb, Fraser, McGeady and Purcell. This is an openaccess article distributed under the terms of the Creative Commons Attribution License (CC BY). The use, distribution or reproduction in other forums is permitted, provided the original author(s) and the copyright owner(s) are credited and that the original publication in this journal is cited, in accordance with accepted academic practice. No use, distribution or reproduction is permitted which does not comply with these terms. 


\section{APPENDIX}

TABLE A1 | Average pain intensity of each single participants before and after NFB training.

\begin{tabular}{|c|c|c|c|c|}
\hline \multirow[b]{2}{*}{ No. } & \multicolumn{2}{|c|}{ Pain Intensity } & \multirow[b]{2}{*}{ Change in Pain Intensity (\%) } & \multirow[b]{2}{*}{$p$-value } \\
\hline & PreNF (VAS) mean \pm SD & PostNF (VAS) mean \pm SD & & \\
\hline 2 & $8.5 \pm 0.7$ & $5.5 \pm 0.7$ & $-35 \%$ & 0.001 \\
\hline 3 & $8.7 \pm 0.6$ & $5.3 \pm 0.6$ & $-38 \%$ & 0.002 \\
\hline 4 & $3.0 \pm 0.5$ & $2.4 \pm 0.5$ & $-18 \%$ & $0.5 e^{-5}$ \\
\hline 7 & $4.5 \pm 0.9$ & $3.5 \pm 0.9$ & $-22 \%$ & 0.001 \\
\hline 8 & - & - & - & - \\
\hline 9 & - & - & - & - \\
\hline 10 & $9.0 \pm 0.7$ & $5.5 \pm 1.8$ & $-38 \%$ & 0.04 \\
\hline 11 & $4.0 \pm 1.0$ & $3.3 \pm 0.6$ & $-15 \%$ & 0.08 \\
\hline 15 & - & - & - & - \\
\hline 16 & 2.85 & $0.65 \pm 1.3$ & $75 \%$ & $0.5 e^{-5}$ \\
\hline 17 & $5.4 \pm 1.0$ & $2.9 \pm 1.3$ & $-46 \%$ & $0.5 e^{-11}$ \\
\hline 18 & $5.3 \pm 2.1$ & $4.7 \pm 2.3$ & $-15 \%$ & 0.09 \\
\hline 19 & $5.8 \pm 1.7$ & $2.3 \pm 1.7$ & $-65 \%$ & 0.0006 \\
\hline 20 & $7.3 \pm 0.6$ & $6.7 \pm 0.6$ & $-9 \%$ & 0.09 \\
\hline
\end{tabular}

VAS: Visual Analogue Scale NAS, 0 = no pain, 10 = worst pain imaginable); PreNF: before each NF session; PostNF: after NF session. 


\section{Exposure in vivo Induced Changes in Neural Circuitry for Pain-Related Fear: A Longitudinal fMRI Study in Chronic Low Back Pain}

OPEN ACCESS

Edited by:

Hannah Madaleine Hobson,

University of Greenwich,

United Kingdom

Reviewed by:

Marcus Grueschow,

University of Zurich, Switzerland

Shinpei Yoshimura,

Otemon Gakuin University, Japan

Atsuo Yoshino,

Hiroshima University, Japan

*Correspondence:

Inge Timmers

itimmers@stanford.edu

Received: 30 May 2019

Accepted: 29 August 2019

Published: 17 September 2019

Citation:

Timmers I, de Jong JR,

Goossens $M$, Verbunt JA, Smeets RJ and Kaas AL (2019) Exposure in vivo Induced Changes in Neural Circuitry for Pain-Related Fear: A Longitudinal fMRI Study in Chronic Low Back Pain.

Front. Neurosci. 13:970

doi: 10.3389/fnins.2019.00970

\author{
Inge Timmers ${ }^{1,2,3 *}$, Jeroen R. de Jong ${ }^{1,4,5}$, Mariëlle Goossens ${ }^{1,6}$, Jeanine A. Verbunt ${ }^{1,4,5}$, \\ Rob J. Smeets ${ }^{1,7}$ and Amanda L. Kaas ${ }^{2}$
}

${ }^{1}$ Department of Rehabilitation Medicine, Maastricht University, Maastricht, Netherlands, ${ }^{2}$ Department of Cognitive Neuroscience, Maastricht University, Maastricht, Netherlands, ${ }^{3}$ Department of Anesthesiology, Perioperative, and Pain Medicine, Stanford University, Palo Alto, CA, United States, ${ }^{4}$ Department of Rehabilitation Medicine, Maastricht University Medical Center, Maastricht, Netherlands, ${ }^{5}$ Adelante Centre of Expertise in Rehabilitation and Audiology, Hoensbroek, Netherlands, ${ }^{6}$ Department of Clinical Psychological Science, Maastricht University, Maastricht, Netherlands, ${ }^{7} \mathrm{CIR}$ Revalidatie, Zwolle/Eindhoven, Netherlands

Exposure in vivo (EXP) is a cognitive-behavioral treatment aimed at reducing pain-related fear in chronic pain, and has proven successful in reducing pain-related disability in patients with chronic low back pain (cLBP). The current longitudinal study aimed to reveal the neural correlates of changes in pain-related fear as a result of EXP. Twentythree patients with CLBP were included in this study. Patients with CLBP underwent MRI scanning pre-treatment (pre-EXP), post-treatment (post-EXP), and 6 months after end of treatment (FU-EXP). Pain-free controls were scanned at two time points. In the scanner, participants were presented with pictures involving back-related movements, evoking pain-related fear in patients. Pre-treatment, functional MRI revealed increased activation in right posterior insula and increased deactivation in medial prefrontal cortex (mPFC) in patients compared to controls. Post-treatment, patients reported reduced fear and pre-EXP group differences were no longer present. Contrasting pre- to postand FU-EXP in patients revealed that stimulus-evoked neural responses changed in sensorimotor as well as cognitive/affective brain regions. Lastly, exploratory analyses revealed a tendency toward an association between changes in neural activation and changes in fear ratings, including the hippocampus and temporal lobe (pre- to post-EXP changes), and mPFC and posterior cingulate cortex (pre- to FU-EXP changes). Taken together, we show evidence that neural circuitry for pain-related fear is modulated by EXP, and that changes are associated with self-reported decreases in pain-related fear.

Keywords: chronic pain, exposure in vivo, neuroimaging, pain-related fear, rehabilitation, chronic low back pain

\section{INTRODUCTION}

While most of us experience acute low back pain at some point in our lives, some will develop chronic low back pain (cLBP), with persistent pain lasting more than 6 months. An estimated one in five adults is currently in chronic pain, with cLBP being the most common (Breivik et al., 2006) and the world's leading cause of disability (GBD 2015 Disease and Injury Incidence and Prevalence Collaborators, 2016; Hartvigsen et al., 2018). 
It is believed that maladaptive cognitions and emotional responses to pain are important factors for developing and maintaining chronic pain, as described by the fear avoidance model (Vlaeyen et al., 1995b, 2016). This model describes how, if immediate pain control is prioritized, pain-catastrophizing and pain-related fear may lead to pain-hypervigilance and avoidance behavior, and in turn increased functional disabilities. This then may amplify the pain experience and paradoxically increases pain-related fear, creating a vicious cycle. A subgroup of patients with CLBP indeed shows pain-related fears, including fear of movement and/or re-injury (Crombez et al., 1999; Vlaeyen and Crombez, 1999; Camacho-Soto et al., 2012; Thibodeau et al., 2013; Bunzli et al., 2015; Hartvigsen et al., 2018). In fact, painrelated fear is more closely linked to disability than pain intensity (Crombez et al., 1999; Zale et al., 2013).

To specifically target pain-related fears in clinical settings, Exposure in vivo (EXP) was developed. EXP is a cognitivebehavioral treatment based on experimental work showing that exposure to fearful activities and movements, rather than avoiding them, challenges catastrophic pain beliefs and can result in the extinction of fears and maladaptive responses (Vlaeyen et al., 1995a; Meulders and Vlaeyen, 2012). In EXP, movements and activities that are perceived as threatening and fearful are first identified using the pictorial tool The Photographic Series of Daily Activities (PHODA) (Leeuw et al., 2007). Then, the patient is repeatedly exposed to these feared movements and activities, while behavioral experiments are performed to challenge catastrophic expectations and interpretations regarding these movements, activities, and/or sensations. EXP has been applied as treatment for patients with chronic pain and elevated pain-related fear in a variety of settings and different pain conditions, including, but not limited to, non-specific cLBP. Ubiquitously, EXP has been successful in reducing pain-related fears and pain-related disability as compared to no treatment and at least as successful, if not more successful, in comparison to other treatments that are proven effective (Vlaeyen et al., 2001; Boersma et al., 2004; de Jong et al., 2005, 2008, 2012; Leeuw et al., 2008; Woods and Asmundson, 2008; den Hollander et al., 2016; Lalouni et al., 2016; Lopez-de-Uralde-Villanueva et al., 2016; Glombiewski et al., 2018).

It would be expected that EXP specifically impacts the neural circuitry involved in pain-related fear and fear extinction learning. Studies examining pain-related fear have identified altered neural responses in patients with cLBP to viewing and imagining activities/movements associated with pain (Taylor et al., 2015; Meier et al., 2016, 2017) - including increased recruitment of the insula, anterior cingulate cortex (ACC), amygdala, orbitofrontal cortex, striatum (i.e., regions involved in attentional/perceptual as well as affective/reappraisive aspects of pain), and altered crosstalk with the periaqueductal gray (PAG; involved in top-down pain modulation). For fear conditioning and extinction, experimental studies identified a core neural network, including the amygdala, insula, and ACC (Sehlmeyer et al., 2009; Fullana et al., 2016, 2018b). Only few imaging studies investigated fear learning and extinction in the context of pain (Kattoor et al., 2013; Labus et al., 2013; Icenhour et al., 2015), reporting altered neural responses in patients, including in the prefrontal cortex (PFC), ACC, insula, amygdala, hippocampus, PAG and thalamus. Further, results of clinical studies in chronic pain investigating treatment-induced functional brain changes show some overlap with neural changes related to pain-related fear and experimental fear extinction (e.g., implicating the amygdala, mPFC, and PAG) (Baliki et al., 2008; Becerra et al., 2014; Erpelding et al., 2014; Simons et al., 2014). The majority of treatment studies focused on intrinsic brain activity, i.e., in rest and without a specific task (Napadow et al., 2012; Harris et al., 2013; Bosma et al., 2018). The effects of EXP specifically have also only been investigated using resting-state fMRI (Zhu et al., 2018), showing that patients with post-traumatic stress disorder showed enhanced post-treatment resting-state functional connectivity between the amygdala, orbitofrontal cortex, hippocampus and the medial PFC. To date, there have been no studies investigating how (EXP) treatment modulates the circuitry underlying painrelated fear in chronic pain.

Therefore, the current longitudinal fMRI study tested the hypothesis that EXP acts upon the neural circuitry involved in pain-related fear, using a task designed to evoke pain-related fear. We compared patients with cLBP with pain-free volunteers preand post-EXP treatment; the cLBP group was also examined 6 months after end of treatment. We evaluated group differences and treatment effects in evoked brain activation. Also, more exploratively, we used (changes in) fear ratings to identify neural correlates specific to (reductions in) pain-related fear. A wholebrain approach was adopted in combination with analyses in a priori defined regions of interest (ROIs) that were considered to be of particular interest due to their involvement in painrelated fear and experimental extinction learning (i.e., amygdala, hippocampus, mPFC, PAG) and/or pain chronification (i.e., mPFC, NAc). We expected (I) pre-treatment group differences in neural circuitry recruited by stimuli evoking pain-related fear, correlated to fear ratings as well as pain-related outcomes in patients; (II) patient-specific pre- to post-treatment changes in regions showing pre-treatment group differences, as well as in other brain regions associated with chronic pain and with extinction (i.e., amygdala, hippocampus, mPFC, NAc, PAG); (III) pre- to post-treatment changes associated with changes in fear and persisting at 6 months follow-up.

\section{MATERIALS AND METHODS}

\section{Overall Study Procedure}

This study presents data of a larger study investigating effects of EXP on chronic pain, "BrainEXPain". BrainEXPain was approved by the Medical Ethical Committee of Maastricht University Hospital/Maastricht University (MUMC+/UM), and the protocol is registered at ClinicalTrials.gov [NCT02347579]. Patient recruitment was done via the department of Rehabilitation Medicine at MUMC+/Adelante rehabilitation center where patients were seen for consultation. If patients were found motivated for rehabilitation treatment and eligible for the multi-disciplinary pain screening program, they were invited by the physiatrist for the study. Recruitment was open between January 2015 and August 2017. 
Participants were then contacted by the research team and were screened for in- and exclusion criteria. Informed consent was obtained at study enrollment. Prior to scanning, all participants filled in questionnaires online (Qualtrics, Provo, United States ${ }^{1}$ ). The first study visit was scheduled prior to any (information on) treatment (i.e., baseline or preEXP). Afterward, patients underwent a multi-disciplinary pain screening and pain education, and started the exposure sessions (if eligible for treatment) - which were all part of standard care. At the end of treatment, patients underwent a post-EXP and a follow-up study visit (6 months after end of treatment; FUEXP). Healthy controls participated in two study visits, with the time in between these visits matching the patients' pre- to post-EXP. Participants received $€ 15$ per study visit and travel reimbursement for their participation.

\section{Participants}

Inclusion criteria for patients were age between 18 and 65 years, stable medication, ${ }^{2}$ experience of non-specific LBP $>6$ months, and no other diagnosis explaining the symptoms. Exclusion criteria were claustrophobia, MRI incompatibility (e.g., pacemaker, pregnancy), and severe psychopathology (Symptom Check List-90). Of the 35 patients with cLBP invited by the physiatrist over the 2.5 years inclusion period, 23 patients with cLBP were included in BrainEXPain (8 patients were not interested in participating, 4 patients were MRI incompatible). Of these, three patients dropped out prior to or during the measurement (due to claustrophobia); of two patients the data analyzed here was not acquired due to technical error; three patients were excluded due to extensive motion (see Data Analysis); and one patient was excluded due to lack of any vision-related (occipital) activity (see Data Analysis). The final sample for this study therefore consisted of 14 patients (Table 1). Post-EXP data is available for 10 patients (three did not start

\footnotetext{
${ }^{1}$ http://www.qualtrics.com

${ }^{2}$ Also, participants were asked not to change anything in their medication use on the day of the MRI, and all confirmed they did not take less or more medication than usual. See Supplementary Table S3 for an overview of the patients' medication use.
}

EXP, one became MRI incompatible), and FU-EXP data is available for 9 patients ( 1 was lost to follow-up due to unrelated medical issues).

The patient group was compared to a sample of 14 painfree healthy volunteers, matched for age, sex and handedness on cohort-level. To match the patient group, 10 controls underwent a second study visit. Controls were recruited through local advertisements. Additional exclusion criteria were: history of a chronic pain syndrome, and seeking treatment for a pain condition in the last 6 months.

\section{Exposure in vivo Treatment}

Within MUMC+/Adelante, EXP is standard care for patients with CLBP presenting with elevated pain-related fear. No additional restrictions or requirements for EXP were set by BrainEXPain. EXP specifically aims to reduce disability by challenging erroneous interpretations and expectancies about pain (e.g., that pain always indicates harm or that activities cause harm). A detailed description of the exposure-protocol for painrelated fear can be found in Vlaeyen et al. (2012). In brief, EXP always started with identifying movements/activities that are perceived as threatening and fearful, education about treatment rationale and that harm or pain does not mean additional injury (i.e., by discussing MR images of the spine by the treating physiatrist). EXP then continued with repeated exposure to feared movements, activities and/or sensations combined with behavioral experiments to challenge catastrophic interpretations by creating violations of expectancies. Patients were furthermore instructed to keep performing the movements and/or activities they performed during their sessions. EXP typically consists of 16 sessions (although it could be shortened to 8 or extended to 20 , per clinicians' decision), which are guided by a psychologist and either a physical or an occupational therapist. To identify movements and activities that are perceived as threatening and fearful, EXP utilizes The Photographic Series of Daily Activities (PHODA) for the low back (Leeuw et al., 2007). The PHODA consists of photographs depicting back-related movements and activities that are rated based on their perceived harmfulness. See Table 2 for more participant and EXP-related characteristics.

TABLE 1 | Demographics of the final sample.

\begin{tabular}{llll}
\hline & $\begin{array}{l}\text { Patients with cLBP } \\
\text { Mean (SD) }\end{array}$ & $\begin{array}{l}\text { Pain-free volunteers } \\
\text { Mean (SD) }\end{array}$ & $\begin{array}{l}\text { Statistics for group } \\
\text { comparison }\end{array}$ \\
\hline Sample size & $n=14$ & $n=14$ & n.a. \\
Age (years) & $42.4(11.6)$ & $41.7(12.5)$ & $F_{(1,26)}=0.02, p=0.89$ \\
Sex & 11 males & 10 males & $X^{2}(1, n=28)=0.19, p=0.66$ \\
& 3 females & 4 females & \\
Handedness & 13 right-handed & 14 right-handed & $X^{2}(1, n=28)=1.04, p=0.31$ \\
& 0 left-handed & 0 left-handed & \\
Pain duration & 1 ambidextrous & 0 ambidextrous & n.a. \\
& $6-12$ months: $n=1$ & n.a. & \\
$1-2$ years: $n=3$ & & \\
& $2-5$ years: $n=8$ & & \\
& $>5$ years: $n=2$ & & \\
\hline
\end{tabular}

n.a., not applicable. 
TABLE 2 | Information about EXP and the repeated measures.

\begin{tabular}{|c|c|c|c|}
\hline & $\begin{array}{l}\text { Patients with cLBP } \\
\text { Mean (SD) }\end{array}$ & $\begin{array}{l}\text { Pain-free volunteers } \\
\text { Mean (SD) }\end{array}$ & $\begin{array}{l}\text { Statistics for group } \\
\text { comparison }\end{array}$ \\
\hline Sample size & $n=10(n=9$ for FU-EXP) & $\mathrm{n}=10$ & n.a. \\
\hline Age (years) & $40.2(11.3)$ & $39.6(12.2)$ & $F_{(1,18)}=0.01, p=0.91$ \\
\hline Sex & $\begin{array}{l}9 \text { males } \\
1 \text { female }\end{array}$ & $\begin{array}{l}8 \text { males } \\
2 \text { females }\end{array}$ & $X^{2}(1, n=20)=0.39, p=0.53$ \\
\hline Pain duration & $\begin{array}{l}6-12 \text { months: } n=1 \\
1-2 \text { years: } n=2 \\
2-5 \text { years: } n=5 \\
>5 \text { years: } n=2\end{array}$ & n.a. & n.a. \\
\hline EXP treatment duration (days) & $45.0(15.9)$ & n.a. & n.a. \\
\hline $\begin{array}{l}\text { Time between pre-EXP session } \\
\text { and start EXP treatment (days) }\end{array}$ & $29.3(12.2)$ & n.a. & n.a. \\
\hline $\begin{array}{l}\text { Time between pre-EXP and } \\
\text { post-EXP session (days) }\end{array}$ & $96.1(42.1)$ & $92.3(33.5)$ & $F_{(1,18)}=0.05, p=0.83$ \\
\hline $\begin{array}{l}\text { Time between post-EXP and } \\
\text { FU-EXP session (days) }\end{array}$ & $186.4(9.6)$ & n.a. & n.a. \\
\hline
\end{tabular}

\section{Assessment of Pain-Related Aspects and Performance Levels}

At all time-points we assessed: pain intensity using a $0-10$ visual analog scale anchored with "no pain at all" and "worst pain imaginable"; pain-related fear using the PHODA short electronic version for low back (Leeuw et al., 2007), and Tampa Scale for Kinesiophobia (TSK; Kori, 1990; Vlaeyen et al., 1995a), Pain Catastrophizing Scale (PCS; Sullivan et al., 1995; Crombez et al., 1999), Pain Disability Index (PDI; Tait et al., 1987; Soer et al., 2013), Physical Activity Rating Scale combined with the Perceived Activity Decline (PARS/PAD; Vercoulen et al., 1997; Verbunt, 2008) questionnaire. Only assessed at baseline as trait measures were: Fear of Pain Questionnaire (PFQ; McNeil and Rainwater, 1998; van Wijk and Hoogstraten, 2006) and State Trait Anxiety Inventory (STAI-Y2; van der Ploeg et al., 1980; Spielberger et al., 1983). In addition, all participants underwent performance testing during all study visits to assess functioning. In the 2 min walking test, participants walked for $2 \mathrm{~min}$ on a standardized track and the covered distance was measured in meters. During staircase walking, participants walked a complete staircase (up and down), after which the average time per step was calculated.

\section{Picture Imagination Task}

In the scanner, the participants were presented with visual stimuli, associated with one of three categories: rest (derived from a web-search - REST), movements and activities perceived as fearful for patients specifically (derived from the extended version of the PHODA, not used in pain assessment and/or treatment MOVEMENT), or pictures implying bodily damage that may be perceived as fearful in general (derived from IAPS (Lang et al., 1997) and a web-search - MEDICAL). Backgrounds were removed to make the physical properties as similar as possible.

Participants were instructed to carefully look at the pictures and imagine that they were the person in the picture (carrying out the movement or activity, if applicable). After a short delay (see Figure 1 for details), participants were asked to rate how they would feel if they were the person on the picture (indirect assessment of fear). Ratings were done by pressing a button that moved a cursor on a horizontal line presented on the screen (later converted to $0-10$ scores). In total, there were 21 trials (7 of each category). Stimuli were presented using Presentation Software (Neurobehavioral Systems Inc.), and were synchronized with MR data acquisition. The total task had a duration of approximately $8 \mathrm{~min}$. The picture imagination task was always performed second, after a resting-state run. The total duration of the scan was approximately 75-90 min (data from other runs will be described elsewhere).

\section{MRI Acquisition}

MRI data were collected using a 3 Tesla whole body MRI scanner (Philips Gyroscan Achieva TX) using a 32-channel head coil, at the department of Radiology at MUMC+.

For the functional images, a $\mathrm{T} 2 *$-weighted standard echoplanar imaging (EPI) sequence was used to acquire 40 axial slices ( $3 \mathrm{~mm}$ isotropic) covering the entire cortical volume, using the following parameters: repetition time (TR) $=2000 \mathrm{~ms}$, echo time $(\mathrm{TE})=25 \mathrm{~ms}$, flip angle $=75^{\circ}$, matrix size $=120 \times 240$, SENSE factor $=2$. In total, 225 functional volumes were collected, of which the first two volumes were dummy volumes that were discarded from subsequent analysis to avoid T1 saturation effects.

T1-weighted anatomical images were acquired using a 3D turbo field echo (TFE) sequence with the following parameters: 170 slices, $1 \mathrm{~mm}$ isotropic, $\mathrm{TR}=8.1 \mathrm{~ms}$, $\mathrm{TE}=3.7 \mathrm{~ms}$, flip angle $=8^{\circ}$, matrix size $=240 \times 240$.

\section{Data Analysis}

\section{Assessment of Pain-Related Outcomes}

Questionnaire and performance test data were analyzed using SPSS (version 24). A general linear model (GLM) with Group (patients, controls) as between-subjects (BS) factor was used to examine group differences pre-EXP, as well as post-EXP. In addition, a repeated measures (rmGLM) with Time [pre-EXP, post-EXP, (FU-EXP)] as a within-subjects (WS) factor was used to investigate changes over time. 


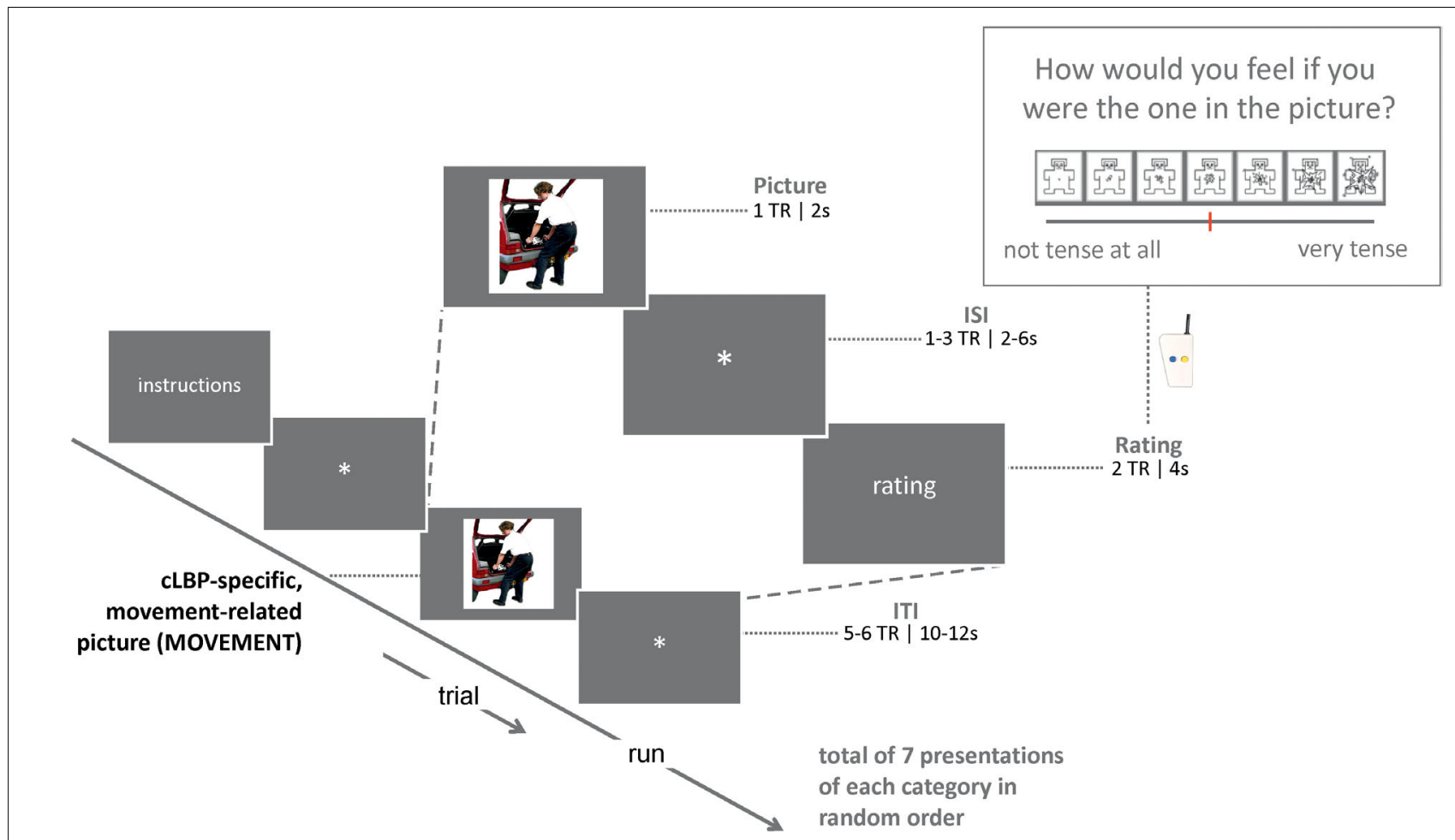

FIGURE 1 | Schematic overview of the design of the picture imagination task, showing the elements plus corresponding timing and a zoom into one example trial; TR, repetition time; ISI, inter-stimulus time; ITI, inter-trial time.

\section{Behavioral Data: Picture Imagination Task}

Group comparisons in in-scanner fear ratings, focusing on MOVEMENT pictures, were evaluated using a rmGLM with Group (patients, controls) as BS factor and Picture Number (7 different Pictures per Category) as WS factor. In addition, the WS factor Time [pre-EXP, post-EXP, (FU-EXP)] was added in a separate analysis.

\section{MRI Data: Pre-processing}

MRI data analysis was performed using BrainVoyager 3.6 (Brain Innovation, Maastricht, the Netherlands). Pre-processing of the functional data included slice scan time correction, 3D head motion correction, linear trend removal, highpass filtering (5 cycles per run; corresponding to $0.1 \mathrm{~Hz}$ ), and spatial smoothing $[4 \mathrm{~mm}$ using a full-width at halfmaximum Gaussian kernel (FWHM)]. Data was then co-registered to the corresponding anatomical image, and normalized to MNI space. The three pictures categories (REST, MOVEMENT, and MEDICAL) plus the delay prior to the rating (i.e., in total $4-8$ s) were used as predictors, convolved with the hemodynamic response function (HRF). Additional information on denoising procedures can be found in Supplementary Information.

\section{MRI Data Analysis: Masking}

Whole-brain analyses were run within a mask that excluded the white matter and cerebral spinal fluid, based on the HarvardOxford atlases (probability threshold 0.25) (Frazier et al., 2005;
Desikan et al., 2006; Makris et al., 2006; Goldstein et al., 2007). To specifically test our hypotheses in brain regions that play important roles in chronic pain and/or fear extinction, additional analyses were run within predefined region-of interest (ROI) masks. ROIs were defined in bilateral medial frontal cortex (mPFC), bilateral amygdala, bilateral nucleus accumbens (NAc), bilateral hippocampus based on the HarvardOxford subcortical atlas (probability threshold 0.25). A ROI corresponding to bilateral PAG was defined by dilating spheres around coordinates from Linnman et al. (2012) $[x=1, y=-$ 29, $z=-10$ (volume $=1612 \mathrm{~mm}^{3}$, diameter $\sim 14.5 \mathrm{~mm}$ )]. In these ROI masks, FDR correction $[q(\mathrm{FDR})<0.05]$ and minimum cluster size of 4 voxels $\left(108 \mathrm{~mm}^{3}\right)$ was used for statistical thresholding.

\section{MRI Data: Group Differences and Treatment Effects}

To compare blood-oxygen-level dependent (BOLD) responses across Groups and Times, a univariate random-effects (RFX) analysis with separate subject predictors was run at the first level, after which this data was fed into a second-level RFX analysis where group maps could be estimated and contrasted. FDR correction $[q(\mathrm{FDR})<0.05]$ was used for map creation. In the whole-brain analysis, an initial threshold of $p<0.001$ was used for contrasts across Groups and Times, after which cluster-size thresholding was performed using MonteCarlo simulations $(n=1000)$ to correct maps at the level of alpha 0.05 . The main contrast of interest was MOVEMENT vs. baseline, plus effects of Group and Time herein, as this 
condition was designed to elicit pain-related fear specifically in the patient group.

\section{MRI Data: Correlations With (Changes in) Pain-Related Outcome Measures and Changes in Fear}

Two types of correlation analyses were performed. From regions in which significant Group and Time differences were observed, betas were extracted in order to perform correlation analyses with measures of pain-related outcomes. An additional, explorative, analysis for the patients was to examine correlations between changes in fear ratings and changes in neural activation patterns at a whole-brain level. For this, we used the percentage of change in fear ratings for MOVEMENT pictures (at post- and FU-EXP compared to pre-EXP), and took a less conservative initial cluster-defining threshold of $p<0.005$ for the clustersize thresholding.

\section{RESULTS}

\section{Pre-treatment (Pre-EXP) Data \\ Patients Show High Levels of Fear, Pain, and Disability Pre-EXP}

Pre-treatment, patients reported significantly higher levels of pain, pain-related fear, catastrophizing and disability compared to controls (Table 3). Groups furthermore differed in trait anxiety, but not in trait fear of pain. Also, patients reported significantly lower levels of physical activity and higher levels of perceived activity decline compared to controls. Lab-assessed performance tests confirmed this: patients covered significantly less distance within 2 min walking, and needed more time to walk stairs, compared to controls.

\section{Patients Report More Fear for MOVEMENT Pictures Pre-EXP}

The in-scanner fear ratings for MOVEMENT pictures showed a significant Group effect $\left[F_{(1,26)}=188.15, p<0.001\right.$, $\left.\eta_{p}{ }^{2}=0.88,95 \% \mathrm{CI}=5.6,7.5\right]$, where patients reported higher fear levels compared to controls (Figure 2 and Supplementary Figure S1 for fear ratings for all Picture Categories). Also, for patients, fear ratings were significantly and strongly correlated with pain-related fear as assessed using the PHODA $(r=0.64$, $p=0.01$ ) (Figure 2).

\section{Patients Show Increased BOLD Activation to MOVEMENT Pictures Pre-EXP}

Figure 3 shows activation maps for the MOVEMENT pictures, per Group (see Supplementary Figure S2 for activation maps of all Picture Categories). Overall, the MOVEMENT pictures elicited activation in a similar network in patients and controls.

The whole-brain analysis showed a significant group difference in the right posterior insula (MNI $x=33, y=-10$, $z=10, k$ cluster size $=206 \mathrm{~mm}^{3}$ ), with patients showed increased BOLD activation compared to controls (Figures 3, 4A). The masked analyses in the pre-defined ROIs additionally showed a difference in mPFC (MNI $x=0, y=41, z=-11$, $k=4 \mathrm{~mm}^{3}$ ), with patients showing increased BOLD deactivation compared to controls (Figure 4A, Supplementary Figure S2, and Supplementary Tables S1, S2).

\section{Patients' Neural Activation to MOVEMENT Pictures Shows Specific Correlation to Pain-Related Outcomes}

Correlation analyses were performed using betas extracted from the right posterior insula and mPFC (i.e., averaged across all voxels in the cluster). When investigating the entire sample, both the activation in the posterior insula and $\mathrm{mPFC}$ was correlated to pain intensity and pain-related fear. Activation in the posterior insula was furthermore correlated to pain catastrophizing, pain disability, and both performance tests (Table 4). When zooming into the patient group, activation during MOVEMENT pictures in the posterior insula was positively correlated with painrelated fear, pain disability and both performance tasks while activation in $\mathrm{mPFC}$ did not correlate with any of the variables (Table 4). For the posterior insula, correlations reflected that increased neural activation was related to

TABLE 3 | Self-reported measures and performance tasks at baseline (pre-EXP).

\begin{tabular}{|c|c|c|c|}
\hline & $\begin{array}{l}\text { Patients with cLBP } \\
\text { Mean (SD) }\end{array}$ & $\begin{array}{c}\text { Pain-free volunteers } \\
\text { Mean (SD) }\end{array}$ & Statistics for group comparison \\
\hline \multicolumn{4}{|l|}{ Self-reported measures } \\
\hline Pain intensity (VAS) (range 0-10) & $5.5(2.4)$ & $0.2(0.5)$ & $F_{(1,26)}=64.39, p<0.001^{* *}, \eta_{p}^{2}=0.71,95 \% \mathrm{Cl}=3.9,6.7$ \\
\hline Pain-related fear (PHODA) (range 0-100, cutoff score 38) & $55.0(23.9)$ & $2.3(3.5)$ & $F_{(1,26)}=66.84, p<0.001^{* *}, \eta_{p}^{2}=0.72,95 \% \mathrm{Cl}=39.5,66.0$ \\
\hline Fear of movement (TSK) (range 17-68) & $40.9(9.0)$ & $27.4(4.8)$ & $F_{(1,26)}=26.33, p<0.001^{* *}, \eta_{p}^{2}=0.48,95 \% \mathrm{Cl}=7.8,19.0$ \\
\hline Pain catastrophizing (PCS) (range 0-52, cutoff score 21) & $24.2(14.2)$ & $3.6(3.7)$ & $F_{(1,26)}=27.71, p<0.001^{* *}, \eta_{p}^{2}=0.52,95 \% \mathrm{Cl}=12.5,28.6$ \\
\hline Pain disability (PDI) (range 0-70) & $39.6(15.9)$ & $1.9(5.1)$ & $F_{(1,26)}=67.04, p<0.001^{* *}, \eta_{p}^{2}=0.73,95 \% \mathrm{Cl}=28.3,47.3$ \\
\hline Perceived activity decline (PAD) (range 0-20) & $12.1(7.4)$ & $0.1(0.5)$ & $F_{(1,26)}=36.53, p<0.001^{* *}, \eta_{p}^{2}=0.58,95 \% \mathrm{Cl}=8.0,16.1$ \\
\hline Physical activity (PARS) (range 0-100) & $35.6(6.7)$ & $45.4(4.6)$ & $F_{(1,26)}=19.51, p<0.001^{* *}, \eta_{p}^{2}=0.43,95 \% \mathrm{Cl}=-13.8,-5.0$ \\
\hline Trait anxiety (STAI-Y2) (range 20-80) & $42.6(10.5)$ & $31.4(5.3)$ & $F_{(1,26)}=21.02, p<0.001^{* *}, \eta_{p}^{2}=0.46,95 \% \mathrm{Cl}=8.2,21.5$ \\
\hline Trait fear of pain (FPQ) (range 0-150) & $47.6(10.4)$ & $55.8(15.2)$ & $F_{(1,26)}=2.71, p=0.11, \eta_{p}^{2}=0.10,95 \% \mathrm{Cl}=-18.5,2.1$ \\
\hline \multicolumn{4}{|l|}{ Performance tasks } \\
\hline Two-min walking test (distance in meters) & $148.1(48.4)$ & $236.9(28.3)$ & $F_{(1,25)}=34.53, p<0.001^{* *}, \eta_{p}^{2}=0.58,95 \% \mathrm{Cl}=-119.9,-57.7$ \\
\hline Stair case walking (average time per step in seconds) & $1.52(0.13)$ & $0.62(0.04)$ & $F_{(1,25)}=19.32, p<0.001^{* *}, \eta_{p}^{2}=0.45,95 \% \mathrm{Cl}=0.48,1.33$ \\
\hline
\end{tabular}

**Survives Bonferroni correction for multiple comparison (alpha =0.05/11 =0.0045); size; 95\% Cl=95\% confidence interval of the difference (patients - controls). 


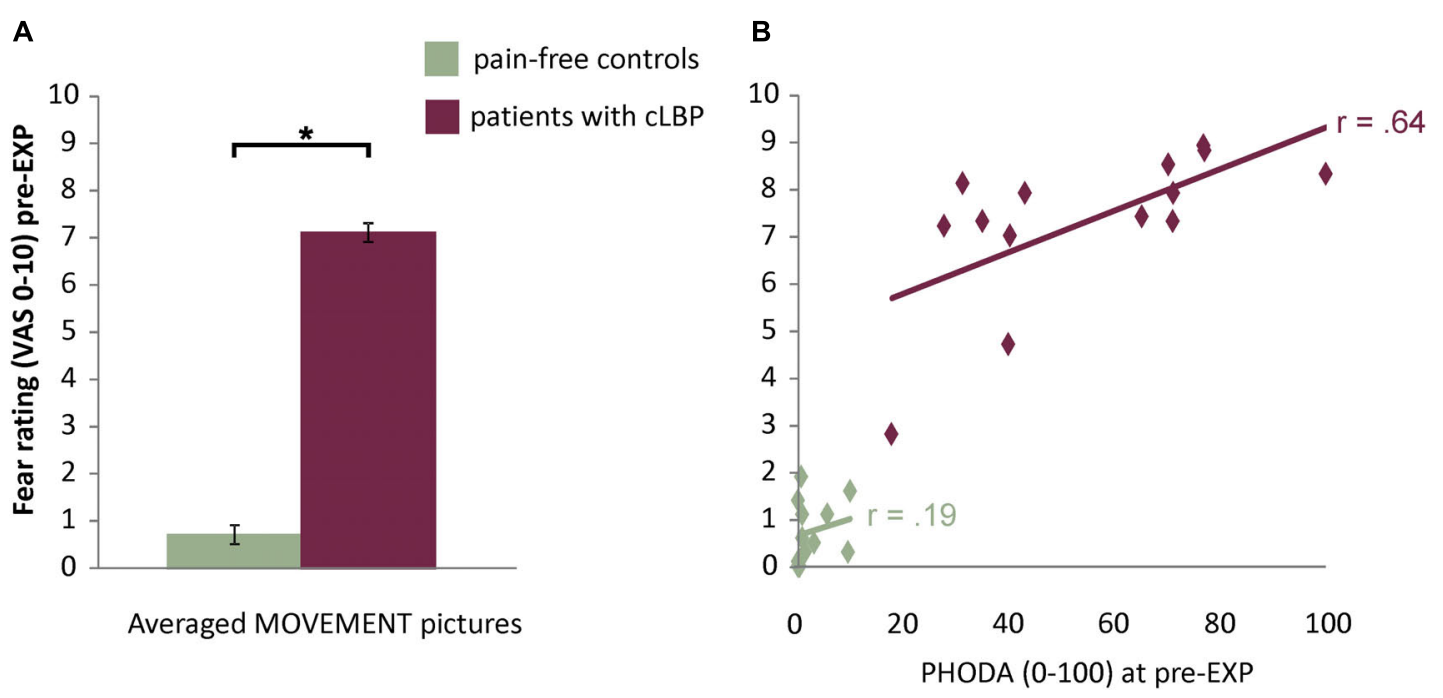

FIGURE 2 | (A) Fear ratings for the MOVEMENT pictures. Presented are means and standard errors for each group. Horizontal lines and asterisks indicate significant effects $\left({ }^{*} p<0.05\right)$. (B) Correlation between fear ratings for MOVEMENT pictures and pain-related fear as assessed using the PHODA.

\section{A Patients with cLBP}

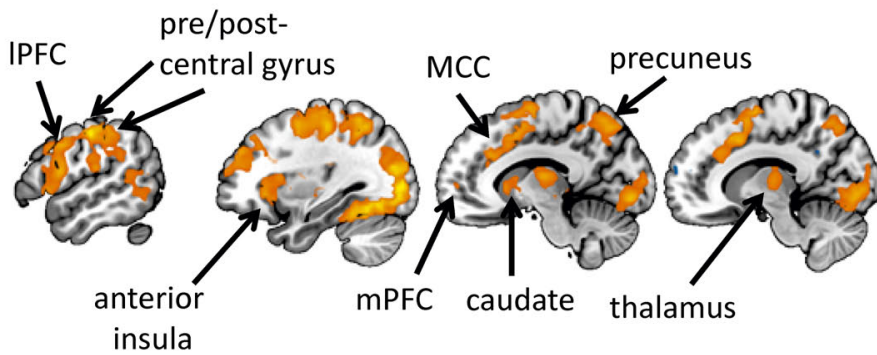

B Pain-free controls
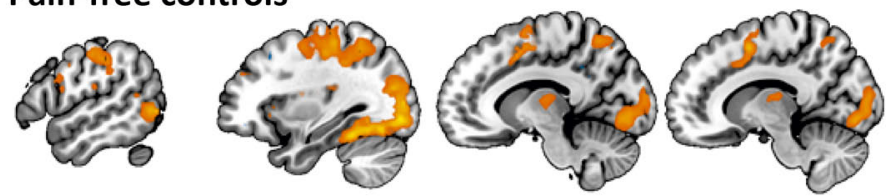

C Group differences
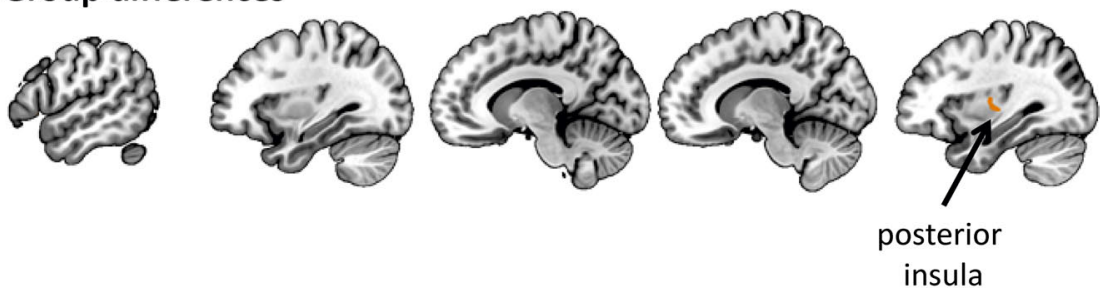
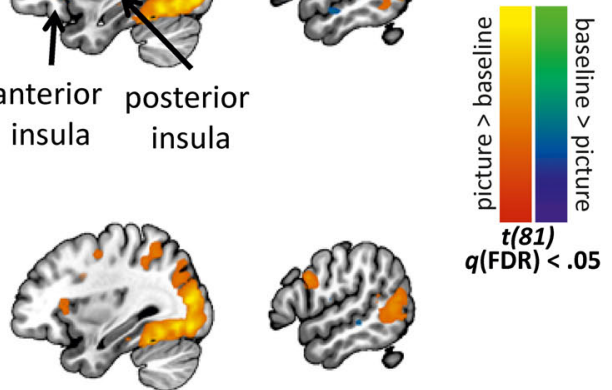

lobe / angular gyrus
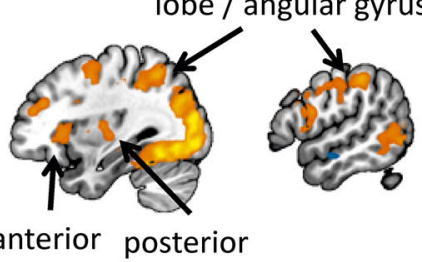

nterior posterior

insula insula
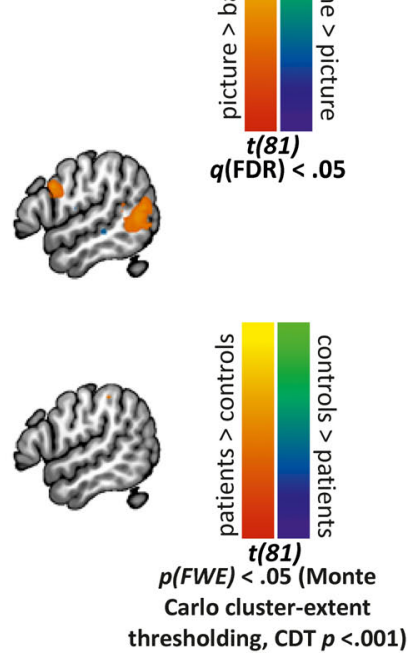

FIGURE 3 | Activation maps for the MOVEMENT Picture Category at pre-EXP, per group. Statistical maps are presented showing the neural activation of the MOVEMENT category relative to baseline for (A) Patients with CLBP, and (B) Pain-free volunteers, (C) Group differences in MOVEMENT condition. Cluster-level correction using $p<0.001$ as initial threshold. CDT, cluster-defining threshold. IPFC, lateral prefrontal cortex; mPFC, medial prefrontal cortex; MCC, mid-cingulate cortex. 

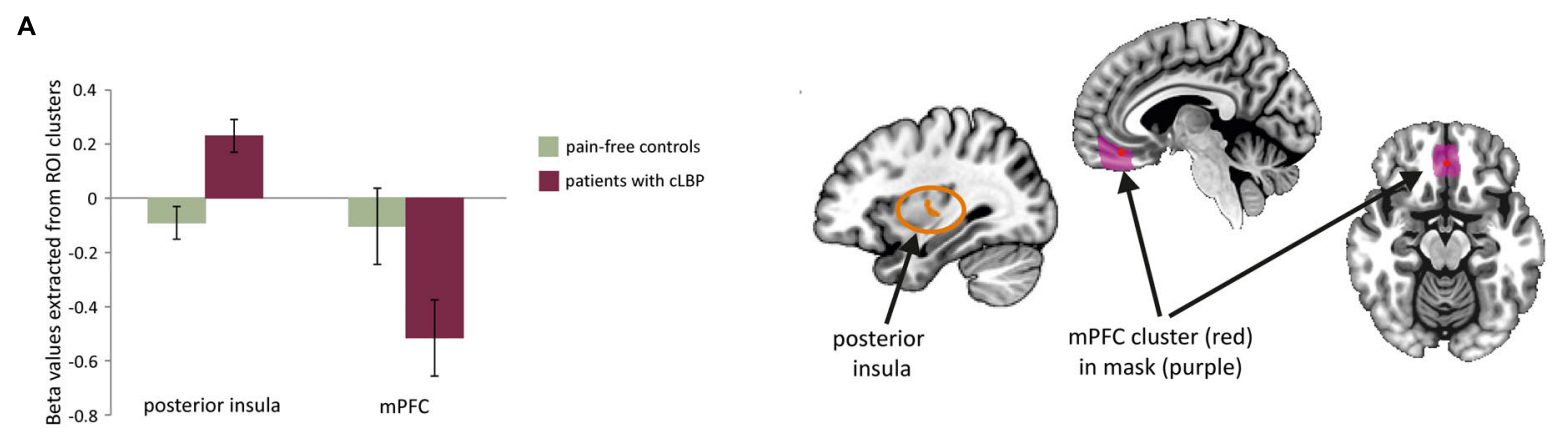

B
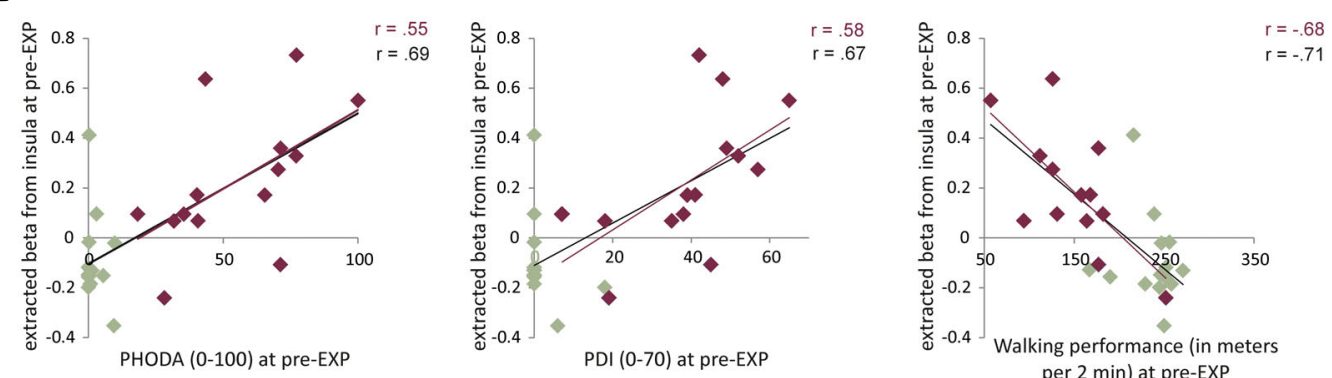

per $2 \mathrm{~min}$ ) at pre-EXP

FIGURE 4 | (A) Left: average beta values and standard errors for the MOVEMENT vs. baseline contrast for each group at pre-EXP, extracted from the two areas showing group differences. Right: Depiction of the location of the identified clusters. (B) Correlations between the posterior insula activation (beta value) and pain-related variables (self-reported and performance tasks). Note that the trendlines and magnitude of the correlations are shown for both the whole group (black) as well as the patient group only (red). mPFC, medial prefrontal cortex; PDI, pain disability index.

TABLE 4 | Correlations at pre-EXP between pain-related variables and activation in the regions displaying a group difference.

\begin{tabular}{|c|c|c|c|c|}
\hline & \multicolumn{2}{|c|}{ Right posterior insula } & \multicolumn{2}{|c|}{ mPFC } \\
\hline & Whole group & Patients only & Whole group & Patients only \\
\hline Pain-related fear (PHODA) & $0.69^{* *}$ & $0.55^{*}$ & $-0.43^{*}$ & -0.23 \\
\hline Fear of movement (TSK) & $0.53^{* *}$ & 0.40 & $-0.39 *$ & -0.14 \\
\hline Pain catastrophizing (PCS) & $0.51^{* *}$ & 0.27 & -0.23 & -0.03 \\
\hline Pain disability (PDI) & $0.67^{* *}$ & $0.58^{*}$ & -0.38 & -0.13 \\
\hline Pain intensity (VAS) & $0.56^{* *}$ & 0.23 & $-0.48^{*}$ & -0.36 \\
\hline Walking performance & $-0.71^{* *}$ & $-0.68^{*}$ & 0.31 & 0.25 \\
\hline Staircase walking performance & $0.73^{* *}$ & $0.71^{*}$ & -0.32 & -0.20 \\
\hline Trait fear of pain (FPQ) & -0.33 & 0.14 & 0.33 & 0.39 \\
\hline Trait anxiety (STAI-Y2) & $0.48^{*}$ & 0.36 & -0.34 & -0.14 \\
\hline
\end{tabular}

${ }^{* *} p<0.005$ (Bonforroni correction for multiple comparison $=0.05 / 9=0.0055$ ), ${ }^{*} p<0.05$, whole group $=$ patients and controls together $n=28$, patients only $n=14$.

increased levels of fear, disability and worse performance (Figure 4B). For the mPFC, the correlations were negative and reflected that decreased neural activation was related to increased levels of fear.

\section{Effects of Exposure in vivo Treatment Patients Show Improvements in Fear and Functioning After EXP Treatment}

Pre- to post- to FU-EXP changes in patients

Patients showed main effects of Time for pain-related fear, painrelated disability, perceived activity decline, and the performance tests (Table 5). There were no main effects for pain intensity, pain catastrophizing and self-reported physical activity, although these measures generally showed a decrease, and showed clinically relevant reductions (defined as reduction of $30 \%$ or more compared to baseline) in 60,60 , and $40 \%$ of patients in these domains, respectively, from pre- to post-EXP (Table 5).

Pre- to post changes in controls

Controls did not show any effects of Time (all p's > 0.05).

\section{Group effects post-EXP}

Post-EXP, groups did not differ anymore in fear of movement, pain catastrophizing, self-reported physical activity, and staircase walking. Patients still reported higher pain intensity and pain-related disability compared to controls, and performed significantly worse on the 2 min walking test (Table 6). 
TABLE 5 | EXP-induced changes in self-reported measures and performance tasks in the patient group.

\begin{tabular}{|c|c|c|c|c|c|}
\hline & $\begin{array}{l}\text { Pre- to post-EXP } \\
\text { change mean } \\
\text { (SE) }\end{array}$ & $\begin{array}{l}\text { Pre- to FU-EXP } \\
\text { change mean } \\
\text { (SE) }\end{array}$ & Stats main effect Session & $\begin{array}{l}\text { Post hoc } \\
\text { comparisons }\end{array}$ & $\begin{array}{c}>30 \% \text { reduction } \\
\text { n: Pre- to post } \\
\text { n: Pre- to FU }\end{array}$ \\
\hline \multicolumn{6}{|l|}{ Self-reported measures } \\
\hline Pain intensity (VAS, range 0-10) & $-1.4(0.79)$ & $-2.0(0.88)$ & $F_{2.0,15.7}=3.12, p=0.07, \eta_{p}^{2}=0.28$ & n.a. & $\begin{array}{l}6 / 10 \\
5 / 9\end{array}$ \\
\hline $\begin{array}{l}\text { Pain-related fear (PHODA; range } \\
0-100 \text {, cutoff score 38) }\end{array}$ & $-40.7(5.6)$ & $-37.3(5.4)$ & $F_{(1.4,10.0)}=44.24, p<0.001^{* *}, \eta_{p}^{2}=0.86$ & $\begin{array}{l}\text { Post- }<\text { Pre-EXP } \\
\text { FU- }<\text { Pre-EXP }\end{array}$ & $\begin{array}{l}9 / 10 \\
8 / 9\end{array}$ \\
\hline $\begin{array}{l}\text { Fear of movement (TSK; range } \\
17-68)\end{array}$ & $-12.2(2.6)$ & $-9.2(3.6)$ & $F_{(1.8,14.2)}=8.49, p=0.005^{* *}, \eta_{p}^{2}=0.52$ & Post- $<$ Pre-EXP & $\begin{array}{l}4 / 10 \\
4 / 9\end{array}$ \\
\hline $\begin{array}{l}\text { Pain catastrophizing (PCS; range } \\
0-52 \text {, cutoff score } 21 \text { ) }\end{array}$ & $-11.7(5.5)$ & $-11.2(5.7)$ & $F_{(1.1,8.5)}=4.05, p=0.08, \eta_{p}^{2}=0.34$ & n.a. & $\begin{array}{l}6 / 10 \\
4 / 9\end{array}$ \\
\hline Pain disability (PDI; range 0-70) & $-27.7(4.2)$ & $-25.6(5.6)$ & $F_{(1.3,10.5)}=24.84, p<0.001^{* *}, \eta_{p}^{2}=0.76$ & $\begin{array}{l}\text { Post- }<\text { Pre-EXP } \\
\text { FU- }<\text { Pre-EXP }\end{array}$ & $\begin{array}{l}10 / 10 \\
7 / 9\end{array}$ \\
\hline $\begin{array}{l}\text { Perceived activity decline (PAD; range } \\
0-20)\end{array}$ & $-6.9(1.7)$ & $-6.8(2.3)$ & $F_{(1.7,12.0)}=8.13, p=0.007, \eta_{p}^{2}=0.54$ & Post- $<$ Pre-EXP & $\begin{array}{l}7 / 10 \\
6 / 8\end{array}$ \\
\hline Physical activity (PARS; range 0-100) & $-8.0(3.3)$ & $-4.4(4.8)$ & $F_{(1.6,13.0)}=2.05, p=0.17, \eta_{p}^{2}=0.20$ & n.a. & $\begin{array}{l}4 / 10 \\
3 / 9\end{array}$ \\
\hline \multicolumn{6}{|l|}{ Performance tasks } \\
\hline $\begin{array}{l}\text { Two-min walking test (distance in } \\
\text { meter) }\end{array}$ & $42.9(8.7)$ & $44.5(13.6)$ & $F_{(1.2,7.1)}=12.42, p=0.008, \eta_{p}^{2}=0.67$ & $\begin{array}{l}\text { Post- }<\text { Pre-EXP } \\
\text { FU- }<\text { Pre-EXP }\end{array}$ & n.a. \\
\hline $\begin{array}{l}\text { Stair case walking (average time per } \\
\text { step in seconds) }\end{array}$ & $0.57(0.12)$ & $0.53(0.13)$ & $F_{(1.1,5.3)}=17.82, p=0.007, \eta_{p}^{2}=0.78$ & $\begin{array}{l}\text { Post- }<\text { Pre-EXP } \\
\text { FU- }<\text { Pre-EXP }\end{array}$ & n.a. \\
\hline
\end{tabular}

** Survives Bonferroni correction for multiple comparison (alpha $=0.05 / 9=0.0055$ ), n.a., not applicable.

\section{Patients Report Less Fear for MOVEMENT Pictures After EXP Treatment}

\section{Pre- to post- to FU-EXP changes in patients}

There was a significant effect of Time for fear ratings for the MOVEMENT pictures $\left[F_{(1.56,12.44)}=24.76, p<001\right.$, $\left.\eta_{p}{ }^{2}=0.76\right]$, with a significant decrease in ratings between preand post-EXP ( $p$-corr $<0.001,95 \% \mathrm{CI}=-7.0,-3.2)$ and between pre- and FU-EXP ( $p$-corr $=0.006,95 \% \mathrm{CI}=-7.2,-1.4)$, but no difference between post-EXP and FU-EXP ( $p$-corr $=0.81,95 \%$ $\mathrm{CI}=-1.3,2.9$ ) (Figure 5 and Supplementary Figure $\mathbf{S} 3$ ratings across all Picture Categories).

Pre- to post changes in controls

There was no significant effect of Time $\left[F_{(1,9)}=0.31, p=0.59\right.$, $\left.\eta_{p}^{2}=0.03\right]$.

\section{Group effects post-EXP}

There was a significant Time $\mathrm{x}$ Group interaction $\left[F_{(1}\right.$, 18) $\left.=55.20, p<0.001, \eta_{p}{ }^{2}=0.78\right]$. Simple effects per time point showed that at post-EXP, there was no longer a Group difference $\left[F_{(1,18)}=1.12, p=0.30, \eta_{p}^{2}=0.06,95 \% \mathrm{CI}=-1.9,0.6\right]$.

\section{Patients Show a Decrease in BOLD Activation to MOVEMENT Pictures After EXP Treatment}

\section{Pre- to post- to FU-EXP changes in patients}

The effect of Time was investigated in the clusters showing a group difference pre-treatment (extracted betas from right posterior insula and mPFC clusters) as well as in a whole-brain analysis and in the predefined ROI masks.

The posterior insula cluster showed a main effect of Time $\left[F_{(1.8,14.8)}=4.06, p=0.04, \eta_{p}^{2}=0.34\right]$, explained by a linearly decreasing response to MOVEMENT pictures over Time
$\left[F_{(1,8)}=7.02, p=0.03, \eta_{p}^{2}=0.40\right]$. The mPFC only showed a marginally significant main effect of Time $\left[F_{2.0}, 158=3.25\right.$, $\left.p=0.07, \eta_{p}{ }^{2}=0.29\right]$, explained by linearly increasing response to MOVEMENT pictures over Time $\left[F_{(1,8)}=8.7878, p=0.02\right.$, $\left.\eta_{p}^{2}=0.41\right]$ (see Figure 6).

The whole-brain analyses showed a decrease in right postcentral/supramarginal gyrus and pre-central gyrus, and an increase in activity in the precuneus from pre- to post-treatment (Figure 7 and Table 7). Comparing pre-treatment to 6 months follow-up, the right angular/inferior parietal lobe, right postcentral, right middle frontal/dorsolateral PFC, right inferior frontal/ventrolateral PFC as well as left middle frontal gyrus showed a significant decrease in activation. Lastly, from posttreatment to 6 months follow-up, the right posterior cingulate cortex showed an additional decrease in activation. When evaluating the effect of Time in the predefined ROIs, there was a significant decrease from pre- to FU-EXP in the NAc (Table 7), but not in the other ROIs.

Pre- to post changes in controls.

There were no effects of Time in the posterior insula and $\mathrm{mPFC}$ cluster. In controls, the whole-brain analysis revealed a change in two regions that do not overlap with the regions identified in patients (Supplementary Table S4). None of the predefined ROIs showed an effect of Time.

\section{BOLD Activation to MOVEMENT Pictures Does Not Differ Anymore Between Patients and Controls After EXP Treatment}

\section{Group effects post-EXP}

Post-treatment, no group differences were present anymore in the whole-brain analysis (also not when being less conservative 
TABLE 6 | Self-reported measures and performance tasks post-EXP.

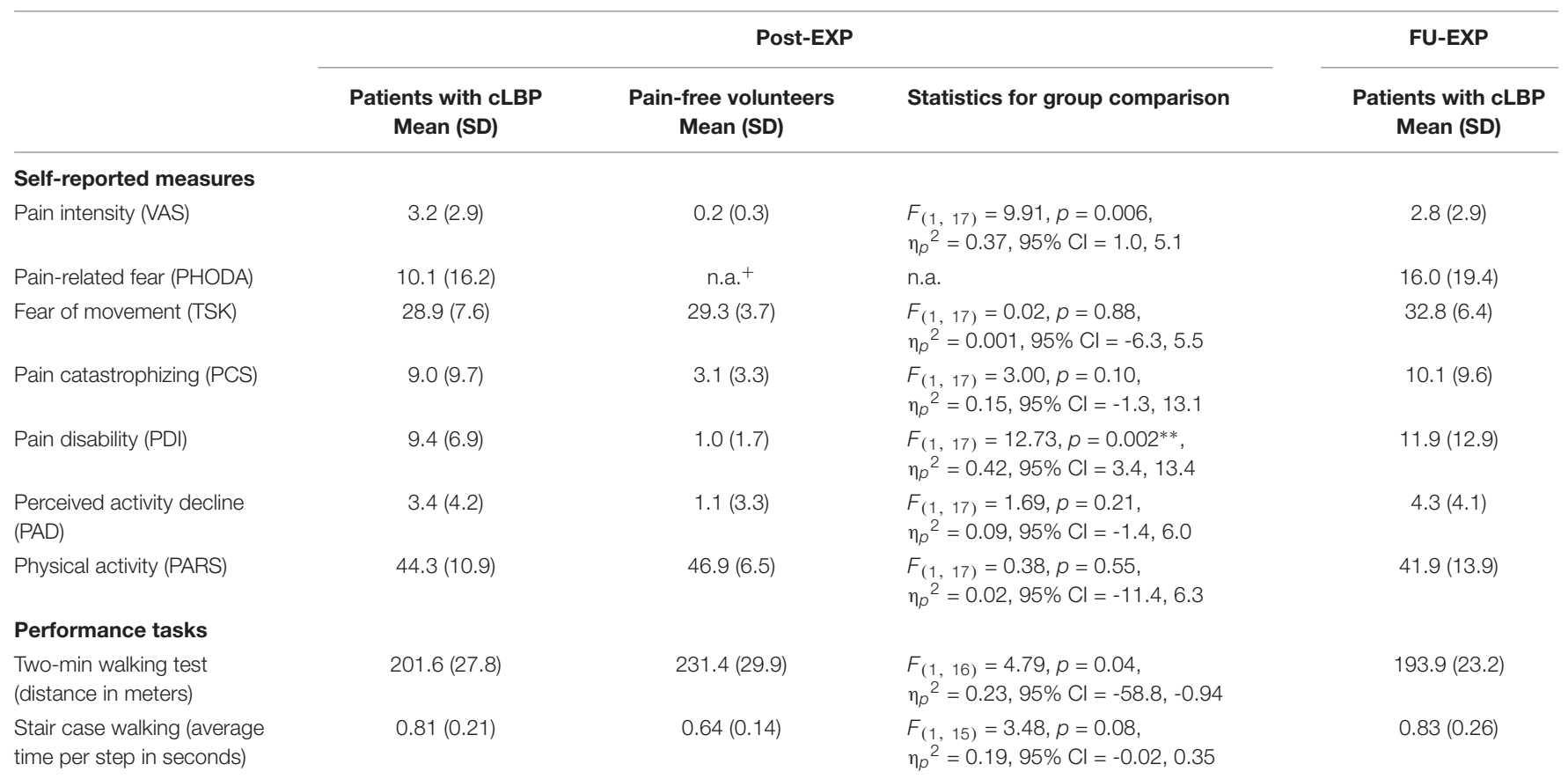

** Survives Bonferroni correction for multiple comparison (alpha $=0.05 / 9=0.0055$ ); ${ }^{+}$Due to a technical error, responses were not recorded for the majority of volunteers, n.a., not applicable; $95 \% \mathrm{Cl}$, 95\% confidence interval of the difference (patients - controls).

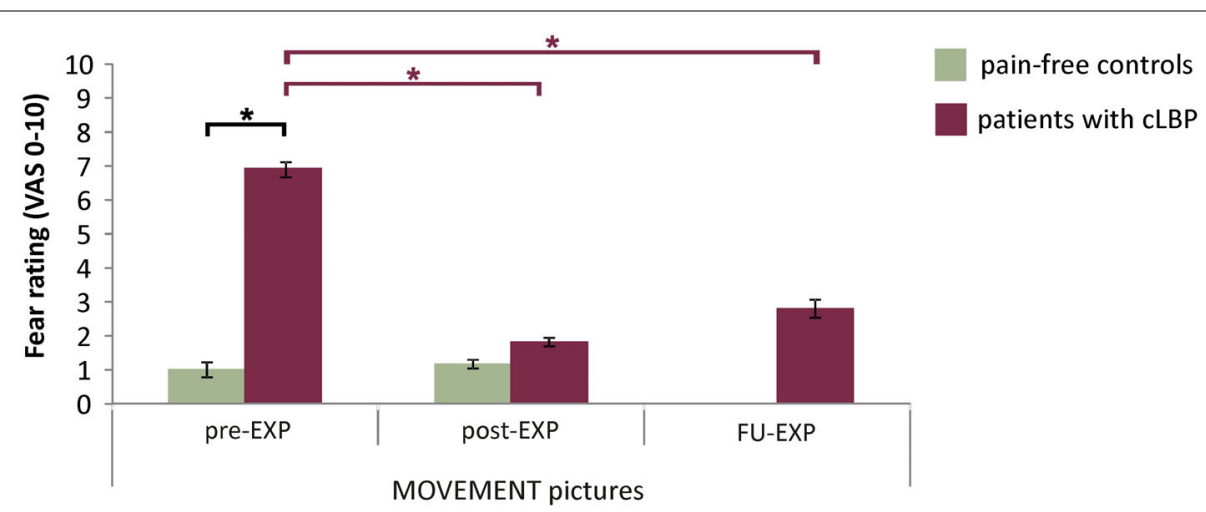

FIGURE 5 | EXP treatment-induced changes in fear ratings. Presented are the means and standard errors for the MOVEMENT pictures for each group across time. Horizontal lines and asterisks indicate significant effects $\left({ }^{*} p<0.05\right)$ : group effects are shown in black, while simple effects of Session, separate per group are shown in color (red for patients with cLBP; there were no significant Session effects for controls).

with an initial threshold of $p<0.005$ for cluster-size thresholding). None of the predefined ROIs showed a group difference post-EXP. In addition, when performing a Group comparison of the extracted betas from these ROIs, no group difference was identified at post-EXP [posterior insula: $F_{(1}$, $18)=2.58, p=0.13, \eta_{p}^{2}=0.13,95 \% \mathrm{CI}=-0.38,0.05$; mPFC: $\left.F_{(1,18)}=2.11, p=0.16, \eta_{p}^{2}=0.11,95 \% \mathrm{CI}=-0.12,0.63\right]$.

\section{Neural Activation Changes to MOVEMENT Pictures in Patients Correlate With Changes in Fear Ratings (Explorative Analyses)}

We explored whether changes in fear ratings for the MOVEMENT pictures from pre- to post-treatment were associated with specific changes in BOLD activation from pre- to post-treatment in patients. We found indications that a decrease in fear ratings from pre- to post-treatment was correlated to an increase of neural activation in the right hippocampus (MNI $x=30, y=-22, z=-17, k=396 \mathrm{~mm}^{3}$ ) and the left temporal pole (MNI $x=-42, y=14, z=-20, k=568 \mathrm{~mm}^{3}$ ) (see Figure 8). When extracting beta values, we found that the increase in BOLD activation in both regions was also related to decreases in painrelated fear from pre- to post-EXP (PHODA; left hippocampus: $r=-0.82, p=0.003$, temporal pole: $r=-0.89, p=0.001)$ and to decreases in pain-related disability from pre- to post-EXP (PDI; left hippocampus: $r=-0.78, p=0.007$, temporal pole: $r=-0.71$, $p=0.02$ ). 
A Posterior insula

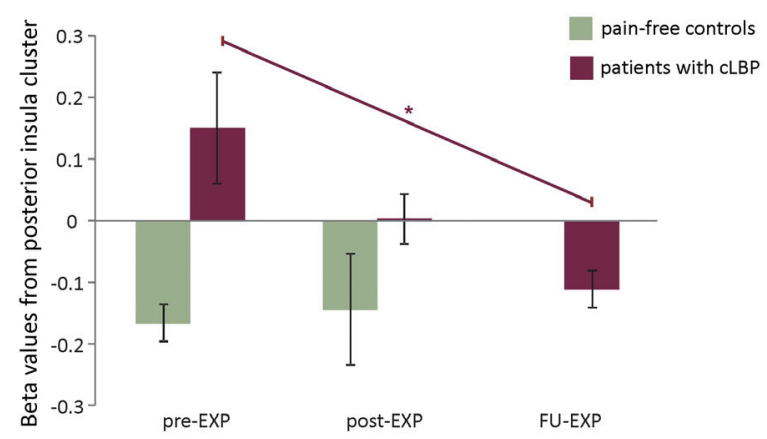

B $\mathrm{MPFC}$

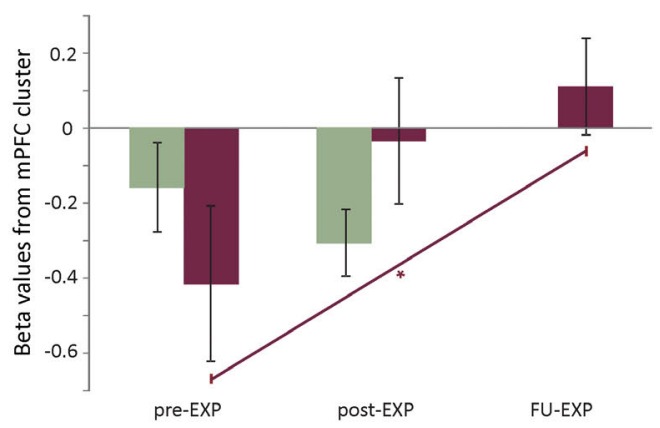

FIGURE 6 | EXP treatment-induced effects in neural activation to MOVEMENT pictures in the posterior insula (A) and mPFC (B). Plotted are averaged beta values and standard errors per time point and per group. Purple lines and asterisks indicate the significant linear effects over Time in patients. ${ }^{*} p<0.05$.

The decrease in fear ratings from pre- to FU-EXP was furthermore related to an increase in right PCC (MNI $x=6$, $y=-55, z=10, k=407 \mathrm{~mm}^{3}$ ) and mPFC (MNI $x=0, y=47$, $z=-5, k=564 \mathrm{~mm}^{3}$ ). The right PCC betas additionally showed significant correlations to decreases in pain-related fear from preto FU-EXP (PHODA; $r=-0.88, p=0.002$ ). Figure 8 shows these relations in more detail.

None of these clusters showed a main effect of Time [hippocampus: $F_{(2.0,15.7)}=0.35, p=0.71, \eta_{p}{ }^{2}=0.04$; temporal pole: $F_{(1.6,12.5)}=0.03, p=0.94, \eta_{p}^{2}=0.004$; PCC: $F_{(1.7}$, $13.9)=2.87, p=0.10, \eta_{p}{ }^{2}=0.26$; mPFC: $F_{(1.5,12.1)}=0.29$, $\left.p=0.69, \eta_{p}^{2}=0.04\right]$.

\section{DISCUSSION}

We provide the first evidence that clinical improvements following EXP in patients with cLBP are mirrored by changes in the neural circuitry for pain-related fear, the main target of EXP. Pre-treatment, we identified group differences in inscanner fear ratings and neural responses to pictures of backspecific movements: compared to pain-free controls, patients with CLBP showed increased activation in the right posterior insula and increased deactivation in mPFC. Post-treatment, group differences were no longer present, and the process of change continued in patients at 6 months follow-up. Apart from general changes across treatment in lateral PFC, PCC, precuneus, NAc, and pre- and post-central gyrus, patients showed neural changes specifically related to changes in in-scanner fear ratings in the temporal pole, mPFC, PCC, and hippocampus. Pain-free volunteers did not show this, indicating that these changes cannot be attributed to general habituation effects. Hence, we provide evidence for treatment-induced neural changes in chronic pain that are specific to and correlate with improvements in selfreported fear.

\section{Replicating the Positive Clinical Effects of EXP}

As expected, after EXP treatment, pain-related fear and disability significantly decreased while the patient's performance (i.e., walking and stair case walking) improved significantly. Changes were maintained, or in some cases even more pronounced, 6 months after the end of treatment. We did not observe a significant effect of EXP on pain intensity, which is not uncommon nor unexpected. EXP focuses on reducing pain-related disabilities and reducing pain intensity is no explicit aim. Some studies, however, have observed significant improvements in pain intensity on a group level (den Hollander et al., 2016; Glombiewski et al., 2018), and also in the current study we observed improvements in some patients (i.e., clinically meaningful reduction in $60 \%$ of the patients). In future studies, it would be interesting to examine why some people respond with a reduction in pain intensity, while others do not. The lack of effect on pain catastrophizing is surprising though and not expected, given previous studies (see e.g., Leeuw et al., 2008; den Hollander et al., 2016; Lopez-de-Uralde-Villanueva et al., 2016) and the focus of EXP on disconfirming negative beliefs (Vlaeyen et al., 2012; den Hollander et al., 2015). Also for pain catastrophizing, however, we did observe a reduction on average as well as clinically meaningful reductions in $60 \%$ of patients (pre to post-EXP), suggesting that there was an effect which did not reach significance due to a relatively small sample size.

\section{Pre-treatment Group Differences in Fear Circuitry}

We identified two brain regions showing a group difference in neural responses to pain-related fear. In the right posterior insula and $\mathrm{mPFC}$, patients with CLBP showed altered neural activation compared to controls in response to our fearevoking task. Focusing on pain-related fear, previous studies have demonstrated increased activation in the insula, as well as in other in regions including the ACC, superior parietal cortex, amygdala, orbitofrontal cortex, and striatum in patients compared to controls (Taylor et al., 2015; Meier et al., 2016). A potential explanation for the difference in extent of findings is our more stringent statistical thresholding (Woo et al., 2014) (i.e., with less stringent parameters, additional brain regions showed group differences; and when taking the picture categories together, a multitude of regions differed 


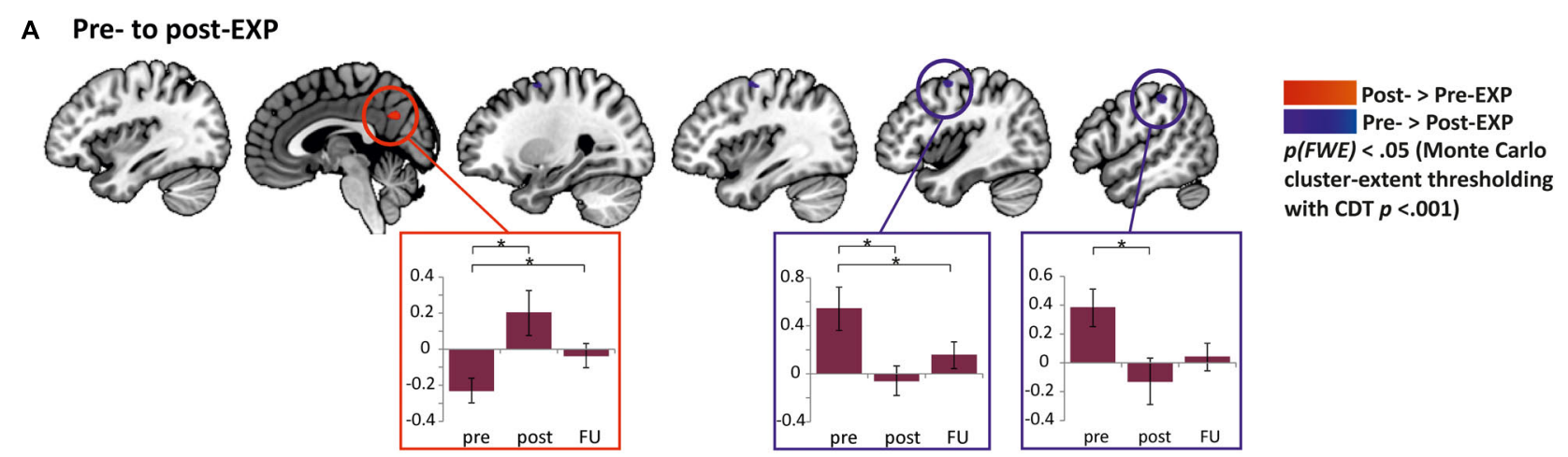

B Pre- to FU-EXP
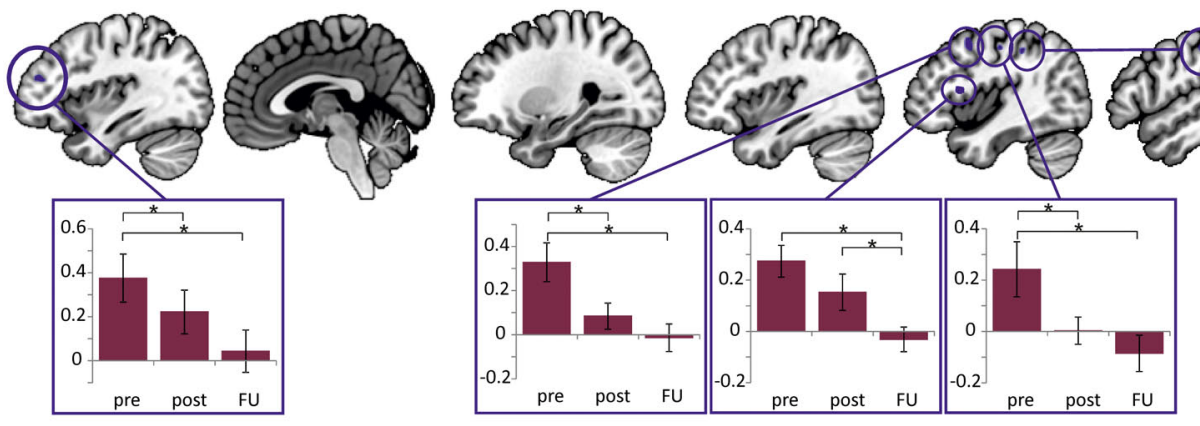
$p$ (FWE) $<.05$ (Monte Carlo cluster-extent thresholding with CDT $p<.001$ )

\section{Post- to FU-EXP}

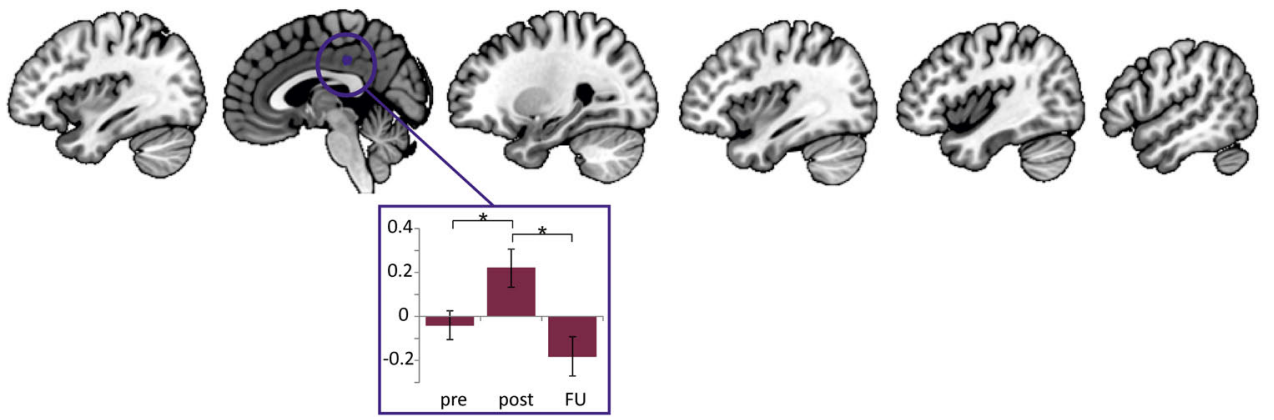

Post- > FU-EXP $p(F W E)<.05$ (Monte Carlo cluster-extent thresholding with CDT $p<.001$ )

FIGURE 7 | Clusters of EXP treatment-induced changes in neural activation to MOVEMENT pictures in patients with cLBP. (A) Differences from pre- to post-EXP were observed in precuneus (increase, red) as well as precentral gyrus and postcentral gyrus/supramarginal gyrus (from left to right; both decreases, blue). In the boxes, the extracted betas from the corresponding cluster are presented seperately for pre-, post- and FU-EXP. Significant differences across Sessions are highlighted by an asterisk $(p<0.05)$. (B) Differences from pre- to FU-EXP along with corresponding beta plots. Significant changes were observed in left middle frontal gyrus, right middle frontal/dorsolateral PFC, right inferior frontal/ventrolateral PFC, right postcentral gyrus, and right angular/inferior parietal lobe (from left to right, all decreases, blue). (C) Differences from post- to FU-EXP changes along with corresponding beta plots. A significant difference was found in the posterior cingulate cortex (PCC, decrease, blue). Cluster-level correction using $p<0.001$ as initial threshold. Presented in the boxes are means and standard errors. CDT, cluster-defining threshold.

across groups, including ACC, superior parietal cortex and striatum, see Supplementary Information). Previous work related activation in insula, amygdala and several other regions to the amount of pain-related fear (Meier et al., 2016). Here, we extend these findings by showing that increased posterior insula activation is furthermore related to pain-related disability and actual physical performance (i.e., walking). In addition, its response was parametrically modulated by in-scanner fear ratings (Supplementary Information), further strengthening its specific involvement in pain-related fear. The insula is a core region involved in fear learning (Sehlmeyer et al., 2009;
Fullana et al., 2016, 2018b), although loci are typically more anterior. The posterior insula, in contrast, has been associated with interoceptive integration (Craig, 2002), sensory aspects of pain/nociception (Garcia-Larrea and Peyron, 2013; Wager et al., 2013; Segerdahl et al., 2015), and experimental rather than clinical pain (Schweinhardt and Bushnell, 2010). This fits with abundant connections between posterior insula and somatosensory cortex (SI/SII; Wiech et al., 2014). Our finding that posterior insula activation was modulated by fear ratings, however, indicates additional involvement in pain-related fear, possibly due to a top-down modulatory effect of fear on this more sensory region. 


\begin{tabular}{|c|c|c|c|c|c|}
\hline & & \multicolumn{4}{|c|}{ MNI } \\
\hline & & $x$ & $y$ & $z$ & Cluster size \\
\hline \multicolumn{6}{|c|}{ Patients: pre- to post-EXP (whole-brain analysis - minimum cluster size $202 \mathrm{~mm}^{3}$ ) } \\
\hline R postcentral gyrus/inferior parietal lobe & Pre $>$ Post & 54 & -28 & 53 & 285 \\
\hline R precentral gyrus & Pre $>$ Post & 42 & -1 & 60 & 738 \\
\hline R Precuneus & Pre $<$ Post & 6 & -62 & 60 & 436 \\
\hline \multicolumn{6}{|l|}{ Patients: pre- to FU-EXP } \\
\hline \multicolumn{6}{|c|}{ Whole-brain analysis (minimum cluster size $210 \mathrm{~mm}^{3}$ ) } \\
\hline $\mathrm{R}$ inferior parietal lobe & Pre $>$ FU & 54 & -31 & 50 & 495 \\
\hline R postcentral gyrus & Pre $>$ FU & 45 & -16 & 47 & 228 \\
\hline R middle frontal gyrus/dIPFC & Pre $>$ FU & 42 & 8 & 50 & 586 \\
\hline R inferior frontal gyrus/vIPFC & Pre $>$ FU & 45 & 11 & 13 & 292 \\
\hline L middle frontal gyrus & Pre $>$ FU & -33 & 47 & 22 & 347 \\
\hline \multicolumn{6}{|c|}{ Masked region of interest analysis (FDR $q<0.05$ ) } \\
\hline Nucleus accumbens & Pre $>$ FU & -15 & 17 & -5 & 4 \\
\hline \multicolumn{6}{|c|}{ Patients: post- to FU-EXP (whole-brain analysis - minimum cluster size 159 mm³) } \\
\hline $\mathrm{R} / \mathrm{L}$ posterior cingulate gyrus & Post $>$ FU & 3 & -28 & 38 & 208 \\
\hline
\end{tabular}

The mPFC, and more specifically its ventromedial part (vmPFC), is also a core region involved in fear acquisition and extinction (Sehlmeyer et al., 2009), and general emotion regulation (Sotres-Bayon et al., 2006; Hartley and Phelps, 2010). $\mathrm{mPFC}$ involvement in pain and chronic pain is furthermore extensive (Ong et al., 2018). Our finding that mPFC showed a decreased (i.e., increased deactivation) response to fear-evoking stimuli in patients could point toward altered inhibitory control, and reduced ability to modulate or self-regulate pain (Tracey, 2010; Woo et al., 2015; Ong et al., 2018). To our surprise, amygdala activation to feared stimuli was not different across groups. Previous studies consistently reported the amygdala as a brain area of interest in (chronic) pain (see e.g., Simons et al., 2012) and fear or more generally threat (LeDoux, 1993). It may be that functional connectivity rather than neural activation distinguishes patients from controls. This will have to be explored in further analyses.

\section{Patient-Specific Neural Changes Across Treatment}

The increased posterior insula response to our stimuli in patients pre-treatment was reduced over the course of EXP, as was the increased $\mathrm{mPFC}$ deactivation. Importantly, we no longer observed group differences post-treatment. This is in accordance with normalizations observed in fear ratings as well as in most clinical measures. Treatment effects were still present or even increased at 6 months follow-up, suggesting generalization to daily life. This is in accordance with a recent RCT in complex regional pain syndrome, where EXP effect sizes were larger at 6 months follow up compared to post-treatment (den Hollander et al., 2016).

Furthermore, several brain regions showed changes in neural responses across treatment, including pre- and post-central gyrus/supramarginal gyrus, precuneus, lateral PFC, and NAc. In pre- and post-central gyrus/supramarginal gyrus, we observed decreases from pre- to post-EXP and from pre-EXP to followup. Recruitment of these areas associated with motor control, sensory properties of somatosensory stimuli (Peyron et al., 2000), as well as sensorimotor imagery (McNorgan, 2012; Hetu et al., 2013) was expected, as participants were imagining performing movements and activities depicted in the stimuli. Functional changes in sensorimotor regions have previously been identified in chronic pain (Flodin et al., 2014; Kregel et al., 2015). The changes over time we observed may reflect normalizations in sensorimotor neurocircuitry, and along similar lines it may also reflect changes in physical performance that go alongside with EXP, as an indirect result of reducing pain-related fear. The precuneus, on the other hand, showed increased activation over the course of treatment. The precuneus is part of the defaultmode network (DMN), involved in interoception, mentalizing, integrating information more than processing it (Cavanna and Trimble, 2006). Its activation has been negatively correlated to pain sensitivity, without contributing to the actual neural representation of pain (Goffaux et al., 2014), the direction of which is in line with our findings. Interestingly, in fibromyalgia, abnormalities in connectivity between the insula (including posterior part) and the DMN have been observed (Napadow et al., 2010), and changes herein and in posterior insula glutamate levels have been observed following treatment-induced pain reductions (Napadow et al., 2012; Harris et al., 2013). Two prefrontal clusters, one in dorsal, one in ventral lateral PFC and a subcortical NAc cluster showed decreased activation from pre-EXP to 6 months follow-up. The NAc is a major reward center of the brain, and has been implicated in the regulation of pain (Woo et al., 2015) and in the chronification of pain (Baliki et al., 2012; Borsook et al., 2016). It is also associated with experiencing pain in the chronic phase (Hashmi et al., 2013), representing its motivational value. Our finding indicates that EXP also induces changes in the motivational component of pain and associated pain-related cues (e.g., reduced motivational 


\section{A Pre- to post-EXP}

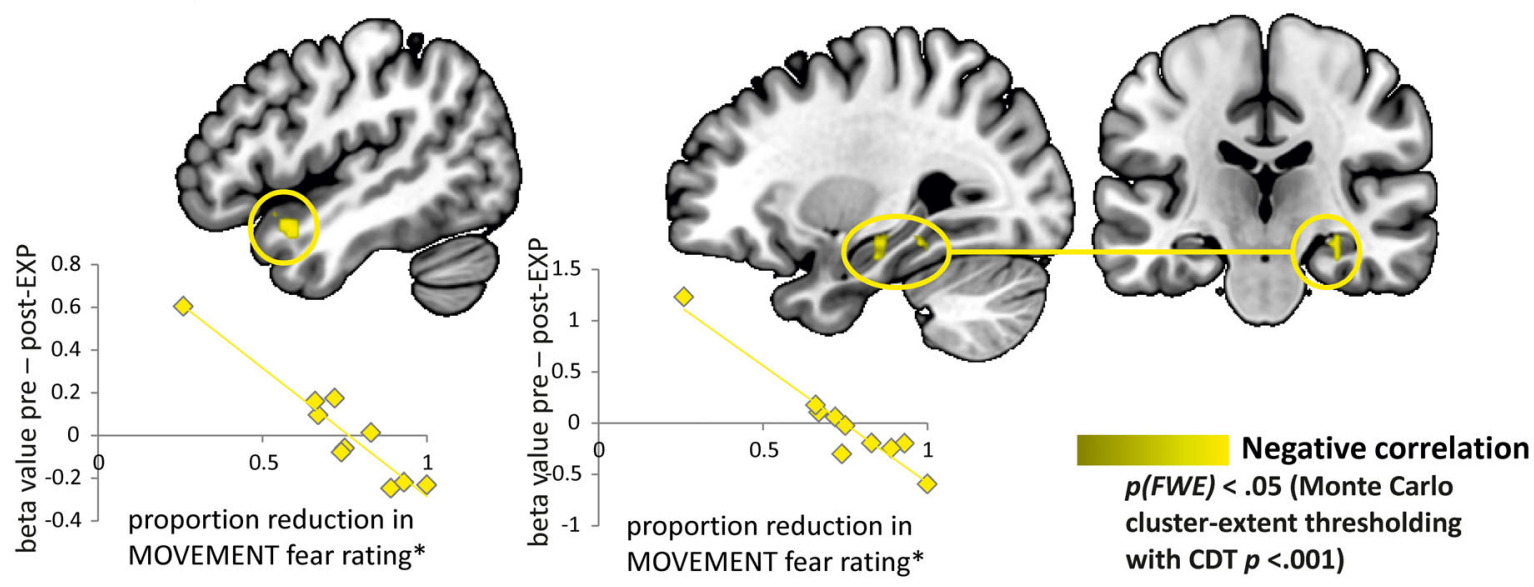

\section{B Pre- to FU-EXP}
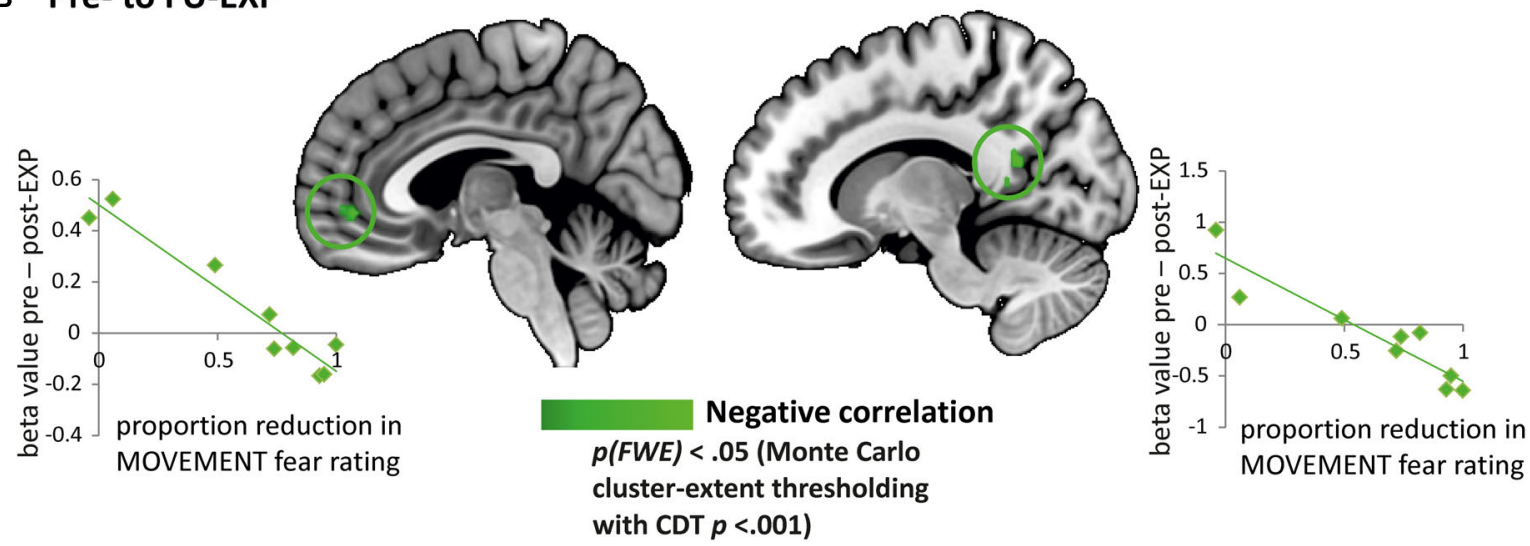

FIGURE 8 | Explorative analyses: EXP treatment-induced changes in fear correlate with changes in neural responses to pain-related fear. (A) Brain regions showing a correlation between change in fear rating and change in neural activation (beta value) from pre- to post-EXP, corresponding to the left temporal pole and the left hippocampus (yellow). The scatterplots present the correlations between the change in neural activation in temporal pole (left) and hippocampus (right) with the proportion of reduction in fear ratings from pre- to post-EXP. *Note that the correlations were evaluated with and without the outlier (i.e., the individual with the lowest reduction in MOVEMENT fear rating). The outlier was not influential, as the correlations were still highly significant. (B) Brain regions showing a correlation between change in fear rating and change in neural activation (beta value) from pre- to FU-EXP, corresponding to the ventromedial prefrontal cortex (vmPFC)/anterior cingulate cortex (ACC) and the posterior cingulate cortex (PCC) (green). The scatterplots present the correlations between the change in neural activation in vmPFC (left) and PCC (right) with the proportion of reduction in fear ratings from pre- to FU-EXP. Cluster-level correction using $p<0.005$ as initial cluster-defining threshold (CDT).

salience of the back-related pictures following EXP). The dlPFC is also involved in the regulation of pain (Lorenz et al., 2003; Seminowicz and Moayedi, 2017), and abnormally increased activation has been observed in chronic pain (Seminowicz and Moayedi, 2017). Interestingly, following treatment, activation in the dlPFC during a cognitively demanding task as well as increases in cortical thickness were normalized (Seminowicz et al., 2011). In contrast, the vlPFC has been associated with affective/motivational processing, and control of goal-directed behavior (Taylor et al., 2004; Sakagami and Pan, 2007). It has extensive connections with orbitofrontal cortex and subcortical areas such as the amygdala, and also interacts with motor regions to orient attention (Corbetta and Shulman, 2002). Neural changes in this region to pain stimuli have been observed following CBT in fibromyalgia, but in opposite directions (Jensen et al., 2012). Importantly, additional analyses show that such changes did not occur in controls (Supplementary Information), suggesting that these time-dependent changes are not due to general habituation effects, but instead specific to the patient group and likely attributable to treatment.

\section{Neural Changes Specific to Reductions in Pain-Related Fear Ratings}

We explored whether fear reduction was associated with specific changes in neural activation to our stimuli. In these explorative analyses, we found indications that pre- to post-EXP decreases in fear ratings were associated with neural activation increases in right hippocampus and left temporal pole. Decreased ratings from pre-EXP to follow-up were associated with increases in the mPFC and PCC. The mPFC, PCC, and hippocampus are associated with fear extinction (Sehlmeyer et al., 2009). Reduced 
hippocampal volumes and abnormal hippocampal connectivity have been reported in chronic pain (Mutso et al., 2012, 2013). Treatment-induced increases in $\mathrm{mPFC}$ neural activation in relation to decreases in fear is in agreement with increased inhibitory control occurring during fear extinction. Cautiously, our findings suggest that extinction during EXP may reflect similar working mechanisms as observed during experimental extinction studies. Noted, the initial cluster-defining statistical threshold (CDT) for cluster-size thresholding was slightly less conservative $(p<0.005)$, which we consider fair given the additional constraints of the analysis. Also note that these regions did not show main effects across treatment, suggesting individual rather than group-level differences. Future analyses will have to investigate whether there are functional connectivity alterations between $\mathrm{mPFC}$ and amygdala, which would be the hypothesized mechanism of extinction (Phelps et al., 2004; but also see Fullana et al., 2018a; Morriss et al., 2018).

\section{Limitations and Future Considerations}

Our findings should be interpreted in light of its limitations. First, there was no control treatment, hence we cannot infer that neural changes are specific to EXP. Though, our pain-free control group did control for effects of practice and time. And as we focused on pain-related fear -the main target of EXP-, related findings to within-session fear ratings as well as to clinical assessments of pain-related outcomes, this adds to the specificity of our findings. Second, the focus here is on the MOVEMENT category, because it is most relevant for our patient group, but also for simplicity reasons. Not all findings were specific to this category (e.g., the other two categories also showed preEXP posterior insula differences). However, most importantly, time-dependent changes in these regions were specific to this category (Supplementary Information). Finally, the relatively small sample size may have comprimised our statistical power, and motivated us to focus on the whole-brain correlation analysis only (i.e., no other correlations with changes over time), limiting the generalizability of our findings. Several participants could not be included in our analyses or were lost to follow-up, partly because our study was conducted amidst clinical standard care (e.g., the patient and/or clinical team decided not to start EXP), and partly due to the challenges of conducting MRI research in clinical pain populations. Despite that, we show strong data of group differences as well as changes across time, all surviving stringent statistical testing. Larger samples will be needed to reproduce the current findings, and to extend to models predicting treatment responses.

\section{CONCLUSION}

We show the first evidence that clinical improvements in chronic pain following EXP treatment are mirrored by changes in pain-related fear neural circuitry. Group differences identified prior to treatment were no longer present after treatment. Time-dependent effects in patients continued up to 6 months after the end of EXP, and involved regions implicated in cognitive/affective, motivational as well as sensory aspects related to pain. This suggests that the effects of EXP are long-term and go above and beyond modulating fear circuitry. Lastly, explorative analyses found indications that brain regions implicated in fear extinction -including the hippocampus, PCC and mPFCchanged their neural response proportionate to the change in self-reported fear, suggesting that extinction during EXP may reflect similar working mechanisms as extinction in experimental settings. Taken together, our findings show that neural circuitry for pain-related fear is modulated by EXP, and that changes are associated with self-reported improvements in pain-related fear.

\section{DATA AVAILABILITY}

The datasets generated for this study are available on reasonable request to the corresponding author.

\section{ETHICS STATEMENT}

This study involving human participants was reviewed and approved by the Medical Ethical Committee of Maastricht University Hospital/Maastricht University (MUMC+/UM). All participants provided their written informed consent to participate in this study.

\section{AUTHOR CONTRIBUTIONS}

IT, JJ, MG, JV, RS, and AK contributed to the conception and design of the study. IT and AK acquired the data and contributed to the data analysis plan. IT performed the data analysis and wrote the manuscript. All authors contributed to the manuscript, and read and approved the final version.

\section{FUNDING}

This work was supported by a grant from the Health Foundation Limburg (Stichting Sint Annadal, Maastricht), Board of Directors of Maastricht University Medical Center (MUMC+), and Esperance Foundation (Stichting Esperance).

\section{ACKNOWLEDGMENTS}

We would like to thank all participants for their time and effort. We would also like to thank the staff of the Department of Rehabilitation Medicine, and in particular Daniëlle Wijnants, and Department of Radiology of MUMC+/Adelante for their help in (coordinating) the study visits and support with scanning, respectively, and Emma Biggs, Judith Eck, and Johan Vlaeyen for fruitful discussions.

\section{SUPPLEMENTARY MATERIAL}

The Supplementary Material for this article can be found online at: https://www.frontiersin.org/articles/10.3389/fnins.2019. 00970/full\#supplementary-material 


\section{REFERENCES}

Baliki, M. N., Geha, P. Y., Jabakhanji, R., Harden, N., Schnitzer, T. J., and Apkarian, A. V. (2008). A preliminary fMRI study of analgesic treatment in chronic back pain and knee osteoarthritis. Mol. Pain 4:47. doi: 10.1186/1744-8069-4-47

Baliki, M. N., Petre, B., Torbey, S., Herrmann, K. M., Huang, L., Schnitzer, T. J., et al. (2012). Corticostriatal functional connectivity predicts transition to chronic back pain. Nat. Neurosci. 15, 1117-1119. doi: 10.1038/nn.3153

Becerra, L., Sava, S., Simons, L. E., Drosos, A. M., Sethna, N., Berde, C., et al. (2014). Intrinsic brain networks normalize with treatment in pediatric complex regional pain syndrome. Neuroimage Clin. 6, 347-369. doi: 10.1016/j.nicl.2014. 07.012

Boersma, K., Linton, S., Overmeer, T., Jansson, M., Vlaeyen, J., and De Jong, J. (2004). Lowering fear-avoidance and enhancing function through exposure in vivo. A multiple baseline study across six patients with back pain. Pain 108, 8-16. doi: 10.1016/j.pain.2003.03.001

Borsook, D., Linnman, C., Faria, V., Strassman, A. M., Becerra, L., and Elman, I. (2016). Reward deficiency and anti-reward in pain chronification. Neurosci. Biobehav. Rev. 68, 282-297. doi: 10.1016/j.neubiorev.2016.05.033

Bosma, R. L., Cheng, J. C., Rogachov, A., Kim, J. A., Hemington, K. S., Osborne, N. R., et al. (2018). Brain dynamics and temporal summation of pain predicts neuropathic pain relief from ketamine infusion. Anesthesiology 129, 1015-1024. doi: 10.1097/ALN.0000000000002417

Breivik, H., Collett, B., Ventafridda, V., Cohen, R., and Gallacher, D. (2006). Survey of chronic pain in Europe: prevalence, impact on daily life, and treatment. Eur. J. Pain 10, 287-333.

Bunzli, S., Smith, A., Schutze, R., and O'sullivan, P. (2015). Beliefs underlying pain-related fear and how they evolve: a qualitative investigation in people with chronic back pain and high pain-related fear. BMJ Open 5:e008847. doi: 10.1136/bmjopen-2015-008847

Camacho-Soto, A., Sowa, G. A., Perera, S., and Weiner, D. K. (2012). Fear avoidance beliefs predict disability in older adults with chronic low back pain. PM R 4, 493-497. doi: 10.1016/j.pmrj.2012.01.017

Cavanna, A. E., and Trimble, M. R. (2006). The precuneus: a review of its functional anatomy and behavioural correlates. Brain 129, 564-583. doi: 10.1093/brain/ awl004

Corbetta, M., and Shulman, G. L. (2002). Control of goal-directed and stimulusdriven attention in the brain. Nat. Rev. Neurosci. 3, 201-215. doi: 10.1038/ nrn755

Craig, A. D. (2002). How do you feel? Interoception: the sense of the physiological condition of the body. Nat. Rev. Neurosci. 3, 655-666. doi: 10.1038/nrn894

Crombez, G., Vlaeyen, J. W. S., Heuts, P. H., and Lysens, R. (1999). Pain-related fear is more disabling than pain itself: evidence on the role of pain-related fear in chronic back pain disability. Pain 80, 329-339. doi: 10.1016/s0304-3959(98) 00229-2

de Jong, J. R., Vangronsveld, K., Peters, M. L., Goossens, M. E. J. B., Onghena, P., Bulté, I., et al. (2008). Reduction of pain-related fear and disability in posttraumatic neck pain: a replicated single-case experimental study of exposure in vivo. J. Pain 9, 1123-1134. doi: 10.1016/j.jpain.2008.06

de Jong, J. R., Vlaeyen, J. W. S., Onghena, P., Goossens, M. E. J. B., Geilen, M., and Mulder, H. (2005). Fear of movement/(re)injury in chronic low back pain: education or exposure in vivo as mediator to fear reduction? Clin. J. Pain 21, 9-17. doi: 10.1097/00002508-200501000-00002

de Jong, J. R., Vlaeyen, J. W. S., Van Eijsden, M., Loo, C., and Onghena, P. (2012). Reduction of pain-related fear and increased function and participation in work-related upper extremity pain (WRUEP): effects of exposure in vivo. Pain 153, 2109-2118. doi: 10.1016/j.pain.2012.07.001

den Hollander, M., Goossens, M. E. J. B., De Jong, J. R., Ruijgrok, J., Oosterhof, J., Onghena, P., et al. (2016). Expose or protect? A randomized controlled trial of exposure in vivo versus physiotherapy in patients with complex regional pain syndrome type 1. Pain 157, 2318-2329. doi: 10.1097/j.pain.0000000000000651

den Hollander, M., Meulders, A., Jakobs, M., and Vlaeyen, J. W. (2015). The effect of threat information on acquisition, extinction, and reinstatement of experimentally conditioned fear of movement-related pain. Pain Med. 16, 2302-2315. doi: 10.1111/pme.12836

Desikan, R. S., Segonne, F., Fischl, B., Quinn, B. T., Dickerson, B. C., Blacker, D., et al. (2006). An automated labeling system for subdividing the human cerebral cortex on MRI scans into gyral based regions of interest. Neuroimage 31, 968-980. doi: 10.1016/j.neuroimage.2006.01.021

Erpelding, N., Simons, L., Lebel, A., Serrano, P., Pielech, M., Prabhu, S., et al. (2014). Rapid treatment-induced brain changes in pediatric CRPS. Brain Struct. Funct. 221, 1095-1111. doi: 10.1007/s00429-014-0957-8

Flodin, P., Martinsen, S., Lofgren, M., Bileviciute-Ljungar, I., Kosek, E., and Fransson, P. (2014). Fibromyalgia is associated with decreased connectivity between pain- and sensorimotor brain areas. Brain Connect. 4, 587-594. doi: 10.1089/brain.2014.0274

Frazier, J. A., Chiu, S., Breeze, J. L., Makris, N., Lange, N., Kennedy, D. N., et al. (2005). Structural brain magnetic resonance imaging of limbic and thalamic volumes in pediatric bipolar disorder. Am. J. Psychiatry 162, 1256-1265. doi: 10.1176/appi.ajp.162.7.1256

Fullana, M. A., Albajes-Eizagirre, A., Soriano-Mas, C., Vervliet, B., Cardoner, N., Benet, O., et al. (2018a). Amygdala where art thou? Neurosci. Biobehav. Rev. $102,430-431$.

Fullana, M. A., Albajes-Eizagirre, A., Soriano-Mas, C., Vervliet, B., Cardoner, N., Benet, O., et al. (2018b). Fear extinction in the human brain: a meta-analysis of fMRI studies in healthy participants. Neurosci. Biobehav. Rev. 88, 16-25. doi: 10.1016/j.neubiorev.2018.03.002

Fullana, M. A., Harrison, B. J., Soriano-Mas, C., Vervliet, B., Cardoner, N., AvilaParcet, A., et al. (2016). Neural signatures of human fear conditioning: an updated and extended meta-analysis of fMRI studies. Mol. Psychiatry 21, 500-508. doi: 10.1038/mp.2015.88

Garcia-Larrea, L., and Peyron, R. (2013). Pain matrices and neuropathic pain matrices: a review. Pain 154(Suppl.), S29-S43. doi: 10.1016/j.pain.2013.09.001

GBD 2015 Disease and Injury Incidence and Prevalence Collaborators (2016). Global, regional, and national incidence, prevalence, and years lived with disability for 310 diseases and injuries, 1990-2015: a systematic analysis for the global burden of disease study 2015. Lancet 388, 1545-1602. doi: 10.1016/ S0140-6736(16)31678-6

Glombiewski, J. A., Holzapfel, S., Riecke, J., Vlaeyen, J. W. S., De Jong, J., Lemmer, G., et al. (2018). Exposure and CBT for chronic back pain: an RCT on differential efficacy and optimal length of treatment. J. Consult. Clin. Psychol. 86, 533-545. doi: 10.1037/ccp0000298

Goffaux, P., Girard-Tremblay, L., Marchand, S., Daigle, K., and Whittingstall, K. (2014). Individual differences in pain sensitivity vary as a function of precuneus reactivity. Brain Topogr. 27, 366-374. doi: 10.1007/s10548-013-0291-0

Goldstein, J. M., Seidman, L. J., Makris, N., Ahern, T., O’brien, L. M., Caviness, V. S., et al. (2007). Hypothalamic abnormalities in schizophrenia: sex effects and genetic vulnerability. Biol. Psychiatry 61, 935-945. doi: 10.1016/j.biopsych. 2006.06.027

Harris, R. E., Napadow, V., Huggins, J. P., Pauer, L., Kim, J., Hampson, J., et al. (2013). Pregabalin rectifies aberrant brain chemistry, connectivity, and functional response in chronic pain patients. Anesthesiology 119, 1453-1464. doi: 10.1097/ALN.0000000000000017

Hartley, C. A., and Phelps, E. A. (2010). Changing fear: the neurocircuitry of emotion regulation. Neuropsychopharmacology 35, 136-146. doi: 10.1038/npp. 2009.121

Hartvigsen, J., Hancock, M. J., Kongsted, A., Louw, Q., Ferreira, M. L., Genevay, S., et al. (2018). What low back pain is and why we need to pay attention. Lancet 391, 2356-2367. doi: 10.1016/S0140-6736(18)30480-X

Hashmi, J. A., Baliki, M. N., Huang, L., Baria, A. T., Torbey, S., Hermann, K. M., et al. (2013). Shape shifting pain: chronification of back pain shifts brain representation from nociceptive to emotional circuits. Brain 136, 2751-2768. doi: 10.1093/brain/awt211

Hetu, S., Gregoire, M., Saimpont, A., Coll, M. P., Eugene, F., Michon, P. E., et al. (2013). The neural network of motor imagery: an ALE meta-analysis. Neurosci. Biobehav. Rev. 37, 930-949. doi: 10.1016/j.neubiorev.2013.03.017

Icenhour, A., Langhorst, J., Benson, S., Schlamann, M., Hampel, S., Engler, H., et al. (2015). Neural circuitry of abdominal pain-related fear learning and reinstatement in irritable bowel syndrome. Neurogastroenterol. Motil. 27, 114-127. doi: 10.1111/nmo.12489

Jensen, K. B., Kosek, E., Wicksell, R., Kemani, M., Olsson, G., Merle, J. V., et al. (2012). Cognitive behavioral therapy increases pain-evoked activation of the prefrontal cortex in patients with fibromyalgia. Pain 153, 1495-1503. doi: 10. 1016/j.pain.2012.04.010 
Kattoor, J., Gizewski, E. R., Kotsis, V., Benson, S., Gramsch, C., Theysohn, N., et al. (2013). Fear conditioning in an abdominal pain model: neural responses during associative learning and extinction in healthy subjects. PLoS One 8:e51149. doi: 10.1371/journal.pone.0051149

Kori, S. H. (1990). Kinisophobia: a new view of chronic pain behavior. Pain Manage 3, 35-43.

Kregel, J., Meeus, M., Malfliet, A., Dolphens, M., Danneels, L., Nijs, J., et al. (2015). Structural and functional brain abnormalities in chronic low back pain: a systematic review. Semin. Arthritis Rheum. 45, 229-237. doi: 10.1016/ j.semarthrit.2015.05.002

Labus, J. S., Hubbard, C. S., Bueller, J., Ebrat, B., Tillisch, K., Chen, M., et al. (2013). Impaired emotional learning and involvement of the corticotropinreleasing factor signaling system in patients with irritable bowel syndrome. Gastroenterology 145, e1-e3. doi: 10.1053/j.gastro.2013.08.016

Lalouni, M., Olen, O., Bonnert, M., Hedman, E., Serlachius, E., and Ljotsson, B. (2016). Exposure-Based cognitive behavior therapy for children with abdominal pain: a pilot trial. PLoS One 11:e0164647. doi: 10.1371/journal.pone.0164647

Lang, P. J., Bradley, M. M., and Cuthbert, B. N. (1997). International Affective Picture System (IAPS): Technical Manual and Affective Ratings. Gainesville, FL: NIMH Center for the Study of Emotion and Attention, 39-58.

LeDoux, J. E. (1993). Emotional memory: in search of systems and synapses. Ann. N. Y. Acad. Sci. 702, 149-157. doi: 10.1111/j.1749-6632.1993.tb17246.x

Leeuw, M., Goossens, M. E., Van Breukelen, G. J., Boersma, K., and Vlaeyen, J. W. (2007). Measuring perceived harmfulness of physical activities in patients with chronic low back pain: the Photograph Series of Daily Activitiesshort electronic version. J. Pain 8, 840-849. doi: 10.1016/j.jpain.2007. 05.013

Leeuw, M., Goossens, M. E. J. B., Van Breukelen, G. J. P., De Jong, J. R., Heuts, P. H. T. G., Smeets, R. J. E. M., et al. (2008). Exposure in vivo versus operant graded activity in chronic low back pain patients: results of a randomized controlled trial. Pain 138, 192-207. doi: 10.1016/j.pain.2007.12.009

Linnman, C., Moulton, E. A., Barmettler, G., Becerra, L., and Borsook, D. (2012). Neuroimaging of the periaqueductal gray: state of the field. Neuroimage 60, 505-522. doi: 10.1016/j.neuroimage.2011.11.095

Lopez-de-Uralde-Villanueva, I., Munoz-Garcia, D., Gil-Martinez, A., PardoMontero, J., Munoz-Plata, R., Angulo-Diaz-Parreno, S., et al. (2016). A systematic review and meta-analysis on the effectiveness of graded activity and graded exposure for chronic nonspecific low back pain. Pain Med. 17, 172-188.

Lorenz, J., Minoshima, S., and Casey, K. L. (2003). Keeping pain out of mind: the role of the dorsolateral prefrontal cortex in pain modulation. Brain 126, 1079-1091. doi: 10.1093/brain/awg102

Makris, N., Goldstein, J. M., Kennedy, D., Hodge, S. M., Caviness, V. S., Faraone, S. V., et al. (2006). Decreased volume of left and total anterior insular lobule in schizophrenia. Schizophr. Res. 83, 155-171. doi: 10.1016/j.schres.2005.11.020

McNeil, D. W., and Rainwater, A. J. (1998). Development of the fear of pain questionnaire-III. J. Behav. Med. 21, 389-410.

McNorgan, C. (2012). A meta-analytic review of multisensory imagery identifies the neural correlates of modality-specific and modality-general imagery. Front. Hum. Neurosci. 6:285. doi: 0.3389/fnhum.2012.00285

Meier, M. L., Stampfli, P., Humphreys, B. K., Vrana, A., Seifritz, E., and Schweinhardt, P. (2017). The impact of pain-related fear on neural pathways of main modulation in chronic low back pain. Pain Rep. 2:e601. doi: 10.1097/ PR9.0000000000000601

Meier, M. L., Stampfli, P., Vrana, A., Humphreys, B. K., Seifritz, E., and HotzBoendermaker, S. (2016). Neural correlates of fear of movement in patients with chronic low Back Pain vs. Pain-Free individuals. Front. Hum. Neurosci. 10:386. doi: 10.3389/fnhum.2016.00386

Meulders, A., and Vlaeyen, J. W. (2012). Reduction of fear of movementrelated pain and pain-related anxiety: an associative learning approach using a voluntary movement paradigm. Pain 153, 1504-1513. doi: 10.1016/j.pain.2012. 04.013

Morriss, J., Hoare, S., and Van Reekum, C. M. (2018). It's time: a commentary on fear extinction in the human brain using fMRI. Neurosci. Biobehav. Rev. 94, 321-322. doi: 10.1016/j.neubiorev.2018.06.025

Mutso, A. A., Petre, B., Huang, L., Baliki, M. N., Torbey, S., Herrmann, K. M., et al. (2013). Reorganization of hippocampal functional connectivity with transition to chronic back pain. J. Neurophysiol. 111, 1065-1076. doi: 10.1152/jn.00611. 2013
Mutso, A. A., Radzicki, D., Baliki, M. N., Huang, L., Banisadr, G., Centeno, M. V., et al. (2012). Abnormalities in hippocampal functioning with persistent pain. J. Neurosci. 32, 5747-5756. doi: 10.1523/JNEUROSCI.0587-12.2012

Napadow, V., Kim, J., Clauw, D. J., and Harris, R. E. (2012). Decreased intrinsic brain connectivity is associated with reduced clinical pain in fibromyalgia. Arthritis Rheum. 64, 2398-2403. doi: 10.1002/art.34412

Napadow, V., Lacount, L., Park, K., As-Sanie, S., Clauw, D. J., and Harris, R. E. (2010). Intrinsic brain connectivity in fibromyalgia is associated with chronic pain intensity. Arthritis Rheum. 62, 2545-2555. doi: 10.1002/art. 27497

Ong, W. Y., Stohler, C. S., and Herr, D. R. (2018). Role of the Prefrontal Cortex in Pain Processing. Mol. Neurobiol. 56, 1137-1166. doi: 10.1007/s12035-0181130-9

Peyron, R., Laurent, B., and Garcia-Larrea, L. (2000). Functional imaging of brain responses to pain: a review and meta-analysis. Neuropsychol. Clin. 30, 263-288. doi: 10.1016/s0987-7053(00)00227-6

Phelps, E. A., Delgado, M. R., Nearing, K. I., and Ledoux, J. E. (2004). Extinction learning in humans: role of the amygdala and vmPFC. Neuron 43, 897-905.

Sakagami, M., and Pan, X. (2007). Functional role of the ventrolateral prefrontal cortex in decision making. Curr. Opin. Neurobiol. 17, 228-233. doi: 10.1016/j. conb.2007.02.008

Schweinhardt, P., and Bushnell, M. C. (2010). Pain imaging in health and disease how far have we come? J. Clin. Investig. 120, 3788-3797. doi: 10.1172/JCI43498

Segerdahl, A. R., Mezue, M., Okell, T. W., Farrar, J. T., and Tracey, I. (2015). The dorsal posterior insula subserves a fundamental role in human pain. Nat. Neurosci. 18, 499-500. doi: 10.1038/nn.3969

Sehlmeyer, C., Schoning, S., Zwitserlood, P., Pfleiderer, B., Kircher, T., Arolt, V., et al. (2009). Human fear conditioning and extinction in neuroimaging: a systematic review. PLoS One 4:e5865. doi: 10.1371/journal.pone.0005865

Seminowicz, D., Wideman, T. H., Naso, L., Hatami-Khoroushahi, Z., Fallatah, S., Ware, M., et al. (2011). Effective treatment of chronic low back pain in humans reverses abnormal brain anatomy and function. J. Neurosci. 31, 7540-7550. doi: 10.1523/JNEUROSCI.5280-10.2011

Seminowicz, D. A., and Moayedi, M. (2017). The dorsolateral prefrontal cortex in acute and chronic pain. J. Pain 18, 1027-1035. doi: 10.1016/j.jpain.2017.03.008

Simons, L. E., Moulton, E. A., Linnman, C., Carpino, E., Becerra, L., and Borsook, D. (2012). The human amygdala and pain: evidence from neuroimaging. Hum. Brain Mapp. 35, 527-538. doi: 10.1002/hbm.22199

Simons, L. E., Pielech, M., Erpelding, N., Linnman, C., Moulton, E., Sava, S., et al. (2014). The responsive amygdala: treatment-induced alterations in functional connectivity in pediatric complex regional pain syndrome. Pain 155, 1727-1742. doi: 10.1016/j.pain.2014.05.023

Soer, R., Koke, A. J., Vroomen, P. C., Stegeman, P., Smeets, R. J., Coppes, M. H., et al. (2013). Extensive validation of the pain disability index in 3 groups of patients with musculoskeletal pain. Spine 38, E562-E568. doi: 10.1097/BRS. 0b013e31828af21f

Sotres-Bayon, F., Cain, C. K., and Ledoux, J. E. (2006). Brain mechanisms of fear extinction: historical perspectives on the contribution of prefrontal cortex. Biol. Psychiatry 60, 329-336. doi: 10.1016/j.biopsych.2005.10.012

Spielberger, C. D., Gorsuch, R. L., Lushene, P. R., Vagg, P. R., and Jacobs, A. G. (1983). Manual for the State-Trait Anxiety Inventory (Form Y). Palo Alto, CA: Consulting Psychologists Press.

Sullivan, M. J., Bishop, S. R., and Pivik, J. (1995). The pain catastrophizing scale: development and validation. Psychol. Assess. 7, 524-532. doi: 10.1037//10403590.7.4.524

Tait, R. C., Pollard, C. A., Margolis, R. B., Duckro, P. N., and Krause, S. J. (1987). The pain disability index: psychometric and validity data. Arch. Phys. Med. Rehabil. 68, 438-441.

Taylor, A. M., Harris, A. D., Varnava, A., Phillips, R., Taylor, J. O., Hughes, O., et al. (2015). A functional magnetic resonance imaging study to investigate the utility of a picture imagination task in investigating neural responses in patients with chronic musculoskeletal pain to daily physical activity photographs. PLoS One 10:e0141133. doi: 10.1371/journal.pone.0141133

Taylor, S. F., Welsh, R. C., Wager, T. D., Phan, K. L., Fitzgerald, K. D., and Gehring, W. J. (2004). A functional neuroimaging study of motivation and executive function. Neuroimage 21, 1045-1054. doi: 10.1016/j.neuroimage.2003.10.032

Thibodeau, M. A., Fetzner, M. G., Carleton, R. N., Kachur, S. S., and Asmundson, G. J. (2013). Fear of injury predicts self-reported and behavioral impairment in 
patients with chronic low back pain. J. Pain 14, 172-181. doi: 10.1016/j.jpain. 2012.10.014

Tracey, I. (2010). Getting the pain you expect: mechanisms of placebo, nocebo and reappraisal effects in humans. Nat. Med. 16, 1277-1283. doi: 10.1038/nm.2229

van der Ploeg, H. M., Defares, P. B., Spielberger, C. D., Defares, P. B., and Spielberger, C. D. (1980). Handleiding bij de Zelf-Beoordelings Vragenlijst ZBV: een nederlandstalige bewerking van de Spielberger State-trait Anxiety Inventory STAI-DY. [Manual for the Self-Assessment Questionnaire ZBV: a Dutch-language adaptation of the Spielberger State-Trait Anxiety Inventory STAI-DY.]. Lisse: Swets \& Zeitlinger.

van Wijk, A. J., and Hoogstraten, J. (2006). Dutch translation of the fear of pain questionnaire: factor structure, reliability and validity. Eur. J. Pain 10, 479-486.

Verbunt, J. A. (2008). Reliability and validity of the PAD questionnaire: a measure to assess pain-related decline in physical activity. J. Rehabil. Med. 40, 9-14. doi: 10.2340/16501977-0126

Vercoulen, J. H., Bazelmans, E., Swanink, C. M., Fennis, J. F., Galama, J. M., Jongen, P. J., et al. (1997). Physical activity in chronic fatigue syndrome: assessment and its role in fatigue. J. Psychiatr. Res. 31, 661-673.

Vlaeyen, J. W., Crombez, G., and Linton, S. J. (2016). The fear-avoidance model of pain. Pain 157, 1588-1589. doi: 10.1097/j.pain.0000000000000574

Vlaeyen, J. W., De Jong, J., Geilen, M., Heuts, P. H., and Van Breukelen, G. (2001). Graded exposure in vivo in the treatment of pain-related fear: a replicated single-case experimental design in four patients with chronic low back pain. Behav. Res. Ther. 39, 151-166. doi: 10.1016/s0005-7967(99)00174-6

Vlaeyen, J. W., Kole-Snijders, A. M., Boeren, R. G., and Van Eek, H. (1995a). Fear of movement/(re)injury in chronic low back pain and its relation to behavioral performance. Pain 62, 363-372. doi: 10.1016/0304-3959(94) 00279-n

Vlaeyen, J. W. S., Kole-Snijders, A. M., Rotteveel, A. M., Ruesink, R., and Heuts, P. H. (1995b). The role of fear of movement/(re)injury in pain disability. J. Occup. Rehabil. 5, 235-252. doi: 10.1007/bf02109988

Vlaeyen, J. W., Morley, S. J., Linton, S. J., Boersma, K., and De Jong, J. (2012). Painrelated Fear: Exposure-Based Treatment for Chronic Pain. Seattle, WA: IASP Press.

Vlaeyen, J. W. S., and Crombez, G. (1999). Fear of movement/(re)injury, avoidance and pain disability in chronic low back pain patients. Man. Ther. 4, 187-195. doi: 10.1054/math.1999.0199
Wager, T. D., Atlas, L. Y., Lindquist, M. A., Roy, M., Woo, C.-W., and Kross, E. (2013). An fMRI-based neurologic signature of physical pain. New Engl. J. Med. 368, 1388-1397. doi: 10.1056/NEJMoa1204471

Wiech, K., Jbabdi, S., Lin, C. S., Andersson, J., and Tracey, I. (2014). Differential structural and resting state connectivity between insular subdivisions and other pain-related brain regions. Pain 155, 2047-2055. doi: 10.1016/j.pain.2014.07. 009

Woo, C. W., Krishnan, A., and Wager, T. D. (2014). Cluster-extent based thresholding in fMRI analyses: pitfalls and recommendations. Neuroimage 91, 412-419. doi: 10.1016/j.neuroimage.2013.12.058

Woo, C. W., Roy, M., Buhle, J. T., and Wager, T. D. (2015). Distinct brain systems mediate the effects of nociceptive input and self-regulation on pain. PLoS Biol. 13:e1002036. doi: 10.1371/journal.pbio.1002036

Woods, M. P., and Asmundson, G. J. (2008). Evaluating the efficacy of graded in vivo exposure for the treatment of fear in patients with chronic back pain: a randomized controlled clinical trial. Pain 136, 271-280. doi: 10.1016/j.pain. 2007.06.037

Zale, E. L., Lange, K. L., Fields, S. A., and Ditre, J. W. (2013). The relation between pain-related fear and disability: a meta-analysis. J. Pain 14, 1019-1030. doi: 10.1016/j.jpain.2013.05.005

Zhu, X., Suarez-Jimenez, B., Lazarov, A., Helpman, L., Papini, S., Lowell, A., et al. (2018). Exposure-based therapy changes amygdala and hippocampus resting-state functional connectivity in patients with posttraumatic stress disorder. Depress. Anxiety 35, 974-984. doi: 10.1002/da. 22816

Conflict of Interest Statement: The authors declare that the research was conducted in the absence of any commercial or financial relationships that could be construed as a potential conflict of interest.

Copyright (C) 2019 Timmers, de Jong, Goossens, Verbunt, Smeets and Kaas. This is an open-access article distributed under the terms of the Creative Commons Attribution License (CC BY). The use, distribution or reproduction in other forums is permitted, provided the original author(s) and the copyright owner(s) are credited and that the original publication in this journal is cited, in accordance with accepted academic practice. No use, distribution or reproduction is permitted which does not comply with these terms. 


\title{
Acupuncture Improves Comorbid Cognitive Impairments Induced by Neuropathic Pain in Mice
}

\author{
Jae-Hwan Jang ${ }^{1,2,3}$, Yu-Kang Kim ${ }^{1,2}$, Won-Mo Jung ${ }^{4}$, Hyung-Kyu Kim ${ }^{5}$, Eun-Mo Song ${ }^{6}$, \\ Hee-Young Kim ${ }^{7}$, Ju-Young Oh ${ }^{1,2,3}$, Ji-Yeun Park ${ }^{8}$, Yeonhee Ryu ${ }^{9}$, Mi-Yeon Song ${ }^{6}$ and \\ Hi-Joon Park ${ }^{1,2,3 *}$

\begin{abstract}
${ }^{1}$ Acupuncture and Meridian Science Research Center, Kyung Hee University, Seoul, South Korea, ${ }^{2}$ Department of Korean Medical Science, Graduate School of Korean Medicine, Kyung Hee University, Seoul, South Korea, ${ }^{3}$ BK21 PLUS Korean Medicine Science Center, College of Korean Medicine, Kyung Hee University, Seoul, South Korea, ${ }^{4}$ VUNO Inc., Seoul, South Korea, ${ }^{5}$ Department of Oral Physiology, School of Dentistry, Kyungpook National University, Daegu, South Korea, ${ }^{6}$ Department of Physical Medicine and Rehabilitation, Graduate School of Korean Medicine, Kyung Hee University, Seoul, South Korea, ${ }^{7}$ College of Korean Medicine, Daegu Haany University, Daegu, South Korea, ${ }^{8}$ College of Korean Medicine, Daejeon University, Daejeon, South Korea, ${ }^{9}$ Korea Institute of Oriental Medicine, Daejeon, South Korea
\end{abstract}

\section{OPEN ACCESS}

Edited by:

Lauren C. Heathcote,

Stanford University, United States

Reviewed by:

Danilo De Gregorio,

McGill University, Canada

Livio Luongo,

Second University of Naples, Italy

*Correspondence:

Hi-Joon Park

acufind@khu.ac.kr

Specialty section:

This article was submitted to

Perception Science,

a section of the journal

Frontiers in Neuroscience

Received: 21 June 2019 Accepted: 03 September 2019 Published: 20 September 2019

Citation: Jang J-H, Kim $Y-K$, Jung $W-M$, Kim H-K, Song E-M, Kim H-Y, Oh J-Y,

Park J-Y, Ryu $Y$, Song $M-Y$ and Park H-J (2019) Acupuncture Improves Comorbid Cognitive Impairments Induced by Neuropathic Pain in Mice. Front. Neurosci. 13:995, doi: 10.3389/fnins.2019.00995
Growing evidence indicates that neuropathic pain is frequently accompanied by cognitive impairments, which aggravate the quality of life of chronic pain patients. Here, we investigated whether acupuncture treatments can improve cognitive dysfunction as well as allodynia induced by neuropathic pain in mice. One week after the left partial sciatic nerve ligation (PSNL), acupuncture treatments on the acupoints GB30-GB34 (AP1), HT7-GV20 (AP2), or control points (CP) were performed for 4 weeks. Notably, the significant attenuations of mechanical allodynia and cognitive impairment were observed in the AP1 group, but not in PSNL, AP2, or CP groups. A random decision forest classifier based on the pain and cognitive functions displayed that the acupuncture group was clearly segregated from the other groups. We also demonstrated that acupuncture restored the reduced field excitatory post-synaptic potentials and was able to elevate the expression levels of glutamate receptors (NR2B and GluR1) in the hippocampus. Moreover, the expressions of $\mathrm{Ca}^{2+} /$ calmodulin-dependent protein kinase II and synaptic proteins (pPSD-95 and pSyn-1) were enhanced by acupuncture treatment. These results suggest that acupuncture can enhance hippocampal long-term action through the regulation of the synaptic efficacy and that acupuncture may provide a viable option for managing both pain and cognitive functions associated with chronic neuropathic pain.

Keywords: acupuncture, partial sciatic nerve ligation, analgesia, cognitive impairment, glutamatergic receptor

\section{INTRODUCTION}

Chronic pain conditions are among the most common causes of disability worldwide. In addition to pain and disability, chronic pain is also associated with cognitive and emotional disorders which further diminish the quality of life (Price, 2000; Nicholson and Verma, 2004; Guida et al., 2015; De Gregorio et al., 2019). Current pharmacological treatments often do not meet patients' needs due to unsatisfactory efficacy and adverse effects. In addition, the comorbidities may cause patients to seek multiple treatments, increasing their financial burdens. Thus, it is essential to enhance therapeutic 
outcomes by taking a holistic approach targeting these multidimensional aspects of chronic pain while controlling for side effects (Bergbom et al., 2011; Hopton et al., 2014).

To date, human studies have indicated that supraspinal structures including the hippocampus, anterior cingulate cortex, medial prefrontal cortex, and dorsal raphe nucleus are involved in chronic pain (Fields et al., 1977; Wang and Nakai, 1994; Valet et al., 2009; Zimmerman et al., 2009; Khan et al., 2014). In particular, the reduced hippocampal volume and changes in hippocampal structures were found in patients with chronic pain, implicating pain-related cognitive dysfunction (Valet et al., 2009; Zimmerman et al., 2009; Ezzati et al., 2014). Many studies have also shown the functional impairment of hippocampus including abnormal cytokine expression, short-term and working memory deficits as well as impairment of long-term potentiation (LTP) in animal models of chronic pain (Kodama et al., 2007, 2011; Ren et al., 2011; Wu et al., 2015).

Nerve injuries also affect synaptic plasticity and induce numerous changes in multiple neurotransmitters and intracellular signal transduction through changes in the expression or function of excitatory and inhibitory transmissions in the hippocampus (Mutso et al., 2012; Wang X.Q. et al., 2015; Liu et al., 2017; Saffarpour et al., 2017). Glutamate is a major excitatory neurotransmitter of the central nervous system, and recent studies have investigated the potential roles of the hippocampal glutamatergic system in the pathophysiology of pain (Wang X.Q. et al., 2015; Saffarpour et al., 2017). It is widely recognized that glutamate regulates excitatory synaptic transmission in the hippocampus via the NMDA receptor (NMDAR) and $\alpha$-amino-3-hydroxy5-methyl-4isoxazolpropionic acid receptor (AMPAR). An NMDAR subunit, $\mathrm{NR} 2 \mathrm{~B}$, is involved in various physiological processes including learning, memory, and synaptic plasticity by regulation of LTP induction. In addition, calcium/calmodulin-dependent protein kinase II (CaMKII) has a critical role in LTP induction. Reduced levels of glutamate receptors and CaMKII were found in the hippocampal area of the neuropathic pain model (Xu et al., 2012).

Both clinical and animal studies have shown that acupuncture significantly improves chronic pain (Kim et al., 2005, 2007). In addition, acupuncture could mitigate pain-related comorbidities such as depression and insomnia in chronic pain patients (Huang et al., 2011; Hopton et al., 2014). Several animal studies showed that acupuncture improves both the nociceptive and cognition-related behaviors in a cobra venom-induced chronic neuropathic pain model (Chen et al., 2017). However, it remains elusive how acupuncture rescues comorbid conditions as well as pain simultaneously.

In the present study, we first examined whether acupuncture could improve both cognitive and pain behaviors in the partial sciatic nerve ligation (PSNL)-induced neuropathic pain model. Next, a random decision forest classifier in machine learning was used to see if the therapeutic effects of acupuncture can be predicted based on pain and cognitive behaviors. Then, we examined the changes in glutamatergic receptors and synaptic proteins as well as LTP in the hippocampus to elucidate the molecular mechanism of therapeutic effects by acupuncture.

\section{MATERIALS AND METHODS}

\section{Animals}

Seven-week-old male C57BL/6 mice (22 to $25 \mathrm{~g}$ in body weight; Samtaco, Seoul, South Korea) and four-week-old male C57BL/6 mice (for patch clamp recording; Samtaco) were individually housed at $24 \pm 2{ }^{\circ} \mathrm{C}$ under a 12/12 h light/dark cycle (light: 08:00 to 20:00, dark: 20:00 to 08:00) for at least 7 days before conducting experiments with free access to food and water.

\section{Induction of Neuropathic Pain by PSNL}

A left hind paw PSNL model was established in accordance with the approach by Malmberg and Basbaum (1998) with a few modifications. Mice were anesthetized with rompun $(100 \mu \mathrm{l}$, intraperitoneally (i.p.); Bayer, Seoul, South Korea) and $2 \%$ zoletil (100 $\mu$ l, i.p.; Virbac S.A., Carros, France). The bilateral hind thigh was shaved, and the sciatic nerve was exposed using scissors. Then, the dorsal $1 / 3$ to $1 / 2$ of the nerve was lightly ligated with 8-0 silk (AILEE, Busan, South Korea), and the open wound was closed. In non-nerve injured group (Sham; $n=10$ ), the nerve was exposed without injury and was closed. Seven days after PSNL surgery, mice were randomly assigned to one of 5 groups: PSNL, acupuncture 1 (AP1), acupuncture 2 (AP2), control points (CP), or amitriptyline groups (each $n=10)$.

\section{Acupuncture and Control Treatments}

Acupuncture treatments were performed at acupoints GB30 and GB34 (AP1 group), HT7 and GV20 (AP2 group), or two control points (CP group) bilaterally, which continued daily for 28 consecutive days starting from day 7 after surgery (Figure 1A). GB30 (at the junction of the lateral $1 / 3$ and medial $2 / 3$ of the greater trochanter with the sacral hiatus) and GB34 (in the depression anterior and distal to the head of the fibula) are combinations of acupoints that are utilized for the treatment of lumber neuropathic pain in the clinic (Cho et al., 2013; Ju et al., 2017). HT7 (radial to the flexor carpi ulnaris tendon on the palmar crease) and GV20 (on the vertex of the head) are combinations of acupoints frequently used for the treatment of cognitive and emotional impairments (Lee et al., 2012; Chen et al., 2016; Fleckenstein et al., 2018; Yu et al., 2018). The detailed locations of acupoints were shown in Figure 1A. For the acupuncture treatments, mice were mildly immobilized, and a sterilized acupuncture needle $(8 \mathrm{~mm}$ in length and $0.18 \mathrm{~mm}$ in diameter; Haenglim-seoweon Acuneedle Co., South Korea) was inserted bilaterally to a depth of $3 \mathrm{~mm}$ and turned bi-directionally for $30 \mathrm{~s}$ at a rate of two spins per second, one $180^{\circ}$ clockwise rotation and $180^{\circ}$ counterclockwise rotation, by bare fingers. In order to rule out the non-specific effects of acupuncture, the same acupuncture stimulation was given to control points (CP group). The two control points were located at the nonacupoints, approximately $3 \mathrm{~mm}$ lateral from the middle of the medial border of the scapula or the tail base on the gluteus muscle (Figure 1A).

Amitriptyline is a tricyclic antidepressant, and is widely used to treat various neuropathic pain (Max et al., 1987; Benbouzid et al., 2008; Palazzo et al., 2016). As a positive 
A
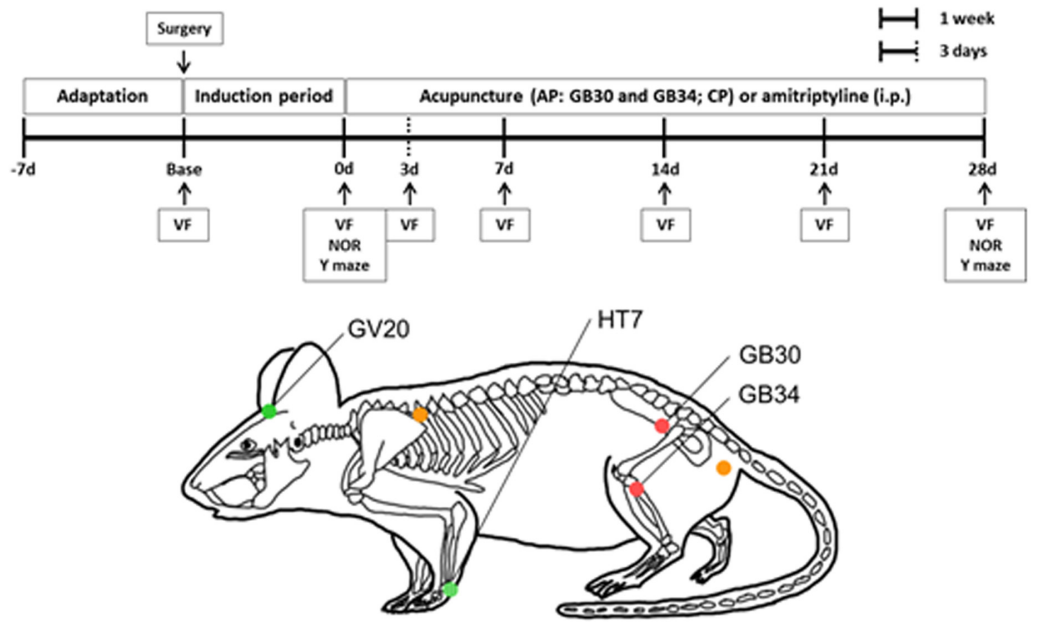

Acupuncture 1 (AP1) Acupuncture 2 (AP2) Control point (CP)

B

\section{Ipsi}

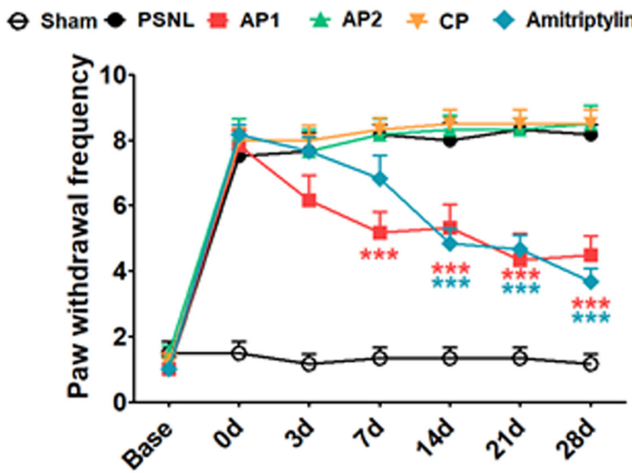

D

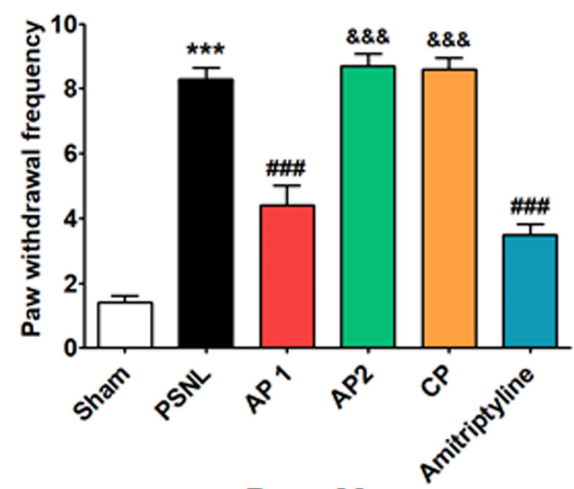

Days 28
C

Contra

$\ominus$ Sham $\bullet$ PSNL $E$ AP1 $\neq$ AP2 $\neq$ CP Amitriptyline

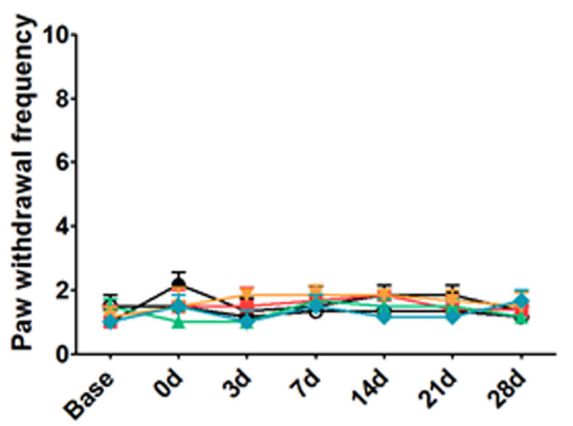

E

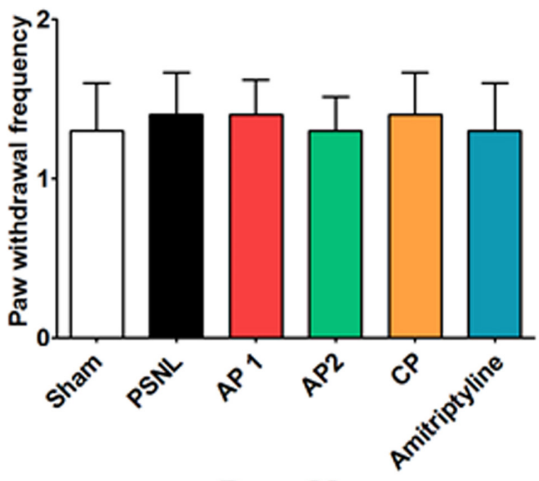

Days 28

FIGURE 1 | Effects of acupuncture on mechanical allodynia evaluated through von Fey test. Experimental design of acupuncture (AP1, AP2, or CP) or amitriptyline (10 mg/kg, i.p.) administration in the PSNL-induced neuropathic pain model. Locations of the acupuncture treatment GB30 (Hwando) and GB34 (Yanglingquan) [AP1 (red circle)], HT7 (Sinmun) and GV20 (Baekhoe) [AP2 (green circle); dapple green circle indicated backside], and control point [CP (orange circle)] (A).

${ }^{* * *} p<0.001$ vs. the PSNL group. Anti-allodynic effects of acupuncture (AP1, AP2, or CP) or amitriptyline was measured on the ipsilateral (B,D) and contralateral (C,E) plantar surfaces $2 \mathrm{~h}$ after the administration (for $0,3,7,14,21$, and 28 days). $n=10 /$ group. ${ }^{* * *} p<0.001$ vs. the Sham group, $\# \# \#<0.001$ vs. the PSNL group, and ${ }^{\& \&} p<0.001$ vs. the AP1 group. Data were analyzed with a two-way repeated measures ANOVA followed by post hoc Bonferroni tests. The results are expressed as the mean \pm SEM. 
control, amitriptyline (amitriptyline group; $10 \mathrm{mg} / \mathrm{kg}$ in $100 \mu \mathrm{l}$, Sigma-Aldrich, St. Louis, MO, United States) was given i.p. daily for 28 consecutive days from day 7 after the PSNL surgery. A solution of amitriptyline was freshly prepared on each treatment day in $0.9 \% \mathrm{NaCl}$ (Berrocoso et al., 2011). To subject animals to the equal stress condition, the Sham, PSNL and amitriptyline group animals were also mildly immobilized as done in AP and CP groups.

\section{Nociceptive Behavior Test}

The electronic von Frey test (IITC, Woodland Hills, CA, United States) was conducted (Jang et al., 2018) to evaluate the mechanical allodynia. This test was performed before surgery and treatment (Base and 0 day), respectively, and was also conducted at $3,7,14,21$, and 28 days after $2 \mathrm{~h}$ of acupuncture and amitriptyline treatment (AM 11:00-PM 06:00).

\section{Cognitive Function Tests}

Spatial working memory was measured by spontaneous alternation behavior in Y-Maze. Each mouse was placed in one of the Y-maze arms and allowed to explore freely through the maze during a 5-min session (Palazzo et al., 2016). The sequence and the total number of arms entered were recorded. An arm entry was considered complete when both hind paws were in the arm. The apparatus was cleaned with water and ethanol between each passage. Percentage of spontaneous alternation was determined by the number of triads containing entries into all three arms/maximum possible alternations (total number of arms entered - 2) × 100 (Palazzo et al., 2016).

The novel object recognition (NOR) test was used to evaluate the short-term memory. The apparatus consisted in a $40 \times 40 \times 27 \mathrm{~cm}$ acrylic box with white walls and floor. The box and objects were cleaned between trials to eliminate olfactory cues. Animals received $5 \mathrm{~min}$ sessions in the empty box for habituation to the apparatus and test room (Palazzo et al., 2016). Twenty-four hours later, each mouse was exposed to two familiar objects (block of round, diameter: $4 \mathrm{~cm}$ ) during $5 \mathrm{~min}$ "training stage" in the box. Next, the animal was placed back in the box and exposed to a novel object (block of rectangle, $4 \times 4 \times 4 \mathrm{~cm}$ ) as well as the familiar object for another 5-min "test stage" at $24 \mathrm{~h}$ after "training stage." The time spent exploring each object was measured. The recognition index reflecting the shortterm memory ability was calculated as the ratio of time spent exploring the novel object over total exploration time (Palazzo et al., 2016). All cognitive function tests were performed before treatment (AP1, AP2, CP, or amitriptyline) and on day 28 of treatment. The tests were conducted from $2 \mathrm{~h}$ after treatment (AM 11:00-PM 06:00).

\section{A Random Decision Forest Classifier in Machine Learning}

Based on the three behavioral data (mechanical allodynia, Y-maze, and NOR), a random forest classifier was used to test whether we could predict which mouse belongs to which experimental group. Random forest classifier is an ensemble method that incoporates decision tree models with multiple randomness and predicts them through the average value of the predictions of these trees (Breiman, 2001; Geurts et al., 2006). The classifier of this study was analyzed for three types of behavioral data that were labeled by the experimental group using Python's scikit learn package ${ }^{1}$ (Pedregosa et al., 2011). The classification accuracy was obtained by applying fourfold cross validation. One hundred iterations were performed to extract the average value. In addition, we randomly permutated the labeling of the experimental group by repeating the procedure 10,000 times to generate a statistical null model for comparison. In order to express the process and meaning of classification, a decision tree was obtained at four depth levels. Finally, mouse data were represented on a scatter plot using two pairs of measurements for pain and cognitive function.

\section{Western Blotting}

After anesthetized, the brain was extracted. Brain tissue samples including the hippocampus were homogenized in $200 \mu \mathrm{L}$ of lysis buffer, containing $20 \mathrm{mM}$ hydroxyethyl piperazineethanesulfonic acid ( $\mathrm{pH}$ 7.5), 1\% NP-40, 10\% glycerol, $150 \mathrm{mM} \mathrm{NaCl}$, $60 \mathrm{mM}$ B-Glucoside, $1 \mathrm{mM}$ phenylmethanesulfonyl fluoride, $0.7 \mu \mathrm{g} / \mathrm{mL}$ Pepstatin, phosphatase and protease inhibitor cocktail tablets. Western blot was performed (Park et al., 2014) to measure the protein expression levels of glutamatergic receptors and synaptic proteins in the hippocampus. Primary antibodies were rabbit anti-phospho-NR2B (pNR2B), rabbit anti-total-NR2B (tNR2B) (diluted 1:1,000; Merck Millipore, Darmstadt, Germany), rabbit anti-phospho-GluR1 (pGluR1), rabbit anti-total-GluR1 (tGluR1), rabbit anti-phospho-CaMKII (pCaMKII), rabbit anti-total-CaMKII (tCaMKII), rabbit antiphospho-protein kinase C- $\gamma$ (pPKC- $\gamma)$, rabbit anti-total- protein kinase $\mathrm{C}-\gamma$ (pPKC- $\gamma)$, rabbit anti-phospho-Syn-1 (pSyn-1), rabbit anti-total-Syn-1 (tSyn-1), rabbit anti-phospho-PSD-95 (pPSD-95), rabbit anti-total-PSD-95 (tPSD-95) (diluted 1:1,000; Cell Signaling Technology, Beverly, MA, United States), and rabbit anti- $\beta$-actin (diluted 1:10,000; Sigma-Aldrich). Then, the membrane was incubated with the secondary horseradish peroxidase-conjugated goat anti-rabbit antibody (diluted 1:1,000; Pierce, Rockford, IL, United States). The membrane was visualized using a chemiluminescence kit (Super Signal West Pico; Pierce), and the signal intensities were analyzed by a densitometry and image QI software. We show all bands before contrast modification (Supplementary Figure 4).

\section{Immunofluorescence}

Immunofluorescence was conducted (Jang et al., 2018) to measure the expression of NR2B and GluR1 in neuron cells in the PFC. Primary antibodies raised against NeuN (mouse, 1:500, MAB377; Chemicon International, Inc., Temecula, CA, United States), GFAP (mouse, 1:500, 14-9892-82; Thermo Fisher Scientific, San Diego, CA, United States), Iba-1 (mouse, 1:500, MABN92; Merck Millipore), NR2B (rabbit, 1:500, 06-600; Merck Millipore), and GluR1 (rabbit, 1:1000, \#8084; Cell Signaling Technology) were diluted in $1 \times$ PBST supplemented with $0.1 \%$ BSA. The incubation was performed in dark at $4^{\circ} \mathrm{C}$ for $72 \mathrm{~h}$.

\footnotetext{
${ }^{1}$ http://scikit-learn.org/stable/
} 
Next, following PBST washes, the sections were incubated for $1 \mathrm{~h}$ with a mixture of Alexa 488-conjugated donkey anti-rabbit secondary antibody (1:1000; Thermo Fisher Scientific) and Alexa 594-conjugated donkey anti-mouse secondary antibody (1:1000; Thermo Fisher Scientific). The numbers of NeuN/NR2B and NeuN/GluR1 double-positive cells within the CA1, CA3, and DG in the hippocampus were counted three times by a researcher blind to each group using a square grid $(300 \times 300 \mu \mathrm{m})$. The mean counts were defined as the numbers of NeuN/NR2B and NeuN/GluR1 double-positive cells. Additionally, tissues incubated without primary antibody were used as a negative control (Supplementary Figure 3).

\section{Electrophysiology}

After post-surgery day 35, electrophysiological recordings were made. The electrophysiology was performed (Citri and Malenka, 2008; Villers and Ris, 2013; Sweet et al., 2015) to measure LTP. Following isoflurane anesthesia, the brain was removed, and the hippocampus was quickly dissected out with a vibratome (VT 1200 S; Leica Microsystems, Wetzlar, Germany). Hippocampal slices $(400 \mu \mathrm{m})$ were incubated in $20 \mathrm{~mL}$ artificial cerebrospinal fluid (aCSF; containing $119 \mathrm{mM} \mathrm{NaCl}, 2.5 \mathrm{mM} \mathrm{KCl}, 1 \mathrm{mM}$ $\mathrm{NaH}_{2} \mathrm{PO}_{4}, 26.2 \mathrm{mM} \mathrm{NaHCO}_{3}, 11 \mathrm{mM}$ D-glucose, $2.5 \mathrm{mM} \mathrm{CaCl}$, and $9 \mathrm{mM} \mathrm{MgCl}_{2} ; \mathrm{pH}=7.2-7.4 ; 4^{\circ} \mathrm{C}$ ) saturated with $5 \% \mathrm{CO}_{2}$ in $\mathrm{O}_{2}$ at $32^{\circ} \mathrm{C}$ for at least $1 \mathrm{~h}$. Then, the prepared slices were transferred to a recording chamber, containing oxygenated aCSF (119 mM NaCl, $2.5 \mathrm{mM} \mathrm{KCl}, 1 \mathrm{mM} \mathrm{NaH} \mathrm{PO}_{4}, 26.2 \mathrm{mM}$ $\mathrm{NaHCO}_{3}, 11 \mathrm{mM}$ D-glucose, $2.5 \mathrm{mM} \mathrm{CaCl}, 1.3 \mathrm{mM} \mathrm{MgCl}$, and $0.4 \mathrm{mM}$ ascorbic acid; $\mathrm{pH}=7.2-7.4 ; 4^{\circ} \mathrm{C}$ ) at a flow rate of $2 \mathrm{~mL} / \mathrm{min}$ at $32^{\circ} \mathrm{C}$. Glass electrode (recording electrode) were filled with recording aCSF and placed in the stratum radiatum of CA1. Electrodes were lowered 75 to $150 \mu \mathrm{m}$ under the surface of the slice using micromanipulator (ROE-200, Sutter Instruments, Novarto, CA, United States) and Controller (MPC200, Sutter Instruments). Then, a bipolar electrode (Stimulation electrode) was placed in the CA3 area to evoke the field excitatory postsynaptic potential (fEPSP) responses (Citri and Malenka, 2008; Sweet et al., 2015). The hippocampal slices were stimulated with $0.5 \mathrm{mV} / 0.5 \mathrm{~ms}$ every $30 \mathrm{~s}$ for at least $10 \mathrm{~min}$, followed by $0.5 \mathrm{mV} / 100 \mathrm{~Hz} / 1 \mathrm{~s}$ for $15 \mathrm{~min}$ of test stimulation. This signal amplified by an amplifier (MultiClamp 700B, Molecular Devices) was then sent to a computer through A/D converter (Digidata 1440A, Molecular Devices, Sunnyvale, CA, United States) and analyzed using Clampex 10.7 Software (Molecular Devices).

\section{Statistical Analysis}

All statistical parameters were calculated using GraphPad Prism 5.0 software (GraphPad Software, San Diego, CA, United States). An unpaired two-tailed $t$-test was used for comparing the difference in emotional and cognitive impairment between sham and PSNL groups. Cognitive impairment behaviors based on the Y-maze and NOR test, electrophysiology, western blot and immunohistochemical data were subjected to one-way analysis of variance (ANOVA) tests followed by Newman-Keuls tests. Analyses of mechanical allodynia at various time points were performed using two-way repeated measures ANOVAs and Bonferroni post hoc tests for pairwise multiple comparisons.
Spearman rank correlation coefficient tests were conducted to analyze whether the von Frey test was correlated with Y-maze and NOR test, and whether the expression levels in NR2B and GluR1 in the hippocampus were correlated with von Frey and NOR test. All data are expressed as the mean \pm standard error of the mean (SEM). For all analyses, $p<0.05$ was considered to indicate statistical significance.

\section{RESULTS}

\section{Acupuncture Improves Mechanical Allodynia in PSNL-Induced Neuropathic Pain}

The anti-allodynic and analgesic effects of acupuncture were measured using the von Frey test in the PSNL-induced neuropathic pain model. The baseline measurement was performed 1 day before PSNL surgery. Then, mice were randomly assigned into five groups. Paw withdrawal frequency in five groups did not show any difference. The effects of treatments with acupuncture (AP1, AP2, or CP) or amitriptyline $(10 \mathrm{mg} / \mathrm{kg}$, i.p.) were investigated for 28 days in the PSNL-induced neuropathic pain model (Figure 1). For mechanical allodynia, a two-way repeated measures ANOVA revealed a significant effect of group $\left(F_{5,246}=175.5, p<0.0001\right)$ and a significant group $\times$ time interaction $\left(F_{30,246}=8.733, p<0.0001\right)$. Bonferroni post hoc tests showed that AP1 treatment reversed the established ipsilateral mechanical allodynia at each time point (each $p<0.001$ vs. PSNL over day 7 after treatment; Figure 1B). Likewise, the paw withdrawal frequency was significantly lower in the amitriptyline group than in the PSNL group (each $p<0.001$ vs. PSNL over day 14 after treatment; Figure 1B). We also observed the effects of acupuncture (AP1, AP2 or CP) or amitriptyline administration in the nociceptive behavior test at day 28 . A one-way ANOVA indicated a significant difference between groups for paw withdrawal frequency on day $28\left(F_{5,54}=68.08\right.$, $p<0.0001)$. Newman-Keuls post hoc tests revealed that the paw withdrawal frequencies were lower in the AP1 $(p<0.001)$ and amitriptyline groups $(p<0.001)$ than the PSNL group (Figure 1D). There were no significant changes in the pain levels in the contralateral hind paws (Figures 1C,E). Our results showed that acupuncture treatment in AP1 had an analgesic effect.

\section{The Effect of Acupuncture on Cognitive Function in PSNL-Induced Pain Model}

To examine the effects of acupuncture treatment on PSNLinduced cognitive impairment, we used Y-maze and NOR test (Figure 2). First, we observed cognitive impairment 8 days after PSNL surgery. Unpaired two-tailed $t$-tests revealed that the spontaneous alternation in PSNL mice was significantly lower than that in sham mice $\left(t_{18}=1.120, p=0.0176\right.$; Figure 2A). In addition, unpaired two-tailed $t$-tests showed that the recognition index in the PSNL group was significantly decreased by PSNL surgery $\left(t_{18}=12.20, p=0.0087\right.$; Figure $\left.2 E\right)$. Next, acupuncture (AP1, AP2, or CP) or amitriptyline $(10 \mathrm{mg} / \mathrm{kg}$, i.p.) treatment was continued for 28 consecutive days and the effects of treatments on cognitive impairment were measured again at day 28. In the 
A

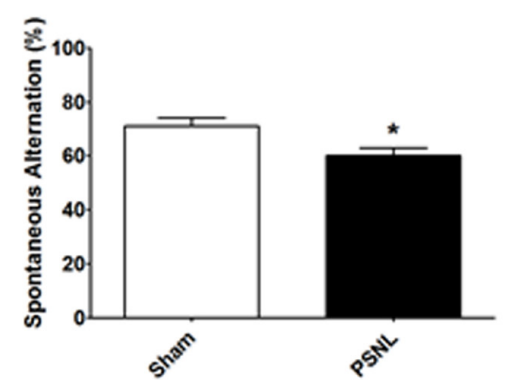

C

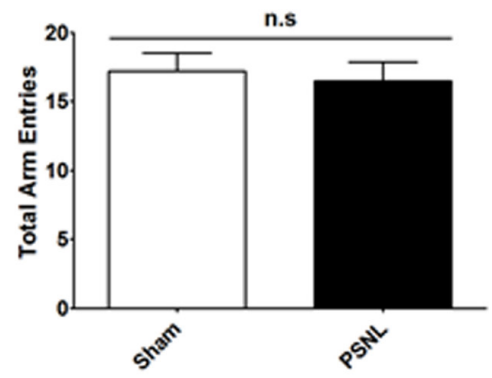

E

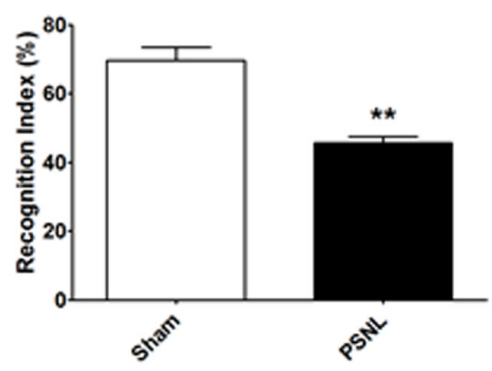

G

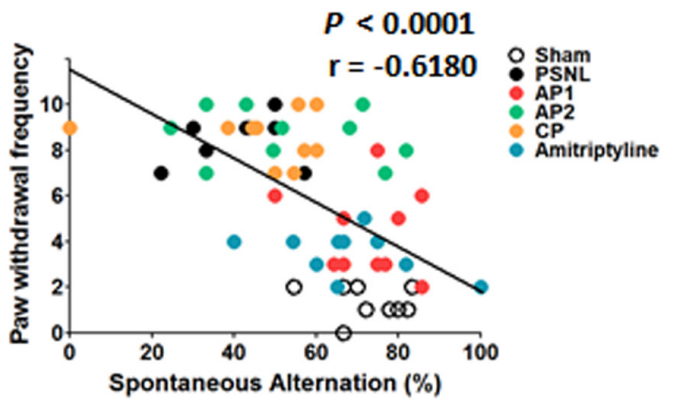

B

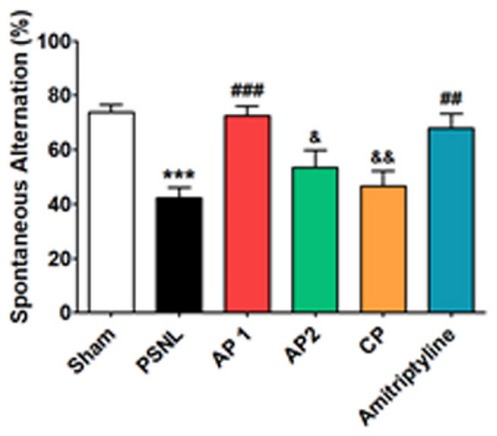

D

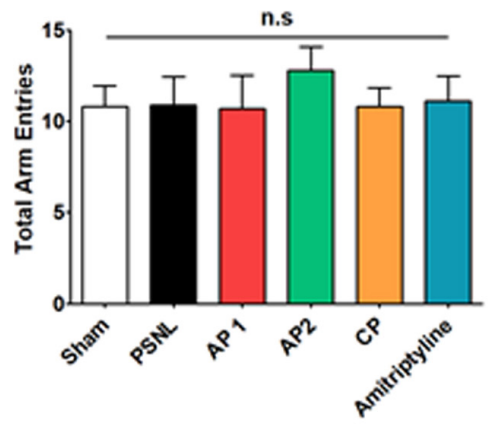

F

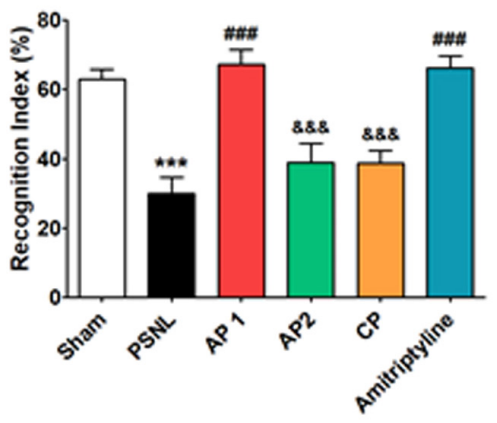

H

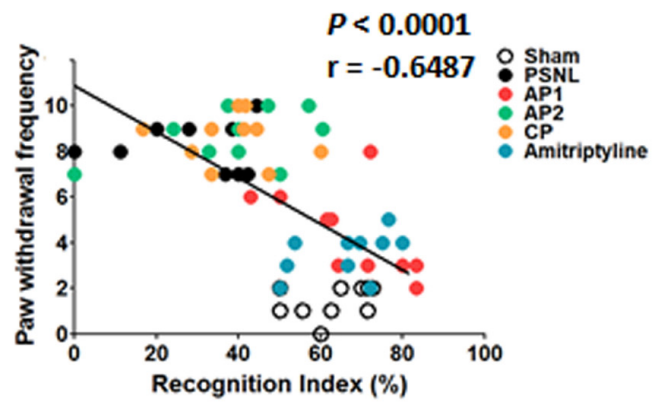

FIGURE 2 | Effects of acupuncture on cognitive functions measured through Y-maze and NOR tests. Effects of acupuncture (AP1, AP2, or CP) or amitriptyline (10 mg/kg, i.p.) treatment on cognitive impairment were investigated using Y-maze (A-D) and NOR (E,F) tests in the PSNL-induced neuropathic pain model. All

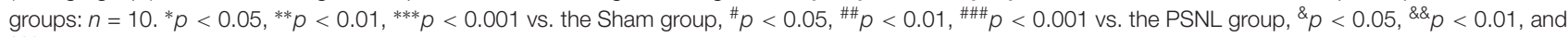
${ }_{8 \& \&} p<0.001$ vs. the AP1 group. The data were analyzed with a one-way ANOVA followed by Newman-Keuls post hoc tests (A-F). Data are expressed as the mean \pm SEM. The cognitive function measures in both $\mathrm{Y}$-maze and NOR tests were correlated with paw withdrawal frequency $(\mathbf{G}, \mathbf{H})$. The $r$-values were analyzed with the Spearman rank correlation coefficient. AP1, acupuncture 1 (GB30 and GB34); AP2, acupuncture 2 (HT7 and GV20); CP, control point; NOR, novel object recognition. 
Y-maze test, one-way ANOVA revealed a significant difference between groups for spontaneous alternation $\left(F_{5,54}=8.450\right.$, $p<0.0001$ ), and Newman-Keuls post hoc tests showed that spontaneous alternation in AP1 group was higher than that in the PSNL group ( $p<0.05$; Figure 2B). There were no significant changes in total arm entries of Y-maze test (Figures 2C,D). In the NOR test, a one-way ANOVA $\left(F_{5,54}=15.78, p<0.0001\right)$ followed by Newman-Keuls post hoc tests showed that the PSNL-induced reduction in recognition index $(p<0.001$ vs. Sham group) was restored by AP1 and amitriptyline treatments (each $p<0.001$ vs. PSNL group), whereas AP2 and CP had no effects (Figure 2F). Furthermore, spontaneous alternation and recognition index were negatively correlated with paw withdrawal frequency $(r=-0.6180, p<0.0001 ; r=-0.6487$, $p=0.0026$, respectively; Figures $2 \mathrm{G}, \mathbf{H}$ ). Therefore, our results showed that cognitive functions are impaired by induced neuropathic pain and that acupuncture has the potential to improve the cognitive impairment in neuropathic pain.

\section{Effects of Acupuncture on Analgesia and Cognitive Function Using Machine \\ Learning}

The decision tree algorithm consists of a random forest algorithm that is internally trained to express the distribution of behavior data values, the classification process, and semantics for each experimental group. It displays the decision boundary of the entire 40 mouse data on the scatter plot. This is presented in Figure 3. The Sham and AP1 groups were segregated from the PSNL and CP groups. The Sham and AP1 groups showed lower pain responses, while the PSNL and CP groups showed higher pain responses. The pain response data appear to provide useful information in distinguishing the Sham and AP1 groups, while the NOR and Y-maze data do not. The pain data between the PSNL and CP groups do not differ significantly, but the Y-maze and NOR data apparently allow a better distinction between them (Figures 3A,B). By applying 4-fold cross validation, we predicted with a $64.4 \%$ accuracy the membership of each mouse in each experimental group through the three threshold values of pain, NOR, and Y-maze.

\section{The Effects of Acupuncture on LTP From CA3 to CA1 Regions of the Hippocampus in PSNL}

LTP recording was performed to investigate the electrophysiological basis of the effects of acupuncture (Figure 4). After 14-28 days of acupuncture treatment (AP1), the time course of fEPSP slopes that were normalized to the $15 \mathrm{~min}$ baseline period was presented in Figure 4B. The fEPSP slopes of the four groups were increased immediately after electronic stimulation and stabilized to different levels above the baseline. The statistical mean value of the last 15 min was shown in Figure 4C. One-way ANOVA showed that there were significant differences in the mean slopes of fEPSP among the four groups $\left(F_{3,120}=19.17, p<0.001\right.$, Figure 4C). Newman-Keuls post hoc tests showed that the mean fEPSP slopes were smaller in the PSNL and CP groups than in the Sham ( $p<0.01$ vs. PSNL) and
AP1 groups ( $p<0.001$ vs. PSNL group; $p<0.001$ vs. CP group), indicating that acupuncture treatment did efficiently reverse the PSNL-induced LTP impairment.

\section{Effects of Acupuncture on Expression Levels of Glutamate Receptors in the Hippocampus of PSNL Mice}

Many studies have shown that NR2B and GluR1 plays important roles in the synaptic plasticity through induction and maintenance of LTP at the Schaffer collateral-CA1 synapses; LTP is important in learning and memory functions (Lisman et al., 2002; Fonseca, 2012; Bliss and Collingridge, 2013; Wang H. et al., 2015; Shang et al., 2017). To investigate the role of glutamate receptors in neuropathic pain, we examined hippocampal NR2B and GluR1 using double-immunostaining (Figures 5, 6). First, NR2B- and GluR1-positive cells were double stained for three cell type markers (GFAP, Iba-1 or NeuN). The results suggest that they are expressed in neurons, but not in astrocytes or microglia (Supplementary Figure 1). Next, double-immunostaining and western blotting were carried on hippocampal NR2B and GluR1 for acupuncture groups (AP1, AP2 or CP) or amitriptyline group $(10 \mathrm{mg} / \mathrm{kg}$, i.p.). Coronal sections of the hippocampus from the three groups (Sham, PSNL and AP1) were subjected to NR2B and NeuN antibodies for double-immunostaining. A one-way ANOVA showed a significant difference between the groups for expression of NR2B (CA1: $F_{2,7}=18.26, p=0.0017$; CA2: $F_{2,7}=0.2718, p=0.7697$; CA3: $F_{2,7}=6.523, p=0.0252 ; \mathrm{DG}:$ $\left.F_{2,7}=11.23, p=0.0065\right)$, and Newman-Keuls post hoc tests showed that expression levels of NR2B were lower in the PSNL group than those in the Sham group (CA1: $p<0.01$, CA3: $p<0.05$, DG: $p<0.01)$. AP1 administration reversed the attenuation of hippocampal NR2B expression resulted from the PSNL surgery (CA1: $p<0.01$, CA3: $p<0.05$, DG: $p<0.01$ vs. PSNL; Figures 5A,B). In western blot analysis, one-way ANOVA showed a significant difference in protein expression of NR2B in the hippocampus between the groups $\left(F_{5,24}=6.521, p=0.0006\right)$. Newman-Keuls post hoc tests showed that hippocampal NR2B expression levels in the AP1 group were significantly higher than those in the PSNL group ( $p<0.01$; Figure 5D). There was a positive correlation between increased expression levels of $\mathrm{NR} 2 \mathrm{~B}$ in the hippocampus and the recognition index in NOR test $(r=0.5837$ and $p=0.0007$; Figure 5E), but a negative correlation between levels of NR2B in the hippocampus and paw withdrawal frequency in von Frey test. $(r=-0.6403$ and $p<0.0001$; Figure 5F).

Next, coronal sections of the hippocampus from the three groups (Sham, PSNL, and AP1) were subjected to GluR1 and NeuN antibodies. A one-way ANOVA showed a significant difference in the expression of GluR1 between the groups (CA1: $F_{2,7}=13.27, p=0.0042 ; \mathrm{CA} 2: F_{2,7}=1.900, p=0.2192$; CA3: $F_{2,7}=6.630, p=0.0242$; DG: $\left.F_{2,7}=17.98, p=0.0017\right)$. Newman-Keuls post hoc tests showed that GluR1 expression levels were lower in the PSNL group than those in the Sham group (CA1: $p<0.01$, CA3: $p<0.05$, DG: $p<0.01$ vs. PSNL). Similar to NR2B, AP1 administration suppressed the reduction of hippocampal GluR1 expression levels induced by sciatic nerve 
A

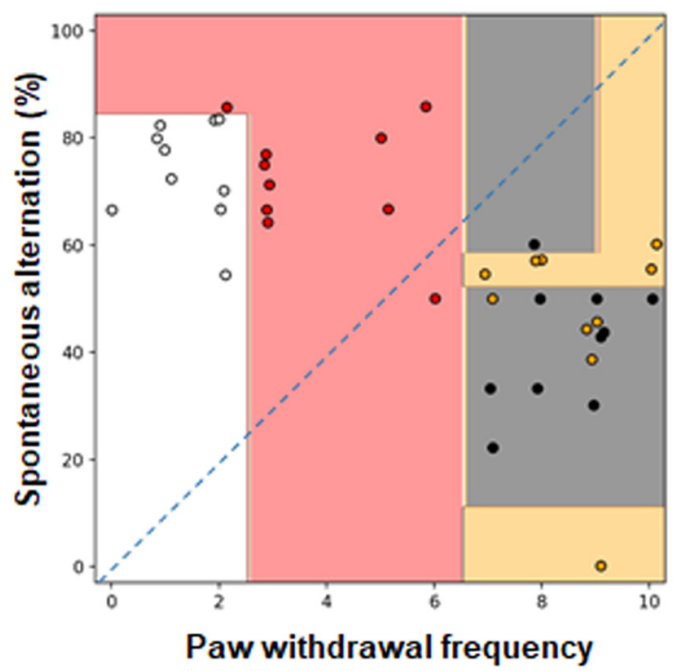

B

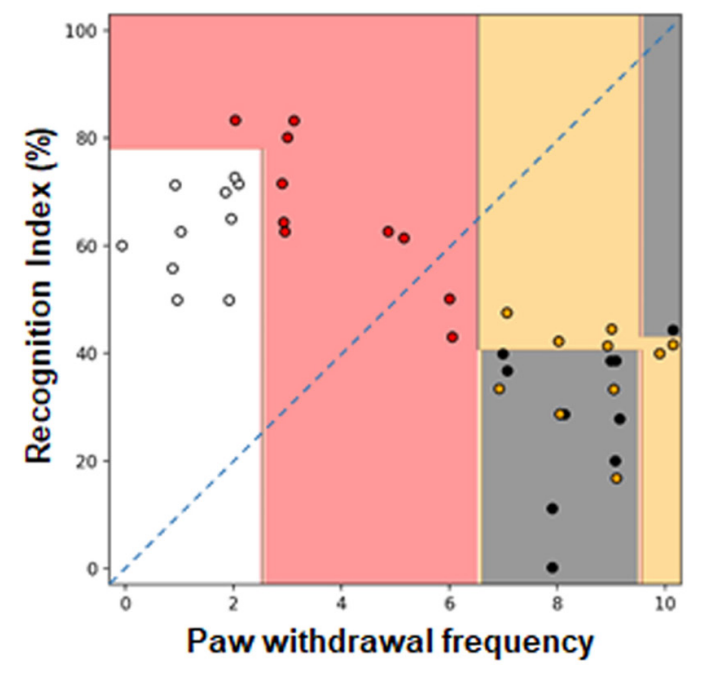

O Sham • PSNL $\bullet \mathrm{AP} \bullet \mathrm{CP}$

FIGURE 3 | Effects of acupuncture on classification using a random forest classifier analysis in machine learning method. Cognitive functions and nociceptive behavior data of the four groups (Sham, PSNL, AP1, and CP) were analyzed by random forest algorithm in machine learning. Representative figures show that the spontaneous alternation in Y-maze (A) and recognition index in NOR test (B) were analyzed against the paw withdrawal frequency in the von Frey test. All groups: $n=10$. AP1, acupuncture 1 (GB30 and GB34); CP, control point.

A

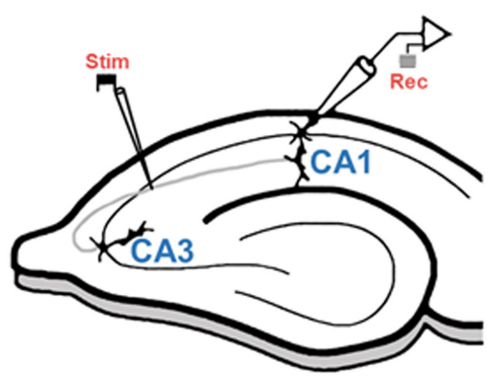

B

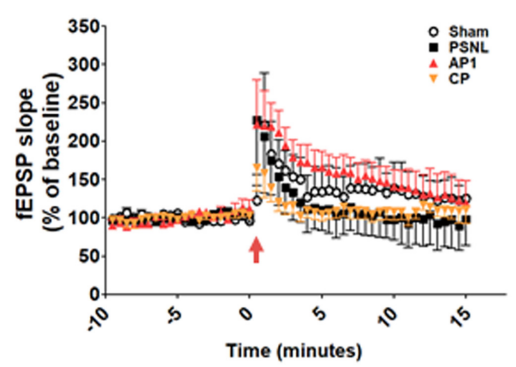

C

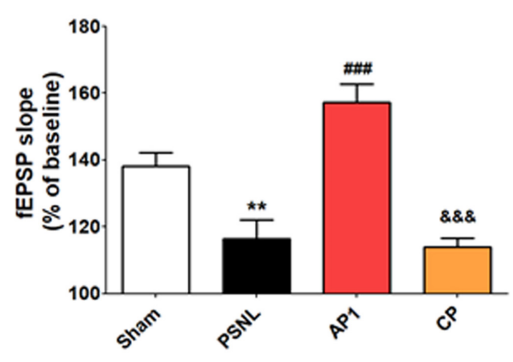

FIGURE 4 | Acupuncture increased fEPSP slopes in the hippocampal CA3 to CA1 areas of neuropathic pain model. A schematic of a transverse hippocampal area used in electrophysiology methods, showing recording (rec) and stimulating (stim) regions (A). Averaged fEPSP data. High-frequency stimulation was given at 0 min, and the signal was followed for up to 15 min (B). Summary of fEPSP slopes (from 0 to 15 min) shown in (C). $n=5$ in all groups. $* * p<0.01$ vs. the Sham groups. \#\#\# $p$ < 0.001 compared with the PSNL group, and \&\&\& $p<0.001$ vs. AP1 group. The data were analyzed with a one-way ANOVA followed by Newman-Keuls post hoctests. The results are expressed as the mean \pm SEM. AP1, acupuncture 1 (GB30 and GB34); AP2, acupuncture 2 (HT7 and GV20); CP, control point.

surgery (CA1: $p<0.01$, CA3: $p<0.05$, DG: $p<0.01$ vs. PSNL; Figures $6 \mathbf{A}, \mathbf{B})$. In western blot analysis, a one-way ANOVA showed a significant difference in protein expression of GluR1 between the groups $\left(F_{5,24}=5.216, p=0.0022\right)$. Newman-Keuls post hoc tests showed that hippocampal GluR1 expression levels were significantly increased in the AP1 group compared to the PSNL group ( $p<0.01$; Figure 6D).

The GluR1 expression levels in the hippocampus were positively correlated with the recognition index in NOR test ( $r=0.5634$ and $p=0.0012$; Figure 6E), and were negatively correlated with the paw withdrawal frequency in von Frey test $(r=-0.6615$ and $p<0.0001$; Figure 6F). These results suggest that acupuncture administration may enhance cognitive functions by restoring glutamate receptors in the hippocampus in neuropathic pain.

\section{Effects of Acupuncture on Expression Levels of CaMKII in the Hippocampus in PSNL Mice}

CaMKII and PKC- $\gamma$ have a critical role in learning and memory functions. Their functions are regulated through $\mathrm{Ca}^{2+}$ influx mediated by NR2B receptors in post synaptic membranes. The reduction of CaMKII expression levels have been found 
A

NR2B
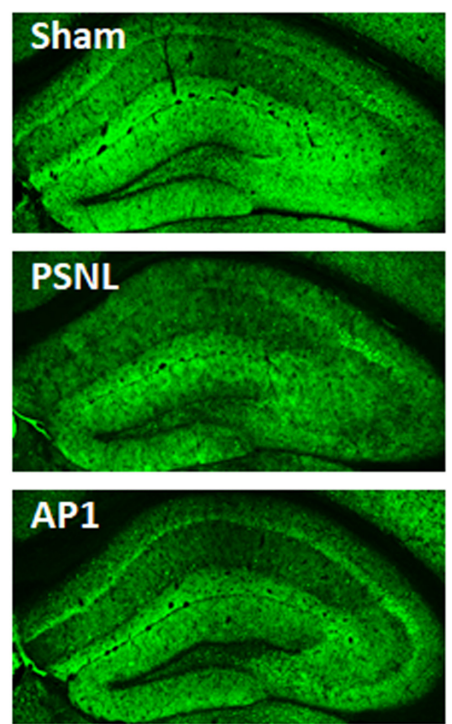

B

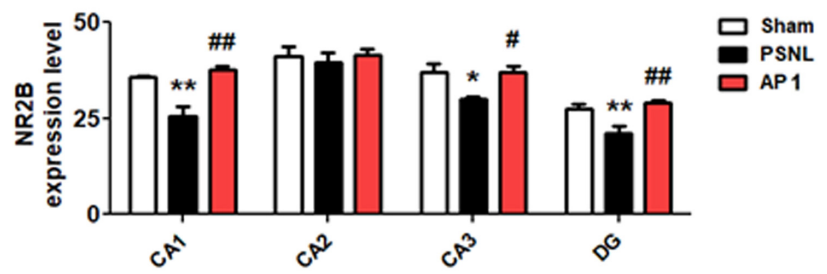

D
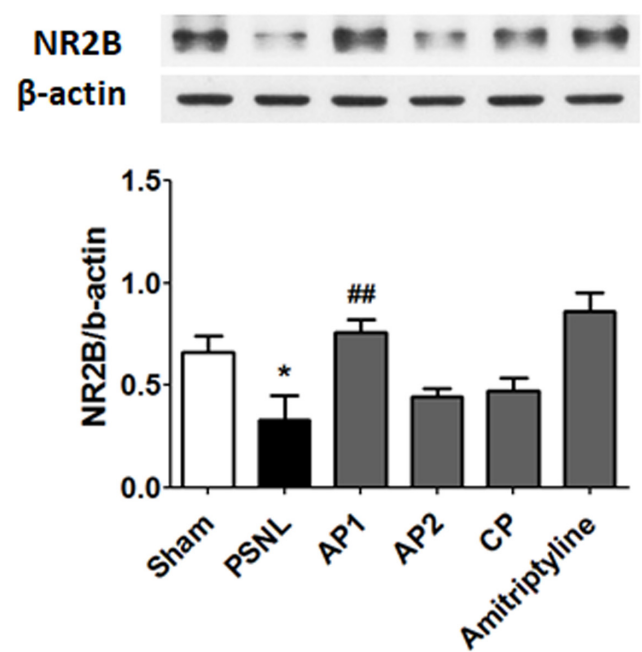

NeuN
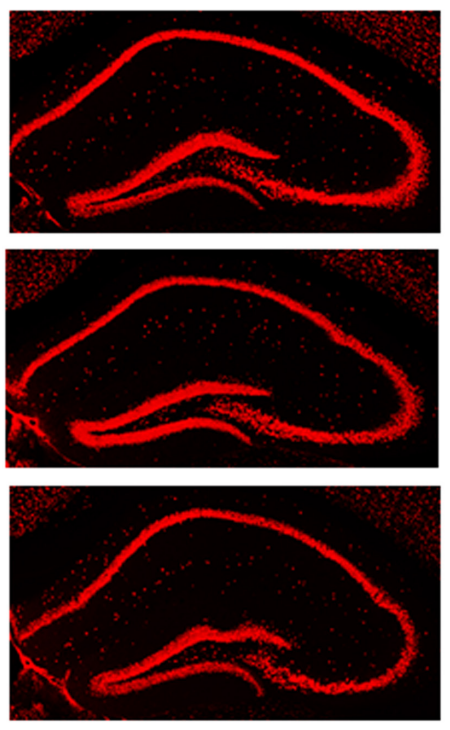

Merge
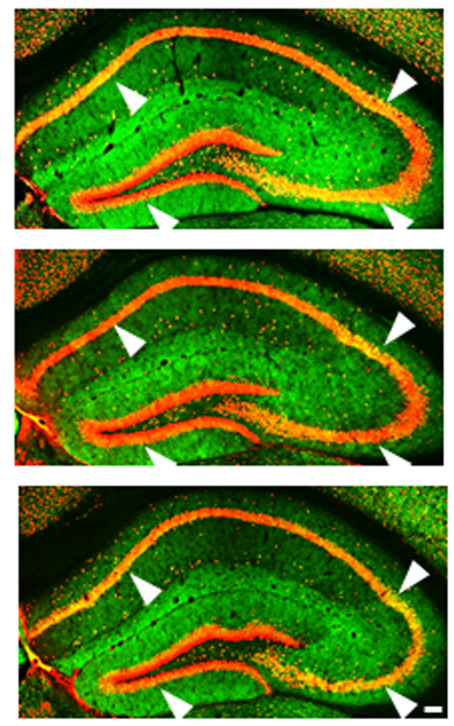

C

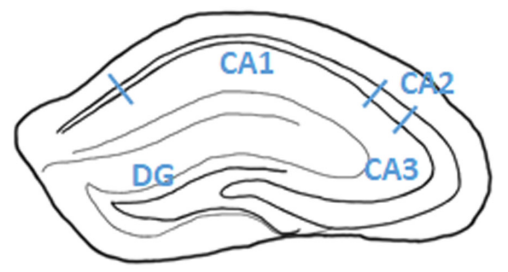

E

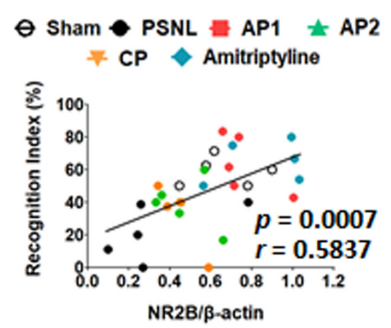

$\mathbf{F}$

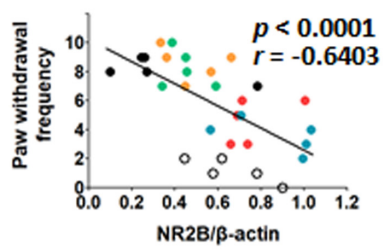

FIGURE 5 | Effects of acupuncture on the expression levels of hippocampal NR2B receptor. These results show the changes in hippocampal NR2B expression levels (CA1, CA2, CA3, and DG) after administration of acupuncture (AP1, AP2, or CP) or amitriptyline (10 mg/kg, i.p.) for 28 consecutive days (A-F). Histological examinations of the hippocampus showing the expression of NR2B (green) and NeuN (red) after AP1 administration in the PSNL-induced neuropathic pain model (A,B) and a representative figure showing the hippocampal regions in the mouse brain (C). $n=3-4 /$ group. Scale bar: $100 \mu \mathrm{m} .{ }^{*} p<0.05$, ${ }^{* *} p<0.01$ compared to the Sham group in each area. ${ }^{\#} p<0.05$, ${ }^{\# \#} p<0.01$ compared to the PSNL group in each area. The NR2B protein levels were measured in the hippocampus (D). $n=5$ /group. ${ }^{* *} p<0.01$ compared to the Sham group. ${ }^{*} p<0.05$ compared to the PSNL group. The results were analyzed with a one-way ANOVA followed by Newman-Keuls post hoc tests. The data are expressed as the mean \pm SEM. The cognitive function values in the NOR test and nociceptive values in the von Frey test were correlated with the hippocampal NR2B protein levels (E,F). The $r$-values were analyzed with the Spearman rank correlation coefficient. AP1, acupuncture 1 (GB30 and GB34); AP2, acupuncture 2 (HT7 and GV20); CP, control point. 
A
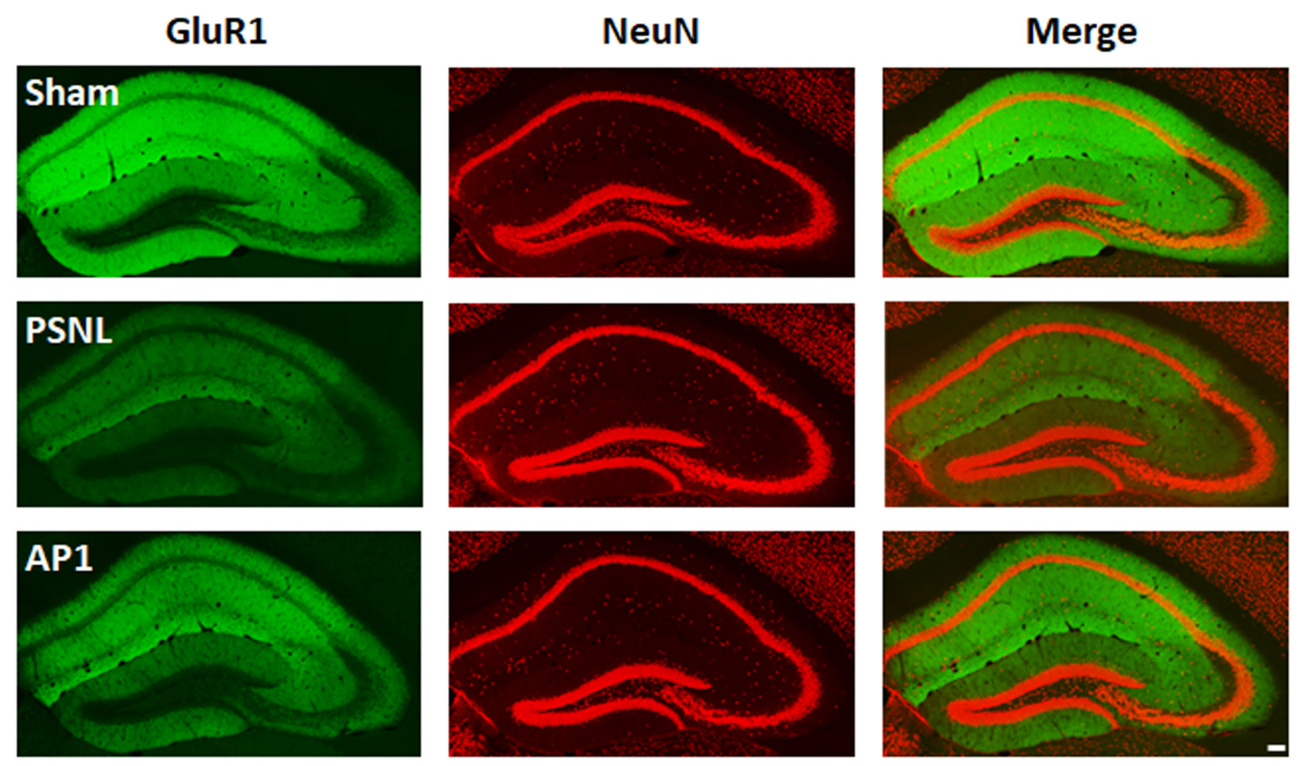

B

C
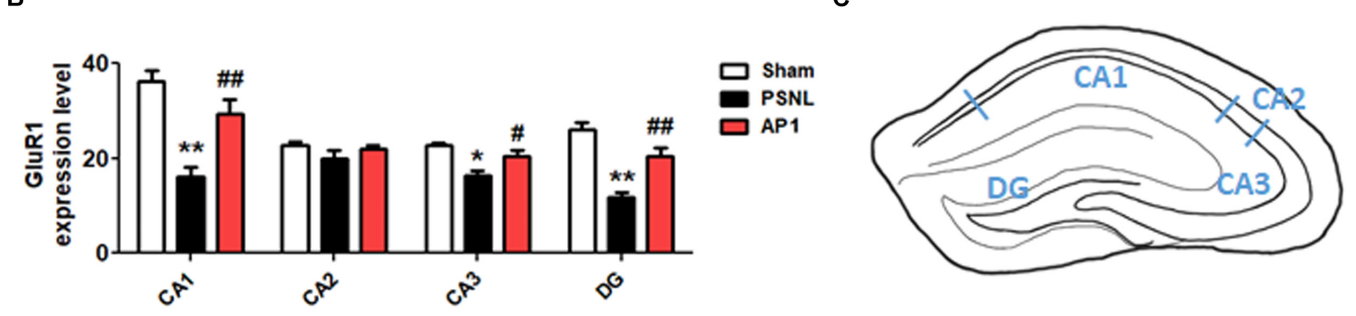

D

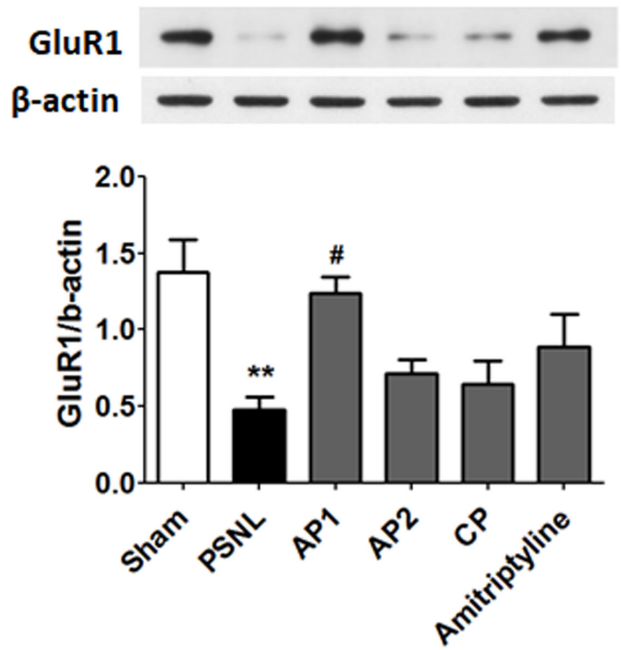

E

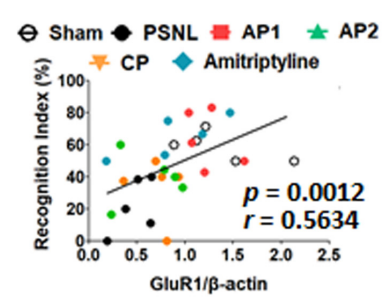

$\mathbf{F}$

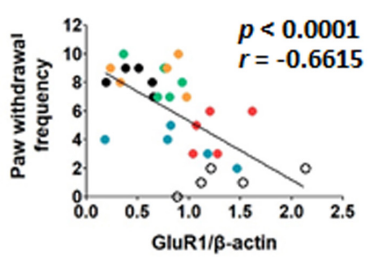

FIGURE 6 | Effects of acupuncture on the expression levels of hippocampal GluR1 receptor. These results show the changes in hippocampal GluR1 expression levels (CA1, CA2, CA3, and DG) after administration of acupuncture (AP1, AP2, or CP) or amitriptyline (10 mg/kg, i.p.) for 28 consecutive days (A-F). Free-floating coronal hippocampus sections from the three groups (Sham, PSNL, and AP1) were subjected to immunofluorescence with GluR1 (green) and NeuN (red) antibodies to label GluR1-positive NeuN in neurons (A). Representative graphs showing the expression levels of GluR1 in the hippocampus (A,B) and a representative figure showing the hippocampal regions in the mouse brain (C). $n=3-4$ /group. Scale bar: $100 \mu \mathrm{m} .{ }^{*} p<0.05,{ }^{* *} p<0.01$ compared to the Sham group in each area. ${ }^{\#} p<0.05, \# p<0.01$ compared to the PSNL group in each area. The expression levels of hippocampal GluR1 protein were examined (D). $n=5 /$ group. ${ }^{* *} p<0.01$ compared to the Sham group. $\# p<0.05$ compared to the PSNL group. All data were analyzed with a one-way ANOVA followed by Newman-Keuls post hoc tests. All data are expressed as the mean \pm SEM. The cognitive function values in the NOR test and nociceptive values in the von Frey test were correlated with the GluR1 protein levels in the hippocampus (E,F). The $r$-values were analyzed with the Spearman rank correlation coefficient. AP1, acupuncture 1 (GB30 and GB34); AP2, acupuncture 2 (HT7 and GV20); CP, control point. 
in the hippocampus of neuropathic pain models ( $\mathrm{Xu}$ et al., 2012). Therefore, we examined the effects of acupuncture (AP1, $\mathrm{AP} 2$, or $\mathrm{CP})$ or amitriptyline $(10 \mathrm{mg} / \mathrm{kg}$, i.p.) treatments on the CaMKII expression levels in the hippocampus of PSNL mice. A one-way ANOVA showed a significant difference in proteins expression of pCaMKII and tCaMKII between the groups (each $F_{5,24}=10.66 ; p<0.0001 ; F_{5,24}=5.156 ; p=0.0024$ ), and Newman-Keuls post hoc tests showed that pCaMKII and tCaMKII expression levels were decreased in the PSNL group compared to the sham group (each $p<0.001, p<0.01$; Figures 7A,B). Notably, AP1 treatment significantly restored pCaMKII and tCaMKII expression levels (each $p<0.01, p<0.05$ vs. PSNL; Figures 7A,B). However, pCaMKII and tCaMKII protein expression levels were not improved in the AP2, CP and amitriptyline groups (Figures 7A,B). PKC- $\gamma$ expression levels were not changed in the hippocampus by acupuncture treatment (Supplementary Figure 2).

\section{Effects of Acupuncture on Expression Levels of Synaptic Proteins in the Hippocampus in PSNL Mice}

Finally, we examined the effects of acupuncture administration on synaptic proteins, such as Syn-1 and PSD-95. A oneway ANOVA showed a significant difference in proteins expression of pSyn-1 $\left(F_{5,18}=5.600 ; p=0.0028\right)$ and pPSD-95 $\left(F_{5,18}=10.11 ; p<0.0001\right)$ between the groups, and NewmanKeuls post hoc tests showed that PSNL of the sciatic nerve induced a significant decrease in pSyn-1 $(p<0.05)$ and pPSD$95(p<0.01)$ compared with sham-operated mice. Acupuncture administration significantly improved pSyn-1 and pPSD-95 protein levels (each $p<0.05, p<0.01$ vs. PSNL). However, pSyn1 and pPSD-95 protein levels were not significantly altered in the AP2 and CP groups (Figures 7C,D). Our data showed that the acupuncture treatment restored impaired synaptic proteins in the hippocampus following neuropathic pain.

\section{DISCUSSION}

Chronic pain is characterized as having a multidimensional aspect, involving nociception and affective or cognitive procession. Acupuncture is known to have an analgesic effect and improve cognitive impairment in chronic pain patients (Paramore, 1997; Couilliot et al., 2013). However, the detailed mechanisms associated with the co-curation effects of acupuncture on pain and cognitive impairment are yet to be revealed. In the present study, we found that the acupuncture treatment at acupoints GB30 and GB34 improved both nociceptive behavior and cognitive impairment associated with a PSNL-induced neuropathic pain model. The acupuncture group was classified as a different one from the PSNL group when analyzed using a decision tree algorithm of machine learning. We also found that acupuncture can enhance synaptic plasticity through increasing LTP as well as expression levels of NR2B and GluR1 in the hippocampus, which were impaired in the PSNL group. In addition, the expressions of CaMKII and synaptic proteins such as pPSD-95 and pSyn-1, indicators of synaptic plasticity, were enhanced by acupuncture treatment.

Central sensitization that develops following peripheral nerve injuries is thought to contribute to chronic pain and the deleterious effects on cognitive functions in preclinical models and in patients (Schnurr and MacDonald, 1995; Nicholson and Verma, 2004; Kodama et al., 2007, 2011). Here, we observed that PSNL mice with prolonged mechanical allodynia had impairments in the working and recognition memory when subjected to $\mathrm{Y}$ maze and NOR tests, consistent with recent studies (Kodama et al., 2011; Dimitrov et al., 2014; Liu et al., 2017). In contrast, acupuncture treatment (AP1) resulted in the improvements of memory-function as well as mechanical allodynia. Other acupoints or non-acupoints did not result in such improvement, which implies that acupoints GB30 and GB34 are specific for these therapeutic effects. Next, a random decision forest classifier in machine learning was used to determine whether the therapeutic effects of acupuncture can be predicted based on the pain and cognitive behaviors. Machine learning classifiers are specific applications of machine learning technology that use individual features to predict the preassigned class to which a given example belongs (Breiman, 2001; Geurts et al., 2006). The success of a given model can be assessed by examining how faithfully it predicts group membership, also allowing for the comparison of performance between feature sets (Pedregosa et al., 2011). We found that the mice treated with acupuncture were not only classified as a different group from the PSNL mice, but also shifted toward the sham mice. In contrast, mice treated with acupuncture at the control points clustered with the PSNL group.

The hippocampus plays a critical role in cognitive functions. Decreased hippocampal volume and alteration of synaptic plasticity have been found in Alzheimer's disease rodent models with cognitive impairment (Tozzi et al., 2015; Jin et al., 2017). Such hippocampal changes were also observed in chronic pain, and the reduced synaptic plasticity might be associated with the cognitive impairment comorbid with pain (Valet et al., 2009; Zimmerman et al., 2009; Mutso et al., 2012). LTP is a cellular model of activity-dependent changes in synaptic strength, underlying information storage and memory creation (Kim et al., 2001). Recently, the decreased hippocampal LTP was reported in several pain models such as the peripheral nerve injury-induced cognition-impaired mice (Kodama et al., 2011; Mutso et al., 2012; Chen et al., 2017; Liu et al., 2017). Consistent with previous studies, we also found that LTP was reduced when measured in the hippocampal CA1 of PSNL mice with cognitive impairment. Moreover, we found that acupuncture enhanced LTP compared to the PSNL-induced neuropathic pain mice. Recent experiments in chronic pain reported that acupuncture may modulate synaptic function and regulate cognitive impairment, but no molecular mechanisms have been proposed (Xu et al., 2012; Chen et al., 2017). Therefore, we investigated the underlying mechanisms associated with the improvements in cognitive function and pain by acupuncture treatment by focusing on the LTP-related molecular mechanisms in the hippocampus.

Induction and maintenance of LTP at the Schaffer collateralCA1 synapses is NMDA and AMPA receptors-dependent 

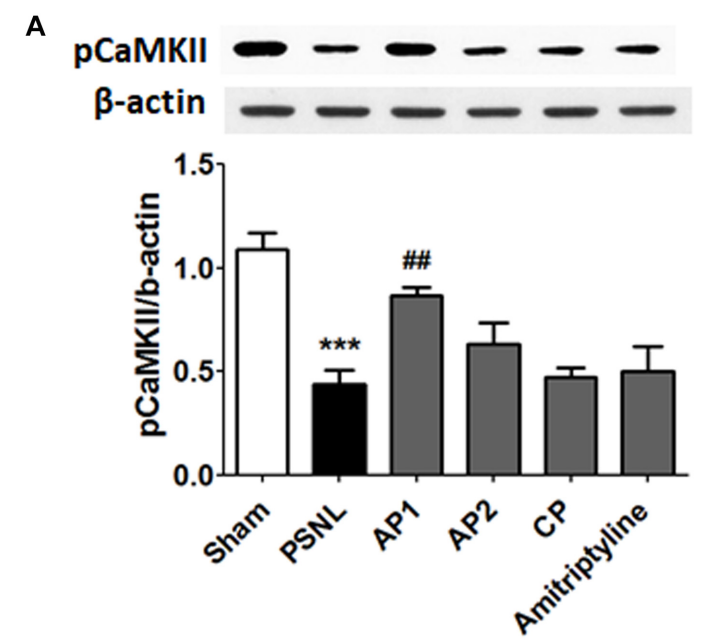

C

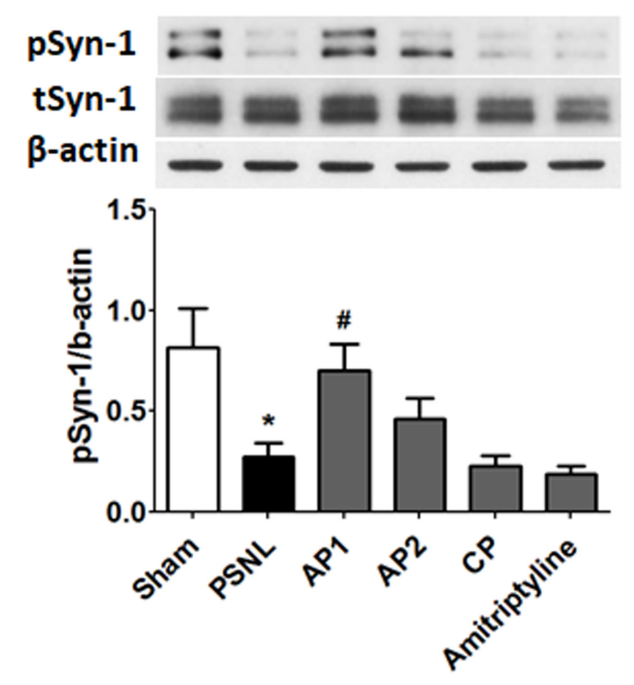

B
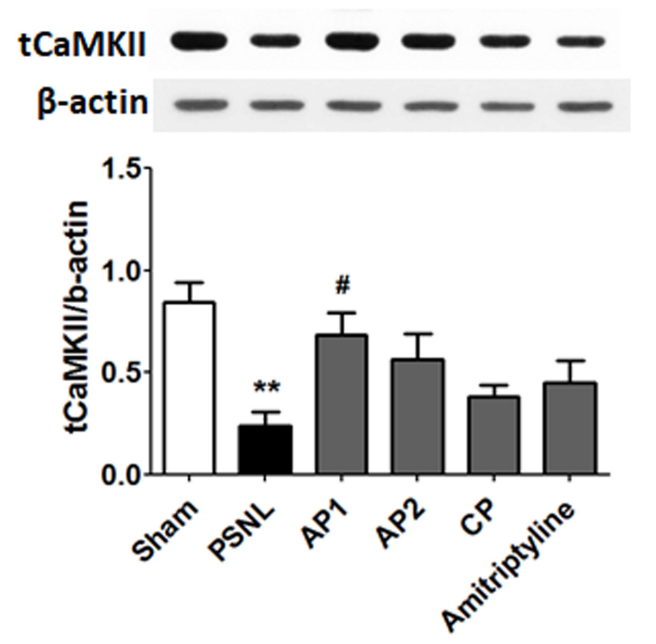

D
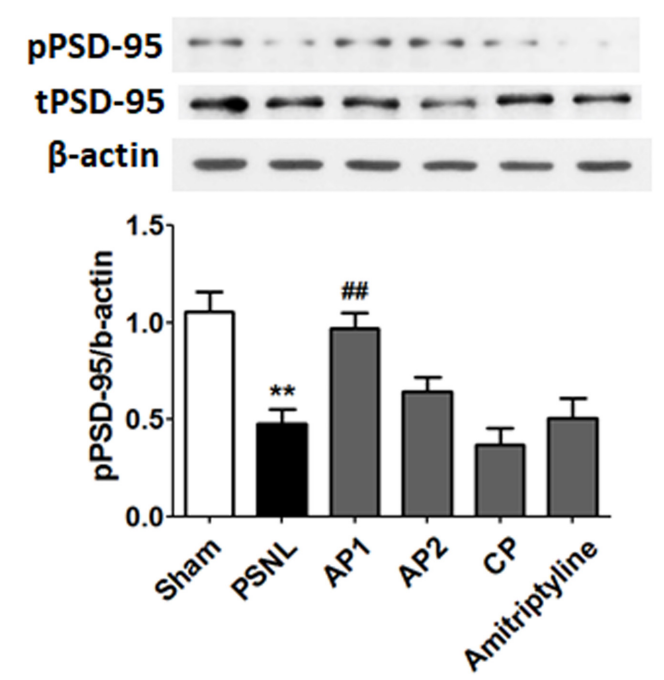

FIGURE 7 | Effects of acupuncture on the expression levels of CaMKII and synaptic proteins. These results show the changes in protein levels of hippocampal CaMKII (A,B; $n=5 /$ group) and synaptic proteins (C,D; $n=4 /$ group) after administration of acupuncture (AP1, AP2, or CP) or amitriptyline (10 mg/kg, i.p.) for 28 consecutive days. ${ }^{* *} p<0.01,{ }^{* * *} p<0.001$ compared to the Sham group. ${ }^{\#} p<0.05,{ }^{\# \#} p<0.01$ compared to the PSNL group. Data are expressed as the mean \pm SEM. The same $\beta$-actin value was used (A,C; B,D). AP1, acupuncture 1 (GB30 and GB34); AP2, acupuncture 2 (HT7 and GV20); CP, control point.

(Holmes and Grover, 2006; Fonseca, 2012; Luscher and Malenka, 2012; Bliss and Collingridge, 2013). NR2B increases CaMKII activity by increasing $\mathrm{Ca}^{2}+$ influx into the synapse, which then increases the expression of GluR1 in the synapse and thus mediates many important brain functions including cognition, learning and memory (Tovar and Westbrook, 1999; Lisman et al., 2002; Goebel et al., 2005; Wang H. et al., 2015; Shang et al., 2017). The down-regulation of NR2B, GluR1, CaMKII and synaptic proteins in the hippocampal neurons are associated with learning-memory deficits and cognitive dysfunctions in Alzheimer's disease (AD) animal models (Liu et al., 2016; Mariani et al., 2017; Zhu et al., 2018). As shown in a recent AD study, the expression of NR2B in the hippocampus was shown to be reduced in a PSNL-induced neuropathic pain model (Wang X.Q. et al., 2015). Zhu et al. reported that the expression of GluR1 as well as the neurons co-expressing brain-derived neurotrophic factor and GluR1 were downregulated in the hippocampal CA3 region of neuropathic pain mice, which was also correlated with the pain-related comorbid conditions (Zhu et al., 2017). Consistent with these studies, our results revealed that hippocampal NR2B and GluR1 were significantly downregulated in the CA1 and CA3 of the PSNL neuropathic pain model, whereas acupuncture treatment rescued them. Interestingly, the correlation analyses revealed that the expression levels of NR2B and GluR1 are positively correlated with the cognitive functions, while negatively correlated with pain behaviors. This implies that the increase in NR2B and GluR1 in the AP1 group can play a pivotal role for the restoration of pain and comorbid cognitive dysfunction. In addition, we showed that acupuncture enhanced the expression levels of pCaMKII and tCaMKII in the hippocampus, while they were decreased in a neuropathic pain model. These results are consistent with a recent study showing 
that acupuncture further reduces CaMKII expression level in the hippocampal CA3 following a peripheral nerve injury (Xu et al., 2012). Activation of CaMKII is crucial for LTP induction in the hippocampus, and it has been implicated in activity-dependent synaptic strengthening (Lisman et al., 2002). PKC- $\gamma$ is activated through increased intracellular $\mathrm{Ca}^{2+}$ influx, but this was not improved by acupuncture administration. Fernández de Sevilla and Buño et al. suggested that CaMKII has an important role in the initiation of the acetylcholine-mediated enhancement of NMDAR- and AMPAR-mediated transmission, but that PKC might be essential in the initiation of the acetylcholine-mediated augment of only NMDAR-mediated transmission (Fernandez de Sevilla and Buno, 2010). Therefore, we postulate that the difference between CaMKII and PKC expression patterns in the hippocampus might be involved in cholinergic mechanisms on the acupuncture treatment, and further studies are required.

Finally, we demonstrated the significant alteration of synaptic proteins such as Syn-1 and PSD-95 in the hippocampus of the PSNL model mice. Syn-1 is a presynaptic terminal specific marker involved in transport, emissions, and recycling of the vesicles, and believed to play an important role in the process of the calcium-dependent neurotransmitter release, such as glutamate (Valtorta et al., 2004). PSD-95 is a structural protein of postsynapse and is increased by the activation of CaMKII, a protein that plays a crucial role in synaptic plasticity (Cheng et al., 2006). In this study, we found that acupuncture treatment recovered the decreased levels of phosphorylated Syn-1 and PSD-95 in the hippocampus of PSNL-induced neuropathic pain. Therefore, our results suggest that acupuncture can improve both nociception and cognitive impairment by regulating glutamate transmission through increasing the expressions of glutamate receptors and synaptic proteins.

It is well known that synapses between neurons are the structural basis of neural connection and neural plasticity. In the recent study, the total dendrite length, the number of dendrite branches, and spine densities in the basal and apical dendrites of CA1 pyramidal neurons were reduced significantly in the neuropathic pain mice (Liu et al., 2017). Therefore, further studies are needed to explore whether acupuncture can change the morphology of CA1 pyramidal neurons related to the synaptic plasticity.

The limitation of this study is that it is not known how changes in the peripheral nervous system affect the brain. Acupuncture showed analgesic effect through increases in adenosine and adenosine monophosphate release in the muscle, and this effect disappeared in the adenosine A1 receptor knockout mice (Goldman et al., 2010). The locally activated ERK signaling pathway was also mediated in the acupuncture analgesia (Park et al., 2014). However, since this study was focused on the CNS, we did not closely observe changes in adenosine, ATP or ERK pathway in the peripheral nervous system following acupuncture treatment. In addition, the stimulation of the metabotropic A1 receptors are known to be involved in modulation of chronic pain at the spinal and supra spinal level, in both neuronal and, at least partly, glial or microglial cells (Luongo et al., 2012; Luongo et al., 2014). Moreover, LTP in hippocampal CA1 area was inhibited in the adenosine A1 receptor knockout mice (Zhou et al., 2018). Further studies are needed to define the relationship between adenosine A1 receptors in the local tissue and glutamate receptors in hippocampus.

In conclusion, we demonstrated that acupuncture treatment can improve both allodynia and comorbid cognitive impairments simultaneously, and that the therapeutic effects of acupuncture were correlated with the increased expressions of NR2B and GluR1 in the hippocampus. Acupuncture also enhanced pCaMKII and tCaMKII as well as the pre- and post-synaptic proteins in this area. Finally, these molecular changes contribute to the increase of LTP in the CA1 of the hippocampus. These results might imply that acupuncture can be one of the potential options for controlling both pain and comorbid cognitive impairments, while further clinical trials are needed to prove it.

\section{DATA AVAILABILITY}

All datasets generated for this study are included in the manuscript and/or the Supplementary Files.

\section{ETHICS STATEMENT}

The animal study was reviewed and approved by Dongguk University Animal Care Committee for Animal Welfare (IACUC-2017-022-1). Written informed consent was obtained from the owners for the participation of their animals in this study.

\section{AUTHOR CONTRIBUTIONS}

J-HJ, E-MS, YR, M-YS, and H-JP conceived the experiments. J-HJ, J-YP, and H-JP contributed to the materials and tools. J-HJ, Y-KK, J-YO, W-MJ, H-KK, and H-YK performed the experiments. J-HJ analyzed and interpreted the data and wrote the main manuscript draft. J-HJ and H-JP designed the experiments and wrote, edited, and revised the manuscript. All authors had input into the manuscript and have approved the manuscript for publication.

\section{FUNDING}

This research was supported by grants from the National Research Foundation of Korea funded by the Korean Government (NRF-2017R1A2B4009963 and NRF2017R1A2B4011707) and from the Korea Institute of Oriental Medicine (Grant K18182).

\section{SUPPLEMENTARY MATERIAL}

The Supplementary Material for this article can be found online at: https://www.frontiersin.org/articles/10.3389/fnins. 2019.00995/full\#supplementary-material 


\section{REFERENCES}

Benbouzid, M., Choucair-Jaafar, N., Yalcin, I., Waltisperger, E., Muller, A., FreundMercier, M. J., et al. (2008). Chronic, but not acute, tricyclic antidepressant treatment alleviates neuropathic allodynia after sciatic nerve cuffing in mice. Eur. J. Pain 12, 1008-1017. doi: 10.1016/j.ejpain.2008.01.010

Bergbom, S., Boersma, K., Overmeer, T., and Linton, S. J. (2011). Relationship among pain catastrophizing, depressed mood, and outcomes across physical therapy treatments. Phys. Ther. 91, 754-764. doi: 10.2522/ptj.20100136

Berrocoso, E., Mico, J. A., Vitton, O., Ladure, P., Newman-Tancredi, A., Depoortere, R., et al. (2011). Evaluation of milnacipran, in comparison with amitriptyline, on cold and mechanical allodynia in a rat model of neuropathic pain. Eur. J. Pharmacol. 655, 46-51. doi: 10.1016/j.ejphar.2011.01.022

Bliss, T. V., and Collingridge, G. L. (2013). Expression of NMDA receptordependent LTP in the hippocampus: bridging the divide. Mol. Brain 6:5. doi: $10.1186 / 1756-6606-6-5$

Breiman, L. (2001). Random forests. Mach. Learn. 45, 5-32. doi: 10.1023/A: 1010933404324

Chen, J., Barrett, D. W., He, Y., and Gonzalez-Lima, F. (2016). Anxiolytic-like behavioural effects of head electroacupuncture in rats susceptible to stress. Acupunct. Med. 34, 235-240. doi: 10.1136/acupmed-2015-010978

Chen, R. W., Liu, H., An, J. X., Qian, X. Y., Jiang, Y. D., Cope, D. K., et al. (2017). Cognitive effects of electro-acupuncture and pregabalin in a trigeminal neuralgia rat model induced by cobra venom. J. Pain Res. 10, 1887-1897. doi: 10.2147/JPR.S140840

Cheng, D., Hoogenraad, C. C., Rush, J., Ramm, E., Schlager, M. A., Duong, D. M., et al. (2006). Relative and absolute quantification of postsynaptic density proteome isolated from rat forebrain and cerebellum. Mol. Cell Proteomics 5, 1158-1170. doi: 10.1074/mcp.D500009-MCP200

Cho, Y. J., Song, Y. K., Cha, Y. Y., Shin, B. C., Shin, I. H., Park, H. J., et al. (2013). Acupuncture for chronic low back pain: a multicenter, randomized, patient-assessor blind, sham-controlled clinical trial. Spine 38, 549-557. doi: 10.1097/BRS.0b013e318275e601

Citri, A., and Malenka, R. C. (2008). Synaptic plasticity: multiple forms, functions, and mechanisms. Neuropsychopharmacology 33, 18-41. doi: 10.1038/sj.npp. 1301559

Couilliot, M. F., Darees, V., Delahaye, G., Ercolano, P., Carcaille, M., Vytopilova, P., et al. (2013). Acceptability of an acupuncture intervention for geriatric chronic pain: an open pilot study. J. Integr. Med. 11, 26-31. doi: 10.3736/ jintegrmed2013005

De Gregorio, D., McLaughlin, R. J., Posa, L., Ochoa-Sanchez, R., Enns, J., LopezCanul, M., et al. (2019). Cannabidiol modulates serotonergic transmission and reverses both allodynia and anxiety-like behavior in a model of neuropathic pain. Pain 160, 136-150. doi: 10.1097/j.pain.0000000000001386

Dimitrov, E. L., Tsuda, M. C., Cameron, H. A., and Usdin, T. B. (2014). Anxietyand depression-like behavior and impaired neurogenesis evoked by peripheral neuropathy persist following resolution of prolonged tactile hypersensitivity. J. Neurosci. 34, 12304-12312. doi: 10.1523/JNEUROSCI.0312-14.2014

Ezzati, A., Zimmerman, M. E., Katz, M. J., Sundermann, E. E., Smith, J. L., Lipton, M. L., et al. (2014). Hippocampal subfields differentially correlate with chronic pain in older adults. Brain Res. 1573, 54-62. doi: 10.1016/j.brainres.2014.05.025

Fernandez de Sevilla, D., and Buno, W. (2010). The muscarinic long-term enhancement of NMDA and AMPA receptor-mediated transmission at schaffer collateral synapses develop through different intracellular mechanisms. J. Neurosci. 30, 11032-11042. doi: 10.1523/JNEUROSCI.1848-10.2010

Fields, H. L., Basbaum, A. I., Clanton, C. H., and Anderson, S. D. (1977). Nucleus raphe magnus inhibition of spinal cord dorsal horn neurons. Brain Res. 126, 441-453. doi: 10.1016/0006-8993(77)90596-0

Fleckenstein, J., Kruger, P., and Ittner, K. P. (2018). Effects of single-point acupuncture (HT7) in the prevention of test anxiety: results of a RCT. PLoS One 13:e0202659. doi: 10.1371/journal.pone.0202659

Fonseca, R. (2012). Activity-dependent actin dynamics are required for the maintenance of long-term plasticity and for synaptic capture. Eur. J. Neurosci. 35, 195-206. doi: 10.1111/j.1460-9568.2011.07955.x

Geurts, P., Ernst, D., and Wehenkel, L. (2006). Extremely randomized trees. Mach. Learn. 63, 3-42. doi: 10.1007/s10994-006-6226-1

Goebel, S. M., Alvestad, R. M., Coultrap, S. J., and Browning, M. D. (2005). Tyrosine phosphorylation of the N-methyl-D-aspartate receptor is enhanced in synaptic membrane fractions of the adult rat hippocampus. Brain Res. Mol. Brain Res. 142, 65-79. doi: 10.1016/j.molbrainres.2005.09.012

Goldman, N., Chen, M., Fujita, T., Xu, Q., Peng, W., Liu, W., et al. (2010). Adenosine A1 receptors mediate local anti-nociceptive effects of acupuncture. Nat. Neurosci. 13, 883-888. doi: 10.1038/nn.2562

Guida, F., Luongo, L., Marmo, F., Romano, R., Iannotta, M., Napolitano, F., et al. (2015). Palmitoylethanolamide reduces pain-related behaviors and restores glutamatergic synapses homeostasis in the medial prefrontal cortex of neuropathic mice. Mol. Brain 8:47. doi: 10.1186/s13041-0150139-5

Holmes, W. R., and Grover, L. M. (2006). Quantifying the magnitude of changes in synaptic level parameters with long-term potentiation. J. Neurophysiol. 96, 1478-1491. doi: 10.1152/jn.00248.2006

Hopton, A., Macpherson, H., Keding, A., and Morley, S. (2014). Acupuncture, counselling or usual care for depression and comorbid pain: secondary analysis of a randomised controlled trial. BMJ Open 4:e04964. doi: 10.1136/bmjopen2014-004964

Huang, W., Kutner, N., and Bliwise, D. L. (2011). Autonomic activation in insomnia: the case for acupuncture. J. Clin. Sleep Med. 7, 95-102.

Jang, J. H., Park, J. Y., Oh, J. Y., Bae, S. J., Jang, H., Jeon, S., et al. (2018). Novel analgesic effects of melanin-concentrating hormone on persistent neuropathic and inflammatory pain in mice. Sci. Rep. 8, 707. doi: 10.1038/s41598-01819145-z

Jin, L., Gao, L. F., Sun, D. S., Wu, H., Wang, Q., Ke, D., et al. (2017). Long-term Ameliorative Effects of the Antidepressant Fluoxetine Exposure on Cognitive Deficits in 3 x TgAD Mice. Mol. Neurobiol. 54, 4160-4171. doi: 10.1007/s12035016-9952-9

Ju, Z. Y., Wang, K., Cui, H. S., Yao, Y., Liu, S. M., Zhou, J., et al. (2017). Acupuncture for neuropathic pain in adults. Cochrane Database Syst. Res. 12:CD012057. doi: 10.1002/14651858.CD012057.pub2

Khan, S. A., Keaser, M. L., Meiller, T. F., and Seminowicz, D. A. (2014). Altered structure and function in the hippocampus and medial prefrontal cortex in patients with burning mouth syndrome. Pain 155, 1472-1480. doi: 10.1016/j. pain.2014.04.022

Kim, J. H., Anwyl, R., Suh, Y. H., Djamgoz, M. B., and Rowan, M. J. (2001). Use-dependent effects of amyloidogenic fragments of (beta)-amyloid precursor protein on synaptic plasticity in rat hippocampus in vivo. J. Neurosci. 21, 1327-1333. doi: 10.1523/jneurosci.21-04-01327.2001

Kim, S. K., Moon, H. J., Park, J. H., Lee, G., Shin, M. K., Hong, M. C., et al. (2007). The maintenance of individual differences in the sensitivity of acute and neuropathic pain behaviors to electroacupuncture in rats. Brain Res. Bull. 74, 357-360. doi: 10.1016/j.brainresbull.2007.07.006

Kim, S. K., Park, J. H., Bae, S. J., Kim, J. H., Hwang, B. G., Min, B. I., et al. (2005). Effects of electroacupuncture on cold allodynia in a rat model of neuropathic pain: mediation by spinal adrenergic and serotonergic receptors. Exp. Neurol. 195, 430-436. doi: 10.1016/j.expneurol.2005.06.018

Kodama, D., Ono, H., and Tanabe, M. (2007). Altered hippocampal long-term potentiation after peripheral nerve injury in mice. Eur. J. Pharmacol. 574, 127-132. doi: 10.1016/j.ejphar.2007.07.054

Kodama, D., Ono, H., and Tanabe, M. (2011). Increased hippocampal glycine uptake and cognitive dysfunction after peripheral nerve injury. Pain 152, 809-817. doi: 10.1016/j.pain.2010.12.029

Lee, B., Sur, B. J., Kwon, S., Jung, E., Shim, I., Lee, H., et al. (2012). Acupuncture stimulation alleviates corticosterone-induced impairments of spatial memory and cholinergic neurons in rats. Evid. Based Complement. Alternat. Med. 2012:670536. doi: 10.1155/2012/670536

Lisman, J., Schulman, H., and Cline, H. (2002). The molecular basis of CaMKII function in synaptic and behavioural memory. Nat. Rev. Neurosci. 3, 175-190. doi: 10.1038/nrn753

Liu, S. J., Yang, C., Zhang, Y., Su, R. Y., Chen, J. L., Jiao, M. M., et al. (2016). Neuroprotective effect of beta-asarone against Alzheimer's disease: regulation of synaptic plasticity by increased expression of SYP and GluR1. Drug Des. Devel. Ther. 10, 1461-1469. doi: 10.2147/DDDT.S93559

Liu, Y., Zhou, L. J., Wang, J., Li, D., Ren, W. J., Peng, J., et al. (2017). TNF-alpha differentially regulates synaptic plasticity in the hippocampus and spinal cord by microglia-dependent mechanisms after peripheral nerve injury. J. Neurosci. 37, 871-881. doi: 10.1523/JNEUROSCI.2235-16.2016 
Luongo, L., Guida, F., Imperatore, R., Napolitano, F., Gatta, L., Cristino, L., et al. (2014). The A1 adenosine receptor as a new player in microglia physiology. Glia 62, 122-132. doi: 10.1002/glia.22592

Luongo, L., Petrelli, R., Gatta, L., Giordano, C., Guida, F., Vita, P., et al. (2012). $5^{\prime}$-Chloro-5'-deoxy-(+/-)-ENBA, a potent and selective adenosine $\mathrm{A}(1)$ receptor agonist, alleviates neuropathic pain in mice through functional glial and microglial changes without affecting motor or cardiovascular functions. Molecules 17, 13712-13726. doi: 10.3390/molecules 171213712

Luscher, C., and Malenka, R. C. (2012). NMDA receptor-dependent long-term potentiation and long-term depression (LTP/LTD.). Cold Spring Harb. Perspect. Biol. 4:a005710. doi: 10.1101/cshperspect.a005710

Malmberg, A. B., and Basbaum, A. I. (1998). Partial sciatic nerve injury in the mouse as a model of neuropathic pain: behavioral and neuroanatomical correlates. Pain 76, 215-222. doi: 10.1016/s0304-3959(98)00045-1

Mariani, M. M., Malm, T., Lamb, R., Jay, T. R., Neilson, L., Casali, B., et al. (2017). Neuronally-directed effects of RXR activation in a mouse model of Alzheimer's disease. Sci. Rep. 7:42270. doi: 10.1038/srep42270

Max, M. B., Culnane, M., Schafer, S. C., Gracely, R. H., Walther, D. J., Smoller, B., et al. (1987). Amitriptyline relieves diabetic neuropathy pain in patients with normal or depressed mood. Neurology 37, 589-596. doi: 10.1212/wnl.37.4.589

Mutso, A. A., Radzicki, D., Baliki, M. N., Huang, L., Banisadr, G., Centeno, M. V., et al. (2012). Abnormalities in hippocampal functioning with persistent pain. J. Neurosci. 32, 5747-5756. doi: 10.1523/JNEUROSCI.0587-12.2012

Nicholson, B., and Verma, S. (2004). Comorbidities in chronic neuropathic pain. Pain Med. 5(Suppl. 1), S9-S27. doi: 10.1111/j.1526-4637.2004.04019.x

Palazzo, E., Luongo, L., Guida, F., Marabese, I., Romano, R., Iannotta, M., et al. (2016). D-Aspartate drinking solution alleviates pain and cognitive impairment in neuropathic mice. Amino Acids 48, 1553-1567. doi: 10.1007/s00726-0162205-4

Paramore, L. C. (1997). Use of alternative therapies: estimates from the 1994 Robert Wood Johnson foundation national access to care survey. J. Pain Symptom Manage. 13, 83-89. doi: 10.1016/s0885-3924(96)00299-0

Park, J. Y., Park, J. J., Jeon, S., Doo, A. R., Kim, S. N., Lee, H., et al. (2014). From peripheral to central: the role of ERK signaling pathway in acupuncture analgesia. J. Pain 15, 535-549. doi: 10.1016/j.jpain.2014.01.498

Pedregosa, F., Varoquaux, G., Gramfort, A., Michel, V., Thirion, B., Grisel, O., et al. (2011). Scikit-learn: machine learning in python. J. Mach. Learn. Res. 12, 2825-2830.

Price, D. D. (2000). Psychological and neural mechanisms of the affective dimension of pain. Science 288, 1769-1772. doi: 10.1126/science.288.5472.1769

Ren, W. J., Liu, Y., Zhou, L. J., Li, W., Zhong, Y., Pang, R. P., et al. (2011). Peripheral nerve injury leads to working memory deficits and dysfunction of the hippocampus by upregulation of TNF-alpha in rodents. Neuropsychopharmacology 36, 979-992. doi: 10.1038/npp.2010.236

Saffarpour, S., Shaabani, M., Naghdi, N., Farahmandfar, M., Janzadeh, A., and Nasirinezhad, F. (2017). In vivo evaluation of the hippocampal glutamate, GABA and the BDNF levels associated with spatial memory performance in a rodent model of neuropathic pain. Physiol. Behav. 175, 97-103. doi: 10.1016/ j.physbeh.2017.03.025

Schnurr, R. F., and MacDonald, M. R. (1995). Memory complaints in chronic pain. Clin. J. Pain 11, 103-111. doi: 10.1097/00002508-199506000-00004

Shang, X., Shang, Y., Fu, J., and Zhang, T. (2017). Nicotine significantly improves chronic stress-induced impairments of cognition and synaptic plasticity in mice. Mol. Neurobiol. 54, 4644-4658. doi: 10.1007/s12035-0160012-2

Sweet, E. S., Saunier-Rebori, B., Yue, Z., and Blitzer, R. D. (2015). The Parkinson's disease-associated mutation LRRK2-G2019S impairs synaptic plasticity in mouse hippocampus. J. Neurosci. 35, 11190-11195. doi: 10.1523/JNEUROSCI. 0040-15.2015

Tovar, K. R., and Westbrook, G. L. (1999). The incorporation of NMDA receptors with a distinct subunit composition at nascent hippocampal synapses in vitro. J. Neurosci. 19, 4180-4188. doi: 10.1523/jneurosci.19-10-04180.1999

Tozzi, A., Sclip, A., Tantucci, M., de Iure, A., Ghiglieri, V., Costa, C., et al. (2015). Region- and age-dependent reductions of hippocampal long-term potentiation and NMDA to AMPA ratio in a genetic model of Alzheimer's disease. Neurobiol. Aging 36, 123-133. doi: 10.1016/j.neurobiolaging.2014.07.002

Valet, M., Gundel, H., Sprenger, T., Sorg, C., Muhlau, M., Zimmer, C., et al. (2009). Patients with pain disorder show gray-matter loss in pain-processing structures: a voxel-based morphometric study. Psychosom. Med. 71, 49-56. doi: 10.1097/PSY.0b013e31818d1e02

Valtorta, F., Pennuto, M., Bonanomi, D., and Benfenati, F. (2004). Synaptophysin: leading actor or walk-on role in synaptic vesicle exocytosis? Bioessays 26, 445-453. doi: 10.1002/bies.20012

Villers, A., and Ris, L. (2013). Improved preparation and preservation of hippocampal mouse slices for a very stable and reproducible recording of long-term potentiation. J. Vis. Exp. 26:50483. doi: 10.3791/50483

Wang, H., Peng, R., Zhao, L., Wang, S., Gao, Y., Wang, L., et al. (2015). The relationship between NMDA receptors and microwave-induced learning and memory impairment: a long-term observation on Wistar rats. Int. J. Radiat. Biol. 91, 262-269. doi: 10.3109/09553002.2014.988893

Wang, Q. P., and Nakai, Y. (1994). The dorsal raphe: an important nucleus in pain modulation. Brain Res. Bull. 34, 575-585. doi: 10.1016/0361-9230(94)90143-0

Wang, X. Q., Zhong, X. L., Li, Z. B., Wang, H. T., Zhang, J., Li, F., et al. (2015). Differential roles of hippocampal glutamatergic receptors in neuropathic anxiety-like behavior after partial sciatic nerve ligation in rats. BMC Neurosci. 16:14. doi: 10.1186/s12868-015-0150-X

Wu, Z., Qian, X. Y., An, J. X., Liu, C. C., Tian, M., Cope, D. K., et al. (2015). Trigeminal neuralgia induced by cobra venom in the rat leads to deficits in abilities of spatial learning and memory. Pain Physician. 18, E207-E216.

Xu, Q., Liu, T., Chen, S., Gao, Y., Wang, J., Qiao, L., et al. (2012). The cumulative analgesic effect of repeated electroacupuncture involves synaptic remodeling in the hippocampal CA3 region. Neural Regen. Res. 7, 1378-1385. doi: 10.3969/j. issn.1673-5374.2012.18.003

Yu, C. C., Wang, Y., Shen, F., Kong, L. H., Wang, Y. W., Zhou, H., et al. (2018). High-frequency $(50 \mathrm{~Hz})$ electroacupuncture ameliorates cognitive impairment in rats with amyloid beta 1-42-induced Alzheimer's disease. Neural Regen. Res. 13, 1833-1841. doi: 10.4103/1673-5374.238620

Zhou, Q., Zhu, S., Guo, Y., Lian, L., Hu, Q., Liu, X., et al. (2018). Adenosine A1 receptors play an important protective role against cognitive impairment and long-term potentiation inhibition in a pentylenetetrazol mouse model of epilepsy. Mol. Neurobiol. 55, 3316-3327. doi: 10.1007/s12035-0170571-x

Zhu, C., Xu, Q., Wang, C., Mao, Z., and Lin, N. (2017). Evidence that CA3 is underling the comorbidity between pain and depression and the Co-curation by wu-tou decoction in neuropathic pain. Sci. Rep. 7:11935. doi: 10.1038/s41598017-12184-y

Zhu, L., Yang, L., Zhao, X., Liu, D., Guo, X., Liu, P., et al. (2018). Xanthoceraside modulates NR2B-containing NMDA receptors at synapses and rescues learning-memory deficits in APP/PS1 transgenic mice. Psychopharmacology 235, 337-349. doi: 10.1007/s00213-017-4775-6

Zimmerman, M. E., Pan, J. W., Hetherington, H. P., Lipton, M. L., Baigi, K., and Lipton, R. B. (2009). Hippocampal correlates of pain in healthy elderly adults: a pilot study. Neurology 73, 1567-1570. doi: 10.1212/WNL.0b013e3181c0 d454

Conflict of Interest Statement: W-MJ was employed by company VUNO Inc.

The remaining authors declare that the research was conducted in the absence of any commercial or financial relationships that could be construed as a potential conflict of interest.

Copyright (c) 2019 Jang, Kim, Jung, Kim, Song, Kim, Oh, Park, Ryu, Song and Park. This is an open-access article distributed under the terms of the Creative Commons Attribution License (CC BY). The use, distribution or reproduction in other forums is permitted, provided the original author(s) and the copyright owner(s) are credited and that the original publication in this journal is cited, in accordance with accepted academic practice. No use, distribution or reproduction is permitted which does not comply with these terms. 


\section{Self-Regulation of SMR Power Led to an Enhancement of Functional Connectivity of Somatomotor Cortices in Fibromyalgia Patients}

\section{OPEN ACCESS}

Edited by:

Lauren C. Heathcote, Stanford University School of Medicine, United States

Reviewed by: Enrico Collantoni,

University of Padova, Italy

Benito de Celis Alonso, Meritorious Autonomous University of Puebla, Mexico

*Correspondence: Pedro Montoya pedro.montoya@uib.es Miguel A. Muñoz mamuoz@ugr.es

Specialty section: This article was submitted to Brain Imaging Methods, a section of the journal Frontiers in Neuroscience

Received: 23 December 2019 Accepted: 03 March 2020 Published: 19 March 2020

Citation: Terrasa JL, Barros-Loscertales A, Montoya $P$ and Muñoz MA (2020) Self-Regulation of SMR Power Led to an Enhancement of Functional Connectivity of Somatomotor Cortices in Fibromyalgia Patients.

Front. Neurosci. 14:236. doi: 10.3389/fnins.2020.00236

\author{
Juan L. Terrasa', Alfonso Barros-Loscertales ${ }^{2}$, Pedro Montoya ${ }^{1 *}$ and Miguel A. Muñoz ${ }^{3 *}$ \\ ${ }^{1}$ Cognitive and Affective Neuroscience and Clinical Psychology, Research Institute of Health Sciences (IUNICS) and Balearic \\ Islands Health Research Institute (IdISBa), University of the Balearic Islands (UIB), Palma, Spain, ${ }^{2}$ Department of Psychology, \\ Universitat Jaume I, Castellón, Spain, ${ }^{3}$ Brain, Mind and Behavior Research Center, University of Granada (CIMCYC-UGR), \\ Granada, Spain
}

Neuroimaging studies have demonstrated that altered activity in somatosensory and motor cortices play a key role in pain chronification. Neurofeedback training of sensorimotor rhythm (SMR) is a tool which allow individuals to self-modulate their brain activity and to produce significant changes over somatomotor brain areas. Several studies have further shown that neurofeedback training may reduce pain and other pain-related symptoms in chronic pain patients. The goal of the present study was to analyze changes in SMR power and brain functional connectivity of the somatosensory and motor cortices elicited by neurofeedback task designed to both synchronize and desynchronize the SMR power over motor and somatosensory areas in fibromyalgia patients. Seventeen patients were randomly assigned to the SMR training $(n=9)$ or to a sham protocol $(n=8)$. All participants were trained during 6 sessions, and $\mathrm{fMRI}$ and EEG power elicited by synchronization and desynchronization trials were analyzed. In the SMR training group, four patients achieved the objective of SMR modulation in more than $70 \%$ of the trials from the second training session (good responders), while five patients performed the task at the chance level (bad responders). Good responders to the neurofeedback training significantly reduced pain and increased both SMR power modulation and functional connectivity of motor and somatosensory related areas during the last neurofeedback training session, whereas no changes in brain activity or pain were observed in bad responders or participants in the sham group. In addition, we observed that good responders were characterized by reduced impact of fibromyalgia and pain symptoms, as well as by increased levels of health-related quality of life during the pre-training sessions. In summary, the present study revealed that neurofeedback training of SMR elicited significant brain changes in somatomotor areas leading to a significant reduction of pain in fibromyalgia patients. In this sense, our research provide evidence that neurofeedback training is a promising tool for a better understanding of brain mechanisms involved in pain chronification.

Keywords: fibromyalgia, neurofeedback, sensorimotor rhythm, fMRI, functional connectivity, somatosensory cortex, motor cortex 


\section{INTRODUCTION}

Fibromyalgia (FM) is a chronic pain syndrome characterized by generalized and enhanced pain sensitivity, as well as by fatigue, morning stiffness, sleep disturbance, affective and cognitive dysfunctions, and a generalized hypersensitivity to pain stimulation (Wolfe et al., 1990, 1995). Its prevalence ranges from 3 to $10 \%$ in the general adult population and is more frequent in women than men (Wolfe et al., 2013; Clauw et al., 2018). Although the underlying etiology of FM still remains unclear, several studies have showed altered brain activation in areas of the so called pain network involved in the emotional and cognitive processing of pain (Gracely, 2004; Burgmer et al., 2009, 2010). Functional magnetic resonance imaging (fMRI) studies have further reported that resting-state functional connectivity of the anterior cingulate cortex (ACC), basal ganglia insula, thalamus, amygdala, medial prefrontal cortex and somatosensory and motor cortices were increased in patients with FM as compared with healthy controls (Cifre et al., 2012; Flodin et al., 2014; Ichesco et al., 2014), reflecting an abnormal hyperexcitability of the central nervous system (Desmeules et al., 2003). In this regard, altered somatosensory and motor cortex activity has been proposed to play a central role in the experience of pain and its chronification (Harris, 1999; Pujol et al., 2014; González-Roldán et al., 2016; Lim et al., 2016; Don et al., 2019). Furthermore, primary motor cortex activity has been suggested to be a major modulator of pain processing (Castillo Saavedra et al., 2014), while primary somatosensory cortex is highly involved in the localization and discrimination of pain experience (Diers, 2019).

EEG neurofeedback is a technique based on learning to selfregulate several parameters of cortical activity such as amplitude, frequency and/or coherence of EEG signal (Gruzelier, 2014). During neurofeedback training, individuals learn to modify their own brain activity by receiving visual or acoustic information about these EEG parameters (Enriquez-Geppert et al., 2017). Neurofeedback has been widely used successfully in chronic pain syndromes, showing potential benefits to reduce pain, anxiety and depression in these patients (Mueller et al., 2001; Kravitz et al., 2006; Kayıran et al., 2010; Nelson et al., 2010; Caro and Winter, 2011). In this sense, it has been highlighted that the efficacy of the treatment could be related to the decrease and/or the increase of somatosensory and motor activity associated with the processing of nociceptive information (Jensen et al., 2008, 2014). Sensorimotor rhythm (SMR) refers to oscillations between 12 and $15 \mathrm{~Hz}$ recorded over somatosensory and motor areas (Pfurtscheller and Lopes da Silva, 1999; Budzynski, 2009; Gruzelier, 2014). Studies about SMR-based neurofeedback training in chronic pain patients have shown significant short-term improvements in pain relief, and other non-pain associated symptoms in patients with complex regional pain syndrome (Jensen et al., 2007), chronic low back pain (Mayaud et al., 2019) and chronic spinal cord injury (Vučković et al., 2019). Furthermore, SMR-based neurofeedback training has provided evidence that it is able to reduce pain and fatigue symptoms in patients with fibromyalgia (Kayiran et al., 2010; Caro and Winter, 2011).
Although several studies have demonstrated that neurofeedback training was able to reduce pain-related symptoms, little is known about the functional changes that the SMR-based neurofeedback training is eliciting in EEG activity and brain connectivity. In this sense, increased resting-state functional connectivity in several pain areas such as ACC (Ros et al., 2013), insula (Kluetsch et al., 2014) or the amygdala (Nicholson et al., 2016) have been reported after neurofeedback training of the alpha EEG. In addition, a significant enhancement of resting-state functional connectivity of somatosensory and motor cortices has been demonstrated after neurofeedback training of SMR in patients with stroke (Várkuti et al., 2013; Young et al., 2014; Mohanty et al., 2018) and in healthy participants (Terrasa et al., 2019). Although all these findings support the notion that neurofeedback training can produce relevant changes in clinical symptoms and brain activity, little is known about the neurophysiological processes involved during brain self-regulation training.

The primary goal of the present study was to analyze changes in SMR activity and brain functional connectivity of the somatosensory and motor cortices in response to neurofeedback training of the SMR in FM patients. For this purpose, a training protocol based on learning to synchronize and desynchronize the SMR power over motor and somatosensory areas was applied during six sessions, and brain changes produced when performing the neurofeedback task were examined. Our hypothesis was that those FM participants achieving a successful self-regulation of the SMR would show increased synchronization and desynchronization modulation of SMR power, enhanced somatomotor functional connectivity and reduced pain during the last neurofeedback training session.

\section{MATERIALS AND METHODS}

\section{Participants}

Seventeen right-handed female patients (aged $54.94 \pm 10.11$ ) with a diagnosis of FM were recruited from the Asociación Granadina de Fibromialgia (AGRAFIM) in Granada (Spain). The diagnosis of FM was confirmed by a professional rheumatologist following the American Rheumatology College 2010 Criteria. Exclusion criteria were: FM diagnosis of less than 1 year, pregnancy, vision or auditory deficits, and neurological or psychiatric diseases (except depression). Thirteen of the seventeen fibromyalgia patients had a diagnosed and medicated depression disorder. No participants with other psychiatric disorders were accepted in the study. All participants were taking regular medication, including analgesic/myorelaxant (88.24\%), antidepressant (76.47\%), and anxiolytic (70.59\%). During the experiment, participants were asked to avoid the use of any other non-pharmacology therapy. The study was conducted in accordance with the Declaration of Helsinki (1991) and approved by the Ethics Committee of the Balearic Islands (Spain). Written informed consents were obtained from the participants after the experimental procedure explanation. 


\section{Procedure and Clinical Assessment}

The patients attended a total of seven sessions and were sequentially assigned to either a SMR neurofeedback training (SMR, $n=9$ ) or a control group that received false feedback during the training task (SHAM, $n=8$ ) following the order of their arrival at the first session. In the first session, a thorough psychological evaluation was conducted under the supervision of a trained and experienced psychologist (MM), including a semi-structured interview on chronic pain and following self-report questionnaires: the McGill Pain Questionnaire (MPQ) (Melzack, 1975), the West Haven-Yale Multidimensional Pain Inventory (WHYMPI) (Kerns et al., 1985), the Tampa Scale for Kinesiophobia (TSK) (Roelofs et al., 2004), the Pain Anxiety Symptoms Scale (PASS) (McCracken et al., 1992), the Pain Vigilance and Awareness Questionnaire (PVAQ) (McCracken, 1997), the MOS Social Support Survey (MOS) (Sherbourne and Stewart, 1991), the Coping Strategies Questionnaire (CSQ) (Rosenstiel and Keefe, 1983), the MOS 36item Short-form Health Survey (SF-36) (Ware and Sherbourne, 1992), the Beck Depression Inventory (BDI-II) (Beck et al., 1996), and the Fibromyalgia Impact Questionnaire (FIQ) (Burckhardt et al., 1991).

After the psychological assessment, patients participated in a six-session neurofeedback training program with 3 sessions per week during 2 weeks. The training protocol was successfully tested in a previous work with healthy participants (Terrasa et al., 2019). During the first (PRE session) and the sixth sessions (POST session), all individuals performed the training in a MRI scanner, while the rest four sessions were performed in a MRI simulator. The simulator reproduced the characteristic disturbing sounds of the real scanner. At the end of the PRE and the POST sessions, patients were asked to rate their pain using a numerical scale ranging from 0 to 100 . Furthermore, given that high anxiety levels can impair neurofeedback training (Hardman et al., 1997; Gruzelier et al., 1999), the level of anxiety was assessed with the State-Trait Anxiety Inventory (STAI) (Spielberger et al., 1970) before the beginning of each assessment session. In addition, all participants were asked to complete a diary three times a day (morning, afternoon, evening) with ratings of pain, fatigue and negative mood on a numerical scale ranging from 0 to 100 .

\section{EEG Neurofeedback Task and Processing}

During the neurofeedback training program, EEG signals were acquired by a QuickAmp amplifier (Brain Products $\mathrm{GmbH}$, Munich, Germany) at $1000 \mathrm{~Hz}$ sampling rate, with high-pass and low-pass filter settings at 0.10 and $70 \mathrm{~Hz}$, respectively. A $50 \mathrm{~Hz}$ notch filter was also applied. EEG was recorded from $64 \mathrm{Ag} / \mathrm{AgCl}$ electrodes placed according of the 1020 International System referenced to FCz. Ground electrode was located at position AFz. Electrode impedance was kept lower than $10 \mathrm{kOhm}$.

The EEG neurofeedback task was to learn to synchronize (i.e., by increasing power amplitudes at specific electrodes) and to desynchronize (i.e., by decreasing power amplitudes at specific electrodes) the SMR. The task was performed using the Cursor Task module of BCI2000 platform (Schalk et al., 2004). Each trial began with the presentation of a target (a gray vertical rectangle) located on the left or right edge of the screen. At the same time, a gray ball appeared in the center of the screen and subjects were asked to control the movement of the ball on the horizontal axis by synchronizing or desynchronizing the SMR for a maximum of $9 \mathrm{~s}$. The goal of the task was to move the ball and impact the target. If the goal was achieved, the ball remained on the screen for a second (reward presentation) and then disappeared; otherwise, the ball simply disappeared. The participants did not receive any instruction other than that they had to learn to control the ball (move it to the right or left according to the position of the target) and hit the target as many times as possible. Two parameters of the task performance were analyzed: number of trials in which the ball hit the target (percentage of hits) and the time to hit the target in successful trials (duration of successful trials).

During each neurofeedback trial, SMR power at C3, CP1, and CP5 electrodes was calculated every $0.5 \mathrm{~s}$ of input data by means of maximum entropy method (autoregressive model order $=16$ ) with $3 \mathrm{~Hz}$ bin resolution. These signal features were translated into output control signal using a linear equation selecting the power of the selected electrodes into $12-15 \mathrm{~Hz}$ frequency bin. Finally, the signal was normalized to make the output control signal zero mean and unit variance. The subjects had to synchronize the SMR power to move the cursor to the left or to desynchronize the power to move the cursor to the right. The greater the power variation was, the greater the cursor movement speed.

Given that the PRE and POST sessions were conducted in the MRI scanner, BrainVision RecView software was applied online to partially remove the gradient artifact (imaging artifact) and the pulse artifact (ballistocardiographic artifact) of MRI from the EEG signal using an automated implementation of the average subtraction method (Allen et al., 1998, 2000). RecView was modified to enable export of the corrected EEG data in real time through a TCP/IP socket to BCI2000. This procedure was optimized with BrainVision Syncbox ensuring an optimal communication between the MRI scanner master clock and Review.

During the PRE and POST sessions, the task consisted of 100 trials (50 trials with the target displayed on each side of the screen) presented in random order with an interval between the $15 \mathrm{~s}$ trials, and all participants (SMR and SHAM) received real feedback on their performance. The remaining four training sessions consisted of four runs with 20 trials (10 trials with the target displayed on each side of the screen) presented in random order within each run and with an interval between trials of 6 s. In these neurofeedback training sessions, only the SMR group received real feedback on the SMR power variations, while the SHAM group received random feedback. For the latter, the movement of the ball was manipulated to reach the target only in $50 \%$ of the trials ( $25 \%$ right, $25 \%$ left).

The preprocessing of EEG data during the PRE and POST sessions was carried out using Matlab R2016b. EEG signals were bandpass filtered within $1-30 \mathrm{~Hz}$ and an algorithm 


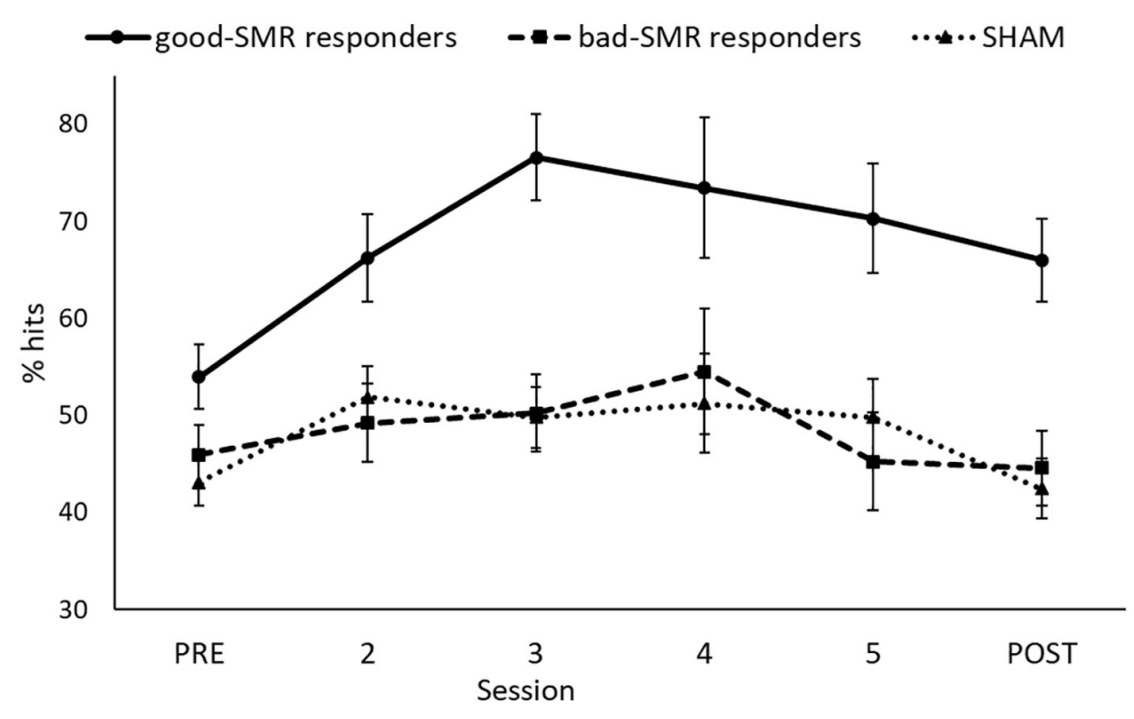

FIGURE 1 | Percentage of successful trials for each group through all the sessions.

for ocular correction (Gratton and Coles) was applied. Data were segmented into epochs of $9 \mathrm{~s}$ and separated by trial type (synchronization or desynchronization). Then, power spectral density was calculated for the interval between 1 and $30 \mathrm{~Hz}$ ( $1 \mathrm{~Hz}$ resolution) for all channels and each trial type (synchronization or desynchronization). The average power density at C3, CP1, and CP5 electrodes within SMR range (12$15 \mathrm{~Hz}$ ) was computed and the difference on SMR power between synchronization and desynchronization trials was calculated as an SMR modulation score, reflecting the degree of self-control over SMR activity.

\section{Functional MRI Data Acquisition}

During the PRE and POST sessions, fMRI images were acquired using a 3.0 Tesla scanner (SIEMENS MAGNETOM TrioTim syngo MR). Echo-planar sequence (EPI) functional images of the whole brain (except the cerebellum) were acquired during the EEG neurofeedback for a maximum time of 40 min and $6 \mathrm{~s}$ (Total volumes $=1200 ; 27$ axial slices per volume interleaved; $\mathrm{TR}=2.0$ $\mathrm{s}$; ET $=23 \mathrm{~ms}$; Flip Angle $=80^{\circ}$; Acquisition Matrix $=66 \times 66$; FOV $=232 \mathrm{~mm}$; Slice Thickness $=3.0 \mathrm{~mm}$; no gap). Furthermore, MPRAGE sequence T1 anatomical images were also acquired for each subject to perform co-register and nuisance pre-analyses $\left(176\right.$ slices; TR $=1900 \mathrm{~ms} ; \mathrm{ET}=2.52 \mathrm{~ms}$; Flip Angle $=9^{\circ}$; FOV $=250 \mathrm{~mm}$; Slice Thickness $=1 \mathrm{~mm}$ ).

\section{Data Analyses}

After the initial statistical analyses, we observed that participants in the SMR group as a whole could not achieve an average performance above the random level, as demonstrated in previous studies with healthy participants (Cincotti et al., 2008; Blankertz et al., 2010; Terrasa et al., 2019). Furthermore, there were no significant differences between the SMR and the SHAM groups on percentage of hits. Therefore, we decided to subdivide the SMR group in good responders (who achieved a mean performance level above $50 \%$ of success during all the sessions) and bad responders (who achieved a mean performance level under $50 \%$ of success during all the sessions). Good responders showed $70 \%$ of success in at least one session of the neurofeedback training. Thus, the study was finally conducted with three groups: good-SMR responders $(n=4)$ with $67.76 \% \pm 15.97$ of successful trials (mean of the six sessions), bad-SMR responders $(n=5)$ with $48.31 \% \pm 7.26$ of successful trials and SHAM group $(n=8)$. The task performance (percentage of hits) for each group through the six sessions are shown in Figure 1.

Statistical analyses were carried out using IBM SPSS Statistics v21. For repeated measures analyses, normal distributions of the used variables were tested and Greenhouse-Geisser epsilon corrections were applied to control for violation of the sphericity assumption. Results are reported with the original degrees of freedom, the $p$-values and the partial eta squared parameters $\left(\eta_{\mathrm{p}}^{2}\right)$. When significant effects were found, post hoc analyses were performed using Bonferroni correction.

For the demographic and psychological data, one-way analyses of variance (ANOVAs) were used to examine differences among groups (good-SMR responders, bad-SMR responders and SHAM) on age, years since FM was diagnosed and self-report questionnaires (MPQ, WHYMPI, TSK, PASS, PVAQ, MOS, CSQ, SF-36, BDI-II, and FIQ). Group differences in depression comorbidity were analyzed with a Chi-Squared test. Differences on pain ratings and STAI-S scores were examined by using an ANOVA with the factors Group and Assessment session (PRE vs. POST). With respect to the diary data, the average of the three data points (morning, afternoon, night) obtained during the day after the PRE assessment session, as well as the average of the data points obtained the previous day to the POST assessment session was computed for pain, fatigue and negative mood. Differences on these ratings were tested by using ANOVAs with the factors Group and Assessment session (PRE vs. POST). 
Task performance scores (percentage of hits and duration of successful trials) during the assessment sessions (PRE vs. POST) were tested by using an ANOVA with the factors Group, Assessment session and Trial type (synchronization vs. desynchronization). Regarding the EEG analyses and to test that good-SMR responders would show significant training effects on SMR modulation scores compared to bad-SMR responders and SHAM group, an ANOVA with the factors Group Assessment Session (PRE vs. POST) was carried out at selected electrodes (C3, CP1, and CP5).

In order to further explore the possible interference of FM symptoms on training effects, those questionnaire scores that showed significant differences among the groups were correlated with task performance scores (percentage of hits and duration of successful trials) and SMR modulation scores during the PRE and POST sessions.

\section{Functional MRI Analyses}

The fMRI connectivity analyses were performed with the CONNfMRI fc toolbox v18a (Whitfield-Gabrieli and Nieto-Castanon, 2012) in conjunction with SPM 12 (Wellcome Department of Imaging Neuroscience, London, United Kingdom $)^{1}$. All structural and functional sequences in both PRE and POST sessions were pre-processed using the CONN's default pipeline for volume-based analysis following these steps: resampling to $2 \times 2 \times 2 \mathrm{~mm}$ voxels and unwarping, centering, slice time correction, normalization to the Montreal Neurological Institute (MNI) template, outlier detection to use as a first-level nuisance covariate (ART-based scrubbing), and smoothing to an $8 \mathrm{~mm}$ Gaussian kernel. Motion parameters (translations in the $\mathrm{x}, \mathrm{y}$, and $\mathrm{z}$ directions) were entered as multiple regressors and images with motion over $2.0 \mathrm{~mm}$ were regressed entirely out of the time course. Furthermore, BOLD data underwent a denoising process by using CompCor method (Behzadi et al., 2007) in a single linear regression step, and applying a band-pass filter $(0.01-0.09 \mathrm{~Hz})$ in order to reduce both noise effects and low frequency drift.

A Seed-to-Voxel parametrical analysis was performed by using four seeds of interest (3 somatomotor and 1 visual brain region, all bilaterally) that were preselected from the HarvardOxford atlas: precentral gyri (PreCG), postcentral gyri (PostCG), supplementary motor area (SMA), and intracalcarine cortex (ICC). The visual area was included as control. Individual correlation maps were generated extracting the mean BOLD time course from the eight preselected seeds and calculating the correlation coefficients with the BOLD time-course of each voxel throughout the whole brain. These correlations were obtained by applying the General Linear Model (GLM) and bivariate correlation analyses weighted for Hemodynamic Response Function (HRF). Only BOLD signals during successful trials were analyzed.

To examine group differences in functional connectivity during the POST compared to the PRE session, we used a $3 \times 2$ factorial analysis with the within-subjects factor Assessment session (PRE vs. POST) and the between-subjects

${ }^{1}$ http://www.fil.ion.ucl.ac.uk/spm/ factor Group (good-SMR responders, bad-SMR responders, SHAM). Furthermore, two-sample $t$-tests between pairs of groups separately for each session were performed. A wholebrain height threshold of $p<0.001$ (uncorrected) was used to identify areas with significant functional connectivity changes, and a family-wise error (FWE)-corrected threshold of $p<0.01$ at this height threshold was applied for all reported clusters.

\section{RESULTS}

\section{Demographic and Psychological Data}

Table 1 displays demographic and psychological data of the three groups. No significant differences among groups were found on age, "years since FM was diagnosed" or depression comorbidity $\left[\chi^{2}(2)=0.049, p=0.976\right]$. One-way ANOVAs revealed slight significant differences on FIQ scores among the three groups $\left[F(2,14)=4.156, p=0.043, \eta_{\mathrm{p}}{ }^{2}=0.409\right]$. However, Bonferroni post hoc analyses only showed a nonsignificant trend between good-SMR and bad-SMR responders on these scores $(40.81 \pm 5.12$ and $68.37 \pm 20.20$, respectively, $p=0.062$ ). Furthermore, one-way ANOVAs revealed significant differences among the three groups on three dimensions of the SF-36: "pain" $\left[F(2,15)=4.116, p=0.041, \eta_{\mathrm{p}}{ }^{2}=0.388\right]$, "general health perception" $[F(2,15)=5.954, p=0.015$, $\left.\eta_{\mathrm{p}}{ }^{2}=0.478\right]$ and "change in health" $[F(2,15)=7.127$, $\left.p=0.008, \eta_{\mathrm{p}}{ }^{2}=0.523\right]$. Bonferroni post hoc analyses of these effects revealed that good-SMR responders had higher score than bad-SMR responders on the dimensions "pain" (39.75 \pm 21.50 and $9.20 \pm 12.60$, respectively, $p=0.049$ ), "general health perception" (41.25 \pm 14.36 and $12.00 \pm 12.55$, respectively, $p=0.013)$ and "change in health" (43.75 \pm 12.50 and $5.00 \pm 12.18$, respectively, $p=0.008$ ). No significant differences among groups were observed on other selfreported questionnaires.

The ANOVA on pain ratings revealed a significant interaction effect of Group $\times$ Assessment Session $[F(2,14)=4.103$, $\left.p=0.040, \eta_{\mathrm{p}}{ }^{2}=0.370\right]$. The Bonferroni post hoc tests showed that good-SMR responders reported lower levels of pain than bad-SMR responders $(27.50 \pm 17.08$ and $74.00 \pm 19.49$, respectively, $p=0.047$ ) after the POST session. No significant group differences were found on pain ratings after the PRE session. Good-SMR responders also reported a significant reduction on pain ratings from the PRE to the POST session $(\mathrm{PRE}=47.50 \pm 20.62$, $\mathrm{POST}=27.50 \pm 17.08$; $p=0.042$ ). No significant differences between the PRE and the POST sessions were observed for either bad-SMR responders $(\mathrm{PRE}=66.00 \pm 5.48$, POST $=74.00 \pm 19.49)$ or SHAM participants $(\mathrm{PRE}=58.13 \pm 36.44, \mathrm{POST}=68.13 \pm 30.46)$ (Figure 2). Thus, neurofeedback training of the SMR was able to elicit a significant average pain reduction of $>40 \%$ in good responders, but not in bad responders. Moreover, it was observed that all good responders ( 4 out of 4 ) reduced pain ratings, whereas neurofeedback training elicited a pain reduction in only 2 of the 5 bad responders and in 2 of the 8 participants of the SHAM group (Supplementary Table S1). 
TABLE 1 | Demographic data and questionnaires scores (mean \pm SD) for each group, including effect sizes of the group differences $\left({ }^{*} p<0.05 ;{ }^{* *} p<0.01\right)$.

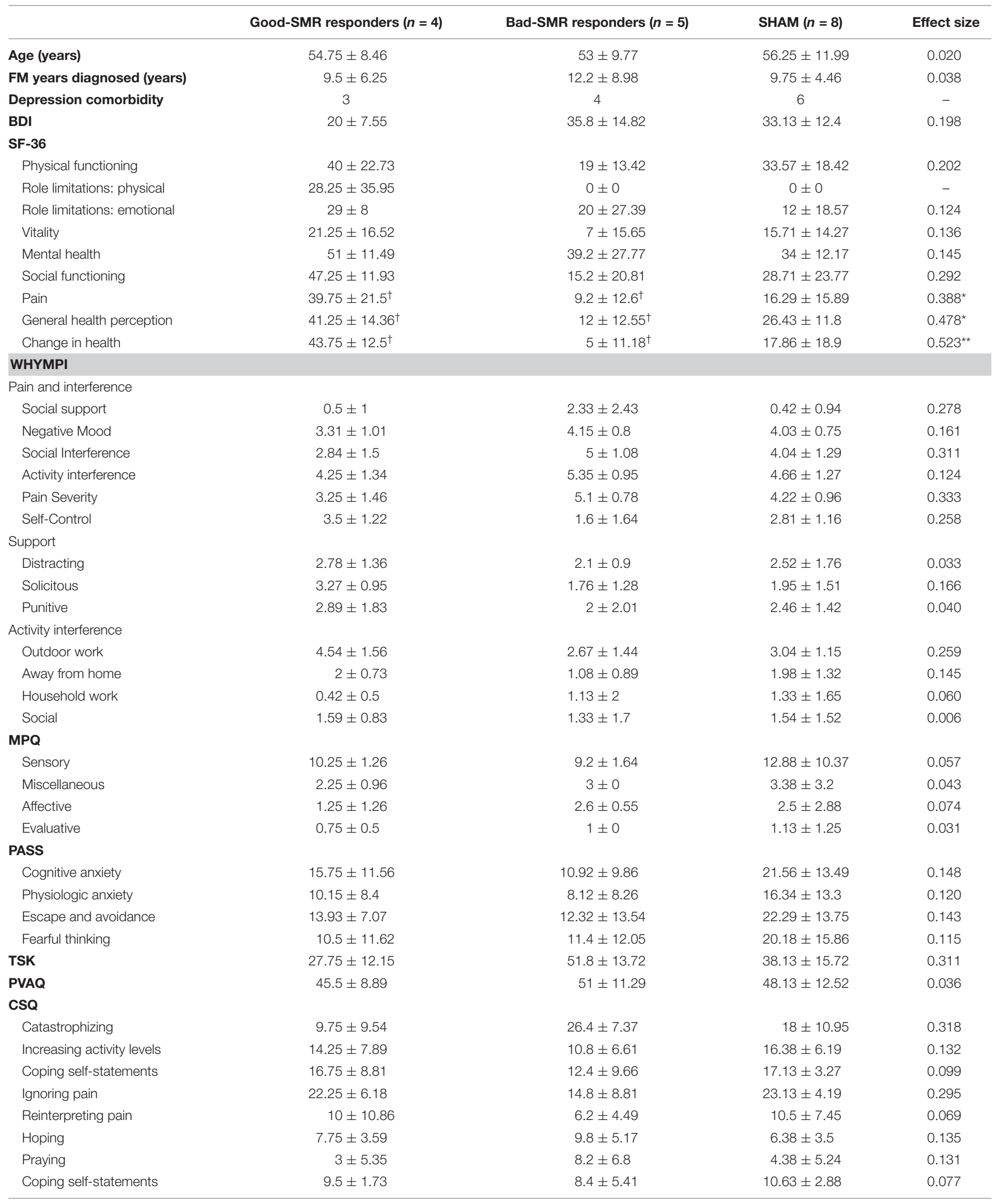


TABLE 1 | Continued

\begin{tabular}{|c|c|c|c|c|}
\hline & Good-SMR responders $(n=4)$ & Bad-SMR responders $(n=5)$ & SHAM $(n=8)$ & Effect size \\
\hline \multicolumn{5}{|l|}{ MOS } \\
\hline Emotional support & $28.25 \pm 13.57$ & $24.4 \pm 11.06$ & $21.75 \pm 6.84$ & 0.077 \\
\hline Tangible support & $14.5 \pm 7.14$ & $12.4 \pm 3.29$ & $11.75 \pm 5.06$ & 0.052 \\
\hline Positive interaction & $14.5 \pm 6.81$ & $13 \pm 5.2$ & $11.75 \pm 4.03$ & 0.054 \\
\hline Affection & $13 \pm 4$ & $11.4 \pm 3.91$ & $9.25 \pm 2.55$ & 0.207 \\
\hline Overall support index & $70.25 \pm 28.81$ & $61.2 \pm 22.71$ & $50.75 \pm 22.78$ & 0.116 \\
\hline FIQ & $40.81 \pm 5.13$ & $68.37 \pm 20.2$ & $65.16 \pm 15.16$ & $0.409^{\star}$ \\
\hline
\end{tabular}

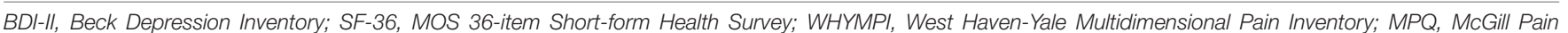

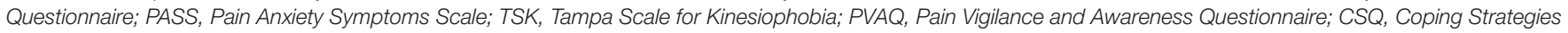
Questionnaire; MOS, MOS Social Support Survey; FIQ, Fibromyalgia Impact Questionnaire; + , good-SMR responders > bad-SMR responders ( $p$ > 0.05).

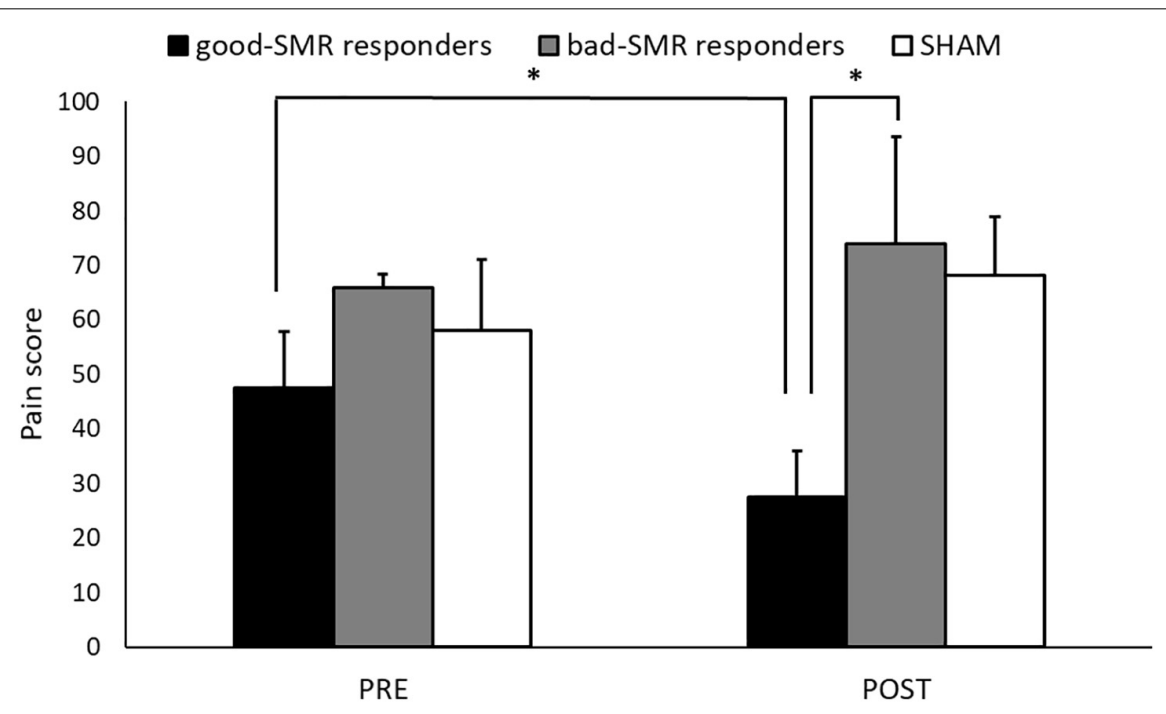

FIGURE 2 | Pain ratings during the Assessment sessions (PRE and POST) for each group (*indicates $p<0.05)$.

No significant differences were found due to Group or Assessment session on anxiety scores (STAI-S) or subjective ratings (pain, fatigue, and negative mood) obtained from the diary (Supplementary Table S1).

\section{Task Performance and EEG Neurofeedback Analyses}

Figure 3 displays the task performance during the PRE and the POST sessions for good-SMR responders, badSMR responders and the SHAM group. The good-SMR responders showed higher percentage of hits than badSMR responders and the SHAM group $(60.25 \% \pm 8.74$, $45.30 \% \pm 5.47$, and $42.81 \% \pm 7.40$, respectively). The ANOVA on task performance revealed significant main effects of Group $\left[F(2,14)=10.865, p=0.001, \eta_{\mathrm{p}}{ }^{2}=0.608\right]$, showing significant differences between the good-SMR responders and the bad-SMR responders, as well as between the good-SMR responders and the SHAM group (Bonferroni post hoc: all ps $<0.01$ ), but not between the bad-SMR responders and the SHAM group. No other significant effects were observed on task performance.
A similar statistical analysis of task performance was computed taking into account the Trial type (synchronization vs. desynchronization). The ANOVA results reveled significant main effects of Group $\left[F(2,14)=10.612, p=0.002, \eta_{p}{ }^{2}=0.603\right]$, as well as a trend effect of Group $\times$ Assessment Session $\times$ Trial type $\left[F(2,14)=3.300, p=0.067, \eta_{\mathrm{p}}{ }^{2}=0.320\right]$. The Bonferroni post hoc test yielded significant differences in bad-SMR responders and the SHAM group between trial types during the POST session (all ps $<0.01$ ). Thus, percentage of hits during synchronization were higher than during desynchronization in bad-SMR responders $(62.80 \% \pm 10.92$ and $26.00 \% \pm 15.75$, respectively) and the SHAM group $(53.75 \% \pm 7.74$ and $31.25 \% \pm 11.16$, respectively) during the POST session. In contrast, good-SMR responders showed no significant differences in percentage of hits between synchronization and desynchronization during the POST session $(67.00 \% \pm 13.11$ and $62.50 \% \pm 15.61$, respectively, $p=0.614$ ). During the PRE session, only the SHAM group showed significant differences in percentage of hits between synchronization and desynchronization $(53.75 \% \pm 9.35$ and $32.25 \% \pm 14.04$, respectively, $p=0.003)$ (Supplementary Figure S1). 


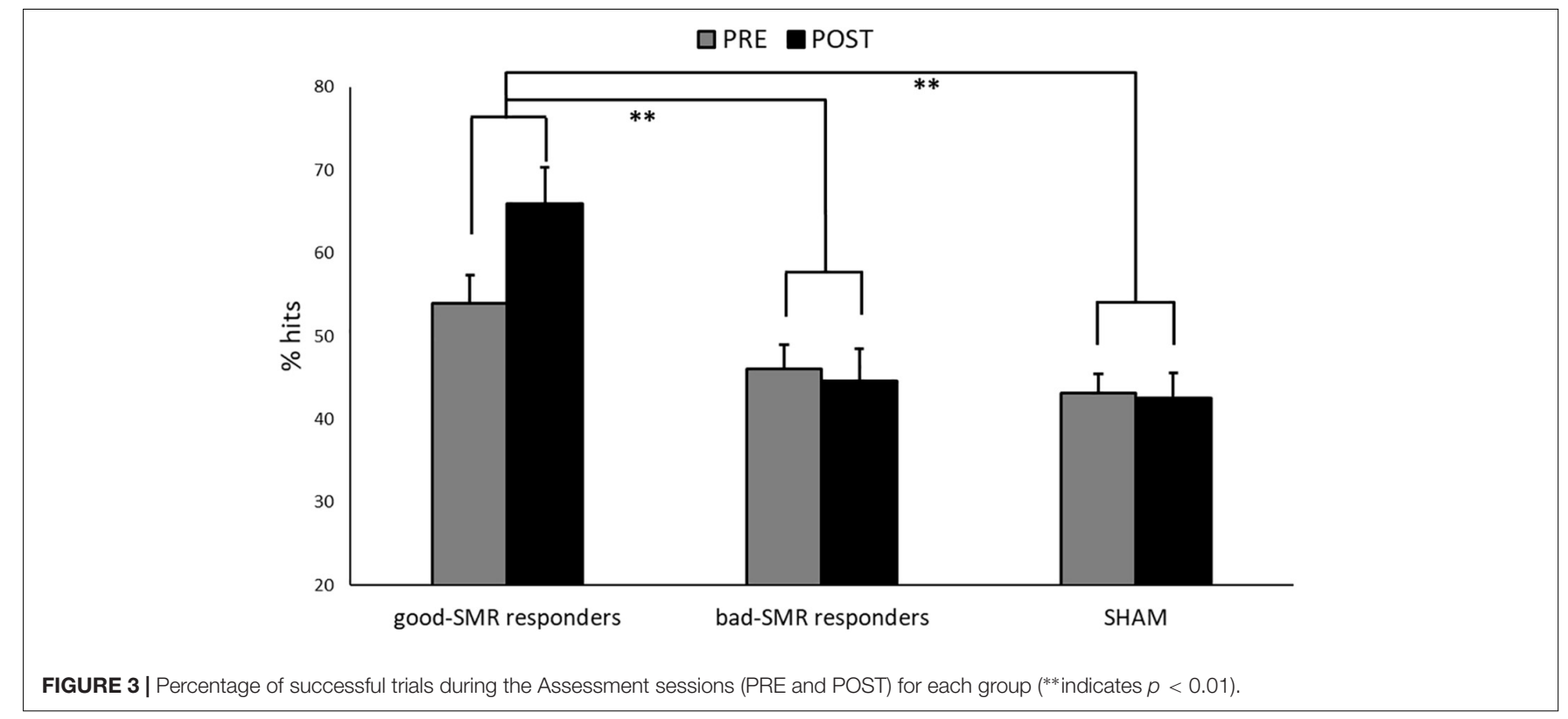

The duration of successful trials during synchronization and desynchronization trials in the assessment sessions are shown for the three groups in Supplementary Figure S2. The ANOVA revealed a significant effect of Group $\times$ Trial type $\left[F(2,14)=6.064, p=0.013, \eta_{\mathrm{p}}^{2}=0.464\right]$. Bonferroni post hoc test showed that desynchronization trials lasted significantly longer than the synchronization trials in bad-SMR responders (4.15 s \pm 0.81 and $3.15 \mathrm{~s} \pm 0.79$, respectively) and the SHAM group (4.03 $\mathrm{s} \pm 0.77$ and $3.33 \mathrm{~s} \pm 0.50$, respectively) (all $p \mathrm{~s}<0.001)$. In contrast, no significant differences were found on duration of successful trials between desynchronization and synchronization in good-SMR responders $(3.83 \mathrm{~s} \pm 0.66$ and $3.82 \mathrm{~s} \pm 0.62$, respectively) $(p=0.958)$. No other significant differences due to group, Assessment session or Trial task were found.

Figure 4 displays changes of SMR power modulation (difference between synchronization and desynchronization trials at electrodes C3, CP1, and CP5 within $12-15 \mathrm{~Hz}$ ) during the Assessment sessions. The ANOVA revealed significant main effects of Group $\left[F(2,14)=11.129, p=0.001, \eta_{\mathrm{p}}{ }^{2}=0.614\right]$, as well as a trend effect of Group $\times$ Assessment Session $[F(2$, 14) $\left.=3.225, p=0.070, \eta_{\mathrm{p}}{ }^{2}=0.315\right]$. The Bonferroni post hoc tests showed significant differences between good-SMR and badSMR responders, as well as between good-SMR responders and the SHAM group during the POST session (all $p$ s $<0.001$ ). SMR modulation score was higher in good-SMR responders $\left(7.91 \mu \mathrm{V}^{2} / \mathrm{Hz} \pm 2.20\right)$ than in bad-SMR responders $(0.04$ $\left.\mu \mathrm{V}^{2} / \mathrm{Hz} \pm 1.06\right)$ and SHAM group $\left(1.63 \mu \mathrm{V}^{2} / \mathrm{Hz} \pm 1.81\right)$. No significant group differences were found during the PRE session. Furthermore, only good-SMR responders displayed a significant enhancement of the SMR modulation score between the PRE $\left(1.57 \mu \mathrm{V}^{2} / \mathrm{Hz} \pm 1.41\right)$ and the POST sessions (7.91 $\left.\mu \mathrm{V}^{2} / \mathrm{Hz} \pm 2.20\right)(p=0.003)$.

Finally, correlational analyses revealed that percentage of successful trials during the POST session was positively correlated with the "pain" $(r=0.688, p=0.003)$ and "change in health" $(r=0.715, p=0.002)$ dimensions of the SF-36 questionnaire. Furthermore, SMR modulation was negatively correlated with FIQ scores $(r=-0.552, p=0.033)$. No correlations were found between performance scores, SMR modulation and questionnaire scores during the PRE session.

\section{Functional MRI Data}

The hypothesis that participants with a successful SMR neurofeedback training would show an increased somatomotor functional connectivity was explored using a seed-to-voxel analysis. No significant group differences were found on functional connectivity from PRE to POST sessions. Nevertheless, significant group differences on the functional connectivity of the somatomotor seed regions with a variety of cortical regions were observed during both the POST and the PRE sessions, separately. Table 2 shows the T-maxima of the significant clusters, as well as MNI coordinates, $P$-values (FWE corrected) and the size of each cluster in contiguous voxels for PRE and POST sessions.

During the PRE session, bad-SMR responders (compared to SHAM group) exhibited increased functional connectivity between the left PreCG seed and the left temporooccipital middle temporal gyrus $[t(11)=7.95, p<0.001]$ and the left central opercular cortex $[t(11)=9.93, p<0.001]$, as well as between the left PostCG seed and the superior lateral occipital cortex $[t(11)=8.20, p<0.001]$ and the left temporooccipital middle temporal gyrus $[t(11)=6.66, p<0.001]$, and between the right ICC seed and the superior lateral occipital cortex $[t(11)=9.19, p<0.001]$ and the left superior frontal gyrus $[t(11)=7.36, p<0.001]$. No significant differences on seed-tovoxel connectivity were found between good-SMR and bad-SMR responders, or between good SMR responders and SHAM in the PRE session. 


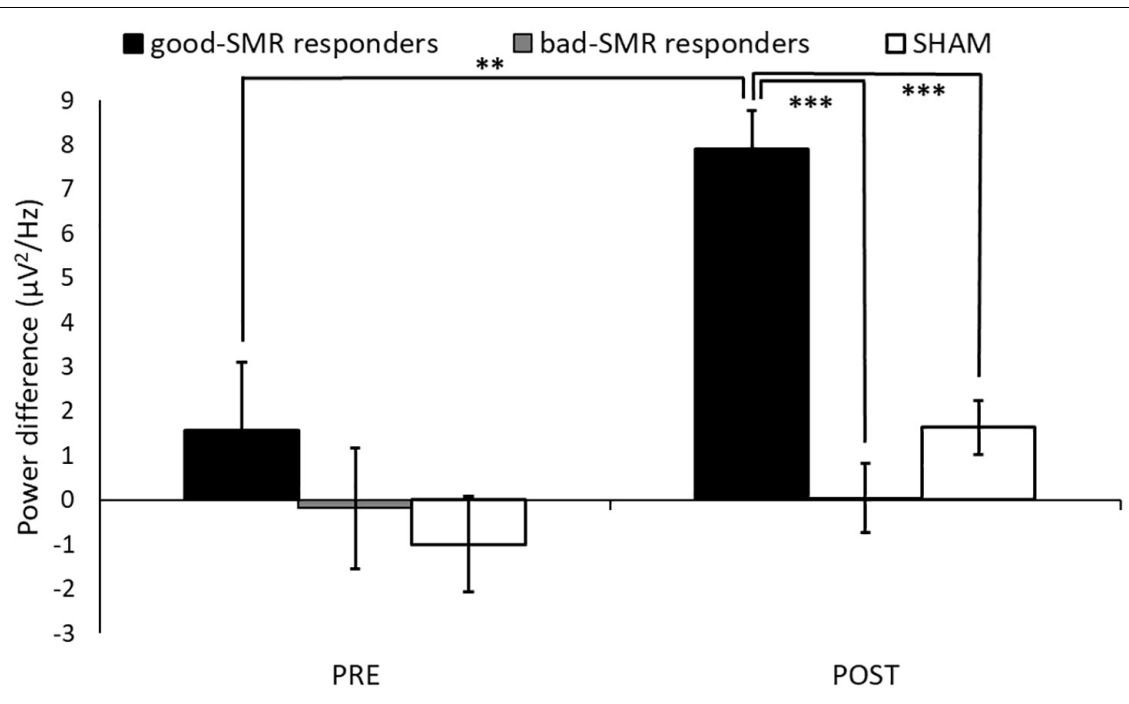

FIGURE 4 | SMR modulation score (power difference between synchronization and desynchronization) over C3, CP1 and CP5 electrodes during the Assessment sessions (** and *** indicate $p<0.01$ and $p<0.001$, respectively).

During the POST session, good-SMR responders (compared to SHAM) exhibited increased functional connectivity between the right PostCG seed ant the left PostCG $[t(10)=6.93$, $p<0.001$ ] and between the left PostCG seed and the right PostCG $[t(10)=11.90, p<0.001]$ and the right temporal occipital fusiform cortex $[t(10)=8.59, p<0.001]$. GoodSMR responders (compared to bad-SMR responders) also demonstrated enhanced connectivity between the left PostCG seed and the right PostCG $[t(7)=14.41, p<0.001]$. Furthermore, bad-SMR responders (compared to good-SMR responders) presented increased functional connectivity between left PreCG seed and the precuneous $[t(7)=14.16, p<0.001]$ and between right PostCG seed and the right superior lateral occipital cortex $[t(7)=11.09, p<0.001]$. Bad-SMR responders (compared to SHAM) also demonstrated enhanced connectivity between the left PostCG seed and the left superior lateral occipital cortex $[t(11)=8.80, p<0.001]$. Thus, it appears that good-SMR responders had improved functional connectivity among somatomotor areas during the POST session; whereas bad-SMR responders showed increased functional connectivity with visual areas. Figure $\mathbf{5}$ displays those brain locations, where the functional connectivity of the left and right PostCG seed was higher in good-SMR than in bad-SMR responders and SHAM.

\section{DISCUSSION}

The aim of the present study was to examine changes in SMR power and functional connectivity of the somatosensory and motor cortices during a neurofeedback training based on synchronization and desynchronization of the SMR power over motor and somatosensory areas in patients with fibromyalgia (FM). In addition, changes in fMRI connectivity of somatosensory and motor cortices elicited when performing the neurofeedback training were also analyzed. Participants were randomly assigned to a SMR training group (real feedback) or to a SHAM group (non-contingent feedback). The analyses of the task performance during the six sessions revealed that only some participants of the SMR group were able to achieve a success rate above $50 \%$ (chance level). Thus, SMR group participants were further subdivided into good (good-SMR responders, those participants who performed the task above the chance level), and bad responders (bad-SMR responders, those participants who performed the task at the chance level). Good responders displayed significant enhancements of power modulation (the difference between SMR synchronization and desynchronization) at electrodes over somatomotor cortices, as well as increased functional connectivity between motor and somatosensory related areas during the last session as compared to the first session of the neurofeedback training. No changes on brain activity or connectivity were observed in bad responders or in the SHAM group. In addition, good responders significantly reduced pain ratings compared to both bad responders and the SHAM group.

Taking together all participants who received SMR neurofeedback training, it was observed that their average percentage of hits during the task was similar to the SHAM group and close to the random probability level. This finding contrasts with previous studies showing that healthy participants can learn to modulate SMR in one session and achieve a successful performance in the neurofeedback task of around 75\% (Popescu et al., 2007; Blankertz et al., 2008, 2010). In the present study, we decided to examine the effects of a sixsession neurofeedback training program (3 sessions per week for 2 weeks) that was already tested in healthy participants with a successful performance of above $75 \%$ (Terrasa et al., 2019). The lack of information about the success rate in previous studies with fibromyalgia patients (Mueller et al., 2001; Kravitz et al., 2006; Kayıran et al., 2010; Nelson et al., 2010; 
TABLE 2 | Seed-to-voxel results of the two-sample $t$-tests comparing pairs of groups during the PRE and POST sessions.

\begin{tabular}{|c|c|c|c|c|c|c|}
\hline Seed & Contrast pair & Cluster $[\mathbf{x}, \mathbf{y}, \mathbf{z}]$ & $\mathbf{k}$ & Cluster p-FWE & Peak p-unc & Result region \\
\hline \multicolumn{7}{|l|}{ PRE } \\
\hline \multirow[t]{2}{*}{ PreCG L } & Bad-SMR responders > SHAM & $-44-44-04$ & 434 & 0.000000 & 0.000003 & $\begin{array}{l}\text { Temporooccipital middle temporal gyrus } L \text { Angular } \\
\text { gyrus } L \text { Inferior lateral occipital cortex } L\end{array}$ \\
\hline & & $-64-20+18$ & 234 & 0.000031 & 0.000000 & $\begin{array}{l}\text { Central opercular cortex } \mathrm{L} \text { Planum temporale } \mathrm{L} \\
\text { Anterior supramarginal gyrus } \mathrm{L} \text { Parietal operculum } \mathrm{L} \\
\text { Anterior superior temporal gyrus } \mathrm{L}\end{array}$ \\
\hline PostCG L & Bad-SMR responders > SHAM & $-32-68+10$ & 1122 & 0.000000 & 0.000003 & $\begin{array}{l}\text { Superior lateral occipital cortex } L \text { Inferior lateral } \\
\text { occipital cortex } L \text { Temporooccipital middle temporal } \\
\text { gyrus } L \text { Angular gyrus } L\end{array}$ \\
\hline \multirow[t]{2}{*}{ ICC R } & Bad-SMR responders > SHAM & $-38-58+48$ & 393 & 0.000000 & 0.000001 & $\begin{array}{l}\text { Superior lateral occipital cortex L Angular gyrus L } \\
\text { Superior parietal lobule L }\end{array}$ \\
\hline & & $-14+38+48$ & 317 & 0.000005 & 0.000007 & Superior frontal gyrus $L$ Middle frontal gyrus $L$ \\
\hline \multicolumn{7}{|l|}{ POST } \\
\hline PreCG L & $\begin{array}{l}\text { Bad-SMR } \\
\text { responders > good-SMR } \\
\text { responders }\end{array}$ & $-06-42+44$ & 301 & 0.000002 & 0.000001 & Precuneous Posterior cingulate gyrus L \\
\hline \multirow[t]{2}{*}{ PostCG R } & $\begin{array}{l}\text { Good-SMR } \\
\text { responders > SHAM }\end{array}$ & $-46-32+58$ & 458 & 0.000002 & 0.000020 & Postcentral gyrus L Anterior supramarginal gyrus L \\
\hline & $\begin{array}{l}\text { Bad-SMR } \\
\text { responders > good-SMR } \\
\text { responders }\end{array}$ & $+42-62+44$ & 399 & 0.000000 & 0.000005 & Angular gyrus R Superior lateral occipital cortex R \\
\hline \multirow[t]{6}{*}{ PostCG L } & $\begin{array}{l}\text { Good-SMR } \\
\text { responders > SHAM }\end{array}$ & $+28-24+56$ & 1426 & 0.000000 & 0.000000 & $\begin{array}{l}\text { Postcentral gyrus R Precentral gyrus R Anterior } \\
\text { supramarginal gyrus R Superior parietal lobule R }\end{array}$ \\
\hline & & $+38-38-14$ & 588 & 0.000000 & 0.000003 & $\begin{array}{l}\text { Temporal occipital fusiform cortex R Lingual gyrus R } \\
\text { Posterior temporal fusiform cortex R } \\
\text { Temporooccipital inferior temporal gyrus R Posterior } \\
\text { parahippocampal gyrus R Posterior inferior } \\
\text { temporal gyrus R }\end{array}$ \\
\hline & & $+52-32+36$ & 198 & 0.000993 & 0.000042 & Posterior supramarginal gyrus R \\
\hline & $\begin{array}{l}\text { Good-SMR } \\
\text { responders > bad-SMR } \\
\text { responders }\end{array}$ & $+50-14+50$ & 1175 & 0.000000 & 0.000001 & $\begin{array}{l}\text { Postcentral gyrus R Precentral gyrus R Superior } \\
\text { parietal lobule R }\end{array}$ \\
\hline & $\begin{array}{l}\text { Bad-SMR } \\
\text { responders > good-SMR } \\
\text { responders }\end{array}$ & $-30-10+34$ & 274 & 0.000003 & 0.000008 & Insular cortex L \\
\hline & Bad-SMR responders > SHAM & $-52-60+24$ & 472 & 0.000000 & 0.000001 & Superior lateral occipital cortex $L$ Angular gyrus $L$ \\
\hline
\end{tabular}

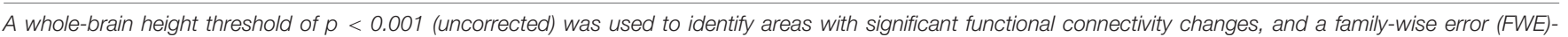

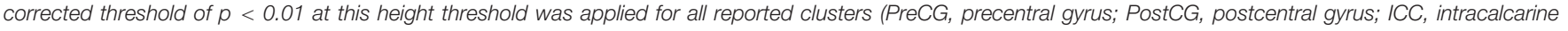
cortex; $L$, left; $R$, right).

Caro and Winter, 2011) makes difficult the comparison, but it is worthy to highlight that about $20 \%$ of healthy individuals cannot modulate their cerebral activity (Allison and Neuper, 2010). In our study, around half of FM participants were not able to perform successfully the neurofeedback task, and we decided to subdivide the participants who received the SMR training in good and bad responders to further explore the differences in brain activity and functional connectivity. Even good responders achieved an average success rate of above $60 \%$ in the neurofeedback training task, below the performance previously observed in healthy subjects. Thus, it seems that the presence of chronic pain could affect the behavioral performance in neurofeedback tasks. In this sense, we also observed that good and bad responders to the SMR neurofeedback training displayed significant differences in several clinical characteristics of pain symptoms before the training program. Thus, for instance, good-SMR responders had significant lower scores in pain impact (FIQ and SF-36 dimension), together with better health perception and health change (SF-36 dimensions) than bad-SMR responders. By contrast, participants in the SHAM group yielded better scores on health status and pain impact than bad responders, but worse than good responders. In addition, significant correlations were observed between successful performance in the neurofeedback task and pain impact and perceived health status, indicating that only those FM participants with less symptom severity were able to perform successfully the neurofeedback training. Other pain-related symptoms such as depression, anxiety or kinesiophobia were not relevant for task performance. These data suggest that the poor performance of chronic pain patients could be related to the direct impact of chronic pain on their health rather than to other pain comorbidities. Future research should further clarify the role of chronic pain in the performance of the neurofeedback task and explore whether 

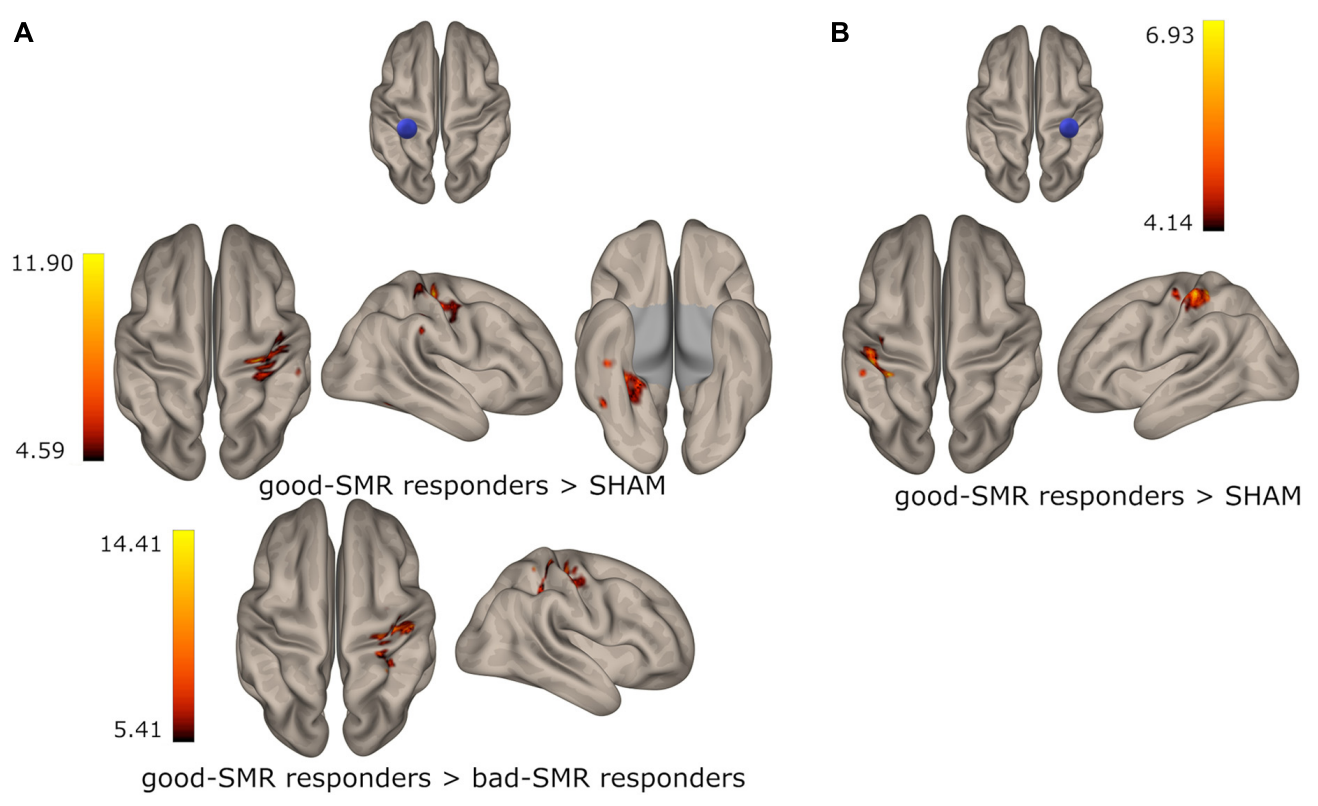

FIGURE 5 | Group differences on functional connectivity of the left (A) and the right (B) postcentral gyrus with somatomotor areas in the POST session. Color indicates the connectivity strength in good-SMR responders when compared to bad-SMR responders and the SHAM group.

there are some patients who could benefit more than others from neurofeedback training.

The best performance of good responders was also reflected in the ability to synchronize and desynchronize SMR with the same success and speed. On the other hand, bad responders and participants in the SHAM group were worse and slower to desynchronize than to synchronize the SMR. Moreover, there was no modulation of SMR power (difference between EEG power in synchronization and desynchronization trials) neither in bad responders nor in the SHAM group. Thus, it appears that the optimal task performance was accompanied by a better power modulation of both synchronization and desynchronization of the SMR over motor and somatosensory related electrodes after the neurofeedback training. Our findings are in agreement with previous data showing, for instance, that FM patients were able to modulate delta, theta and alpha EEG power (Mueller et al., 2001), or the theta/SMR ratio after the neurofeedback training (Kayıran et al., 2010). Considering that most neurofeedback studies have reported significant but unspecific changes in brain activity over the cortex, the present study provides further evidence that only some FM participants were able to selfregulate their brain activity over somatomotor cortices and that these modulatory changes were followed by a significant reduction in pain.

Previous studies have suggested that neurofeedback training may increase resting-state functional connectivity in several pain areas such as ACC (Ros et al., 2013), insula (Kluetsch et al., 2014) or the amygdala (Nicholson et al., 2016). In addition, significant enhancements of resting-state functional connectivity of somatosensory and motor cortices have been demonstrated in patients with stroke (Várkuti et al., 2013; Young et al., 2014; Mohanty et al., 2018) and healthy participants
(Terrasa et al., 2019). In the present study, significant changes in functional brain connectivity of motor and somatosensory areas were observed when performing the neurofeedback task in good responders to the neurofeedback training of the SMR, but not in bad responders or participants in the SHAM group. Indeed, good responders showed higher functional connectivity of the bilateral PostCG with other somatosensory and motor areas than bad-SMR responders and the SHAM group. Therefore, it appears that a successful neurofeedback training based on the modulation of the SMR may lead to a greater interconnectivity between somatosensory and other somatomotor areas. In contrast, bad responders displayed increased functional connectivity between somatomotor areas and several brain areas during both before and after the neurofeedback training. Most of these areas (precuneous, angular gyrus and superior lateral occipital cortex) are involved in visuospatial processing and object recognition (Grill-Spector et al., 2001; Cavanna and Trimble, 2006; Seghier, 2013), suggesting that bad responders were trying to use some visual strategy to solve the neurofeedback task. Interestingly, functional connectivities of somatomotor areas and the insula, as well as visual processing and pain-related areas, were also increased in bad responders at the beginning and the end of the neurofeedback training. It is well known that the insula is involved in sensory and affective dimensions of pain processing and its functional connectivity seems to be impaired in chronic pain leading to a disruption of modulatory circuits involved in pain ( $\mathrm{Lu}$ et al., 2016). Thus, our findings of an enhanced functional connectivity of these pain-related brain areas in bad responders suggest that patients could be more focused on pain perception, rather than on the neurofeedback task. 
Together with changes in activity and functional connectivity within motor and somatosensory brain areas, neurofeedback training of the SMR was able to elicit a significant average pain reduction of $>40 \%$ ( $2 \mathrm{~cm}$ on the VAS) in good responders, but not in bad responders. In addition, it was observed that all good responders (4 out of 4) reduced pain ratings, whereas neurofeedback training elicited a pain reduction in only 2 of the 5 bad responders. Although the sample of the present study was small, our findings are in agreement with previous studies showing that neurofeedback training of SMR can produce pain reduction in chronic pain patients (Mueller et al., 2001; Kayıran et al., 2010; Nelson et al., 2010; Caro and Winter, 2011). Furthermore, our neurofeedback training protocol consisted of six sessions, while other studies with relevant clinical effects have used at least 10 (Kayiran et al., 2010), or even more than 30 sessions (Caro and Winter, 2011). Indeed, there is significant variability in terms of study design and intervention procedures (duration and number of treatment sessions) with respect to neurofeedback intervention in patients with fibromyalgia (Santoro and Cronan, 2014). In the present study, we decided to examine the effects of a sixsession neurofeedback training program (3 sessions per week for 2 weeks) that was already tested in healthy participants (Terrasa et al., 2019). Although patients with chronic pain achieved poorer performance than healthy controls in this program, our findings seem to indicate that 4-6 training sessions may be enough to produce positive clinical results. Thus, this short program could be used as a marker to examine the long-term suitability of neurofeedback training to reduce pain in patients with chronic pain.

Nevertheless, the design of the present study has some shortcomings and its findings should be taken with caution. First and most important, the sample size was small and this makes the findings only preliminary, especially in the fMRI analyses. Second, the fact that all participants took regular medication during neurofeedback training could have biased the results, so their possible effects on the brain changes observed in this study should be further explored. Third, all subjects were women and, therefore, further studies should include male participants with FM to assess the possible influence of gender on the effects of neurofeedback training. Fourth, this was not a double-blind study and, therefore, our findings could be affected by factors that were not related to the neurofeedback intervention. And finally, our psychological assessment was designed to characterize patients and ensure that the groups were comparable in those measures before the training. Further analyzing the effects of neurofeedback training on self-report questionnaires (including depression) is of great interest and should be examined in subsequent studies.

\section{REFERENCES}

Allen, P. J., Josephs, O., and Turner, R. (2000). A method for removing imaging artifact from continuous EEG recorded during functional MRI. Neuroimage 12, 230-239. doi: 10.1006/nimg.2000.0599

Allen, P. J., Polizzi, G., Krakow, K., Fish, D. R., and Lemieux, L. (1998). Identification of EEG events in the mr scanner: the problem of pulse artifact
In summary, the present study revealed that neurofeedback training based on the synchronization and the desynchronization of the SMR led to an augmented functional connectivity between areas associated with the somatosensory and motor activity, as well as to an enhancement of power modulation in fibromyalgia patients. Nevertheless, this result was only obtained in those participants with less impact of the fibromyalgia symptoms. Moreover, these changes in EEG power and functional brain connectivity were mirrored by a reduction in pain. In this sense, our research provide evidence that neurofeedback training is a promising tool for a better understanding of brain mechanisms involved in pain chronification.

\section{DATA AVAILABILITY STATEMENT}

The datasets generated for this study are available on request to the corresponding author.

\section{ETHICS STATEMENT}

The studies involving human participants were reviewed and approved by the Ethics Committee of the Balearic Islands (Spain). The patients/participants provided their written informed consent to participate in this study.

\section{AUTHOR CONTRIBUTIONS}

JT, AB-L, PM, and MM contributed significantly to the design of the study. JT collected the data. JT and AB-L performed the data analyses. JT and MM wrote most of the manuscript. AB-L and PM critically revised the manuscript.

\section{FUNDING}

This work was supported by the grants from PSI2017-88388-C41-R (AEI/FEDER, UE), PSI2017-88388-C4-3-R (AEI/FEDER, UE), and the Spanish Ministerio de Economía, Industria y Competitividad (ref: PSI2013-48260-C3-1-R and PSI201457231-R).

\section{SUPPLEMENTARY MATERIAL}

The Supplementary Material for this article can be found online at: https://www.frontiersin.org/articles/10.3389/fnins. 2020.00236/full\#supplementary-material

and a method for its subtraction. Neuroimage 8, 229-239. doi: 10.1006/nimg. 1998.0361

Allison, B. Z., and Neuper, C. (2010). “Could anyone use a BCI?" in BrainComputer Interfaces, eds D. S. Tan and A. Nijholt (London: Springer), 35-54. doi: 10.1007/978-1-84996-272-8_3

Beck, A. T., Steer, R. A., Ball, R., and Ranieri, W. F. (1996). Comparison of beck depression inventories-IA and-II in psychiatric 
outpatients. J. Pers. Assess. 67, 588-597. doi: 10.1207/s15327752jpa67 $03 \_13$

Behzadi, Y., Restom, K., Liau, J., and Liu, T. T. (2007). A component based noise correction method (CompCor) for BOLD and perfusion based fMRI. Neuroimage 37, 90-101. doi: 10.1016/j.neuroimage.2007.04.042

Blankertz, B., Losch, F., Krauledat, M., Dornhege, G., Curio, G., and Muller, K.R. (2008). The Berlin Brain-Computer Interface: accurate performance from first-session in BCI-naive subjects. IEEE Trans. Biomed. Eng. 55, 2452-2462. doi: 10.1109/TBME.2008.923152

Blankertz, B., Sannelli, C., Halder, S., Hammer, E. M., Kübler, A., Müller, K.-R., et al. (2010). Neurophysiological predictor of SMR-based BCI performance. Neuroimage 51, 1303-1309. doi: 10.1016/j.neuroimage.2010.03.022

Budzynski, T. (ed.) (2009). Introduction to Quantitative EEG and Neurofeedback: Advanced Theory and Applications, 2nd Edn. Amsterdam: Academic Press.

Burckhardt, C. S., Clark, S. R., and Bennett, R. M. (1991). The fibromyalgia impact questionnaire: development and validation. J. Rheumatol. 18, 728-733.

Burgmer, M., Pogatzki-Zahn, E., Gaubitz, M., Stüber, C., Wessoleck, E., Heuft, G., et al. (2010). Fibromyalgia unique temporal brain activation during experimental pain: a controlled fMRI Study. J. Neural Transm. 117, 123-131. doi: 10.1007/s00702-009-0339-1

Burgmer, M., Pogatzkizahn, E., Gaubitz, M., Wessoleck, E., Heuft, G., and Pfleiderer, B. (2009). Altered brain activity during pain processing in fibromyalgia. Neuroimage 44, 502-508. doi: 10.1016/j.neuroimage.2008.09.008

Caro, X. J., and Winter, E. F. (2011). EEG biofeedback treatment improves certain attention and somatic symptoms in fibromyalgia: a pilot study. Appl. Psychophysiol. Biofeedback 36, 193-200. doi: 10.1007/s10484-011-9159-9

Castillo Saavedra, L., Mendonca, M., and Fregni, F. (2014). Role of the primary motor cortex in the maintenance and treatment of pain in fibromyalgia. Med. Hypotheses 83, 332-336. doi: 10.1016/j.mehy.2014.06.007

Cavanna, A. E., and Trimble, M. R. (2006). The precuneus: a review of its functional anatomy and behavioural correlates. Brain 129, 564-583. doi: 10.1093/brain/ awl004

Cifre, I., Sitges, C., Fraiman, D., Munoz, M. A., Balenzuela, P., Gonzalez-Roldan, A., et al. (2012). Disrupted functional connectivity of the pain network in fibromyalgia. Psychosom. Med. 74, 55-62. doi: 10.1097/PSY.0b013e3182408f04

Cincotti, F., Mattia, D., Aloise, F., Bufalari, S., Schalk, G., Oriolo, G., et al. (2008). Non-invasive brain-computer interface system: towards its application as assistive technology. Brain Res. Bull. 75, 796-803. doi: 10.1016/j.brainresbull. 2008.01.007

Clauw, D. J., D’Arcy, Y., Gebke, K., Semel, D., Pauer, L., and Jones, K. D. (2018). Normalizing fibromyalgia as a chronic illness. Postgrad. Med. 130, 9-18. doi: 10.1080/00325481.2018.1411743

Desmeules, J. A., Cedraschi, C., Rapiti, E., Baumgartner, E., Finckh, A., Cohen, P., et al. (2003). Neurophysiologic evidence for a central sensitization in patients with fibromyalgia. Arthritis Rheum. 48, 1420-1429. doi: 10.1002/art. 10893

Diers, M. (2019). Neuroimaging the pain network - Implications for treatment. Best Pract. Res. Clin. Rheumatol. 33:101418. doi: 10.1016/j.berh.2019.05.003

Don, S., Venema, M., De Kooning, M., van Buchem, B., Nijs, J., and Voogt, L. (2019). Does sensorimotor incongruence trigger pain and sensory disturbances in people with chronic low back pain? A randomized cross-over experiment. J. Pain 20, 315-324. doi: 10.1016/j.jpain.2018.09.011

Enriquez-Geppert, S., Huster, R. J., and Herrmann, C. S. (2017). EEGneurofeedback as a tool to modulate cognition and behavior: a review tutorial. Front. Hum. Neurosci. 11:51. doi: 10.3389/fnhum.2017.00051

Flodin, P., Martinsen, S., Löfgren, M., Bileviciute-Ljungar, I., Kosek, E., and Fransson, P. (2014). Fibromyalgia is associated with decreased connectivity between pain- and sensorimotor brain areas. Brain Connect. 4, 587-594. doi: 10.1089/brain.2014.0274

González-Roldán, A. M., Cifre, I., Sitges, C., and Montoya, P. (2016). Altered dynamic of EEG oscillations in fibromyalgia patients at rest. Pain Med. 17, 1058-1068. doi: 10.1093/pm/pnw023

Gracely, R. H. (2004). Pain catastrophizing and neural responses to pain among persons with fibromyalgia. Brain 127, 835-843. doi: 10.1093/brain/ awh098

Grill-Spector, K., Kourtzi, Z., and Kanwisher, N. (2001). The lateral occipital complex and its role in object recognition. Vision Res. 41, 1409-1422. doi: 10.1016/s0042-6989(01)00073-6
Gruzelier, J., Hardman, E., Wild, J., and Zaman, R. (1999). Learned control of slow potential interhemispheric asymmetry in schizophrenia. Int. J. Psychophysiol. 34, 341-348. doi: 10.1016/s0167-8760(99)00091-4

Gruzelier, J. H. (2014). EEG-neurofeedback for optimising performance. I: A review of cognitive and affective outcome in healthy participants. Neurosci. Biobehav. Rev. 44, 124-141. doi: 10.1016/j.neubiorev.2013.09.015

Hardman, E., Gruzelier, J., Cheesman, K., Jones, C., Liddiard, D., Schleichert, H., et al. (1997). Frontal interhemispheric asymmetry: self regulation and individual differences in humans. Neurosci. Lett. 221, 117-120. doi: 10.1016/ s0304-3940(96)13303-6

Harris, A. J. (1999). Cortical origin of pathological pain. Lancet 354, 1464-1466. doi: 10.1016/s0140-6736(99)05003-5

Ichesco, E., Schmidt-Wilcke, T., Bhavsar, R., Clauw, D. J., Peltier, S. J., Kim, J., et al. (2014). Altered resting state connectivity of the insular cortex in individuals with fibromyalgia. J. Pain 15, 815-826.e1. doi: 10.1016/j.jpain.2014.04.007

Jensen, M. P., Day, M. A., and Miró, J. (2014). Neuromodulatory treatments for chronic pain: efficacy and mechanisms. Nat. Rev. Neurol. 10, 167-178. doi: 10.1038/nrneurol.2014.12

Jensen, M. P., Grierson, C., Tracy-Smith, V., Bacigalupi, S. C., and Othmer, S. (2007). Neurofeedback treatment for pain associated with complex regional pain syndrome type I. J. Neurother. 11, 45-53. doi: 10.1300/J184v11n01_04

Jensen, M. P., Hakimian, S., Sherlin, L. H., and Fregni, F. (2008). New insights into neuromodulatory approaches for the treatment of pain. J. Pain 9, 193-199. doi: 10.1016/j.jpain.2007.11.003

Kayıran, S., Dursun, E., Dursun, N., Ermutlu, N., and Karamürsel, S. (2010). Neurofeedback intervention in fibromyalgia syndrome; a randomized, controlled, rater blind clinical trial. Appl. Psychophysiol. Biofeedback 35, 293302. doi: 10.1007/s10484-010-9135-9

Kerns, R. D., Turk, D. C., and Rudy, T. E. (1985). The west haven-yale multidimensional pain inventory (WHYMPI). Pain 23, 345-356. doi: 10.1016/ 0304-3959(85)90004-1

Kluetsch, R. C., Ros, T., Théberge, J., Frewen, P. A., Calhoun, V. D., Schmahl, C., et al. (2014). Plastic modulation of PTSD resting-state networks and subjective wellbeing by EEG neurofeedback. Acta Psychiatr. Scand. 130, 123-136. doi: 10.1111/a.12229

Kravitz, H. M., Esty, M. L., Katz, R. S., and Fawcett, J. (2006). Treatment of fibromyalgia syndrome using low-intensity neurofeedback with the flexyx neurotherapy system: a randomized controlled clinical trial. J. Neurother. 10, 41-58. doi: 10.1300/J184v10n02_03

Lim, M., Roosink, M., Kim, J. S., Kim, H. W., Lee, E. B., Son, K. M., et al. (2016). Augmented pain processing in primary and secondary somatosensory cortex in fibromyalgia: a magnetoencephalography study using intra-epidermal electrical stimulation. PLoS One 11:e0151776. doi: 10.1371/journal.pone.0151776

Lu, C., Yang, T., Zhao, H., Zhang, M., Meng, F., Fu, H., et al. (2016). Insular cortex is critical for the perception, modulation, and chronification of pain. Neurosci. Bull. 32, 191-201. doi: 10.1007/s12264-016-0016-y

Mayaud, L., Wu, H., Barthélemy, Q., Favennec, P., Delpierre, Y., Congedo, M., et al. (2019). Alpha-phase synchrony EEG training for multi-resistant chronic low back pain patients: an open-label pilot study. Eur. Spine J. 28, 2487-2501. doi: 10.1007/s00586-019-06051-9

McCracken, L. M. (1997). “Attention” to pain in persons with chronic pain: a behavioral approach. Behav. Ther. 28, 271-284. doi: 10.1016/s0005-7894(97) 80047-0

McCracken, L. M., Zayfert, C., and Gross, R. T. (1992). The pain anxiety symptoms scale: development and validation of a scale to measure fear of pain. Pain 50, 67-73. doi: 10.1016/0304-3959(92)90113-P

Melzack, R. (1975). The McGill Pain Questionnaire: major properties and scoring methods. Pain 1, 277-299. doi: 10.1016/0304-3959(75)90044-5

Mohanty, R., Sinha, A. M., Remsik, A. B., Dodd, K. C., Young, B. M., Jacobson, T., et al. (2018). Early findings on functional connectivity correlates of behavioral outcomes of brain-computer interface stroke rehabilitation using machine learning. Front. Neurosci. 12:624. doi: 10.3389/fnins.2018.00624

Mueller, H. H., Donaldson, C. C., Nelson, D. V., and Layman, M. (2001). Treatment of fibromyalgia incorporating EEG-Driven stimulation: a clinical outcomes study. J. Clin. Psychol. 57, 933-952. doi: 10.1002/jclp.1060

Nelson, D. V., Bennett, R. M., Barkhuizen, A., Sexton, G. J., Jones, K. D., Esty, M. L., et al. (2010). Neurotherapy of Fibromyalgia? Pain Med. 11, 912-919. doi: $10.1111 /$ j.1526-4637.2010.00862.x 
Nicholson, A. A., Ros, T., Frewen, P. A., Densmore, M., Théberge, J., Kluetsch, R. C., et al. (2016). Alpha oscillation neurofeedback modulates amygdala complex connectivity and arousal in posttraumatic stress disorder. Neuroimage Clin. 12, 506-516. doi: 10.1016/j.nicl.2016.07.006

Pfurtscheller, G., and Lopes da Silva, F. H. (1999). Event-related EEG/MEG synchronization and desynchronization: basic principles. Clin. Neurophysiol. 110, 1842-1857. doi: 10.1016/s1388-2457(99)00141-8

Popescu, F., Fazli, S., Badower, Y., Blankertz, B., and Müller, K.-R. (2007). Single trial classification of motor imagination using 6 dry EEG electrodes. PLoS One 2:e637. doi: 10.1371/journal.pone.0000637

Pujol, J., Macià, D., Garcia-Fontanals, A., Blanco-Hinojo, L., López-Solà, M., Garcia-Blanco, S., et al. (2014). The contribution of sensory system functional connectivity reduction to clinical pain in fibromyalgia. Pain 155, 1492-1503. doi: 10.1016/j.pain.2014.04.028

Roelofs, J., Goubert, L., Peters, M. L., Vlaeyen, J. W. S., and Crombez, G. (2004). The Tampa Scale for Kinesiophobia: further examination of psychometric properties in patients with chronic low back pain and fibromyalgia. Eur. J. Pain 8, 495-502. doi: 10.1016/j.ejpain.2003.11.016

Ros, T., Théberge, J., Frewen, P. A., Kluetsch, R., Densmore, M., Calhoun, V. D., et al. (2013). Mind over chatter: Plastic up-regulation of the fMRI salience network directly after EEG neurofeedback. Neuroimage 65, 324-335. doi: 10. 1016/j.neuroimage.2012.09.046

Rosenstiel, A. K., and Keefe, F. J. (1983). The use of coping strategies in chronic low back pain patients: relationship to patient characteristics and current adjustment. Pain 17, 33-44. doi: 10.1016/0304-3959(83)90125-2

Santoro, M., and Cronan, T. (2014). A systematic review of neurofeedback as a treatment for fibromyalgia syndrome symptoms. J. Musculoskelet. Pain 22, 286-300. doi: 10.3109/10582452.2014.883037

Schalk, G., McFarland, D. J., Hinterberger, T., Birbaumer, N., and Wolpaw, J. R. (2004). BCI2000: a general-purpose brain-computer interface (BCI) system. IEEE Trans. Biomed. Eng. 51, 1034-1043. doi: 10.1109/TBME.2004.827072

Seghier, M. L. (2013). The Angular Gyrus: multiple functions and multiple subdivisions. Neuroscientist 19, 43-61. doi: 10.1177/1073858412440596

Sherbourne, C. D., and Stewart, A. L. (1991). The MOS social support survey. Soc. Sci. Med. 32, 705-714. doi: 10.1016/0277-9536(91)90150-b

Spielberger, C. D., Gorsurch, R. L., and Lushene, R. E. (1970). The State-Trait Anxiety Inventory (STAI): Test Manual. Palo Alto, CA: Consulting Psychologists Press.

Terrasa, J. L., Alba, G., Cifre, I., Rey, B., Montoya, P., and Muñoz, M. A. (2019). Power spectral density and functional connectivity changes due to a sensorimotor neurofeedback training: a preliminary study. Neural Plast. 2019:7647204. doi: 10.1155/2019/7647204
Várkuti, B., Guan, C., Pan, Y., Phua, K. S., Ang, K. K., Kuah, C. W. K., et al. (2013). Resting state changes in functional connectivity correlate with movement recovery for BCI and robot-assisted upper-extremity training after stroke. Neurorehabil. Neural Repair 27, 53-62. doi: 10.1177/154596831244 5910

Vučković, A., Altaleb, M. K. H., Fraser, M., McGeady, C., and Purcell, M. (2019). EEG correlates of self-managed neurofeedback treatment of central neuropathic pain in chronic spinal cord injury. Front. Neurosci. 13:762. doi: 10.3389/fnins. 2019.00762

Ware, J. E., and Sherbourne, C. D. (1992). The MOS 36-item short-form health survey (SF-36). I. Conceptual framework and item selection. Med. Care 30, 473-483. doi: 10.1097/00005650-199206000-00002

Whitfield-Gabrieli, S., and Nieto-Castanon, A. (2012). Conn: a functional connectivity toolbox for correlated and anticorrelated brain networks. Brain Connect. 2, 125-141. doi: 10.1089/brain.2012.0073

Wolfe, F., Brähler, E., Hinz, A., and Häuser, W. (2013). Fibromyalgia prevalence, somatic symptom reporting, and the dimensionality of polysymptomatic distress: results from a survey of the general population. Arthritis Care Res. 65, 777-785. doi: 10.1002/acr.21931

Wolfe, F., Ross, K., Anderson, J., and Russell, I. J. (1995). Aspects of fibromyalgia in the general population: sex, pain threshold, and fibromyalgia symptoms. J. Rheumatol. 22, 151-156.

Wolfe, F., Smythe, H. A., Yunus, M. B., Bennett, R. M., Bombardier, C., Goldenberg, D. L., et al. (1990). The american college of rheumatology 1990 criteria for the classification of fibromyalgia. Arthritis Rheum. 33, 160-172. doi: 10.1002/art.1780330203

Young, B. M., Nigogosyan, Z., Walton, L. M., Song, J., Nair, V. A., Grogan, S. W., et al. (2014). Changes in functional brain organization and behavioral correlations after rehabilitative therapy using a brain-computer interface. Front. Neuroeng. 7:26. doi: 10.3389/fneng.2014.00026

Conflict of Interest: The authors declare that the research was conducted in the absence of any commercial or financial relationships that could be construed as a potential conflict of interest.

Copyright (c) 2020 Terrasa, Barros-Loscertales, Montoya and Muñoz. This is an open-access article distributed under the terms of the Creative Commons Attribution License (CC BY). The use, distribution or reproduction in other forums is permitted, provided the original author(s) and the copyright owner(s) are credited and that the original publication in this journal is cited, in accordance with accepted academic practice. No use, distribution or reproduction is permitted which does not comply with these terms. 


\title{
Neurofeedback for Pain Management: A Systematic Review
}

\author{
Rubén Roy ${ }^{1}$, Rocío de la Vega ${ }^{2}$, Mark P. Jensen ${ }^{3}$ and Jordi Miró ${ }^{1 *}$ \\ 1 Universitat Rovira i Virgili, Unit for the Study and Treatment of Pain-ALGOS, Department of Psychology, Research Center for \\ Behavior Assessment (CRAMC), Tarragona, Spain, ${ }^{2}$ Center for Child Health, Behavior and Development, Children's \\ Research Institute, Seattle, WA, United States, ${ }^{3}$ Department of Rehabilitation Medicine, University of Washington, Seattle, \\ WA, United States
}

OPEN ACCESS

Edited by:

Trevor Thompson,

University of Greenwich,

United Kingdom

Reviewed by:

Tony C. Steffert,

The Open University, United Kingdom

Aleksandra Vuckovic,

University of Glasgow,

United Kingdom

${ }^{*}$ Correspondence:

Jordi Miró

jordi.miro@urv.cat

Specialty section:

This article was submitted to

Perception Science,

a section of the journal

Frontiers in Neuroscience

Received: 04 February 2020

Accepted: 02 June 2020

Published: 16 July 2020

Citation:

Roy $R$, de la Vega $R$, Jensen MP and Miró J (2020) Neurofeedback for Pain

Management: A Systematic Review.

Front. Neurosci. 14:671.

doi: 10.3389/fnins.2020.00671
Background: Chronic pain is a significant global health issue. For most individuals with chronic pain, biomedical treatments do not provide adequate relief. Given the evidence that neurophysiological abnormalities are associated with pain, it is reasonable to consider treatments that target these factors, such as neurofeedback (NF). The primary objectives of this review were to summarize the current state of knowledge regarding: (1) the different types of NF and NF protocols that have been evaluated for pain management; (2) the evidence supporting each NF type and protocol; (3) if targeted brain activity changes occur with NF training; and (4) if such brain activity change is associated with improvements on treatment outcomes.

Methods: Inclusion criteria were intentionally broad to encompass every empirical study using NF in relation to pain. We considered all kinds of NF, including both electroencephalogram- (EEG-) and functional magnetic resonance imagining- (fMRI-) based. We searched the following databases from inception through September 2019: Pubmed, Ovid, Embase, Web of Science, PsyclNFO. The search strategy consisted of a combination of key terms referring to all NF types and pain conditions (e.g., neurofeedback, rt-fMRI-NF, BOLD, pain, migraine).

Results: A total of 6,552 citations were retrieved; 24 of these that were included in the review. Most of the studies were of moderate quality, included a control condition and but did not include a follow-up. They focused on studying pain intensity (83\%), pain frequency, and other variables (fatigue, sleep, depression) in samples of adults ( $n$ $=7-71)$ with headaches, fibromyalgia and other pain conditions. Most studies (79\%) used EEG-based NF. A wide variety of NF types and protocols have been used for pain management aiming to either increase, decrease or regulate brain activity in certain areas theoretically associated with pain.

Conclusions: Given the generally positive results in the studies reviewed, the findings indicate that NF procedures have the potential for reducing pain and improving other related outcomes in individuals with chronic pain. However, the current evidence does not provide definitive conclusions or allow for reliable recommendations on which protocols or methods of administration may be the most effective. These findings support the need for continued - but higher quality - research in this area.

Keywords: systematic review, neurofeedback, neuromodulation, pain management, treatment outcome 


\section{INTRODUCTION}

\section{Rationale}

Chronic pain is a major global health issue (Goldberg and Mcgee, 2011), affecting about one in four adults (Schopflocher et al., 2011; van Hecke et al., 2013; Nahin, 2015) and a similar number of youths (Huguet and Miró, 2008; King et al., 2011). Chronic pain has a number of negative physical, psychological and social consequences in the life for those with this condition (Institute of Medicine (U.S) Committee on a National Agenda for the Prevention of Disabilities, 1991; Bair et al., 2003; Finan et al., 2013; De Ruddere and Craig, 2016). The costs of chronic pain to society are enormous, and include both direct (e.g., medical expenses) as well as indirect costs [e.g., expenses associated with work absenteeism, hiring somebody to take care of the patients, or travel costs to receive treatment (Gaskin and Richard, 2012; Groenewald et al., 2014)]. For most individuals with chronic pain, the available treatments do not provide adequate relief and are generally unable to prevent new episodes (Williams et al., 2012).

The brain, an organ influenced by biological, psychological, and social factors, plays a central role in the onset and maintenance of pain (Chapin et al., 2012). For example, a growing body of evidence indicates that there are structural and functional neurophysiological brain abnormalities in individuals with chronic pain (May, 2008; Apkarian et al., 2011; Davis and Moayedi, 2013). Likewise, individuals with chronic pain evidence patterns of brain activity (as measured by electroencephalography; EEG) that differ from those without chronic pain (Pinheiro et al., 2016). It is possible that some of these brain abnormalities may be reversible with treatment (May, 2008; Flor, 2014). Thus, it would be reasonable to consider treatments that target brain activity directly as viable interventions for reducing the severity and impact of chronic pain.

Neurofeedback (NF) is a non-invasive treatment that targets brain activity. It is a type of biofeedback that provides real-time information to patients about their brain activity, allowing them to learn how to directly change this activity in ways that may lead to improved health and comfort. NF can be performed either by using brain activity measured via EEG or functional Magnetic Resonance Imaging (fMRI). The EEG approach is used much more often, because EEG biofeedback technology is more accessible and less expensive. With EEG-based NF, one or more electrodes are placed on the patient's scalp to measure the amplitude (also referred to as "power") of oscillatory activity in different frequency bandwidths. The raw electrical signal represents the collective activity of millions of neurons in the cortex, just below the electrode. This signal is analyzed and aspects of that electrical brain activity are fed back to the patient (Jensen et al., 2014). Normally, EEG-based NF targets a change in the power of activity in specific oscillation bandwidths whereas fMRI-based NF targets changes in the blood oxygenlevel dependent (BOLD) activity in regions of interest in the brain (Sulzer et al., 2013; Thibault et al., 2018).

Whether NF is conducted with EEG or fMRI, measured changes in brain activity are fed back to the patient. Often, but not always, the feedback is provided via a game. For example, a program might allow the patient to "fly" a plane when he or she makes a change in the targeted brain activity (e.g., an increase in alpha power as measured over the sensory cortex). The plane will fly smoothly as long as the targeted brain activity is in the direction of the training criteria established by the therapist, whereas the plane might drop or otherwise malfunction if the brain activity falls outside of the training range. This feedback influences and progressively helps the patient learn to change brain activity via operant conditioning (Heinrich et al., 2007; Sherlin et al., 2011). It is important to note that although operant conditioning is the principle underlying the most common NF treatments, there are some types of NF that operate via different principles (Sherlin et al., 2011). Also, changes in brain activity often take a relatively long time to occur with NF treatment; a full course of NF treatment is normally comprised of 15-50 sessions of 20-40 min each (Heinrich et al., 2007; Hammond, 2011).

In the context of pain treatment, NF aims to change brain activity that is thought to underlie or influence the experience of pain (Ibric and Dragomirescu, 2009). The findings from a number of research studies provide preliminary support for the efficacy of NF for reducing pain in clinical samples (Jensen et al., 2014; Miró et al., 2016). However, some investigators have questioned whether NF has any beneficial effect for pain or other problems over and above placebo or outcome expectancy effects (Thibault et al., 2017). Thus, a critical summary of the available evidence regarding the efficacy of NF interventions targeting pain as an outcome is needed in order to better understand the current state of knowledge regarding this potentially promising pain intervention.

\section{Objectives}

Given the considerations discussed above, the primary objectives of this review were to summarize the current state of knowledge regarding (1) the efficacy of NF for reducing pain and (2) the effects of NF on pain-related brain activity in individuals experiencing pain.

\section{Research Questions}

Specifically, we aimed to: (1) describe the different types of NF and NF protocols, and how NF has been used for pain management; (2) summarize the evidence regarding the efficacy of each type of NF and different NF protocols for modulating pain and for improving pain-related outcomes; (3) determine the level of evidence regarding the effect of NF training on measures of brain activity thought to be related to pain, and if changes in measures of this brain activity are associated with improvements in pain-related outcomes; and (4) asses the quality of the studies included in the review.

\section{METHODS}

\section{Study Design}

The current systematic review was conducted and reported following the Preferred Reporting Items for Systematic reviews and Meta-Analyses for Protocols 2015 (PRISMA$P$ 2015) guidelines (Moher, 2015) and was preregistered at the PROSPERO International Prospective Register of 
Systematic Reviews (https://www.crd.york.ac.uk/prospero/; with registration number CRD42018115335).

\section{Participants, Interventions, Comparators}

We included studies using samples of children or adults, either healthy or with clinical pain conditions, where neurofeedback was used to influence pain outcomes. The inclusion criteria were intentionally broad in order to include in the review every empirical study using NF to treat pain. All types of studies were included, regardless of sample size or study design. We also considered all kinds of NF, both EEG- and fMRI-based NF, and included studies combining the use of NF with other interventions or using NF to enhance the efficacy of other pain treatments. We also aimed to include studies on all types of pain, including chronic pain, acute pain, and laboratory (induced) pain. Any study that assessed at least pain intensity or pain frequency was included. The only exclusion criterion was if a given paper under consideration was written in a language other than Spanish or English.

We considered studies that included the assessment of pre- to post- treatment changes in pain intensity and/or pain frequency, as measured using questionnaires or rating scales with support for their reliability and validity (Jensen and Karoly, 2001). When available, we also examined the extent to which any changes noted after NF training did or did not maintain at follow-up.

When assessed, we noted the effects of NF on pain-related outcomes, including fatigue, sleep problems/sleep quality, psychological function (anxiety or depression), perceived health-related quality of life and pain-related interference or disability. We also considered pre- to post-treatment changes in measures of brain activity; that is, pre- to posttreatment changes in the power of different brain oscillation bandwidths or pre- to post-treatment changes in BOLD activity. When possible, we also examined if any pre- to post-treatment improvements in these outcomes maintained at follow-up.

\section{Search Strategy}

We searched the following databases from inception through September 2019: PubMed, Ovid, Embase, Web of Science, PsycINFO and Scopus. The search strategy consisted of a combination of key terms referring to all neurofeedback types and pain conditions (e.g., neurofeedback, rt-fMRI-NF, pain, migraine, fibromyalgia). To see the full Pubmed strategy please see https://www.crd.york.ac.uk/PROSPEROFILES/115335_ STRATEGY_20181031.pdf. We also searched the reference lists of all articles reviewed in order to identify any additional studies to include. In addition, we performed a search of ClinicalTrials.gov to identify ongoing or completed studies with unpublished results and asked the corresponding authors to allow us to include their results in the review. Finally, we attempted to contact the authors of any papers included in the review that did not provide all the data needed for our synthesis to request these data.

\section{Data Sources, Studies Sections, and Data Extraction}

Two of the authors (RR and RdlV) independently assessed the eligibility of the articles retrieved after the database search for inclusion in the review. If any disagreement emerged, they were resolved in consultation with a third author (JM). Next, a deduplication process was conducted via a reference manager (Mendeley). Once a final list of selected articles was identified, their reference lists were reviewed to identify additional studies that could be of interest.

We extracted the following study characteristics from each article identified for inclusion: article title, author(s), publication year, country, sample characteristics (sample size, age, sex, education level, household income, pain problem), intervention protocols (i.e., scalp positions and bandwidths targeted for EEG-based NF, brain regions being targeted in fMRI-based NF, number, duration and frequency of sessions), primary study outcomes (i.e., pain intensity, pain frequency), and secondary outcomes (i.e., fatigue, sleep quality, psychological function [anxiety, depression], perceived health-related quality of life and pain-related disability). If available, we extracted EEG or BOLD activity in whichever way it was reported.

When more than one measure was used to assess the same construct, we planned to inform about the one that is reported most often in the literature as the primary outcome for that study. If data from the same study were reported in different papers, we only retrieved the data from the paper that was published first, unless there was a subsequent study that added additional participants or provided additional data.

\section{Data Analysis}

Given the paucity of research on the topic, as evidenced by preliminary searches as well as the disparity of methods and outcomes reported, we anticipated that a meta-analytical approach would not be feasible. As this was confirmed after the search, here we present a systematic narrative synthesis summarizing the characteristics and findings of the studies included in the review. We included all studies identified irrespective of their risk of bias. In addition, we organized the narrative synthesis by study design, starting with those with stronger designs and continuing from there to the studies using lower-quality designs. We describe separately EEG-based NF (and its subtypes) and fMRI-based NF. We report on the outcomes (clinical and neurophysiological) as a function of the type of NF (EEG- or fMRI-based) and protocol used. We also summarize the different uses of NF in pain management. Next, we summarize NF's effects on pain intensity and pain frequency, as well as on measures of the pain-related variables mentioned above. We also note whether the studies provided EEG- or fMRI-assessed physiological data, and if they reported changes in measures of physiological activity following NF. If so, we assessed whether these changes in brain activity were associated with changes in the brain activity targeted by the intervention. If presented by the study authors, we also report on the extent to which changes in measured brain activity change were associated with observed improvements in treatment outcomes. 
In addition, we rated and describe study quality using the Quality Assessment Tool for Quantitative Studies from the Effective Public Health Practice Project [EPHPP; (Thomas et al., 2004)], as this tool allows for a comparison of study quality between studies using different designs. The EPHPP tool consists of six quality components to be rated as "strong" (coded as "1") "moderate" (coded as "2"), or "weak" (coded as " 3 "): selection bias, study design, confounders, blinding, data collection methods, and withdrawals and drop-outs. We did not compute a final score for each study as relevant methodological aspects of the studies appear to be better assessed individually (Jüni et al., 1999). Again, two authors (RR and RdlV) conducted this evaluation independently. In the event of any disagreements, these were resolved in consultation with a third author (JM).

\section{RESULTS}

\section{Study Selection and Characteristics}

Our initial search retrieved 6,552 citations. After eliminating duplicates, 3,560 articles were assessed based on their title and abstract. A total of 3,513 articles were excluded because they did not meet the inclusion criteria and 47 were read in full. A total of 11 authors were contacted for additional data. However, only one of these responded to us, and this author did not provide the additional data needed. One completed project that could be potentially eligible was found in ClinicalTrials.gov. We contacted the corresponding author for that project but did not receive an answer. The final number of studies included in the review was 24. See Figure 1 for a flow diagram of the article selection process.

The vast majority of the studies we identified for inclusion in this review were conducted in the last decade. A plurality of the studies $(k=12,50 \%)$ were conducted in the United States, four (17\%) were conducted in Germany, and the rest were conducted in six other countries. The quality of the study designs was rated as "moderate" for the most part. Two studies (9\%) were case series, 19 (79\%) were non-randomized trials, and only three (13\%) were randomized controlled trials (RCTs). The sample sizes in the studies that were not case series ranged from $n=7-71$. Only seven (29\%) studies included follow-up assessments. Most of the studies (19,79\%) included only adults, four (17\%) included both adults and youths, and one (4\%) used a pediatric sample only. The pain type most frequently studied was headache (including migraines; $k=5,21 \%$ ). The rest of the studies evaluated the effects of NF in individuals with a variety of pain conditions: fibromyalgia (two studies), spinal cord injury (SCI) and chronic pain (three studies), a variety of chronic pain problems (two studies), pain associated with radiation therapy for cancer (one study), chemotherapy-induced peripheral neuropathy (CIPN; one study), postherpetic neuralgia (one study), Complex Regional Pain Syndrome Type I (CRPSI; one study), and chronic paraplegia (one study). Two studies (8\%) used NF to enhance hypnotic analgesia in individuals with multiple sclerosis. Also, a total of four studies (17\%) assessed the effects of NF on laboratory (induced) pain in healthy individuals.

In addition to pain intensity $(k=20,83 \%)$ and pain frequency $(k=4,17 \%)$, the studies assessed a number of other pain-related outcomes such as: fatigue $(k=6,25 \%)$, sleep quality/problems $(k=3,13 \%)$, anxiety $(k=2,8 \%)$, depression $(k=2,8 \%)$, and pain-related interference $(k=4,17 \%)$. Seventeen $(71 \%)$ of the studies assessed changes in brain activity after the intervention. Of these, $11(46 \%)$ performed analyses to determine if preto post-treatment changes in measures of brain activity were associated with pre- to post-treatment changes in one or more study outcomes.

Regarding the NF type, most studies $(k=19 ; 79 \%)$ used EEG-based NF; five $(k=5,21 \%)$ used fMRI-based NF. Among the studies that were conducted with EEG, 15 (63\%) used brain oscillation power-based NF, two (8\%) used surface and/or lowresolution electromagnetic tomography (LORETA) Z-score NF, and two (8\%) used event related potentials (ERPs) NF. A total of 21 studies (88\%) used NF as a single intervention, one (4\%) used it in addition to other interventions and two (8\%) used it to enhance the effects of another intervention.

A variety of control conditions were used in the controlled studies: one study (4\%) tested NF provided to a clinical sample against the same NF intervention provided to a control sample of healthy individuals and a waitlist-control condition, one (4\%) used an active control condition and a waitlist-control condition, two (8\%) used a waitlist-control condition, one (4\%) used a sham condition, four studies (17\%) used an active control condition, one $(4 \%)$ used three active control conditions and a sham condition, and one (4\%) used four sham control groups and one active control condition.

Participants in the studies reviewed received between one to 98 sessions. For those who received more than one session, frequency ranged from once a week to daily, and duration ranged from 16-120 min. See Tables 1, 2 for details about the interventions and participants in the studies reviewed.

\section{Synthesized Findings Description of the Different NF Types and NF Protocols}

A variety of NF types and protocols have been used for pain management. Most of them attempted to decrease brain activity hypothesized to be associated with the processing of nociceptive information (Siniatchkin et al., 2000; Emmert et al., 2014) and/or increase brain activity hypothesized to be inconsistent with pain information processing (Mathew et al., 1987; Jensen et al., 2014). Others aimed to normalize brain activity, relative to available normative data on brain activity (Koberda et al., 2013; Prinsloo et al., 2019). Here, we briefly describe the main characteristics of each type of NF used before discussing their effects on treatment outcomes.

We identified five different types of NF: four EEG-based and one fMRI-based. EEG-based NF asses and aim to modify the power of brain oscillation activity in different bandwidths from electrodes placed on the scalp. Brain oscillations are traditionally grouped in different bandwidths, expressed in cycles per second $(\mathrm{Hz})$. The traditional bandwidths most often used for bandwidth classification, from slower to more rapid are: delta $(\delta, 0.5-4 \mathrm{~Hz})$, theta $(\theta, 4-8 \mathrm{~Hz})$, alpha $(\alpha, 8-13 \mathrm{~Hz})$, beta $(\beta, 13-30 \mathrm{~Hz})$, and gamma $(\gamma, 30+\mathrm{Hz})$. Other bandwidths that are sometimes used in NF studies are most often subclassifications of these primary ones, such as low $\beta(12-15 \mathrm{~Hz})$ and high $\beta(21-30 \mathrm{~Hz})$ (Marzbani 


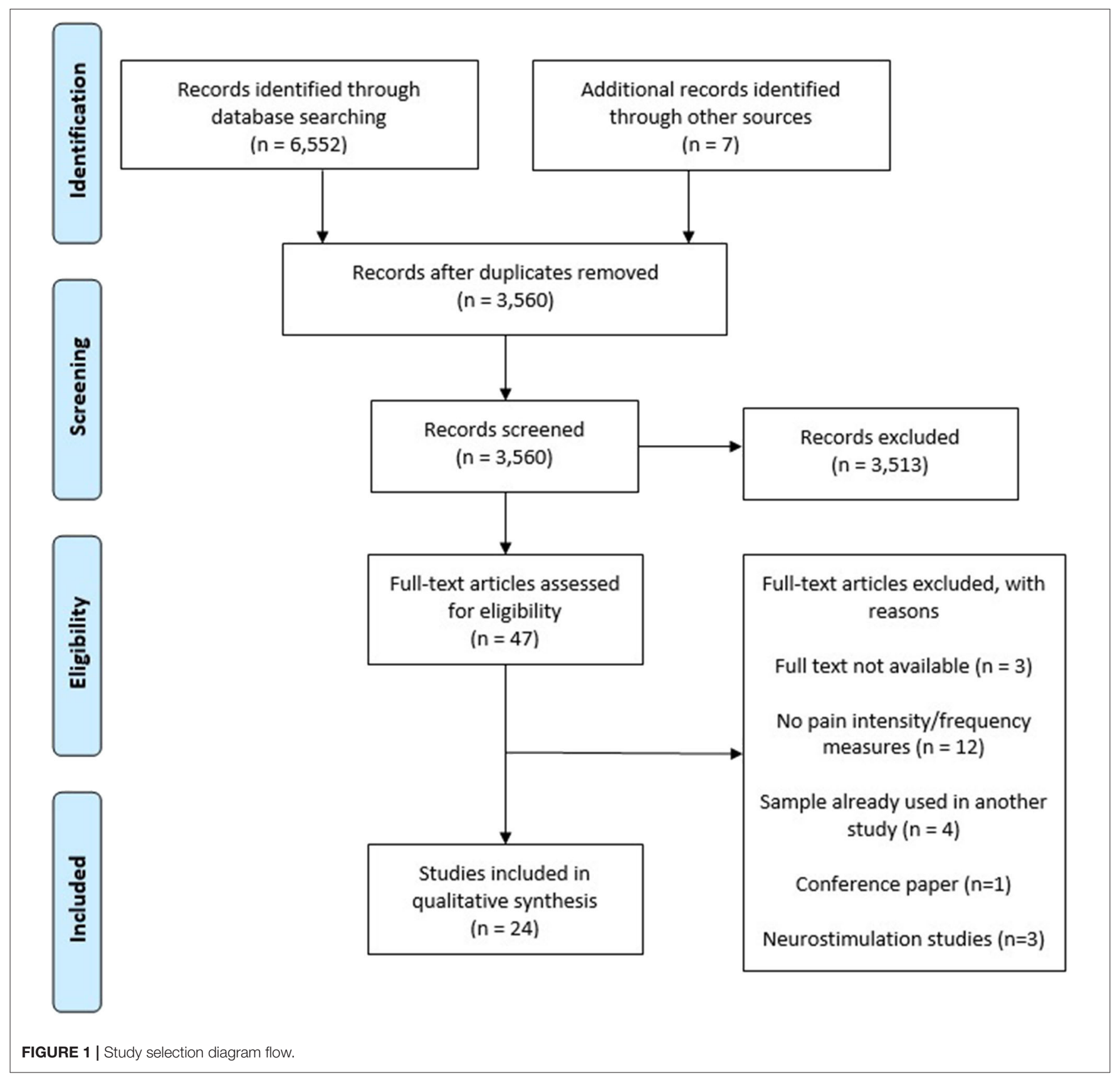

et al., 2016). Another common bandwidth used in NF studies is called "sensorimotor rhythm" (SMR) frequency $(12-15 \mathrm{~Hz})$. The SMR bandwidth is the same frequency as low $\beta$, but is a common frequency found in the sensorimotor areas of the cortex (Hoedlmoser et al., 2008).

\section{Brain oscillation power-based NF}

This type of NF that has been used most frequently in research in this area (Krigbaum and Wigton, 2014). This approach aims to increase or decrease the power of specific oscillation bandwidths as assessed from electrodes placed on different parts of the scalp. There is a large variety of protocols that have been used when treating patients with this procedure; in fact, we were unable to identify any studies that used the same NF protocol. That said, many of the protocols were quite similar. The protocols are often named based on the frequencies they seek to alter (e.g., an "alpha protocol" would be one seeking to alter - often increase $-\alpha$ power). This approach normally involves three electrodes: one for the active training site, one for the reference site, and one for ground. Some protocols using this approach are theory-based; that is, they intend to alter a frequency theorized to be associated with a behavioral outcome [e.g., increased $\alpha$ is associated with increased relaxation; (Hammond, 2011)]. Other protocols are data-based; that is, based on an initial 
TABLE 1 | Description of participant characteristics.

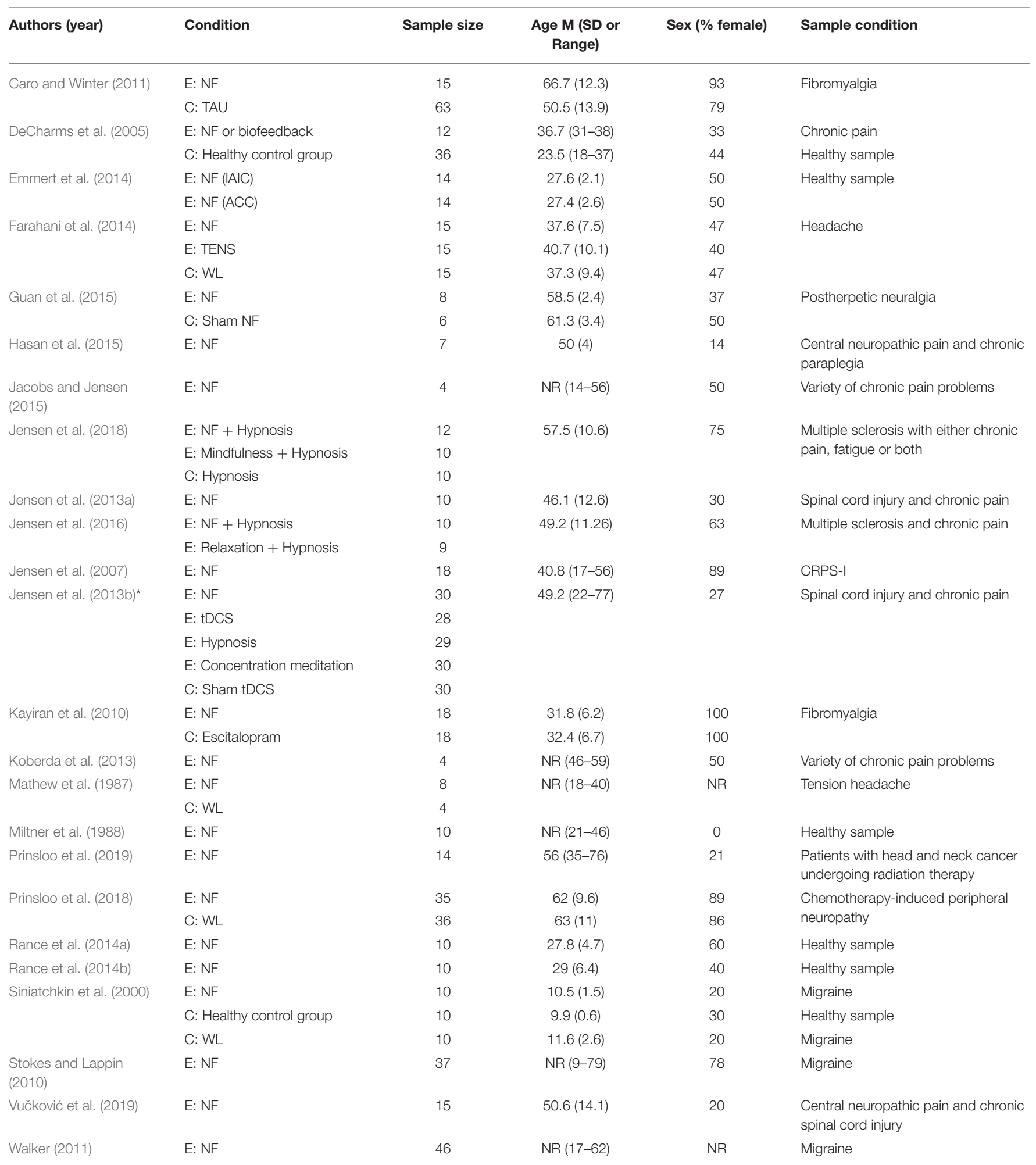

NR, not reported; E, Experimental; C, Control; NF, neurofeedback; TAU, Treatment as usual; WL, Wait-list control group; TENS, Transcutaneous electrical nerve stimulation; CRPS-I, Complex regional pain syndrome type I; TBI, Traumatic brain injury; CIPN, Chemotherapy-induced peripheral neuropathy. IAIC, Left anterior insular cortex; ACC, Anterior cingulate cortex; $t D C S$, transcranial Direct Current Stimulation. "In this study the same participants received up to a single session of all four active procedures and the sham control procedure. 
TABLE 2 | Description of study and intervention characteristics.

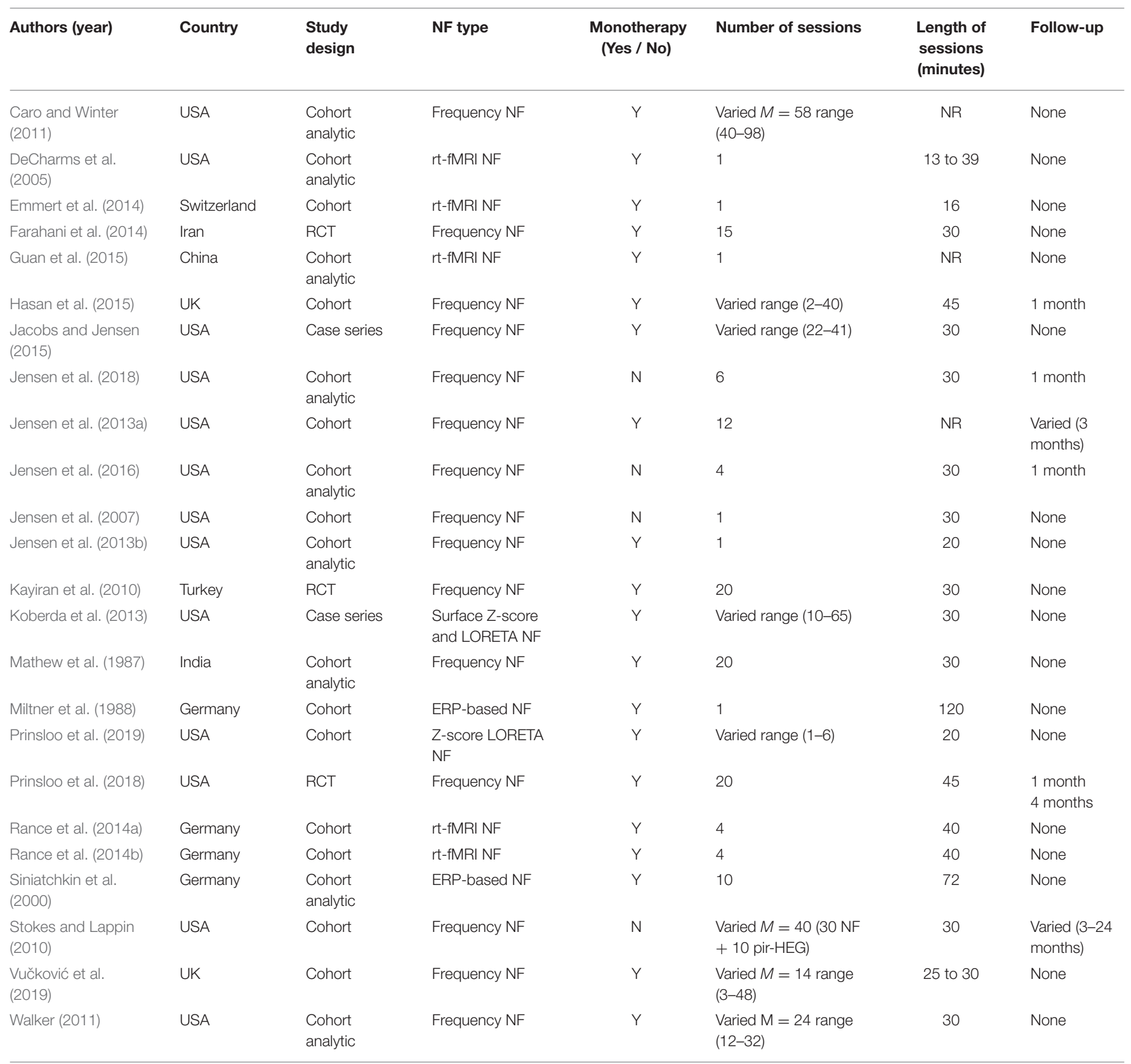

NR, not reported; NF, neurofeedback; RCT, randomized controlled trial; fMRI, functional magnetic resonance imaging; ERP, event related potential; LORETA, low-resolution electromagnetic tomography.

quantitative electroencephalogram (qEEG) assessment of the patient that is then used to select the electrode positions and bandwidths to be targeted. Using the data-based approach, the participant is first administered a qEEG assessment to evaluate his or her unique EEG pattern, relative to a normative database. "Excesses" (power at bandwidths that are substantially greater than normative values) or "deficits" (power at bandwidths that are substantially lower than normative values) for any bandwidth activity at specific electrode sites are then identified, relative to healthy individuals. Once this assessment is conducted, an individualized treatment protocol is then designed to target any
EEG "abnormalities" (i.e., deviations from the norm). The goal is to "normalize" the brain activity.

\section{Surface Z-score NF and LORETA Z-score NF}

To discuss the LORETA Z-score NF approach it is necessary to explain what LORETA imaging is. LORETA is a functional imaging procedure that seeks to estimate EEG bandwidth activity in deeper (intracranial) regions of the brain, based on data collected from surface electrodes (Pascual-Marqui et al., 1994, 2002). Similar to EEG data collected from specific electrodes, data from LORETA imaging can be compared with normative 
LORETA data, and then used to develop a treatment protocol (e.g., to reduce $\theta$ power in the thalamus, if a specific patient's pretreatment LORETA assessment indicates excessive thalamic $\theta$ ). Alternatively, it is possible to simply determine that more (or less) power of a specific bandwidth at a certain intracranial site might decrease an individual's pain and, based on that information, to develop a protocol to use LORETA Z-score NF to alter activity in that bandwidth at that location. It is also possible to use a "normalizing" protocol in real time, such that the qEEG or LORETA-based data are compared to the norms directly, allowing to reinforce responses in the direction of the normative database. The use of qEEG and LORETA data in real time NF are commonly referred to as "surface Z-score NF" and “LORETA Z-score NF”, respectively (Wigton, 2013).

\section{ERP-based NF}

Event-related potential (ERP) assessments allow the study of stereotypical brain activity responses that occur at different specific time points following a specific stimulating cognitive, sensory or motor event (such as a response to an aversive stimuli; Luck, 2014). These time-locked brain responses to the aforementioned events are called components, which are believed to reflect the activity of postsynaptic potentials produced when thousands or millions of pyramidal neurons fire in synchrony while processing information (Sur and Sinha, 2009). ERP-based NF seeks to alter these components. One common ERP-based NF approach targets slow cortical potentials (SCP), which are slow event-related electrical shifts in the EEG of less than $1 \mathrm{~Hz}$, that alternate between being electrically positive and negative (Wyckoff and Strehl, 2011; Krigbaum and Wigton, 2014). A distinctive component central to SCPs is the contingent negative variation (CNV), a negative potential that is recorded from the scalp during response anticipation, while the subject is anticipating and preparing for task performance (i.e., when they are told to press a button when a warning appears on the monitor). The aim of SCPs NF is to either increase or suppress the CNV by means of feedback, in order to regulate the excitation threshold (Strehl, 2009). Increased negativity is related to increased neural activity and a lower excitation threshold, whereas increased positivity is related to less neural activity and a higher excitation threshold (Strehl et al., 2006). Another ERPbased protocol that has been used for pain management targets changes in the amplitude of the N150-P260 complex, as this complex is sensitive to nociceptive stimulation (Miltner et al., 1988). The N150 is an early negative component that occur 150 milliseconds after the presentation of a stimulus, whereas the P260 is an early positive component that can be observed 260 milliseconds after the presentation of a stimulus.

\section{Real-time fMRI NF}

rt-fMRI NF allows patients to regulate brain activity in specific brain areas (including deeper areas of the brain) by targeting changes in the BOLD activity in the regions of interest. The most commonly used procedure in this type of NF involves an anatomical scan combined with a localizer task to identify the voxels of the region of interest to be trained (Sulzer et al., 2013; Thibault et al., 2018). Following this, the level of BOLD activity in the targeted area is fed back to the patient in order to facilitate their ability to increase or decrease that activity, as appropriate. The goal is to teach the individual to deliberately control the activation of the brain areas thought to be involved in pain perception and regulation.

\section{Evidence Regarding the Effects of EEG-Based NF Brain Oscillation Power-Based NF}

We identified 15 articles that evaluated the effects of brain oscillation power-based NF on pain and pain-related outcomes. In the first of these, a RCT was conducted to evaluate the efficacy of a SMR protocol in individuals with fibromyalgia (Kayiran et al., 2010). Participants were randomly allocated to either the NF group $(n=18)$ or an active control group $(n=18)$ receiving $10 \mathrm{mg}$ of escitalopram per day for 8 weeks. The NF treatment was comprised of 2030 -min sessions aiming to increase SMR bandwidth activity assessed over the right-central area of the scalp (C4 in the international 10-20 system). In addition to assessing pain intensity, the authors assessed resting state EEG activity in the participants who received NF during an eyes-open condition at baseline, 2 weeks, 4 weeks (end of treatment), 8 weeks (1-month follow-up), 16 weeks (3-month follow-up) and 24 weeks (5-month follow-up) after treatment started. Although they found no changes in the mean amplitudes of resting state bandwidth power over time, there was a statistically significant decrease in the $\theta / \mathrm{SMR}$ ratio at the end of the treatment, compared to baseline. Participants in both treatment conditions reported significant pre- to post-treatment reductions in pain intensity (measured with a $10-\mathrm{cm}$ Visual Analog Scale), fatigue, anxiety and depression. The improvements were maintained at all the follow-up assessment points (i.e., up to 5 months after treatment started, or 4 months after treatment ended). In the NF group, the maximum reductions in both pain intensity and fatigue were reached at the 4 th week of treatment (i.e., at the end of NF treatment), whereas in the active control group the greatest reduction in pain intensity was reported at the 8 th week of treatment (i.e., at the end of active treatment for the control group). Moreover, the improvements in pain intensity, fatigue, anxiety and depression were significantly greater for the NF group than the control group at every assessment point. See Tables 3, 4 for a summary of the pain and brain activity outcomes for all the studies.

In another RCT, a sample of 71 cancer survivors with CIPN were randomly allocated to the NF group $(n=35)$ or to a waitlist control group $(n=36)$ (Prinsloo et al., 2018). A qEEG was conducted and used to develop patient-specific NF protocols to normalize EEG-assessed oscillation power. The NF treatment consisted in 20 45-min sessions. The average pain intensity and pain interference ratings for the NF group were significantly lower at the end of the treatment compared to the wait-list control group; these differences were still statistically significant at 1-month and 4-month follow-up assessment points. Although there was also a significant difference in fatigue ratings between groups at the end of treatment, these differences were no longer statistically significant at 1 -month and 4 -month follow-up. There 
TABLE 3 | Pre-treatment, post-treatment and follow-up pain intensity and frequency ratings.

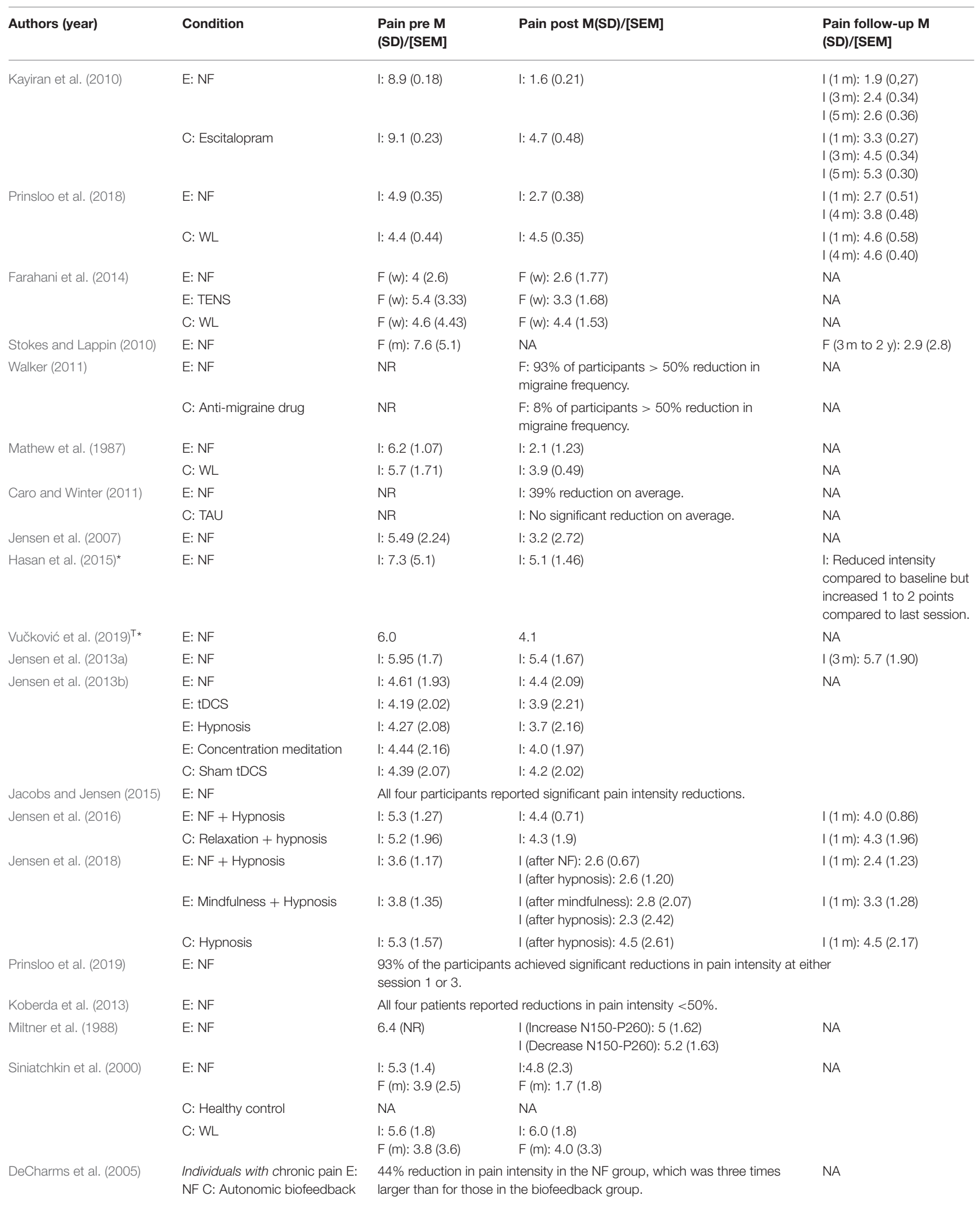


TABLE 3 | Continued

\begin{tabular}{|c|c|c|c|c|}
\hline Authors (year) & Condition & $\begin{array}{l}\text { Pain pre M } \\
\text { (SD)/[SEM] }\end{array}$ & Pain post $M(S D) /[S E M]$ & $\begin{array}{l}\text { Pain follow-up M } \\
\text { (SD)/[SEM] }\end{array}$ \\
\hline & $\begin{array}{l}\text { Healthy individuals E: NF (rACC) } \\
\text { C: } 4 \text { control groups with no } \\
\text { feedback from rACC }\end{array}$ & \multicolumn{2}{|c|}{$\begin{array}{l}\text { In the experimental group, increasing or decreasing the BOLD activity in the } \\
\text { rACC resulted in the noxious stimuli to be rated as more or less painful, } \\
\text { respectively. The changes in pain intensity in the experimental were } \\
\text { significantly larger than for any of the four control groups. }\end{array}$} & \\
\hline \multirow[t]{2}{*}{ Guan et al. (2015) } & $\mathrm{E}: \mathrm{NF}$ & I: $4.13[0.55]$ & $\begin{array}{l}\text { I (Up-training): increase in NRS scores of } \\
1.8 \text { [0.31] points. } \\
\text { I (Down-training): decrease in NRS scores } \\
\text { of } 1.5 \text { [0.33] points }\end{array}$ & NA \\
\hline & C: Sham NF & I: $5.0(0.52)$ & $\begin{array}{l}\text { I (Up-training): increase in NRS scores of } \\
0.1 \text { [0.01] points. } \\
\text { I (Down-training): decrease in NRS scores } \\
\text { of } 0.5 \text { [0.22] points. }\end{array}$ & \\
\hline \multirow[t]{2}{*}{ Emmert et al. (2014)* } & $\mathrm{E}: \mathrm{NF}(\mathrm{IAIC})$ & I: $7.7(1.20)$ & I: $6.0(1.63)$ & NA \\
\hline & E: NF (ACC) & I: $7.0(1.15)$ & I: $6.2(1.76)$ & \\
\hline Rance et al. (2014a) & E: NF & \multicolumn{2}{|c|}{ None of the four conditions reported a significant decrease in pain intensity. } & NA \\
\hline Rance et al. (2014b) & E: NF & \multicolumn{2}{|c|}{ None of the two conditions reported a significant decrease in pain intensity. } & NA \\
\hline
\end{tabular}

E, Experimental; C, Control; NF, neurofeedback; NA, Not assessed; NR, Not reported; TAU, Treatment as usual; WL, Wait-list control group; TENS, Transcutaneous electrical nerve stimulation; I, Intensity, F, Frequency; W, week; M, month; Y, year; [SEM] standard error of the mean; IAIC, Left anterior insular cortex; ACC, Anterior cingulate cortex; BOLD, blood oxygen-level dependent. * Pain intensity scores calculated from participants individual's data presented in the study. ${ }^{\top}$ Average pre- and post-session scores.

were no significant between-group differences in sleep quality or sleep disturbances at any assessment point. Results showed that brain activity, that is, the EEG frequencies targeted in the scalp positions chosen by the protocol, changed significantly from preto post-treatment toward a more "normal" EEG activity and that it was significantly different for the NF group compared to the waitlist group. Specifically, the NF group showed a significant increase in $\alpha$ relative power and a significant decrease in $\beta$ relative power as averaged over all the electrodes.

Another RCT compared the efficacy of NF and transcutaneous electrical nerve stimulation (TENS) in a group of 45 healthcare practitioners with primary headaches (Farahani et al., 2014). Participants were randomly allocated to either a NF group $(n=$ $15)$, a TENS group $(n=15)$ or a waitlist-control group $(n=15)$. The NF treatment consisted of 2030 -min sessions and aimed to increase SMR and decrease $\theta$ and high $\beta$ over the right and left temporal cortex (T3 and T4 in the international 10-20 system). Both the NF and TENS groups experienced significant reductions in headache frequency compared to the waitlist-control group. However, the NF group achieved a significantly greater reduction in headache frequency than the TENS group.

In an uncontrolled study (Stokes and Lappin, 2010), 37 patients with migraine were treated with a combination of NF, passive infrared hemo-encephalography (pIR-HEG; a form of neurofeedback based on thermal outputs in response to changes in blood flow dynamics rather than brain electrical activity Carmen, 2004), and thermal biofeedback (i.e., a type of biofeedback that aims to change body temperature). The treatment consisted of an average of 40 sessions and included an average of 30 frequency-based NF sessions and an average of 10 pIR-HEG or hand-warming biofeedback sessions. NF training aimed to reduce the amplitude of the frequencies which were assessed at baseline and determined to be "excessive;" that is, treatment was tailored to each participant and was not standardized. The scalp positions where NF was conducted were primarily 5 sets of homologous sites (including over the prefrontal, frontal, temporal, central and parietal areas; FP1FP2, F3-F4, T3-T4, C3-C4, and P3-P4 in the international 1020 system). Compared with baseline scores, patients reported a significant reduction in the number of migraines per month at follow-up (a post-treatment assessment was not conducted), which was conducted three months to two years after the end of the treatment.

Walker studied the effects of NF as a treatment for recurrent migraine headaches (Walker, 2011). Of the 76 individuals entering the study, 46 chose to follow the NF treatment and 25 chose to remain with anti-migraine medication (the specific medication used by the study participants was not reported). The qEEG analysis at baseline showed an excess of power in the high $\beta$ frequency band at a number of electrode sites - excesses that were most pronounced in the frontal, central and parietal regions. The NF protocol consisted in five 30-min sessions targeting a reduction in high $\beta$ activity and an increase in $10 \mathrm{~Hz}$ activity at each electrode where an excessive high $\beta$ activity had been identified. At post-treatment, $98 \%$ and $32 \%$ of the participants in the NF and control condition reported reductions in headache frequency, respectively. Specifically, in the NF group, $54 \%$ experienced a complete cessation of migraine headaches, $39 \%$ experienced a reduction in migraine headaches greater than 50 , and $4 \%$ experienced a reduction of $<50 \%$. In the control group, none of the participants experienced a complete cessation of migraine headaches, $8 \%$ experienced a reduction in migraine headaches greater than 50 , and $20 \%$ experienced a reduction of less than $50 \%$.

The oldest study included in this review (Mathew et al., 1987) assessed the efficacy of NF as a treatment for 
TABLE 4 | Brain activity outcomes.

\begin{tabular}{ll}
\hline Authors (year) & NF protocol \\
\hline $\begin{array}{l}\text { Kayiran et al. } \\
\text { (2010) }\end{array}$ & $\nearrow$ SMR at C4. \\
& Normalize EEG at several unreported \\
$\begin{array}{l}\text { Prinsloo et al. } \\
\text { (2018) }\end{array}$ & $\begin{array}{l}\nearrow \text { SMR, } \searrow \theta \text { and high } \beta \text { at T3 and T4. } \\
\text { Farahani et al. } \\
\text { (2014) }\end{array}$ \\
$\begin{array}{l}\text { Stokes and Lappin } \\
\text { (2010) }\end{array}$ & $\begin{array}{l}\text { NF, pir-HEG, hand-warming biofeedback. } \\
\text { locations, mainly at: T3, T4, C3, C4, F3, }\end{array}$ \\
& $\begin{array}{l}\text { F4, FP1, FP2, P3, P4. } \\
\text { Walker (2011) }\end{array}$ \\
& $\begin{array}{l}\nearrow \alpha \text { high } \beta \text { and } \nearrow 10 \text { Hz activity at each } \\
\text { electrode with excessive high } \beta .\end{array}$ \\
$\begin{array}{l}\text { Mathew et al. } \\
\text { (1987) }\end{array}$ & $\begin{array}{l}\text { locations. } \\
\end{array}$
\end{tabular}

Caro and Winter

(2011)

Jensen et al.

(2007)

Hasan et al. (2015)

Vučković et al. (2019) $\nearrow \mathrm{SMR}, \searrow \theta$ and high $\beta$ at $\mathrm{Cz}$.

Tailored to each patient and adapted depending on patient's improvement. Normally started by $\nearrow$ SMR at T3 and T4.

First part: $\nearrow \alpha$ at $\mathrm{Oz}$

Second part: combination of 4 protocols: $\overline{\mathrm{A}: \nearrow \mathrm{SMR}, \searrow} \theta$ and high $\beta$ at Cz. B: $\nearrow \alpha$, $\searrow \theta$ and high $\beta$ at P4. C: $\nearrow \alpha, \searrow \theta$ and high $\beta$ at C3. D: $\nearrow \alpha, \searrow \theta$ and high $\beta$ at $\mathrm{C} 4$.

Placebo testing protocol: Either prerecorded session or $\nearrow \alpha$ at $\mathrm{Oz}$.

$\nearrow \alpha, \searrow \theta$ and high $\beta$ between C2 and C4.

3 protocols: $\mathrm{A}: \nearrow \alpha$ and $\searrow \beta$ at T3 and T4. B: $\nearrow \mathrm{SMR}, \searrow \theta$ and $\beta$ at C3 and C4. C: $\nearrow$ SMR, $\searrow \theta$ and $\beta$ at $\mathrm{P} 3$ and P4.

$\nearrow \alpha$ and $\searrow$ high $\beta$ at T3 and T4.
Effects in brain activity (pre- to post-treatment or during)

No changes in the mean amplitudes of EEG rhythms.

A significant decrease in the $\theta / S M R$ ratio at the end of the treatment compared to baseline.

After treatment, the NF group significantly increased $\alpha$ activity and decreased $\beta$ activity.

NA

NA

Association between brain activity change and pain improvements

NA

NA

NA

NA

NA

NA

The NF group showed an increase in the amount of time spent with a preponderance of $\alpha$ activity. In the NF group, there was no change in overall $\alpha$ amplitude.

The wait-list control group did not evidence any significant brain activity change after treatment. NA

NA

\section{First part:}

All participants successfully increased at $\mathrm{Oz}$, with no effect on pain intensity.

Second part:

All five participants decreased frontal $\theta$ during training.

a power increased in the central cortex in four patients during training.

Four patients decreased frontal high $\beta$ during training.

The largest long-term changes were in the high $\beta$ band of the insular cortex, the cingulate cortex and the dorsolateral prefrontal cortex.

Placebo testing protocol:

During the placebo prerecorded session, the brain activity was not different from baseline. Participants were successful increasing $\alpha$ power at $\mathrm{Oz}$, but this had no effect on pain intensity.

With respect to baseline power:

9/15 participants significantly increased $\alpha$ power.

$7 / 15$ significantly decreased $\theta$ power.

$-6 / 15$ participants significantly decreased high

$\beta$ power.

Pre- to post-treatment decrease in $\theta$ and increase in $\alpha$, that were no longer significant at 3-month

follow-up.

No changes in $\beta$ activity.

No significant pre- to post-session change in any of the five EEG bandwidths ( $\delta, \theta, \alpha, \beta$ and $\gamma$ ).
NA

NA

NA meaningful reduction in pain intensity were the ones that successfully increased $\alpha$ power and to some degree, decreased $\beta$.

Brain activity changes after NF were partially associated with pain improvements.

Eight of the 12 participants that achieved pain improvements successfully increased $\alpha$ during NF.

Three of the remaining four participants who achieved pain improvements with NF but did not increase $\alpha$, did achieve a significant decrease in $\theta$, high $\beta$ or both. NA

There was no association between brain activity change with NF and pain changes. 
TABLE 4 | Continued

\begin{tabular}{|c|c|c|c|}
\hline Authors (year) & NF protocol & $\begin{array}{l}\text { Effects in brain activity (pre- to post-treatment } \\
\text { or during) }\end{array}$ & $\begin{array}{l}\text { Association between brain activity } \\
\text { change and pain improvements }\end{array}$ \\
\hline $\begin{array}{l}\text { Jacobs and } \\
\text { Jensen (2015) }\end{array}$ & $\begin{array}{l}\text { Tailored to each patient, but all received at } \\
\text { some point a protocol involving } \nearrow \alpha, \text { low } \\
\beta, \searrow \theta \text { and high } \beta \text {. Several scalp locations } \\
\text { used. }\end{array}$ & NA & NA \\
\hline $\begin{array}{l}\text { Jensen et al. } \\
\text { (2016) }\end{array}$ & $\begin{array}{l}\text { Before hypnosis: Increase } \theta \text { (by } \nearrow 5-9 \mathrm{~Hz} \\
\text { and } 8-11 \mathrm{~Hz} \text { ) at FP1 and F3. } \\
\text { After hypnosis: } \nearrow \text { low } \beta, \searrow \gamma \text {, high } \beta \text { and } \theta \\
\text { at } \mathrm{Cz} \text {. }\end{array}$ & NA & NA \\
\hline $\begin{array}{l}\text { Jensen et al. } \\
\text { (2018) }\end{array}$ & $\nearrow \theta$ at AFz. & $\begin{array}{l}\text { There was no significant time effect in the NF group } \\
\text { for any of the EEG bandwidths }(\delta, \theta, \alpha, \beta \text { and } \gamma) \text {. }\end{array}$ & NA \\
\hline $\begin{array}{l}\text { Prinsloo et al. } \\
\text { (2019) }\end{array}$ & $\begin{array}{l}\text { Normalize electrical activity in the } \\
\text { Brodmann's areas } 3,4,5,13,24,32 \text {, and } \\
33 .\end{array}$ & $\begin{array}{l}\text { EEG changed toward EEG activity more } \\
\text { representative of the normal population in all } \\
\text { targeted Brodmann's areas but the } 32 \text {. }\end{array}$ & $\begin{array}{l}\text { Changes in the current source density in } \\
\text { Brodmann's areas } 24 \text { and } 33 \text { accounted } \\
\text { completely for the variance in pain } \\
\text { changes with NF }\left(R^{2}=1, p=0.012\right) \text {. }\end{array}$ \\
\hline $\begin{array}{l}\text { Koberda et al. } \\
\text { (2013) }\end{array}$ & $\begin{array}{l}\text { Tailored protocols aimed at normalizing } \\
\text { EEG activity. }\end{array}$ & $\begin{array}{l}\text { The four participants evidenced changes toward a } \\
\text { more normal brain activity pattern. }\end{array}$ & NA \\
\hline $\begin{array}{l}\text { Siniatchkin et al. } \\
(2000)\end{array}$ & $\nearrow$ and $\searrow$ the amplitude of the SCPs at Cz. & $\begin{array}{l}\text { Children with migraine were only able to decrease } \\
\text { the amplitude of their SCPs; they were unable to } \\
\text { increase cortical negativity. } \\
\text { The control group of healthy children learned to both } \\
\text { increase and decrease the amplitude of their SCPs. }\end{array}$ & $\begin{array}{l}\text { No association between the change in the } \\
\text { amplitude of the SCPS and the reduction } \\
\text { of migraines. }\end{array}$ \\
\hline Miltner et al. (1988) & $\begin{array}{l}\nearrow \text { and } \searrow \text { the size of the N150-P260 } \\
\text { complex at Cz. }\end{array}$ & $\begin{array}{l}\text { Participants learned to increase and decrease the } \\
\text { size of the N150-P260 complex. -Subjective pain } \\
\text { intensity reports were slightly higher in the } \\
\text { up-training condition compared to the } \\
\text { down-training condition. }\end{array}$ & NA \\
\hline $\begin{array}{l}\text { DeCharms et al. } \\
\text { (2005) }\end{array}$ & $\nearrow$ and $\searrow$ BOLD activity in the rACC. & $\begin{array}{l}\text { The experimental healthy group learned to both } \\
\text { increase and decrease BOLD activity in the rACC. } \\
\text { The experimental group of patients with chronic } \\
\text { pain learned to regulate BOLD activity in the rACC. }\end{array}$ & $\begin{array}{l}\text { For the } 6 \text { patients with chronic pain that } \\
\text { completed at least two training runs, there } \\
\text { was a significant and strong association } \\
\text { between the extent to which they were } \\
\text { able to regulate BOLD activity in the rACC } \\
\text { and pain intensity reductions }(r=0.9) \text {. }\end{array}$ \\
\hline Guan et al. (2015) & $\nearrow$ and $\searrow$ BOLD activity in the rACC. & $\begin{array}{l}\text { The experimental group was able to both increase } \\
\text { and decrease BOLD activity in the rACC. }\end{array}$ & $\begin{array}{l}\text { No association between the changes in } \\
\text { BOLD activity and changes in pain ratings. }\end{array}$ \\
\hline $\begin{array}{l}\text { Emmert et al. } \\
\text { (2014) }\end{array}$ & $\begin{array}{l}\searrow \text { BOLD activity in ACC. } \\
\searrow \text { BOLD activity in IAIC. }\end{array}$ & $\begin{array}{l}\text { Eight of the } 14 \text { participants were able to decrease } \\
\text { the BOLD activity in the ACC. } \\
\text { Nine of the } 14 \text { participants were able to decrease } \\
\text { BOLD activity in the IAIC. }\end{array}$ & $\begin{array}{l}\text { There were no differences in pain ratings } \\
\text { between those who were able to decrease } \\
\text { BOLD activity in IAIC and ACC and those } \\
\text { who were not. }\end{array}$ \\
\hline $\begin{array}{l}\text { Rance et al. } \\
\text { (2014a) }\end{array}$ & $\begin{array}{l}4 \text { conditions: } \nearrow \text { the BOLD activity in the } \\
\text { rACC. } \nearrow \text { the BOLD activity in the plnsL. } \\
\searrow \text { the BOLD activity in the rACC. } \searrow \text { the } \\
\text { BOLD activity in the plnsL. }\end{array}$ & $\begin{array}{l}\text { Participants were able to increase BOLD activity in } \\
\text { the plnsL and decrease BOLD activity in the rACC } \\
\text { and plnsL. }\end{array}$ & NA \\
\hline $\begin{array}{l}\text { Rance et al. } \\
\text { (2014b) }\end{array}$ & $\begin{array}{l}\text { Increase the difference in activation levels } \\
\text { between the rACC and plnsL. } \\
2 \text { conditions: } \\
\text { [1] higher activation in rACC than in plnsL. } \\
\text { [2] higher activation in plnsL than in rACC. }\end{array}$ & $\begin{array}{l}\text { Participants were successful in achieving the } \\
\text { training goals for the two conditions. }\end{array}$ & $\begin{array}{l}\text { The achieved difference in activation } \\
\text { between the rACC and the plnsL was not } \\
\text { associated to changes in pain intensity } \\
\text { ratings. }\end{array}$ \\
\hline
\end{tabular}

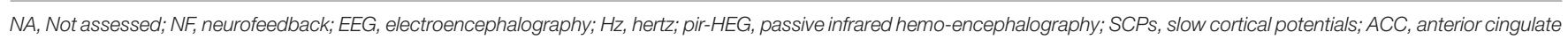
cortex; rACC, rostral anterior cingulate cortex; IAIC, left anterior insular cortex; plnsL, left posterior insula; BOLD, blood oxygen-level dependent.

eight individuals with tension-type headache compared to a waitlist control group $(n=4)$. The NF participants received $2030-\mathrm{min}$ sessions of a protocol aiming to increase $\alpha$ assessed from one or more (unreported) electrode sites. The treatment group reported a significant increase in the amount of time spent with a preponderance of $\alpha$ activity, but not in its overall amplitude. The NF group also reported statistically significant reductions in pain intensity and anxiety from pre- to post-treatment. The waitlist control group, on the other hand, did not evidence any significant changes in brain activity, pain intensity or anxiety from preto post-assessment.

Caro and colleagues conducted an uncontrolled study assessing the use of NF to reduce attention difficulties and 
somatic symptoms in patients with fibromyalgia (Caro and Winter, 2011). Fifteen patients were treated with NF and compared with a historical control group comprised of 63 individuals receiving standard medical care. The NF group received 58 sessions on average (ranging from 40 to 98 ) aiming to increase SMR oscillation power, while inhibiting both $\theta$ and high $\beta$ oscillations at the same time. The training electrode was placed over the center of the scalp ( $\mathrm{Cz}$ in the international 10-20 system). The NF group reported significant mean reductions in global pain and fatigue severity ( 39 and 40\%, respectively). The 63 control participants did not report any significant improvements in either outcome variable.

Another study reported on changes after a single session of NF in 18 individuals with CRPS-I participating in a 20-day multidisciplinary treatment program (Jensen et al., 2007). The treatment protocol used varied over the course of each 30-min session, and was tailored to each patient, depending on their reports of pain reduction (or not) as the session progressed. For example, if training at a specific site to increase the power of a specific bandwidth was associated with improvements, that training continued. Training usually began by reinforcing SMR activity at sites over temporal areas (T3 and T4 in the international 10-20 system) to "stabilize" brain activity. If the patient reported no improvement with this protocol, different electrode sites or training frequencies were used until (and if) the patient reported improvements. Participants reported an average pre- to post-session reduction of 2.3 points in pain intensity (on a 0-10 Numerical Rating Scale) of their primary pain. Half of the participants reported a pain intensity reduction that was clinically meaningful, that is, a reduction of $30 \%$ or more from pre- to post-session (Rowbotham, 2001).

A pilot study (Hasan et al., 2015) aimed to investigate the potential mechanisms underlying NF efficacy to treat central neuropathic pain in seven patients with chronic paraplegia. Four patients received 40 sessions, one received 20 and two received only three sessions. The first $10 \mathrm{~min}$ of the NF treatment aimed to increase $\alpha$ at occipital regions ( $\mathrm{Oz}$ in the international 10 20 system) with a goal of increasing general relaxation. The remainder of the NF training session had a goal of pain reduction. In this second component of each training session, each patient received a combination of one of four different protocols (all in a 30- to 35-min period), depending on their response to each. Protocol A reinforced SMR and suppressed $\theta$ and high $\beta$ assessed from the central area of the scalp $(\mathrm{Cz}$ in the international $10-$ 20 system). Protocol $\mathrm{B}$ reinforced $\alpha$ and suppressed $\theta$ and high $\beta$ from an electrode placed over the right parietal area ( $\mathrm{P} 4$ in the international 10-20 system). Protocol $\mathrm{C}$ reinforced $\alpha$ and suppressed $\theta$ and high $\beta$ from an electrode placed over the left central area (C3 in the international 10-20 system). Protocol D reinforced $\alpha$ and suppressed $\theta$ and high $\beta$ at from an electrode placed over the right central area (C4 in the international 10-20 system). It is important to note that the $\alpha$ range targeted in this study was slightly higher than usual, that is, $9-12 \mathrm{~Hz}$ instead of the general $8-12 \mathrm{~Hz}$, as lower $\alpha$ frequencies have been found to be associated with central neuropathic pain (Boord et al., 2008). Also, each participant received two "placebo" sessions at some point between sessions 10 and 20 (the specific sessions that were "placebo" sessions differed for each participant), with the goal of testing for placebo responses. One placebo protocol "fed back" pre-recorded data from a different NF session, and the other provided feedback aiming to increase $\alpha$ at the occipital area $(\mathrm{Oz}$ in the 10-20 system). Both placebo protocols were hypothesized to not have any impact on pain. Resting state EEG in both open eyes and closed eyes conditions and sLORETA imaging (a newer and more accurate LORETA) was recorded before and after treatment. In addition, the researchers assessed and recorded EEG activity before and during NF training. All participants received a different number of sessions of each protocol, and the sequence of protocols used also differed for each patient and changed depending on their initial response. The five patients that received at least 20 sessions reported statistically significant pre- to post-treatment reductions in pain intensity; four $(80 \%)$ reported pain reductions that were clinically meaningful (>30\%). The patients that achieved clinically meaningful reductions in pain intensity were the ones that successfully increased $\alpha$ power and, to some degree, decreased high $\beta$ power. At one-month follow-up assessment the participants who reported reductions in pain still reported lower pain intensity, relative to baseline, although they also reported an increase in pain intensity of one to two points (on a $0-10$ scale), relative to baseline. Additionally, regarding pre- to post-session effects, protocols $\mathrm{C}$ and $\mathrm{D}$ were associated with the greatest reductions in pain intensity, although three patients had strong muscle spasms with protocol C. Protocol B yielded a moderate reduction in pain intensity whereas protocol A did not decrease pain intensity for any of the patients. Also, in the two sessions used to test for placebo effects, participants successfully increased $\alpha$ power at the central occipital area ( $\mathrm{Oz}$ in the international 10-20 system), but this had no impact in pain intensity. The largest long-term changes were in the high $\beta$ band of the insular cortex, the cingulate cortex and the dorsolateral prefrontal cortex, assessed via SLORETA.

Another study conducted by the same research team tested the use of self-administered NF to treat central neuropathic pain in 15 patients with chronic SCI (Vučković et al., 2019). Participants were offered up to four training sessions at the hospital before they had to self-administer the treatment at home. They were instructed to use NF on demand but at least once a week for two months, and to record pain intensity before and after each session. The NF session protocol consisted in reinforcing $\alpha$ power and suppressing $\theta$ and high $\beta$ power as measured at a central site (specifically between C2 and C4 in the international 10-20 system). As in the previous study conducted by the same research team, the $\alpha$ range targeted was slightly higher than usual (i.e., $9-12 \mathrm{~Hz}$ ). Each session lasted 30 to $35 \mathrm{~min}$. In total, participants received or self-administered an average of 14 sessions, ranging from 3 to 48 sessions. Statistically significant pre- to post-session improvements in average pain intensity were found in 12 of the 15 participants, with eight participants achieving clinically meaningful reductions in each session on average. With respect to brain activity changes, each NF session was preceded by 2-min baseline EEG recording in the eyes-opened condition. Of the 15 participants, nine significantly increased $\alpha$ power with treatment, whereas seven and six participants significantly 
decreased $\theta$ and high $\beta$ power, respectively. These changes were partially associated with pain improvements. Specifically, eight of the 12 participants that achieved pain improvements successfully increased $\alpha$ during NF. Three of the remaining four participants who achieved pain improvements with NF but did not increase $\alpha$, did achieve a significant decrease in $\theta$, high $\beta$ or both.

Another study tested the efficacy of three different NF protocols in 10 individuals with SCI and chronic pain (Jensen et al., 2013a). Each individual received 4 sessions of each of the following protocols in random order. Protocol A reinforced $\alpha$ and suppressed $\beta$ activity measured from electrodes at the temporal sites frequently used in NF treatment for pain management (i.e., T3 and T4 in the international 10-20 system). Protocol B reinforced SMR activity and suppressed $\beta$ and $\theta$ power assessed from electrodes at central sites ( $\mathrm{C} 3$ and $\mathrm{C} 4$ in the international 10-20 system). Protocol C reinforced SMR activity and suppressed $\beta$ and $\theta$ power at parietal sites (P3 and $\mathrm{P} 4$ in the international $10-20$ system). There were similar pre- to post-session reductions in pain intensity for all three protocols. However, statistically significant pre- to post-treatment (i.e., after the 12 sessions) reductions were not found in average pain intensity. In addition, there were not statistically significant preto post-treatment improvements in fatigue, sleep quality and pain interference. The investigators also assessed and reported resting EEG in eyes closed condition at pretreatment, post-treatment and 3 -month follow-up. In line with the protocols, there were both an increase of $\alpha$ power and a decrease in $\theta$ power from pre- to posttreatment. These changes in $\alpha$ and $\theta$ power were not sustained and were no longer different from baseline levels at the 3-month follow-up. $\beta$ power did not change significantly over time, despite the fact that all three protocols aimed to decrease it.

Another study (Jensen et al., 2013b) assessed the effects of a single 20-min session of four different interventions [NF, hypnosis, concentration-meditation and transcranial Direct Current Stimulation (tDCS)] on pain intensity in thirty patients with SCI and chronic pain, compared to a tDCS sham procedure. Each intervention session took place in a different day. The NF session protocol consisted in reinforcing $\alpha$ and suppressing high $\beta$ power measured at right and left temporal sites (T3 and T4 in the international $10-20$ system). In addition, resting state EEG was recorded for $10 \mathrm{~min}$ in eyes closed before and after each of the five procedures. Neither pain intensity nor EEG activity in any of the five bandwidths (i.e., $\delta, \theta, \alpha, \beta$, and $\gamma$ ) changed significantly after a single session of NF. Also, the associations between changes in EEG power at the different bandwidths and changes in pain intensity were not significant.

Jacobs and Jensen (Jacobs and Jensen, 2015) published a case series reporting the use of NF as a treatment for four individuals with a variety of chronic pain problems. The first patient was a 19-year-old girl with abdominal pain. She received $41 \mathrm{NF}$ sessions. The second patient was a 56 -year-old woman with migraine headaches who received 32 sessions. The third patient was a 14-year-old young man with chronic testicular pain who received $22 \mathrm{NF}$ sessions, and the fourth patient was a 47 year-old man with severe gastrointestinal pain who received 26 sessions of NF treatment. The treatment protocols were tailored for each patient based on standard practice recommendations for addressing the presenting problems of the patients. Given the common practice of rewarding increases in $\alpha$ and low $\beta$ power for chronic pain management, all the patients received training that involved these components for at least some of the sessions. Specifically, at some point, they all received a protocol that involved rewarding increases in $\alpha$ and low $\beta$ power and decreases in $\theta$ and high $\beta$ power. A number of electrode positions were used as training sites, with the goal of identifying the sites and protocols that would be most effective for each patient. All four patients achieved clinically meaningful reductions in pain intensity or pain frequency at some point during treatment, although one of the patients reported that his pain intensity returned to baseline levels by the end of the treatment.

Two pilot studies were conducted to explore the possibility that NF might be used for enhancing the effect of hypnosis for chronic pain management in individuals with multiple sclerosis. In the first of these (Jensen et al., 2016), participants were randomly allocated to receive five sessions of self-hypnosis (one face-to-face session and four prerecorded sessions), preceded by either four 30-min sessions of NF $(n=10)$ or four 20-min sessions of relaxation training, which served as a control group $(n=9)$. After each session, all the individuals received one selfhypnosis session. The NF protocol aimed to increase $\theta$ power by reinforcing slow wave power $(5-9$ and $8-11 \mathrm{~Hz})$ at frontal sites (FP1 and F3 in the international 10-20 system), based on evidence suggesting that higher levels of $\theta$ power are associated with greater response to hypnosis (Jensen et al., 2015). These investigators had a concern that an excess of $\theta$ power might result in negative effects, given the association between $\theta$ activity and having a diagnosis of attention deficit disorder (Arns et al., 2013). To address this possibility, after each hypnosis session, the participants received 10 additional minutes of a NF protocol aiming to reverse any enhanced $\theta$ with a protocol reinforcing low $\beta$ while inhibiting $\gamma$, high $\beta$, and $\theta$ at a central site $(\mathrm{Cz}$ in the international 10-20 system). The participants who received the hypnosis treatment preceded by either the NF or relaxation treatment reported statistically significant reductions in average pain intensity, pain interference and fatigue severity. In the group receiving NF treatment, participants reported larger decreases in average pain intensity from pre- to post-treatment and from pretreatment to 1-month follow up, compared to the participants receiving the relaxation treatment. No differences between the $\mathrm{NF}$ and relaxation groups were found regarding improvements in pain interference or fatigue severity.

In the second study (Jensen et al., 2018), individuals with multiple sclerosis and either chronic pain, chronic fatigue or both pain and fatigue, were randomly allocated to receive five sessions of self-hypnosis (one face-to-face session and four prerecorded sessions), preceded by either six 30-min sessions of NF ( $n=$ 12), six 30-min sessions of mindfulness meditation (MM; $n=$ $10)$ or no intervention $(n=10)$. After this, all participants received one face-to-face hypnosis session, and then four prerecorded hypnosis sessions (recorded by the same clinicians who provided the single face-to-face hypnosis session), targeting pain reduction, fatigue reduction, or both, depending on the presenting problem(s) of the participants. The NF group received in addition four sessions of NF immediately before the recorded 
hypnosis treatment sessions, and the MM group received an additional four sessions of MM immediately before the recorded hypnosis treatment sessions. Therefore, the NF and MM groups received 11 sessions in total (six NF or MM sessions alone, one face-to-face hypnosis session, and then four "combined" NF with hypnosis or MM with hypnosis sessions), and the control group received five sessions in total (the single face-toface hypnosis session and four pre-recorded hypnosis sessions. The NF protocol reinforced an increase in $\theta$ power at the frontal midline region of the scalp (AFz in the international 10-20 system). Participants in all three conditions reported statistically significant reductions in pain intensity from pretreatment to 1month follow-up, which were the highest for the NF group. Both the NF and MM groups reported similar significant pain intensity reductions with six sessions of each treatment alone. At 1-month follow-up, the NF group had maintained the gains made during treatment, whereas the pain intensity ratings in the MM group returned to baseline levels. Fatigue severity ratings improved similarly for the three groups, with a small decrease from baseline to before the hypnotic treatment and an additional decrease after the hypnotic treatment. Nevertheless, fatigue severity increased slightly from post-treatment to followup. With respect to the secondary outcomes (sleep disturbance, pain interference and depression), only the NF group reported significant improvements from pretreatment to 1-month followup. EEG data were recorded for both the NF and MM groups at baseline, after the first six sessions (pre-hypnosis) and at the last NF or MM session (post-treatment). Although there were some differences in the mean amplitudes of the five EEG bandwidths from baseline to pre-hypnosis or from pre-hypnosis to posttreatment, there was no significant time effect for neither the NF nor the MM groups.

\section{Surface Z-Score NF and LORETA Z-Score NF}

Prinsloo and colleagues conducted an exploratory study to assess the use of LORETA Z-score NF (i.e., with a goal toward normalizing brain activity) to treat pain in patients with head and neck cancer undergoing radiation therapy (Prinsloo et al., 2019). In this study, pain intensity and resting eyes-open EEG activity was measured at three time points: baseline (i.e., before starting radiation therapy), after starting radiation therapy and when and if patients reported a pain intensity score of 4 or higher, and after the NF treatment. Pain intensity was also assessed and reported before and after NF sessions 1 and 3. Fourteen patients received one to six 20-min sessions of LORETA Z-score NF targeting a normalization of the activity in the Brodmann's areas number three, four, five, 13, 24, 32, and 33, in real time. As reported by the investigators, 14 patients received one or more sessions, 12 received at least three sessions and five received six NF sessions. Significant pre- to post-session reductions in pain intensity was reported by $93 \%$ of the participants at either session one $(n=9)$, with an average mean reduction of 2.1 points $(\mathrm{SD}=1.54$; on a $0-$ 10 NRS scale) or session three $(n=8)$, with an average reduction of 1.13 points $(S D=0.35$; it was not clear based on the data presented by the investigators how many of these participants reported significant pain reductions in both sessions). With respect to brain activity changes, there was a change toward normality in the current source density of all targeted brain areas but one (i.e., Brodmann's area 32). Interestingly, regression analysis found that changes in the current source density in Brodmann's area 24 accounted for $\sim 92 \%$ of pain variance, and current source density in Brodmann's area 33 accounted for the rest. Specifically, lower levels of current source density in Brodmann's area 24 and higher levels of current source density in Brodmann's area 33 were significant predictors of pain intensity.

Another case series (Koberda et al., 2013) reported the use of both 19-channel Surface Z-score and 19-channel LORETA Zscore NF to decrease pain in four patients with different pain problems. The first patient had neuropathic pain and received 65 sessions. At the initial assessment, his qEEG showed an excess of $\beta$ activity at temporal locations whereas LORETA imaging showed an excess in $\theta$ and $\beta$ activity at the left insular cortex. The second patient had chronic pain associated with depression and received 25 sessions. Her initial qEEG showed an excess of $\delta$ and $\beta$ power in frontal and central areas, and the LORETA imaging showed "dysregulation" in the anterior cingulate cortex. The third patient had both postherpetic neuropathy and sensory motor polyneuropathy, and received 45 sessions. His qEEG showed an excess of $\delta$ power in frontal areas, and the LORETA imaging showed "dysregulation" in the left insular cortex. The fourth and final patient had trigeminal neuralgia and received 10 sessions of NF. Her qEEG showed an excess of $\delta$ and $\theta$ power in fronto-temporal areas and an excess of $\beta$ in frontal areas, whereas the LORETA imaging showed "dysregulation" in the left insular cortex. The investigators did not specify the number of sessions that each patient received of each treatment approach (i.e., surface Z-score or LORETA Z-score NF). Compared with the pre-treatment pain levels, all the patients reported substantial reductions in pain intensity, ranging from $50 \%$ reduction to complete remission. With respect to brain activity changes, and whether assessed with qEEG or LORETA, all patients evidenced changes in the direction of more normal brain activity patterns over the course of treatment.

\section{ERP-Based NF}

Two studies used ERP-based NF to modulate pain: one was a clinical study whereas the other was an experimental study with laboratory induced pain.

\section{Clinical Pain Study}

The first study (Siniatchkin et al., 2000) was a controlled trial that examined the efficacy of Slow Cortical Potentials NF in a small sample $(n=10)$ of children with migraine without aura. Participants in this study were compared with two control groups: a wait-list control group of children with migraines ( $n$ $=10)$ and a control group of healthy children who also received the NF treatment $(n=10)$. This latter control group was used to compare the ability to self-regulate slow cortical potentials in children with migraine compared to healthy children. The NF protocol was conducted with brain activity measures from the central region of the scalp ( $\mathrm{Cz}$ in the international 10-20 system) and consisted in two different tasks that were trained during the same session: each task was to either increase or decrease the amplitude of the SCPs. Additionally, EEG was recorded at frontal 
and central sites ( $\mathrm{Fz}$ and $\mathrm{Cz}$ in the international 10-20 system). Children in the treatment group and in the healthy control group were able to control the amplitude of their SCPs after the 10 sessions. However, the group of children with migraine was only able to decrease cortical negativity (i.e., decrease the amplitude of their SCPs). After 10 sessions, the treatment group showed significant reductions in the number of days with migraine per month; effects that were not found in the wait-list control group. There was no association between the extent of decrease in the amplitude of the SCPs with NF and the reduction in the number of days with migraine.

\section{Laboratory Induced Pain Study}

The second study aimed to test whether it was possible to modify pain intensity via increasing the ability to alter the N150-P260 complex evoked by aversive stimulation (Miltner et al., 1988). In this study, 10 otherwise healthy male individuals underwent a single 120-min experimental session. First, the individual's pain threshold and the amount of noxious stimulation required for the participant to experience a pain intensity at $20 \%$ above his or her pain threshold were measured. Then, the baseline ERPs and subjective pain intensity in response to the simulation with an intensity of $20 \%$ above the threshold were measured. The last part of the session was devoted to the NF training in the form of two different tasks when presented with the same noxious stimulation used at baseline (i.e., $20 \%$ above threshold): one in which the subjects were reinforced for increasing the size of the N150-P260 complex and one in which they were reinforced for decreasing the size of this complex. Both tasks were randomly presented during the session. EEG was recorded at central areas of the scalp (i.e., $\mathrm{C} z$ according the international $10-20$ system), where the $\mathrm{NF}$ intervention was conducted. With respect to brain activity, the subjects were able to learn to alter the size of the N150-P260 complex consistent with the training. Also, pain intensity reports were different in the up-training and down-training conditions; when presented with identical noxious stimuli, those in the up-training condition reported slightly higher pain intensity reports than those in the down-training condition. Despite the differences in pain intensity reports between both conditions, however, the decrease after the whole session in pain intensity ratings was not statistically significant.

\section{Evidence Regarding the Effects of fMRI-Based NF}

To date, five studies have evaluated the efficacy of rt-fMRI NF to modulate pain: two were clinical studies whereas the other three were experimental studies with laboratory induced pain.

\section{Clinical Pain Studies}

DeCharms and colleagues tested whether it was possible for individuals to learn to control brain activation in the rostral anterior cingulate cortex ( $\mathrm{rACC}$ ) in a single session of rt-fMRI NF (DeCharms et al., 2005). This study used seven groups. Of these, two were experimental groups that received the rt-fMRI NF and five were control groups. The first experimental group, which was comprised of eight healthy individuals, was compared to four healthy control groups (three of them had eight individuals and one had four individuals) that underwent similar procedures but without valid feedback from rACC (i.e., training using sham rt-fMRI data belonging to another subject recorded session, or training using rt-fMRI data from a brain area other than the rACC). These four control groups were used to determine if the effects of the rt-fMRI NF were due to the ability to modulate the activation in the rACC rather than due to non-specific (i.e., placebo) effects. The second experimental group, which was comprised of eight patients with chronic pain, was compared to a control group of four patients with chronic pain that were trained with autonomic biofeedback. The rt-fMRI NF protocol consisted of training runs (i.e., a specific training period within a training session) in which participants were asked to both increase and decrease BOLD activity in the region of interest within the rACC, hypothesized to be an important area underlying the experience of pain. Each training run lasted $13 \mathrm{~min}$ and was comprised by five 60-second increase cycles and five 60-s decrease cycles. A thermal noxious stimulus was presented for $30 \mathrm{~s}$ to the healthy participants only in each cycle. All the healthy subjects went through a localizer scan, three training runs and a posttest scan, whereas, patients with chronic pain also had the localizer and posttest scan but could choose the number of training runs they were willing to do. Thus, four patients had three training runs, two patients had two training runs and two patients had one training run. After each training run, all study participants were asked to report pain intensity.

The experimental healthy group learned to modulate the BOLD activity in the rACC, whereas the control groups did not. The experimental healthy group learned to both increase and decrease BOLD activity in the rACC, affecting pain perception differently. That is, noxious stimuli presented when subjects were trying to increase BOLD activity in the rACC were rated as significantly more painful than when subjects were trying to do the opposite; that is, to decrease BOLD activity in the rACC activation. The control over pain intensity achieved by the healthy experimental group (who trained with valid feedback from rACC) was significantly larger than for any of the four healthy control groups (who underwent similar training but without valid feedback from rACC). With respect to the experimental group of patients with chronic pain, they reported a $44 \%$ pre- to post-session decrease in pain intensity. There was a strong association between the level of control over the BOLD activity in the rACC achieved by the patients with chronic pain after rt-fMRI NF and the change in pain ratings $(r=0.9, p<$ 0.01 ). Also, the pain intensity reductions in this group were three times greater than those reported by participants who received the autonomic biofeedback intervention.

A more recent study evaluated the effects of a single session of rt-fMRI NF to teach voluntary control over activation in the rACC (Guan et al., 2015). The participants in this study had postherpetic neuralgia, and were randomly allocated to either an experimental group, which received real information from the rACC, or to a control group, which received sham information from a different brain region (i.e., the posterior cingulate cortex). In this experiment, both the experimental $(n=8)$ and the control $(n=6)$ groups were reinforced at different times for increasing and decreasing activation in the respective regions of interest. 
The experimental group was able to both up- and down-regulate BOLD activity in the rACC significantly better than the control group, suggesting that rACC activity may be more amendable to control than activity in the posterior cingulate cortex. Moreover, the experimental group achieved significantly greater changes in pain intensity compared to the control group. In the upregulation condition, pain intensity ratings increased 1.8 and 0.1 (on a $0-10$ scale) for the experimental and control groups, respectively. In the down-regulation condition, pain intensity ratings decreased 1.5 for the experimental group and 0.5 in the control group. However, the associations between changes in BOLD activity and changes in pain intensity for either the upand down-regulation conditions were not statistically significant.

\section{Laboratory Induced Pain Studies}

Emmert et al. (2014) assessed the use of a single session of rtfMRI NF in healthy individuals targeting two different regions hypothesized to be associated with the processing of pain information: the ACC and the left anterior insular cortex (IAIC). Both groups were first asked to participate in a localizer task with noxious heat stimulation to establish the specific pain-sensitive target region in the AIC or ACC for each participant. Next the NF training was conducted, during which participants received feedback to decrease the BOLD activity during pain stimulation in the brain area identified during the localizer task for that participant. Over half of the participants in each group were able to successfully decrease BOLD activity in either the ACC or lAIC. Both the lAIC $(n=14)$ and ACC $(n=14)$ groups significantly reduced pain ratings in the feedback task compared to the localizer task. Moreover, there was no significant difference in the reduction of pain intensity between the IAIC and the ACC groups, nor there was a significant difference in pain ratings between those who successfully decreased BOLD activity and those who did not.

The final two studies were conducted by a single research team and used similar procedures. Both studies included 10 healthy individuals. The investigators conducted an anatomical scan, a baseline run, and 24 training runs over four consecutive days. Each of the training runs was comprised of six regulation phases (where the individuals received electrical noxious stimulation along with rt-fMRI NF training) and six non-regulation phases (where participants engaged in mental arithmetic tasks).

The first study (Rance et al., 2014a) aimed to evaluate the effect of separately increasing and decreasing the BOLD activity in the rACC and left posterior insula (pInsL) on pain intensity. The study had four conditions: increase BOLD activity in rACC, decrease BOLD activity in $\mathrm{rACC}$, increase BOLD activity in $\mathrm{PInsL}$ and decrease BOLD activity in pInsL. Three of the conditions (all except the condition that aimed to increase activity in the rACC) resulted in brain activity changes in the intended directions. However, none of the four conditions resulted in significant changes in pain intensity ratings.

In the second study, the investigators (Rance et al., 2014b) aimed to assess the effect of disrupting a part of the pain processing network by training participants to increase the difference in activation levels between two brain regions: the rACC and pInsL. Participants received rt-fMRI NF training with the goal to achieve two states: one where the activation of the rACC was higher than the activation of the pInsL, and a second state where the activation of the pInsL was higher than the activation of the rACC. Although the participants were successful in achieving the training goals, pain intensity ratings did not change significantly from the first to the last training trial.

\section{Risk of Bias}

The details of the quality ratings according to the Quality Assessment Tool for Quantitative Studies are presented in Table 5. It is noteworthy that none of the studies received a "strong" rating for the components of selection bias and confounders. All but two of the studies were rated as either "strong" or "moderate" in study design. Most $(k=17,71 \%)$ of the studies were rated as weak in the blinding component, and just one study (4\%) was double-blinded (i.e., it received a "strong" rating for the blinding component). Seventeen studies (71\%) used reliable and valid measures to assess outcomes and 14 studies (58\%) were rated as "strong" with respect to withdrawals and drop-outs.

\section{DISCUSSION}

In this review we summarized the available evidence regarding the efficacy of NF as a treatment for pain and its effects on painrelated brain activity. To our knowledge, this is the first review to systematically summarize the use and effects of NF as an intervention for any type of pain and pain-related outcomes.

\section{NF Protocols Studied}

The first aim of this review was to describe the different types of NF and NF protocols that have been used in pain research and how NF has been used for pain management. Most of the 24 studies that were included and reviewed were EEG-based and focused mostly on adults with migraines or headache and other chronic pain conditions, such as fibromyalgia or cancer-related pain. Of the five types of NF that we identified and described, brain oscillation power-based NF was evaluated the most often.

Within each type of NF studied, the specific protocols used varied from study to study. Although some NF protocols shared some features, no two studies used the exact same protocol. To the extent that several high-quality clinical trials are needed to draw conclusions regarding the efficacy of a clinical intervention, the lack of consistency in the NF protocols studied means that the field has not advanced enough to be able to draw strong conclusions regarding the efficacy of specific NF protocols for pain management.

\section{Efficacy of NF}

The second aim of the study was to summarize the evidence regarding NF and different NF protocols for modulating pain and improving pain-related outcomes. As a whole, and given the generally positive results in the studies reviewed, the findings indicate that NF procedures have the potential for reducing pain and improving other outcomes in individuals with chronic pain. Most of the studies reviewed found significant pre- to post-treatment improvements in pain intensity and/or pain 
TABLE 5 | Quality ratings for the included studies.

\begin{tabular}{|c|c|c|c|c|c|c|}
\hline Authors, year & Selection bias & Study design & Confounders & Blinding & $\begin{array}{l}\text { Data collection } \\
\text { methods }\end{array}$ & $\begin{array}{l}\text { Withdrawals and } \\
\text { drop-outs }\end{array}$ \\
\hline Caro and Winter (2011) & 3 & 2 & $\mathrm{~N} / \mathrm{A}$ & 3 & 2 & 3 \\
\hline Emmert et al. (2014) & 3 & 1 & 2 & 3 & 1 & 1 \\
\hline Farahani et al. (2014) & 2 & 1 & 2 & 3 & 1 & 1 \\
\hline Jacobs and Jensen (2015) & 3 & 3 & $\mathrm{~N} / \mathrm{A}$ & $\mathrm{N} / \mathrm{A}$ & 3 & N/A \\
\hline Jensen et al. (2018) & 2 & 1 & 2 & 3 & 1 & 1 \\
\hline Jensen et al. (2013a) & 3 & 2 & $\mathrm{~N} / \mathrm{A}$ & 3 & 1 & 1 \\
\hline Jensen et al. (2016) & 3 & 1 & 3 & 3 & 1 & 1 \\
\hline Jensen et al. (2007) & 3 & 2 & $\mathrm{~N} / \mathrm{A}$ & 3 & 2 & 1 \\
\hline Miltner et al. (1988) & 2 & 2 & $\mathrm{~N} / \mathrm{A}$ & 3 & 1 & 1 \\
\hline Prinsloo et al. (2019) & 3 & 2 & $\mathrm{~N} / \mathrm{A}$ & 3 & 1 & 2 \\
\hline Prinsloo et al. (2018) & 2 & 1 & 2 & 3 & 1 & 2 \\
\hline Rance et al. (2014a) & 3 & 2 & 2 & 3 & 1 & 1 \\
\hline Rance et al. (2014b) & 3 & 2 & 2 & 3 & 1 & 1 \\
\hline Siniatchkin et al. (2000) & 3 & 1 & 2 & 2 & 1 & 3 \\
\hline Stokes and Lappin (2010) & 3 & 2 & $\mathrm{~N} / \mathrm{A}$ & 3 & 3 & 1 \\
\hline Vučković et al. (2019) & 3 & 2 & $\mathrm{~N} / \mathrm{A}$ & $\mathrm{N} / \mathrm{A}$ & 1 & 2 \\
\hline Walker (2011) & 3 & 2 & $\mathrm{~N} / \mathrm{A}$ & 3 & 3 & 3 \\
\hline
\end{tabular}

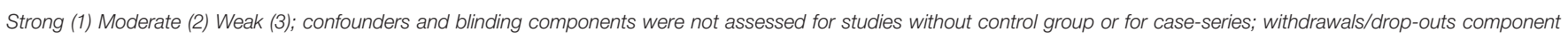
was not assessed for case-series.

frequency, with some of these improvements being maintained at follow-up (when follow-up was evaluated). Also, most of these studies found significant improvements in other pain-related variables such as fatigue, sleep problems/sleep quality, anxiety, depression, and pain-related interference. NF was also found to enhance the effects of hypnosis for chronic pain management and to reduce the perception of experimentally induced pain in healthy individuals.

However, and as alluded to previously, the high level of protocol heterogeneity and the heterogeneity in the characteristics of the samples studied do not allow us to draw conclusions regarding the efficacy of NF types and specific NF protocols. That said, there were some patterns in the study findings that could be used for hypothesis generation for future research. For example, the brain oscillation power-based NF protocols often included some combination of protocols that increased $\alpha$ and SMR power, and decreased $\beta$ and $\theta$ power. Another commonly used protocol was to tailor NF treatment to each individual participant based on their baseline qEEG assessment, with a goal of bringing their qEEG in line with normative values. Most of these studies found positive results for the NF interventions evaluated. These preliminary findings raise the possibility that the beneficial effects of NF may be due to (1) NF's effects on the power of one or more specific bandwidths or (2) NF's ability to normalize bandwidth power across the spectrum. The need for more research in this area is discussed in more detail in the next section.

\section{The Mechanisms That Underlie NF Treatment}

As mentioned previously, in the context of pain treatment, NF aims to change brain activity that is thought to underlie or influence the experience of pain (Ibric and Dragomirescu, 2009). The third aim of the current review was to determine the level of evidence regarding the effect of NF training on targeted brain activity, and the associations of these with improvements in pain outcomes. Unfortunately, almost a third of the studies included in the review did not assess changes in brain activity. Moreover, those studies that did include some measure of brain activity studied different domains of brain activity. For example, some studies evaluated whether there were any brain activity changes during a training session or training sessions, whereas others evaluated pre- to post-treatment changes in resting state activity.

An important question that remains unanswered is how exactly NF works to reduce pain intensity. Although a given NF protocol usually seeks to alter brain activity in a specific way, as noted previously, researchers do not always include a manipulation check to determine if (and how much) brain 
activity changed as intended. Moreover, when such checks are performed, the findings indicate that even if the treatment protocol was effective for reducing pain, it was not always effective for changing brain activity as originally intended (Rogala et al., 2016; Omejc et al., 2018). In fact, in many studies the changes in pain intensity or frequency occurred irrespective of whether the targeted brain activity modulation occurred (Siniatchkin et al., 2000; Jensen et al., 2013b; Emmert et al., 2014; Rance et al., 2014b; Guan et al., 2015). It remains possible that much, if not all, of the beneficial effects of many NF protocols are due to their non-specific effects (e.g., effects on patient outcome expectancies, or effects on mechanisms that may be shared across different NF protocols, such as perceived self-efficacy), as argued by Thibault and colleagues (Thibault et al., 2017).

Mechanism research is needed to address the specificity of NF treatment. For example, participants in a clinical trial could be randomly assigned to one of three conditions: (1) a condition targeting an increase in a specific bandwidth power; (2) a condition that seeks to normalize power across all bandwidths, based on the results of a pre-treatment qEEG assessment; or (3) a control condition (e.g., sham EEG or a protocol that seeks to decrease $\alpha$ power). qEEG could be assessed before and after treatment sessions, during one or more of the treatment sessions and before and after treatment. A finding that participants in one or the other of the experimental conditions report larger improvements in pain than participants in the control condition could be used as evidence for the potential specificity of NF's effects. Even more importantly, additional evidence for treatment specificity could come from mediation analyses to determine the extent to which pre- to post-treatment changes in the power of one or more bandwidths or the ability of an individual to alter bandwidth power during a treatment session mediates the beneficial effects of the experimental conditions relative to the control condition. One example of such a mediation analyses performed in the context of an exploratory study was recently published by Prinsloo and colleagues (Prinsloo et al., 2019). They found that changes in the current source density in two of the targeted Brodmann's areas (the ventral and the dorsal parts of the ACC) completely mediated the reduction in pain intensity achieved with LORETA Z-score NF in patients with cancer undergoing radiation therapy (Prinsloo et al., 2019). This finding provides preliminary support for the specific effects of the NF protocol examined, and points to the activity in the ACC as a potential mechanism for NF interventions that should be examined in future NF studies.

\section{Study Quality}

The fourth and final aim of this review was to assess the quality of the studies included. The results of the quality analysis were mixed. On one hand, all but two of the studies were rated as either "strong" or "moderate" with respect to study design. It is important to note that "strong" study quality is a rating assigned to RCTs or controlled clinical trials, whereas the "moderate" study quality is a rating assigned to studies with a pre-post design, with either just one cohort or with a control group, or casecontrol studies. It is also important to note that only three studies included in the review were RCTs. Also, more than half of the studies used reliable and valid measures to assess outcomes and were rated as "strong" with respect to withdrawals and drop-outs. On the other hand, most of the studies reviewed had relatively small sample sizes and were pilot studies.

In order to maximize the quality of future clinical trials in this area, so that future systematic reviews could draw more definitive conclusions regarding the efficacy of NF for pain management, researchers should ponder several important study quality considerations. First, future studies would benefit from more robust experimental designs and a more homogeneous and clearer reporting of the protocols and outcomes of the study. In order to achieve this, consensus recommendations on the reporting and experimental design of clinical and cognitivebehavioral neurofeedback studies was recently published (Ros et al., 2020). These recommendations could serve as a framework for the design, conduct, and reporting NF studies.

Second, it is necessary for future studies to estimate sample sizes a priori, ensuring they are adequate for the planned statistical analyses. To be on the safe side, given that pilot studies often over-estimate effect sizes, researchers should seriously consider exceeding the estimated sample size. This would also help to ensure that the samples are large enough to allow for drop-outs or potential missing data.

Third, less than a third of the studies included in this review conducted follow-up assessments. This issue does not allow us to determine if the gains in the studies that did find a reduction in pain intensity or pain frequency at posttreatment were maintained for any period of time after treatment. For NF to be recommended, future studies should consistently report NF effects after treatment and in successive follow-ups.

Fourth, with rare exceptions, most of the studies included in this review used adult samples. As chronic pain is also highly prevalent in children and adolescents (Huguet and Miró, 2008), it would be essential to include these segments of the population in future studies in order to ascertain NF's efficacy in youths.

Fifth, most of the studies did not report several confounding factors, such as medication intake and duration of the problem. Thus, we were not able to take into account the moderating effect of these factors in the effects of NF on pain.

Finally, detailed information about the studies was often lacking. For example, the interventions were often not described in enough detail to allow for replicability. Moreover, detail was sometimes lacking in the description of the outcomes (e.g., reporting decrease or increase percentages only, rather than specific baseline and post-treatment numbers in addition to percentages). In addition to the fact that some studies did not report brain activity information, those which did reported a large variety of variables; it appears that there are no standards yet for reporting basic brain activity information. All of these limitations prevented us from encapsulating and drawing firm conclusions on the efficacy of NF to modulate pain.

\section{Future Studies}

In order to improve on the quality and utility of clinical trials, future studies should seek to identify the protocols that work best for each pain condition, the number of sessions needed to see improvements, the brain mechanisms involved, and how long 
the improvements are maintained after treatment (Van Boxtel and Gruzelier, 2014), both in youths and adults. However, it is possible that determining fixed protocols for each condition might not be the best path, and instead, tailored protocols for each individual might be better to improve the efficacy of NF studies (Rogala et al., 2016). Also, and in light of some researchers questioning the benefits of NF over and above placebo (Thibault et al., 2017), future studies should consider including a placebo condition. In our review, only three studies controlled for possible placebo effects; for example, by targeting a brain region or a frequency band assumed to be unrelated to pain processing.

\section{Limitations}

There are a number of limitations to this review that should be acknowledged. Because we sought to summarize the evidence of NF used to modulate any type of pain, inclusion criteria were broad. As a result, the included studies were highly heterogeneous, so that we were not able to conduct a metaanalysis. Another limitation is that our data search was limited to studies published in either English or Spanish. It is possible that we overlooked some additional relevant contributions to the field published in journals written in additional languages.

\section{Summary and Conclusions}

This review provides positive preliminary evidence of NF as a potential treatment for chronic pain. However, higher quality studies using similar procedures and outcome measures are still needed to: (1) determine the extent to which promising preliminary studies replicate in order to determine if NF is

\section{REFERENCES}

Apkarian, A. V., Hashmi, J. A., and Baliki, M. N. (2011). Pain and the brain: specificity and plasticity of the brain in clinical chronic pain. Pain 152 (Suppl. 3), S49-S64. doi: 10.1016/j.pain.2010.11.010

Arns, M., Conners, C. K., and Kraemer, H. C. (2013). A decade of EEG theta/beta ratio research in ADHD: a meta-analysis. J. Atten. Disord. 17, 374-383. doi: $10.1177 / 1087054712460087$

Bair, M. J., Robinson, R. L., Katon, W., and Kroenke, K. (2003). Depression and pain comorbidity. Arch. Intern. Med. 163, 2433-2445. doi: 10.1001/archinte.163.20.2433

Boord, P., Siddall, P. J., Tran, Y., Herbert, D., Middleton, J., and Craig, A. (2008). Electroencephalographic slowing and reduced reactivity in neuropathic pain following spinal cord injury. Spinal Cord. 46, 118-123. doi: 10.1038/sj.sc.3102077

Carmen, J. A. (2004). Passive infrared hemoencephalography: four years and 100 migraines. J. Neurother. 8, 23-51. doi: 10.1300/J184v08n03_03

Caro, X. J., and Winter, E. F. (2011). EEG biofeedback treatment improves certain attention and somatic symptoms in fibromyalgia: a pilot study. Appl. Psychophysiol. Biofeedback 36, 193-200. doi: 10.1007/s10484-011-9159-9

Chapin, H., Bagarinao, E., and Mackey, S. (2012). Real-time fMRI applied to pain management. Neurosci. Lett. 520, 174-181. doi: 10.1016/j.neulet.2012.02.076

Davis, K. D., and Moayedi, M. (2013). Central mechanisms of pain revealed through functional and structural MRI. J. Neuroimmune Pharmacol. 8, 518-534. doi: 10.1007/s11481-012-9386-8

De Ruddere, L., and Craig, K. D. (2016). Understanding stigma and chronic pain. Pain 157, 1607-1610. doi: 10.1097/j.pain.0000000000000512

DeCharms, R. C., Maeda, F., Glover, G. H., Ludlow, D., Pauly, J. M., Soneji, D., et al. (2005). Control over brain activation and pain learned by using effective, (2) elucidate the mechanisms of NF treatments on pain, and (3) determine the best NF approach(es) for individuals with chronic pain.

\section{AUTHOR CONTRIBUTIONS}

RR conceptualized the study, created the research strategy, selected the studies, and extracted the data. RV contributed to the previous tasks and independently selected the studies. JM and MJ supervised all the steps of the process. All authors participated in the interpretation of the data, drafted the work and revised it critically for important intellectual content, and provided approval for publication of the content.

\section{FUNDING}

This work was partly funded by grants from Spanish Ministry of Economy and Competitiveness (MINECO; PSI201570966-P; RTI2018-09870-B-I00; RED2018-102546), the European Regional Development Fund (ERDF) and Obra Social de Caixabank. JM's work is supported by Fundación Grünenthal. RR is supported by a doctoral grant from MINECO. This research was supported in part by Grant No. P01 HD33988 from the National Institutes of Health, National Institute of Child Health and Human Development (National Center for Medical Rehabilitation Research) awarded to $\mathrm{MJ}$.

real-time functional MRI. Proc. Natl. Acad. Sci. U.S.A. 102, 18626-18631. doi: 10.1073/pnas.0505210102

Emmert, K., Breimhorst, M., Bauermann, T., Birklein, F., Van De Ville, D., Haller, S., et al. (2014). Comparison of anterior cingulate vs. insular cortex as targets for real-time fMRI regulation during pain stimulation. Front Behav Neurosci. 8:350. doi: 10.3389/fnbeh.2014.00350

Farahani, D. M., Tavallaie, S. A., Ahmadi, K., and Ashtiani, A. F. (2014). Comparison of neurofeedback and transcutaneous electrical nerve stimulation efficacy on treatment of primary headaches: a randomized controlled clinical trial. Iran Red Crescent Med. J. 16:e17799. doi: 10.5812/ircmj. 17799

Finan, P. H., Goodin, B. R., and Smith, M. T. (2013). The association of sleep and pain: an update and a path forward. J. Pain 14, 1539-1552. doi: 10.1016/j.jpain.2013.08.007

Flor, H. (2014). Psychological pain interventions and neurophysiology: Implications for a mechanism-based approach. Am. Psychol. 69, 188-196. doi: 10.1037/a0035254

Gaskin, D. J., and Richard, P. (2012). The economic costs of pain in the United States. J. Pain 13, 715-724. doi: 10.1016/j.jpain.2012.03.009

Goldberg, D. S., and Mcgee, S. J. (2011). Pain as a global public health priority. BMC Public Health 11:770. doi: 10.1186/1471-2458-11-770

Groenewald, C. B., Essner, B. S., Wright, D., Fesinmeyer, M. D., and Palermo, T. M. (2014). The economic costs of chronic pain among a cohort of treatment-seeking adolescents in the United States. J. Pain 15, 925-933. doi: 10.1016/j.jpain.2014.06.002

Guan, M., Ma, L., Li, L., Yan, B., Zhao, L., Tong, L., et al. (2015). Selfregulation of brain activity in patients with postherpetic neuralgia: a doubleblind randomized study using real-time fMRI neurofeedback. PLoS ONE 10:e0123675. doi: 10.1371/journal.pone.0123675 
Hammond, D. C. (2011). What is neurofeedback: an update. J. Neurother. 15, 305-336. doi: 10.1080/10874208.2011.623090

Hasan, M. A., Fraser, M., Conway, B. A., Allan, D. B., and Vuckovic, A. (2015). The mechanism of neurofeedback training for treatment of central neuropathic pain in paraplegia: a pilot study. BMC Neurol. 15:200. doi: 10.1186/s12883-015-0445-7

Heinrich, H., Gevensleben, H., and Strehl, U. (2007). Annotation : neurofeedback - train your brain to train behaviour. J. Child Psychol. Psychiatry 1, 3-16. doi: 10.1111/j.1469-7610.2006.01665.x

Hoedlmoser, K., Pecherstorfer, T., Gruber, G., Anderer, P., Doppelmayr, M., Klimesch, W., et al. (2008). Instrumental conditioning of human sensorimotor rhythm $(12-15 \mathrm{~Hz})$ and its impact on sleep as well as declarative learning. Sleep 31, 1401-1408. doi: 10.5665/sleep/31.10.1401

Huguet, A., and Miró, J. (2008). The severity of chronic pediatric pain: an epidemiological study. J. Pain 9, 226-236. doi: 10.1016/j.jpain.2007.10.015

Ibric, V. L., and Dragomirescu, L. G. (2009). "Neurofeedback in pain management," In Introduction to Quantitative EEG and Neurofeedback. (New York, NY: Elsevier Inc.), 417-51. doi: 10.1016/B978-0-12-374534-7.00016-2

Institute of Medicine (U.S) Committee on a National Agenda for the Prevention of Disabilities (1991). Disability in America: Toward a National Agenda for Prevention-Committee on a National Agenda for the Prevention of Disabilities, Institute of Medicine. editors A. Pope, A. Tarlov. Washington, DC: National Academy Press.

Jacobs, E. H., and Jensen, M. P. (2015). EEG neurofeedback in the treatment of chronic pain: a case series. NeuroRegulation 2, 86-102. doi: 10.15540/nr.2.2.86

Jensen, M., and Karoly, P. (2001). "Self-report scales and procedures for assessing pain in adults," in Handbook of Pain Assessment, 3 Edn. eds D. Turk, R. Melzack (New York, NY: Guilford Press), 15-34.

Jensen, M. P., Adachi, T., and Hakimian, S. (2015). Brain oscillations, hypnosis, and hypnotizability. Am. J. Clin. Hypn. 57, 230-253. doi: 10.1080/00029157.2014.976786

Jensen, M. P., Battalio, S. L., Chan, J. F., Edwards, K. A., Day, M. A., Sherlin, L. H., et al. (2018). Use of neurofeedback and mindfulness to enhance response to hypnosis treatment in individuals with multiple sclerosis: results from a pilot randomized clinical trial. Int. J. Clin. Exp. Hypn. 66, 231-264. doi: 10.1080/00207144.2018.1460546

Jensen, M. P., Day, M. A., and Miró J. (2014). Neuromodulatory treatments for chronic pain: efficacy and mechanisms. Nat. Rev. Neurol. 10, 167-178. doi: 10.1038/nrneurol.2014.12

Jensen, M. P., Gertz, K. J., Kupper, A. E., Braden A. L., Howe, J. D., Hakimian, S., et al. (2013a). Steps toward developing an EEG biofeedback treatment for chronic pain. Appl. Psychophysiol. Biofeedback 38, 101-108. doi: 10.1007/s10484-013-9214-9

Jensen, M. P., Gianas, A., George, H. R., Sherlin, L. H., Kraft, G. H., Ehde, D. M., et al. (2016). Use of neurofeedback to enhance response to hypnotic analgesia in individuals with multiple sclerosis. Int. J. Clin. Exp. Hypn. 64, 1-23. doi: 10.1080/00207144.2015.1099400

Jensen, M. P., Grierson, C., Tracy-Smith, V., Bacigalupi, S. C. S. C., Othmer, S. S. F., Jensen, M. P., et al. (2007). Neurofeedback treatment for pain associated with complex regional pain syndrome type I. J. Neurother. 11, 45-53. doi: 10.1300/J184v11n01_04

Jensen, M. P., Sherlin, L. H., Askew, R. L., Fregni, F., Witkop, G., Gianas, A., et al. (2013b). Effects of non-pharmacological pain treatments on brain states. Clin. Neurophysiol. 124, 2016-2024. doi: 10.1016/j.clinph.2013.04.009

Jüni, P., Witschi, A., Bloch, R., and Egger, M. (1999). The hazards of scoring the quality of clinical trials for meta-analysis. J. Am. Med. Assoc. 282, 1054-1060. doi: 10.1001/jama.282.11.1054

Kayiran, S., Dursun, E., Dursun, N., Ermutlu, N., and Karamürsel, S. (2010). Neurofeedback intervention in fibromyalgia syndrome; a randomized, controlled, rater blind clinical trial. Appl. Psychophysiol. Biofeedback 35, 293-302. doi: 10.1007/s10484-010-9135-9

King, S., Chambers, C. T., Huguet, A., MacNevin, R. C., McGrath, P. J., Parker, L., et al. (2011). The epidemiology of chronic pain in children and adolescents revisited: a systematic review. Pain 152, 2729-2738. doi: 10.1016/j.pain.2011.07.016

Koberda, J. L., Koberda, P., Bienkiewicz, A. A., Moses, A., and Koberda, L. (2013). Pain management using 19-electrode $\mathrm{Z}$-score LORETA neurofeedback. J. Neurother. 17, 179-190. doi: 10.1080/10874208.2013.813204
Krigbaum, G., and Wigton, N. L. (2014). When discussing neurofeedback, does modality matter? NeuroRegulation 1, 48-60. doi: 10.15540/nr.1.1.48

Luck, S. J. (2014). An Introduction to the Event-Related Potential Technique. Massachusetts: MIT Press, 406.

Marzbani, H., Marateb, H. R., and Mansourian, M. (2016). Methodological note: neurofeedback: a comprehensive review on system design, methodology and clinical applications. Basic Clin Neurosci. 7, 143-158. doi: 10.15412/J.BCN.03070208

Mathew, A., Mishra, H., and Kumaraiah, V. (1987). Alpha feedback in the treatment of tension headache. J Personal Clin Stud. 3, 17-22.

May, A. (2008). Chronic pain may change the structure of the brain. Pain 137, 7-15. doi: 10.1016/j.pain.2008.02.034

Miltner, W., Larbig, W., and Braun, C. (1988). Biofeedback of somatosensory event-related potentials: can individual pain sensations be modified by biofeedback-induced self-control of event-related potentials? Pain 35, 205-213. doi: 10.1016/0304-3959(88)90228-X

Miró, J., Castarlenas, E., de la Vega, R., Roy, R., Sole, E., Tome-Pires, C., et al. (2016). Psychological neuromodulatory treatments for young people with chronic pain. Children 3:41. doi: 10.3390/children3040041

Moher, D. (2015). Preferred reporting items for systematic review and metaanalysis protocols (PRISMA-P) 2015 statement david. Syst. Rev. 207, 1-9. doi: 10.1186/2046-4053-4-1

Nahin, R. L. (2015). Estimates of pain prevalence and severity in adults: United States, 2012. J Pain. 16, 769-80. doi: 10.1016/j.jpain.2015.05.002

Omejc, N., Rojc, B., Battaglini, P. P., and Marusic, U. (2018). Review of the therapeutic neurofeedback method using electroencephalography: EEG neurofeedback. Bosn. J. Basic Med. Sci. 213-20. doi: 10.17305/bjbms.2018.3785

Pascual-Marqui, R. D., Esslen, M., Kochi, K., and Lehmann, D. (2002). Functional imaging with low-resolution brain electromagnetic tomography (LORETA): a review. Methods Find. Exp. Clin. Pharmacol. 24 (Suppl. C), 91-95.

Pascual-Marqui, R. D., Michela, M., and Lehmannb, D. (1994). Low resolution electromagnetic tomography: a new method for localizing electrical activity in the brain. Int. J. Psychophysiol 18, 49-65. doi: 10.1016/0167-8760(84) 90014-X

Pinheiro, E. S. D. S., Queirós, F. C., de Montoya, P., Santos, C. L., Nascimento, M. A., do Ito, C. H., et al. (2016). Electroencephalographic patterns in chronic pain: a systematic review of the literature. PLoS ONE 11:e0149085. doi: 10.1371/journal.pone.0149085

Prinsloo, S., Novy, D., Driver, L., Lyle, R., Ramondetta, L., Eng, C., et al. (2018). The long-term impact of neurofeedback on symptom burden and interference in patients with chronic chemotherapy-induced neuropathy: analysis of a randomized controlled trial. J. Pain Symptom Manage. 55, 1276-1285. doi: 10.1016/j.jpainsymman.2018.01.010

Prinsloo, S., Rosenthal, D. I., Lyle, R., Garcia, S. M., Gabel-Zepeda, S., Cannon, R., et al. (2019). Exploratory study of low resolution electromagnetic tomography (LORETA) real-time Z-score feedback in the treatment of pain in patients with head and neck cancer. Brain Topogr. 32, 283-285. doi: $10.1007 /$ s10548-018-0686-z

Rance, M., Ruttorf, M., Nees, F., Schad, L. R., and Flor, H. (2014a). Real time fMRI feedback of the anterior cingulate and posterior insular cortex in the processing of pain. Hum. Brain Mapp. 35, 5784-5798. doi: 10.1002/hbm.22585

Rance, M., Ruttorf, M., Nees, F., Schad, L. R., and Flor, H. (2014b). Neurofeedback of the difference in activation of the anterior cingulate cortex and posterior insular cortex: two functionally connected areas in the processing of pain. Front. Behav. Neurosci. 8, 1-12. doi: 10.3389/fnbeh.2014.00357

Rogala, J., Jurewicz, K., Paluch, K., Kublik, E., Cetnarski, R., and Wróbel, A. (2016). The do's and don'ts of neurofeedback training: a review of the controlled studies using healthy adults. Front. Hum. Neurosci. 10:301. doi: $10.3389 /$ fnhum. 2016.00301

Ros, T., Enriquez-Geppert, S., Zotev, V., Young, K. D., Wood, G., WhitfieldGabrieli, S., et al. (2020). Consensus on the reporting and experimental design of clinical and cognitive-behavioural neurofeedback studies (CRED-nf checklist). Brain 143, 1674-1685. doi: 10.1093/brain/awaa009

Rowbotham, M. C. (2001). What is a 'clinically meaningful' reduction in pain? Pain 94, 131-132. doi: 10.1016/S0304-3959(01)00371-2

Schopflocher, D., Taenzer, P., and Jovey, R. (2011). The prevalence of chronic pain in Canada. Pain Res. Manag. 16, 445-450. doi: 10.1155/2011/8 76306 
Sherlin, L. H., Arns, M., Lubar, J., Heinrich, H., Kerson, C., Strehl, U., et al. (2011). Neurofeedback and basic learning theory: implications for research and practice. J. Neurother. 15, 292-304. doi: 10.1080/10874208.2011.623089

Siniatchkin, M., Hierundar, A., Kropp, P., Kuhnert, R., Gerber, W.-D., and Stephani, U. (2000). Self-regulation of slow cortical potentials in children with migraine: an exploratory study. Appl. Psychophysiol. Biofeedback 25, 13-32. doi: 10.1023/A:1009581321624

Stokes, D. A., and Lappin, M. S. (2010). Neurofeedback and biofeedback with 37 migraineurs: a clinical outcome study. Behav. Brain Funct. 6:9. doi: 10.1186/1744-9081-6-9

Strehl, U. (2009). Slow cortical potentials neurofeedback. J. Neurother. 13, 117-126. doi: $10.1080 / 10874200902885936$

Strehl, U., Leins, U., Goth, G., Klinger, C., Hinterberger, T., and Birbaumer, N. (2006). Self-regulation of slow cortical potentials: a new treatment for children with attention-deficit/hyperactivity disorder. Pediatrics 118, e1530e1540. doi: 10.1542/peds.2005-2478

Sulzer, J., Haller, S., Scharnowski, F., Weiskopf, N., Birbaumer, N., Blefari, M. L., et al. (2013). Real-time fMRI neurofeedback: progress and challenges. Neuroimage 76, 386-399. doi: 10.1016/j.neuroimage.2013.03.033

Sur, S., and Sinha, V. (2009). Event-related potential: an overview. Ind. Psychiatry J. 18, 70-73. doi: 10.4103/0972-6748.57865

Thibault, R. T., Lifshitz, M., and Raz, A. (2017). The climate of neurofeedback: scientific rigour and the perils of ideology. Brain 141:e11. doi: 10.1093/brain/awx330

Thibault, R. T., MacPherson, A., Lifshitz, M., Roth, R. R., and Raz, A. (2018). Neurofeedback with fMRI: a critical systematic review. Neuroimage 172, 786-807. doi: 10.1016/j.neuroimage.2017.12.071

Thomas, B. H., Ciliska, D., Dobbins, M., and Micucci, S. (2004). A process for systematically reviewing the literature: providing the research evidence for public health nursing interventions. Worldviews Evid. Based Nurs. 1, 176-184. doi: $10.1111 / j .1524-475 X .2004 .04006 . x$

Van Boxtel, G. J. M., and Gruzelier, J. H. (2014). Neurofeedback: introduction to the special issue. Biol. Psychol. 95, 1-3. doi: 10.1016/j.biopsycho.2013.11.011 van Hecke, O., Torrance, N., and Smith, B. H. (2013). Chronic pain epidemiology and its clinical relevance. Br. J. Anaesth. 111, 13-18. doi: 10.1093/bja/ aet123

Vučković, A., Altaleb, M. K. H., Fraser, M., McGeady, C., and Purcell, M. (2019). EEG correlates of self-managed neurofeedback treatment of central neuropathic pain in chronic spinal cord injury. Front. Neurosci. 13:762. doi: $10.3389 /$ fnins.2019.00762

Walker, J. E. (2011). QEEG-guided neurofeedback for recurrent migraine headaches. Clin. EEG Neurosci. 42, 59-61. doi: 10.1177/155005941104 200112

Wigton, N. L. (2013). Clinical perspectives of 19-channel Z-score neurofeedback: benefits and limitations. J. Neurother. 17, 259-264. doi: 10.1080/10874208.2013.847142

Williams, A., Eccleston, C., and Morley, S. (2012). Psychological therapies for the management of chronic pain (excluding headache) in adults (Review). Cochrane Database Syst. Rev. 11:CD007407. doi: 10.1002/14651858.CD007407.pub3

Wyckoff, S., and Strehl, U. (2011). "Neurofeedback and neuromodulation techniques and applications," in Neurofeedback and Neuromodulation Techniques and Applications. eds R. Coben., J. R. Evans (London: Academic Press), 205-22. doi: 10.1016/B978-0-12-382235-2.00008-1

Conflict of Interest: The authors declare that the research was conducted in the absence of any commercial or financial relationships that could be construed as a potential conflict of interest.

Copyright (c) 2020 Roy, de la Vega, Jensen and Miró. This is an open-access article distributed under the terms of the Creative Commons Attribution License (CC BY). The use, distribution or reproduction in other forums is permitted, provided the original author(s) and the copyright owner(s) are credited and that the original publication in this journal is cited, in accordance with accepted academic practice. No use, distribution or reproduction is permitted which does not comply with these terms. 


\section{OPEN ACCESS}

Edited by:

Trevor Thompson,

University of Greenwich,

United Kingdom

Reviewed by:

Lu Zhang,

Georgia Institute of Technology,

United States

Quoc Vuong,

Newcastle University, United Kingdom

${ }^{*}$ Correspondence:

Laura J. Arendsen

laura.arendsen@uni-tuebingen.de

Specialty section:

This article was submitted to

Perception Science,

a section of the journal

Frontiers in Neuroscience

Received: 01 May 2020

Accepted: 15 July 2020

Published: 20 August 2020

Citation:

Arendsen LJ, Henshaw J, Brown CA, Sivan M, Taylor JR, Trujillo-Barreto NJ, Casson AJ and Jones AKP (2020) Entraining Alpha

Activity Using Visual Stimulation

in Patients With Chronic

Musculoskeletal Pain: A Feasibility

Study. Front. Neurosci. 14:828.

doi: 10.3389/fnins.2020.00828

\section{Entraining Alpha Activity Using Visual Stimulation in Patients With Chronic Musculoskeletal Pain: A Feasibility Study}

\author{
Laura J. Arendsen ${ }^{*}$, James Henshaw ${ }^{2}$, Christopher A. Brown²,3, Manoj Sivan ${ }^{2,4}$, \\ Jason R. Taylor ${ }^{2}$, Nelson J. Trujillo-Barreto ${ }^{2}$, Alexander J. Casson ${ }^{5}$ and \\ Anthony K. P. Jones ${ }^{2}$
}

\begin{abstract}
${ }^{1}$ Division of Functional and Restorative Neurosurgery, Eberhart Karls University of Tübingen, Tübingen, Germany, ${ }^{2}$ Human Pain Research Group, Division of Neuroscience and Experimental Psychology, University of Manchester, Manchester, United Kingdom, ${ }^{3}$ Department of Psychological Sciences, University of Liverpool, Liverpool, United Kingdom, ${ }^{4}$ Leeds Institute of Rheumatology and Musculoskeletal Medicine, University of Leeds, Leeds, United Kingdom, ${ }^{5}$ Department of Electrical and Electronic Engineering, University of Manchester, Manchester, United Kingdom
\end{abstract}

Entraining alpha activity with rhythmic visual, auditory, and electrical stimulation can reduce experimentally induced pain. However, evidence for alpha entrainment and pain reduction in patients with chronic pain is limited. This feasibility study investigated whether visual alpha stimulation can increase alpha power in patients with chronic musculoskeletal pain and, secondarily, if chronic pain was reduced following stimulation. In a within-subject design, 20 patients underwent 4-min periods of stimulation at $10 \mathrm{~Hz}$ (alpha), $7 \mathrm{~Hz}$ (high-theta, control), and $1 \mathrm{~Hz}$ (control) in a pseudo-randomized order. Patients underwent stimulation both sitting and standing and verbally rated their pain before and after each stimulation block on a 0-10 numerical rating scale. Global alpha power was significantly higher during $10 \mathrm{~Hz}$ compared to $1 \mathrm{~Hz}$ stimulation when patients were standing $(t=-6.08, p<0.001)$. On a more regional level, a significant increase of alpha power was found for $10 \mathrm{~Hz}$ stimulation in the right-middle and left-posterior region when patients were sitting. With respect to our secondary aim, no significant reduction of pain intensity and unpleasantness was found. However, only the alpha stimulation resulted in a minimal clinically important difference in at least $50 \%$ of participants for pain intensity (50\%) and unpleasantness ratings $(65 \%)$ in the sitting condition. This study provides initial evidence for the potential of visual stimulation as a means to enhance alpha activity in patients with chronic musculoskeletal pain. The brief period of stimulation was insufficient to reduce chronic pain significantly. This study is the first to provide evidence that a brief period of visual stimulation at alpha frequency can significantly increase alpha power in patients with chronic musculoskeletal pain. A further larger study is warranted to investigate optimal dose and individual stimulation parameters to achieve pain relief in these patients.

\footnotetext{
Keywords: alpha activity, chronic pain, electroencephalography, entrainment, visual stimulation
} 


\section{INTRODUCTION}

Chronic pain is a prevalent and debilitating condition that has a wide-reaching impact on physical and mental well-being (Breivik et al., 2006; Van Hecke et al., 2013). Opioids and other medications are commonly prescribed to treat chronic pain (Turk et al., 2011). However, most medications have considerable sideeffects and evidence for their long-term effectiveness is limited (Turk, 2002; Chou et al., 2015). In Europe, 40\% of people with chronic pain report that their pain was inadequately managed (Breivik et al., 2006). Therefore, the development of alternative therapies to relieve pain is warranted.

Chronic musculoskeletal pain, regardless of the specific diagnostic classification, is influenced by multifactorial mechanisms including central sensitization, and is associated with changes in brain structure and function (Kulkarni et al., 2007; Gwilym et al., 2010; Baliki et al., 2014; Brown et al., 2014). These observations justify greater focus on the development of brain-based treatments that have generalizable efficacy across musculoskeletal conditions. A promising target for such treatments comes from evidence that chronic pain is associated with changes in oscillatory neural activity in the brain. Most commonly, an increase of theta power and beta power has been found (Sarnthein et al., 2006; Lim et al., 2016; Ploner et al., 2017), as well as a slowing of the peak alpha frequency that was found in patients with neurogenic pain, abdominal pain, and fibromyalgia (Sarnthein et al., 2006; Boord et al., 2008; De Vries et al., 2013; Lim et al., 2016). Thus, the brain's response to pain provides a promising target for the development of novel pain therapies (Jensen et al., 2008).

A brain signal of particular interest as a therapeutic target is alpha activity, oscillatory neural activity in the frequency range of $8-12 \mathrm{~Hz}$. Alpha activity gates the processing of incoming sensory information via a mechanism of functional inhibition (Jensen and Mazaheri, 2010). Incoming information is gated via the inhibition of brain regions processing irrelevant information (high alpha power), which routes the processing of information to task-relevant regions (low alpha power). This mechanism has been linked to top-down control and attention (Foxe and Snyder, 2011; Klimesch, 2012) and is also involved in pain processing. Alpha activity is decreased during experimental pain stimulation and has been found to encode subjective pain experience (Shao et al., 2012; Michail et al., 2016). Somatosensory alpha activity during pain (Hauck et al., 2015) and the anticipation of pain (May et al., 2012) is modulated by attention, and frontal alpha activity is increased following a placebo-induced expectation of pain relief (Huneke et al., 2013). Importantly, pre-stimulus somatosensory alpha power is inversely related to perceived pain: higher alpha power is associated with lower pain intensity and vice versa, both for experimental pain (Babiloni et al., 2006; Tu et al., 2016) and chronic pain (Camfferman et al., 2017; Ahn et al., 2019). Thus, neurotherapies that increase alpha power may have potential in reducing chronic pain.

Alpha activity can be enhanced through the application of rhythmic stimulation, including visual, auditory, and electrical stimulation (Thut et al., 2011). When presented with an external stimulation at a certain frequency, oscillatory neural activity at this same frequency synchronizes in phase with the external stimulation, a phenomenon often referred to as entrainment. This ultimately leads to an increase of power at the stimulation frequency at the population level (de Graaf et al., 2013; Helfrich et al., 2014; Spaak et al., 2014; Vossen et al., 2015).

Alpha entrainment has been successfully implemented to reduce experimentally induced pain using rhythmic visual (Ecsy et al., 2018), auditory (Ecsy et al., 2017), and transcranial alternating current stimulation (tACS) (Arendsen et al., 2018). However, to date, only one study successfully induced an increase in somatosensory alpha power that was correlated with a reduction in pain intensity in patients with chronic low-back pain (CLBP), using tACS at alpha frequency over somatosensory regions (Ahn et al., 2019). More work is required to understand the efficacy of alpha entrainment and the relationship between entrainment and analgesia across different clinical pain populations and using different stimulation modalities.

In particular, there is a lack of evidence that visual stimulation can entrain alpha activity in patients with varying diagnoses of chronic musculoskeletal pain. The effectiveness of external stimulation to entrain neural oscillations can be influenced by background activity in the brain (Feurra et al., 2013; Ruhnau et al., 2016; Gulbinaite et al., 2017) and an impairment of entrainment has been shown in patients with Parkinson's disease (Te Woerd et al., 2017) and schizophrenia (Lakatos et al., 2013). Cerebral neuroplasticity in chronic pain may change the intrinsic or natural frequencies of affected areas of the brain, meaning that the gain (increased/decreased response) to external stimulation at a specific frequency might be changed, possibly to the extent of making entrainment to a specific frequency impossible. Therefore, evidence of alpha entrainment in chronic pain is critical in order to identify a treatment mechanism, should any analgesic effects follow from treatment, and is essential to justify the general application of alpha entrainment as a treatment.

This feasibility study primarily investigated whether visual alpha stimulation increased global alpha power in patients with chronic musculoskeletal pain. A secondary aim was to explore whether a brief period of alpha stimulation was also associated with reduced clinical pain. We used a withinsubject design to compare alpha stimulation $(10 \mathrm{~Hz})$ to $1 \mathrm{~Hz}$ control stimulation. Furthermore, we also compared $7 \mathrm{~Hz}$ stimulation (high theta) to $1 \mathrm{~Hz}$ stimulation. An important confounding factor to take into account in the use of visual stimulation to entrain alpha activity is that synchronization of alpha oscillations can also be induced indirectly via the engagement of attentional mechanisms by the visual stimulus (Thut et al., 2011; Klimesch, 2012; Brüers and Vanrullen, 2018). Whereas $7 \mathrm{~Hz}$ visual stimulation could lead to indirect synchronization of alpha activity via attentional engagement similar to $10 \mathrm{~Hz}$ stimulation, it should not lead to direct alpha entrainment. Thus, the $7 \mathrm{~Hz}$ stimulation was included to address the confounding factor of attentional mechanisms related to the rhythmic visual stimulation. A correlation between alpha power and chronic pain intensity has been shown for frontal 
and somatosensory regions specifically ( $\mathrm{Tu}$ et al., 2016; Ahn et al., 2019), suggesting that any reduction of chronic pain by alpha entrainment might be related to an increase of frontal and somatosensory alpha activity in particular. Therefore, this study also explored more regional changes in alpha power. Finally, this study also included a manipulation of the level of discomfort the patients experienced during stimulation, as previous studies have shown that background alpha activity (endogenous alpha) has an influence on alpha entrainment. Patients underwent stimulation both in a more uncomfortable condition (standing) and a resting condition (sitting). These different levels of discomfort might affect endogenous alpha activity, because chronic pain levels are negatively correlated with alpha power. In line with the finding that entraining alpha activity with tACS at alpha frequency is effective during a state of low endogenous alpha (eyes open), but to a lesser extent when endogenous alpha is high (eyes closed) (Neuling et al., 2013; Ruhnau et al., 2016), we might expect that visual alpha entrainment is more effective during the condition of stronger discomfort (lower endogenous alpha). However, it should be noted that another study found, in contrast, that higher levels of resting-state alpha power before stimulation were associated with more effective entrainment of alpha oscillations (Mathewson et al., 2012).

\section{MATERIALS AND METHODS}

\section{Participants}

Twenty-two participants were recruited from local pain and musculoskeletal clinics (Salford Royal NHS Trust and North West CATS NHS) and support groups and from the University of Manchester. All participants gave written informed consent to take part in the study and received a reimbursement for their time and travel expenses. The study was approved by the North West-Liverpool East Research Ethics Committee (NHS Health Research Authority; reference number 17/NW/0255).

The inclusion criterion was a diagnosis of chronic musculoskeletal pain, i.e., presence of pain for at least 3 months. To promote generalizability of the findings, we did not focus on any particular diagnostic subgroup. Using opportunity sampling, the study resulted in the recruitment of 14 patients with fibromyalgia; 2 patients with osteoarthritis; 1 patient with fibromyalgia and osteoarthritis; 2 patients with low back pain; 1 patient with stenosis of the lower back; and 2 patients with widespread chronic pain (no specific diagnosis). All participants took part in a telephone interview to complete a screening questionnaire prior to participation to ensure they (1) were aged 18 or older; (2) did not have any difficulty understanding verbal or written English; (3) were not involved in any clinical trials at the time of testing; and (4) were not hospitalized/scheduled to be hospitalized during their participation in the study. To ensure that it was safe to undergo rhythmic visual stimulation, participants were excluded if they (1) were diagnosed with epilepsy or had ever had a convulsion or seizure; (2) had any first-degree relative with epilepsy; or (3) had ever experienced discomfort when exposed to flashing lights.
The datasets of two participants were removed from the final analysis as they were not able to complete the entire study due to high levels of pain and discomfort. These two participants both had a diagnosis of fibromyalgia. This resulted in a total of 20 participants who were included in the statistical analysis (mean age $\pm \mathrm{SD}=43.45 \pm 16.82$ years; 13 females) (Table 1). The employment status of these 20 participants was as follows: 6 were employed full-time; 6 were employed part-time; 4 were unemployed; 3 were retired; and 1 was a student. The annual income bracket for the participants was $6, £ 0-14.999 ; 10$, $£ 15.000-29.999 ; 1, £ 30.000-44.999$; and $2, \geq 60.000$ (data missing for 1 participant).

\section{Visual Stimulation}

All participants underwent visual stimulation at $10 \mathrm{~Hz}$ to entrain alpha activity and at the two control frequencies of 1 and $7 \mathrm{~Hz}$. The visual stimulation was administered using goggles with eight LEDs, four around each eye (bespoke equipment-made by Medical Physics, Salford Royal NHS Foundation Trust; Figure 1). Rhythmic flashes were generated using bespoke software run in Matlab 2017a (The Mathworks, Inc., Natick, MA, United States; Matlab). Participants were asked to close their eyes during the stimulation, and brightness was adjusted for each individual participant to ensure that stimulation was administered at a comfortable brightness. After participants closed their eyes, they were presented with a $30-\mathrm{s} 1-\mathrm{Hz}$ stimulation sample to identify the brightness at which they could clearly perceive the visual stimulation without experiencing any discomfort.

The three different stimulation frequencies were delivered in separate blocks (Figure 2). During each block a 1-min baseline period was followed by $4 \mathrm{~min}$ of rhythmic visual stimulation, while EEG was recorded. During the 1-min baseline, non-rhythmic visual stimulation was applied with a jittered interstimulus interval (ISI) between flashes. During this nonrhythmic stimulation period the signal phase would change by 180 degrees frequently, in a semi-random manner, so as not to cause any long-term entrainment effects. These phase changes would occur either every $1.6 \mathrm{~s}$ ( $50 \%$ of the time), every $1.15 \mathrm{~s}$ ( $25 \%$ of the time) or every $1.9 \mathrm{~s}$ ( $25 \%$ of the time). These nonrhythmic baseline periods before each stimulation period were later used for the EEG analysis to provide a standardized baseline for each stimulation condition. It was decided to include the non-rhythmic visual stimulation during the baseline period to ensure that the baseline and entrainment period were kept as similar as possible, e.g., with respect to luminance, with the only difference being that the stimulation during the baseline period was not rhythmic and would therefore not induce any direct alpha entrainment.

\section{Pain Assessment}

To quantify chronic pain experience, participants were asked to verbally rate their pain intensity and pain unpleasantness using two 11-point numerical rating scales (NRS) ranging from 0 to $10(0=$ not at all intense/unpleasant, $10=$ extremely intense/unpleasant).

To assess the effect of visual alpha stimulation on chronic pain, changes in chronic pain intensity and unpleasantness ratings 
TABLE 1 | Demographic details for the 20 participants included in the statistical analysis of the study.

\begin{tabular}{|c|c|c|c|c|c|c|}
\hline Patient number & Age & Gender & Pain condition & Pain history & HADS anxiety & HADS depression \\
\hline 1 & 25 & $\mathrm{~F}$ & FM & Diagnosed 5 years ago & 13 & 7 \\
\hline 3 & 66 & $M$ & $\mathrm{FM}$ and $\mathrm{AO}$ & Diagnosed $>40$ years ago & 6 & 6 \\
\hline 4 & 51 & $\mathrm{~F}$ & FM & Diagnosed 14 years ago & 12 & 12 \\
\hline 5 & 51 & M & FM & Diagnosed 7 years ago & 18 & 12 \\
\hline 6 & 51 & $\mathrm{M}$ & $\mathrm{FM}$ & Diagnosed 5 years ago & 9 & 7 \\
\hline 7 & 62 & $\mathrm{~F}$ & CLBP & - & 5 & 8 \\
\hline 8 & 50 & $\mathrm{~F}$ & $\mathrm{OA}$ & Diagnosed 9 years ago & 9 & 4 \\
\hline 9 & 26 & $\mathrm{~F}$ & FM & Diagnosed this year (2018), pain present > 3 years & 13 & 13 \\
\hline 10 & 41 & M & FM & Diagnosed this year (2018), symptoms started 10 years ago & 9 & 10 \\
\hline 12 & 19 & $\mathrm{~F}$ & CWP & Pain present for 3 years & 6 & 1 \\
\hline 13 & 56 & $\mathrm{~F}$ & FM & Diagnosed $4-5$ years ago & 8 & 6 \\
\hline 14 & 71 & M & Stenosis & Pain present $>30$ years & 4 & 4 \\
\hline 15 & 25 & $\mathrm{~F}$ & FM & Diagnosed this year (2018), pain present for 2 years & 13 & 14 \\
\hline 16 & 47 & $\mathrm{~F}$ & $\mathrm{OA}$ & - & 12 & 5 \\
\hline 17 & 46 & $\mathrm{~F}$ & FM & Diagnosed this year (2018), pain present for $4-5$ years & 9 & 13 \\
\hline 18 & 70 & M & CLBP & Pain present $>2$ years & 2 & 2 \\
\hline 19 & 23 & $\mathrm{~F}$ & FM & - & 14 & 15 \\
\hline 20 & 22 & $\mathrm{~F}$ & $\mathrm{FM}$ & Diagnosed 2 years ago & 17 & 12 \\
\hline 21 & 35 & $\mathrm{~F}$ & FM & Diagnosed this year (2018) & 15 & 8 \\
\hline 22 & 32 & M & CWP & Pain present $>9$ years & 8 & 8 \\
\hline
\end{tabular}

The pain history column contains a summary of the verbal response of the participants when asked to describe their pain history of as part of the screening procedure.

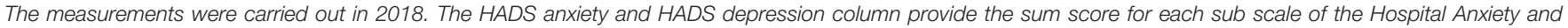

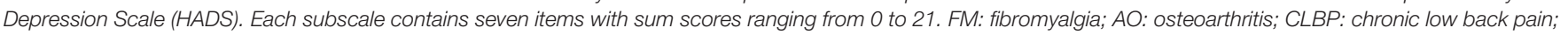
CWP: chronic widespread pain, no specific diagnosis; F: female; M: male.

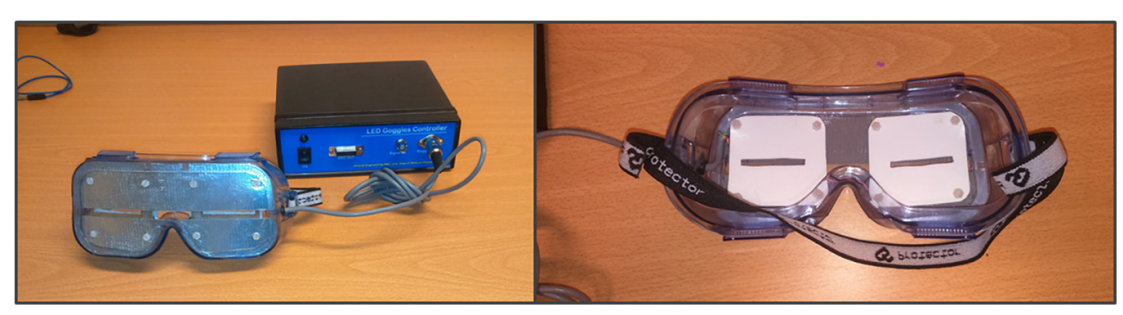

FIGURE 1 | Goggles used for the visual stimulation at the three different stimulation frequencies (bespoke equipment-Medical Physics, Salford Royal NHS Foundation Trust). Eight LEDs were used in total, four around each eye. The goggles were kept in place with an elasticated headband.

were assessed in two settings associated with different levels of discomfort: (1) when participants were seated in a comfortable chair (sitting position); and (2) when participants were standing while holding the back of the chair for support (standing position). Participants were asked to rate their subjective level of discomfort as a result of the position they were in at the start and end of each stimulation block on an NRS ranging from 0 to 10 $(0=$ not at all uncomfortable, $10=$ extremely uncomfortable). On average (mean \pm SD) patients rated their discomfort at the start of each stimulation block as $3.94 \pm 2.14$ when sitting and as $4.78 \pm 2.07$ when standing. At the end of each stimulation block average discomfort was $3.80 \pm 2.34$ when patients were sitting and $5.53 \pm 2.30$ when standing. Stimulation was applied both in a setting of higher and lower discomfort to assess whether the level of ongoing discomfort might influence the effect of the visual stimulation.

Pain ratings were collected before and directly after each visual stimulation block, each block including a 1-min baseline and a 4-min entrainment period (at 1,7 , and $10 \mathrm{~Hz}$ ), both in the sitting and standing condition (Figure 2).

\section{Questionnaires}

Chronic pain and the outcome of chronic pain treatment are influenced by personality factors and pain-related cognition and beliefs (Keefe et al., 1989; Crombez et al., 1999; Granot and Ferber, 2005). Therefore, a series of questionnaires were included in this study to assess if the questionnaire variables were related to the effect of the visual alpha stimulation. This could potentially inform the design of a future, larger trial, e.g., whether to balance participants for these variables between treatment and control arms.

Participants were asked to complete a set of four questionnaires once, during the breaks between stimulation blocks: the Hospital Anxiety and Depression Scale (HADS) (Zigmond and Snaith, 1983); the Pain Self-Efficacy Questionnaire (PSEQ) (Nicholas, 1989); the Brief Pain Inventory (BPI) 


\section{General study procedure}

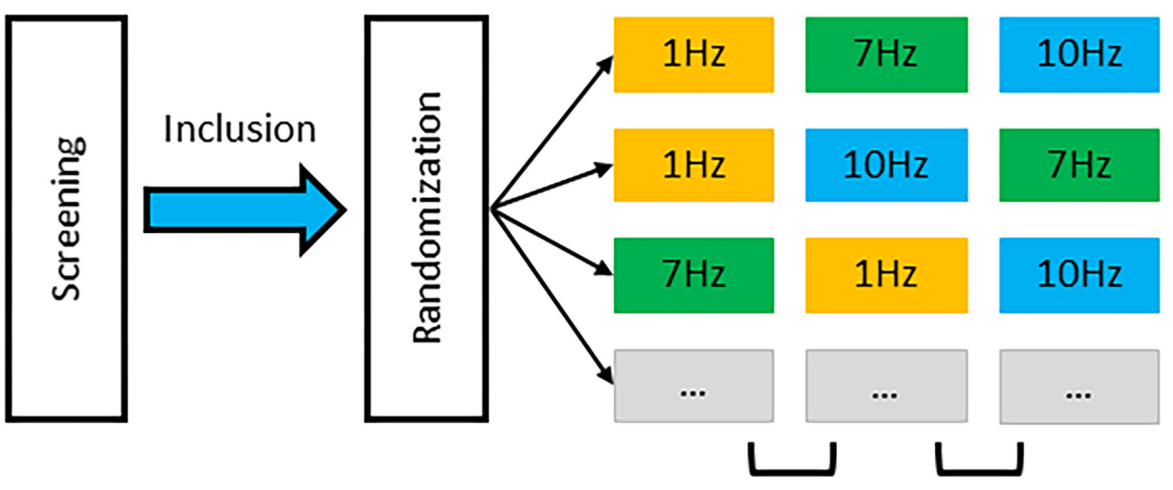

10-minute break between

each stimulation block

\section{Procedure for each stimulation block}

Sitting position

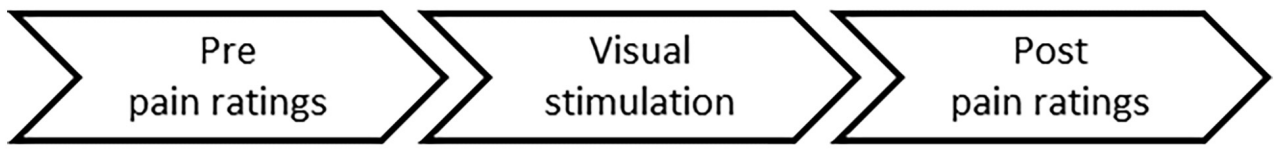

Standing position

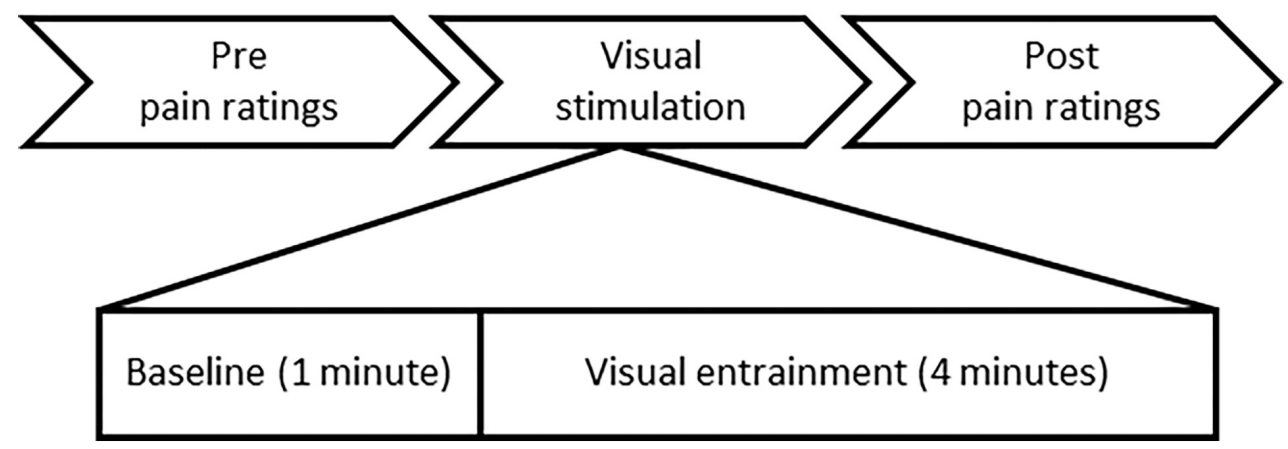

FIGURE 2 | Overview of study procedure. During a single study visit each participant completed three stimulation blocks that each contained stimulation at one particular frequency $(1,7$, or $10 \mathrm{~Hz})$, with stimulation both in a sitting and standing position. For each stimulation block half of the participants always started with stimulation while sitting, the other half while standing. Each participant was randomly allocated to one of six possible stimulation frequency orders: $1,7,10 \mathrm{~Hz} ; 1,10$, $7 \mathrm{~Hz} ; 7,1,10 \mathrm{~Hz} ; 7,10,1 \mathrm{~Hz} ; 10,1,7 \mathrm{~Hz}$; or 10, 7, $1 \mathrm{~Hz}$. There was a break of at least 10 min between each stimulation block with a specific stimulation frequency to minimize any potential carryover effects. Thus, each participant received equal numbers of stimulation blocks at the different frequencies but randomized to a different order of stimulation blocks.

(Cleeland and Ryan, 1994); and the Multidimensional Health Locus of Control scale (MHLC) (Wallston et al., 1978).

Both anxiety and depression have been found to frequently co-occur with chronic pain conditions (Mcwilliams et al., 2003). Moreover, a positive association between pain experience and depression and anxiety has been found, both in an experimental pain setting (Walsh, 1998; Tang and Gibson, 2005) and a clinical pain setting (Geisser et al., 2000; Granot and Ferber, 2005). To assess anxiety and depression in the present study we used the HADS. The HADS is a valid self-assessment scale 
originally developed as a tool to reliably detect states of anxiety and depression in patients attending a general medical clinic (Zigmond and Snaith, 1983; Herrmann, 1997). The HADS comprises seven items to assess anxiety and seven items to assess depression. Participants are asked to tick the box that most closely represents how they were feeling in the past week on a 4-point scale ranging from 0 to 3 . For example, "I feel tense or "wound up"': (0) not at all; (1) occasionally; (2) a lot of the time; or (3) most of the time.

The BPI, PSEQ, and MHLC were used to gain further insight into the pain experience of the participants and their pain-related beliefs and cognitions.

The BPI is a tool to assess both pain intensity (sensory dimension) and pain interference (reactive dimension) in patients with chronic pain. The BPI was originally developed to assess cancer-related pain (Cleeland and Ryan, 1994) but is also a widely used and valid measure for patients with non-malignant chronic pain (Tan et al., 2004). Participants are asked to rate their worst and least pain intensity over the last $24 \mathrm{~h}$, their average pain intensity, and their current pain intensity on a scale of 0 to 10 (sensory dimension). Participants are also asked to rate the degree to which pain interferes with seven domains of functioning on a scale of 0 to 10 , for instance walking ability and relationships with other people (reactive dimension).

The PSEQ is a questionnaire designed to assess self-efficacy beliefs in people experiencing chronic pain (Nicholas, 1989), by assessing the confidence participants have in their ability to perform certain tasks and activities despite their pain. The PSEQ contains 10 items describing different settings/activities, such as "I can do most of the household chores (e.g., tidying-up, washing dishes, etc.), despite the pain" and "I can live a normal lifestyle, despite the pain." Participants are asked to rate how confident they are that they can do these things at present despite the pain on a scale from 0 to 6 , with $0=$ not at all confident and $6=$ completely confident.

The MHLC was developed to assess three dimensions of internal health locus of control, powerful others' locus of control, and chance health of control (Wallston et al., 1978). The MHLC contains 18 items with a belief statement about the participant's health, for example, "Whatever goes wrong with my pain condition is my own fault," and "Other people play a big role in whether my pain condition improves, stays the same, or gets worse." Participants are asked to rate to what extent they agree with each item on a scale of 1 to $6(1=$ strongly disagree and 6 = strongly agree).

\section{EEG Acquisition}

An EEG was recorded during all visual stimulation blocks using $64 \mathrm{Ag} / \mathrm{AgCl}$ electrodes attached to a cap according to the extended standard 10-20 system, using the BrainCap MR, BrainAmp DC/MR amplifiers, and the EEG data recording software BrainVision Recorder (Brain Products $\mathrm{GmbH}$, Germany). The $\mathrm{FCz}$ electrode was used as a reference electrode and $\mathrm{AFz}$ as the ground electrode. EEG was recorded with a sampling rate of $500 \mathrm{~Hz}$ and band-pass filter settings of DC-100 Hz.

\section{Procedure}

All participants attended the lab for a single study visit during which they underwent visual stimulation at all 3 frequencies (1, 7, and $10 \mathrm{~Hz}$ ), once while sitting down and once while standing up. This resulted in a total of 6 stimulation conditions: 3 visual stimulation frequencies $(1,7,10 \mathrm{~Hz}) \times 2$ positions (sitting and standing). For each condition, a 1-min baseline period was followed by a 4-min stimulation period (Figure 2).

After obtaining written informed consent, completing the EEG setup, and identifying the individual stimulation brightness, each participant was pseudo-randomly allocated to 1 of the 6 possible stimulation frequency orders. All participants completed 3 stimulation blocks, with each block containing 1 specific stimulation frequency. Participants experienced each stimulation frequency both sitting and standing. Half of the participants completed each stimulation block in the sitting position first, and the other half started with the standing position first. Pain intensity and unpleasantness were assessed before and after each stimulation condition, i.e., directly before and after the stimulation in the sitting position and also directly before and after the stimulation in the standing position. After each stimulation block participants had a break of at least $10 \mathrm{~min}$ before carrying on with the next block, to limit potential carryover effects of the previous block of stimulation. Although both the experimenter and the participants were blinded to the order of visual stimulation frequencies, due to the nature of the stimulation (visual) the frequency of stimulation for each block became apparent as soon as the stimulation was started. However, importantly, participants were not provided with any clues as to which was the expected therapeutic condition.

\section{EEG Analysis}

The EEG recordings were imported into Matlab (The Mathworks, Inc., Natick, MA, United States; Matlab version R2017a). A number of pre-processing and artifact removal steps were carried out on the continuous EEG data using the EEGLAB toolbox (Delorme and Makeig, 2004) in the following order: (1) interpolation of any bad channels (spherical interpolation); (2) re-referencing to the common average; (3) and high-pass $(0.05 \mathrm{~Hz})$ and low-pass filtering $(30 \mathrm{~Hz})$. A median of 0.5 channels were interpolated with a range from 0 to 5 . Next, the continuous data were segmented into 2-s consecutive epochs to accommodate later visual inspection of the data post-independent component analysis (ICA). Finally, as an EEG for each of the different stimulation conditions was recorded and saved in separate files, the data from the different stimulation conditions were combined into one single data file per participant. These data were decomposed into independent signals using ICA in order to remove components reflecting artifactual sources, with components from frontal sources reflecting eyeblinks and eye movements selected for removal. The number of ICs to be calculated was adjusted for the number of interpolated channels ( $N$ channels $-N$ interpolated channels). The median number of components removed was 2.5 with a range of 1 to 6 . The reconstructed EEG data were then visually inspected to remove any remaining muscle artifacts and any other 
remaining large artifacts, i.e., large spikes and jumps present in the EEG data (on average $4.79 \%$ of trials were removed per participant).

Frequency analysis was performed using the Fieldtrip Toolbox (Oostenveld et al., 2011). Average alpha power (8-12 Hz) was calculated for each visual stimulation condition (1, 7, and $10 \mathrm{~Hz}$ ) using FFT with a single Hanning taper and nonoverlapping windows. All individual alpha power outcomes were log-transformed.

Global alpha power was calculated by averaging the logtransformed alpha power from $8-12 \mathrm{~Hz}$ across all 2-s epochs per condition across all electrodes, resulting in a single average alpha power outcome per visual stimulation condition. The same was applied to the baseline periods preceding each stimulation condition. Next, the average log-transformed alpha power during each stimulation condition was standardized against its respective baseline period (subtraction method: log alpha power entrainment - log alpha power baseline). To assess changes in alpha activity on a more regional level, alpha power was also calculated for nine regions of interest (ROIs) by averaging over the electrodes in each region only (Figure 3 ).

\section{Statistical Analysis}

Statistical analysis was performed using SPSS version 22 (IBM Corp, Armonk, NY, United States). To assess the effect of the visual stimulation on global alpha power, i.e., alpha power averaged across all electrodes, a repeated-measures ANOVA with the factors stimulation $(1,7$, and $10 \mathrm{~Hz}$ ) and position (sitting and standing) was applied.

Next, to further explore the effect of alpha stimulation on a more regional level, changes in alpha power were also compared for the nine ROIs (Figure 3) using a repeated-measures ANOVA with the factors stimulation $(1,7$, and $10 \mathrm{~Hz}$ ), position (sitting and standing), the left-to-right (L-R) ROI factor (left, central, and right), and the anterior-to-posterior (A-P) ROI (anterior, middle, and posterior).

Finally, two repeated-measures ANOVAs with the factors stimulation (1, 7, and $10 \mathrm{~Hz}$ ), position (sitting and standing), and time (pre- and post-stimulation) were applied to assess a change in pain intensity and unpleasantness ratings respectively.

For all ANOVAs, in the case of a violation of sphericity, Greenhouse-Geisser corrected outcomes were used. To correct for multiple comparisons, the Bonferroni correction was applied.

\section{Minimal Clinically Important Difference in Pain Ratings}

In line with the recommendations of the Initiative on Methods, Measurement, and Pain Assessment in Clinical Trials (IMMPACT) consensus statement (Dworkin et al., 2005), we also assessed what percentage of participants showed a minimally important clinical difference (MCID) in pain intensity and unpleasantness. A MCID is considered the smallest difference in pain rating that patients perceive as important and is reflected by a $15 \%$ reduction in pain intensity/unpleasantness rating compared to baseline [(pain rating post-stimulation - pain rating pre-stimulation)/pain rating pre-stimulation] (Dworkin et al., 2008).

\section{Correlations}

To assess the potential relationship between alpha activity and changes in pain due to the alpha stimulation, correlations between standardized global alpha power and the change in pain intensity/unpleasantness rating were calculated. In detail, the average log-transformed alpha power during $10 \mathrm{~Hz}$ stimulation standardized against its baseline period was used (subtraction method: alpha power entrainment alpha power baseline). To calculate the change in the pain intensity/unpleasantness ratings we used the formula ratings post-stimulation - ratings pre-stimulation. Thus, a negative correlation would reflect that higher alpha power during stimulation (compared to baseline) was associated with lower pain ratings post-stimulation and vice versa.

To assess the relationship between changes in pain following alpha stimulation and personality factors and pain-related cognitions and beliefs, correlations between the different questionnaire scores and the change in pain intensity and unpleasantness rating were calculated. The following questionnaire outcomes were used: the sum score for the depression subscale and anxiety subscale separately (HADS); the individual rating for average, worst, and least pain intensity over the last $24 \mathrm{~h}$ and a sum score for the seven pain interference items (BPI); a single sum score for all PSEQ items; and a separate sum score for each of the three subscales of the MHLC.

\section{RESULTS}

\section{Global Alpha Power}

The repeated-measures ANOVA with the factors Stimulation (1, 7 , and $10 \mathrm{~Hz}$ ) and Position (sitting and standing) showed a significant main effect of Stimulation $\left(F_{2,38}=34.88 ; p<0.001\right.$; partial $\left.\eta^{2}=0.65\right)$ on global alpha power and a significant interaction between Stimulation and Position $\left(F_{2,38}=13.48\right.$; $p<0.001$; partial $\eta^{2}=0.42$; Figure 4). Post hoc repeated-measures $t$-tests showed that alpha power was significantly higher during $10 \mathrm{~Hz}$ stimulation compared to the $1 \mathrm{~Hz}$ control stimulation during the standing condition $(t=-6.08, p<0.001)$. There was no significant increase of global alpha power during the sitting condition $(t=-1.30, p=0.21)$. No increase of alpha power was found for the $7 \mathrm{~Hz}$ condition compared to $1 \mathrm{~Hz}$ condition, only a significant decrease of global alpha power for $7 \mathrm{~Hz}$ compared to $1 \mathrm{~Hz}$ stimulation in the sitting condition $(t=2.51$, $p=0.021)$. However, this effect did not survive correction of multiple comparisons (corrected significance level of 0.0125). Finally, alpha power was also significantly higher during $10 \mathrm{~Hz}$ stimulation compared to $7 \mathrm{~Hz}$ stimulation, both for the sitting condition $(t=-3.69, p=0.002)$ and the standing condition $(t=-6.03, p<0.001)$.

As a significant increase of alpha power for $10 \mathrm{~Hz}$ compared to $1 \mathrm{~Hz}$ stimulation was only found for the standing condition, a further post hoc repeated-measures $t$-test was calculated to assess whether there was a significant difference in global alpha 


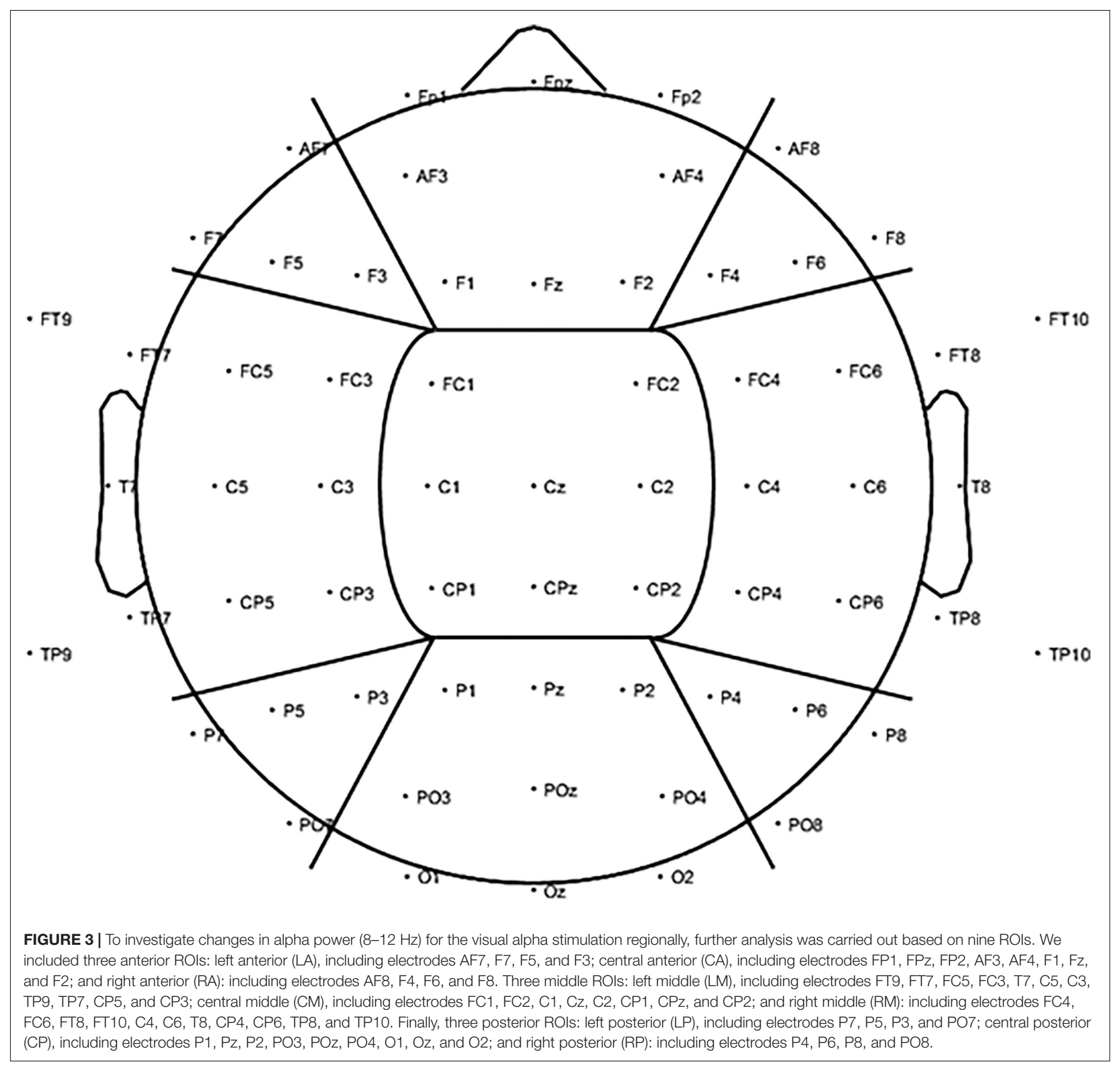

power comparing sitting and standing for the $10 \mathrm{~Hz}$ stimulation. This $t$-test showed that global alpha power was significantly higher during the $10 \mathrm{~Hz}$ stimulation in the standing condition $(t=-6.40, p<0.001)$.

\section{ROI Alpha Power}

A repeated-measures ANOVA was calculated to assess more regional changes in alpha power with the factors Stimulation (1, 7, and $10 \mathrm{~Hz}$ ), Position (sitting and standing), L-R ROI (left, central, and right) and A-P ROI (anterior, middle, and posterior). A significant main effect was found for the A-P ROI factor $\left(F_{1.34,25.49}=11.43 ; p=0.001\right.$; partial $\eta^{2}=0.38$; Greenhouse-Geisser corrected). Moreover, a significant interaction between Stimulation, Position, and A-P ROI was found $\left(F_{2 \cdot 48,47 \cdot 07}=6.65 ; p<0.002\right.$; partial $\eta^{2}=0.26$; Greenhouse-Geisser corrected). Similarly, a significant main effect was found for the L-R ROI factor $\left(F_{1.25,23.76}=4.50 ; p=0.037\right.$; partial $\eta^{2}=0.19$; GreenhouseGeisser corrected), accompanied by a significant interaction between Stimulation and L-R ROI $\left(F_{1 \cdot 25,23.68}=11.87 ; p=0.001\right.$; partial $\eta^{2}=0.39$; Greenhouse-Geisser corrected) and a significant interaction between Stimulation, Position, and the L-R ROI $\left(F_{1.91,36 \cdot 20}=12.24 ; p<0.001\right.$; partial $\eta^{2}=0.39$; Greenhouse-Geisser corrected). Thus, the effect of visual stimulation on alpha power was different depending on position and on scalp region. 


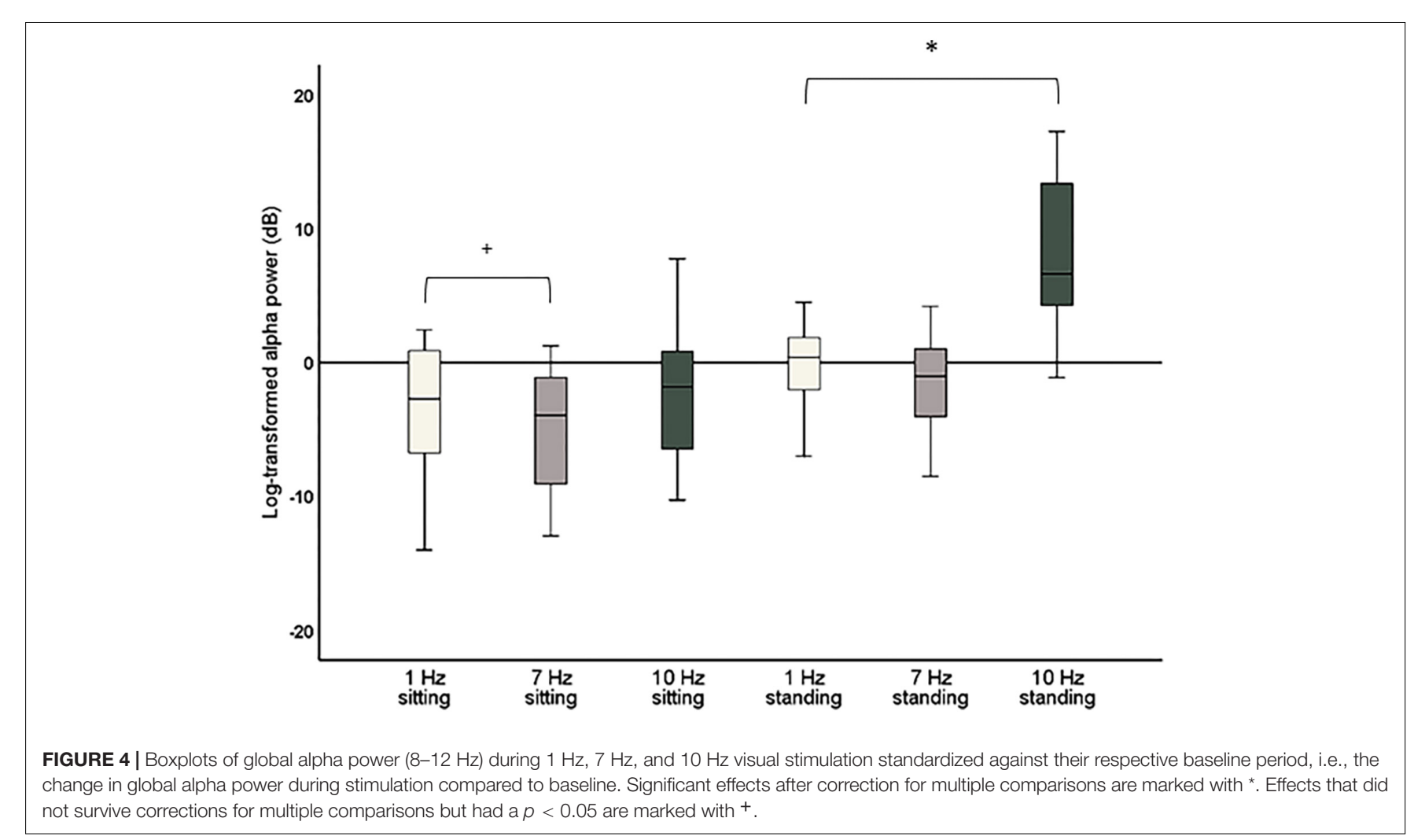

Post hoc repeated-measures $t$-tests showed that alpha power was significantly higher during $10 \mathrm{~Hz}$ stimulation compared to $1 \mathrm{~Hz}$ stimulation in the right-middle region (RM) and the leftposterior region (LP) in particular when participants were sitting (Tables 2, 4 and Figure 5). In the standing condition, alpha power was higher during $10 \mathrm{~Hz}$ compared to $1 \mathrm{~Hz}$ stimulation across a wider range of mostly middle-anterior regions; however, these effects did not survive correction for multiple comparisons (corrected significance level of 0.0056).

Post hoc t-tests comparing 7 and $1 \mathrm{~Hz}$ visual stimulation showed that alpha power was significantly lower during $7 \mathrm{~Hz}$ stimulation compared to $1 \mathrm{~Hz}$ stimulation, in particular in the central-middle region (CM) and the central-posterior region

TABLE 2 | Outcomes of the post hoc repeated measures $t$-tests comparing 1 and $10 \mathrm{~Hz}$ stimulation for the nine ROls separately.

\begin{tabular}{lcc}
\hline ROI & \multicolumn{1}{c}{ Sitting } & Standing \\
\hline Left-anterior (LA) & $t=-1.66, p=0.11$ & $t=-2.43, p=0.025$ \\
Central-anterior (CA) & $t=-1.51, p=0.15$ & $t=-2.22, p=0.039$ \\
Right-anterior (RA) & $t=-1.96, p=0.065$ & $t=-2.26, p=0.036$ \\
Left-middle (LM) & $t=-1.93, p=0.069$ & $t=-2.34, p=0.031$ \\
Central-middle (CM) & $t=-1.08, p=0.29$ & $t=-1.23, p=0.24$ \\
Right-middle (RM) & $t=-4.44, p<0.001^{*}$ & $t=-1.62, p=0.12$ \\
Left-posterior (LP) & $t=-4.33, p<0.001^{*}$ & $t=-2.32, p=0.032$ \\
Central-posterior (CP) & $t=-1.19, p=0.25$ & $t=-1.66, p=0.11$ \\
Right-posterior (RP) & $t=-0.61, p=0.55$ & $t=-1.04, p=0.31$ \\
\hline
\end{tabular}

Significant $t$-tests are marked with ${ }^{*}$ (Bonferroni-corrected significance level).
(CP; Tables 3, 4 and Figure 5), when participants were sitting. A similar pattern was present when participants were standing; however, this did not survive correction for multiple comparisons for the central-middle region.

\section{Intensity Ratings}

The repeated-measures ANOVA demonstrated a significant main effect of Position (sitting and standing) on intensity ratings $\left(F_{1,19}=12.32 ; p=0.002\right.$; partial $\left.\eta^{2}=0.39\right)$, but no significant main effect of Stimulation $\left(F_{2,38}=1.80\right.$; $p=0.18$; partial $\left.\eta^{2}=0.087\right)$. There was a significant

TABLE 3 | Outcomes of the post hoc repeated measures $t$-tests comparing 1 and $7 \mathrm{~Hz}$ stimulation for the nine ROls.

\begin{tabular}{|c|c|c|}
\hline ROI & Sitting & Standing \\
\hline Left-anterior (LA) & $t=2.44, p=0.025$ & $t=2.01, p=0.059$ \\
\hline Central-anterior (CA) & $t=3.08, p=0.006$ & $t=1.73, p=0.099$ \\
\hline Right-anterior (RA) & $t=2.70, p=0.014$ & $t=1.93, p=0.068$ \\
\hline Left-middle (LM) & $t=2.87, p=0.010$ & $t=2.23, p=0.038$ \\
\hline Central-middle (CM) & $t=3.66, p=0.002^{\star}$ & $t=3.03, p=0.007$ \\
\hline Right-middle (RM) & $t=2.76, p=0.012$ & $t=2.57, p=0.019$ \\
\hline Left-posterior (LP) & $t=-1.82, p=0.085$ & $t=1.99, p=0.062$ \\
\hline Central-posterior (CP) & $t=4.44, p<0.001^{*}$ & $t=3.45, p=0.003^{\star}$ \\
\hline Right-posterior (RP) & $t=2.66, p=0.016$ & $t=2.73, p=0.013$ \\
\hline
\end{tabular}


TABLE 4 | Standardized alpha power (dB) per stimulation condition (1, 7, and $10 \mathrm{~Hz}$ ) for each $\mathrm{ROI}$ and for sitting (top) and standing (bottom) separately.

\begin{tabular}{|c|c|c|c|}
\hline ROI & $1 \mathrm{~Hz}$ & $7 \mathrm{~Hz}$ & $10 \mathrm{~Hz}$ \\
\hline \multicolumn{4}{|l|}{ Sitting } \\
\hline Left-anterior (LA) & $0.43(2.46)$ & $-0.92(1.90)$ & $1.38(2.33)$ \\
\hline Central-anterior (CA) & $0.81(2.75)$ & $-1.10(2.08)$ & $1.80(2.71)$ \\
\hline Right-anterior (RA) & $0.70(2.73)$ & $-1.04(2.06)$ & $1.91(2.35)$ \\
\hline Left-middle (LM) & $0.83(2.01)$ & $-0.50(1.99)$ & $1.57(1.95)$ \\
\hline Central-middle (CM) & $1.35(2.38)$ & $-1.09(2.49)$ & $1.87(2.62)$ \\
\hline Right-middle (RM) & $0.84(2.07)$ & $-0.82(2.12)$ & $7.13(5.15)$ \\
\hline Left-posterior (LP) & $-4.65(8.36)$ & $-1.63(2.66)$ & 1.55 (3.35) \\
\hline Central-posterior (CP) & $0.68(2.94)$ & $-2.48(2.33)$ & $1.56(3.14)$ \\
\hline Right-posterior (RP) & $-0.016(3.25)$ & $-2.17(2.88)$ & $0.38(2.92)$ \\
\hline \multicolumn{4}{|l|}{ Standing } \\
\hline Left-anterior (LA) & $0.75(1.78)$ & $-0.74(2.45)$ & $2.22(1.91)$ \\
\hline Central-anterior (CA) & $0.79(1.75)$ & $-0.53(2.62)$ & $2.32(2.43)$ \\
\hline Right-anterior (RA) & $0.82(1.59)$ & $-0.54(2.33)$ & $2.16(2.42)$ \\
\hline Left-middle (LM) & $0.67(1.69)$ & $-0.69(2.26)$ & $1.83(1.66)$ \\
\hline Central-middle (CM) & $1.12(1.78)$ & $-1.01(2.59)$ & $1.86(2.13)$ \\
\hline Right-middle (RM) & $0.91(1.77)$ & $-0.74(2.31)$ & $1.75(1.59)$ \\
\hline Left-posterior (LP) & $0.55(1.69)$ & $-0.99(2.96)$ & $2.21(2.81)$ \\
\hline Central-posterior (CP) & $0.95(1.76)$ & $-1.88(2.87)$ & $2.36(2.95)$ \\
\hline Right-posterior (RP) & $0.45(2.12)$ & $-2.14(3.55)$ & $1.26(2.88)$ \\
\hline
\end{tabular}

interaction between Stimulation and Time (pre- and poststimulation) $\left(F_{1 \cdot 46,27 \cdot 80}=0.065 ; p=0.89\right.$; partial $\eta^{2}=0.003$; Greenhouse-Geisser corrected), nor a significant interaction between Stimulation, Position, and Time $\left(F_{2,38}=0.59 ; p=0.56\right.$; partial $\eta^{2}=0.030$ ) (Table 5 and Figure 6). Moreover, the repeated-measures $t$-tests further assessing an effect on pain intensity for the alpha stimulation $(10 \mathrm{~Hz})$, specifically, did not find a significant change in pain intensity ratings comparing pre- and post-alpha stimulation (sitting: $t=1.54, p=0.14$; and standing: $t=-1.11, p=0.28$ ).

\section{Unpleasantness Ratings}

The repeated measures ANOVA demonstrated a significant main effect of Position (sitting and standing) on unpleasantness ratings $\left(F_{1,19}=12.61 ; p=0.002\right.$; partial $\left.\eta^{2}=0.40\right)$, but no significant main effect of Stimulation $\left(F_{2,38}=1.78 ; p=0.18\right.$; partial $\left.\eta^{2}=0.085\right)$. There was also not a significant interaction between Stimulation and Time $\left(F_{2,38}=0.73 ; p=0.49\right.$; partial $\left.\eta^{2}=0.037\right)$, nor a significant interaction between Stimulation, Position, and Time $\left(F_{2,38}=2.63 ; p=0.085\right.$; partial $\left.\eta^{2}=0.12\right)$ (Table 6 and Figure 7). Moreover, the repeated-measures $t$-tests assessing an effect on pain unpleasantness for the alpha stimulation $(10 \mathrm{~Hz})$ specifically did not find a significant change of pain unpleasantness ratings comparing pre- and post-alpha stimulation (sitting: $t=1.77, p=0.093$; and standing: $t=-1.32$, $p=0.20)$.

\section{Minimal Clinically Important Difference in Pain Ratings Sitting Condition}

We assessed the number of participants that showed a MCID (percentage change $>15 \%$ ) in pain ratings, for the three different

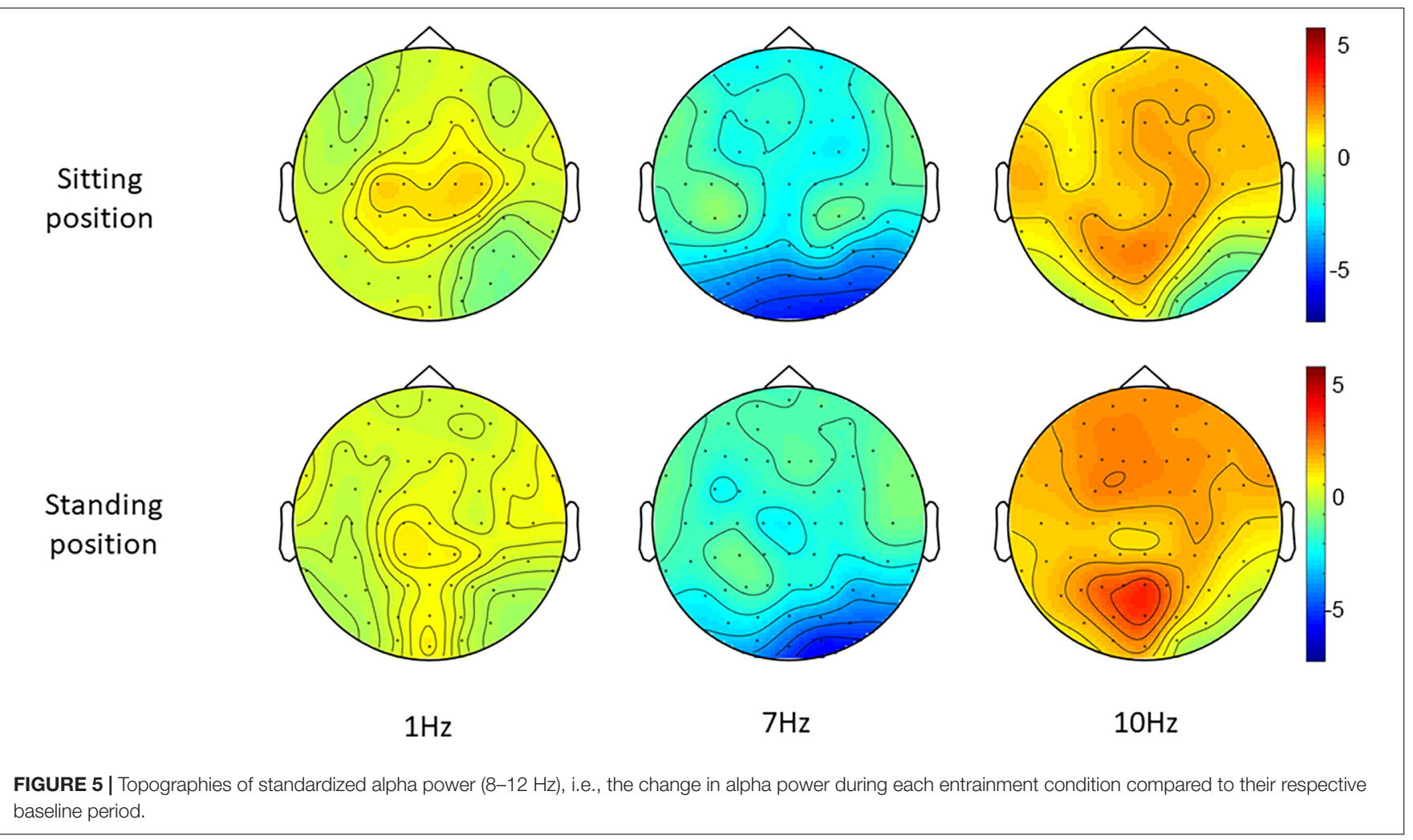


TABLE 5 | Pain intensity ratings (Mean \pm SD) pre- and post-stimulation, for the 1 , 7 , and $10 \mathrm{~Hz}$ stimulation condition and for the sitting and standing positions.

\begin{tabular}{lcccc}
\hline \multicolumn{5}{c}{ Intensity ratings } \\
\hline Frequency & Sitting pre & Sitting post & Standing pre & Standing post \\
\hline $1 \mathrm{~Hz}$ & $4.70 \pm 1.95$ & $4.15 \pm 2.20$ & $5.18 \pm 1.84$ & $5.28 \pm 1.97$ \\
$7 \mathrm{~Hz}$ & $4.43 \pm 1.70$ & $4.03 \pm 2.07$ & $4.75 \pm 1.99$ & $4.60 \pm 2.47$ \\
$10 \mathrm{~Hz}$ & $4.39 \pm 2.12$ & $3.73 \pm 2.01$ & $4.45 \pm 1.87$ & $4.78 \pm 2.07$ \\
\hline
\end{tabular}

stimulation conditions separately. For the intensity ratings, 50\% of participants demonstrated a MCID for the $10 \mathrm{~Hz}$ stimulation. A similar value was found for the $1 \mathrm{~Hz}$ condition (45\%). For the $7 \mathrm{~Hz}$ condition an MCID was found for 35\%. For the unpleasantness ratings, $65 \%$ of participants demonstrated an MCID for the $10 \mathrm{~Hz}$ stimulation. This percentage was lower for the $1 \mathrm{~Hz}$ condition (40\%) and the $7 \mathrm{~Hz}$ condition (30\%). Only the alpha stimulation led to an MCID in pain intensity and unpleasantness for $\geq 50 \%$ of participants.

\section{Standing Condition}

Next, we assessed the number of participants who showed an MCID (percentage change $>15 \%$ ) in pain ratings, for the three different stimulation conditions separately when the participants were standing. For the intensity ratings, $25 \%$ of participants demonstrated an MCID (percentage change $>15 \%$ ) for the $10 \mathrm{~Hz}$ stimulation. A similar value was found for the $1 \mathrm{~Hz}$ condition (20\%) and the $7 \mathrm{~Hz}$ condition (30\%). For the unpleasantness ratings, $30 \%$ of participants demonstrated an MCID for the $10 \mathrm{~Hz}$ stimulation. This percentage was also found for the $1 \mathrm{~Hz}$ condition (30\%) and the $7 \mathrm{~Hz}$ condition
(30\%). None of the stimulation conditions led to an MCID in pain intensity or unpleasantness for $\geq 50 \%$ of participants in the standing condition.

\section{Correlations}

The correlations between standardized global alpha power during $10 \mathrm{~Hz}$ stimulation (log alpha power during $10 \mathrm{~Hz}$ stimulation - baseline log alpha power) and the difference in intensity/unpleasantness ratings comparing pre- and poststimulation were calculated (ratings post-stimulation - ratings pre-stimulation) (Table 7). No significant correlation was found for the intensity ratings (sitting: $r=0.34 ; p=0.14 ; N=20$; standing: $r=0.16 ; p=0.51 ; N=20$ ). For the unpleasantness ratings no significant correlation with global alpha power was found either for the standing condition $(r=0.11 ; p=0.65$; $N=20)$. A correlation was found for the sitting condition $(r=0.46 ; p=0.04 ; N=20)$; however, this did not survive correction for multiple comparisons (corrected significance level $=0.0125$ ).

Post hoc it was decided also to explore the correlations between the change in pain intensity/unpleasantness ratings and standardized alpha power for the two ROIs that showed a significant increase of alpha power during $10 \mathrm{~Hz}$ stimulation compared to $1 \mathrm{~Hz}$ stimulation in the sitting condition, the rightmiddle (RM) and left-posterior (LP) ROI (Table 7). For the RM ROI, there was no significant correlation between change in pain ratings (ratings post-stimulation - ratings pre-stimulation) and alpha power (intensity ratings: $r=-0.40 ; p=0.082 ; N=20$; unpleasantness ratings: $r=-0.41 ; p=0.073 ; N=20)$. For the LP ROI, no significant correlation was found either for the intensity ratings $(r=0.43 ; p=0.060 ; N=20)$. A correlation was identified

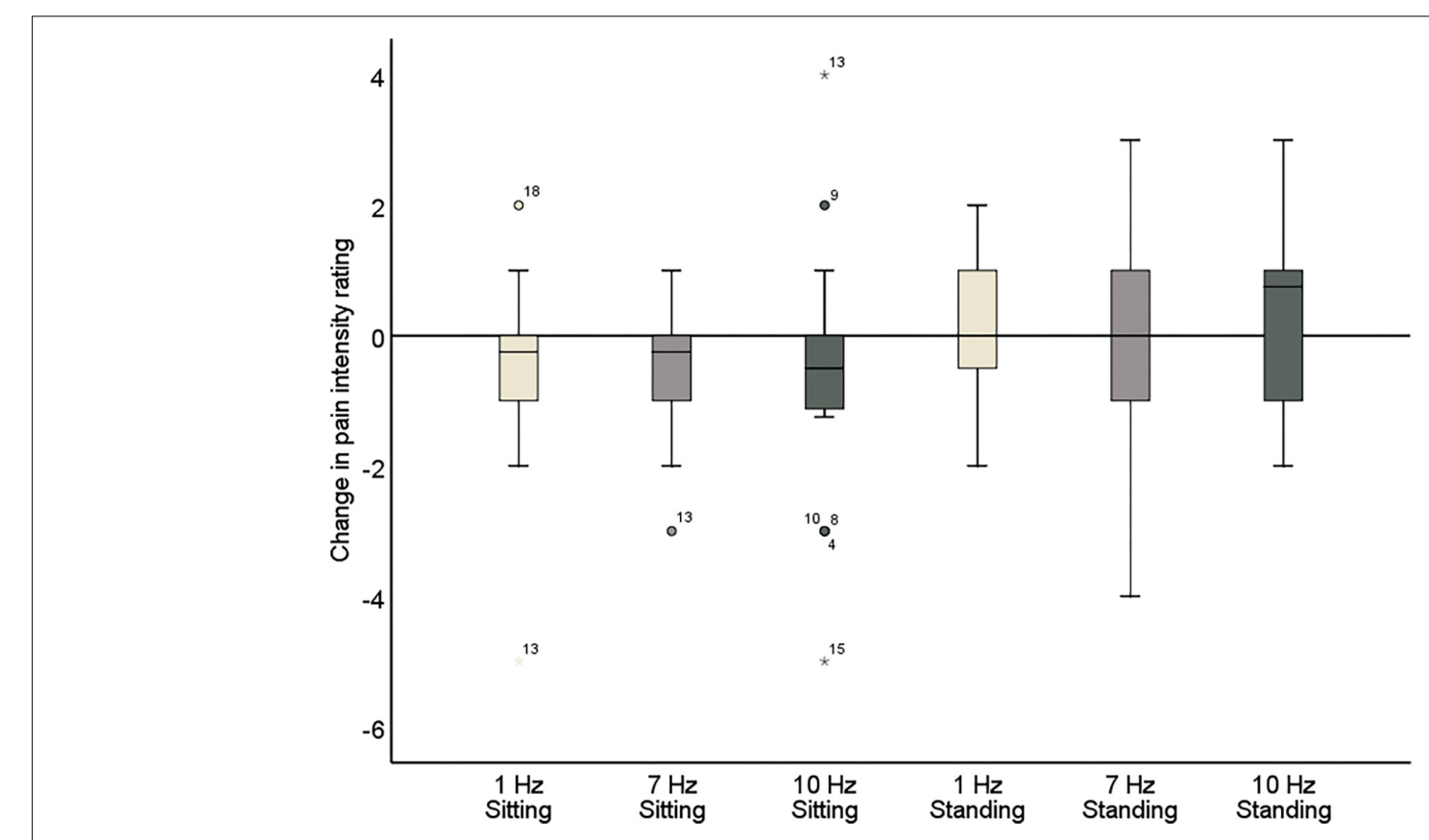

FIGURE 6 | Change in pain intensity ratings comparing pre- and post-stimulation for 1, 7, and $10 \mathrm{~Hz}$ stimulation, both in the sitting and standing condition. A negative score reflects a reduction in pain and a positive score reflects an increase of pain following the stimulation. 
TABLE 6 | Pain unpleasantness ratings (Mean \pm SD) pre- and post-stimulation, for the 1,7 , and $10 \mathrm{~Hz}$ stimulation condition and for the sitting and standing positions.

\begin{tabular}{lcccc}
\hline \multicolumn{5}{c}{ Unpleasantness ratings } \\
\hline Frequency & Sitting pre & Sitting post & Standing pre & Standing post \\
\hline $1 \mathrm{~Hz}$ & $4.55 \pm 2.04$ & $4.10 \pm 2.37$ & $5.40 \pm 1.98$ & $5.20 \pm 2.02$ \\
$7 \mathrm{~Hz}$ & $4.20 \pm 1.82$ & $4.33 \pm 2.34$ & $4.63 \pm 1.75$ & $4.65 \pm 2.24$ \\
$10 \mathrm{~Hz}$ & $4.38 \pm 2.31$ & $3.50 \pm 2.07$ & $4.28 \pm 1.89$ & $4.80 \pm 2.17$ \\
\hline
\end{tabular}

for the unpleasantness ratings $(r=0.54 ; p=0.015 ; N=20)$, but this did not survive correction for multiple comparisons ( corrected significance level $=0.0125$ ).

Finally, correlations were assessed between the change in intensity/unpleasantness ratings and the questionnaire outcomes (Table 7). No significant correlation between ratings and any of the questionnaire outcomes was found. A correlation between pain unpleasantness ratings and the HADS Depression subscale was identified in the standing condition $(r=0.50$; $p=0.026 ; N=20)$. However, this did not survive correction for multiple comparisons.

\section{DISCUSSION}

Emerging evidence shows an inverse relationship between alpha power and chronic pain (Camfferman et al., 2017; Ahn et al., 2019). Therefore, alpha activity has been proposed as a key target for novel neuromodulatory therapies to manage chronic pain (Jensen et al., 2008). This feasibility study primarily aimed to assess the efficacy of visual alpha stimulation to enhance alpha activity in patients with chronic musculoskeletal pain. Secondarily, it was evaluated whether a brief period of alpha stimulation was also sufficient to reduce chronic pain. The main finding of this study was that visual alpha stimulation can effectively enhance alpha activity in patients with chronic musculoskeletal pain. Global alpha power was significantly higher during alpha stimulation compared to the $1 \mathrm{~Hz}$ control stimulation when patients were experiencing stronger discomfort (standing condition). On a more regional level, a significant increase of alpha activity was also found in the right-middle and left-posterior region when patients were sitting. With respect to our secondary aim, $4 \mathrm{~min}$ of alpha stimulation was not sufficient to significantly reduce chronic pain. However, only the alpha stimulation resulted in an MCID in at least 50\% of participants for the pain intensity (50\%) and unpleasantness ratings (65\%). This study is the first to demonstrate the efficacy of rhythmic visual stimulation to modulate alpha activity in patients with chronic pain. However, further study is warranted to investigate the optimal dose and individual stimulation parameters (Krause and Cohen Kadosh, 2014), such as duration and frequency of entrainment to achieve significant pain relief.

Whereas both 7 and $10 \mathrm{~Hz}$ stimulation can result in an indirect entrainment of alpha activity via attentional mechanisms (Thut et al., 2011), only the $10 \mathrm{~Hz}$ stimulation should lead to a direct entrainment of alpha. No evidence for entrainment of alpha activity during $7 \mathrm{~Hz}$ stimulation was found, i.e., global alpha power was not significantly higher during $7 \mathrm{~Hz}$ stimulation compared to $1 \mathrm{~Hz}$ stimulation. Only a significant decrease of alpha power was found for $7 \mathrm{~Hz}$ stimulation compared to $1 \mathrm{~Hz}$ stimulation in central-middle and central-posterior regions. In addition, global alpha power was significantly higher during $10 \mathrm{~Hz}$ stimulation compared to $7 \mathrm{~Hz}$ stimulation, both for the sitting condition and the standing condition. Together, this suggests that the effect of alpha $(10 \mathrm{~Hz})$ stimulation on alpha power found in this study is likely the result of direct entrainment, and does not only reflect a non-specific effect of attention being directed away from the pain by visual stimulation.

The present study's findings build on the findings by Ecsy et al. (2018), who previously demonstrated that visual alpha stimulation can increase alpha power and reduce pain in an experimental pain setting, albeit in healthy individuals experiencing acute laser pain rather than in patients with chronic pain. Qualitatively, when we compare the analyses of regional changes, the scalp regions showing increases in alpha activity are similar between the two studies, with a posterior dominance in both cases and weaker (in the current study, statistically nonsignificant) evidence for additional fronto-central increases. In the absence of a more robust quantitative comparison between patients and healthy controls (which would require a further controlled study), we cannot conclude that alpha entrainment differs in patients with chronic musculoskeletal pain, but we cannot rule out this possibility either.

Ahn et al. (2019) provided the first evidence that alpha stimulation can be used successfully in a clinical pain setting. They demonstrated that alpha tACS applied over somatosensory regions enhances somatosensory alpha power in patients with CLBP. Here we demonstrate that rhythmic visual stimulation can also modulate alpha activity in patients. Moreover, as this study included patients with various chronic musculoskeletal pain conditions, it also offers a first indication that the modulation of alpha activity with alpha stimulation can be generalized across different chronic pain populations.

The effect of visual alpha stimulation may be influenced by the level of discomfort experienced by the patients. Only when a patient was standing-a setting of stronger discomfort possibly related to lower endogenous alpha-did stimulation result in a global entrainment of alpha activity. In addition, global alpha power was significantly higher during standing compared to sitting during the $10 \mathrm{~Hz}$ stimulation. This would be in line with previous studies showing that alpha entrainment with tACS at alpha frequency is most effective when endogenous alpha is low (Neuling et al., 2013; Ruhnau et al., 2016). When the patient was sitting (lower discomfort), the stimulation did not result in a significant increase of global alpha power. However, a significant increase was found for two regions of interest, suggesting a more regional entrainment of alpha activity. Previously, a negative correlation has been found between somatosensory alpha power and perceived pain intensity for experimentally induced pain (Babiloni et al., 2006; Tu et al., 2016) and between frontal and somatosensory alpha power and chronic pain intensity (Camfferman et al., 2017). Ahn et al. (2019) also found that the increase of frontal and somatosensory alpha power by alpha tACS was associated with pain relief. 


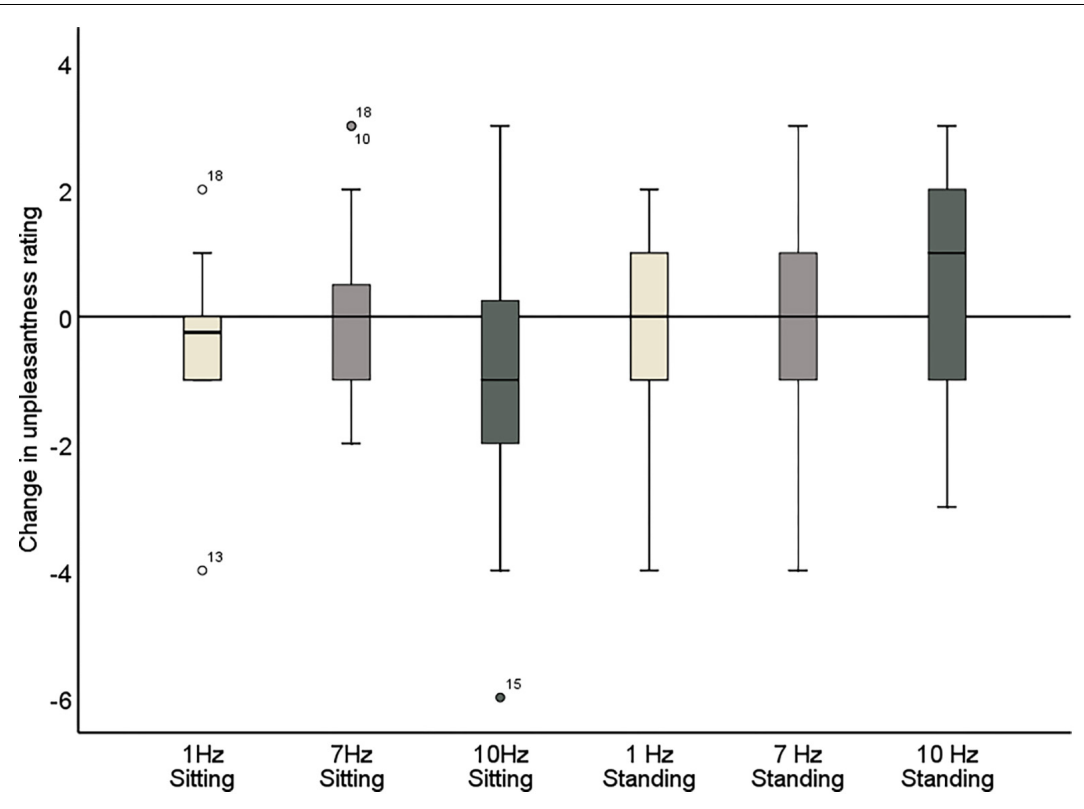

FIGURE 7 | Change in pain unpleasantness ratings comparing pre- and post-stimulation for 1, 7, and $10 \mathrm{~Hz}$ stimulation, in both the sitting and standing conditions. A negative score reflects a reduction in pain and a positive score reflects an increase of pain following the stimulation.

TABLE 7 | Overview of the results of the correlation analysis.

\begin{tabular}{|c|c|c|c|c|}
\hline & \multicolumn{2}{|c|}{ Intensity ratings } & \multicolumn{2}{|c|}{ Unpleasantness ratings } \\
\hline & Sitting & Standing & Sitting & Standing \\
\hline Global alpha & $r=0.34 ; p=0.14$ & $r=0.16 ; p=0.51$ & $r=0.46 ; p=0.04^{+}$ & $r=0.11 ; p=0.65$ \\
\hline RM-alpha & $r=-0.40 ; p=0.082$ & & $r=-0.41 ; p=0.073$ & \\
\hline LP-alpha & $r=0.43 ; p=0.060$ & & $r=0.54 ; p=0.015^{+}$ & \\
\hline HADS-A & $r=0.048 ; p=0.84$ & $r=-0.11 ; p=0.64$ & $r=0.10 ; p=0.68$ & $r=-0.038 ; p=0.87$ \\
\hline HADS-D & $r=0.081 ; p=0.73$ & $r=0.30 ; p=0.20$ & $r=0.12 ; p=0.60$ & $r=0.50 ; p=0.026^{+}$ \\
\hline BPI-average & $r=-0.18 ; p=0.47$ & $r=-0.042 ; p=0.87$ & $r=-0.041 ; p=0.87$ & $r=0.13 ; p=0.60$ \\
\hline BPI-worst & $r=-0.25 ; p=0.30$ & $r=0.15 ; p=0.54$ & $r=-0.057 ; p=0.82$ & $r=0.19 ; p=0.43$ \\
\hline BPI-least & $r=0.11 ; p=0.65$ & $r=-0.29 ; p=0.23$ & $r=0.20 ; p=0.41$ & $r=-0.23 ; p=0.35$ \\
\hline BPI-I & $r=-0.18 ; p=0.45$ & $r=0.21 ; p=0.39$ & $r=-0.12 ; p=0.63$ & $r=0.32 ; p=0.18$ \\
\hline PSEQ & $r=-0.055 ; p=0.82$ & $r=-0.35 ; p=0.14$ & $r=0.040 ; p=0.87$ & $r=-0.40 ; p=0.077$ \\
\hline MHLC-I & $r=0.19 ; p=0.43$ & $r=-0.014 ; p=0.96$ & $r=0.23 ; p=0.34$ & $r=0.077 ; p=0.75$ \\
\hline MHLC-O & $r=0.090 ; p=0.71$ & $r=0.14 ; p=0.56$ & $r=-0.036 ; p=0.88$ & $r=0.097 ; p=0.68$ \\
\hline MHLC-C & $r=-0.41 ; p=0.075$ & $r=-0.040 ; p=0.87$ & $r=-0.29 ; p=0.21$ & $r=-0.020 ; p=0.93$ \\
\hline
\end{tabular}

In the present study, increasing global alpha power with visual stimulation did not result in a significant reduction of pain intensity and unpleasantness. Moreover, the present study found only a non-significant negative correlation between standardized somatosensory alpha power (right-middle ROI) and the change in pain intensity $(r=-0.40 ; p=0.082)$ and unpleasantness $(r=-0.41 ; p=0.073)$ following alpha stimulation (sitting condition). As these correlations were only marginally significant and based on a relatively small sample $(N=20)$, no confident conclusions can be drawn from these findings. However, where this study only included brief periods of stimulation, Ahn et al. (2019) applied alpha tACS for $40 \mathrm{~min}$. In an experimental pain setting with pain-free volunteers, Ecsy et al. $(2017,2018)$ achieved a significant reduction in pain ratings using $10 \mathrm{~min}$ of auditory and visual stimulation and Arendsen et al. (2018) applied alpha tACS for $15-20 \mathrm{~min}$. This feasibility study focused primarily 
on the entrainment of alpha activity, where it has been shown that even very short periods of stimulation can entrain alpha oscillations (Herrmann, 2001; Mathewson et al., 2012; Notbohm and Herrmann, 2016). However, to also reduce chronic pain, longer stimulation periods might be required. Moreover, this feasibility study included a small and heterogenous group of patients with chronic musculoskeletal pain, which introduces the possibility that the study is simply underpowered to find an effect of the stimulation on chronic pain. Further investigation with a larger sample size is needed to confirm whether a longer period of visual alpha stimulation leads to a significant reduction of chronic pain.

Further inspection of the individual changes in pain intensity and unpleasantness in response to the alpha stimulation showed that a wide variability in pain response was present (Figure 7 and Tables 5, 6). Whereas some patients showed a reduction of several points on the 11-point NRS, others did not improve at all or even showed an increase of pain. Large variability in response is a problem for neurostimulation techniques in general. To improve the efficacy of neurostimulation interventions to manage chronic pain, it is important to take into account interand intra-individual factors such as cognitive, psychological, and neurophysiological state, and methodological factors that might contribute to this variability (Li et al., 2015; Fertonani and Miniussi, 2017). In this study we did not identify a relationship between patient characteristics (as assessed with the questionnaires) and the pain response. However, larger sample sizes (e.g., 80-100) are likely needed for such analyses to be adequately powered for medium effect sizes. Another important source of variability in the effects of neurostimulation is brain-state dependency, i.e., the effect of neurostimulation depends on the timing of stimulation with respect to the underlying brain state. A number of studies have shown that applying neurostimulation in a brain-state dependent manner can enhance the modulation of corticospinal excitability (Saito et al., 2013; Kaneko et al., 2014; Kraus et al., 2016). Ultimately, taking into account these factors in the application of neurostimulation should lead to a more personalized and adaptive neuromodulatory therapy to reduce chronic pain.

Evidence shows that the efficacy of alpha entrainment depends on the distance between the stimulation frequency and the individual alpha peak frequency (IAF) (Herrmann et al., 2016; Notbohm et al., 2016; Gulbinaite et al., 2017). Thus, tailoring the frequency of the visual alpha stimulation to each individual could improve the effect of alpha stimulation in patients with chronic pain. Moreover, recent studies have also explored the potential of combined stimulation. Anodal tDCS over the primary motor cortex (M1) combined with peripheral electrical stimulation led to an enhanced, long-lasting, and clinically important reduction in chronic pain (Boggio et al., 2009; Schabrun et al., 2014; Hazime et al., 2017). Together, these recent developments in the application of neurostimulation offer promising future directions for application of alpha stimulation to reduce chronic pain.

To successfully implement visual alpha stimulation to reduce chronic pain, it is also important to better understand the relationship between alpha activity and chronic pain. So far, most studies have focused on the role of alpha activity in the perception of experimentally induced pain in pain-free individuals. Although there are some initial findings showing a negative correlation between frontal and somatosensory alpha power and chronic pain (Camfferman et al., 2017; Ahn et al., 2019), the functional role of alpha activity in the perception of chronic pain remains unclear. Experimental pain studies have demonstrated that the relationship between alpha activity and pain is influenced by attention (May et al., 2012; Hauck et al., 2015) and expectations about pain (Huneke et al., 2013; Arendsen et al., 2018), and that pain expectations can influence the effect of neuro-stimulation on pain perception (Arendsen et al., 2018). However, the relationship between attention, expectation, and alpha activity in a setting of chronic pain is little understood. It is important to better understand how these factors influence the relationship between alpha activity and chronic pain and the effectiveness of alpha stimulation to reduce chronic pain.

The present study showed that visual alpha stimulation offers a means to modulate alpha activity in patients with chronic pain in a lab-based environment. Whereas this is an important first step, further development is required to transform this lab-based application into a therapeutic technique that patients can use in their own home with therapeutic benefit. In a parallel study (Locke et al., 2020), a first qualitative assessment of a smartphonebased alpha entrainment technology was carried out. Individuals with chronic pain were asked about their experience with using the technology at home, using a virtual-reality headset for rhythmic visual stimulation and headphones for rhythmic auditory stimulation (binaural beats). The study provided initial support for the acceptability and usability of this smartphonebased technology as an affordable and accessible alternative to manage chronic pain. An important next step is to investigate the effectiveness of longer periods and multiple sessions of alpha stimulation to reduce chronic pain in the lab and at home, to translate these initial findings into a technology that can effectively reduce pain in a home-based setting.

\section{CONCLUSION}

To conclude, this study provides first evidence that visual stimulation at alpha frequency can be used to increase alpha power in patients with musculoskeletal pain. However, a brief 4min period of stimulation was not sufficient to reduce chronic pain. This study is a first step in the development of a novel neurostimulation approach to reducing chronic pain. Further study is warranted to investigate individual stimulation and optimal dose parameters (Krause and Cohen Kadosh, 2014) to achieve significant pain relief in a larger group of patients. Together with the further development of a homebased neurostimulation platform, this could ultimately lead to the implementation of alpha stimulation as an affordable and accessible neurotherapy to manage chronic pain.

\section{AUTHOR'S NOTE}

This manuscript has been released as a pre-print at BioRxiv (Arendsen et al., 2020). 


\section{DATA AVAILABILITY STATEMENT}

The raw data supporting the conclusions of this article will be made available by the authors, without undue reservation.

\section{ETHICS STATEMENT}

The studies involving human participants were reviewed and approved by the North West - Liverpool East Research Ethics Committee (NHS Health Research Authority; reference number 17/NW/0255). The patients/participants provided their written informed consent to participate in this study.

\section{REFERENCES}

Ahn, S., Prim, J. H., Alexander, M. L., McCulloch, K. L., and Fröhlich, F. (2019). Identifying and engaging neuronal oscillations by transcranial alternating current stimulation in patients with chronic low back pain: a randomized, crossover, double-blind, sham-controlled pilot Study . J. Pain 20, 277.e1277.e11. doi: 10.1016/j.jpain.2018.09.004

Arendsen, L. J., Henshaw, J., Brown, C. A., Sivan, M., Taylor, J. R., Trujillo-barreto, N. J., et al. (2020). Entraining alpha activity using visual stimulation in patients with chronic musculoskeletal pain. A feasibility study. BioRxiv [Preprint]. doi: 10.1101/2020.04.27.063339

Arendsen, L. J., Hugh-Jones, S., and Lloyd, D. M. (2018). Transcranial alternating current stimulation at alpha frequency reduces pain when the intensity of pain is uncertain. J. Pain 19, 807-818.

Babiloni, C., Brancucci, A., Del Percio, C., Capotosto, P., Arendt-Nielsen, L., Chen, A. C. N., et al. (2006). Anticipatory electroencephalography alpha rhythm predicts subjective perception of pain intensity. J. Pain 7, 709-717. doi: 10.1016/ j.jpain.2006.03.005

Baliki, M. N., Mansour, A. R., Baria, A. T., and Apkarian, A. V. (2014). Functional reorganization of the default mode network across chronic pain conditions. PLoS One 9:e106133. doi: 10.1371/journal.pone.0106133

Boggio, P. S., Amancio, E. J., Correa, C. F., Cecilio, S., Valasek, C., Bajwa, Z., et al. (2009). Transcranial DC stimulation coupled with TENS for the treatment of chronic pain. Clin. J. Pain 25, 691-695.

Boord, P., Siddall, P. J., Tran, Y., Herbert, D., Middleton, J., and Craig, A. (2008). Electroencephalographic slowing and reduced reactivity in neuropathic pain following spinal cord injury. Spinal Cord 46, 118-123. doi: 10.1038/sj.sc. 3102077

Breivik, H., Collett, B., Ventafridda, V., Cohen, R., and Gallacher, D. (2006). Survey of chronic pain in Europe: prevalence, impact on daily life, and treatment. Eur. J. Pain 10, 287-333. doi: 10.1016/j.ejpain.2005.06.009

Brown, C. A., El-Deredy, W., and Jones, A. K. P. (2014). When the brain expects pain: common neural responses to pain anticipation are related to clinical pain and distress in fibromyalgia and osteoarthritis. Eur. J. Neurosci. 39, 663-672. doi: 10.1111 /ejn. 12420

Brüers, S., and Vanrullen, R. (2018). Alpha power modulates perception independently of endogenous factors. Front. Neurosci. 12:279. doi: 10.3389/ fnins.2018.00279

Camfferman, D., Moseley, G. L., Gertz, K., Pettet, M. W., and Jensen, M. P. (2017). Waking EEG cortical markers of chronic pain and sleepiness. Pain Med. 18, 1921-1931. doi: 10.1093/pm/pnw294

Chou, R., Turner, J. A., Devine, E. B., Hansen, R. N., Sullivan, S. D., Blazina, I., et al. (2015). The effectiveness and risks of long-term opioid therapy for chronic pain: a systematic review for a national institutes of health pathways to prevention Workshop. Ann. Intern. Med. 162, 276-286. doi: 10.7326/M142559

Cleeland, C. S., and Ryan, K. M. (1994). Pain assessment: global use of the brief pain inventory. Ann. Acad. Med. Singapore 23, 129-138.

\section{AUTHOR CONTRIBUTIONS}

AC, AJ, CB, JH, JT, LA, MS, and NT-B: conceptualization, study design, and writing - review and editing. AJ, LA, and MS: recruitment. LA: data collection and writing - original draft preparation. $\mathrm{CB}, \mathrm{JH}$, and LA: analysis. All authors contributed to the article and approved the submitted version.

\section{FUNDING}

This research was funded by MRC $\mathrm{CiC}$ (confidence in concept), grant number MC_PC_16053 and EPSRC Fellowship EP/N006771/1.

Crombez, G., Vlaeyen, J. W. S., Heuts, P. H. T. G., and Lysens, R. (1999). Pain-related fear is more disabling than pain itself: evidence on the role of pain-related fear in chronic back pain disability. Pain 80, 329-339. doi: 10.1016/ S0304-3959(98)00229-2

de Graaf, T. A., Gross, J., Paterson, G., Rusch, T., Sack, A. T., and Thut, G. (2013). Alpha-band rhythms in visual task performance: phase-locking by rhythmic sensory stimulation. PLoS One 8:e60035. doi: 10.1371/journal.pone.0060035

De Vries, M., Wilder-Smith, O. H. G., Jongsma, M. L. A., Van Den Broeke, E. N., Arns, M., Van Goor, H., et al. (2013). Altered resting state EEG in chronic pancreatitis patients: toward a marker for chronic pain. J. Pain Res. 6, 815-824.

Delorme, A., and Makeig, S. (2004). EEGLAB: an open source toolbox for analysis of single-trial EEG dynamics including independent component analysis. J. Neurosci. Methods 134, 9-21. doi: 10.1016/j.jneumeth.2003.10.009

Dworkin, R. H., Turk, D. C., Farrar, J. T., Haythornthwaite, J. A., Jensen, M. P., Katz, N. P., et al. (2005). Core outcome measures for chronic pain clinical trials: IMMPACT recommendations. Pain 113, 9-19. doi: 10.1016/j.pain.2004.09.012

Dworkin, R. H., Turk, D. C., Wyrwich, K. W., Beaton, D., Cleeland, C. S., Farrar, J. T., et al. (2008). Interpreting the clinical importance of treatment outcomes in chronic pain clinical trials: IMMPACT recommendations. J. Pain 9, 105-121. doi: 10.1016/j.jpain.2007.09.005

Ecsy, K., Brown, C. A., and Jones, A. K. P. (2018). Cortical nociceptive processes are reduced by visual alpha-band entrainment in the human brain. Eur. J. Pain 22, 538-550. doi: 10.1002/ejp.1136

Ecsy, K., Jones, A. K. P., and Brown, C. A. (2017). Alpha-range visual and auditory stimulation reduces the perception of pain. Eur. J. Pain 21, 562-572. doi: 10. 1002/ejp.960

Fertonani, A., and Miniussi, C. (2017). Transcranial electrical stimulation: what we know and do not know about mechanisms. Neuroscientist 23, 109-123. doi: 10.1177/1073858416631966

Feurra, M., Pasqualetti, P., Bianco, G., Santarnecchi, E., Rossi, A., and Rossi, S. (2013). State-dependent effects of transcranial oscillatory currents on the motor system: what you think matters. J. Neurosci. 33, 17483-17489. doi: 10.1523/ JNEUROSCI.1414-13.2013

Foxe, J. J., and Snyder, A. C. (2011). The role of alpha-band brain oscillations as a sensory suppression mechanism during selective attention. Front. Psychol. 2:154. doi: 10.3389/fpsyg.2011.00154

Geisser, M. E., Roth, R. S., Theisen, M. E., Robinson, M. E., and Riley, J. L. (2000). Negative affect, self-report of depressive symptoms, and clinical depression: relation to the experience of chronic pain. Clin. J. Pain 16, $110-120$.

Granot, M., and Ferber, S. G. (2005). The roles of pain catastrophizing and anxiety in the prediction of postoperative pain intensity: a prospective study. Clin. J. Pain 21, 439-445.

Gulbinaite, R., van Viegen, T., Wieling, M., Cohen, M. X., and VanRullen, R. (2017). Individual alpha peak frequency predicts $10 \mathrm{~Hz}$ flicker effects on selective attention. J. Neurosci. 37, 10173-10184. doi: 10.1523/jneurosci.1163-17.2017

Gwilym, S. E., Filippini, N., Douaud, G., Carr, A. J., and Tracey, I. (2010). Thalamic atrophy associated with painful osteoarthritis of the hip is reversible after 
arthroplasty: a longitudinal voxel-based morphometric study. Arthr. Rheum. 62, 2930-2940. doi: 10.1002/art.27585

Hauck, M., Domnick, C., Lorenz, J., Gerloff, C., and Engel, A. K. (2015). Top-down and bottom-up modulation of pain-induced oscillations. Front. Hum. Neurosci. 9:375. doi: $10.3389 /$ fnhum.2015.00375

Hazime, F. A., Baptista, A. F., de Freitas, D. G., Monteiro, R. L., Maretto, R. L., Hasue, R. H., et al. (2017). Treating low back pain with combined cerebral and peripheral electrical stimulation: a randomized, double-blind, factorial clinical trial. Eur. J. Pain 21, 1132-1143. doi: 10.1002/ejp.1037

Helfrich, R. F., Schneider, T. R., Rach, S., Trautmann-Lengsfeld, S. A., Engel, A. K., and Herrmann, C. S. (2014). Entrainment of brain oscillations by transcranial alternating current stimulation. Curr. Biol. 24, 333-339. doi: 10.1016/j.cub.2013. 12.041

Herrmann, C. (1997). International experiences with the hospital anxiety and depression scale-a review of validation data and clinical results. J. Psychos. Res. 42, 17-41. doi: 10.1016/S0022-3999(96)00216-4

Herrmann, C. S. (2001). Human EEG responses to 1-100 Hz flicker: resonance phenomena in visual cortex and their potential correlation to cognitive phenomena. Exp. Brain Res. 137, 346-353. doi: 10.1007/s002210100682

Herrmann, C. S., Murray, M. M., Ionta, S., Hutt, A., and Lefebvre, J. (2016). Shaping intrinsic neural oscillations with periodic stimulation. J. Neurosci. 36, 5328-5337. doi: 10.1523/JNEUROSCI.0236-16.2016

Huneke, N. T. M., Brown, C. A., Burford, E., Watson, A., Trujillo-Barreto, N. J., ElDeredy, W., et al. (2013). Experimental placebo analgesia changes resting-state alpha oscillations. PLoS One 8:e78278. doi: 10.1371/journal.pone.0078278

Jensen, M. P., Hakimian, S., Sherlin, L. H., and Fregni, F. (2008). New insights into neuromodulatory approaches for the treatment of pain. J. Pain 9, 193-199. doi: $10.1016 /$ j.jpain.2007.11.003

Jensen, O., and Mazaheri, A. (2010). Shaping functional architecture by oscillatory alpha activity: gating by inhibition. Front. Hum. Neurosci. 4:186. doi: 10.3389/ fnhum.2010.00186

Kaneko, F., Hayami, T., Aoyama, T., and Kizuka, T. (2014). Motor imagery and electrical stimulation reproduce corticospinal excitability at levels similar to voluntary muscle contraction. J. Neuroeng. Rehabil. 11:94. doi: 10.1186/17430003-11-94

Keefe, F. J., Brown, G. K., Wallston, K. A., and Caldwell, D. S. (1989). Coping with rheumatoid arthritis pain: catastrophizing as a maladaptive strategy. Pain 37, 51-56. doi: 10.1016/0304-3959(89)90152-8

Klimesch, W. (2012). A-band oscillations, attention, and controlled access to stored information. Trends Cogn. Sci. 16, 606-617. doi: 10.1016/j.tics.2012.10.007

Kraus, D., Naros, G., Bauer, R., Khademi, F., Leão, M. T., Ziemann, U., et al. (2016). Brain state-dependent transcranial magnetic closed-loop stimulation controlled by sensorimotor desynchronization induces robust increase of corticospinal excitability. Brain Stimulation 9, 415-424. doi: 10.1016/j.brs.2016.02.007

Krause, B., and Cohen Kadosh, R. (2014). Not all brains are created equal: the relevance of individual differences in responsiveness to transcranial electrical stimulation. Front. Syst. Neurosci. 8:25. doi: 10.3389/fnsys.2014.00025

Kulkarni, B., Bentley, D. E., Elliott, R., Julyan, P. J., Boger, E., Watson, A., et al. (2007). Arthritic pain is processed in brain areas concerned with emotions and fear. Arthr. Rheum. 56, 1345-1354. doi: 10.1002/art.22460

Lakatos, P., Schroeder, C. E., Leitman, D. I., and Javitt, D. C. (2013). Predictive suppression of cortical excitability and its deficit in schizophrenia. J. Neurosci. 33, 11692-11702. doi: 10.1523/JNEUROSCI.0010-13.2013

Li, L. M., Uehara, K., and Hanakawa, T. (2015). The contribution of interindividual factors to variability of response in transcranial direct current stimulation studies. Front. Cell. Neurosci. 9:181. doi: 10.3389/fncel.2015.00181

Lim, M., Kim, J. S., Kim, D. J., and Chung, C. K. (2016). Increased low- and highfrequency oscillatory activity in the prefrontal cortex of fibromyalgia patients. Front. Hum. Neurosci. 10:111. doi: 10.3389/fnhum.2016.00111

Locke, H. N., Brooks, J., Arendsen, L. J., Jacob, N. K., Casson, A., Jones, A. K. P., et al. (2020). Acceptability and usability of smartphone-based brainwave entrainment technology used by individuals with chronic pain in a home setting. Br. J. Pain 1-10. doi: 10.1177/2049463720908798

Mathewson, K. E., Prudhomme, C., Fabiani, M., Beck, D. M., Lleras, A., and Gratton, G. (2012). Making waves in the stream of consciousness: entraining oscillations in EEG alpha and fluctuations in visual awareness with rhythmic visual stimulation. J. Cogn. Neurosci. 24, 2321-2333. doi: 10.1162/jocn_a_00288
May, E. S., Butz, M., Kahlbrock, N., Hoogenboom, N., Brenner, M., and Schnitzler, A. (2012). Pre- and post-stimulus alpha activity shows differential modulation with spatial attention during the processing of pain. NeuroImage 62, 1965-1974. doi: 10.1016/j.neuroimage.2012.05.071

Mcwilliams, L. A., Cox, B. J., and Enns, M. W. (2003). Mood and anxiety disorders associated with chronic pain: an examination in a nationally representative sample. Pain 106, 127-133. doi: 10.1016/S0304-3959(03)00301-4

Michail, G., Dresel, C., Witkovskl, V., Stankewitz, A., and Schulz, E. (2016). Neuronal oscillations in various frequency bands differ between pain and touch. Front. Hum. Neurosci. 10:180. doi: 10.3389/fnhum.2016.00182

Neuling, T., Rach, S., and Herrmann, C. S. (2013). Orchestrating neuronal networks: sustained after-effects of transcranial alternating current stimulation depend upon brain states. Front. Hum. Neurosci. 7:161. doi: 10.3389/fnhum. 2013.00161

Nicholas, M. K. (1989). "Self-efficacy and chronic pain," in Paper Presented at the Annual Conference of the British Psychological Society, (St Andrews: University of St Andrews).

Notbohm, A., and Herrmann, C. S. (2016). Flicker regularity is crucial for entrainment of alpha oscillations. Front. Hum. Neurosci. 10:503. doi: 10.3389/ fnhum.2016.00503

Notbohm, A., Kurths, J., and Herrmann, C. S. (2016). Modification of brain oscillations via rhythmic light stimulation provides evidence for entrainment but not for superposition of event-related responses. Front. Hum. Neurosci. 10:10. doi: 10.3389/fnhum.2016.00010

Oostenveld, R., Fries, P., Maris, E., and Schoffelen, J. M. (2011). FieldTrip: open source software for advanced analysis of MEG, EEG, and invasive electrophysiological data. Comput. Intell. Neurosci. 2011:156869. doi: 10.1155/ 2011/156869

Ploner, M., Sorg, C., and Gross, J. (2017). Brain rhythms of pain. Trends Cogn. Sci. 21, 100-110. doi: 10.1016/j.tics.2016.12.001

Ruhnau, P., Neuling, T., Fuscá, M., Herrmann, C. S., Demarchi, G., and Weisz, N. (2016). Eyes wide shut: transcranial alternating current stimulation drives alpha rhythm in a state dependent manner. Sci. Rep. 6:27138. doi: 10.1038/srep27138

Saito, K., Yamaguchi, T., Yoshida, N., Tanabe, S., Kondo, K., and Sugawara, K. (2013). Combined effect of motor imagery and peripheral nerve electrical stimulation on the motor cortex. Exp. Brain Res. 227, 333-342. doi: 10.1007/ s00221-013-3513-5

Sarnthein, J., Stern, J., Aufenberg, C., Rousson, V., and Jeanmonod, D. (2006). Increased EEG power and slowed dominant frequency in patients with neurogenic pain. Brain 129, 55-64. doi: 10.1093/brain/awh631

Schabrun, S. M., Jones, E., Elgueta Cancino, E. L., and Hodges, P. W. (2014). Targeting chronic recurrent low back pain from the top-down and the bottomup: a combined transcranial direct current stimulation and peripheral electrical stimulation intervention. Brain Stimulation 7, 451-459. doi: 10.1016/j.brs.2014. 01.058

Shao, S., Shen, K., Yu, K., Wilder-Smith, E. P. V., and Li, X. (2012). Frequencydomain EEG source analysis for acute tonic cold pain perception. Clin. Neurophysiol. 123, 2042-2049. doi: 10.1016/j.clinph.2012.02.084

Spaak, E., de Lange, F. P., and Jensen, O. (2014). Local entrainment of alpha oscillations by visual stimuli causes cyclic modulation of perception. J. Neurosci. 34, 3536-3544. doi: 10.1523/JNEUROSCI.4385-13.2014

Tan, G., Jensen, M. P., Thornby, J. I., and Shanti, B. F. (2004). Validation of the brief pain inventory for chronic nonmalignant pain. J. Pain 5, 133-137. doi: 10.1016/j.jpain.2003.12.005

Tang, J., and Gibson, S. J. (2005). A psychophysical evaluation of the relationship between trait anxiety, pain perception, and induced state anxiety. J. Pain 6 , 612-619. doi: 10.1016/j.jpain.2005.03.009

Te Woerd, E. S., Oostenveld, R., De Lange, F. P., and Praamstra, P. (2017). Impaired auditory-to-motor entrainment in Parkinson's disease. J. Neurophysiol. 117, 1853-1864. doi: 10.1152/jn.00547.2016

Thut, G., Schyns, P. G., and Gross, J. (2011). Entrainment of perceptually relevant brain oscillations by non-invasive rhythmic stimulation of the human brain. Front. Psychol. 2:170. doi: 10.3389/fpsyg.2011.00170

Tu, Y., Zhang, Z., Tan, A., Peng, W., Hung, Y. S., Moayedi, M., et al. (2016). Alpha and gamma oscillation amplitudes synergistically predict the perception of forthcoming nociceptive stimuli. Hum. Brain Mapp. 37, 501-514. doi: 10. $1002 / \mathrm{hbm} .23048$ 
Turk, D. C. (2002). Clinical effectiveness and cost-effectiveness of treatments for patients with chronic pain. Clin. J. Pain 18, 355-365.

Turk, D. C., Wilson, H. D., and Cahana, A. (2011). Treatment of chronic noncancer pain. Lancet 377, 2226-2235. doi: 10.1016/S0140-6736(11)60402-9

Van Hecke, O., Torrance, N., and Smith, B. H. (2013). Chronic pain epidemiology and its clinical relevance. Br. J. Anaesth. 111, 13-18. doi: 10.1093/bja/aet123

Vossen, A., Gross, J., and Thut, G. (2015). Alpha power increase after transcranial alternating current stimulation at alpha frequency (a-tACS) reflects plastic changes rather than entrainment. Brain Stimulation 8, 499-508. doi: 10.1016/ j.brs.2014.12.004

Wallston, K. A., Strudler Wallston, B., and DeVellis, R. (1978). Development of the multidimensional health locus of control (MHLC) scales. Heath Educ. Monogr. 6, 160-170. doi: 10.1002/jgm

Walsh, T. M. (1998). Pain correlates of depressed mood in young adults. Pain Res. Manag. 3, 135-144.
Zigmond, A. S., and Snaith, R. P. (1983). The hospital anxiety and depression scale. Acta Psychiatr. Scand. 67, 361-370. doi: 10.1111/j.1600-0447.1983.tb0 9716.x

Conflict of Interest: The authors declare that the research was conducted in the absence of any commercial or financial relationships that could be construed as a potential conflict of interest.

Copyright $\odot 2020$ Arendsen, Henshaw, Brown, Sivan, Taylor, Trujillo-Barreto, Casson and Jones. This is an open-access article distributed under the terms of the Creative Commons Attribution License (CC BY). The use, distribution or reproduction in other forums is permitted, provided the original author(s) and the copyright owner(s) are credited and that the original publication in this journal is cited, in accordance with accepted academic practice. No use, distribution or reproduction is permitted which does not comply with these terms. 


\title{
Non-invasive Brain and Spinal Stimulation for Pain and Related Symptoms in Multiple Sclerosis: A Systematic Review
}

\author{
Chiara Zucchella ${ }^{1 \dagger}$, Elisa Mantovani ${ }^{2 \dagger}$, Roberto De Icco ${ }^{3,4}$, Cristina Tassorelli ${ }^{3,4}$, \\ Giorgio Sandrini ${ }^{3,4}$ and Stefano Tamburin ${ }^{1,2 *}$
}

${ }^{1}$ Section of Neurology, Department of Neurosciences, Verona University Hospital, Verona, Italy, ${ }^{2}$ Department of Neurosciences, Biomedicine and Movement Sciences, University of Verona, Verona, Italy, ${ }^{3}$ Neurorehabilitation Unit, IRCCS Mondino Foundation, Pavia, Italy, ${ }^{4}$ Department of Brain and Behavioral Sciences, University of Pavia, Pavia, Italy

OPEN ACCESS

Edited by:

Marco Solmi,

University of Padua, Italy

Reviewed by:

Enrico Collantoni,

University of Padua, Italy

Francesco Monaco,

Azienda Sanitaria Locale Salerno, Italy

${ }^{*}$ Correspondence:

Stefano Tamburin

stefano.tamburin@univr.it orcid.org/0000-0002-1561-2187

†These authors have contributed equally to this work

Specialty section: This article was submitted to

Perception Science,

a section of the journa

Frontiers in Neuroscience

Received: 31 March 2020

Accepted: 09 October 2020

Published: 20 November 2020

Citation:

Zucchella C, Mantovani E, De Icco $R$

Tassorelli C, Sandrini $G$ and

Tamburin S (2020) Non-invasive Brain and Spinal Stimulation for Pain and

Related Symptoms in Multiple

Sclerosis: A Systematic Review.

Front. Neurosci. 14:547069.

doi: 10.3389/fnins.2020.547069
Background: Neuropathic and nociceptive pain frequently affect patients with multiple sclerosis (MS), with a prevalence close to $90 \%$ and significant impact on general health and quality of life. Pharmacological strategies are widely used to treat pain in MS, but their effectiveness and side-effects are controversial. Among non-pharmacological treatments for pain, non-invasive brain and spinal stimulation (NIBSS) has shown promising preliminary results in MS.

Objective: Systematic review to investigate the effect of NIBSS for the management of pain in MS.

Methods: A literature search using Pubmed, Science Direct and Web of Science was conducted from databases inception to February 21, 2020 for studies assessing the analgesic effect of NIBSS on pain in MS.

Results: A total of 279 records were title- and abstract-screened, nine were assessed for full text and included. The NIBSS techniques explored were transcranial direct current stimulation $(N=5)$, transcranial magnetic stimulation $(N=2)$, transcranial random noise stimulation $(N=1)$, transcutaneous spinal direct current stimulation $(N=1)$. The targets were the primary motor cortex $(\mathrm{M} 1 ; N=4)$, the left dorsolateral pre-frontal cortex (DLPFC; $N=3)$, the spinal cord $(N=1)$, unspecified brain target $(N=1)$. The study designs were randomized $(N=7)$, open label $(N=1)$, single case report $(N=1)$. Despite the differences in study design, target and NIBSS technique that impeded a meta-analysis, all the studies converge in showing a significant improvement of pain after active NIBSS with less consistent effects on other symptoms of the pain-related cluster (depression, fatigue, cognition) and quality of life.

Conclusions: Excitatory NIBSS over M1, left DLPFC and spinal cord appear to be the most effective protocols for pain in MS. Open questions include the use of neurophysiological or neuroimaging surrogate outcome measures, the stratification of 
patients according to the clinical profiles and underlying pathogenetic mechanisms and the combination of NIBSS to pharmacological treatment, neurorehabilitation, or psychotherapy to improve the clinical effect. The duration of the effect to NIBSS and the feasibility and efficacy of telemedicine NIBSS protocols are other open key questions.

Keywords: depression, fatigue, multiple sclerosis, non-invasive brain stimulation (NIBS), non-invasive spinal stimulation, pain, systematic review

\section{INTRODUCTION}

Pain is common in patients with multiple sclerosis (MS; O'Connor et al., 2008) and has a significant burden on general and psychological health, quality of life (QoL), work and social role (Kalia and O'Connor, 2005; Foley et al., 2013). Pain was reported in $29-86 \%$ of MS patients, such a wide range being due to different diagnostic criteria and assessment methods, heterogeneity of the samples, and different disease stages span when pain was assessed (Nurmikko et al., 2010; Thompson et al., 2010; Foley et al., 2013; Solaro et al., 2013).

MS-related pain may present with high variability in terms of clinical presentation, severity, onset (Feinstein et al., 2015) and may be reported at any stage of the disease including the early ones (Solaro et al., 2004), but its prevalence increases over time because of the disease process itself, MS-related complications and aging (Khan et al., 2013).

The most common types of pain in MS are classified as nociceptive or neuropathic pain (Magrinelli et al., 2013). Nociceptive pain is a physiological response secondary to the activation of nociceptors aimed to warn the brain of real or potential tissue damage. In contrast, neuropathic pain is due to a lesion or disease of the peripheral or central parts of the somatosensory system (Finnerup et al., 2016). Pain associated with MS stands amongst the most common causes of chronic neuropathic pain (Scholz et al., 2019).

Nociceptive pain in MS patients includes (a) musculoskeletal system, which is often related to abnormal or forced posture, (b) headache, which may predate or be unrelated to MS, (c) post-traumatic pain, and (d) pain secondary to treatment, e.g., painful pathological fractures secondary to long-term steroid use and immobilization causing osteoporosis (Solaro et al., 2018). MS patients may experience both pain and spasticity, and pain secondary to spasticity or painful tonic spasms is a subtype of nociceptive pain frequently reported in MS (Solaro et al., 2018). Among primary headaches, migraine was reported to be three times more frequent in MS patients than in the general population, to carry a considerable disability, and to be associated with a more symptomatic course and an increased contrast enhancing lesion activity compared to MS patients without headache (Kister et al., 2010; Graziano et al., 2015).

Central neuropathic pain in MS includes (a) ongoing neuropathic pain of limbs, (b) Lhermitte's phenomenon, (c) trigeminal neuralgia, and (d) pain associated with optic neuritis, all of which are associated with inflammation and secondary degeneration of central nervous system sensory pathways (Truini et al., 2013; Solaro et al., 2018).
MS patients may also report, to a variable extent, psychogenic, idiopathic or mixed pain (Truini et al., 2013). Psychogenic pain is defined as somatoform pain associated with psychiatric conditions (i.e., depression, or anxiety), or as pain behaviors associated with chronic refractory pain; idiopathic pain includes poorly understood and to some extent controversial chronic pain conditions, such as fibromyalgia, or persistent idiopathic facial pain, while mixed pain includes different pain types, often difficult to separate and quantify, caused by the same disease through different pathophysiological mechanisms (Truini et al., 2013).

According to the neuropathic pain definition and grading system (Finnerup et al., 2016), neuropathic pain can be separated from nociceptive and other types of pain based on the clinical or instrumental demonstration of a lesion or disease involving the somatosensory system (La Cesa et al., 2015; Porro et al., 2016), but this task may be difficult in MS patients, because of the frequent clinical or subclinical involvement of posterior columns of the spinal cord and/or brain somatosensory pathways.

Pain can interfere with daily functioning by reducing mobility, working activities, and engagement in recreational activities, and may cause a consistent impairment of participation in home, social, and other activities (Ehde et al., 2003; Svendsen et al., 2003, 2005; Kalia and O'Connor, 2005; Grasso et al., 2008; Gromisch et al., 2019). MS is one of the most common causes of neurological disability in young adults, and MS-related pain may impact this population of working-age patients and represent an independent risk factor for social disadvantage (Shahrbanian et al., 2013).

Pain is frequently associated to fatigue, depression and cognitive complaints in MS patients (Penner et al., 2007; Trojan et al., 2007), and these three symptoms may influence each other (Harrison et al., 2015; Marck et al., 2017), being considered a symptom cluster with some shared pathogenetic mechanisms and that should be targeted together to improve patients' QoL (Shahrbanian et al., 2015).

Despite its high prevalence and severe burden, MS-related pain is still an ongoing and challenging issue with no established treatment. Pharmacological treatment of pain in MS patients is based on guidelines derived from other clinical conditions (Finnerup et al., 2015) and includes (a) non-steroidal antiinflammatory drugs for nociceptive pain, (b) anticonvulsant, (c) antidepressants, and (d) botulinum toxin for neuropathic pain, (e) cannabinoids, (f) muscle relaxants and (g) intrathecally administered baclofen for pain secondary to spasticity or to painful tonic spasms, (h) opioid analgesics for mixed pain (Solaro et al., 2013). However, results of pharmacological approaches, 
even with complex therapeutic schemes, are often poor and disturbing side effects, such in the case of opioids, frequently cause the patients to drop-out (Urits et al., 2019).

The need of more effective treatments with safer profiles and fewer adverse effects has paved the way to non-pharmacological interventions for pain in MS (Amatya et al., 2018; Aboud and Schuster, 2019). In this field, evidence on neuromodulation through non-invasive brain and spinal stimulation (NIBSS) has been published in recent years, and preliminary results appear to be promising (Abboud et al., 2017; Iodice et al., 2017). NIBBS techniques can be grouped into two categories, namely electrical and magnetic stimulation, according to the differential way of inducing their neurobiological effects. Electrical stimulation is the application of current/voltage to two or more surface electrodes, whereas magnetic stimulation results from a current passing through a coil positioned on the head to generate a magnetic field, inducing in turn an electrical field and a current density field in the brain (Peterchev et al., 2012). Electrical stimulation techniques include, but are not limited to, transcranial direct current stimulation (tDCS), transcranial alternating current stimulation, transcranial random noise stimulation (tRNS), and transcutaneous spinal direct current stimulation (tsDCS).

tDCS and repetitive transcranial magnetic stimulation (rTMS) are the most widely used types of non-invasive neuromodulation techniques.

tDCS is based on a battery-powered device connected to two electrodes that deliver low-amplitude direct currents that induce neuronal membrane depolarization or hyperpolarization leading to changes in the excitability of specific brain areas being stimulated (Nitsche and Paulus, 2000). In healthy subjects, anodal tDCS delivered to the motor cortex causes neurons depolarization and increased cortical excitability, while cathodal tDCS hyperpolarizes neurons, thus reducing cortical excitability (Nitsche and Paulus, 2000). However, the effect of tDCS polarity on motor cortex excitability might not be generalized to other cortices, and several factors (e.g., stimulation duration, current intensity, tDCS setup) may affect the direction of the induced effects (Lefaucheur et al., 2017).

tDCS induces sustained changes in cortical excitability if applied for a sufficient period of time, (Nitsche and Paulus, 2001). tDCS is safe and has been reported to cause only mild side effects, e.g., skin irritation or burning sensation, especially when used daily and/or with higher current intensity (Antal et al., 2017). This side effect could be minimized when using saline-soaked electrodes (Antal et al., 2017).

rTMS is delivered to the brain by a phasic electrical current that circulates through an insulated wire coil placed over the skull and generates a transient high-intensity magnetic field, which propagates in space and induces a secondary current that depolarizes neurons in targeted brain regions, finally leading to neuroplastic changes (Paulus et al., 2013). High- and lowfrequency rTMS have short-lasting excitatory and inhibitory effects on the motor cortex, respectively (Paulus et al., 2013), but the effect of rTMS frequency cannot be generalized to all cortical sites (Lefaucheur et al., 2020). Theta-burst stimulation (TBS) is a novel modified rTMS technique that causes consistent, long-lasting facilitatory and inhibitory effects on synaptic transmission according to the TBS protocol used (Huang et al., 2005). Intermittent TBS (iTBS) causes prevalent facilitation, while continuous TBS leads to prevalent inhibition (Huang et al., 2005). rTMS side effects are transient and include headache, scalp discomfort and hearing disorders, more commonly after highfrequency treatments, while epileptic seizures very seldom occur if appropriate guidelines are applied and patients are accurately selected (Rossi et al., 2009; Lefaucheur et al., 2020).

New NIBSS protocol have been recently introduced in the clinical setting, including tRNS (Terney et al., 2008) and tsDCS (Berra et al., 2019).

tRNS is a non-invasive transcranial electrical stimulation technique that produces a random electrical oscillation spectrum within defined thresholds, following the Gaussian curve around an offset midpoint (Terney et al., 2008). tRNS was reported to induce consistent excitability increases lasting $60 \mathrm{~min}$ after stimulation when applied to the primary motor cortex (M1), with higher frequencies $(100-640 \mathrm{~Hz})$ being responsible for generating this hyperexcitability probably through repeated opening of sodium channels. tRNS was found to have similar effects than tDCS without the constraint of current flow direction sensitivity characteristic of the latter (Terney et al., 2008; Palm et al., 2016).

Anodal tsDCS may represent a potentially self-administered NIBSS technique in those clinical conditions that are characterized by changes in spinal cord interneurons, and was found to inhibit nociceptive specific responses, such as the nociceptive withdrawal reflex (NWR; Cogiamanian et al., 2011) and the NWR temporal summation threshold (TST; Perrotta et al., 2016), which may contribute to the pathogenesis of pain in MS (Berra et al., 2019).

Very recent evidence-based guidelines indicated level A evidence (definite efficacy) for high-frequency rTMS of M1 contralateral to the painful side for neuropathic pain and level B evidence (probable efficacy) for high-frequency rTMS of the left M1 or DLPFC for improving quality of life or pain, respectively, in fibromyalgia (Lefaucheur et al., 2020). Despite the evidence of the efficacy of high frequency rTMS for the treatment of some types of pain, NIBSS is not routinely used in patients with MS and pain. The aim of this systematic review is to collect and report data on the role of NIBSS for the management of MS-related pain.

\section{METHODS}

This systematic review was conducted according to the Preferred Reporting Items for Systematic Reviews and Meta-Analyses (PRISMA) recommendations (Liberati et al., 2009; Moher et al., 2015).

\section{Eligibility Criteria}

Studies assessing the effect of NIBSS on MS-related neuropathic and/or nociceptive pain as primary or secondary outcome were considered eligible for this systematic review. Both controlled and exploratory studies were eligible and included and no restrictions were placed on the publication date of the studies.

We excluded reviews, commentaries, abstracts, conference papers, and studies on animal models or healthy subjects. 
Studies exploring NIBSS without therapeutic goals, e.g., aiming to assess neurophysiological measures to explore MS-related pathophysiology were also excluded. We also excluded studies that explored the effect of NIBSS on other MS outcomes (e.g., fatigue, motor function, spasticity, sensory function, bladder function, cognition) but did not include pain.

Outcomes of interest were pain measured with clinically validated tools (e.g., Visual Analog Scale, Numerical Rating Scale, Short Form McGill Pain Questionnaire; Brief Pain Inventory) and other MS symptoms related or secondary to pain (e.g., fatigue, spasticity, psychosocial outcomes, QoL).

According to the PICOS model, the Participants were MS patients, the Intervention was NIBSS, the Comparison was sham NIBSS, other pain treatment or no treatment, the Outcome was pain either neuropathic or nociceptive, the Study design was controlled and exploratory studies.

\section{Search Strategy}

The Pubmed, Science Direct and Web of Science databases were searched for peer-reviewed papers exploring the therapeutic role of NIBSS for MS-related pain, published from database inception until February 21, 2020. Only studies written in English were considered. Different search strings were used according to the maximum number of Boolean operators that are allowed in each of the selected databases.

The search string for Pubmed and Web of Science was: (pain OR chronic pain OR pain management OR pain intractable OR pain measurement OR pain threshold OR nociceptors OR neuropathic pain OR neuralgia) AND (multiple sclerosis OR demyelinating disease) AND (transcranial magnetic stimulation OR TMS OR r-TMS OR theta burst stimulation OR theta burst OR TBS OR c-TBS OR i-TBS OR NIBS OR non-invasive brain stimulation OR brain stimulation OR transcranial direct current stimulation OR tDCS OR tES OR transcranial electrical stimulation OR tCS OR transcranial current stimulation).

The search strategy for Science Direct database included: (pain OR nociceptors OR neuralgia) AND (multiple sclerosis OR demyelinating disease) AND (transcranial magnetic stimulation OR TMS OR r-TMS OR theta burst stimulation), then (pain OR nociceptors OR neuralgia) AND (multiple sclerosis OR demyelinating disease) AND (theta burst OR TBS OR c-TBS OR i-TBS), (pain OR nociceptors OR neuralgia) AND (multiple sclerosis OR demyelinating disease) AND (NIBS OR noninvasive brain stimulation OR brain stimulation OR transcranial direct current stimulation), and (pain OR nociceptors OR neuralgia) AND (multiple sclerosis OR demyelinating disease) AND (tDCS OR tES OR transcranial electrical stimulation OR transcranial current stimulation).

\section{Study Selection}

Search results were uploaded to Rayyan software, a web-based app to facilitate collaborations among reviewers during the study selection phase (Ouzzani et al., 2016). Two authors (CZ, EM) independently screened titles and abstracts. The reference lists of relevant papers were manually checked to identify additional significant studies potentially missed in the databases search.
Any disagreement was planned to be solved by consensus or consulting a third reviewer (ST).

\section{Data Collection Procedure}

Two authors (CZ, EM) independently extracted the following data from the included papers: study design (i.e., randomized, cross-over, parallel, open label, single arm trials), type of MS, sample size, gender and age of included patients, type of pain targeted by the NIBSS intervention, type of NIBSS applied, targeted central nervous system area, NIBSS protocol features, primary and secondary outcome measures, follow-up duration, side effects, and results.

\section{Risk of Bias Assessment}

Risk of bias was assessed independently by two authors ( $C Z$ and EM) using the revised tool for Risk of Bias in randomized trials (RoB 2.0), which consists of five domains and an overall judgement (Sterne et al., 2019). The five domains are: (1) bias arising from the randomization process; (2) bias due to deviations from the intended interventions; (3) bias due to missing outcome data; (4) bias in measurement of the outcome; (5) bias in selection of the reported result (Sterne et al., 2019).

Any disagreement was planned to be solved via consensus or by consulting a third author (ST). Risk of bias was classified as "low," "some concerns" "high."

\section{Data Analysis}

A systematic and descriptive analysis of the results was provided with information presented in the text and Tables 1-3 to summarize and explain the characteristics and findings of the included studies. A meta-analysis was not feasible due to the small number of studies and subjects, as well as to the methodological, clinical and statistical heterogeneity of the included studies.

\section{RESULTS}

\section{Identification and Selection of the Studies}

A total of 279 records were identified. After removal of duplicates, 186 papers were screened through title and abstract and 9 papers were obtained for full-text screening. The reference lists of relevant papers were inspected for additional studies potentially missed in the databases search, but no significant papers were further added. Two authors (CZ, EM) independently evaluated the 9 papers selected for the full-text examination. Disagreement was solved by consensus between the two reviewers, therefore the advice of a third reviewer (ST) was not required.

Nine studies fulfilled the criteria and were included in the systematic review (Figure 1).

\section{Description of the Included Studies}

The included papers evaluated the efficacy of NIBSS on neuropathic or nociceptive pain in MS patients. Studies were grouped according to the NIBSS technique (i.e., tDCS, rTMS, tRNS, tsDCS). 
TABLE 1 | Overview of the tDCS studies included in the review.

\begin{tabular}{|c|c|c|c|c|c|c|c|c|c|c|c|}
\hline \multicolumn{2}{|r|}{ Study } & \multicolumn{3}{|c|}{ Patients features } & \multicolumn{2}{|r|}{ tDCS features } & \multicolumn{5}{|c|}{ Findings } \\
\hline Ref & Design & MS type & $\begin{array}{l}\text { Sample size } \\
\text { (gender, age) }\end{array}$ & Pain type & Target & Protocol & $\begin{array}{l}\text { Primary } \\
\text { outcomes }\end{array}$ & $\begin{array}{l}\text { Secondary } \\
\text { outcomes }\end{array}$ & Follow up & Side effects & Results \\
\hline $\begin{array}{l}\text { Mori et al. } \\
\text { (2010) }\end{array}$ & $\begin{array}{l}\text { Randomized, } \\
\text { parallel, double-blind } \\
\text { sham-controlled }\end{array}$ & $\mathrm{RR}$ & $\begin{array}{c}19(\mathrm{~W}: 11, \mathrm{M}: 8 \\
\text { age } 44.8 \pm 27.5)\end{array}$ & Central NP & M1 & $\begin{array}{l}\text { Five daily sessions, } 2 \mathrm{~mA} \text {, } \\
20 \text { min; anode: C3/C4, } \\
\text { cathode: contralateral } \\
\text { supraorbital area }\end{array}$ & $\begin{array}{l}\text { Pain VAS, } \\
\text { PMQ-SF }\end{array}$ & $\begin{array}{l}\text { QoL } \\
\text { (MSQOL-54), } \\
\text { depression (BDI), } \\
\text { anxiety (VAS) }\end{array}$ & 4 weeks & None & $\begin{array}{l}\text { Pain and QoL } \\
\text { significantly } \\
\text { improved to active } \\
\text { tDCS; effects lasted } \\
3 \text { weeks }\end{array}$ \\
\hline $\begin{array}{l}\text { Ayache et al. } \\
\text { (2016) }\end{array}$ & $\begin{array}{l}\text { Randomized, } \\
\text { double-blind, } \\
\text { cross-over } \\
\text { sham-controlled }\end{array}$ & $\begin{array}{l}\text { RR: 11, SP: } \\
\text { 4, PP: } 1\end{array}$ & $\begin{array}{l}16(\mathrm{~W}: 13, \mathrm{M}: 3 \\
\text { age } 48.9 \pm 10.0)\end{array}$ & NP & Left DLPFC & $\begin{array}{l}\text { Three daily sessions, } \\
2 \mathrm{~mA}, 20 \text { min; active- } \\
\text { sham } 3 \text { weeks washout; } \\
\text { anode: } \mathrm{F3} \text {, cathode: right } \\
\text { supraorbital region }\end{array}$ & $\begin{array}{l}\text { Pain VAS, } \\
\text { BPI }\end{array}$ & $\begin{array}{l}\text { Mood (HADS), } \\
\text { attention (ANT), } \\
\text { fatigue (MFIS) }\end{array}$ & None & $\begin{array}{l}\text { Insomnia, nausea, } \\
\text { headache (both } \\
\text { arms), phosphene } \\
\text { (sham) }\end{array}$ & $\begin{array}{l}\text { Pain improved to } \\
\text { active tDCS; no } \\
\text { effect on secondary } \\
\text { outcomes }\end{array}$ \\
\hline $\begin{array}{l}\text { Kasschau et al. } \\
\text { (2016) }\end{array}$ & Feasibility pilot & $\begin{array}{l}\text { SP: 12, RR: } \\
\text { 6, PP: } 2 \\
\text { (EDSS: } 1-8)\end{array}$ & $\begin{array}{l}20(\mathrm{~W}: 17, \mathrm{M}: 3 \\
\text { age } 51 \pm 9.25)\end{array}$ & NS & Left DLPFC & $\begin{array}{l}\text { Ten remotely supervised } \\
\text { tDCS sessions, } 20 \text { min } \\
\text { and cognitive } \\
\text { rehabilitation; uniform } \\
\text { bilateral DLPFC (left } \\
\text { anodal) montage }\end{array}$ & $\begin{array}{l}\text { Completion } \\
\text { of at least } 8 \\
\text { tDCS } \\
\text { sessions }\end{array}$ & $\begin{array}{l}\text { Pain (PROMIS, } \\
\text { VAS), fatigue } \\
\text { (PROMIS, VAS), } \\
\text { affect (PANAS), } \\
\text { cognitive speed } \\
\text { (ANT-I) }\end{array}$ & None & None relevant & $\begin{array}{l}\text { Nineteen patients } \\
\text { completed } 8 \text { tDCS } \\
\text { sessions; all } \\
\text { outcomes } \\
\text { consistently } \\
\text { improved }\end{array}$ \\
\hline $\begin{array}{l}\text { Rudroff et al. } \\
\text { (2019) }\end{array}$ & Case report & $\mathrm{RR}$ & 1 (M, 52 years) & NP & Left M1 & $\begin{array}{l}\text { Five daily sessions, } 2 \text { mA, } \\
20 \text { min; anode: C3, } \\
\text { cathode: contralateral } \\
\text { supraorbital area }\end{array}$ & $\begin{array}{l}\text { Pain VAS, } \\
\text { NPSI }\end{array}$ & {$\left[{ }^{18} \mathrm{~F}\right]-\mathrm{FDG}$ PET } & None & None & $\begin{array}{l}\text { Pain improved and } \\
\left.{ }^{18} \mathrm{~F}\right]-\mathrm{FDG} \text { PET } \\
\text { uptake increased in } \\
\text { the thalamus after } \\
\text { tDCS }\end{array}$ \\
\hline $\begin{array}{l}\text { Workman et al. } \\
\text { (2020) }\end{array}$ & $\begin{array}{l}\text { Randomized, } \\
\text { double-blind } \\
\text { sham-controlled } \\
\text { cross-over pilot }\end{array}$ & $\mathrm{RR}$ & $\begin{array}{c}6(\mathrm{~W}: 3, \mathrm{M}: 3 \\
\text { age } 46.7 \pm 14.1)\end{array}$ & NS & M1 & $\begin{array}{l}\text { Five daily sessions, } 2 \mathrm{~mA} \text {, } \\
20 \text { min; anode: C3/C4, } \\
\text { cathode: contralateral } \\
\text { supraorbital area }\end{array}$ & $\begin{array}{l}\text { Pain VAS, } \\
\text { fatigue (FSS), } \\
\text { depression } \\
\text { (BDI) }\end{array}$ & $\begin{array}{l}\text { Isokinetic leg } \\
\text { strength, } \\
\text { fatigability } \\
\text { testing }\end{array}$ & None & None & $\begin{array}{l}\text { Pain, fatigability and } \\
\text { fatigue improved to } \\
\text { active tDCS }\end{array}$ \\
\hline
\end{tabular}

ANT, Attention Network Test; ANT-I, Attention Network Test-Interaction; BDI, Beck Depression Inventory; BPI, Brief Pain Inventory; DLPFC, dorsolateral pre-frontal cortex; EDSS, Expanded Disability Status Scale; ${ }^{18}$ F] FDG-PET, [18F fluorodeoxyglucose positron emission tomography; FSS, Fatigue Severity Scale; HADS, 14-item Hospital Anxiety and Depression Scale; M, men; mA, milliampere; MFIS, Multidimensional Fatigue Inventory Score; MPQ-SF, McGill pain questionnaire short form; MS, multiple sclerosis; MSQOL-54, Multiple Sclerosis Quality of Life-54 scale; M1, primary motor cortex; NP, neuropathic pain; NPSI, Neuropathic Pain Symptom Inventory; NS, not specified; PANAS, Positive and Negative Affect Schedule; PP, primary progressive; PROMIS, Patient Reported Outcomes Measurement Information System; QoL, quality of life; Ref, reference; RR, relapsing remitting; SP, secondary progressive; tDCS, transcranial direct current stimulation; VAS, Visual Analog Scale; W, women. 
TABLE 2 | Overview of the rTMS studies included in the review.

\begin{tabular}{|c|c|c|c|c|c|c|c|c|c|c|c|}
\hline \multicolumn{2}{|c|}{ Study } & \multicolumn{3}{|c|}{ Patients features } & \multicolumn{2}{|r|}{ rTMS features } & \multicolumn{5}{|c|}{ Findings } \\
\hline Ref & Design & MS type & $\begin{array}{l}\text { Sample size } \\
\text { (gender, age) }\end{array}$ & Pain type & Target & Protocol & $\begin{array}{l}\text { Primary } \\
\text { outcomes }\end{array}$ & $\begin{array}{l}\text { Secondary } \\
\text { outcomes }\end{array}$ & Follow up & $\begin{array}{l}\text { Side } \\
\text { effects }\end{array}$ & Results \\
\hline Seada et al. (2013) & $\begin{array}{l}\text { Randomized, } \\
\text { parallel (control } \\
\text { group: LLT) }\end{array}$ & NS & $\begin{array}{c}30 \text { (age } 56.4 \pm \\
6.6)\end{array}$ & $\mathrm{TN}$ & NS & $10 \mathrm{~Hz}, 50 \mathrm{~mA}, 20 \mathrm{~min}$ & Pain NRS & $\begin{array}{l}\text { Oral mouth } \\
\text { opening, } \\
\text { masseter and } \\
\text { temporalis } \\
\text { muscle tension } \\
\text { and CMAP }\end{array}$ & None & NS & $\begin{array}{l}\text { Both groups } \\
\text { improved, no } \\
\text { statistical } \\
\text { comparison } \\
\text { between the two } \\
\text { groups }\end{array}$ \\
\hline Korzhova et al. (2019) & $\begin{array}{l}\text { Randomized, } \\
\text { parallel, single blind } \\
\text { sham-controlled }\end{array}$ & SP & $\begin{array}{c}34 \text { (W: 20, M: } \\
14)\end{array}$ & $\begin{array}{l}\text { Spasticity } \\
\text { pain }\end{array}$ & M1 & $\begin{array}{l}\text { Ten sessions for } 5 \text { days for } \\
2 \text { weeks; HF rTMS }(20 \mathrm{~Hz} \text {, } \\
30 \mathrm{~min}) \text {; iTBS ( } 35 \mathrm{~Hz}, 1,200 \\
\text { pulses, } 10 \mathrm{~min})\end{array}$ & $\begin{array}{l}\text { Spasticity } \\
\text { (MAS, NAS, } \\
\text { SESS) }\end{array}$ & $\begin{array}{l}\text { Pain, fatigue } \\
\text { (MFIS) }\end{array}$ & $\begin{array}{l}2 \text { and } 12 \\
\text { weeks }\end{array}$ & None & $\begin{array}{l}\text { MAS significantly } \\
\text { improved to HF } \\
\text { rTMS and iTBS; } \\
\text { SESS significantly } \\
\text { improved to iTBS } \\
\text { and lasted at } \\
\text { follow-up; pain and } \\
\text { fatigue significantly } \\
\text { improved to HF } \\
\text { rTMS }\end{array}$ \\
\hline
\end{tabular}

CMAP, compound muscle action potential; HF, high frequency; Hz, hertz; iTBS, intermittent theta-burst stimulation; LLT, low-level laser therapy; M, men; mA, milliampere; MAS, Modified Ashworth Scale; MFIS, Multidimensional Fatigue Inventory Score; MS, multiple sclerosis; M1, primary motor cortex; NAS, numerical analog scale; NRS, numerical rating scale; NS, not specified; Ref, reference; rTMS, repetitive transcranial magnetic stimulation; SESS, Subjective Evaluating Spasticity Scale; SP, secondary progressive; tDCS, transcranial direct current stimulation; TNP, trigeminal neuropathy; W, women.

TABLE 3 | Overview of the other NIBSS studies included in the review.

\begin{tabular}{|c|c|c|c|c|c|c|c|c|c|c|c|}
\hline \multicolumn{2}{|c|}{ Study } & \multicolumn{3}{|c|}{ Patients features } & \multicolumn{2}{|r|}{ NIBSS features } & \multicolumn{5}{|c|}{ Findings } \\
\hline Ref & Design & MS type & $\begin{array}{l}\text { Sample size } \\
\text { (gender, age) }\end{array}$ & Pain type & Target & NIBSS type and protocol & $\begin{array}{l}\text { Primary } \\
\text { outcomes }\end{array}$ & $\begin{array}{l}\text { Secondary } \\
\text { outcomes }\end{array}$ & Follow up & $\begin{array}{l}\text { Side } \\
\text { effects }\end{array}$ & Results \\
\hline Palm et al. (2016) & $\begin{array}{l}\text { Randomized, } \\
\text { double-blind, } \\
\text { sham-controlled } \\
\text { cross-over }\end{array}$ & $\begin{array}{l}\text { RR: } 11, \mathrm{SP}: \\
\quad \text { 4, PP: } 1\end{array}$ & $\begin{array}{l}16(\mathrm{~W}: 3, \mathrm{M}: 13 \\
\text { age } 47.4 \pm 8.9)\end{array}$ & NP & Left DLPFC & $\begin{array}{l}\text { tRNS: } 3 \text { consecutive days, } \\
2 \mathrm{~mA}, 20-30 \text { min; } 3 \text { weeks }\end{array}$ & $\begin{array}{l}\text { Pain VAS, } \\
\text { BPI; } \\
\text { attention } \\
\text { (ANT) }\end{array}$ & $\begin{array}{l}\text { Depression and } \\
\text { anxiety (HADS); } \\
\text { fatigue (MFIS); } \\
\text { PREPS; frontal } \\
\text { midline theta }\end{array}$ & None & None & $\begin{array}{l}\text { Pain scores and } \\
\text { PREPS N2-P2 } \\
\text { amplitude } \\
\text { decreased to real } \\
\text { tRNS }\end{array}$ \\
\hline Berra et al. (2019) & $\begin{array}{l}\text { Pilot randomized, } \\
\text { parallel, double-blind } \\
\text { sham-controlled }\end{array}$ & $\begin{array}{l}\text { SP: 24, PP: } 5 \text {, } \\
\text { RR: } 4 \text { (EDSS: } \\
5.9 \pm 1.3)\end{array}$ & $\begin{array}{l}33 \text { (W: } 25, \mathrm{M}: 8 \\
\text { age: real } 57.6 \pm \\
9.1 \text {, sham: } 54.0 \\
\quad \pm 7.8)\end{array}$ & NP & Spinal cord & $\begin{array}{l}\text { tsDCS: } 10 \text { sessions in } 2 \\
\text { weeks, } 2 \text { mA, } 20 \text { min; } \\
\text { anode: thoracic spinal cord; } \\
\text { cathode: right shoulder } \\
\text { (suprascapular region) }\end{array}$ & $\begin{array}{l}\text { NPSI, } \\
\text { spasticity } \\
\text { (AS), fatigue } \\
\text { (FSS) }\end{array}$ & NWR, NWR TST & 1 month & None & $\begin{array}{l}\text { NPSI significantly } \\
\text { reduced to real } \\
\text { tsDCS with effect } \\
\text { lasting up to } 1 \\
\text { month; trend toward } \\
\text { inhibition of NWR } \\
\text { responses to real } \\
\text { tsDCS }\end{array}$ \\
\hline
\end{tabular}

ANT, Attention Network Test; AS, Ashworth scale; BPI, Brief Pain Inventory; DLPFC, dorsolateral pre-frontal cortex; EDSS, Expanded Disability Status Scale; FSS, Fatigue Severity Scale; HADS, 14-item Hospital Anxiety and Depression Scale; M, men; mA, milliampere; MFIS, Multidimensional Fatigue Inventory Score; MS, multiple sclerosis; NIBSS, non-invasive brain and spinal stimulation; NP, neuropathic pain; NPSI, Neuropathic Pain Symptom Inventory; NWR, nociceptive withdrawal reflex; PP, primary progressive; PREPS, pain related evoked potentials; Ref, reference; RR, relapsing remitting; SP, secondary progressive; tRNS, transcranial random noise stimulation; tsDCS, transcutaneous spinal direct current stimulation; TST, temporal summation threshold; VAS, Visual Analog Scale; W, women. 


\section{tDCS Studies}

We found five studies that explored tDCS for the treatment of pain in MS (Mori et al., 2010; Ayache et al., 2016; Kasschau et al., 2016; Rudroff et al., 2019; Workman et al., 2020).

Mori et al. (2010) investigated whether anodal tDCS may be effective in reducing central drug-resistant chronic neuropathic pain in MS with a randomized, parallel, double blind, shamcontrolled study. Nineteen patients with relapsing-remitting MS received a 5 day treatment with sham or real tDCS over M1 contralateral to the painful body region. Real tDCS resulted in significant reduction of pain and improvement of QoL in comparison to sham tDCS and the effects lasted up to 3 weeks after the stimulation period. The Authors hypothesized that pain reduction was the result of functional plastic changes in brain structures involved in the pathogenesis of chronic neuropathic pain (Mori et al., 2010).

Ayache et al. (2016) reported a prospective, randomized, cross-over, sham-controlled study to evaluate the effect of tDCS over the DLPFC in sixteen MS patients with a history of neuropathic pain since $>3$ months. The primary outcome was pain intensity, and secondary outcomes included mood, attention and fatigue. Patients received real or sham anodal tDCS blocks in a random order, each consisting of tDCS sessions in 3 consecutive days, separated by a 3 week washout period. Real tDCS yielded significant analgesic effects compared to sham, but no effects on mood, attention, or fatigue. The Authors suggested that analgesia might have occurred through specific modulation of the emotional pain network by tDCS over the left DLPFC (Ayache et al., 2016).

Since repeated tDCS sessions are needed to obtain a therapeutic effect, but for many MS patients visiting the clinic daily for the treatment is not feasible, Kasschau et al. (2016) performed a pilot study to test the feasibility and safety of a remotely supervised tDCS protocol for home delivery using a specially designed equipment and a telemedicine platform. Twenty MS (any subtype) patients with an extended range of disability (Expanded Disability Status Scale $=1-$ 8) underwent 10 tDCS sessions over the left DLPFC, each lasting $20 \mathrm{~min}$, across 2 weeks. Nineteen of them (95\%) completed at least eight sessions, meeting the compliance criteria, while 17 (85\%) completed the full 10 study sessions. Improvement of all secondary clinical outcomes (cognitive measures, pain, fatigue, mood) was reported. Despite the limitations of the study, i.e., lack of a control group, and patient economic compensation that might have increased the attendance, this telemedicine tDCS protocol suggests that access to tDCS can be expanded in MS patients (Kasschau et al., 2016).

Rudroff et al. (2019) reported a 52 year old man with a 13 year history of relapsing-remitting MS, moderate disability and central neuropathic pain, treated with anodal tDCS $(20 \mathrm{~min}$, 5 consecutive days) over the left M1. Pain scores improved and metabolism, assessed with $\left[{ }^{18} \mathrm{~F}\right]$ fluorodeoxyglucose positron emission tomography, increased in both thalami, suggesting at a very preliminary stage that $\mathrm{tDCS}$ may induce functional changes in brain structures critical in the pathogenesis of neuropathic pain (Rudroff et al., 2019).
Workman et al. (2020) reported the results of a double blind, sham-controlled, randomized cross-over pilot study to investigate whether tDCS may improve the MS symptom cluster of pain, fatigue and depression. Six moderately disabled MS patients underwent two randomly ordered blocks of stimulation (real or sham tDCS), each block composed of five daily 20 min sessions, with the anode placed over the M1 representation of the more-affected leg and the cathode over the contralateral supraorbit. Real tDCS improved performance fatigability, perceived fatigue and pain but not depression in comparison to sham (Workman et al., 2020).

\section{rTMS Studies}

We found two studies that explored rTMS in MS with pain as primary (Seada et al., 2013) or secondary outcome (Korzhova et al., 2019).

Seada et al. (2013) reported a randomized, parallel study that compared rTMS and low-level laser therapy for trigeminal neuralgia in thirty MS patients. Patients were randomly divided into rTMS group (age $46.6 \pm 9.6)$ who received rTMS $(10 \mathrm{~Hz}$, $50 \mathrm{~mA}, 20 \mathrm{~min}$ ) with the coil placed tangentially over the head of the patient contralateral to trigeminal pain and the laser group (age $48.8 \pm 6.3$ ) who received low-level laser therapy (15 mW helium-neon laser, $830 \mathrm{~A}$ wave length, $150-170 \mathrm{mw} / \mathrm{cm}^{2}$ laser beam density, $10 \mathrm{~min}$ ). Both groups reported improvements, but no statistical comparison between the two groups was performed (Seada et al., 2013). Indeed, some methodological issues should be noted, such as poor description of rTMS targeting, absence of a sham group, unclear significance of outcome measures and some poorly reported data (e.g., the overall mean age was $56.4 \pm$ 6.6 that is in contrast with the age of the two groups, see above).

Korzhova et al. (2019) performed a parallel, randomized controlled trial to compare the effects of two rTMS protocols, i.e., high frequency $(20 \mathrm{~Hz})$ and iTBS in comparison to a sham group on the level of spasticity (primary outcome) and associated symptoms, including pain that was a secondary outcome, in thirty-four secondary progressive MS patients. All patients underwent real (high frequency rTMS: twelve patients, iTBS: twelve patients) or sham rTMS (ten patients) once a day for 5 consecutive days for 2 weeks. Concurrently with rTMS, all patients received a course of 10 physical therapy sessions. Both high frequency rTMS and iTBS significantly reduced spasticity with some evidence favoring a longer-lasting effect of iTBS and a reduction of pain and fatigue to high frequency rTMS (Korzhova et al., 2019).

\section{Other NIBSS Studies}

We found two studies (Table 3), one dealing with tRNS (Palm et al., 2016) and one with tsDCS (Berra et al., 2019).

Palm et al. (2016) explored the effect of tRNS over the left DLPFC on affective symptoms, attention, fatigue, and pain by exploring pain perception and attentional resources in a prospective randomized, cross-over, sham-controlled study of sixteen MS patients with neuropathic pain. Each patient randomly received two tRNS blocks (i.e., real, sham), each consisting of three consecutive 20 min daily sessions, separated by a 3 week washout interval. All patients were evaluated for 


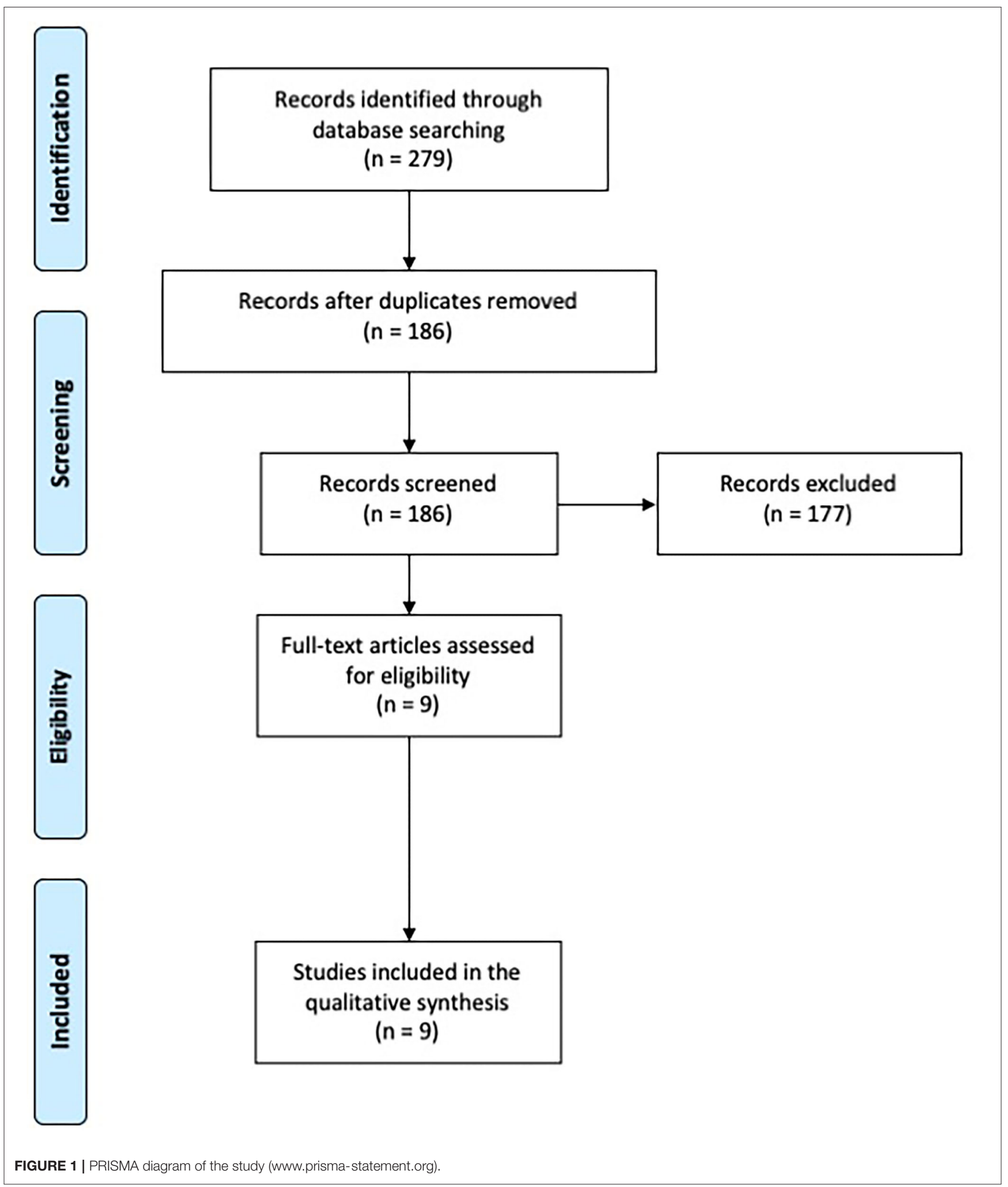

pain, attention and mood and underwent a neurophysiological evaluation using pain related evoked potentials. Compared to sham, real tRNS showed a trend toward decreased N2-P2 amplitude of pain related evoked potentials and improvement of pain ratings, while attention performance and mood scales did not change (Palm et al., 2016). 


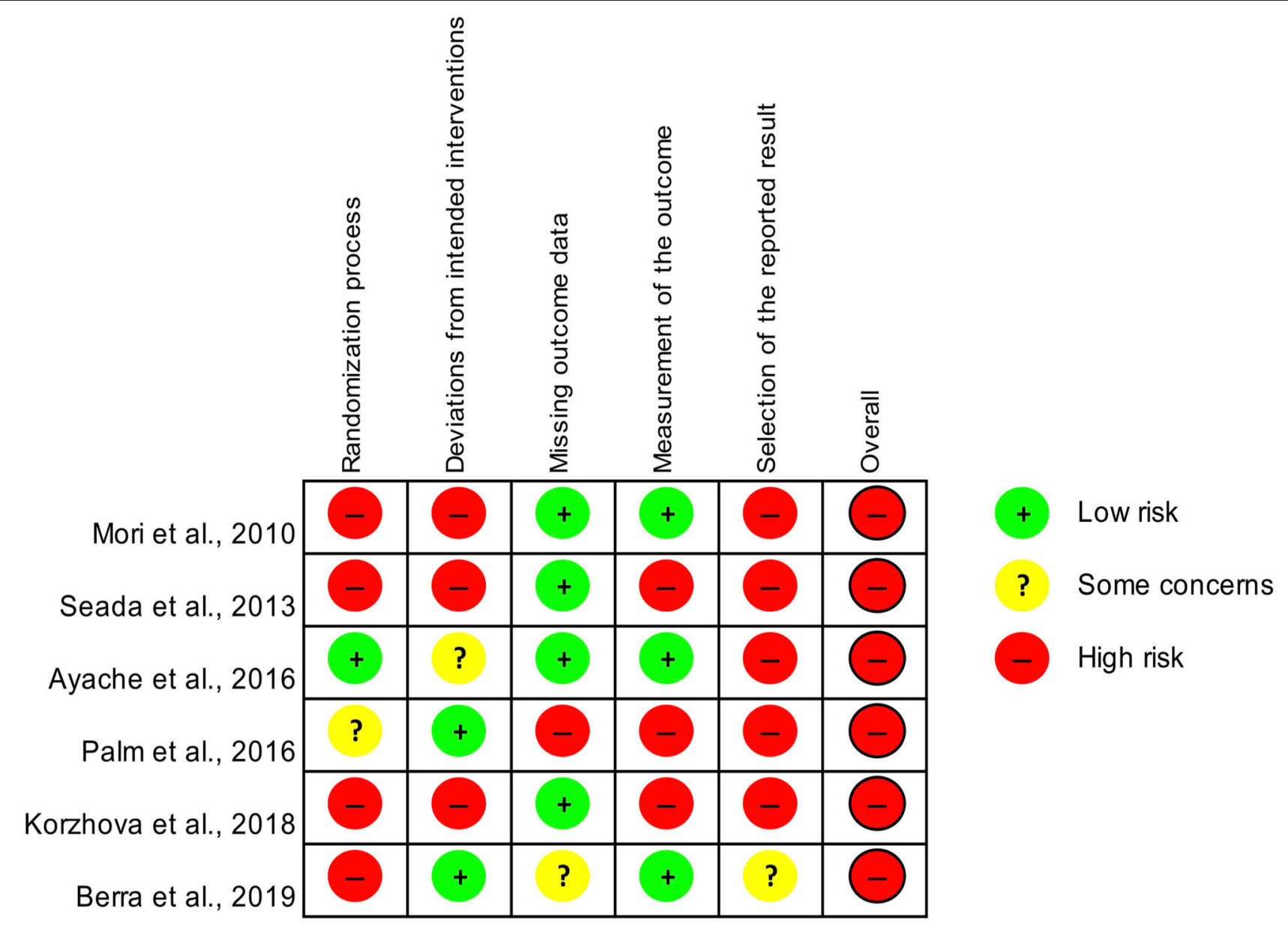

FIGURE 2 | Assessment of the risk of bias for controlled studies included in the systematic review according to the RoB 2.0 tool.

Berra et al. (2019) explored whether anodal tsDCS could represent an effective, safe and well-tolerated treatment for neuropathic pain in MS in a double-blind sham-controlled, parallel design study involving thirty-three patients. Real tsDCS resulted in a significant improvement in neuropathic pain scores at the end of treatment that persisted 1 month later, but no changes in spasticity and fatigue. In a subgroup of patients, who underwent NWR and NWR TST, a nonsignificant trend toward an inhibition of NWR responses to real tsDCS was found, suggesting the effect of tsDCS was related to modulation of spinal nociception (Berra et al., 2019).

\section{Risk of Bias Assessment}

Only controlled studies with samples of at least ten patients (Mori et al., 2010; Seada et al., 2013; Ayache et al., 2016; Palm et al., 2016; Berra et al., 2019; Korzhova et al., 2019) were assessed for the risk of bias according to the RoB 2.0 tool, which yielded an overall high risk for all of them (Figure 2).

\section{DISCUSSION}

The present systematic review, which was aimed to collect and report evidence on the role of NIBSS for the management of MS-related pain, yielded nine studies, of whom five on tDCS (Mori et al., 2010; Ayache et al., 2016; Kasschau et al., 2016; Rudroff et al., 2019; Workman et al., 2020), two on rTMS (Seada et al., 2013; Korzhova et al., 2019), one on tRNS (Palm et al., 2016), and one on tsDCS (Berra et al., 2019).

Four studies targeted M1, all of them using excitatory protocols, i.e., anodal tDCS in three of them (Mori et al., 2010; Rudroff et al., 2019; Workman et al., 2020), and high-frequency rTMS in another one (Korzhova et al., 2019), with the targeted side being the one contralateral to the most affected limbs in three of them (Mori et al., 2010; Korzhova et al., 2019; Workman et al., 2020), and the left side in another one (Rudroff et al., 2019). Three studies targeted the left DLPFC with excitatory protocols, i.e., anodal tDCS in two of them (Ayache et al., 2016; Kasschau et al., 2016) and tRNS in another one (Palm et al., 2016). One study targeted the spinal cord with anodal tsDCS (Berra 
et al., 2019) and the brain target was not specified in one study (Seada et al., 2013).

Seven studies had a randomized design, either double-blind (Mori et al., 2010; Ayache et al., 2016; Palm et al., 2016; Berra et al., 2019; Workman et al., 2020), single-blind (Korzhova et al., 2019), or with blinding not specified (Seada et al., 2013), one had an open design (Kasschau et al., 2016), and another one was a single case report (Rudroff et al., 2019). Sham NIBSS was the control group in six of the seven randomized studies (Mori et al., 2010; Ayache et al., 2016; Palm et al., 2016; Berra et al., 2019; Korzhova et al., 2019; Workman et al., 2020), while lowlevel laser therapy was used as control condition in one study (Seada et al., 2013).

Only three studies reported a follow-up that ranged from 4 to 12 weeks (Mori et al., 2010; Berra et al., 2019; Korzhova et al., 2019).

Apart from the single case report, the sample size ranged from 6 to 34 with a total of 175 patients (women: 92, men: 53; gender not specified: 30 ) included in the nine studies we found; among them there were 58 relapsing-remitting patients, 78 secondary progressive patients, 9 primary progressive patients, while MS type was not specified in 30 patients.

Pain was the primary outcome in seven of the included studies (Mori et al., 2010; Seada et al., 2013; Ayache et al., 2016; Palm et al., 2016; Berra et al., 2019; Rudroff et al., 2019; Workman et al., 2020) and secondary outcome in the other two studies, one being on feasibility of a telemedicine tDCS protocol (Kasschau et al., 2016), and the other one having spasticity as the primary outcome (Korzhova et al., 2019). The type of MS-related pain addressed in the study, either as primary or secondary outcome measures was neuropathic pain in seven studies (Mori et al., 2010; Seada et al., 2013; Ayache et al., 2016; Palm et al., 2016; Berra et al., 2019; Rudroff et al., 2019; Workman et al., 2020), more specifically central neuropathic pain in one of them (Mori et al., 2010) and trigeminal neuralgia in another one (Seada et al., 2013), while pain type was not specified in one study (Kasschau et al., 2016), and another study was focused on spasticity-related pain (Korzhova et al., 2019).

One or more of the other symptoms belonging to the symptoms cluster associated with pain in MS, i.e., fatigue, depression and cognitive complaints (Penner et al., 2007; Trojan et al., 2007; Harrison et al., 2015; Marck et al., 2017), and QoL were assessed as primary or secondary outcomes in six studies (Mori et al., 2010; Ayache et al., 2016; Kasschau et al., 2016; Palm et al., 2016; Berra et al., 2019; Korzhova et al., 2019).

The variability of NIBSS techniques, central nervous system targets, study designs including sham control and blinding, patient populations, outcomes, and the presence of follow-up data in a minority of the studies included did not allow a meta-analysis of the findings. However, the results of all the included studies converge in showing a significant improvement in pain after active NIBSS with less consistent effects on the other symptoms of the pain-related cluster and QoL (Mori et al., 2010; Seada et al., 2013; Ayache et al., 2016; Kasschau et al., 2016; Palm et al., 2016; Berra et al., 2019; Korzhova et al., 2019; Rudroff et al., 2019; Workman et al., 2020).

Most studies used validated scales to measure pain, such as the Visual Analog Scale, the Numerical Rating Scale, the Short
Form McGill Pain Questionnaire, the Brief Pain Inventory or similar outcomes (Jensen and Karoly, 2001). However, despite most of the reports addressed neuropathic pain, only one study (Berra et al., 2019) used an outcome measure that was specific for this type of pain, i.e., the Neuropathic Pain Symptom Inventory (Bouhassira et al., 2004), which can be used to characterize subgroups of neuropathic pain patients and verify whether they respond differentially to a therapeutic intervention (Magrinelli et al., 2013). Moreover, because of the complexity of pain experience, and the coexistence of psychiatric and cognitive symptoms (Chiaravalloti and De Luca, 2008), MS patients may have difficulty in reporting their experience through a single intensity scale (Amatya et al., 2018), but only one study used a multidimensional pain scale, i.e., the Brief Pain Inventory (Ayache et al., 2016).

Some studies explored the MS symptom cluster related to pain. Depression, anxiety, mood, and affect were explored in five studies (Mori et al., 2010; Ayache et al., 2016; Kasschau et al., 2016; Palm et al., 2016; Workman et al., 2020), but found to improve to NIBSS only in one of them whose design was unblinded and open (Kasschau et al., 2016) and thus prone to placebo effect. The absence of changes in mood outcomes in the studies targeting the DLPFC (Ayache et al., 2016; Palm et al., 2016), might be due to the short stimulation period, since the effect on mood to DLPFC non-invasive stimulation is known to be dose-dependent (Palm et al., 2016).

Fatigue was an outcome measure in six studies (Ayache et al., 2016; Kasschau et al., 2016; Palm et al., 2016; Berra et al., 2019; Korzhova et al., 2019; Workman et al., 2020), with one of them performing a fatigability test with isokinetic leg strength in addition to subjective measures (Workman et al., 2020). Apart from the open feasibility study that found an unspecific improvement of all outcomes (Kasschau et al., 2016), the other studies converge in showing improvement of fatigue and fatigability to M1 excitatory NIBSS (Korzhova et al., 2019; Workman et al., 2020), but no effect to either targeting DLPFC (Ayache et al., 2016; Palm et al., 2016), or the spinal cord (Berra et al., 2019), thus suggesting that M1 may represent an interesting target for this MS symptom, which is very bothersome, may heavily impact on QoL and functioning, and has no established treatment (Miller and Soundy, 2017). However, it is worth noting that M1 reports applied 5 (Workman et al., 2020) and 10 sessions (Korzhova et al., 2019), respectively, whereas DLPFC studies (Ayache et al., 2016; Palm et al., 2016) applied only 3 sessions. Studies that targeted primary fatigue in MS documented significant effects by stimulating the DLPFC for $\geq 5$ sessions and suggest that targeting the MS fatigue loop with 5 or more NIBSS sessions could improve the symptom (Ayache and Chalah, 2018). In MS patients with pain, fatigue is probably secondary to pain rather than representing primary fatigue, and future studies should better explore this topic.

Two studies explored the effect of NIBSS on spasticity (Berra et al., 2019; Korzhova et al., 2019) and showed improvement of this outcome to high-frequency rTMS over M1 (Korzhova et al., 2019), but not to anodal tsDCS (Berra et al., 2019), offering some ground to future studies aimed to explore excitatory NIBBS over M1 as a therapeutic strategy in MS-related spasticity. 
Three studies explored attentional changes to left DLPFC anodal tDCS (Ayache et al., 2016; Kasschau et al., 2016) and tRNS (Palm et al., 2016). Only one of them (Kasschau et al., 2016) reported improvement of attentional outcomes, but the open design of the study, absence of blinding, and coexisting treatment with web-based cognitive rehabilitation might have represented potential bias factors. The left DLPFC might not represent the best target for this cognitive domain, which is frequently impaired in MS (Chiaravalloti and De Luca, 2008), as anodal tDCS over the right posterior parietal cortex was reported to be more effective on attentional measures than the left DLPFC (Roy et al., 2015).

QoL was explored in one study only, with no evidence of efficacy of NIBSS (Mori et al., 2010), probably because this outcome is likely to improve in response to change of a symptom cluster instead of a single symptom (Ehde et al., 2003; Svendsen et al., 2003, 2005; Kalia and O'Connor, 2005).

Three studies presented follow-up data and were consistent in showing that NIBSS effects outlasted the period of stimulation; in particular, pain reduction lasted up to 1 month after the end of NIBSS treatment (Mori et al., 2010; Berra et al., 2019; Korzhova et al., 2019).

Some interesting pieces of information can be derived from the instrumental outcomes reported in some of the studies we collected. Rudroff et al. (2019) documented increased $\left[{ }^{18} \mathrm{~F}\right]$ fluorodeoxyglucose positron emission tomography uptake in the thalamus after anodal tDCS and suggested that NIBSS may modulate sensory discriminative and affective-motivational pain pathways. However, this finding was derived from a single case report and should be replicated in larger patient populations. Moreover, validated and clinically reliable neuroimaging markers of MS-related pain are still lacking (Seixas et al., 2014). Palm et al. (2016) reported reduced amplitude of the N2-P2 component of pain related evoked potentials. This finding might be related to a change in cortical processing of pain, but should be taken with care, because of the uncertainties on the fibers stimulated by the electrode they used (Perchet et al., 2012) and the presence of saliency and habituation effects that may represent bias factors for the interpretation of the significance of the cortical components to pain stimuli (Iannetti et al., 2008). Berra et al. (2019) reported a trend for a change in NWR in parallel to neuropathic pain improvement to tsDCS. NWR is a reliable neurophysiological tool for the assessment of the spinal and supraspinal mechanisms of pain processing but is sensitive to physiological changes and to some drugs (Sandrini et al., 2005) and not widely used in the clinical setting.

M1 and the DLPFC were the two most common NIBSS sites for the treatment of MS-related pain in the studies we reviewed. M1 stimulation is supposed to induce analgesic effects trough an antidromic top-down modulation of thalamo-cortical pathways (Nguyen et al., 2011). The DLPFC plays a pivotal role in pain processing, as well as cognitive and emotional pain-related behaviors, and its stimulation may act through a descending modulation of opioidergic pathways and in the affective and attentional aspects of pain (Seminowicz and Moayedi, 2017). However, the underlying brain networks that mediate pain relief to these brain targets are only partially understood, and they may be partially disrupted in patients with MS.
In conclusion, the results of the studies included in this systematic review indicate overall a positive effect of various NIBSS techniques on pain and some related symptoms in patients with MS. These results are promising but far from being conclusive, because of the small sample size in the included studies, the variability in NIBSS technique, targeted area, patient population, outcomes, the absence of follow-up for many of the studies, and the overall high risk of bias. It is worth noting that the assessment of the risk of bias in our study differs from that in a recent Cochrane review focused on the management of chronic pain in MS patients (Amatya et al., 2018), because of the different risk of bias tools used in the two studies. Excitatory NIBSS over M1, the left DLPFC and the spinal cord appear to be the most promising protocols to be used in future larger therapeutic studies for MS-related pain.

Open questions include the use of neurophysiological or neuroimaging surrogate outcome measures and the stratification of patients according to the clinical profiles and underlying pathogenetic mechanisms (Magrinelli et al., 2013). Future studies should explore whether NIBSS protocols associated to pharmacological treatment, neurorehabilitation, or psychotherapy (Arewasikporn et al., 2018) may be more effective than NIBSS alone on pain, related symptoms and/or QoL.

The duration of the effect to NIBSS is another key question. Unlike other clinical conditions such as depression, there is still no consensus regarding the treatment of MS related pain with maintenance sessions of NIBBS beyond the normal treatment duration. Studies on other neuropathic pain conditions suggest that the analgesic effect to rTMS of M1 is favored by longer session duration and serial treatment, i.e., greater number of sessions (Lefaucheur et al., 2020). Additional studies are thus needed to address this important question.

Regarding the last point, the use of telemedicine NIBSS techniques may be promising (Kasschau et al., 2016), but results are still contradictory, in that a randomized controlled pilot study documented that patient-delivered tDCS was not effective on mixed types of neuropathic pain in prior responders to rTMS (O'Neill et al., 2018). Telemedicine could also lead to advantages for designing future NIBBS clinical trials to test more appropriate stimulation parameters, treatment duration and follow-ups. The remote provision of NIBSS (e.g., tDCS), safely administered at home may be an interesting option to provide accessible maintenance protocol treatments, to explore the effects of NIBSS in an ecological context, and to overcome the limitations of notportable NIBBS devices. The use of telemedicine could also be helpful for research purposes, allowing for a better control of experimental variables and thus increasing the reproducibility of studies' findings.

\section{AUTHOR CONTRIBUTIONS}

The study has been designed by CZ, EM, RD, CT, GS, and ST. Data have been gathered by CZ and EM, under the supervision of ST. Data have been analyzed by $\mathrm{CZ}$ and EM. The manuscript has been drafted by CZ, EM, and ST. RD, CT, and GS revised the manuscript for important intellectual content. All authors approved the final version of the manuscript. 


\section{REFERENCES}

Abboud, H., Hill, E., Siddiqui, J., Serra, A., and Walter, B. (2017). Neuromodulation in multiple sclerosis. Mult. Scler. 23, 1663-1676. doi: $10.1177 / 1352458517736150$

Aboud, T., and Schuster, N. M. (2019). Pain management in multiple sclerosis: a review of available treatment options. Curr. Treat. Options Neurol. 21:62. doi: 10.1007/s11940-019-0601-2

Amatya, B., Young, J., and Khan, F. (2018). Non-pharmacological interventions for chronic pain in multiple sclerosis. Cochrane Database Syst. Rev. 12:CD012622. doi: 10.1002/14651858.CD012622.pub2

Antal, A., Alekseichuk, I., Bikson, M., Brockmöller, J., Brunoni, A. R., Chen, R., et al. (2017). Low intensity transcranial electric stimulation: safety, ethical, legal regulatory and application guidelines. Clin. Neurophysiol. 128, 1774-1809. doi: 10.1016/j.clinph.2017.06.001

Arewasikporn, A., Turner, A. P., Alschuler, K. N., Hughes, A. J., and Ehde, D. M. (2018). Cognitive and affective mechanisms of pain and fatigue in multiple sclerosis. Health Psychol. 37, 544-552. doi: 10.1037/hea0000611

Ayache, S. S., and Chalah, M. A. (2018). Transcranial direct current stimulation: a glimmer of hope for multiple sclerosis fatigue? J. Clin. Neurosci. 55, 10-12. doi: 10.1016/j.jocn.2018.06.002

Ayache, S. S., Palm, U., Chalah, M. A., Al-Ani, T., Brignol, A., Abdellaoui, M., et al. (2016). Prefrontal tDCS decreases pain in patients with multiple sclerosis. Front. Neurosci. 10:147. doi: 10.3389/fnins.2016.00147

Berra, E., Bergamaschi, R., De Icco, R., Dagna, C., Perrotta, A., Rovaris, M., et al. (2019). The effects of transcutaneous spinal direct current stimulation on neuropathic pain in multiple sclerosis: clinical and neurophysiological assessment. Front. Hum. Neurosci. 13:31. doi: 10.3389/fnhum.2019.00031

Bouhassira, D., Attal, N., Fermanian, J., Alchaar, H., Gautron, M., Masquelier, E., et al. (2004). Development and validation of the neuropathic pain symptom inventory. Pain 108, 248-257. doi: 10.1016/j.pain.2003.12.024

Chiaravalloti, N. D., and De Luca, J. (2008). Cognitive impairment in multiple sclerosis. Lancet Neurol. 7, 1139-1151. doi: 10.1016/S1474-4422(08)70259-X

Cogiamanian, F., Vergari, M., Schiaffi, E., Marceglia, S., Ardolino, G., Barbieri, S., et al. (2011). Transcutaneous spinal cord direct current stimulation inhibits the lower limb nociceptive flexion reflex in human beings. Pain 152, 370-375. doi: 10.1016/j.pain.2010.10.041

Ehde, D. M., Gibbons, L. E., Chwastiak, L., Bombardier, C. H., Sullivan, M. D., and Kraft, G. H. (2003). Chronic pain in a large community sample of persons with multiple sclerosis. Mult. Scler. 9, 606-611. doi: 10.1191/1352458503ms939oa

Feinstein, A., Freeman, J., and Lo, A. C. (2015). Treatment of progressive multiple sclerosis: what works, what does not, and what is needed. Lancet Neurol. 14, 194-207. doi: 10.1016/S1474-4422(14)70231-5

Finnerup, N. B., Attal, N., Haroutounian, S., McNicol, E., Baron, R., Dworkin, R. H., et al. (2015). Pharmacotherapy for neuropathic pain in adults: a systematic review and meta-analysis. Lancet Neurol. 14, 162-173. doi: 10.1016/S1474-4422(14)70251-0

Finnerup, N. B., Haroutounian, S., Kamerman, P., Baron, R., Bennett, D. L., Bouhassira, D., et al. (2016). Neuropathic pain: an updated grading system for research and clinical practice. Pain 157, 1599-1606. doi: 10.1097/j.pain.0000000000000492

Foley, P. L., Vesterinen, H. M., Laird, B. J., Sena, E. S., Colvin, L. A., Chandran, S., et al. (2013). Prevalence and natural history of pain in adults with multiple sclerosis: systematic review and meta-analysis. Pain 154, 632-642. doi: 10.1016/j.pain.2012.12.002

Grasso, M. G., Clemenzi, A., Tonini, A., Pace, L., Casillo, P., Cuccaro, A., et al. (2008). Pain in multiple sclerosis: a clinical and instrumental approach. Mult. Scler. 14, 506-513. doi: 10.1177/1352458507085553

Graziano, E., Hagemeier, J., Weinstock-Guttman, B., Ramasamy, D. P., and Zivadinov, R. (2015). Increased contrast enhancing lesion activity in relapsingremitting multiple sclerosis migraine patients. Neuroimage Clin. 9, 110-116. doi: 10.1016/j.nicl.2015.07.013

Gromisch, E. S., Kerns, R. D., and Beauvais, J. (2019). Pain-related illness intrusiveness is associated with lower activity engagement among persons with multiple sclerosis. Mult. Scler. Relat. Disord. 38:101882. doi: 10.1016/j.msard.2019.101882

Harrison, A. M., McCracken, L. M., Bogosian, A., and Moss-Morris, R. (2015). Towards a better understanding of MS pain: a systematic review of potentially modifiable psychosocial factors. J. Psychosom. Res. 78, 12-24. doi: 10.1016/j.jpsychores.2014.07.008

Huang, Y. Z., Edwards, M. J., Rounis, E., Bhatia, K. P., and Rothwell, J. C. (2005). Theta burst stimulation of the human motor cortex. Neuron 45, 201-206. doi: 10.1016/j.neuron.2004.12.033

Iannetti, G. D., Hughes, N. P., Lee, M. C., and Mouraux, A. (2008). Determinants of laser-evoked EEG responses: pain perception or stimulus saliency? J. Neurophysiol. 100, 815-828. doi: 10.1152/jn.00097.2008

Iodice, R., Manganelli, F., and Dubbioso, R. (2017). The therapeutic use of noninvasive brain stimulation in multiple sclerosis - a review. Restor. Neurol. Neurosci. 35, 497-509. doi: 10.3233/RNN-170735

Jensen, M. P., and Karoly, P. (2001). "Self-report scales and procedures for assessing pain in adults," in Handbook of Pain Assessment, eds D. C. Turk and R. Melzack (New York, NY: Guilford Press), 15-34.

Kalia, L. V., and O'Connor, P. W. (2005). Severity of chronic pain and its relationship to quality of life in multiple sclerosis. Mult. Scler. 11, 322-327. doi: 10.1191/1352458505ms1168oa

Kasschau, M., Reisner, J., Sherman, K., Bikson, M., Datta, A., and Charvet, L. E. (2016). Transcranial direct current stimulation is feasible for remotely supervised home delivery in multiple sclerosis. Neuromodulation 19, 824-831. doi: $10.1111 /$ ner. 12430

Khan, F., Amatya, B., and Kesselring, J. (2013). Longitudinal 7-year follow- up of chronic pain in persons with multiple sclerosis in the community. J. Neurol. 206, 2005-2031. doi: 10.1007/s00415-013-6925-z

Kister, I., Caminero, A. B., Monteith, T. S., Soliman, A., Bacon, T. E., Bacon, J. H., et al. (2010). Migraine is comorbid with multiple sclerosis and associated with a more symptomatic MS course. J. Headache Pain 11, 417-425. doi: 10.1007/s10194-010-0237-9

Korzhova, J., Bakulin, I., Sinitsyn, D., Poydasheva, A., Suponeva, N., Zakharova, M., et al. (2019). High-frequency repetitive transcranial magnetic stimulation and intermittent theta-burst stimulation for spasticity management in secondary progressive multiple sclerosis. Eur. J. Neurol. 26, 680-e44. doi: 10.1111/ene.13877

La Cesa, S., Tamburin, S., Tugnoli, V., Sandrini, G., Paolucci, S., Lacerenza, M., et al. (2015). How to diagnose neuropathic pain? The contribution from clinical examination, pain questionnaires and diagnostic tests. Neurol. Sci. 36, 2169-2175. doi: 10.1007/s10072-015-2382-z

Lefaucheur, J. P., Aleman, A., Baeken, C., Benninger, D. H., Brunelin, J., Di Lazzaro, V., et al. (2020). Evidence-based guidelines on the therapeutic use of repetitive transcranial magnetic stimulation (rTMS): an update (2014-2018). Clin. Neurophysiol. 131, 474-528. doi: 10.1016/j.clinph.2020.02.003

Lefaucheur, J. P., Antal, A., Ayache, S. S., Benninger, D. H., Brunelin, J., Cogiamanian, F., et al. (2017). Evidence-based guidelines on the therapeutic use of transcranial direct current stimulation (tDCS). Clin. Neurophysiol. 128, 56-92. doi: 10.1016/j.clinph.2016.10.087

Liberati, A., Altman, D. G., Tetzlaff, J., Mulrow, C., Gøtzsche, P. C., Ioannidis, J. P., et al. (2009). The PRISMA statement for reporting systematic reviews and meta-analyses of studies that evaluate health care interventions: explanation and elaboration. PLoS Med. 6:1000100. doi: 10.1371/journal.pmed.1000100

Magrinelli, F., Zanette, G., and Tamburin, S. (2013). Neuropathic pain: diagnosis and treatment. Pract. Neurol. 13, 292-307. doi: 10.1136/practneurol-2013-000536

Marck, C. H., De Livera, A. M., Weiland, T. J., Jelinek, P. L., Neate, S. L., Brown, C. R., et al. (2017). Pain in people with multiple sclerosis: associations with modifiable lifestyle factors, fatigue, depression, anxiety, and mental health quality of life. Front. Neurol. 8:461. doi: 10.3389/fneur.2017. 00461

Miller, P., and Soundy, A. (2017). The pharmacological and non-pharmacological interventions for the management of fatigue related multiple sclerosis. $J$. Neurol. Sci. 381, 41-54. doi: 10.1016/j.jns.2017.08.012

Moher, D., Shamseer, L., Clarke, M., Ghersi, D., Liberati, A., Petticrew, M., et al. (2015). Preferred reporting items for systematic review and meta-analysis protocols (PRISMA-P) 2015 statement. Syst. Rev. 4:1. doi: 10.1186/2046-4053-4-1

Mori, F., Codecà, C., Kusayanagi, H., Monteleone, F., Buttari, F., Fiore, S., et al. (2010). Effects of anodal transcranial direct current stimulation on chronic neuropathic pain in patients with multiple sclerosis. Pain 11, 436-442. doi: 10.1016/j.jpain.2009.08.011 
Nguyen, J. P., Nizard, J., Keravel, Y., and Lefaucheur, J. P. (2011). Invasive brain stimulation for the treatment of neuropathic pain. Nat. Rev. Neurol. 7, 699-709. doi: $10.1038 /$ nrneurol.2011.138

Nitsche, M. A., and Paulus, W. (2000). Excitability changes induced in the human motor cortex by weak transcranial direct current stimulation. J. Physiol. 527, 633-639. doi: 10.1111/j.1469-7793.2000.t01-1-00633.x

Nitsche, M. A., and Paulus, W. (2001). Sustained excitability elevations induced by transcranial DC motor cortex stimulation in humans. Neurology 57, 1899-1901. doi: 10.1212/WNL.57.10.1899

Nurmikko, T. J., Gupta, S., and Maclver, K. (2010). Multiple sclerosisrelated central pain disorders. Curr. Pain Headache Rep. 14, 189-195. doi: 10.1007/s11916-010-0108-8

O'Connor, A. B., Schwid, S. R., Herrmann, D. N., Markman, J. D., and Dworkin, R. H. (2008). Pain associated with multiple sclerosis: systematic review and proposed classification. Pain 137, 96-111. doi: 10.1016/j.pain.2007.08.024

O’Neill, F., Sacco, P., Bowden, E., Asher, R., Burnside, G., Cox, T., et al. (2018). Patient-delivered tDCS on chronic neuropathic pain in prior responders to TMS (a randomized controlled pilot study). J. Pain Res. 11, 3117-3128. doi: 10.2147/JPR.S186079

Ouzzani, M., Hammady, H., Fedorowicz, Z., and Elmagarmid, A. (2016). Rayyan-a web and mobile app for systematic reviews. Syst. Rev. 5:210. doi: 10.1186/s13643-016-0384-4

Palm, U., Chalah, M. A., Padberg, F., Al-Ani, T., Abdellaoui, M., Sorel, M., et al. (2016). Effects of transcranial random noise stimulation (tRNS) on affect, pain and attention in multiple sclerosis. Restor. Neurol. Neurosci. 34, 189-199. doi: 10.3233/RNN-150557

Paulus, W., Peterchev, A. V., and Ridding, M. (2013). Transcranial electric and magnetic stimulation: technique and paradigms. Handb. Clin. Neurol. 116, 329-342. doi: 10.1016/B978-0-444-53497-2.00027-9

Penner, I. K., Bechtel, N., Raselli, C., Stöcklin, M., Opwis, K., Kappos, L., et al. (2007). Fatigue in multiple sclerosis: relation to depression, physical impairment, personality and action control. Mult. Scler. 13, 1161-1167. doi: $10.1177 / 1352458507079267$

Perchet, C., Frot, M., Charmarty, A., Flores, C., Mazza, S., Magnin, M., et al. (2012). Do we activate specifically somatosensory thin fibres with the concentric planar electrode? A scalp and intracranial EEG study. Pain 153, 1244-1252. doi: 10.1016/j.pain.2012.03.004

Perrotta, A., Bolla, M., Anastasio, M. G., Serrao, M., Sandrini, G., and Pierelli, F. (2016). Modulation of temporal summation threshold of the nociceptive withdrawal reflex by transcutaneous spinal direct current stimulation in humans. Clin. Neurophysiol. 127, 755-761. doi: 10.1016/j.clinph.2015.01.031

Peterchev, A. V., Wagner, T. A., Miranda, P. C., Nitsche, M. A., Paulus, W., Lisanby, S. H., et al. (2012). Fundamentals of transcranial electric and magnetic stimulation dose: definition, selection, and reporting practices. Brain Stimul. 5 , 435-453. doi: 10.1016/j.brs.2011.10.001

Porro, C. A., Sandrini, G., Truini, A., Tugnoli, V., Alfonsi, E., Berliocchi, L., et al. (2016). Diagnosing and assessing pain in neurorehabilitation: from translational research to the clinical setting. Evidence and recommendations from the Italian Consensus Conference on Pain in Neurorehabilitation. Eur. J. Phys. Rehabil. Med. 52, 717-729.

Rossi, S., Hallett, M., Rossini, P. M., Pascual-Leone, A., Safety of T. M. S. and Consensus Group. (2009). Safety, ethical considerations, and application guidelines for the use of transcranial magnetic stimulation in clinical practice and research. Clin. Neurophysiol. 120, 2008-2039. doi: 10.1016/j.clinph.2009.08.016

Roy, L. B., Sparing, R., Fink, G. R., and Hesse, M. D. (2015). Modulation of attention functions by anodal tDCS on right PPC. Neuropsychologia 74, 96-107. doi: 10.1016/j.neuropsychologia.2015.02.028

Rudroff, T., Proessl, F., Kamholz, J., and Ponto, L. L. (2019). Increased thalamic activity and less neuropathic pain after tDCS observed with PET in a patient with multiple sclerosis: a case report. Brain Stimul. 12, 198-199. doi: 10.1016/j.brs.2018.10.006

Sandrini, G., Serrao, M., Rossi, P., Romaniello, A., Cruccu, G., and Willer, J. C. (2005). The lower limb flexion reflex in humans. Prog Neurobiol. 77, 353-395. doi: 10.1016/j.pneurobio.2005.11.003

Scholz, J., Finnerup, N. B., Attal, N., Aziz, Q., Baron, R., Bennett, M. I., et al. (2019). The IASP classification of chronic pain for ICD-11: chronic neuropathic pain. Pain 160, 53-59. doi: 10.1097/j.pain.0000000000001365
Seada, Y. I., Nofel, R., and Sayed, H. M. (2013). Comparison between trans-cranial electromagnetic stimulation and low-level laser on modulation of trigeminal neuralgia. J. Phys. Ther. Sci. 25, 911-914. doi: 10.1589/jpts.25.911

Seixas, D., Foley, P., Palace, J., Lima, D., Ramos, I., and Tracey, I. (2014). Pain in multiple sclerosis: a systematic review of neuroimaging studies. Neuroimage Clin. 5, 322-331. doi: 10.1016/j.nicl.2014.06.014

Seminowicz, D. A., and Moayedi, M. (2017). The dorsolateral prefrontal cortex in acute and chronic pain. J. Pain 18, 1027-1035. doi: 10.1016/j.jpain.2017.03.008

Shahrbanian, S., Auais, M., Duguette, P., Anderson, K., and Mayo, N. (2013). Does pain in individuals with multiple sclerosis affect employment? A systematic review and meta-analysis. Pain Res. Manag. 18, 94-100. doi: $10.1155 / 2013 / 829464$

Shahrbanian, S., Duquette, P., Kuspinar, A., and Mayo, N. E. (2015). Contribution of symptom clusters to multiple sclerosis consequences. Qual. Life Res. 24, 617-629. doi: 10.1007/s11136-014-0804-7

Solaro, C., Brichetto, G., Amato, M. P., Cocco, E., Colombo, B., D'Aleo, G., et al. (2004). The prevalence of pain in multiple sclerosis: a multicenter cross-sectional study. Neurology 63, 919-921. doi: 10.1212/01.WNL.0000137047.85868.D6

Solaro, C., Cella, M., Signori, A., Martinelli, V., Radaelli, M., Centonze, D., et al. (2018). Identifying neuropathic pain in patients with multiple sclerosis: a cross-sectional multicenter study using highly specific criteria. J. Neurol. 265, 828-835. doi: 10.1007/s00415-018-8758-2

Solaro, C., Trabucco, E., and Messmer Uccelli, M. (2013). Pain and multiple sclerosis: pathophysiology and treatment. Curr. Neurol. Neurosci. Rep. 13:320. doi: 10.1007/s11910-012-0320-5

Sterne, J. A. C., Savović, J., Page, M. J., Elbers, R. G., Blencowe, N. S., Boutron, I., et al. (2019). RoB 2: a revised tool for assessing risk of bias in randomised trials. BMJ 366:14898. doi: 10.1136/bmj.14898

Svendsen, K. B., Jensen, T. S., Hansen, H. J., and Bach, F. W. (2005). Sensory function and quality of life in patients with multiple sclerosis and pain. Pain 114, 473-481. doi: 10.1016/j.pain.2005.01.015

Svendsen, K. B., Jensen, T. S., Overvad, K., Hansen, H. J., Koch - Henderson, N., and Bach, F. W. (2003). Pain in patients with multiple sclerosis: a population based study. Arch. Neurol. 60, 1089-1094. doi: 10.1001/archneur.60.8.1089

Terney, D., Chaieb, L., Moliadze, V., Antal, A., and Paulus, W. (2008). Increasing human brain excitability by transcranial highfrequency random noise stimulation. J. Neurosci. 28, 14147-14155. doi: 10.1523/JNEUROSCI.4248-08.2008

Thompson, A. J., Toosy, A. T., and Ciccarelli, O. (2010). Pharmacological management of symptoms in multiple sclerosis: current approaches and future directions. Lancet Neurol. 9, 1182-1199. doi: 10.1016/S1474-4422(10)70249-0

Trojan, D. A., Arnold, D., Collet, J. P., Shapiro, S., Bar-Or, A., Robinson, A., et al. (2007). Fatigue in multiple sclerosis: association with diseaserelated, behavioural and psychosocial factors. Mult. Scler. 13, 985-995. doi: $10.1177 / 1352458507077175$

Truini, A., Barbanti, P., Pozzilli, C., and Cruccu, G. (2013). A mechanismbased classification of pain in multiple sclerosis. J. Neurol. 260, 351-367. doi: 10.1007/s00415-012-6579-2

Urits, I., Adamian, L., Fiocchi, J., Hoyt, D., Ernst, C., Kaye, A. D., et al. (2019). Advances in the understanding and management of chronic pain in multiple sclerosis: a comprehensive review. Curr. Pain Headache Rep. 23:59. doi: 10.1007/s11916-019-0800-2

Workman, C. D., Kamholz, J., and Rudroff, T. (2020). Transcranial direct current stimulation (tDCS) for the treatment of a Multiple Sclerosis symptom cluster. Brain Stimul. 13, 263-264. doi: 10.1016/j.brs.2019.09.012

Conflict of Interest: The authors declare that the research was conducted in the absence of any commercial or financial relationships that could be construed as a potential conflict of interest.

Copyright (c) 2020 Zucchella, Mantovani, De Icco, Tassorelli, Sandrini and Tamburin. This is an open-access article distributed under the terms of the Creative Commons Attribution License (CC BY). The use, distribution or reproduction in other forums is permitted, provided the original author(s) and the copyright owner(s) are credited and that the original publication in this journal is cited, in accordance with accepted academic practice. No use, distribution or reproduction is permitted which does not comply with these terms. 
OPEN ACCESS

Edited by:

Marco Solmi,

University of Padua, Italy

Reviewed by:

Kenji Hashimoto,

Chiba University, Japan

Qian Ren,

Hebei Medical University, China

${ }^{*}$ Correspondence:

Ulderico Freo

ulderico.freo@unipd.it

Specialty section:

This article was submitted to

Perception Science,

a section of the journal

Frontiers in Neuroscience

Received: 17 July 2020

Accepted: 04 March 2021

Published: 11 May 2021

Citation:

Freo U, Brugnatelli V, Turco $F$ and

Zanette G (2021) Analgesic

and Antidepressant Effects of the

Clinical Glutamate Modulators

Acetyl-L-Carnitine and Ketamine.

Front. Neurosci. 15:584649.

doi: 10.3389/fnins.2021.584649

\section{Analgesic and Antidepressant Effects of the Clinical Glutamate Modulators Acetyl-L-Carnitine and Ketamine}

\author{
Ulderico Freo $^{1 *}$, Viola Brugnatelli2, Fabio Turco ${ }^{3}$ and Gastone Zanette ${ }^{2}$ \\ ${ }^{1}$ Section of Anesthesiology and Intensive Care, Department of Medicine-DIMED, University of Padua, Padua, Italy, ${ }^{2}$ Section \\ of Dentistry, Department of Neurosciences-DNS, University of Padua, Padua, Italy, ${ }^{3}$ Molecular Biology and Biochemistry \\ Laboratory, Department of Neurogastroenterology, University of Naples Federico II, Naples, Italy
}

Pain and depression are leading causes of disability and of profound social and economic burden. Their impact is aggravated by their chronicity and comorbidity and the insufficient efficacy of current treatments. Morphological and functional metabolism studies link chronic pain and depressive disorders to dysfunctional neuroplastic changes in fronto-limbic brain regions that control emotional responses to painful injuries and stressful events. Glutamate modulators are emerging new therapies targeting dysfunctional brain areas implicated in the generation and maintenance of chronic pain and depression. Here, we report the effects of two clinically approved glutamate modulators: acetyl-L-carnitine (ALCAR) and S,R( \pm )ketamine (KET). ALCAR is a natural neurotrophic compound currently marketed for the treatment of neuropathies. KET is the prototypical non-competitive antagonist at $\mathrm{N}$-methyl-D-aspartate glutamate receptors and a clinically approved anesthetic. Although they differ in pharmacological profiles, ALCAR and KET both modulate aminergic and glutamatergic neurotransmissions and pain and mood. We assessed in rats the effects of ALCAR and KET on cerebral metabolic rates for glucose ( $\mathrm{rCMRglc}$ ) and assessed clinically the effects of ALCAR in chronic pain and of KET in post-operative pain. ALCAR and KET increased rCMRglc at similar degrees in prefrontal, somatosensory, and cingulate cortices, and KET increased rCMRglc at a different, much larger, degree in limbic and dopaminergic areas. While rCMRglc increases in prefrontal cortical areas have been associated with analgesic and antidepressant effects of ALCAR and KET, the marked metabolic increases KET induces in limbic and dopaminergic areas have been related to its psychotomimetic and abuse properties. In patients with chronic neuropathic pain, ALCAR (1,000 mg/day) yielded to a fast ( 2 weeks) improvement of mood and then of pain and quality of life. In day-surgery patients, KET improved dischargeability and satisfaction. In obese patients undergoing bariatric surgery, a single, low dose of KET $(0.5 \mathrm{mg} / \mathrm{kg})$ at induction of anesthesia determined a very fast (hours) amelioration of post-operative depression and pain and an opioid-sparing effect. These findings indicate that ALCAR and KET, two non-selective glutamate modulators, still offer viable therapeutic options in comorbid pain and depression.

Keywords: acetyl-L-carnitine, ketamine, regional cerebral metabolic rates for glucose, pain, depression 


\section{INTRODUCTION}

Chronic pain and depression are leading causes of disability that are frequently encountered comorbidly in a variety of clinical conditions, sharing genetic and psychological risk factors, a relapsing-chronic course, and neurobiological features (Bair et al., 2003; Meyer et al., 2007; Kroenke et al., 2011; Global Burden of Disease Study 2013 Collaborators, 2015; D'Amato et al., 2016; Freo et al., 2019a,b). In the chronic pain patient, the presence of a major depression is associated with reduced function, poorer outcome, and expanded health-care costs; in the depressed patient, pain is a frequent presenting symptom and a predictor of treatment response (Bair et al., 2003; Meyer et al., 2007; Kroenke et al., 2011; D’Amato et al., 2016).

Because of global aging, the prevalence of pain, depression, and comorbid pain and depression is expected to increase (Molton and Terrill, 2014; Chui et al., 2015). As currently available therapies do not work for many patients, new pharmacological approaches are deemed essential. Glutamate drugs are emerging treatments for pain and depression (Henter et al., 2017; Pereira and Goudet, 2019). Newly developed, receptor-selective glutamate compounds are often hampered by the uncertain toxicity and the tolerability profile; older, nonselective agents are available for different routes of administration [i.e., oral (PO), intramuscular (IM), and intravenous (IV)] and continue to be investigated actively (Henter et al., 2017; Pereira and Goudet, 2019).

We assessed the effects of two clinical glutamate modulators, acetyl-L-carnitine (ALCAR) and ketamine (KET), on regional glucose cerebral metabolism and on patients with comorbid pain and depression. This review summarizes our pre-clinical and clinical research on ALCAR and KET.

\section{PAIN AND DEPRESSION}

According to the International Association for the Study of Pain, pain can be roughly classified on the basis of mechanism as nociceptive, neuropathic, or nociplastic pain. Nociceptive pain reflects the normal functioning of the somatosensory systems responding in a stimulus-dependent manner to an actual or potential damage of non-neuronal tissue and is treated with conventional non-steroidal anti-inflammatory and/or opioid analgesics (Freynhagen et al., 2019). In contrast to nociceptive pain, neuropathic pain is induced by a lesion or disease of the somatosensory nervous system that generates and maintains spontaneous pain and positive and negative sensory disturbances, independently from stimuli (Freynhagen et al., 2019; Scholz et al., 2019). Neuropathic pain worsens cognitive and mood functions and quality of life and is treated with antiepileptic and/or antidepressant drugs targeting the abnormal somatosensory nervous systems (Nicholson and Verma, 2004; Fornasari, 2017). Recently, the International Association for the Study of Pain defined nociplastic pain as pain occurring from an altered nociception in spite of no evidence of any tissue damage (Freynhagen et al., 2019). Multiple pain mechanisms may be active at the same time in the single patient, making diagnosis and treatment more difficult (Freynhagen et al., 2019). Finally, pain is considered chronic if it lasts longer than the 3 months' healing time.

Neuropathic pain is often chronic, and neuropathic symptoms (i.e., "component") are frequently reported and aggravate painful, non-primary neurological conditions in spite of no demonstrable neuronal injury (Freo et al., 2019b, 2020; Freynhagen et al., 2019; Scholz et al., 2019). Because of the loss of protective features and the damage they cause, chronic and neuropathic pain are viewed as "disease states" (Costigan et al., 2009).

Although they often coexist and complicate each other's outcome, the exact relation between chronic pain and depression has yet to be elucidated (Bair et al., 2003; Sheng et al., 2017). Most studies report an increased sensitivity to experimental pain and, therefore, a decreased pain threshold in depressed compared to non-depressed subjects especially when emotional aspects of experimental pain are taken into account (Ushinsky et al., 2013); probably because of an altered sensation, unexplained pain is common in depression and is often the presenting and prevailing symptom (Bair et al., 2003). Stressful events may facilitate chronification of both pain and of negative/depressed mood; personal experiences such emotional strain, childhood traumatic experiences and post-traumatic stress disorder, and negative social and work experiences are associated with a higher risk of developing depression and/or chronic pain; personal attitudes such as catastrophizing and low self-efficacy are also risk factors of developing either or both states (O'Sullivan, 2004; Edwards et al., 2016). In a large genetic study, different pain phenotypes presented robust and positive genetic correlations with each other as well as with depression, suggesting common underlying genetic factors between pain and depression (Meng et al., 2020). Because it has a much higher incidence, approximately tenfold, than other mental disorders, evolutionary and pain psychologists have attempted to explain pain and depression in terms of behavioral adaptiveness. In this contest, Baliki and Apkarian (2015) support that nociception is essential to protect individuals from injury not only by inducing conscious pain and active avoidance behaviors but also by modulating automatic motor behaviors continuously and in the absence of overt pain. Similarly, a negative mood may be reconceptualized as a psychic pain that may be protective against environmental dangers in complex and hierarchical societies and promote healing; even persistent pain after an injury may have an adaptational value in that it favors survival after injuries that impair motor functions and increase vulnerability (Gałecki and Talarowska, 2017). In contrast, within the evolutionary framework, chronic pain beyond normal healing and chronic or relapsing depression are viewed as maladaptive processes which are maintained by neuropathological abnormalities.

\section{GLUTAMATE DRUGS}

\section{Neuroplasticity}

Neuroplasticity indicates the brain's ability to change over time and, more specifically, the ability of strengthening or weakening the synaptic signals between neurons in response to a variety of 
physiological stimuli such as behavioral, cognitive, and motor activities, as well as after pathological events such as painful or stressful conditions and neurological diseases (Lucassen et al., 2014; Pelletier et al., 2015). In chronic pain and depression, morphological and functional neuroplastic changes were found most pronounced in fronto-limbic regions (Mutso et al., 2012; Khan et al., 2014; Ezzati et al., 2019). The human prefrontal cortex is phylogenetically a recent brain area that matures late during development and is pivotal in the acquisition of motivational properties of different types of rewarding and aversive stimuli which include self-reference, self-appraisal, and emotion and mood control (Teffer and Semendeferi, 2012).

In chronic pain and depression, chronic exposure to stress is a common factor that may produce long-lasting changes (i.e., maladaptive neuroplasticity) in highly sensitive brain areas such as the prefrontal cortex and the hippocampus and in their functional connections, which may underlie the cognitive and behavioral impairments accompanying these conditions (Teffer and Semendeferi, 2012; Lucassen et al., 2014). For example, in comparison to healthy controls, a group of patients with chronic low back pain performing a simple visual attention task presented a reduced deactivation in regions of the default mode network; similarly, patients with a major depressive or a bipolar disorder while performing a $n$-back working memory task failed to deactivate the medial prefrontal cortex (Baliki et al., 2008; Rodríguez-Cano et al., 2017). The default mode, attention, and salience networks are all disrupted in pain and depression (Shao et al., 2018; van Ettinger-Veenstra et al., 2019). Conversely, the deep brain stimulation of the cingulate cortex relieved patients suffering from an intense neuropathic pain as well as patients with a severe treatment-resistant depression (Boccard et al., 2016; Zhang et al., 2018).

Treating pain improves depressive symptoms and vice versa (Skolasky et al., 2012; Rahman et al., 2020). Because the incidence of depressive and pain symptoms is increasing in parallel with the aging of the world population, it is particularly important to develop strategies that target both disorders to minimize polypharmacy and optimize therapeutic outcomes (Solhaug et al., 2012; Molton and Terrill, 2014). Although therapeutic options are available for chronic pain and depression, less than $50 \%$ of all patients treated for chronic pain report a clinically meaningful (i.e., $\geq 50 \%$ ) pain relief with current analgesic treatments, and only about $50-60 \%$ of patients with major depressive disorders achieved remission after an adequate course with conventional antidepressants. The efficacy of treatments of comorbid pain and depression has been less studied, but it is well known that these two conditions worsen each other's severity and therapeutic response.

Glutamate is the most abundant excitatory neurotransmitter in the central nervous system of adult mammals and has a major role in neuroplasticity (Henter et al., 2017; Pereira and Goudet, 2019). Glutamate acts through eight ionotropic and metabotropic receptor subtypes (mGluR1-mGluR8) that have been classified into three groups: Group I receptors (mGluR1 and mGluR5) are coupled to $\mathrm{G}_{\alpha \mathrm{q}}$ proteins and phospholipase $\mathrm{C}$ and are involved in central sensitization and pain chronification; Group II receptors (mGluR2/mGluR3) and Group III receptors
(mGluR4 and mGluR6-mGluR8) are coupled to $\mathrm{G}_{\alpha \mathrm{i} / \mathrm{o}}$ proteins and inhibit adenylate cyclase; their activation is effective against nociceptive and neuropathic pain (Zammataro et al., 2011; Henter et al., 2017; Pereira and Goudet, 2019).

Glutamate competitive and non-competitive ligands, binding to the same or to a different receptor site of the endogenous ligand, have been on development for at least three decades. Trials on stroke and traumatic brain injuries with competitive glutamate or glycine antagonists (i.e., selfotel, aptiganel, eliprodil, licostinel, and gavestinel) have failed (Ikonomidou and Turski, 2002). Recent trials with glutamate agents to treat pain and depression yielded promising but sometimes inconsistent results; non-selective glutamate modulators such as ALCAR and KET can still be of interest to glutamate research (Gould et al., 2019; Pereira and Goudet, 2019).

\section{ALCAR}

Acetyl-L-carnitine $\quad(\gamma$-trimethyl- $\beta$-acetylbutyrrobetaine $) \quad$ is an acetyl ester of carnitine, an endogenous molecule with pleiotropic biological and pharmacological activities on central and peripheral nervous systems (Chiechio et al., 2017). ALCAR has a key role in neuronal metabolism (i.e., $\beta$-oxidation, glycogen production, glucose utilization, and ammonia cycle), growth, plasticity, and regeneration; ALCAR is actively taken up by the brain and modulates the release of aminergic neurotransmitters and the biosynthesis and release of glutamate (Kuratsune et al., 2002; Tolu et al., 2002; Tanaka et al., 2003; Freo et al., 2009; Smeland et al., 2012; Chiechio et al., 2017; Wang et al., 2014; Burks et al., 2019). Exogenous ALCAR may increase neurogenesis in prefrontal-limbic areas via a selective upregulation of mGluR2 receptors, by acting as histone acetylator on transcription factors of the nuclear factor (NF)-kappa B family (Chiechio et al., 2006; Nasca et al., 2013).

In experiment animals, ALCAR is neuroprotective against hypoxia, nerve and spinal cord injury, and neurotoxins such as amphetamines and 1-methyl-4-phenyl-1,2,3,6tetrahydropyridine (Chiechio et al., 2017; Burks et al., 2019). In humans, low plasma levels of ALCAR have been associated with an increased vulnerability to chronic pain and depression. Specifically, circulating ALCAR was found to be reduced in patients with severe osteoarthritis pain that did not improve after a total joint replacement (Costello et al., 2020). ALCAR was found to be reduced also in the plasma and the brain of patients with chronic fatigue or major depressive disorder and, more markedly, when depression was severe, treatment resistant or associated with history of childhood trauma or neglect (Kuratsune et al., 2002; Nasca et al., 2018; Post, 2018; Pu et al., 2020).

Given its excellent, long-term safety profile, ALCAR has been studied in several neurological and psychiatric conditions, confirming a strong antineuropathic activity in toxic and traumatic painful neuropathies (Onofrj et al., 2013; Chiechio et al., 2017). Specifically, ALCAR improved the function of peripheral nerves by reducing sensory neuronal loss and by enhancing nerve regeneration and conduction velocity (Onofrj et al., 2013; Cruccu et al., 2017). Administration of ALCAR has consistently shown good tolerability and 
efficacy in dysthymic disorder and on depressive symptoms associated with fibromyalgia or minimal hepatic encephalopathy (Wang et al., 2014).

\section{Ketamine}

Ketamine is an arylcycloalkylamine structurally analogue to phencyclidine (PCP, angel dust), an approved anesthetic and the prototypical, non-competitive $N$-methyl-D-aspartate receptorglutamate (NMDA) antagonist.

In addition to NMDA antagonism, however, KET has a myriad of effects on neurotransmitters which include the following: increase of synaptic concentrations of acetylcholine in the spinal cortex, hippocampus, and neocortices; increase of glutamate, serotonin, and noradrenaline in the prefrontal cortex; increase of dopamine in the basal ganglia and neocortices; activation of synaptogenic $\alpha$-amino-3-hydroxy-5-methyl-4isoxazolepropionic acid (AMPA) receptors; and activation of synaptogenic intracellular signaling, including mammalian target of rapamycin complex (TORC1) (Freo and Ori, 2002b, 2003; Cohen et al., 2018). Behaviorally, KET has almost unique, dose-dependent effects on the central nervous system. Different from most general anesthetics, at high, anesthetic doses, KET and few other congeners produce a "dissociative anesthesia" during which patients may appear awake and maintain spontaneous eye movements and respiratory drive although they are relatively insensitive to sensory stimulation (Cohen et al., 2018). At lower subanesthetic doses, KET promotes arousal from anesthesia and has strong antidepressant and analgesic activities (Hambrecht-Wiedbusch et al., 2017; Cohen et al., 2018). After a low intravenous dose of KET $(0.5 \mathrm{mg} / \mathrm{kg})$, most patients with a major depressive disorder reported a very fast, within hours, improvement of depressive symptoms that lasted for 7-10 days (Cohen et al., 2018). The antidepressant effects of KET were replicated in patients with treatment-resistant or bipolar depression, suggesting that KET may be effective on a wider range of depressed patients (Romeo et al., 2015).

In addition, low-dose KET has been beneficial on large numbers of patients suffering from oncological and nononcological pain (Cohen et al., 2018; Orhurhu et al., 2019). The consensus guidelines recently elaborated by the American Society of Regional Anesthesia and Pain Medicine, the American Academy of Pain Medicine, and the American Society of Anesthesiologists support the use of KET for chronic pain, but with different degrees of evidence for different conditions and dose ranges (Cohen et al., 2018). Adverse events of KET were similar to those of placebo, with higher dosages and more frequent infusions being associated with greater risks (Cohen et al., 2018). However, not all authors agree and dismiss KET adverse events as anecdotal. In clinical practice, using KET for chronic pain or depression is still limited by intravenous administration and its potential neurotoxic and toxic effects (Jevtovic-Todorovic et al., 2001; Liao et al., 2010; Orhurhu et al., 2020). However, intranasal and oral administration as well as the discovery of antidepressant activity and lower psychotomimetic effect of isomers and metabolites of KET may foster a wider and longer-term clinical use of KET in the future (Zanos et al., 2016). Because $\mathrm{S}(+)$ KET has higher affinity for NMDA receptors than
$\mathrm{R}(-) \mathrm{KET}, \mathrm{S}(+) \mathrm{KET}$ has been developed and later approved by the Food and Drug Administration as a clinical antidepressant (Hashimoto, 2019). However, in animal models of depression, $\mathrm{R}(-)$ KET has shown longer-lasting antidepressant effects and lesser adverse effects than $\mathrm{S}(+) \mathrm{KET}$ and is currently being investigated as a promising antidepressant (Hashimoto, 2019).

\section{CEREBRAL METABOLISM STUDIES}

The regional cerebral metabolic rates for glucose (rCMRglc) were measured using the quantitative autoradiographic $\left[{ }^{14} \mathrm{C}\right] 2$ deoxy-D-glucose technique in groups of five to seven male, Fischer-344, conscious rats at $30 \mathrm{~min}$ after IV administration of saline or ALCAR $250-750 \mathrm{mg} / \mathrm{kg}$ and at $20 \mathrm{~min}$ after IV saline or KET $20 \mathrm{mg} / \mathrm{kg}$ (Ori et al., 2002; Freo and Ori, 2004). The $\left[{ }^{14} \mathrm{C}\right] 2$-deoxy-D-glucose procedure has been detailed previously (Freo and Ori, 2002a, 2004, 2009).

Acetyl-L-carnitine dose-dependently increased rCMRglc in the prefrontal, cingulate, and somatosensory cortices, in the cortical amygdala and in the accumbens, diagonal band, dorsal raphe, and locus coeruleus nuclei (ANOVA and unpaired $t$-test, $P<0.05)$ (Figure 1; Ori et al., 2002). Acetate and carnitine alone had no effect on cerebral metabolism, indicating that rCMRglc increases by ALCAR are independent from its effects on mitochondrial metabolism (Ori et al., 2002). KET $[S, R( \pm)$ ketamine] increased rCMRglc similarly to ALCAR in cortical areas, to a lesser extent in serotoninergic raphe nuclei and to a much greater extent in hippocampal regions and dopaminergic nuclei (average percentage increase $32.2 \pm 11.4$ vs. $20.0 \pm 23.0$; $P<0.01$ ) (Freo and Ori, 2004). Using functional magnetic resonance imaging in paralyzed, mechanically ventilated rats, Masaki and coworkers reported that $S, R( \pm) K E T 10 \mathrm{mg} / \mathrm{kg}$ and $\mathrm{S}(+) \mathrm{KET} 10 \mathrm{mg} / \mathrm{kg}$ increased the regional cerebral blood flow signal in the basal ganglia and cortical regions in a similar fashion to that in MK801 (Masaki et al., 2019); in contrast, the same dose of $\mathrm{R}(-)$ KET produced no noticeable behavioral change and a widespread decrease of regional cerebral blood flow (Freo and Ori, 2004; Masaki et al., 2019). In GluN2D-knockout mice, KET failed to increase the $\left[{ }^{14} \mathrm{C}\right]-2$-deoxy-D-glucose uptake as well as the cortical gamma-band power, suggesting that prefrontal cortical activations are mediated by mGluR2 receptors (Sapkota et al., 2016). Furthermore, in humans, KET increased the $\left[{ }^{18} \mathrm{~F}\right]$-fluorodeoxy-D-glucose uptake in the prefrontal cortex in a correlative fashion to post-treatment antidepressant effects (Li et al., 2016). Finally, the antidepressant effects of KET continuing beyond its pharmacokinetic half-life was associated with persistent activation of the frontal supplementary motor and cingulate cortices (Chen et al., 2018).

While the rCMRglc effects of KET were similar to those of other non-competitive NMDA antagonists, they differ markedly from the small rCMRglc changes induced by competitive NMDA receptor antagonists (i.e., AP7, CGP39551, CPP, and CGS19755), which have demonstrated relatively modest antidepressant effects and to actually counteract KET-induced dopamine activations (French, 1992; Sharkey et al., 1996; Iadarola et al., 2015). Preclinical and (Freo and Ori, 2002a,b) human studies have reported 


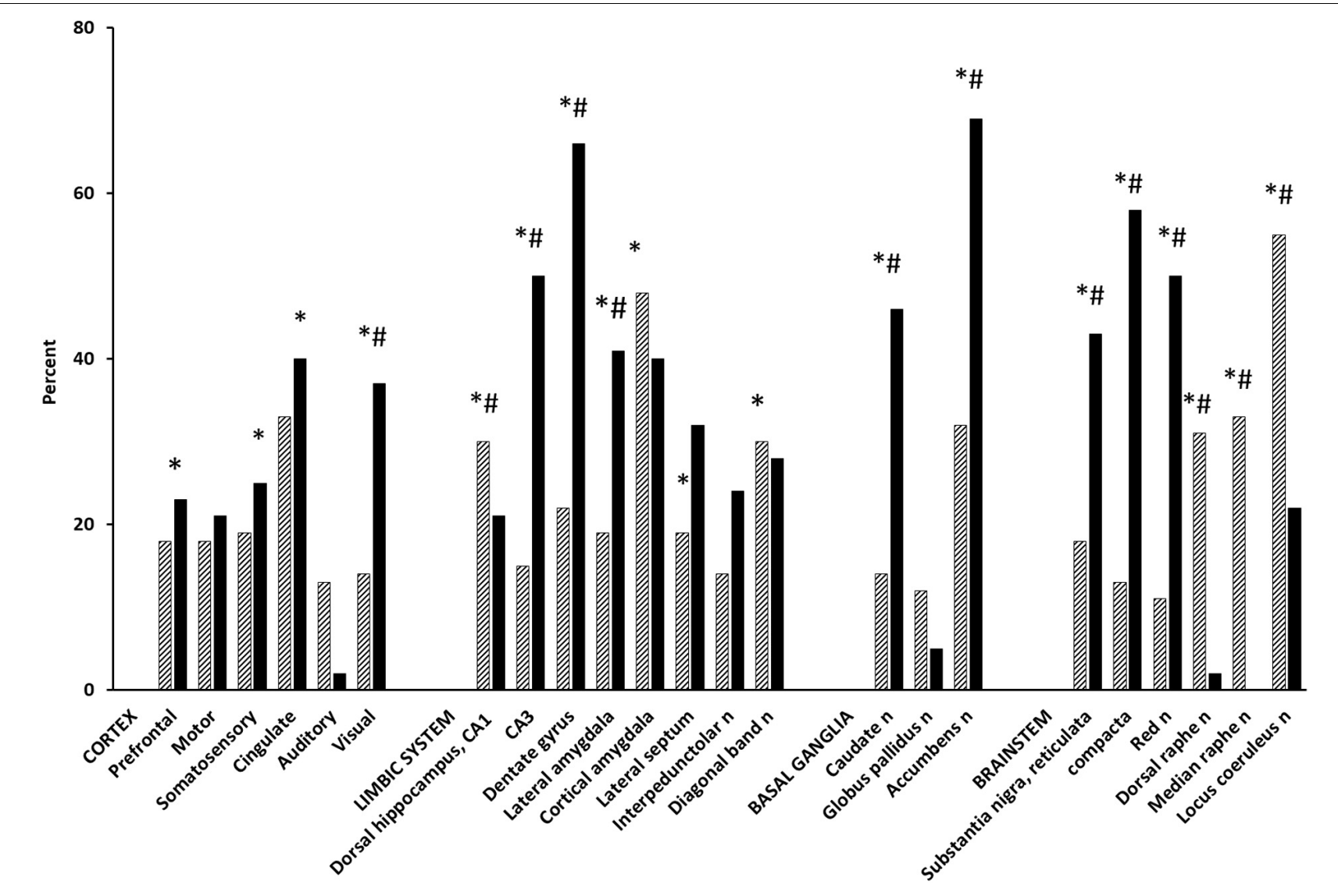

FIGURE 1 | Effect of ALCAR and KET on rCMRglc. Bars are mean rCMRglc differences (percent) from saline controls in groups of 5-7 Fischer-344, male rats at 30 min after IV administration of ALCAR 500 mg (hatched) and 20 min after IV KET 20 mg (solid). ALCAR difference from saline control: *P < 0.05; ALCAR difference from KET: ${ }^{\#} P<0.05$.

abnormalities of glutamatergic systems in depression (JiménezSánchez et al., 2016); conversely, enhancement of glutamate neurotransmission in the prefrontal cortex is considered necessary and sufficient for the antidepressant properties of glutamate drugs (Fukumoto et al., 2016; Highland et al., 2019). However, ALCAR and KET differ in their mechanisms of action and can lead to prefrontal activation in two different manners: ALCAR may do so directly via upregulation of mGluR2 receptors, KET may act indirectly via an NMDA antagonism on GABA inhibitory neurons, and the subsequent disinhibition of pyramidal cortical neurons (Moghaddam et al., 1997). Enhanced AMPA/glutamate transmission by KET stimulates, in turn, release of adrenaline and serotonin, which may contribute to KET antidepressant effects (Fukumoto et al., 2016; Jiménez-Sánchez et al., 2016).

Compared to ALCAR, KET determined greater rCMRglc increases in dopaminergic nuclei (i.e., accumbens and substantia nigra, pars reticulata and compacta, nuclei: 233, 138, and $346 \%$, respectively, $P<0.01$ ) and in hippocampal areas (i.e., dorsal $\mathrm{CA}_{3}$ and dentate gyrus: 233 and 200\%, respectively, $P<0.01$ ), which are among the largest metabolic activations ever reported (Sharkey et al., 1996). However, they are consistent with the marked increases KET elicits also on electrical activity in the ventral tegmental area, extracellular concentrations of dopamine in the nucleus accumbens and prefrontal cortex, and hyperlocomotion by the dopamine $\mathrm{D}_{2 / 3}$ receptor agonist quinpirole (Witkin et al., 2016). KET dopaminergic activations were prevented by dopaminergic neuroleptics and an AMPA receptor antagonist, indicating AMPA-dependent effects (Duncan et al., 2003; Witkin et al., 2016). While the role of dopaminergic and glutamatergic activations in KET antidepressant actions remains questionable, the large rCMRglc increase KET induces in mesolimbic areas likely reflects dopamine "surges" that mediate natural and drug rewards and, possibly, the abuse liability of KET (Kokkinou et al., 2018; Volkow et al., 2019). In contrast, ALCAR increases much less dopamine release and rCMRglc; although it is faster than conventional selective serotonin and/or norepinephrine reuptake inhibitor and tricyclic antidepressants, ALCAR is a less potent and slower antidepressant than KET and is devoid of abuse risk (Tolu et al., 2002; Romeo et al., 2015; Chiechio et al., 2017).

In experiment animals, KET has potential neuroprotective properties in stroke, neurotrauma, subarachnoid hemorrhage, and status epilepticus; however, KET has been reported to cause also some worrisome neurotoxic damage, which, interestingly, can be counteracted by ALCAR (Jevtovic-Todorovic et al., 2001; 
Liao et al., 2010; Robinson et al., 2016; Bell, 2017; Orhurhu et al., 2020).

Acetyl-L-carnitine increased rCMRglc to a similar extent in most brain areas in young and aged rats and to a larger extent in the limbic regions of aged rats (Freo et al., 2009). Following its chronic administration, ALCAR determined larger rCMRglc increases in hippocampal areas, which are crucial to attention and memory functions (Freo et al., 2009). Because ALCAR is endowed with cholinomimetic properties, its positive effects on attention and memory were ascribed to ALCAR cholinergic agonism (Battistin et al., 1989; Jeong et al., 2017). However, as the limbic regions are electrically and metabolically hyporesponsive to acute cholinergic muscarinic stimulation and to chronic cholinergic treatment, a noncholinergic mechanism for memory-enhancing effects of ALCAR is likely (Freo et al., 2009). During aging, the glutamate neurotransmission undergoes complex changes within the hippocampus, which include increases of glutamate-induced phosphoinositol hydrolysis, of densities of glutamate receptors (i.e., mGluR2, mGluR3, and mGluR5) and of their mRNAs, all of which have been interpreted as compensatory for agerelated alteration of glutamate neurotransmission (Griego and Galván, 2020). The aging cognitive decline has been associated with weakened synaptic strength in prefrontal and hippocampal regions. Interestingly, riluzole, a glutamate release inhibitor and glutamate antagonist, was shown to increase glutamatergic activity in the hippocampus, preventing thus cognitive decline during aging (Pereira et al., 2014). Hence, ALCAR may also have a positive effect on cognitive functions by activating the hippocampal glutamatergic mechanisms.

\section{CLINICAL STUDIES}

\section{Effects of ALCAR on Chronic Pain}

Chronic pain and depression often coexist, requiring frequent or continuous treatments (Freo et al., 2019a). Multi-pathologies and multi-therapies make it challenging especially in the elderly and frail population. In comorbid chronic pain and depression, ALCAR may be useful because of its analgesic and antidepressant properties and high long-term tolerability (Chiechio et al., 2017).

We investigated the effects of ALCAR in painful neuropathies and radiculopathies that were unresponsive or poorly responsive (i.e., $\leq 30 \%$ pain relief) to previous therapies in 28 patients (17 females and 11 males; age $66.4 \pm 10.1$ years; pain duration $16 \pm 21$ months) (Freo et al., 2019a). The primary outcome was pain intensity after a 4-month treatment with ALCAR $500 \mathrm{mg}$ BID that was given initially IM for an average of $57 \pm 9$ days and then PO. Patients were assessed for the 24$\mathrm{h}$ average pain with a $0-10$ numerical rating scale (NRS) (pain rating, $0=$ no pain, $1-3=$ mild, $4-6=$ moderate, and $7-$ 10 = severe pain), for neuropathic pain symptoms with the painDETECT questionnaire $[<12=$ negative (nociceptive pain), 13-18 = uncertain (mixed pain), $\geq 19=$ positive (neuropathic pain)], for depressive symptoms with the Hospital Anxiety and Depression Scale (HADS) $(<7=$ no depression, $8-10=$ mild, $11-$ $15=$ moderate, and 16-21 = severe depression), and for quality of life with the 12-item Short Form Health Survey (SF-12), physical and mental components (Freo et al., 2019a,b).

At baseline, all patients reported a moderate-to-severe, 24-h average pain (NRS $\geq 4 / 10$ ), $60 \%$ of patients reported symptoms of a mild-to-moderate depression (HADS $\geq 8$ ), and $57 \%$ had a positive painDETECT score $(\geq 12)$ for neuropathic pain (Freo et al., 2019a). The 4-month treatment with ALCAR was associated with a reduction of pain and depression (Figure 2). Pain intensity significantly improved from baseline to month 1 of treatment (pain NRS from $7.4 \pm 1.5$ to $5.6 \pm 1.7$; means \pm standard deviation, Kruskal-Wallis and Wilcoxon's test, $P<0.01$ ) and depressive symptoms improved already at week 2 of treatment (HADS scores from $8.8 \pm 4.4$ to $6.1 \pm 3.4$, $P<0.01$ ) (Figure 2; Freo et al., 2019a). Compared to baseline, at month 4 outcome, a moderate (30-49\%) pain improvement was observed in 11 patients and a substantial $(\geq 50 \%)$ improvement in 8 patients. The painDETECT score for neuropathic pain decreased from baseline to month 4 outcome from $12.6 \pm 6.0$ to $5.0 \pm 0.9(P<0.01)$; the SF-12 mental component increased from $44.1 \pm 4.5$ to $53.5 \pm 5.1(P<0.01)$ and the SF-12 physical component from $35.3 \pm 3.5$ to $39.4 \pm 5.2$ (not significant). Five patients discontinued treatment because of lack of efficacy or unwillingness to continue treatment; no adverse effect was recorded (Freo et al., 2019a).

Reportedly, ALCAR improved pain and nerve function in experimental and clinical neuropathies of different etiologies with therapeutic effects being ascribed mainly to ALCAR neuroprotective and neuroregenerative properties ( $\mathrm{Li}$ et al., 2015). However, although in peripheral neuropathies, depression is common and noradrenaline-serotonin reuptake inhibitor and tricyclic antidepressants are first-line treatments, depressive symptoms are not always measured. Pain and depression have a biunivocal relation with worsening or improvement in one variable predicting subsequent changes in severity of the other (Kroenke et al., 2011; D’Amato et al., 2016). As such, rapidacting antidepressant and analgesic drugs have been a major breakthrough (Chiechio et al., 2017). In our patients, ALCAR improved depressive symptoms earlier than pain symptoms, suggesting that the antidepressant activity of ALCAR may anticipate and contribute to its analgesic properties.

Chronic neuropathic pain and depression are age-dependent, highly comorbid disorders that complicate courses and outcomes (Dworkin et al., 2003; Brouwer et al., 2015; Hanewinckel et al., 2016; Freo et al., 2019b). Therapeutic responses are often poor and limited by concurrent therapies. The elderly population is at increased risk for adverse events from antidepressants and anticonvulsants that may worsen stability, balance, and cognition (Dworkin et al., 2003; Brouwer et al., 2015; Hanewinckel et al., 2016). Because of its high tolerability and the positive effect it has on pain, depression, and cognition, ALCAR should be in the therapeutic armamentarium for treating comorbid pain and depression, especially in the elderly population.

\section{Effects of KET on Post-operative Pain}

Almost unique among general anesthetics, the NMDA antagonist KET has anesthetic properties with low cardiovascular and respiratory depression (Cohen et al., 2018). KET is also clinically 

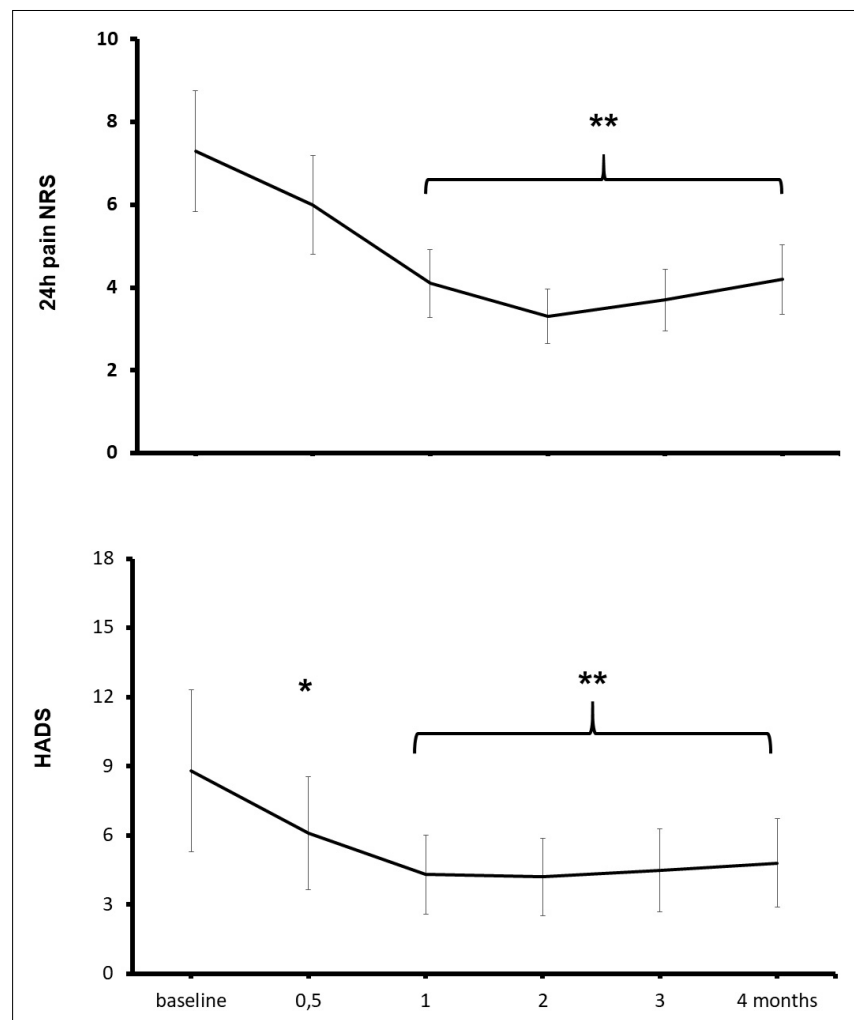

FIGURE 2 | Effects of ALCAR on chronic pain and depression. Points are means \pm standard deviation of 24-h average pain NRS scores (above) and depression HADS scores (below) from pre-treatment baseline to month 4 treatment in 28 patients receiving ALCAR $500 \mathrm{mg}$ BID IM/PO for chronic neuropathy or radiculopathy pain. Significantly different from baseline: ${ }^{\star} P<0.05 ;{ }^{\star \star} P<0.01$.

attractive because it has strong analgesic and antidepressant activities and may prevent central sensitization and hyperalgesia (Freo and Ori, 2003; Ori et al., 2003; García-Henares et al., 2018). Hence, KET is of interest for patients prone to anesthesia-induced respiratory impairment or suffering from chronic pain and/or depression or, more, for patients presenting with all these clinical features such as the morbidly obese (Freo and Ori, 2003; Ori et al., 2003; Carron et al., 2012; García-Henares et al., 2018).

In fact, overweight and obesity are frequently associated with an obstructive apnea syndrome and/or to a depressive disorder that places patients at risk, respectively, of post-operative critical events and of post-operative complications and prolonged stay (Luppino et al., 2010; Ghoneim and O'Hara, 2016; Subramani et al., 2017; Nijland et al., 2020). Obesity is associated with higher rates of chronic pain and higher scores of post-operative pain, which are both challenging to treat in this patient population (Belcaid and Eipe, 2019; Mills et al., 2019). As opioids may induce ventilatory impairment, multimodal opioid- and muscle relaxant-sparing techniques are being investigated to improve the safety of analgesia in obese patients; in this regard, KET may present specific advantages (Ori et al., 2003; Dalsasso et al., 2005; Freo et al., 2011; Carron et al., 2012; García-Henares et al., 2018; Aronsohn et al., 2019).
The effects of KET as the main anesthetic agent were determined in 500 patients (172 males and 328 females, ASA III, age $53.9 \pm 12.2$ years, weight $76.1 \pm 22.5 \mathrm{~kg}$ ) undergoing an opioid-free anesthesia for day surgery including breast surgery, laparoscopy, superficial excision of minor lesions, thoracoscopy, appendectomy, and proctology (Dalsasso et al., 2005). At induction, patients received IV midazolam $0.03-0.05 \mathrm{mg} / \mathrm{kg}$, clonidine $150 \mu \mathrm{g}$, and KET $0.4 \mathrm{mg} / \mathrm{kg}$; the latter was repeated as needed during surgery (mean total dose $0.6 \pm 0.2 \mathrm{mg} / \mathrm{kg}$ ). Anesthesia was maintained with nitrous oxide and sevoflurane. Seventy-four percent of patients were eligible to discharge from the operating theater by $30 \mathrm{~min}$, and all patients were dischargeable by $1 \mathrm{~h}$. Patients did not complain of hallucinations, while presenting a high rate of satisfaction at the Iowa Satisfaction with Anesthesia Scale (Dalsasso et al., 2005).

The post-operative effects of KET were assessed in 41 obese patients (26 females and 15 males; age $42.7 \pm 10.7$ years; body mass index $44.5 \pm 7.2$ ) undergoing laparoscopic gastric banding or sleeve gastrectomy with the primary outcomes being post-operative pain and depression (Freo, 2020). All patients were pre-medicated with midazolam and induced with IV propofol $1.5 \mathrm{mg} / \mathrm{kg}$ and fentanyl $1-2 \mu \mathrm{g} / \mathrm{kg}$ and maintained with sevoflurane 1-2\%; patients were randomized to receive at induction either saline or $\mathrm{KET}[\mathrm{S}, \mathrm{R}( \pm)$ ketamine $0.5 \mathrm{mg} / \mathrm{kg}$ by ideal body weight]. Baseline demographic features (i.e., age, education, body mass index, comorbidities, and medical therapies), times of anesthesia and surgery, and average propofol dosages were similar between groups; average fentanyl dosage was higher in the saline control than in the KET group (i.e., $341 \pm 109$ and $192 \pm 67 \mathrm{mg}, P<0.01$ ) (Freo, 2020).

At pre-operative baseline, in the control and the KET groups, seven and eight patients reported moderate-to-severe pain (NRS $\geq 4 / 10$ ), and 9 and 10 patients presented mild-to-moderate depressive symptoms in the Hamilton Depression Rating Scaling (HAMD), respectively (Freo, 2020). Pain scores were significantly lower at post-operative hours 6 and 12 in KET-treated patients than in controls and then subsided in both groups (Figure 3). Pain improvement was less in non-depressed than depressed patients (mean intergroup difference at post-operative day 1 , -33 and $-51 \%, P<0.05$ ) (Freo, 2020). HAMD scores were significantly lower in the KET-pre-treated patients at the postoperative days 1 and 3 (HAMD scores in saline and KET groups at baseline: $6.7 \pm 5.7$ and $7.1 \pm 5.7$; at post-operative day $1: 6.7 \pm 5.7$ and $3.4 \pm 2.6$; at post-operative day $3: 7.0 \pm 5.6$ and $3.7 \pm 2.9$; Friedman's and Mann-Whitney $U$ tests, $P<0.01$ ) (Figure 4; Freo, 2020).

A recent Cochrane meta-analysis review concluded that perioperative intravenous KET reduces post-operative pain and nausea and analgesic consumption (Brinck et al., 2017); however, not all studies are consistent with these findings. In a recent randomized controlled study (RCT) on 100 obese patients, of whom 22 with history of depression and 13 with history of chronic pain, undergoing laparoscopic gastric bypass or gastrectomy, post-operative infusion of KET $(0.4 \mathrm{mg} / \mathrm{kg}$, ideal body weight) was not superior to placebo on postoperative pain and mood assessed with a pain Visual Analogue 


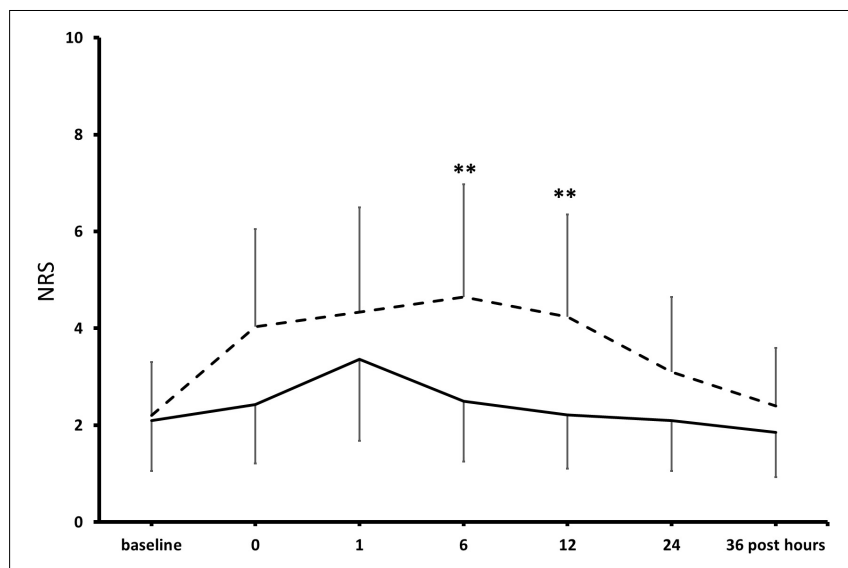

FIGURE 3 | Effects of KET on post-operative pain in obese bariatric patients. Points are means \pm standard deviations of NRS scores of pain in the first $36 \mathrm{~h}$ after bariatric surgery in 41 obese patients who had received either saline (broken line) or KET $0.5 \mathrm{mg} / \mathrm{kg}$ (continuous line) at induction of anesthesia. Significantly different from saline controls: ${ }^{\star \star} P<0.01$.

Scale, the Beck Depression Inventory and the MontgomeryAsberg Depression Rating Scale; KET, however, improved the affective and the total score of the short-form McGill Pain Questionnaire starting on post-operative day 2 (Wang et al., 2019). In the PODCAST multicenter RCT on 672 older adults (i.e., $>60$ years) undergoing cardiac and noncardiac surgery, pre-incisional KET $(0.5$ or $1 \mathrm{mg} / \mathrm{kg})$ did not decrease post-operative pain, delirium, or depressed mood (Avidan et al., 2017).

Most negative studies focused on post-operative pain. Analgesic effects of KET are considered use dependent: the worst the pain, the more efficient KET will be as analgesic (Robu and Lavand'homme, 2019). The same may hold true for its antidepressant effect. KET has a plasma half-life of $2.3 \pm 0.5 \mathrm{~h}$ with a duration of action of IV bolus of 5-10 min (Cohen et al., 2018). KET has a rapid and potent antidepressant effect that peaks at 24-48 $\mathrm{h}$ after administration and could have a larger impact on patients with mood disorders (Cohen et al., 2018). Consistently, Kudoh et al. (2002) reported that KET significantly improved mood and pain on post-operative day 1 in depressed patients undergoing orthopedic surgery. In a second double-blind RCT, KET $0.5 \mathrm{mg} / \mathrm{kg}$ IV bolus followed by a $30 \mathrm{~min}$ infusion of $0.25 \mathrm{mg} / \mathrm{kg} / \mathrm{h}$ increased mood and serum brain-derived neurotrophic factor, which is a marker of major depressive disorders and treatment response (Jiang et al., 2016). It is therefore possible that the analgesic effect of KET is contributed at least in part by its antidepressant activities. In our sample, 15 patients (37\%) had at least a mild-to-moderate depression, and nine patients were on chronic antidepressant therapy; large-cohort studies indicated that the prevalence of subclinical depression ranges from 1 to $17 \%$ (Heo et al., 2006), which suggests that obese patients with mood disorders may especially benefit from KET treatment.

Ketamine is endowed with a peculiar profile with multiple pharmacological activities that may result from different

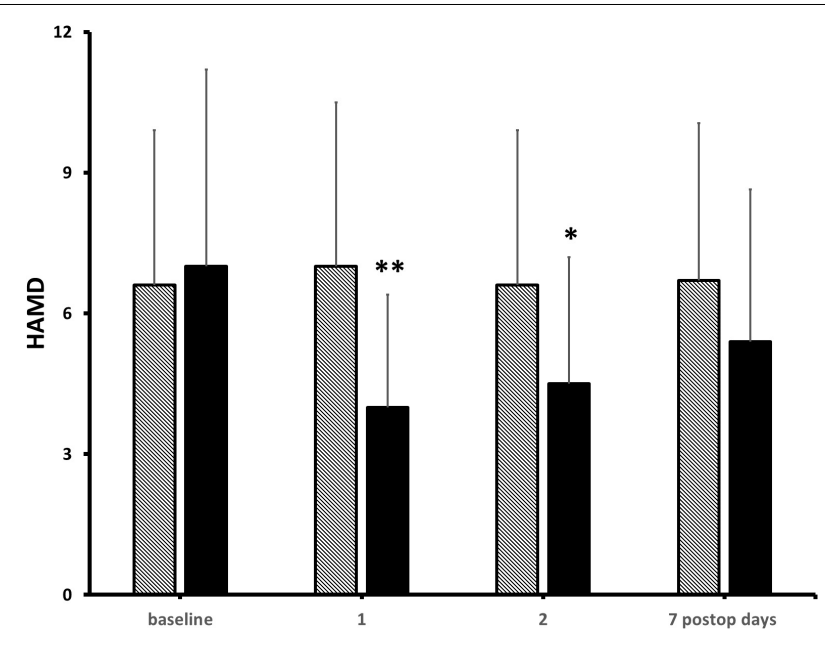

FIGURE 4 | Effects of KET on depression in obese bariatric patients. Columns are means \pm standard deviations of HAMD scores from baseline to post-operative day 7 in 41 bariatric patients who had received either saline (hatched columns) or KET $0.5 \mathrm{mg} / \mathrm{kg}$ (solid columns) at induction of anesthesia. Significantly different from saline controls: ${ }^{*} P<0.05 ;{ }^{* *} P<0.01$.

underlying mechanisms. Other NMDA antagonists (i.e., MK801 and memantine) do not have the same anesthetic, analgesic, and antidepressant effects of KET, leaving open the question of its mechanisms of action (Kelland et al., 1993; Gould et al., 2019; Robu and Lavand'homme, 2019). Besides the glutamate system, KET interacts with several other neurotransmitter systems (Jelen et al., 2020). For example, the administration of the insulin growth factor small-interfering RNA blocks KET antidepressant effects in the mouse learned helplessness model of depression (Grieco et al., 2016). In rodents, activation of AMPA receptors by the KET metabolite (2R,6R)-hydroxynorketamine has initially been thought essential to replicate KET antidepressant effects while administration of 2,3-dihydroxy-6-nitro-7-sulfamoylbenzo[f]quinoxaline-2,3-dione (NBQX), an AMPA receptor antagonist, blocks KET effects (Zanos et al., 2016). However, in subsequent investigations in rodent models of depression, (2R,6R)-hydroxynorketamine did not exhibit antidepressant-like effects and increased aggressive behavior (Yang et al., 2019). The brain-derived neurotrophic factor (BDNF) and its receptor, tyrosine kinase receptor B (TrkB), are essential and common mechanisms for the antidepressant effects of both the parent molecules of ketamines [i.e., $\mathrm{S}, \mathrm{R}( \pm)$ ketamine, $\mathrm{S}(+)$ ketamine, and $\mathrm{R}(-)$ ketamine] and their active metabolites [i.e., $(2 \mathrm{R}, 6 \mathrm{R})-$ hydroxynorketamine and of $\mathrm{S}(+)$ norketamine] (Yang et al., 2019). In patients with treatment-resistant depression, naltrexone $50 \mathrm{mg}$ blocked the antidepressant but not the dissociative effects of KET, suggesting that the opioid system may also be required for KET antidepressant activities (Williams et al., 2018). Other works, however, have shown that naltrexone pre-treatment did not affect the antidepressant activities of KET in depressed individuals (Marton et al., 2019; Yoon et al., 2019; Zhang and Hashimoto, 2019). 


\section{ALCAR AND KET AS GLUTAMATE DRUGS FOR PAIN AND DEPRESSION}

The excitatory actions of glutamate in the central nervous system have been recognized in the early 1950s (Curtis and Watkins, 1960). Since the discovery of neurotoxic effects of massive glutamate release, glutamate antagonists have been trialed in massive neuronal damage (i.e., stroke and brain and spinal cord trauma), with disappointing results (Ikonomidou and Turski, 2002). Later, it was shown that glutamate is actually essential to physiological neuroplasticity underlying learning and memory, as well recovery from brain stroke or trauma (Ikonomidou and Turski, 2002).

The report of the fast antidepressant effects of KET, a non-selective glutamate agent, fostered interest in the role of glutamate in specific neurological functions (Henter et al., 2017; Pereira and Goudet, 2019). Reportedly, glutamate is involved in maladaptive neuroplastic processes contributing to generation and maintenance of pain and mood disorders (Riggs and Gould, 2021). Frontal areas are critical for topdown cognitive modulation of pain and pain-related emotions (Thompson and Neugebauer, 2019). A lower level of frontal activity has been linked to higher pain and low mood, and conversely, an increased frontal activity has been linked to analgesic and antidepressant effects (Thompson and Neugebauer, 2019). Furthermore, glutamate concentrations are reduced in frontal areas in experimental and clinical pain (Thompson and Neugebauer, 2019), and a frontal glutamatergic dysfunction has been implicated in depression as well (Moriguchi et al., 2019). Therefore, modulation of glutamate neurotransmission is a current research target for pain and depression (Pereira and Goudet, 2019; Riggs and Gould, 2021).

The number of glutamate-receptor-selective agents has been fast growing, but the clinical safety still remains an issue for most new agents (Henter et al., 2017; Pereira and Goudet, 2019). Also, more generally, development of non-glutamate drugs for pain and depression has been plagued by failures in advanced human trials. As a consequence, older glutamate drugs are being reassessed.

Acetyl-L-carnitine and KET are non-selective, clinical glutamate modulators which have been shown to improve pain

\section{REFERENCES}

Aronsohn, J., Orner, G., Palleschi, G., and Gerasimov, M. (2019). Opioid-free total intravenous anesthesia with ketamine as part of an enhanced recovery protocol for bariatric surgery patients with sleep disordered breathing. J. Clin. Anesth. 52, 65-66. doi: 10.1016/j.jclinane.2018.09.014

Avidan, M. S., Maybrier, H. R., Abdallah, A. B., Jacobsohn, E., Vlisides, P. E., Pryor, K. O., et al. (2017). Intraoperative ketamine for prevention of postoperative delirium or pain after major surgery in older adults: an international, multicentre, double-blind, randomised clinical trial. Lancet (Lond. Engl.) 390, 267-275. doi: 10.1016/S0140-6736(17)31467-8

Bair, M. J., Robinson, R. L., Katon, W., and Kroenke, K. (2003). Depression and pain comorbidity: aliterature review. Arch. Intern. Med. 10, 2433-2445. doi: 10.1001/archinte.163.20.2433

Baliki, M. N., and Apkarian, A. V. (2015). Nociception, pain, negative moods, and behavior selection. Neuron 87, 474-491. doi: 10.1016/j.neuron.2015.06.005 and depressed mood in different experimental and clinical settings; they share the common properties of increasing brain glutamate concentration and neurotransmission and of activating rCMRglc in frontal areas and subcortical aminergic nuclei (Toth et al., 1993; Moghaddam et al., 1997; Fukumoto et al., 2016; Jiménez-Sánchez et al., 2016). These findings are consistent with frontal and brainstem activations occurring during opioid, placebo (Petrovic et al., 2002), and other types of analgesia and in individuals resilient to depression (Ong et al., 2019; Fischer et al., 2021). Prefrontal and frontal cortices are pivotal components of the "pain matrix" and of the frontolimbic, frontostriatal, and default-mode networks that regulate pain perception, emotionally driven behaviors, and attention allocation (Li et al., 2018; Ong et al., 2019). A prefrontal dysfunction/hypofunction has been associated with abnormal pain processing and with loss of pleasure, motivational energy, cognitive abilities, and speed. In contrast, increased frontal activation and/or normalization of abnormal connectivity have been associated with improvement of pain and of sad mood (Li et al., 2018; Ong et al., 2019). ALCAR and KET also activated brainstem nuclei (locus coeruleus, diagonal band, and raphe nuclei) which send aminergic projections to cortical areas and spinal dorsal horns that are involved in pain and mood control (Freo et al., 2010; Khan and Stroman, 2015).

Altogether, the findings indicate that non-selective, clinical glutamate modulators such as ALCAR and KET can still provide therapeutic benefits and generate hypotheses on glutamate drug actions in neuropsychiatric conditions.

\section{AUTHOR CONTRIBUTIONS}

UF and GZ contributed to the conception and design of the study and wrote sections of the manuscript. UF wrote the first draft of the manuscript. All authors revised, read, and approved the submitted version.

\section{FUNDING}

This research was supported by departmental funds only.

Baliki, M. N., Geha, P. Y., Apkarian, A. V., and Chialvo, D. R. (2008). Beyond feeling: chronic pain hurts the brain, disrupting the default-mode network dynamics. J. Neurosci. 28, 1398-1403. doi: 10.1523/jneurosci.4123-07.2008

Battistin, L., Pizzolato, G., Dam, M., Da Col, C., Perlotto, N., Saitta, B., et al. (1989). Single-photon emission computed tomography studies with 99mTc-hexamethylpropyleneamine oxime in dementia: effects of acute administration of L-acetylcarnitine. Eur. Neurol. 29, 261-265. doi: 10.1159/ 000116423

Belcaid, I., and Eipe, N. (2019). Perioperative pain management in morbid obesity. Drugs 79, 1163-1175. doi: 10.1007/s40265-019-01156-3

Bell, J. D. (2017). In vogue: ketamine for neuroprotection in acute neurologic injury. Anesth. Analg. 124, 1237-1243. doi: 10.1213/ANE.0000000000001856

Boccard, S. G. J., Prangnell, S. J., Pycroft, L., Cheeran, B., Moir, L., Pereira, E. A. C., et al. (2016). Long-term results of deep brain stimulation of the anterior cingulate cortex for neuropathic pain. World Neurosurg. 106, 625-637. doi: 10.1016/j.wneu.2017.06.173 
Brinck, E. C., Tiippana, E., Heesen, M., Bell, R. F., Straube, S., Moore, R. A., et al. (2018). Perioperative intravenous ketamine for acute postoperative pain in adults. Cochr. Datab. Syst. Rev. 12:CD012033. doi: 10.1002/14651858. CD012033.pub4

Brouwer, B. A., de Greef, B. T., Hoeijmakers, J. G., Geerts, M., van Kleef, M., Merkies, I. S., et al. (2015). Neuropathic pain due to small fiber neuropathy in aging: current management and future prospects. Drugs Aging 32, 611-621. doi: 10.1007/s40266-015-0283-8

Burks, S., Raymick, J., Robinson, B., Hanig, J., and Sarkar, S. (2019). Neuroprotective effects of acetyl-l-carnitine (ALC) in a chronic MPTP-induced Parkinson's disease mouse model: endothelial and microglial effects. Neurosci. Lett. 703, 86-95. doi: 10.1016/j.neulet.2019.03.015

Carron, M., Veronese, S., Gomiero, W., Foletto, M., Nitti, D., Ori, C., et al. (2012). Hemodynamic and hormonal stress responses to endotracheal tube and ProSeal laryngeal mask airway ${ }^{\mathrm{TM}}$ for laparoscopic gastric banding. Anesthesiology 117, 309-320. doi: 10.1097/aln.0b013ef31825b6a80

Chen, M. H., Li, C. T., Lin, W. C., Hong, C. J., Tu, P. C., and Bai, Y. M. (2018). Persistent antidepressant effect of low-dose ketamine and activation in the supplementary motor area and anterior cingulate cortex in treatment-resistant depression: a randomized control study. J. Affect. Disord. 225, 709-714. doi: 10.1016/j.jad.2017.09.008

Chiechio, S., Canonico, P. L., and Grilli, M. (2017). L-Acetylcarnitine: a mechanistically distinctive and potentially rapid-acting antidepressant drug. Int. J. Mol. Sci. 19:11. doi: 10.3390/ijms19010011

Chiechio, S., Copani, A., De Petris, L., Morales, M. E., Nicoletti, F., and Gereau, R. W. IV (2006). Transcriptional regulation of metabotropic glutamate receptor $2 / 3$ expression by the NF-kappaB pathway in primary dorsal root ganglia neurons: a possible mechanism for the analgesic effect of l-acetylcarnitine. Mol. Pain 2:20.

Chui, H., Gerstorf, D., Hoppmann, C. A., and Luszcz, M. A. (2015). Trajectories of depressive symptoms in old age: integrating age-, pathology-, and mortality-related changes. Psychol. Aging 30, 940-951. doi: 10.1037/pag000 0054

Cohen, S. P., Bhatia, A., Buvanendran, A., Schwenk, E. S., Wasan, A. D., Hurley, R. W., et al. (2018). Consensus guidelines on the use of intravenous ketamine infusions for chronic pain from the American Society of Regional Anesthesia and pain medicine, the American Academy of Pain Medicine, and the American Society of Anesthesiologists. Reg. Anesth. Pain Med. 43, 521-546.

Costello, C. A., Hu, T., Liu, M., Zhang, W., Furey, A., and Fan, Z. (2020). Metabolomics signature for non-responders to total joint replacement surgery in primary osteoarthritis patients: the Newfoundland osteoarthritis study. J. Orthop. Res. 38, 793-802. doi: 10.1002/jor.24529

Costigan, M., Scholz, J., and Woolf, C. J. (2009). Neuropathic pain: a maladaptive response of the nervous system to damage. Annu. Rev. Neurosci. 32, 1-32. doi: 10.1146/annurev.neuro.051508.135531

Cruccu, G., Di Stefano, G., Fattapposta, F., Jann, S., Padua, L., Schenone, A., et al. (2017). L-Acetyl-carnitine in patients with carpal tunnel syndrome: effects on nerve protection, hand function and pain. CNS Drugs 31, 1103-1111. doi: 10.1007/s40263-017-0476-2

Curtis, D. R., and Watkins, J. C. (1960). The excitation and depression of spinal neurones by structurally related amino acids. J. Neurochem. 6, 117-141. doi: 10.1111/j.1471-4159.1960.tb13458.x

Dalsasso, M., Tresin, P., Innocente, F., Veronese, S., and Ori, C. (2005). Lowdose ketamine with clonidine and midazolam for adult day care surgery. Eur. J. Anaesthesiol. 22, 67-68. doi: 10.1017/s0265021505210141

D’Amato, C., Morganti, R., Greco, C., Di Gennaro, F., Cacciotti, L., and Longo, S. (2016). Diabetic peripheral neuropathic pain is a stronger predictor of depression than other diabetic complications and comorbidities. Diabetes Vasc. Dis. Res. 2016, 418-428. doi: 10.1177/1479164116653240

Duncan, G. E., Miyamoto, S., and Lieberman, J. A. (2003). Chronic administration of haloperidol and olanzapine attenuates ketamine-induced brain metabolic activation. J. Pharmacol. Exp. Ther. 305, 999-1005. doi: 10.1124/jpet.102. 048140

Dworkin, R. H., Backonja, M., Rowbotham, M. C., Allen, R. R., Argoff, C. R., Bennett, G. J., et al. (2003). Advances in neuropathic pain: diagnosis, mechanisms, and treatment recommendations. Arch. Neurol. 60, 1524-1534.

Edwards, R. R., Dworkin, R. H., Sullivan, M. D., Turk, D. C., and Wasan, A. D. (2016). The role of psychosocial processes in the development and maintenance of chronic pain. J. Pain 17(9 Suppl), T70-T92. doi: 10.1016/j.jpain.2016. 01.001

Ezzati, A., Zammit, A. R., Lipton, M. L., and Lipton, R. B. (2019). The relationship between hippocampal volume, chronic pain, and depressive symptoms in older adults. Psychiatry Res. Neuroimaging 289, 10-12. doi: 10.1016/j.pscychresns. 2019.05.003

Fischer, A. S., Hagan, K. E., and Gotlib, I. H. (2021). Functional neuroimaging biomarkers of resilience in major depressive disorder. Curr. Opin. Psychiatry 34, 22-28. doi: 10.1097/YCO.0000000000000662

Fornasari, D. (2017). Pharmacotherapy for neuropathic pain: a review. Pain Ther. 6S1, 25-33. doi: 10.1007/s40122-017-0091-4

French, E. D. (1992). Competitive NMDA receptor antagonists attenuate phencyclidine-induced excitations of A10 dopamine neurons. Eur. J. Pharmacol. 217, 1-7. doi: 10.1016/0014-2999(92)90503-v

Freo, U. (2020). "New pain modulating drugs," in XIV Course of Neuromodulations, Venezia.

Freo, U., Carron, M., Innocente, F., Foletto, M., Nitti, D., and Ori, C. (2011). Effects of a-line autoregression index (AAI) monitoring on recovery after sevoflurane anesthesia for bariatric surgery. Obes. Surg. 21, 850-857. doi: 10.1007/s11695010-0150-y

Freo, U., Dam, M., and Ori, C. (2009). Cerebral metabolic effects of acetyl-1carnitine in rats during aging. Brain Res. 1259, 32-39. doi: 10.1016/j.brainres. 2008.12.025

Freo, U., Furnari, M., Ambrosio, F., Calcarella, G., and Briani, C. (2019a). AcetylL-carnitine in the treatment of chronic lumbar radiculopathy pain. J. Peripher. Nerv. Syst. 24, 22-23.

Freo, U., Furnari, M., Ambrosio, F., and Navalesi, P. (2020). Efficacy and tolerability of tapentadol for the treatment of chronic low back pain in elderly patients. Aging Clin. Exp. Res. doi: 10.1007/s40520-020-01586-0 [Epub ahead of print].

Freo, U., Merico, A., Ermani, M., and Ori, C. (2010). Chronic treatment with fluoxetine decreases cerebral metabolic responses to the 5-HT1A agonist 8-hydroxy-2(di-N-propylamino)tetralin and increases those to the 5HT2A/2C agonist 1-(2,5-dimethoxy-4-iodophenyl)-2-aminopropane and to the dopaminergic agonist apomorphine. Brain Res. 1335, 24-34. doi: 10.1016/j. brainres.2010.03.090

Freo, U., and Ori, C. (2002a). "Mapping cerebral metabolic and blood flow effects of general anesthetics," in Proceedings of the 17th Postgraduate Course in Critical Care Medicine, ed. A. Gullo (Milano: Springer), 877-891. doi: 10.1007/978-88470-2215-7_19

Freo, U., and Ori, C. (2002b). Opioid pharmacology of ketamine. Acta Anaesth. Ital. 53, 149-163.

Freo, U., and Ori, C. (2003). "Neuroimaging studies on ketamine," in Proceedings of the $18^{\text {th }}$ Postgraduate Course in Critical Care Medicine, ed. A. Gullo (Milano: Springer), 881-893. doi: 10.1007/978-88-470-2189-1_18

Freo, U., and Ori, C. (2004). Effects of anesthesia and recovery from ketamine racemate and enantiomers on regional cerebral glucose metabolism in rats. Anesthesiology 100, 1172-1178. doi: 10.1097/00000542-20040500000020

Freo, U., and Ori, C. (2009). "Ketamine for $3^{\text {rd }}$ millenium," in Proceedings of the STAT Meeting, Udine (Italy), Udine, 23-26.

Freo, U., Romualdi, P., and Kress, H. G. (2019b). Tapentadol for neuropathic pain: a review of clinical studies. J. Pain Res. 12, 1537-1551. doi: 10.2147/JPR. S190162

Freynhagen, R., Parada, H. A., Calderon-Ospina, C. A., Chen, J., Rakhmawati Emril, D., and Fernández-Villacorta, F. J. (2019). Current understanding of the mixed pain concept: a brief narrative review. Curr. Med. Res. Opin. 35, 1011-1018. doi: 10.1080/03007995.2018.1552042

Fukumoto, K., Iijima, M., and Chaki, S. (2016). ). The Antidepressant effects of an mGlu2/3 receptor antagonist and ketamine require AMPA receptor stimulation in the $\mathrm{MPFC}$ and subsequent activation of the 5-HT neurons in the DRN. Neuropsychopharmacology 41, 1046-1056. doi: 10.1038/npp. 2015.233

Gałecki, P., and Talarowska, M. (2017). The evolutionary theory of depression. Med. Sci. Monit. 23, 2267-2274. doi: 10.12659/msm.90 1240

García-Henares, J. F., Moral-Munoz, J. A., Salazar, A., and Del Pozo, E. (2018). Effects of ketamine on postoperative pain after remifentanil-based anesthesia 
for major and minor surgery in adults: a systematic review and meta-Analysis. Front. Pharmacol. 9:921. doi: 10.3389/fphar.2018.00921

Ghoneim, M. M., and O'Hara, M. W. (2016). Depression and postoperative complications: an overview. BMC Surg. 16:5. doi: 10.1186/s12893-016-0120-y

Global Burden of Disease Study 2013 Collaborators (2015). Global, regional, and national incidence, prevalence, and years lived with disability for 301 acute and chronic diseases and injuries in 188 countries, 1990-2013: a systematic analysis for the Global Burden of Disease Study 2013. The Lancet 386, 743-800. doi: 10.1016/S0140-6736(15)60692-4

Gould, T. D., Zarate, C. A. Jr., and Thompson, S. M. (2019). Molecular pharmacology and neurobiology of rapid-acting antidepressants. Annu. Rev. Pharmacol. Toxicol. 59, 213-236. doi: 10.1146/annurev-pharmtox-010617052811

Grieco, S. F., Cheng, Y., Eldar-Finkelman, H., Jope, R. S., and Beurel, E. (2016). Up-regulation of insulin-like growth factor 2 by ketamine requires glycogen synthase kinase-3 inhibition. Prog. Neuropsychopharmacol. Biol. Psychiatry 72, 49-54. doi: 10.1016/j.pnpbp.2016.08.008

Griego, E., and Galván, E. J. (2020). Metabotropic glutamate receptors at the aged mossy fiber - CA3 synapse of the hippocampus. Neuroscience 456, 95-105.

Hambrecht-Wiedbusch, V. S., Li, D., and Mashour, G. A. (2017). Paradoxical emergence: administration of subanesthetic ketamine during isoflurane anesthesia induces burst suppression but accelerates recovery. Anesthesiology 126, 482-494. doi: 10.1097/ALN.0000000000001512

Hanewinckel, R., Drenthen, J., van Oijen, M., Hofman, A., van Doorn, P. A., and Ikram, M. A. (2016). Prevalence of polyneuropathy in the general middleaged and elderly population. Neurology 87, 1892-1898. doi: 10.1212/WNL. 0000000000003293

Hashimoto, K. (2019). Rapid-acting antidepressant ketamine, its metabolites and other candidates: a historical overview and future perspective. Psychiatry Clin. Neurosci. 73, 613-627. doi: 10.1111/pcn.12902

Henter, I. D., de Sousa, R. T., Gold, P. W., Brunoni, A. R., Zarate, C. A. Jr., and Machado-Vieira, R. (2017). Mood therapeutics: novel pharmacological approaches for treating depression. Expert. Rev. Clin. Pharmacol. 10, 153-166. doi: 10.1080/17512433.2017.1253472

Heo, M., Pietrobelli, A., Fontaine, K., Sirey, J. A., and Faith, M. S. (2006). Depressive mood and obesity in US adults: comparison and moderation by sex, age, and race. Int. J. Obes. 30, 513-519. doi: 10.1038/sj.ijo.0803122

Highland, J. N., Zanos, P., Georgiou, P., and Gould, T. D. (2019). Group II metabotropic glutamate receptor blockade promotes stress resilience in mice. Neuropsychopharmacology 44, 1788-1796. doi: 10.1038/s41386-019-0380-1

Iadarola, N. D., Niciu, M. J., Richards, E. M., Vande Voort, J. L., Ballard, E. D., et al. (2015). Ketamine and other N-methyl-D-aspartate receptor antagonists in the treatment of depression: a perspective review. Ther. Adv. Chronic. Dis. 6, 97-114. doi: 10.1177/2040622315579059

Ikonomidou, C., and Turski, L. (2002). Why did NMDA receptor antagonists fail clinical trials for stroke and traumatic brain injury? Lancet Neurol. 1, 383-386. doi: 10.1016/s1474-4422(02)00164-3

Jelen, L. A., Young, A. H., and Stone, J. M. (2020). Ketamine: a tale of two enantiomers. J. Psychopharmacol. 35, 109-123. doi: 10.1177/0269881120959644

Jeong, H. S., Park, J. S., Yang, Y., Na, S. H., Chung, Y. A., and Song, I. U. (2017). Cerebral perfusion changes after acetyl-L-carnitine treatment in early Alzheimer's disease using single photon emission computed tomography. Dement. Neurocogn. Disord. 16, 26-31. doi: 10.12779/dnd.2017.16.1.26

Jevtovic-Todorovic, V., Wozniak, D. F., Benshoff, N. D., and Olney, J. W. (2001). A comparative evaluation of the neurotoxic properties of ketamine and nitrous oxide. Brain Res. 895, 264-267. doi: 10.1016/s0006-8993(01)02079-0

Jiang, M., Wang, M. H., Wang, X. B., Liu, L., Wu, J. L., Yang, X. L., et al. (2016). Effect of intraoperative application of ketamine on postoperative depressed mood in patients undergoing elective orthopedic surgery. J. Anesth. 30, 232237. doi: 10.1007/s00540-015-2096-7

Jiménez-Sánchez, L., Castañé, A., Pérez-Caballero, L., Grifoll-Escoda, M., LópezGil, X., Campa, L., et al. (2016). Activation of AMPA receptors mediates the antidepressant action of deep brain stimulation of the infralimbic prefrontal cortex. Cereb. Cortex 26, 2778-2789. doi: 10.1093/cercor/bhv133

Kelland, M. D., Soltis, R. P., Boldry, R. C., and Walters, J. R. (1993). Behavioral and electrophysiological comparison of ketamine with dizocilpine in the rat. Physiol. Behav. 54, 547-554. doi: 10.1016/0031-9384(93)90248-e
Khan, H. S., and Stroman, P. W. (2015). Inter-individual differences in pain processing investigated by functional magnetic resonance imaging of the brainstem and spinal cord. Neuroscience 307, 231-241. doi: 10.1016/j. neuroscience.2015.08.059

Khan, S. A., Keaser, M. L., Meiller, T. F., and Seminowicz, D. A. (2014). Altered structure and function in the hippocampus and medial prefrontal cortex in patients with burning mouth syndrome. Pain 155, 1472-1480. doi: 10.1016/j. pain.2014.04.022

Kokkinou, M., Ashok, A. H., and Howes, O. D. (2018). The effects of ketamine on dopaminergic function: meta-analysis and review of the implications for neuropsychiatric disorders. Mol. Psychiatry 23, 59-69. doi: 10.1038/mp. 2017.190

Kroenke, K., Wu, J., Bair, M. J., Krebs, E. E., Damush, T. M., and Tu, W. (2011). Reciprocal relationship between pain and depression: a 12-month longitudinal analysis in primary care. J. Pain 12, 964-973. doi: 10.1016/j.jpain.2011.03.003

Kudoh, A., Takahira, Y., Katagai, H., and Takazawa, T. (2002). Small-dose ketamine improves the postoperative state of depressed patients. Anesthes. Anal. 95, 114-118. doi: 10.1097/00000539-200207000-00020

Kuratsune, H., Yamaguti, K., Lindh, G., Evengård, B., Hagberg, G., Matsumura, K., et al. (2002). Brain regions involved in fatigue sensation: reduced acetylcarnitine uptake into the brain. Neuroimage 17, 1256-1265. doi: 10.1006/nimg.2002. 1260

Li, B. J., Friston, K., Mody, M., Wang, H. N., Lu, H. B., and Hu, D. W. (2018). A brain network model for depression: from symptom understanding to disease intervention. CNS Neurosci. Therap. 24, 1004-1019. doi: 10.1111/cns.12998

Li, C. T., Chen, M. H., Lin, W. C., Hong, C. J., Yang, B. H., and Liu, R. S. (2016). The effects of low-dose ketamine on the prefrontal cortex and amygdala in treatment-resistant depression: a randomized controlled study. Hum. Brain Mapp. 37, 1080-1090. doi: 10.1002/hbm.23085

Li, S., Li, Q., Li, Y., Li, L., Tian, H., and Sun, X. (2015). Acetyl-L-carnitine in the treatment of peripheral neuropathic pain: a systematic review and meta-analysis of randomized controlled trials. PLoS One 10:e0119479. doi: 10.1371/journal. pone.0119479

Liao, Y., Tang, J., Ma, M., Wu, Z., Yang, M., and Wang, X. (2010). Frontal white matter abnormalities following chronic ketamine use: a diffusion tensor imaging study. Brain 133(Pt 7), 2115-2122. doi: 10.1093/brain/ awq131

Lucassen, P. J., Pruessner, J., Sousa, N., Almeida, O. F., Van Dam, A. M., Rajkowska, G., et al. (2014). Neuropathology of stress. Acta Neuropathol. 127, 109-135.

Luppino, F. S., de Wit, L. M., Bouvy, P. F., Stijnen, T., Cuijpers, P., and Penninx, B. W. (2010). Overweight, obesity, and depression: a systematic review and meta-analysis of longitudinal studies. Arch. Gen. Psychiatry 67, 220-229. doi: 10.1001/archgenpsychiatry.2010.2

Marton, T., Barnes, D. E., Wallace, A., and Woolley, J. D. (2019). Concurrent use of buprenorphine, methadone, or naltrexone does not inhibit ketamine's antidepressant activity. Biol. psychiatry 85, e75-e76. doi: 10.1016/j.biopsych. 2019.02.008

Masaki, Y., Kashiwagi, Y., Watabe, H., and Abe, K. (2019). (R)- and (S)-ketamine induce differential fMRI responses in conscious rats. Synapse 73:e22126. doi: 10.1002/syn.22126

Meng, W., Adams, M. J., Reel, P., Rajendrakumar, A., Huang, Y., and Deary, I. J. (2020). Genetic correlations between pain phenotypes and depression and neuroticism. Eur. J. Hum. Genet. EJHG 28, 358-366. doi: 10.1038/s41431-0190530-2

Meyer, T., Cooper, J., and Raspe, H. (2007). Disabling low back pain and depressive symptoms in the community-dwelling elderly: a prospective study. Spine 32, 2380-2386. doi: 10.1097/brs.0b013e3181557955

Mills, S. E. E., Nicolson, K. P., and Smith, B. H. (2019). Chronic pain: a review of its epidemiology and associated factors in population-based studies. Br. J. Anaesth. 123, e273-e283.

Moghaddam, B., Adams, B., Verma, A., and Daly, D. (1997). Activation of glutamatergic neurotransmission by ketamine: a novel step in the pathway from NMDA receptor blockade to dopaminergic and cognitive disruptions associated with the prefrontal cortex. J. Neurosci. 17, 2921-2927. doi: 10.1523/jneurosci. 17-08-02921.1997

Molton, I. R., and Terrill, A. L. (2014). Overview of persistent pain in older adults. Am. Psychol. 69, 197-207. doi: 10.1037/a0035794 
Moriguchi, S., Takamiya, A., Noda, Y., Horita, N., Wada, M., Tsugawa, S., et al. (2019). Glutamatergic neurometabolite levels in major depressive disorder: a systematic review and meta-analysis of proton magnetic resonance spectroscopy studies. Mol. Psychiatry 24, 952-964. doi: 10.1038/s41380-0180252-9

Mutso, A. A., Radzicki, D., Baliki, M. N., Huang, L., Banisadr, G., and Centeno, M. V. (2012). Abnormalities in hippocampal functioning with persistent pain. J. Neurosci. 32, 5747-5756. doi: 10.1523/jneurosci.0587-12.2012

Nasca, C., Bigio, B., Lee, F. S., Young, S. P., Kautz, M. M., and Albright, A. (2018). Acetyl-L-carnitine deficiency in patients with major depressive disorder. Proc. Natl. Acad. Sci. U.S.A. 115, 8627-8632.

Nasca, C., Xenos, D., Barone, Y., Caruso, A., Scaccianoce, S., Matrisciano, F., et al. (2013). L-acetylcarnitine causes rapid antidepressant effects through the epigenetic induction of mGlu2 receptors. Proc. Natl. Acad. Sci. U.S.A. 110, 4804-4809. doi: 10.1073/pnas.1216100110

Nicholson, B., and Verma, S. (2004). Comorbidities in chronic neuropathic pain. Pain Med. 5S1, S9-S27.

Nijland, L. M. G., de Castro, S. M. M., and van Veen, R. N. (2020). Risk factors associated with prolonged hospital stay and readmission in patients after primary bariatric surgery. Obes. Surg. 30, 2395-2402. doi: 10.1007/s11695-02004507-2

Ong, W. Y., Stohler, C. S., and Herr, D. R. (2019). Role of the prefrontal cortex in pain processing. Mol. Neurobiol. 56, 1137-1166. doi: 10.1007/s12035-0181130-9

Onofrj, M., Ciccocioppo, F., Varanese, S., di Muzio, A., Calvani, M., and Chiechio, S. (2013). Acetyl-L-carnitine: from a biological curiosity to a drug for the peripheral nervous system and beyond. Expert. Rev. Neurother. 13, 925-936. doi: 10.1586/14737175.2013.814930

Orhurhu, V., Claus, L. E., Vashisht, R., and Cohen, S. P. (2020). Ketamine Toxicity. Available online at: https://www.ncbi.nlm.nih.gov/books/NBK541087 (accessed July 8, 2020).

Orhurhu, V., Orhurhu, M. S., Bhatia, A., and Cohen, S. P. (2019). Ketamine infusions for chronic pain: a systematic review and meta-analysis of randomized controlled rrials. Anesth. Analg. 129, 241-254. doi: 10.1213/ANE. 0000000000004185

Ori, C., Dalsasso, M., and Freo, U. (2003). "Ketamine, a general anaesthetic agent and more. A review on its use in and out the clinical setting," in Anaesthesia, Pain, Intensive Care and Emergency Medicine. $18^{\text {th }}$ Postgraduate Course in Critical Care Medicine, ed. A. Gullo (Milano: Spinger), 863-979. doi: 10.1007/ 978-88-470-2189-1_17

Ori, C., Freo, U., Pizzolato, G., and Dam, M. (2002). Effects of acetyl-L-carnitine on regional cerebral glucose metabolism in awake rats. Brain Res. 951, 330-335. doi: 10.1016/s0006-8993(02)03290-0

O'Sullivan, C. (2004). The psychosocial determinants of depression: a lifespan perspective. J. Nerv. Ment. Dis. 192, 585-594. doi: 10.1097/01.nmd.0000138225. 19549.dd

Pelletier, R., Higgins, J., and Bourbonnais, D. (2015). Is neuroplasticity in the central nervous system the missing link to our understanding of chronic musculoskeletal disorders? BMC Musculoskelet. Disord. 12:16-25. doi: 10.1186/ s12891-015-0480-y

Pereira, A. C., Lambert, H. K., Grossman, Y. S., Dumitriu, D., Waldman, R., and Jannetty, S. K. (2014). Glutamatergic regulation prevents hippocampaldependent age-related cognitive decline through dendritic spine clustering. Proc. Natl. Acad. Sci U.S.A. 111, 18733-18738. doi: 10.1073/pnas.1421285111

Pereira, V., and Goudet, C. (2019). Emerging trends in pain modulation by metabotropic glutamate receptors. Front. Mol. Neurosci. 11:464. doi: 10.3389/ fnmol.2018.00464

Petrovic, P., Kalso, E., Petersson, K. M., and Ingvar, M. (2002). Placebo and opioid analgesia- imaging a shared neuronal network. Science 295, 1737-1740. doi: $10.1126 /$ science. 1067176

Post, R. M. (2018). Myriad of implications of acetyl-L-carnitine deficits in depression. Proc. Natl. Acad. Sci. U.S.A. 115, 8475-8477. doi: 10.1073/pnas. 1811389115

Pu, J., Liu, Y., Zhang, H., Tian, L., Gui, S., and Yu, Y. (2020). An integrated meta-analysis of peripheral blood metabolites and biological functions in major depressive disorder. Mol. Psychiatry 20. doi: 10.1038/s41380-0200645-4
Rahman, R., Ibaseta, A., Reidler, J. S., Andrade, N. S., Skolasky, R. L., and Riley, L. H. (2020). Changes in patients' depression and anxiety associated with changes in patient-reported outcomes after spine surgery. J. Neurosurg. Spine 31, 1-20. doi: 10.3171/2019.11.SPINE19586

Riggs, L. M., and Gould, T. D. (2021). Ketamine and the future of rapidacting antidepressants. Annu. Rev. Clin. Psychol. doi: 10.1146/annurev-clinpsy072120-014126 [Epub ahead of print].

Robinson, B. L., Dumas, M., Cuevas, E., Gu, Q., Paule, M. G., and Ali, S. F. (2016). Distinct effects of ketamine and acetyl L-carnitine on the dopamine system in zebrafish. Neurotoxicol. Teratol. 54, 52-60. doi: 10.1016/j.ntt.2016.02. 004

Robu, B., and Lavand'homme, P. (2019). Targeting the affective component of pain with ketamine: a tool to improve the postoperative experience? Eur. J. Anaesthesiol. 36, 4-5. doi: 10.1097/eja.0000000000000915

Rodríguez-Cano, E., Alonso-Lana, S., Sarró, S., Fernández-Corcuera, P., Goikolea, J. M., Vieta, E., et al. (2017). Differential failure to deactivate the default mode network in unipolar and bipolar depression. Bipolar Disord. 19, 386-395. doi: 10.1111/bdi.12517

Romeo, B., Choucha, W., Fossati, P., and Rotge, J. Y. (2015). Meta-analysis of short- and mid-term efficacy of ketamine in unipolar and bipolar depression. Psychiatry Res. 230, 682-688. doi: 10.1016/j.psychres.2015.10.032

Sapkota, K., Mao, Z., Synowicki, P., Lieber, D., Liu, M., Ikezu, T., et al. (2016). GluN2D N-methyl-d-aspartate receptor subunit contribution to the stimulation of brain activity and gamma oscillations by ketamine: implications for schizophrenia. J. Pharmacol. Exp. Ther. 356, 702-711. doi: 10.1124/jpet.115. 230391

Scholz, J., Finnerup, N. B., Attal, N., Aziz, Q., Baron, R., and Bennett, M. I. (2019). The IASP classification of chronic pain for ICD-11: chronic neuropathic pain. Pain 160, 53-59.

Shao, J., Meng, C., Tahmasian, M., Brandl, F., Yang, Q., Luo, G., et al. (2018). Common and distinct changes of default mode and salience network in schizophrenia and major depression. Brain Imaging Behav. 12, 1708-1719. doi: 10.1007/s11682-018-9838-8

Sharkey, J., Ritchie, I. M., Butcher, S. P., and Kelly, J. S. (1996). Comparison of the patterns of altered cerebral glucose utilisation produced by competitive and non-competitive NMDA receptor antagonists. Brain Res. 735, 67-82. doi: 10.1016/0006-8993(96)00574-4

Sheng, J., Liu, S., Wang, Y., Cui, R., and Zhang, X. (2017). The link between depression and chronic pain: neural mechanisms in the brain. Neural Plast. 2017:9724371. doi: 10.1155/2017/9724371

Skolasky, R. L., Riley, L. H. III, Maggard, A. M., and Wegener, S. T. (2012). The relationship between pain and depressive symptoms after lumbar spine surgery. Pain 153, 2092-2096. doi: 10.1016/j.pain.2012.06.026

Smeland, O. B., Meisingset, T. W., Borges, K., and Sonnewald, U. (2012). Chronic acetyl-1-carnitine alters brain energy metabolism and increases noradrenaline and serotonin content in healthy mice. Neurochem. Int. 61, 100-107. doi: 10. 1016/j.neuint.2012.04.008

Solhaug, H. I., Romuld, E. B., Romild, U., and Stordal, E. (2012). Increased prevalence of depression in cohorts of the elderly: an 11-year follow-up in the general population - the HUNT study. Int. Psychogeriatr. 24, 151-158. doi: 10.1017/S1041610211001141

Subramani, Y., Nagappa, M., Wong, J., Patra, J., and Chung, F. (2017). Death or near-death in patients with obstructive sleep apnoea: a compendium of case reports of critical complications. Br. J. Anaesth. 119, 885-899. doi: 10.1093/bja/ aex341

Tanaka, M., Nakamura, F., Mizokawa, S., Matsumura, A., Matsumura, K., and Watanabe, Y. (2003). Role of acetyl-L-carnitine in the brain: revealed by bioradiography. Biochem. Biophys. Res. Commun. 306, 1064-1069. doi: 10. 1016/s0006-291x(03)01103-3

Teffer, K., and Semendeferi, K. (2012). Human prefrontal cortex: evolution, development, and pathology. Prog. Brain Res. 195, 191-218. doi: 10.1016/B9780-444-53860-4.00009-X

Thompson, J. M., and Neugebauer, V. (2019). Cortico-limbic pain mechanisms. Neurosci. Lett. 702, 15-23. doi: 10.1016/j.neulet.2018.11.037

Tolu, P., Masi, F., Leggio, B., Scheggi, S., Tagliamonte, A., De Montis, M. G., et al. (2002). Effects of long-term acetyl-L-carnitine administration in rats: I. increased dopamine output in mesocorticolimbic areas and protection toward 
acute stress exposure. Neuropsychopharmacology 27, 410-420. doi: 10.1016/ s0893-133x (02)00306-8

Toth, E., Harsing, L. G. Jr., Sershen, H., Ramacci, M. T., and Lajtha, A. (1993). Effect of acetyl-L-carnitine on extracellular amino acid levels in vivo in rat brain regions. Neurochem. Res. 18, 573-578. doi: 10.1007/BF00966933

Ushinsky, A., Reinhardt, L. E., Simmons, A. N., and Strigo, I. A. (2013). Further evidence of emotional allodynia in unmedicated young adults with major depressive disorder. PLoS One 8:e80507. doi: 10.1371/journal.pone.0080507

van Ettinger-Veenstra, H., Lundberg, P., Alföldi, P., Södermark, M., GravenNielsen, T., Sjörs, A., et al. (2019). Chronic widespread pain patients show disrupted cortical connectivity in default mode and salience networks, modulated by pain sensitivity. J. Pain Res. 12, 1743-1755. doi: 10.2147/JPR. S189443

Volkow, N. D., Michaelides, M., and Baler, R. (2019). The neuroscience of drug reward and addiction. Physiol. Rev. 99, 2115-2140. doi: 10.1152/physrev.00014. 2018

Wang, J., Echevarria, G. C., Doan, L., Ekasumara, N., Calvino, S., Chae, F., et al. (2019). Effects of a single subanaesthetic dose of ketamine on pain and mood after laparoscopic bariatric surgery: a randomised double-blind placebo controlled study. Eur. J. Anaesthesiol. 36, 16-24. doi: 10.1097/EJA. 0000000000000860

Wang, S. M., Han, C., Lee, S. J., Patkar, A. A., Masand, P. S., and Pae, C. U. (2014). A review of current evidence for acetyl-l-carnitine in the treatment of depression. J. Psychiatr. Res. 53, 30-37. doi: 10.1016/j.jpsychires.2014.02.005

Williams, N. R., Heifets, B. D., Blasey, C., Sudheimer, K., Pannu, J., Pankow, H., et al. (2018). Attenuation of antidepressant effects of ketamine by opioid receptor antagonism. Am. J. Psychiatry 175, 1205-1215. doi: 10.1176/appi.ajp. 2018.18020138

Witkin, J. M., Monn, J. A., Schoepp, D. D., Li, X., Overshiner, C., Mitchell, S. N., et al. (2016). The rapidly acting antidepressant ketamine and the mGlu2/3 receptor antagonist LY341495 rapidly engage dopaminergic mood circuits. J. Pharmacol. Exp. Therap. 358, 71-82. doi: 10.1124/jpet.116.233627
Yang, C., Yang, J., Luo, A., and Hashimoto, K. (2019). Molecular and cellular mechanisms underlying the antidepressant effects of ketamine enantiomers and its metabolites. Trans. Psychiatry 9:280. doi: 10.1038/s41398-019-0624-1

Yoon, G., Petrakis, I. L., and Krystal, J. H. (2019). Association of combined naltrexone and ketamine with depressive symptoms in a case series of patients with depression and alcohol use disorder. JAMA Psychiatry 76, 337-338. doi: 10.1001/jamapsychiatry.2018.3990

Zammataro, M., Chiechio, S., Montana, M. C., Traficante, A., Copani, A., and Nicoletti, F. (2011). ). mGlu2 metabotropic glutamate receptors restrain inflammatory pain and mediate the analgesic activity of dual mGlu2/mGlu3 receptor agonists. Mol. Pain 7:6.

Zanos, P., Moaddel, R., Morris, P. J., Georgiou, P., Fischell, J., Elmer, G. I., et al. (2016). NMDAR inhibition-independent antidepressant actions of ketamine metabolites. Nature 533, 481-486. doi: 10.1038/nature 17998

Zhang, K., and Hashimoto, K. (2019). Lack of opioid system in the antidepressant actions of ketamine. Biol. Psychiatry, 85, e25-e27. doi: 10.1016/j.biopsych.2018. 11.006

Zhang, Y., Mao, Z., Pan, L., Ling, Z., Liu, X., Zhang, J., et al. (2018). Dysregulation of pain- and emotion-related networks in trigeminal neuralgia. Front. Hum. Neurosci. 12:107. doi: 10.3389/fnhum.2018.00107

Conflict of Interest: The authors declare that the research was conducted in the absence of any commercial or financial relationships that could be construed as a potential conflict of interest.

Copyright (c) 2021 Freo, Brugnatelli, Turco and Zanette. This is an open-access article distributed under the terms of the Creative Commons Attribution License (CC BY). The use, distribution or reproduction in other forums is permitted, provided the original author(s) and the copyright owner(s) are credited and that the original publication in this journal is cited, in accordance with accepted academic practice. No use, distribution or reproduction is permitted which does not comply with these terms. 


\section{OPEN ACCESS}

Edited by:

Gregor Thut,

University of Glasgow, United Kingdom

Reviewed by: Michele Dileone, Hospital Virgen del Puerto, Spain David Haslacher,

Charité-Universitätsmedizin Berlin, Germany

*Correspondence:

Charlotte Ide-Walters charlotte.ide@cancer.org.uk; c.v.ide@gre.ac.uk

Trevor Thompson

t.thompson@gre.ac.uk

Specialty section: This article was submitted to

Perception Science, a section of the journal Frontiers in Neuroscience

Received: 03 August 2020 Accepted: 25 June 2021 Published: 26 July 2021

Citation:

Ide-Walters $C$ and Thompson $T$ (2021) A Sham-Controlled Study of Neurofeedback for Pain Management. Front. Neurosci. 15:591006. doi: 10.3389/fnins.2021.591006

\section{A Sham-Controlled Study of Neurofeedback for Pain Management}

\author{
Charlotte Ide-Walters ${ }^{1,2 *}$ and Trevor Thompson ${ }^{1 *}$ \\ ${ }^{1}$ Centre for Chronic Illness and Ageing, University of Greenwich, London, United Kingdom, ${ }^{2}$ Cancer Research UK, London, \\ United Kingdom
}

Background: Neurofeedback (NFB) attempts to alter the brain's electrophysiological activity and has shown potential as a pain management technique. Existing studies, however, often lack appropriate control groups or fail to assess whether electrophysiological activity has been successfully regulated. The current study is a randomized controlled trial comparing changes in brain activity and pain during NFB with those of a sham-control group.

Methods: An experimental pain paradigm in healthy participants was used to provide optimal control of pain sensation. Twenty four healthy participants were blind randomized to receive either $10 \times \mathrm{NFB}$ (with real EEG feedback) or $10 \times$ sham (with false EEG feedback) sessions during noxious cold stimulation. Prior to actual NFB training, training protocols were individually determined for each participant based on a comparison of an initial 32-channel qEEG assessment administered at both baseline and during an experimental pain task. Each individual protocol was based on the electrode site and frequency band that showed the greatest change in amplitude during pain, with alpha or theta up-regulation at various electrode sites (especially Pz) the most common protocols chosen. During the NFB sessions themselves, pain was assessed at multiple times during each session on a $0-10$ rating scale, and ANOVA was used to examine changes in pain ratings and EEG amplitude both across and during sessions for both NFB and sham groups.

Results: For pain, ANOVA trend analysis found a significant general linear decrease in pain across the 10 sessions $(p=0.015)$. However, no significant main or interaction effects of group were observed suggesting decreases in pain occurred independently of NFB. For EEG, there was a significant During Session X Group interaction ( $p=0.004$ ), which indicated that EEG amplitude at the training site was significantly closer to the target amplitude for the NFB compared to the sham group during painful stimulation, but this was only the case at the beginning of the cold task.

Conclusion: While these results must be interpreted within the context of an experimental pain model, they underline the importance of including an appropriate comparison group to avoid attributing naturally occurring changes to therapeutic effects.

Keywords: EEG-biofeedback, neurofeedback, experimental pain in humans, neuromodulation, sham-controlled design, acute pain, pain, sham-controlled 


\section{INTRODUCTION}

Chronic pain is one of the leading causes of disability (Van Hecke et al., 2013) and negatively impacts wellbeing, sleep, and physical health (Hadi et al., 2019; Murray et al., 2020), as well as costing billions in health care and lost work productivity (American Geriatrics Society Panel on the Pharmacological Management of Persistent Pain in Older Persons, 2009; Langley et al., 2010; Abdulla et al., 2013; Gaskin et al., 2017; Saxen and Rosenquist, 2020). Pain can also persist well after any physical injury has healed suggesting the central nervous system may play a significant role in the experience and maintenance of pain. Although the cortical signature of pain is complex, reliable evidence suggests involvement of a neuromatrix of cortical pathways (Melzack, 1999, 2001, 2005; Fitzgerald, 2020) including the anterior cingulate, prefrontal cortex, insular cortex, and primary and secondary cortices (Casey, 1980; Brooks and Tracey, 2005). These pathways may be mediated by several physiological (Apkarian et al., 2005) and psychological components (Wall et al., 1994; Thompson et al., 2012; Herbert et al., 2014; Riva et al., 2014; Dave et al., 2015). In theory, if the activity of the brain structures involved in pain processing can be regulated, this could in turn influence our experience of pain (Ros et al., 2010, 2013). This has led to an increased interest in novel interventions for pain management, such as neurofeedback (NFB).

NFB involves the real-time feedback of a person's cortical activity by translating an EEG signal measured at the scalp to a changing audio or visual display (e.g., moving bars) in line with changes in the EEG. The basic aim of NFB is to provide a reward [e.g., auditory (music, chime), visual (point, token)] for a change in EEG activity that it is believed to be associated with a positive emotional or behavioral change, in line with basic principles of instrumental conditioning (Schabus et al., 2017). However, a recent review by Enriquez-Geppert et al. (2013) studied the role of NFB in the improvement of executive function (EF). Executive function is an important function that mediates learning, which highlights the link between both behavioral and cognitive elements involved in NFB training, and that there may be multiple factors that drive treatment efficacy (EnriquezGeppert et al., 2013; Ros et al., 2020). There is a debate to the extent of which NFB can alter brain activity, and the exact mechanisms to how it operates (Gruzelier, 2014a,b; Ros et al., 2020), and reinforces the importance to conduct controlled studies in order to evaluate efficacy (Thibault et al., 2017).

Several studies have generated some promising findings supporting the potential of NFB for pain management (Roy et al., 2020). Some of the earliest studies were case studies (Sime, 2004; Kayiran et al., 2007). Sime (2004) studied a single female patient suffering from trigeminal neuralgia. It was concluded that the patient had experienced a large reduction in pain to the extent she canceled her planned neurosurgery, and even reduced her use of prescribed analgesic. Kayiran et al. (2007) conducted a case study of three patients who were diagnosed with Fibromyalgia (FMS) and also found pain decreased with NFB.

Later research examined larger cohorts of patients (Jensen et al., 2008, 2013a, 2014; Stokes and Lappin, 2010) and found promising findings. Jensen et al. (2007) conducted a study with
18 patients with complex regional pain syndrome and found a statistically significant decrease in pain reported pre to post training, with over half of participants reporting a clinically meaningful decrease of $>30 \%$ (Moore et al., 2013). Stokes and Lappin (2010) and Jensen et al. (2013a, 2014) found similar results, however, Jensen et al. (2013a) found their decrease in pain was not clinically meaningful. In addition to single-arm designs, some studies have included control comparison groups (Kayiran et al., 2010; Caro and Winter, 2011; Hassan et al., 2015). Kayiran et al. (2010) implemented an active control group and Caro and Winter (2011) implemented an historic control group, and both found that their NFB groups demonstrated greater success than the control group for pain reduction. Hassan et al. (2015) attempted a placebo style control group, however, they did not have a dedicated control group and instead applied a sham style procedure to the 10th and 20th session for the same participants. The challenges of a cross over control design means that the participants will have possibly already learnt NFB and it isn't an isolated controlled condition and the training they received previously may still influence how they respond during the non-trained session. However, Hassan et al. (2015) found promising findings with decreases in pain noted for the NFB sessions, and no reported decrease in pain for the sham conditions. All studies found results that offered support for the efficacy of NFB with EEG, however, it was recommended further controlled studies were required.

Importantly, the majority of these supporting studies lack an optimal placebo control groups and only a few studies have assessed whether cortical regulation (the key putative mechanism) has actually occurred Roy et al. (2020). Rogala et al. (2016) identified little evidence for desired changes in EEG frequency power. Some studies have found pain changed regardless of whether changes in EEG activity occurred (Jensen et al., 2013b). A sham-control group is the most appropriate research design to study a NFB intervention as it provides a method of controlling for several components that are fundamentally unrelated to EEG regulation but may nevertheless affect pain or EEG (Schabus et al., 2017; Roy et al., 2020), including attention and expectancy effects (Loo and Barkley, 2005, Thompson et al., 2011). If distraction, for example, is a key putative mechanism that underlies any analgesic effects of NFB treatment, it might be possible that such effects could be more simply and easily achieved using a simple distraction task (Thompson et al., 2011).

Most studies of NFB and pain have used protocols that focus on regulation of EEG activity at a target site expected to reflect cortical activity at key areas of the "pain matrix." Imaging studies have established that the anterior cingulate, insular, primary/secondary cortices and the thalamus are active during naturally occurring and experimentally induced pain, with additional involvement of the prefrontal cortex in chronic pain reflecting it's greater cognitive-emotional component (Apkarian et al., 2005). The variation of areas involved in pain processing is mimicked by considerable variation in the protocols used in previous studies. These have ranged from up-regulation of SMR at C4 (Kayiran et al., 2010) and theta at AFz (Jensen et al., 2018) to down-regulation of theta at $\mathrm{Cz}$ (Caro and Winter, 2011) or C2-C4 
(Vučković et al., 2019), with details not reported in several other studies (Roy et al., 2020). A recent systematic review published in this Frontiers Research Topic (Roy et al., 2020) provides an extensive review of these protocols and concludes that no two protocols used across the examined studies were identical.

Most commonly, studies of NFB and pain have used a single fixed protocol applied to all participants. While such a generalized approach would be expected to be most beneficial for conditions with a predictable and homogenous pattern of EEG activity, a more effective approach where heterogeneity is present may be the use of individualized protocols based on the pattern of EEG dysregulation for that individual. Such data-driven protocols are typically based on an individual initial quantitative electroencephalogram (qEEG) assessment, which is compared to EEG from a normative database to identify the electrode positions and bandwidths to be targeted with the overarching aim of "normalizing" brain activity (Roy et al., 2020). This approach has recently been successfully adopted in NFB therapy for Chronic Tinnitus (Güntensperger et al., 2019) and ADHD (Dobrakowski and Łebecka, 2020). Despite the existence of an established cortical pain matrix, variation in EEG activity at different frequency bands and scalp sites is still likely as a result of heterogenous pain aetiology and individual factors such as head shape, and a few studies have utilized individualized protocols. Jensen et al. (2007), for example, used initial SMR up-regulation at T3/T4 in individuals with Complex Regional Pain Syndrome, but then employed progressively different protocols if the patient failed to report improvement until an optimal individual protocol was found. Prinsloo et al. (2018) used patient-specific protocols and found NFB to reduce pain in cancer survivors (although the authors did not report details of the electrode sites or frequencies that were trained).

In the current study, we will examine the effect of NFB within an experimentally induced cold pain paradigm applied to healthy participants. This approach has the advantage of facilitating the identification of an individualized protocol by comparing each participant's EEG during a pain-free state with their EEG during noxious stimulation to identify the most relevant target site and frequency band. The use of experimentally induced pain also offers a level of control that can help overcome some of the difficulties encountered in clinical settings, such as dayto-day variation in chronic pain and use of pain medications that can complicate interpretation (Staahl et al., 2009). The two primary aims of the current study are to assess the ability of NFB to: (1) modulate cortical activity during pain; and (2) produce reductions in pain that exceed those of a sham-control group.

\section{MATERIALS AND METHODS}

\section{Participants}

The sample consisted of 24 healthy volunteers with a mean age of 27.9 years $(S D=12.3$, range $=18-56)$ with 9 males and 15 females. There was a mixture of 11 students (from the hosting University) and 13 non-student volunteers. Students were compensated with course credit for their participation, non-students were volunteers who received no payment or compensation for their time. Participants were required to confirm they did not violate any of the exclusion criteria, which were any long-term pain condition, Raynaud's disease or any other condition that might affect the perception of or cause an adverse reaction to pain. Participants were also requested to abstain from the following prior to experimentation to prevent potential disruption of pain processing or EEG activity: analgesics $(48 \mathrm{~h})$; alcohol (12 h); caffeine $(2 \mathrm{~h})$; and nicotine $(1 \mathrm{~h})$. Participants were asked if they had ever taken part in a cold pain experiment or an experiment with EEG. All participants $(n=24)$ stated that they had not.

Participants were assigned to either the sham control group $(n=12)$ or the NFB group $(n=12)$ using block randomization to ensure equal group sizes. Participants were aware that they may be allocated to either the NFB or the placebo group but were blinded to group assignment until the end of the study. The researcher was not blinded as this was part of doctoral research and therefore it was the same researcher designing the study and implementing the research and analysis. Despite randomization of participants there was some difference between groups in the distribution of sex and age, with more females in the placebo group $(n=9)$ than the NFB group $(n=6)$, and with a lower mean age for the placebo (22 years) relative to the NFB group (31 years). As only healthy participants were used no "standard of care" equivalent was possible, however for all baselines the participants not in pain data was used as a baseline to both inform protocol selection and to set the target threshold for the NFB protocols.

\section{Sham Control Group}

The sham-control group and the NFB intervention group underwent identical procedures, including assessment of individualized training sites for their NFB training (see section "Individualized Neurofeedback Protocols"), except for the feedback provided to them during the session. Specifically, the NFB intervention group were presented with genuine real time EEG activity and the sham-control group were given false EEG feedback using pre-recorded EEG data from participants who had undergone genuine NFB training.

\section{Pain Induction and Assessment}

For pain induction, we employed the cold pressor task. A thermostatically controlled tank was used, which housed a circulating motor that ensured the water was consistently and continuously circulated around the tank to avoid localized heating of the hand. This method of pain induction is simple to administer with no long-term adverse effects (Mitchell et al., 2004). Further to this, the cold pressor test scores high in terms of validity and is widely used across several research areas to induce pain (Rebbeck et al., 2015). As shown in Figure 1, $3 \times 4$ min cold water pain inductions were administered within each session of NFB. Participants were told to take their hand out of the water at any point if they found the pain unbearable.

For pain assessment, a 0-10 numerical rating scale (NRS) was used to assess pain in response to cold stimulation and was administered at multiple time points during each NFB session. Specifically, participants were asked to verbally report pain ratings at $15 \mathrm{~s}$ (for a baseline measure) and at 2, 3, and 4 min during each of the three 4-min cold trials that took place 


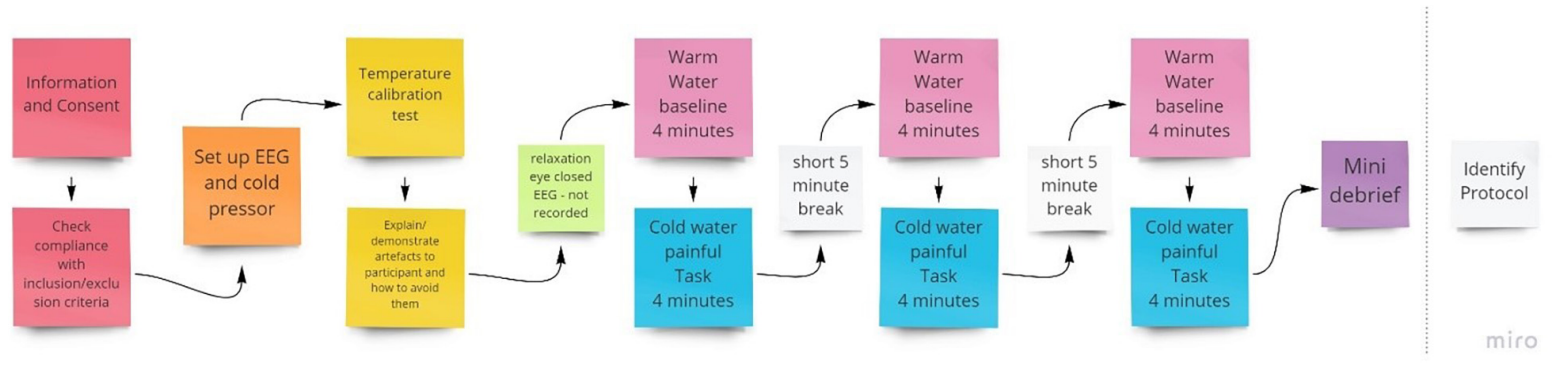

FIGURE 1 | Procedure for the initial qEEG session to identify individual training protocols to be used in subsequent NFB sessions.

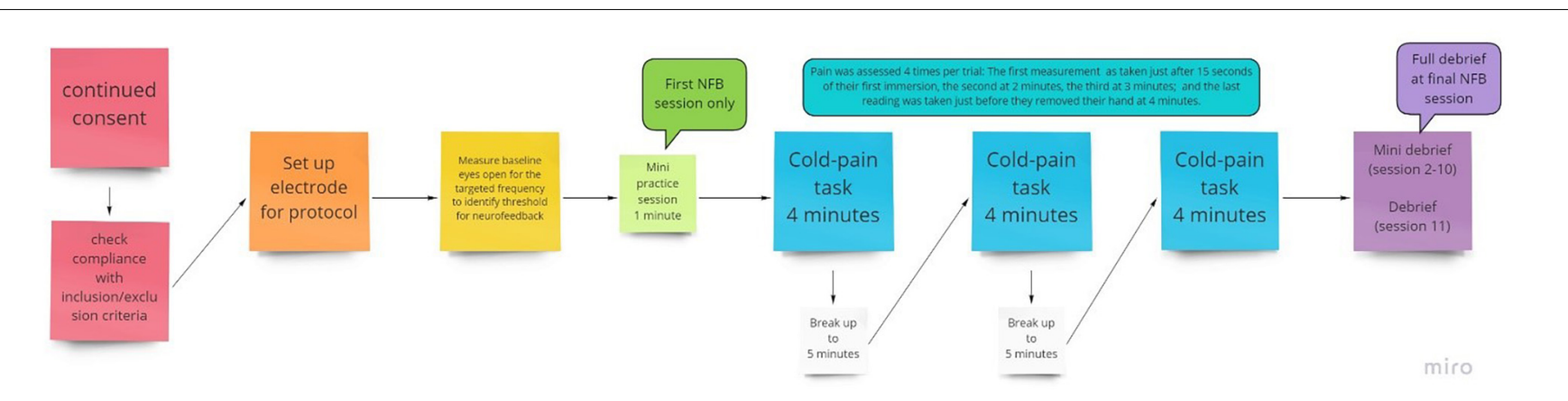

FIGURE 2 | Flow chart of the procedure for an individual session, with NFB or sham given during the 3 pain tasks provided.

during each session, for a total of 12 ratings per session (see Figure 2). The NRS is a well-established pain measure with a low administration time of just a few seconds, which was necessary to minimize disruption during the NFB session. For the purposes of analysis, NRS pain scores were averaged across the 3 trials that occurred with a session to produce a single pain rating at each of $15 \mathrm{~s}, 2,3$, and 4 min intervals.

\section{Individualized Neurofeedback Protocols}

To inform electrode placement for the individualized sessions, a full brain EEG was first performed during a 4-min baseline warm $\left(37^{\circ} \mathrm{C}\right)$ followed by a 4 -min noxious cold (initially $10^{\circ} \mathrm{C}$ ) trial, with this procedure conducted three times (Figure 1). The purpose of this was to identify optimal training electrode sites which best reflect changes in EEG during pain by comparing the individual's EEG activity (amplitude) when in pain, subject to cold thermal noxious stimulation, and when not in pain (qEEG). For EEG recording, we used a Mitsar 202-32 EEG amplifier (REF), which has 31 active channels and 1 reference channel. Electrodes were placed according to the extended 10-20 system (channels included were: FPz, FP1, FP2, F7, F3, Fz, F4, F8, FT7, FC3, FCz, FC4, FT8, T3, C3, Cz, C4, T4, TP7, CP3, CPz, CP4, TP8, T5, P3, Pz, P4, T6, O1, Oz, O2; Thompson et al., 2008) and EEG recorded at $256 \mathrm{~Hz}$, with all electrode impedances maintained at $<5 \mathrm{k} \Omega$ throughout. A common reference of the average signal across both ears was used during EEG acquisition.

After removal of EEG artifact, peak amplitude was computed for the painful cold and the non-painful warm condition for each electrode, for each of the three 8-min trials. The electrode site and frequency band for NFB training for a participant was selected based on the placement site for each individual and frequency that appeared to demonstrate the largest amplitude difference between the painful and non-painful stimuli, whilst also demonstrating consistency across the three trials. This is a similar method implemented by Stokes and Lappin (2010) who collected data from 10 sites to determine peak amplitudes and used this information along with prior experience to determine 5 homologous protocols to target their feedback. See Table 1 for the final protocols used.

\section{Cold Pressor Temperature Calibration}

A single fixed noxious cold temperature can produce a wide variation in subjective pain intensity (Graven-Nielsen et al., 2001) and may produce no pain in some participants and pain that cannot be tolerated for more than a short period in others, we used an individualized temperature to provide a relatively homogenous level of baseline pain across participants. Specifically, we used a stimulus intensity that was tolerable for that individual for $4 \mathrm{~min}$ (the duration of the pain stimulus) and that resulted in a rating of $4-6$ on a $0-10$ pain rating scale as this roughly approximates a moderate and clinically meaningful level of pain (Boonstra et al., 2016). For the first trial, a $10^{\circ} \mathrm{C}$ temperature was initially used. If a pain rating $<4$ was given, the procedure was repeated with a $1^{\circ} \mathrm{C}$ reduction in temperature (i.e., made colder), to make the task more painful, and was continued until a 4-6 pain rating was reported. If the trial was terminated before the $4 \mathrm{~min}$ had elapsed or pain ratings were $>6$, the temperature was increased by $1^{\circ} \mathrm{C}$ in a subsequent trial. 
TABLE 1 | Protocols identified as optimal for the intervention and the (untrained) sham control groups.

\begin{tabular}{|c|c|c|c|}
\hline Group & Frequency & Direction & Target site \\
\hline NFB & Alpha & Up & $\mathrm{Pz}$ \\
\hline NFB & Theta & Up & Fpz \\
\hline NFB & Alpha & Up & C4 \\
\hline NFB & Alpha & Up & $\mathrm{Pz}$ \\
\hline NFB & Theta & Down & $\mathrm{Pz}$ \\
\hline NFB & Alpha & Up & $\mathrm{FCz}$ \\
\hline NFB & Theta & Down & $\mathrm{Cz}$ \\
\hline NFB & Alpha & Up & $\mathrm{Pz}$ \\
\hline NFB & Theta & Up & $\mathrm{FCz}$ \\
\hline NFB & Theta & Up & $\mathrm{FCz}$ \\
\hline NFB & Theta & Up & $\mathrm{Fz}$ \\
\hline NFB & Alpha & Down & C3 \\
\hline Control & Alpha & Down & CP3 \\
\hline Control & Alpha & Up & $\mathrm{Pz}$ \\
\hline Control & Alpha & Up & Fp1 \\
\hline Control & Alpha & Up & P4 \\
\hline Control & Alpha & Up & $\mathrm{Pz}$ \\
\hline Control & Alpha & Up & C3 \\
\hline Control & Alpha & Down & Cp3 \\
\hline Control & Theta & Down & $\mathrm{F} 4$ \\
\hline Control & Alpha & Up & Cp3 \\
\hline Control & Alpha & Down & C4 \\
\hline Control & Alpha & Up & T3 \\
\hline Control & Alpha & Down & $\mathrm{FCz}$ \\
\hline
\end{tabular}

The final individual temperature reached was applied for that participant in all the following NFB sessions. A comparison of NFB experimental and sham control groups with independent $t$-tests found no group differences in either temperature $[t=0.65$, $p=0.52, M(\mathrm{NFB})=10.2^{\circ} \mathrm{C}, M($ Sham $\left.)=9.8^{\circ} \mathrm{C}\right]$ or baseline pain intensity $[t=0.04, p=0.97, M(\mathrm{NFB})=4.52, M($ Sham $)=4.54]$.

The entire first session took between 90 and $120 \mathrm{~min}$ to complete. This included time to brief and debrief, set up the equipment, and conduct assessments and the trials. Participants were also shown their EEG and the experimenter explained the implications of artifacts and showed how they are caused and how to avoid them to help reduce avoidable artifacts (Thompson et al., 2008).

\section{Neurofeedback}

NFB was performed using a Nexus-4, which has up to 2 EEG channels recorded at a sampling rate of $256 \mathrm{~Hz}$. Electrode placement was determined by an individual assessment of each participant's EEG during pain as previously described (see section "Individualized Neurofeedback Protocols"). Biotrace software was used for the application of the NFB protocol and to build the biofeedback screen used (Figure 3 provides a screenshot). The reward protocol was to feedback a reward to the participant of 1 point for every second they were able to maintain their EEG within the targeted range.

The threshold (target) for the NFB protocol was calculated by recording $4 \mathrm{~min}$ of eyes open activity using the feedback electrode. The participants were not subject to any pain, but their hand was immersed into warm water as done above for the first baseline to identify the NFB protocol. This was to give a target to reinforce that was related to the subject non pain state on that day and at that time. The average EEG amplitude from the 4-min was used. This was repeated at the start of every NFB session to account for variations (e.g., different times of day) and the same threshold was used for all three trials within that session. This baseline was recorded with the participant's non-dominant hand immersed into the warm water. This identified what their normal frequency for the targeted protocol frequency was for that electrode site when not in pain and was the benchmark for which they are attempting to train their cortical activity to return to during training. In addition to the training protocol, an EMG (muscle/movement artifact) control was added to the NFB screen to encourage participants to keep their EMG artifact low. The inhibit EMG bar was displayed to the left of the NFB protocol and showed green when the participant kept this at the correct level, and red when EMG increased. To further encourage participants to keep their EMG low, participants would only achieve points/rewards for achieving their protocol when their EMG was also at the correct level. This control helped to prevent moving, EMG artifact, and participants from training themselves to move to achieve the NFB protocol.

Participants took part in 10 NFB sessions, which were conducted 3-7 times per week subject to participants' availability, with no more than one session per day to avoid fatigue but to ensure it was regular. Each session lasted around 45$60 \mathrm{~min}$, which included set up time, briefing/debriefing, baseline measurement and three cold trials (Figure 2). Each session consisted of $3 \times 4$ min cold pain tasks at the participant's individualized temperature.

In the first of the ten NFB sessions, participants were given a minute to practice and understand the objective of the session. Participants were not provided with a strategy, only to be observant of when their cortical activity fed back that they had been successful and to try to replicate that. Their understanding was checked, and they were reminded about artifacts and how to avoid them. In each of the 4-min cold trials, pain ratings were recorded at $15 \mathrm{~s}, 2,3$, and 4 min or when they removed their hand. A break of up to 5 min was added in between each cold pain task in order to allow participants to recover from the numbing effects of the cold water.

\section{EEG Data Pre-processing}

The data was visually inspected and if any large EMG artifacts were found these were removed manually before running independent component analysis (ICA) to identify EOG artifact. ICA was conducted using the WinEEG software with eye blink or lateral eye movement components removed automatically (Gao et al., 2010).

In the initial assessment of data for determining protocols, WinEEG was used to conduct Fast Fourier Transform (FFT) for Delta $(0-4 \mathrm{~Hz})$, Theta $(4-8 \mathrm{~Hz})$, Alpha $(8-12 \mathrm{~Hz})$, and Beta (12-40 Hz), visually comparing all spectral output (with a similar approach to Stokes and Lappin, 2010), by subtracting the baseline 


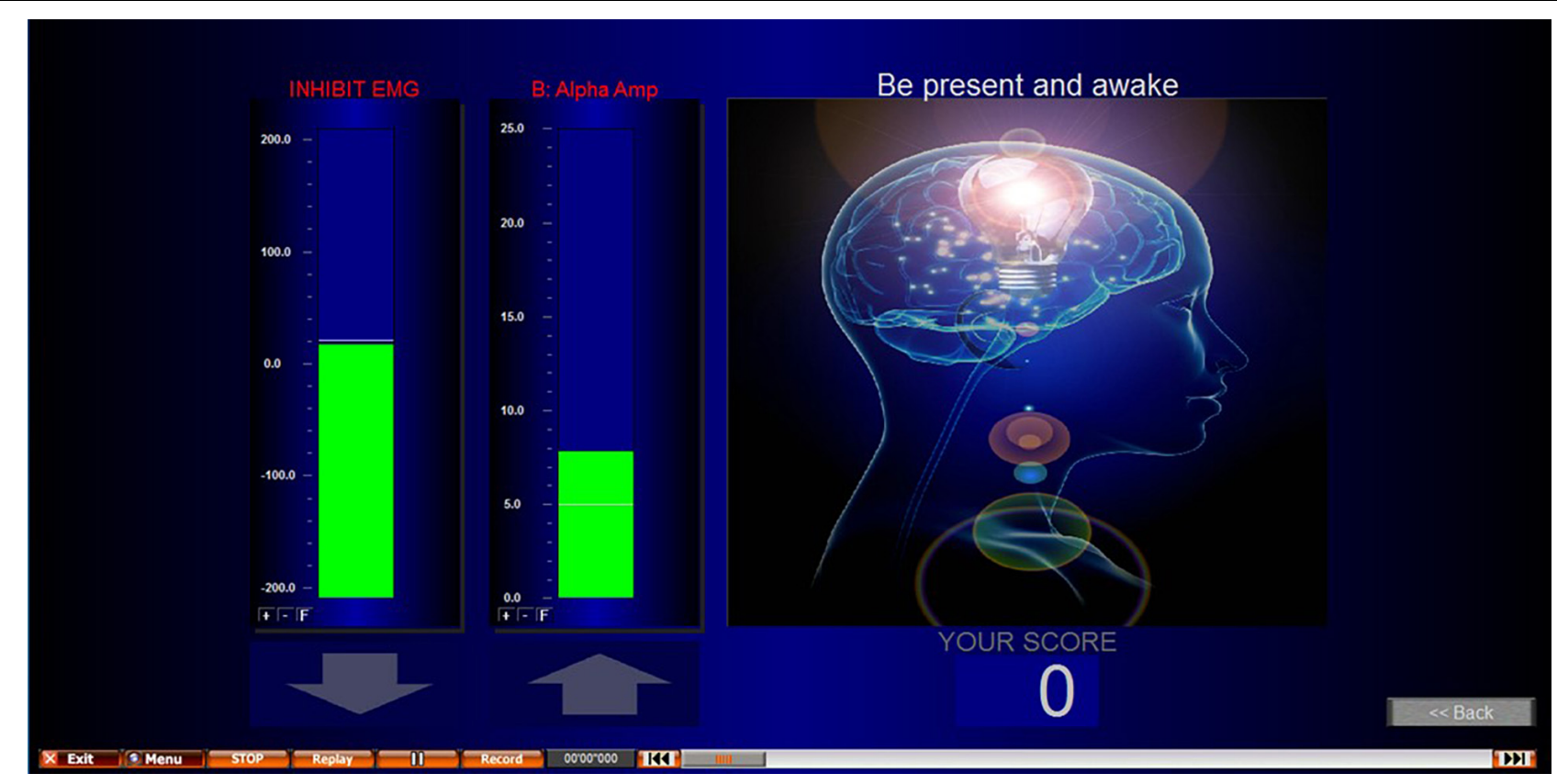

FIGURE 3 | Screenshot of NFB screen presented during NFB/sham. EMG inhibit bar encouraged participants to keep their movement artifact low, and to prevent conditioning participants to achieve the desired outcome through movement. This second column shows an alpha uptraining example. However, alpha up, alpha down, theta up, and theta down were all protocols used in this study.

from the painful condition. For the main analysis, amplitudes for the target frequency bands (alpha or theta) were computed using FFT from the first $30 \mathrm{~s}$ and the last $30 \mathrm{~s}$ for each of the three trials within a session. These were then averaged across the three trials, at each time point within a session, to give a single mean amplitude for the first $30 \mathrm{~s}$ and the last $30 \mathrm{~s}$ of each of the 10 sessions.

\section{Statistical Analysis Plan}

The Effect of Neurofeedback on Pain and Regulation of EEG

Mixed ANOVAs were used to examine whether changes in EEG occurred during sessions (first $30 \mathrm{~s}$ vs. last $30 \mathrm{~s}$ ) and across sessions (1-10), and whether this differed by group (NFB vs. control). Given that the target EEG amplitude (established from the individual's EEG activity during their pain-free baseline state) required up-regulation for some participants and downregulation for others, change was coded such that a positive sign indicated a change in the desired direction (i.e., toward the target amplitude) while a negative sign indicated a change in the opposite direction. Although there are different ways in which training "success" could be potentially assessed, we computed the size of the difference between the participant's target amplitude and the amplitude during the pain task (at the chosen training site and frequency band). Smaller differences therefore represent greater "success," as reward protocols were designed to return amplitude to the target frequency associated with a baseline pain-free state.

To assess whether NFB resulted in lower pain than the control procedure during sessions (baseline $15 \mathrm{~s}, 2,3$, and $4 \mathrm{~min}$ ) or across sessions (1-10), the same analysis was conducted as described above, but with pain ratings as the outcome variable. In all instances, trend analysis was also conducted to examine whether any progressive changes in EEG or pain across sessions followed a linear or quadratic trend.

\section{RESULTS}

\section{Data Screening}

EEG data were missing for 5 of the 240 (2\%) sessions due to equipment failure, and these values were imputed using the mean value from non-missing participants for the equivalent trial and session number. For pain rating data, $<2 \%$ of data were missing so we imputed missing values with the participant's trial average. No outliers were identified $(z>3.29$ or $<-3.29$, Tabachnick and Fidell, 2013) for pain ratings. Z scores for EEG amplitudes suggested 5 outliers which were again substituted with linear interpolations of that participant's adjacent sessions. Alpha $(S D=2.9)$ and theta $(S D=2.1)$ frequencies showed similar dispersion.

\section{Regulation of EEG}

Individual protocols for each participant in the NFB groups are shown in Table 1 (which also shows the protocols identified to be optimal for the sham-control participants although no actual NFB was performed). Training protocols for the NFB group consisted of a mixture of alpha and theta training (at different electrode sites), with six participants receiving alpha training 
(down-regulation $=1$, up-regulation $=5$ ) and six receiving theta regulation (down-regulation $=2$, up-regulation $=4$ ).

ANOVA revealed a significant overall deviation from target EEG during a session $[F(1,22)=10.35, p=0.004]$, with this deviation greater during the first $30 \mathrm{~s}(M=3.01)$ compared to the last $30 \mathrm{~s}(M=2.08)$ of the pain task. There was also a significant During-session X Group interaction $[F(1,22)=10.31, p=0.004]$, with Figure 4 suggesting EEG amplitude was closer to the target value during NFB than the control procedure during the first $30 \mathrm{~s}$ of the pain task, but with little difference near the end of the task. No other effects approached significance $(p=0.13-0.46)$, with all results shown in Table 2.

As different protocols were used, we conducted an exploratory investigation of whether alpha training or theta training produced a greater impact on EEG parameters by rerunning ANOVA on the NFB experimental group only (a protocol was not implemented for the sham group), but including frequency band (alpha vs. theta) as an additional variable. Results found no significant differences between alpha $(M=2.06 \mu \mathrm{V})$ and theta $(M=2.24 \mu \mathrm{V})$ frequency bands $(p=0.798)$ or any interactive effects $(p=0.342-0.813)$. We did not compare up-regulation $(M=2.35 \mu \mathrm{V})$ vs. down-regulation $(M=1.55 \mu \mathrm{V})$ with inferential statistical tests given the fact only three participants were given down-regulation training.

\section{Pain Modulation}

ANOVA revealed a significant difference in mean pain ratings during the session $F(3,66)=7.34, p<0.001)$, with Figure 5 showing a sharp rise in pain from $15 \mathrm{~s}$ to that at 2,3 , and 4 min period, with a quadratic trend identified $[F(1,22)=24.31$, $p<0.001]$. Neither the control nor the experimental groups saw a clinically meaningful reduction in average pain ratings (>30\%).

With respect to changes in overall pain across sessions, significant differences across the 10 sessions were also observed $[F(9,198)=3.09, p=0.002]$, with trend analysis indicating a progressive linear decrease in pain overall across sessions $[F(1,22)=6.91, p=0.015]$. There was no significant interaction of during session changes and group, with both NFB and controls demonstrating a similar decrease in pain ratings across sessions as shown in Figure 6. Table 3 shows the complete table of results and with no other effects significant.

TABLE 2 | Results of the Mixed ANOVA for EEG amplitude with $\eta^{2}$ indicating the partial-eta squared effect size.

\begin{tabular}{lcccl}
\hline & $\boldsymbol{d f}$ & $\mathbf{F}$ & $\boldsymbol{\eta}^{2}$ & $\boldsymbol{p}$-value \\
\hline Group & 1,22 & 2.39 & 0.098 & 0.137 \\
Across $^{\mathrm{a}}$ sessions & $5.8,127.9$ & 0.96 & 0.042 & 0.455 \\
During $^{b}$ session & 1,22 & 10.35 & 0.320 & $0.004^{*}$ \\
Group $\times$ Across sessions & $5.8,127.9$ & 1.67 & 0.071 & 0.135 \\
Group $\times$ During session & 1,22 & 10.31 & 0.319 & $0.004^{*}$ \\
Across sessions $\times$ During session & $6.2,136.9$ & 0.98 & 0.043 & 0.441 \\
Group $\times$ Across sessions $\times$ During session & $6.2,136.9$ & 1.38 & 0.059 & 0.226
\end{tabular}

${ }^{a}$ Across sessions (1-10).

${ }^{b}$ During a session (first $30 \mathrm{vs}$. last $30 \mathrm{~s}$ ). *Significant $<0.05$.
TABLE 3 | Results of the Mixed ANOVA for pain ratings with $\eta^{2}$ indicating the partial-eta squared effect size.

\begin{tabular}{lcccc}
\hline & $\boldsymbol{d f}$ & $\mathbf{F}$ & $\eta^{2}$ & $\boldsymbol{p}$-value \\
\hline Group & 1,22 & 0.23 & 0.010 & 0.637 \\
Across $^{\text {a }}$ sessions & 9,198 & 3.09 & 0.123 & $0.002^{*}$ \\
During $^{\text {b session }}$ & 3,66 & 7.34 & 0.250 & $<0.001^{*}$ \\
Group $\times$ Across sessions & 9,198 & 0.43 & 0.019 & 0.919 \\
Group $\times$ During session & 3,66 & 0.92 & 0.040 & 0.437 \\
Across sessions $\times$ During session & 27,594 & 01.45 & 0.062 & 0.069 \\
Group $\times$ Across sessions $\times$ During session & 27,594 & 0.73 & 0.032 & 0.838
\end{tabular}

across sessions (1-10).

${ }^{b}$ During a session (baseline $15 \mathrm{~s}, 2,3$, and $4 \mathrm{~min}$ ). *Significant $<0.05$.

We also conducted an exploratory investigation of whether pain ratings were impacted by the type of frequency band trained (alpha vs. theta) as described in the previous section. Results indicated no main $(p=0.853)$ or interactive effect of frequency band on pain ratings $(p=0.315-0.699)$.

\section{DISCUSSION}

Several key results emerged from the present study. First, although we individualized neurofeedback protocols based on each participant's EEG response to painful stimulation, a general pattern of altered EEG activity in certain regions of the scalp at specific frequency bands emerged. In particular, alterations in alpha and theta frequency bands in mostly frontal, and some central, regions were observed, suggesting these may be key target areas for NFB for pain management. This is largely consistent with cortical areas found to be active during pain in imaging studies (Apkarian et al., 2005, 2009; Fitzgerald, 2020), but also supports the complex nature of pain and the need to better understand the relationship between the brain and pain on a cortical level (EEG) to develop efficacious NFB protocols with EEG.

Second, in terms of the ability of participants to regulate EEG activity when in pain, results indicated EEG amplitude at the training site during pain was closer to the target amplitude for the NFB than the sham group in the early stages of the pain task. Importantly, however, this was only observed during the first 30 s of the training task, with no differences between NFB and sham in the latter stages of the task (final $30 \mathrm{~s}$ ). This pattern of results does not provide a clear-cut picture of whether EEG regulation during pain is possible. One possible interpretation is that EEG can be more successfully regulated (compared to the natural EEG occurs during pain) during the early stages of pain, but that this becomes more difficult after sustained exposure to noxious stimulation. However, it seems unlikely that any substantive training effects would be present so early on in the task (30 s), and any differences at this stage of the task seem more likely to simply reflect group differences in reaction to an initial noxious stimulus. This ambiguous pattern of findings does little to resolve the question of whether EEG can be regulated during pain, but does underline the complex nature of EEG changes that occur during pain and clearly indicates that more convincing evidence 


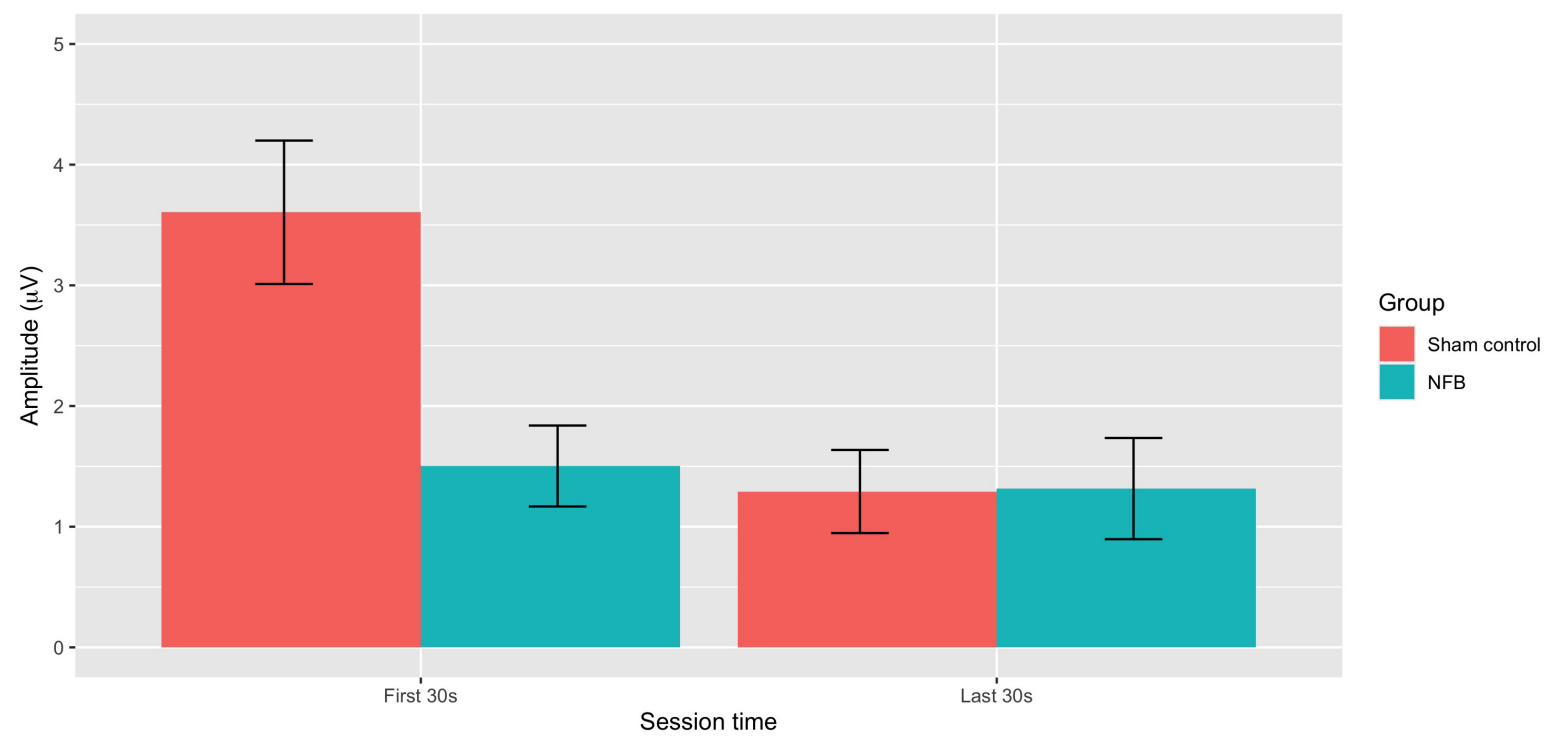

FIGURE 4 | Mean absolute deviation from baseline EEG amplitude for NFB and sham control groups during the first 30 and last 30 s of the pain tasks (error bars represent $\pm 1 \mathrm{SE})$.

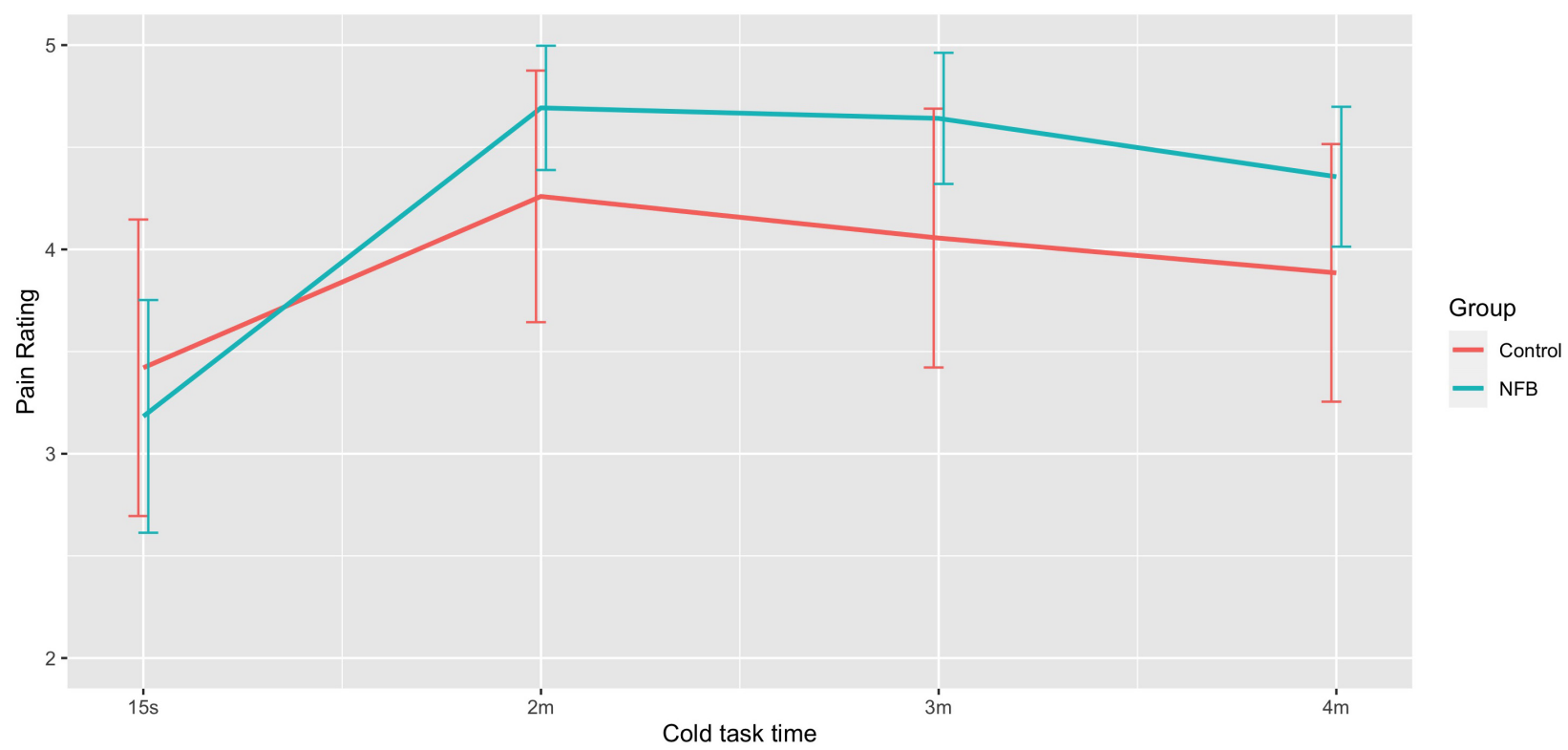

FIGURE 5 | Mean pain rating at different stages of the 4-min pain trial for NFB and sham control groups (error bars represent \pm 1 SE).

is required before this claims that EEG can be regulated during pain can be made.

These findings are consistent with some previous work. Hassan et al. (2015) reported that in control sessions of NFB, changes in power spectral density (PSD) did not differ across the placebo controlled condition to before the session (pre-NFB vs. during NFB), suggesting that training did not take place in the controlled condition; however, there doesn't appear to be a direct comparison between groups to test for statistical significance. Kayiran et al. (2010) identified no significant changes in EEG amplitude overall, however, they identified a statistically significant difference for session 4 . This suggests that further research is needed to better understand this, for example Aliño et al. (2016) and Roy et al. (2020) suggest additional variations of sham-procedures that could be run in parallel with an intervention to further study changes between intervention and sham groups, as well as double blinding (Ros et al., 2020), which future research could attempt with pain. Overall, these results are consistent with the possibility that NFB can elicit changes in EEG during pain that are different from those that 


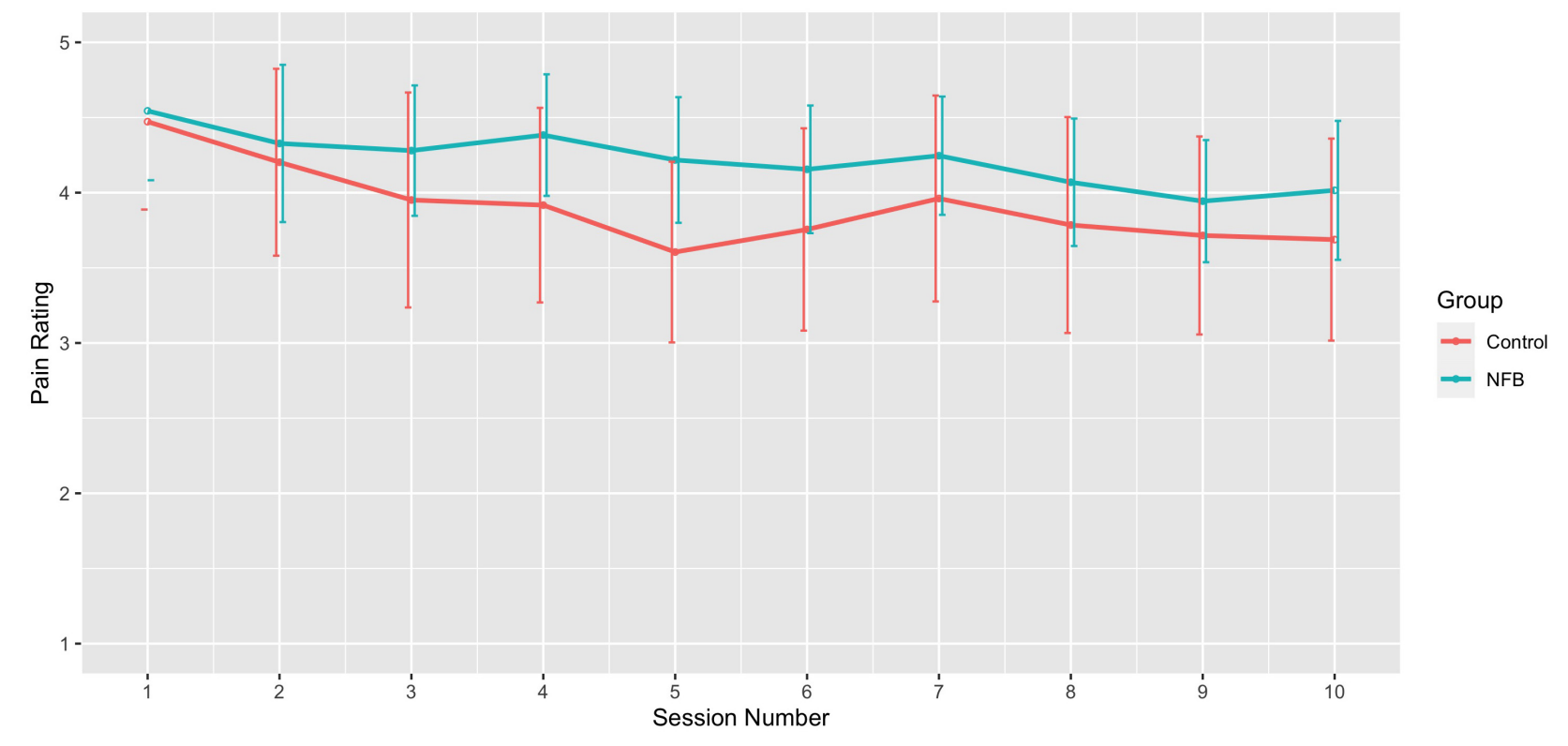

FIGURE 6 | Mean pain rating across sessions for NFB and sham control groups (error bars represent \pm 1 SE).

occur with a sham procedure, although the fact that this seemed to occur only in the early stages of noxious stimulation does underline the need for further corroborating evidence.

With respect to the potential for NFB as a technique for pain management, we found no differences in pain ratings between NFB and sham control either during the sessions or across sessions. We did, however, find a decrease in average pain intensity with progressive sessions that were very similar for both the NFB and sham control groups. This could provocatively suggest that any putatively beneficial effects on pain before and after a series of NFB session observed in previous studies that do not provide a sham comparator may have been attributable simply to non-EEG regulatory mechanisms such as placebo expectation effects, demand characteristics or a number of other potential explanations (Loo and Barkley, 2005; Thompson et al., 2011; Gollub et al., 2018).

Although our study involved experimentally induced pain in healthy participants, rather than clinical pain, these findings do nevertheless demonstrate that changes in reported pain can occur in the absence of real NFB and this may be critical to the interpretation of any possible therapeutic effects. There appears to be little previous research that has compared changes in pain during NFB to a sham procedure, and the current findings endorse the view of Aliño et al. (2016) that a suitable sham procedure is essential to identify any clear benefits of NFB. One study by Hassan et al. (2015) has attempted to test for a placebo effect in two ways. One involved displaying a pre-recorded NFB session and the other involved displaying visual feedback and targeting $\mathrm{Oz}$ (the occipital area), which is not normally associated with pain. This was done on the 10th and 20th training session for participants so there was no independent sham-control group. As mentioned previously this limitation means that learning or other carryover effects cannot be ruled out. Hassan et al. (2015) found that the targeted activity for participants did not change in the placebo-controlled group studying PSD, nor was a reduction in pain reported. The alpha placebo control did note a shift in alpha, but again no change in pain reported. Although these findings seem promising, this design does not allow conclusions of the efficacy of NFB to be made and further controlled studies were required. This study has attempted to address this and is able to make direct comparisons between a sham-control group and a NFB intervention group, including a manipulation check to not only study if EEG changed, but if it was changing as was intended/trained.

The current study has several limitations. First, it is impossible to say from our results whether NFB per se is ineffective for reducing pain, or simply that there is limited evidence for the effectiveness of the NFB approach we examined. Given the almost endless variation in how neurofeedback can be administered (e.g., a differing number and length of sessions, single or multiple channel approaches, outcomes targeted, different reward protocols etc.), it would be unwise to dismiss the possibility of therapeutic effects of NFB. Given encouraging findings reported elsewhere, further work is clearly required to identify which of these factors might be important in potentially affecting the success of NFB. Second, while the use of an experimental pain paradigm offers a high degree of control, results do not necessarily generalize to clinical pain which differs on intensity, perceived control and frontal cortex involvement (Apkarian et al., 2005). Despite this, there are many cortical areas that are commonly active during the processing of both chronic and acute pain and therefore does have the potential to be effective, these findings suggest that the most likely target is the frontal and central areas area (e.g., $\mathrm{FCz}, \mathrm{Pz}$ ). Third, if a genuine analgesic effect of NFB does exist, it may simply have been that the current study was underpowered to detect this. As there are few if any 
studies that have examined the impact of NFB on experimentally induced pain we were not able to estimate an effect size that could be used a priori to perform power analysis calculations. Although the $p$-value for pain indicated a reliable decrease in pain ratings across sessions $(p=0.015)$ and the present sample size is similar to others in the field, a larger sample size in future could provide greater power for group comparisons. Fourth, despite random group allocation there were some chance differences in age and gender across groups, with the NFB group 9 years older on average with more females compared to the sham group (9 vs. 6). As we examined NFB effects within a repeatedmeasures design, we would not expect these differences to have had any substantive impact on an evaluation of therapeutic effects, in the absence of any previous robust evidence of any major moderating effects of these variables. Nevertheless, stratification may be a sensible option in future studies to avoid this possibility given it is often difficult to recruit large numbers of people in NFB studies needed for randomization to be most effective.

Overall, these findings underline the importance of a robust study design which includes an appropriate sham control group in order to reliably evaluate the possible therapeutic effects of NFB. In particular, the fact that an analysis of the NFB group in isolation would have indicated a successful reduction in pain across progressive sessions and thus a treatment benefit, despite a similar benefit being observed in the sham group, does prompt extreme caution in interpreting previous studies indicating NFB benefits where no sham control group is present. Further studies including those with clinical pain populations that include an appropriately designed sham control group are needed to clarify the therapeutic potential for NFB as a pain intervention.

\section{REFERENCES}

Abdulla, A., Adams, N., Bone, M., Elliott, A. M., Gaffin, J., Jones, D., et al. (2013). Guidance on the management of pain in older people. Age Ageing 42, i1-i57.

Aliño, M., Gadea, M., and Espert, R. (2016). A critical view of neurofeedback experimental designs: sham and control as necessary conditions. Int. J. Neurol. Neurother. 3:041.

American Geriatrics Society Panel on the Pharmacological Management of Persistent Pain in Older Persons (2009). Pharmacological management of persistent pain in older persons. Pain Med. 10, 1062-1083. doi: 10.1111/j.15264637.2009.00699.x

Apkarian, A. V., Baliki, M. N., and Geha, P. Y. (2009). Towards a theory of chronic pain. Prog. Neurobiol. 87, 81-97.

Apkarian, A. V., Bushnell, M. C., Treede, R. D., and Zubieta, J. K. (2005). Human brain mechanisms of pain perception and regulation in health and disease. Eur. J. Pain 9, 463-463. doi: 10.1016/j.ejpain.2004. 11.001

Boonstra, A. M., Stewart, R. E., Köke, A. J., Oosterwijk, R. F., Swaan, J. L., Schreurs, K. M., et al. (2016). Cut-off points for mild, moderate, and severe pain on the numeric rating scale for pain in patients with chronic musculoskeletal pain: variability and influence of sex and catastrophizing. Front. Psychol. 7:1466.

Brooks, J., and Tracey, I. (2005). REVIEW: from nociception to pain perception: imaging the spinal and supraspinal pathways. J. Anat. 207, 19-33. doi: 10.1111/ j.1469-7580.2005.00428.x

Caro, X. J., and Winter, E. F. (2011). EEG biofeedback treatment improves certain attention and somatic symptoms in fibromyalgia: a pilot study.

\section{DATA AVAILABILITY STATEMENT}

The raw data supporting the conclusions of this article will be made available by the authors, without undue reservation.

\section{ETHICS STATEMENT}

The studies involving human participants were reviewed and approved by Secretary, University Research Ethics Committee c/o Vice Chancellor's Office, Queen Anne Court, University of Greenwich, Old Royal Naval College, London. The patients/participants provided their written informed consent to participate in this study.

\section{AUTHOR CONTRIBUTIONS}

TT and CI-W designed the study. CI-W recruited participants, collected and analyzed the data, and wrote the initial manuscript. TT conceived the study, contributed to the writing of the manuscript, provided statistical advice, and provided a critical review of the final draft. Both authors contributed to the article and approved the submitted version.

\section{FUNDING}

CI-W was awarded the Vice Chancellors scholarship at the University of Greenwich from 2014-2017 to undertake the doctoral research that resulted in this present report.

Appl. Psychophysiol. Biofeedback 36, 193-200. doi: 10.1007/s10484-0119159-9

Casey, K. L. (1980). Supraspinal Mechanisms in Pain: the Reticular Formation. Pain and Society. Weinheim: Chemie Verlag, 188-200.

Dave, V., Roberts, R., Griffo, Y., Moryl, N., Tickoo, R., Cubert, K., et al. (2015). Association of severe pain with poor response to opioids, psychological distress, and aberrant drug taking behaviors in a large cohort of cancer patients. J. Clin. Oncol. 33(15_suppl), 9600. doi: 10.1200/jco.2015.33.15_suppl. 9600

Dobrakowski, P., and Łebecka, G. (2020). Individualized neurofeedback training may help achieve long-term improvement of working memory in children with ADHD. Clin. EEG Neurosci. 51, 94-101. doi: 10.1177/15500594198 79020

Enriquez-Geppert, S., Huster, R. J., and Herrmann, C. S. (2013). Boosting brain functions: improving executive functions with behavioral training, neurostimulation, and neurofeedback. Int. J. Psychophysiol. 88, 1-16. doi: 10. 1016/j.ijpsycho.2013.02.001

Fitzgerald, J. L. (2020). Extending the Neuromatrix. In Life in Pain. Singapore: Springer, 23-43.

Gao, J. F., Yang, Y., Lin, P., Wang, P., and Zheng, C. X. (2010). Automatic removal of eye-movement and blink artifacts from EEG signals. Brain Topogr. 23, 105-114. doi: 10.1007/s10548-009-0131-4

Gaskin, D. J., Richard, P., and Walburn, J. (2017). The Economical Impact of Pain. In Neuroimaging of Pain. Cham: Springer, 1-17.

Gollub, R. L., Kirsch, I., Maleki, N., Wasan, A. D., Edwards, R. R., Tu, Y., et al. (2018). A functional neuroimaging study of expectancy effects on pain response 
in patients with knee osteoarthritis. J. Pain 19, 515-527. doi: 10.1016/j.jpain. 2017.12.260

Graven-Nielsen, T., Sergerdahl, M., Svensson, P., and Arendt-Nielsen, L. (2001). Methods for induction and assessment of pain in humans with clinical and pharmacological examples. Methods Pain Res. 1, 264-304.

Gruzelier, J. H. (2014a). EEG-neurofeedback for optimising performance. I: a review of cognitive and affective outcome in healthy participants. Neurosci. Biobehav. Rev. 44, 124-141. doi: 10.1016/j.neubiorev.2013. 09.015

Gruzelier, J. H. (2014b). EEG-neurofeedback for optimising performance. III: a review of methodological and theoretical considerations. Neurosci. Biobehav. Rev. 44, 159-182. doi: 10.1016/j.neubiorev.2014.03.015

Güntensperger, D., Thüring, C., Kleinjung, T., Neff, P., and Meyer, M. (2019). Investigating the efficacy of an individualized alpha/delta neurofeedback protocol in the treatment of chronic tinnitus. Neural Plast. 2019: 3540898.

Hadi, M. A., McHugh, G. A., and Closs, S. J. (2019). Impact of chronic pain on patients' quality of life: a comparative mixed-methods study. J. Patient Exp. 6, 133-141. doi: 10.1177/2374373518786013

Hassan, M. A., Fraser, M., Conway, B. A., Allan, D. B., and Vučković, A. (2015). The mechanism of neurofeedback training for treatment of central neuropathic pain in paraplegia: a pilot study. BMC Neurol. 15:200.

Herbert, M. S., Goodin, B. R., Pero, S. T. IV, Schmidt, J. K., Sotolongo, A., Bulls, H. W., et al. (2014). Pain hypervigilance is associated with greater clinical pain severity and enhanced experimental pain sensitivity among adults with symptomatic knee osteoarthritis. Ann. Behav. Med. 48, 50-60. doi: 10.1007/ s12160-013-9563-x

Jensen, M. P., Battalio, S. L., Chan, J. F., Edwards, K. A., Day, M. A., Sherlin, L. H., et al. (2018). Use of neurofeedback and mindfulness to enhance response to hypnosis treatment in individuals with multiple sclerosis: results from a pilot randomized clinical trial. Int. J. Clin. Exp. Hypnos. 66, 231-264. doi: 10.1080/00207144.2018.1460546

Jensen, M. P., Day, M. A., and Miró, J. (2014). Neuromodulatory treatments for chronic pain: efficacy and mechanisms. Nat. Rev. Neurol. 10, 167-178. doi: 10.1038/nrneurol.2014.12

Jensen, M. P., Gertz, K. J., Kupper, A. E., Braden, A. L., Howe, J. D., Hakimian, S., et al. (2013a). Steps toward developing an EEG biofeedback treatment for chronic pain. Appl. Psychophysiol. Biofeedback 38, 101-108. doi: 10.1007/ s10484-013-9214-9

Jensen, M. P., Grierson, C., Tracy-Smith, V., Bacigalupi, S. C., and Othmer, S. (2007). Neurofeedback treatment for pain associated with complex regional pain syndrome type I. J. Neurother. 11, 45-53.

Jensen, M. P., Hakimian, S., Sherlin, L. H., and Fregni, F. (2008). New insights into neuromodulatory approaches for the treatment of pain. J. Pain 9, 193-199. doi: 10.1016/j.jpain.2007.11.003

Jensen, M. P., Sherlin, L. H., Askew, R. L., Fregni, F., Witkop, G., Gianas, A., et al. (2013b). Effects of non-pharmacological pain treatments on brain states. Clin. Neurophysiol. 124, 2016-2024. doi: 10.1016/j.clinph.2013. 04.009

Kayıran, S., Dursun, E., Dursun, N., Ermutlu, N., and Karamürsel, S. (2010). Neurofeedback intervention in fibromyalgia syndrome; a randomized, controlled, rater blind clinical trial. Appl. Psychophysiol. Biofeedback 35, 293302. doi: 10.1007/s10484-010-9135-9

Kayiran, S., Dursun, E., Ermutlu, N., Dursun, N., and Karamürsel, S. (2007). Neurofeedback in fibromyalgia syndrome. Agri: Agri (Algoloji) Dernegi'nin Yayin organidir=. J. Turkish Soc. Algol. 19, 47-53.

Langley, P., Müller-Schwefe, G., Nicolaou, A., Liedgens, H., Pergolizzi, J., and Varrassi, G. (2010). The impact of pain on labor force participation, absenteeism and presenteeism in the European Union. J. Med. Econom. 13, 662-672. doi: $10.3111 / 13696998.2010 .529379$

Loo, S. K., and Barkley, R. A. (2005). Clinical utility of EEG in attention deficit hyperactivity disorder. Appl. Neuropsychol. 12, 64-76.

Melzack, R. (1999). Pain-an overview. Acta Anaesthesiol. Scand. 43, 880-884. doi: 10.1034/j.1399-6576.1999.430903.x

Melzack, R. (2001). Pain and the neuromatrix in the brain. J, Dent. Educ. 65, 1378-1382. doi: 10.1002/j.0022-0337.2001.65.12.tb03497.x
Melzack, R. (2005). Evolution of the neuromatrix theory of pain. the prithvi raj lecture: presented at the third world congress of world institute of pain, barcelona 2004. Pain Pract. 5, 85-94. doi: 10.1111/j.1533-2500.2005. 05203.x

Mitchell, L. A., MacDonald, R. A., and Brodie, E. E. (2004). Temperature and the cold pressor test. J. Pain 5, 233-237.

Moore, R., Straube, S., and Aldington, D. (2013). Pain measures and cut-offs-'no worse than mild pain'as a simple, universal outcome. Anaesthesia 68, 400-412. doi: 10.1111/anae.12148

Murray, C. B., Groenewald, C. B., de la Vega, R., and Palermo, T. M. (2020). Longterm impact of adolescent chronic pain on young adult educational, vocational, and social outcomes. Pain 161, 439-445. doi: 10.1097/j.pain.000000000000 1732

Prinsloo, S., Novy, D., Driver, L., Lyle, R., Ramondetta, L., Eng, C., et al. (2018). The long-term impact of neurofeedback on symptom burden and interference in patients with chronic chemotherapy-induced neuropathy: analysis of a randomized controlled trial. J. Pain Symptom Manag. 55, 1276-1285. doi: 10.1016/j.jpainsymman.2018.01.010

Rebbeck, T., Moloney, N., Azoory, R., Hübscher, M., Waller, R., Gibbons, R., et al. (2015). Clinical ratings of pain sensitivity correlate with quantitative measures in people with chronic neck pain and healthy controls: cross-sectional study. Phys. Ther. 95, 1536-1546. doi: 10.2522/ptj.20140352

Riva, P., Williams, K. D., and Gallucci, M. (2014). The relationship between fear of social and physical threat and its effect on social distress and physical pain perception. $P A I N^{\circledR} 155,485-493$. doi: 10.1016/j.pain.2013. 11.006

Rogala, J., Jurewicz, K., Paluch, K., Kublik, E., Cetnarski, R., and Wróbel, A. (2016). The do's and don'ts of neurofeedback training: a review of the controlled studies using healthy adults. Front. Hum. Neurosci. 10:301.

Ros, T., Enriquez-Geppert, S., Zotev, V., Young, K. D., Wood, G., WhitfieldGabrieli, S., et al. (2020). Consensus on the reporting and experimental design of clinical and cognitive-behavioural neurofeedback studies (CRED-nf Checklist). Brain 143, 1674-1685. doi: 10.1093/brain/awaa009

Ros, T., Munneke, M. A., Ruge, D., Gruzelier, J. H., and Rothwell, J. C. (2010). Endogenous control of waking brain rhythms induces neuroplasticity in humans. Eur. J. Neurosci. 31, 770-778. doi: 10.1111/j.1460-9568.2010. 07100.x

Ros, T., Théberge, J., Frewen, P. A., Kluetsch, R., Densmore, M., Calhoun, V. D., et al. (2013). Mind over chatter: plastic up-regulation of the fMRI salience network directly after EEG neurofeedback. Neuroimage 65, 324-335. doi: 10. 1016/j.neuroimage.2012.09.046

Roy, R., de la Vega, R., Jensen, M. P., and Miró, J. (2020). Neurofeedback for pain management: a systematic review. Front. Neurosci. 14:671.

Saxen, M., and Rosenquist, R. W. (2020). The Epidemiology and Economic Impact of Spine Pain. In Spine Pain Care. Cham: Springer, 7-11.

Schabus, M., Griessenberger, H., Gnjezda, M. T., Heib, D. P., Wislowska, M., and Hoedlmoser, K. (2017). Better than sham? A double-blind placebo-controlled neurofeedback study in primary insomnia. Brain 140, 1041-1052. doi: 10.1093/ brain/awx011

Sime, A. (2004). Case study of trigeminal neuralgia using neurofeedback and peripheral biofeedback. J. Neurother. 8, 59-71. doi: 10.1300/j184v08n 01_05

Staahl, C., Olesen, A. E., Andresen, T., Arendt-Nielsen, L., and Drewes, A. M. (2009). Assessing analgesic actions of opioids by experimental pain models in healthy volunteers-an updated review. Br. J. Clin. Pharmacol. 68, 149-168. doi: 10.1111/j.1365-2125.2009.03456.x

Stokes, D. A., and Lappin, M. S. (2010). Neurofeedback and biofeedback with 37 migraineurs: a clinical outcome study. Behav. Brain Funct. 6:1. doi: 10.5298/ 1081-5937-37.1.1

Tabachnick, B. G., and Fidell, L. S. (2013). Using Multivariate Statistics, 6th Edn. Boston MA: Allyn and Bacon.

Thibault, R. T., Lifshitz, M., and Raz, A. (2017). Neurofeedback or neuroplacebo? Brain 140, 862-864. doi: 10.1093/brain/awx033

Thompson, T., Keogh, E., Chen, M. J.-L., and French, C. C. (2012). Emotionfocused coping and distraction: sex differences in the influence of anxiety sensitivity during noxious heat stimulation. Eur. J. Pain 16, 410-420. doi: 10. 1002/j.1532-2149.2011.00028.x 
Thompson, T., Keogh, E., and French, C. C. (2011). Sensory focusing versus distraction and pain: moderating effects of anxiety sensitivity in males and females. J. Pain 12, 849-858. doi: 10.1016/j.jpain.2011. 01.004

Thompson, T., Steffert, T., Ros, T., Leach, J., and Gruzelier, J. (2008). EEG applications for sport and performance. Methods 45, 279-288. doi: 10.1016/j. ymeth.2008.07.006

Van Hecke, O., Torrance, N., and Smith, B. H. (2013). Chronic pain epidemiology and its clinical relevance. Br. J. Anaesthesia 111, 13-18. doi: 10.1093/bja/ aet123

Vučković, A., Altaleb, M. K. H., Fraser, M., McGeady, C., and Purcell, M. (2019). EEG correlates of self-managed neurofeedback treatment of central neuropathic pain in chronic spinal cord injury. Front. Neurosci. 13:762. doi: 10.3389/fnins. 2019.00762

Wall, P. D., Melzack, R., and Bonica, J. J. (1994). Textbook of Pain. London: Churchill Livingstone.
Conflict of Interest: The authors declare that the research was conducted in the absence of any commercial or financial relationships that could be construed as a potential conflict of interest.

Publisher's Note: All claims expressed in this article are solely those of the authors and do not necessarily represent those of their affiliated organizations, or those of the publisher, the editors and the reviewers. Any product that may be evaluated in this article, or claim that may be made by its manufacturer, is not guaranteed or endorsed by the publisher.

Copyright (c) 2021 Ide-Walters and Thompson. This is an open-access article distributed under the terms of the Creative Commons Attribution License (CC BY). The use, distribution or reproduction in other forums is permitted, provided the original author(s) and the copyright owner(s) are credited and that the original publication in this journal is cited, in accordance with accepted academic practice. No use, distribution or reproduction is permitted which does not comply with these terms. 


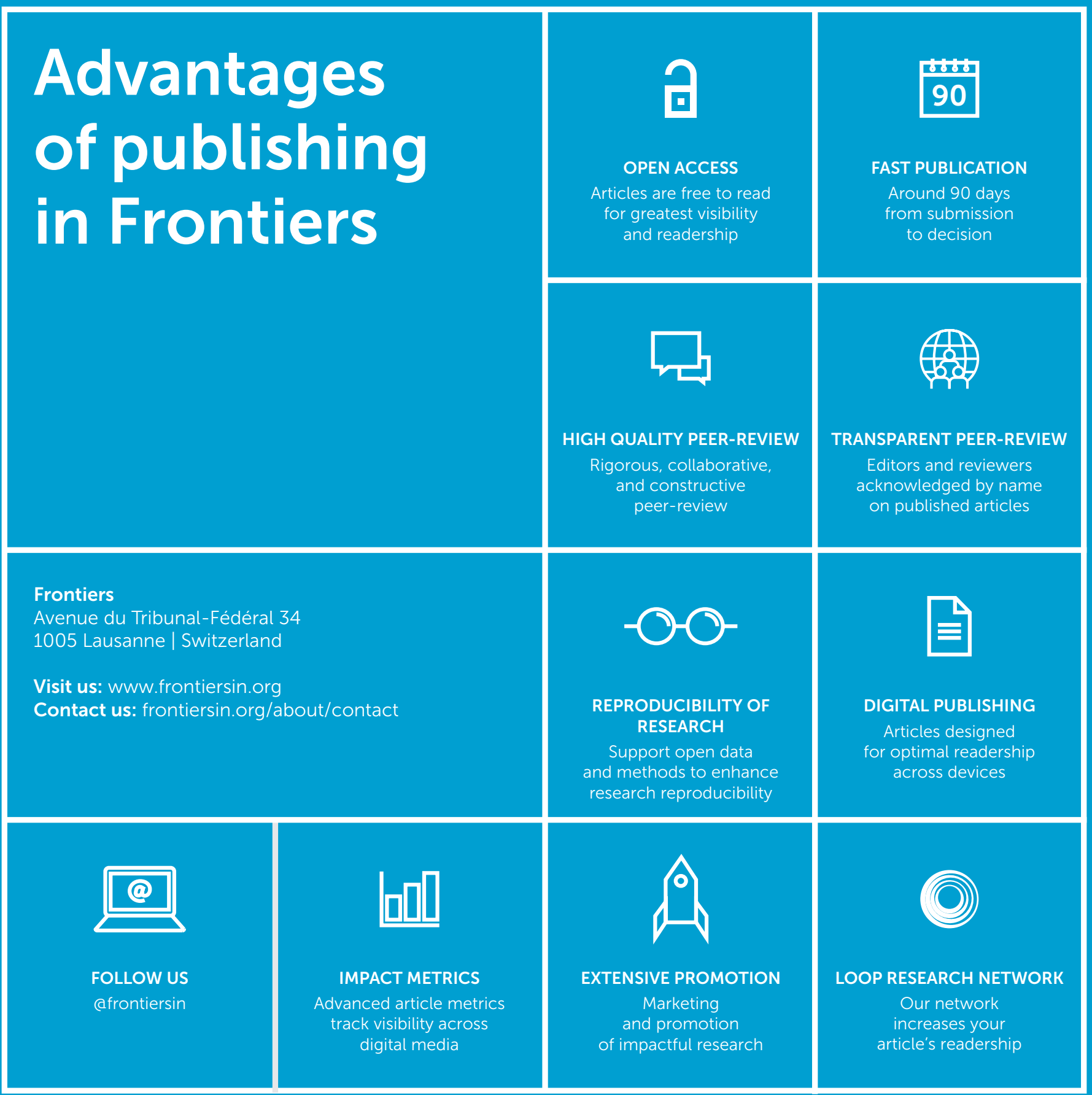

